


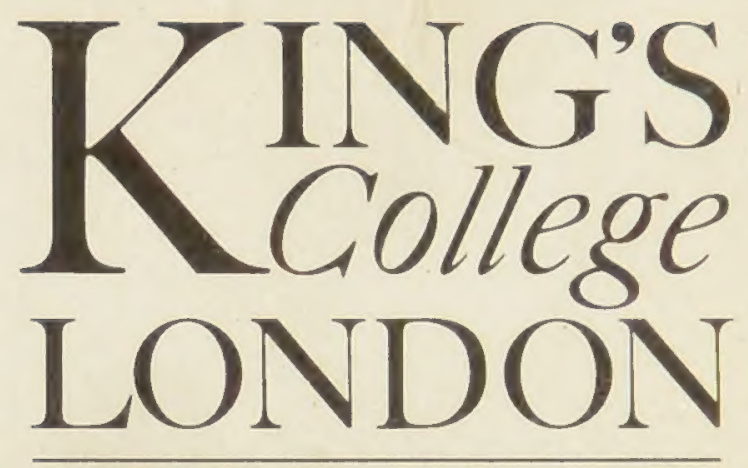

GUYPR QL8O5 M46

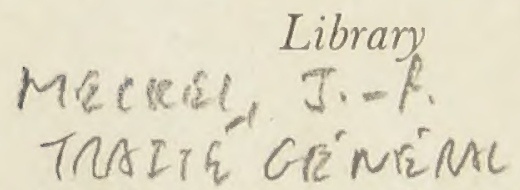

Didwatomée comparáe $1828-1728$

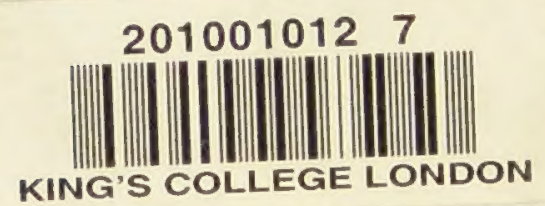


Digitized by the Internet Archive in 2015 



\section{TRAITÉ GÉNÉRAL}

\section{D'A NATOMIE COMPARÉE,}

PAR

\section{J.-F. MECKEL,}

TRADUIT DE L'ALLEMAND

ET AUGMENTÉ DE NOTES

PAR MM. RIISTER, ITAIPH. SANSON, DOCTEOH EN CHIRUGGIE DE LA FACULTḰ DE PARIS.

PRÉCÉDÉ D'UNE LETTRE DQE L'AUTEUR.

TOME DEUXIË ME.

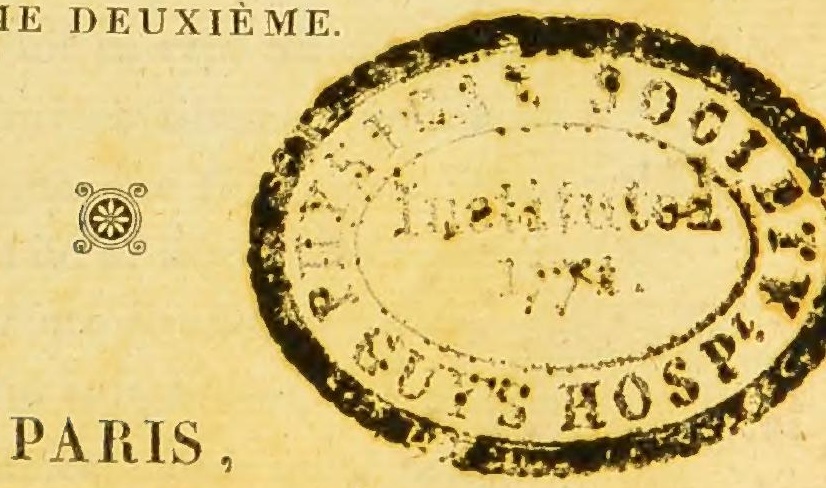

VILLERET ET CIE, LIBRAIRES - ÉDITEURS,

RUE DE L'ÉCOLE-DE-MÉDECINE, No I3,

VIS-A-VIS LA RUE HAUTEFEUILLE.

1828. 



\section{A VERTISSEM ENT.}

Les naturalistes n'ayant en vue que la recherche de caractères propres à faire reconnaître un animal donné, entre tous les autres, se bornaient, avant l'époque actuelle, à saisir des signes différentiels; les ressemblances étaient une sorte d'obstacle qu'on s'efforçait d'aplanir, quoiqu'on en fit usage pour grouper, sous les noms de classes, d'ordres, etc., les êtres qu'elles rapprochaient. On admettait, sans s'en être rendu un compte exact, un type unique de composition pour tous les animaux, et on se proposait non pas de définir ce type unique, mais de caractériser les formes diverses qu'offrait l'être animé, tout vague que fût encore l'idée que l'on était en droit de se former de l'animalité. Or, la multiplicité des êtres dont les découvertes de chaque siècle enrichirent la zoologie nécessita d'observer autre chose que la forme superficielle; on pénétra dans l'organisation; des rapports de conformation et d'usage entre les parties de l'animal situées au dehors et celles 
que cachait son enveloppe, furent successivement saisis et étudiés; les appareils organiques des divers animaux furent placés en regard sous ce double point de vue ; l'anatomie et la physiologie comparées étaient dès lors fondées. Nées du besoin zoologique de différencier, elles n'eurent pour but dans leurs premiers pas que d'établir des dissemblances ; mais le grand nombre de faits, que des investigations laborieuses amassèrent de toutes parts, permit bientôt à des génies du premier ordre d'imprimer à ces sciences une direction plus hardie et plus indépendante. Des considérations de la plus haute élévation, des lois, des principes, furent proposés et accueillis avec plus ou moins de faveur. Ces dernières années ont été surtout fécondes en créations de ce genre; sur cette base toute matérielle ont été même fondés des systèmes qui ne le cèdent en rien à ce que la métaphysique imagina jamais de plus abstrait, et l'on peut dire que l'état actuel de la science est caractérisé par une extrême tendance à généraliser.

La lecture du premier volume de cet ouvrage a confirmé le public dans l'idée qu'il avait déjà pu concevoir, par la connaissance d'autres tra- 
vaux de M. MecreL, que cet auteur appartenait au nombre de ceux qui se renferment dans les limites de l'observation la plus rigoureuse.

Le sujet plus spécial, qui est traité dans ce second volume, convaincra les lecteurs de plus en plus que M. Meckel n'accorde aux vues théoriques qu'une confiance fort ménagée.

Il est cependant un certain nombre de conséquences philosophiques, tirées de l'observation de pièces anatomiques préparées avec soin, que les traducteurs ont cru devoir rappeler lorsqu'il s'est agi des faits particuliers auxquels ces conséquences se rattachent.

Ainsi a été exposé le système crânien tel qu'il est conçu par M. Geoffroy-Sarnt-Hulaire et par M. DE Blainville; ainsi a été esquissée l'idée philosophique qui domine les travaux de M. Audouin, ceux de M. Robineau Desvoidy, ceux de M. DE Savigny sur l'organisation des crustacés, insectes, annelides, etc., en analysant les recherches spéciales et les découvertes publiées par ces derniers naturalistes.

Telle est la rapidité de la marche parcourue par la science, que ces travaux, édits depuis la publication de ce second volume en Allemagne,

a. 
ont fourni matière à des notes longues el nombreuses, quoique insuffisantes encore your la matière qui en fait le sujet.

Ce n'est pas sans un grand regret que les traducteurs se sont vus privés des lumières qu'ils auraient pu puiser dans l'ouvrage de M. Straus, qui ne l'avait pas encore mis au jour, lorsque la partie de ce volume qui traite du squeletle. des articulés était déjà imprimée; mais ils y reviendront lorsqu'il sera question, dans le quatrième volume de cet ouvrage, de la partie active de la locomotion de ces animaux.

Les traducteurs ont cherchć auprès des auteurs eux-mêmes des conseils sur les notes qu'ils cnt extraites de leurs travaux; ainsi ils doivent particulièrement à MM. GEOFFroYSaint-Hilaire, Audouin, de Biainville, des avis dontils ont profité avec empressement; ces savans ont mis à les guider une obligeance qui n'étonnera aucune des personnes ayant cil: dans l'occasion d'y avoir recours; mais elle est pour les traducteurs l'objel d'une reconnaissance qu'ils aiment à proclamer. L'extrême bienveillance de M. le baron Cuvier les a mis, d'autre part, à même de constater sur les piòces 
déposées au cabinet d'anatomie comparée de Paris les descriptions renfermées dans le texte.

Ils ne doivent pas moins d'actions de grâces à M. MeckeL, dont la complaisance a été assez grande pour leur adresser, à Paris, les feuilles du dernier volume de son ouvrage, à mesure qu'il fut imprimé en Allemagne, et qui leur a réitéré récemment encore, dans une lettre datée des derniers jours d'avril r 828 , de continuer cet envoi obligeant jusqu'à la fin du septième volume, de telle sorte que l'ouvrage pût paraître à la fois en France et à Halle.

M. Robineau-Desvoidy s'est aussi montré plein de bonne volonté à leur égard.

Aucune recherche ne sera évitée pour placer cet ouvrage, si digne déjà de l'attention publique et qui en a reçu un accueil si favorable, lout-à-fait au niveau des acquisitions quotidiennes de la science.

L'anatomie comparée est devenue d'ailleurs cultivée si généralement, que chaque année voit éclore de nouvelles découvertes, ce qui rend incessamment utiles des additions nouvelles. C'est particulièrement sur les travaux faits suivant l'impulsion de la recherche des ressem- 
blances ou des analogies que les traducteurs ont porté leur choix, afin de faire ressortir surtout la marche philosophique qui en caractérise l'esprit. Ils ont lieu d'espérer que l'impatience avec laquelle ce volume a été demandé, accrô̂tra, par l'effet de sa lecture, celle avec laquelle on réclamera ceux qui doivent le suivre, et qui ne seront d'ailleurs que peu altendus.

M. Meckel annonce dans sa préface au quatrième volume, qui a paru à la fin du mois de février dernier, que l'impression du cinquième volume, qui traite des organes de l'appareil digestif, touche à sa fin, et que les autres volumes., pour lesquels tout est prêt, vont se succéder rapidement et sans interruption.

LES TRADUCTEURS. 


\section{TABLE DES MATIERES.}

\section{SECONDE PARTIE. ANATOMIE SPÉCiale.}

\section{LIVRE PREMIIER. - organes passifs de la}

\section{Pages.}

LOCOMOTION. . . . . . . . . . . .

CHAPITRE PREMIER. - CONSIDÉRATIONS GÉNÉ-

RALES. ..................... 3

CHAPITRE DEUXIEME. - DESCRIPTION SPÉ-

CIAIE DU SQUELETTE DANS LES DIFEERENTES

CLASSES D'ANIMAUX. .................. 12

rREMIÉrE SECTION. - Squelelte des zoophyles... id. DEUXIÈIE SEGTION. - Squelette des échinodermes. 2 I

1. Stellérides...................... 25

2. Echinides.......................... 39

3. Holothuries.................... 53

TROISIÈME SECTION. - Squelette des annelides. . . 56 QUatrième section. - Squelelle des insectes, des arachnides et des crustacés. ........ 64 I. Squenete des insectes.............. 77 CONDITIONS GÉnÉRALES. . . . . . . . . id.

1. Insectes à métamorphoses........... 86

A. Diptères................... id.

B. Hémiplères................. 88

C. Lépidoptères................ 93

D. Hyménoptères............. $9^{8}$

E. Névroptères.............. 102

F. Orthoptères................. 103

G. Coléoptères............... $\$ 11$ 
Pages.

2. Insectes sans métamorphoses......... 124

I. SQuflette nes arachinide... . . . . . 127

A. Arachnides proprement dites.......... id.

B. Scorpionides.................. 130

II. Seuelette nfs chustacés.......... 156 CINQUIÈme section. - Squelette des cirripedes. . 157 sixième section. - Squelelle des mollusques. . I6 I SEPTIEMF. SECTION - Squelette des céphalopodes. 180 HUITIEME SECTIDN. - Sipueletle des vertébrés ou système osseux. .................. I99

I. Caractères généraux du système osseux ...... 200 1. Position.................. id.

2. Forme extérieure............... id.

5. Forme intérieure ou texture......... 205

4. Composition................... 204

5. Propriétés physiques.............. 205

6. Propriétés vitales............... id.

II. Caractères particulicrs ou différences du système osseux........................ 207

A. Différences relatives à la région....... irl.

B. Différences qui dépendent des âges...... 210

C. Différences de classes............. 213

1. Forme extérieure.............. 314

2. Volume.................... 222

5. Nombre..................... 223

4. Texture.................. 227

5. Consistance................. 228

6. Composition................ 229

7. Coloration.................. 230

8. Modes d'articulation............ 231

9. Différences périodaires........... 234

NeUViEME SECTION. - Squelelle des poissons. . . 243 
A. Os du tronc................................ 243

A. Colonne vertébrale............. id.

1. Poissons cartilagineux........... id.

3 Poissons osseux.............. 282

B. Côtes.......................... 354

1. Poissons cartilagineux............ id.

2. Poissons osseux................ 347

c. Sternum.................... 355

B. Os des membres................. 363

A. Os des membres antérieurs......... 364

I. Poissons cartilagineux............ id.

2. Poissons osseux.............. 38 i

a. Epaule................ 384

b. Nageoire................ 414

B. Os des membres postérieurs......... 423

a. Poissons cartilagineux........... 427

I. Hanche................. id.

2. Nageoire................ id.

b. Poissons osseux.............. 430

1. Hanche.................. id.

2. Nageoire.................. 435

C. Os de la tête................... id.

A. Poissons catilagineux............ 445

a. Cyclostômes................. id.

b. Plagiostômes................ 448

n. Poissons osseux................ 460

1. Portion occipitale de l'os basilaire... 462

2. P’ortion sphénoïdale de l'os basilaire. (17 1

3. Temporal................. $47^{8}$

a. Yortion crånienne du temporal.... 479

๖. Portion articulaire du temporal. . 4,85 
4. Pariétal.................... $44^{0}$

5. Ethmoïde. ................ 492

6. Coronal.................... 493

7. Os sus-orbitaire............... 495

8. Vomer...................... 496

9. Palatin...................... 497

10. Unguis ..................... 498

I . Nasal..................... id.

12. Maxillaire supérieur............. 499

a. Pièce sus-maxillaire........... 500

b. Pièce inter-maxillaire.......... 502

13. Malaire.................... 505

14. Maxillaire inférieur............. 507

15. Forme de la tête des poissons osseux en général.................... 514

16. Disposition particulière et insolite des os de la têlc de quelques poissons osseux..

17 Comparaison de quelques cartilages de la tête des poissons cartilagineux avec des os correspondans chez les poissons osseux. $\quad 528$

DIXIÈme SEcTION. - Squelette des reptiles. . . . 534

A. Os $d u$ tronc..................... 538

1. Batraciens..................... 539

a. Colonne vertébrale............. id.

1. Batraciens urodèles............... 540

2. Batraciens anoures............... 54 I

3. Différences qui distinguent les vertèbres les plus antérieures des autres......... 545

b. Côtes....................... 548

c. Sternum................... 550

a. Batraciens urodèles........... id.

b. Batraciens anoures.......... 555

2. Ophidiens................... 555 
a. Colonne vertébrale.............. 556

b. Côtes......................... 568

c. Sternum. ................... $55_{7 I}$

5. Chéloniens....................... id.

a. Colonne vertébrale.............. 572

b. Cotes....................... 584

c. Sternum................... 590

4. Sauriens...................... 597

a. Colonne vertébrale............... id.

b. Côtes....................... 6o3

c. Sternum.................... 6o 6

B. Os des membres. ................. 610

I. Menbaes attértedrs. ................. 611

1. Os de l'épaule................. 6 13

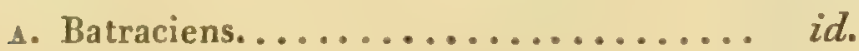

a. Batraciens urodèles............ id.

b. Id, anoures............ 6 I5

B. Chéloniens................. 618

c. Ophidiens.................. 62

D. Sauriens................. 623

2. Humérus..................... $6{ }_{2} 6$

3. Os de l'avant-bras................ 629

4. Os de la main.................. 634

4. Carpe..................... 635

a. Batraciens.................. 656

I. Batraciens urodèles........... id. 2. Id. anoures.......... 638

b. Chéloniens................. 640

c. Sauriens................... . 64 642

в. Métacarpe.................... 643

c. Doigts....................... 646

1. Batraciens................. 647 
2. Chéloniens.

3. Sauriens.

I1. Membres postériedras............ 655

1: Os $\mathrm{du}$ bassin................. id.

A. Batraciens................ 65/

a. Batraciens urodèles. ............ id.

b. Id. anoures............ 656

B. Ophidiens.................. 658

C. Sauriens.....................66 $66_{1}$

D. Chéloniens................... 663

2. Fémur....................... 666

万. Os de la jambe................. 669

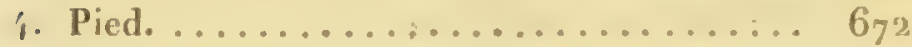

ร. Tarse................... 675

a. Batraciens................ id.

b. Chéloniens................ 979

c. Sauriens................ 68 68

2. Métatarse ............... (6...2

3. Orteils................... 684

C. Os de la tête................... 686 ;

1. Batraciens.................... 6.

1. Basilaire. ................. id.

2. Temporal..................... óg!

3. Pariétal................... $69^{3}$

4. Ethmoïde.................. 6. 69-1

5. Coronal..................... $69_{95}$

6. Maxillaire supérieur............... id.

7. Vomer..................... $6 g_{6} 6$

8. Palatin..................... 697

9. Nasal....................... id.

$10-11$. Malaire et Unguis................ 698

12. Maxillaire inférieur.............. id 
II. Chriloviess.

Pages.

1. Basilaire.

(ing)

2. Tenporal. ................. 702

3. Pariétal.................... 704

4. Ethmoïde................... 705

5. Frontal. .................... id.

6. Maxillaire supérieur. . . . . . . . . . 706

7. Vomer..................... id.

8. Palatin. .................. 707

9-10: Nasal et Unguis................ id.

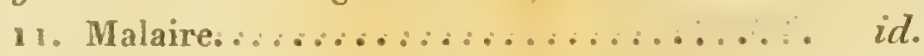

12. Maxillaire inférieur............ 708

III. Ophidiens. ................... 711

1. Basilaire................... id.

2. Temporal..................... 715

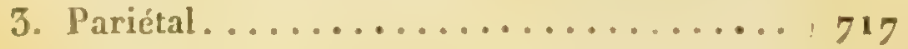

4 Ethmoïde.................. 718

5. Coronal..................... id.

6 Maxiliaire supérieur.............. 719

7. Vomer.......................... 720

8. Palatin...................... $72 x$

9. Nasal...................... id.

ro. Malaire..................... id.

11. Unguis. ...................... 722

12. Maxillaire inférieur.............. 723

IV. SAI RiENS..................... 725

I. Basilaire. ................... id.

2. Temporal. .................. 750

3. Pariétal..................... 752

f. Jithmoide. ................. 755

5. Coronal..................... $7^{34}$

6. Vaxillaire supciricur.............

7. Vomer..................... $7_{7}$ 
8. Palatin................... 738

9. Nasal...................... id.

10. Malaire..................... 739

1r. Unguis...................... id.

12. Maxillaire inférieur.............. 240

D. Tête des REPTILes CONSIDÉRÉE EN GÉNÉkAL... 742

1. Batraciens.................. id.

2. Chéloniens.................. 744

3. Ophidiens.................. $=46$

4. Sauriens.................... 748

Errata.................... $7^{52}$

FIN DE LA 'YABLE. 


\section{TRAITÉ GÉNÉRAL}

\section{DANATOMIE COMPAREE.}

\section{SECONDE PARTIE.}

ANATOMIE SPÉCIALE.

\section{LIVRE PREMIER.}

ORGANES PASSIFS DE LA LOCOMOTION.

$$
\text { S. } 1 .
$$

Nous avons considéré, dans le premier volume de cet ouvrage, les conditions et les circonstances générales de l'organisation animale: l'objet de ceux qui vout suivre est la description particulière des différens appareils dont l'assemblage constitue le corps de l'être animé. Les premiers organes dont il sera question serout les pièces locomrives, surtout celles qui sont soumises à l'empire de lí volonté. Le motif de ce choix est quelles constituent très généralement la plus grande masse du corps, lui donnent par conséquent la forme, et déterminent en majeure partie 11. 
la position et la formation des autres organes de l'économie.

Nous ne traiterons cependant pas dans cette portion de notre ouvrage des organes qui, quoique se mouvant sous l'influence de la volonté, sont des parties subordonnées d'autres appareils. Ceux qui ont pour but de déterminer le rapport de situation du corps animal avec les autres corps de la nature, feront seuls ici le sujet de nos études. Une autre marche nécessiterait de morceler la description des autres appareils; il en résulterait une sorte de confusion, quoique ce procédé dût conduire à obtenir une image complète de l'appareil de la locomotion en particulier. 


\section{CHAPITRE PREMIER.}

\section{CONSIDERATIONS GENERALES.}

\section{S. 2 .}

Les organes de la locomotion se divisent en actifs et en passifs.

Les premiers sont les muscles qui, par leur contraction, changent la situation des seconds lorsqu'ils existent; premier effet suivi, ou d'une modification bornée à la forme des fractions du corps sur lesquelles ils agissent, on d'un déplacement de la lotalité de l’ètre qui est transporté dans un autre lieu.

Les organes passifs de la locomotion consistent principalement en parties qui se distinguent des autres par une consistance et une dureté susceptibles de plusieurs degrés; propriété qui les rend aptes à diverses fonctions, telles que de constituer des leviers sur lesquels agissent les muscles, ou des moyens protecteurs destinés à abriter des organes plus ou moins importans à la vie, autour desquels ils forment, en se réunissant, des cavités perfectionnées à des degrés divers. I.a position relative de ces parties solides et dures, quise composent de gélatine endurcie (I), et très-

(1) M. Odier, en reprenant les analyses faites par M.M. Thonvencl, Beanplé et Robiquet, a reconnu que le II. 
généralement de carbonate ou de phosphate de chaux, varie; elles sont situées ou à l'extérieur, et recouvrent les muscles, ou plus profondément, et en sont alors entourées avec une exactitude qui a des mesures différentes. Elles occupent ainsi souvent la région la plus intérieure de certaines portions du corps, surtout de celles qui, comme les membres, sont presque uniquement destinées à la locomotion. Les organes locomoteurs passifs, situés profondément, se rencontrent chez les animaux supérieurs; ceux qui sont superficiels sont propres aux animaux inférieurs. Il y a cependant des animaux, même moins parfaits que ceux munis d'un test, dont le corps a pour base des parties dures intérieures.

Les organes passifs de la locomotion situés à l'extérieur, sont des parties de peau endurcie; on peut les désigner en général sous le nom de test; les instrumens passifs de la locomotion, pla-

quart en poids de l'enveloppe des insectes était formé par une substance très-analogue au ligneux; il l'a nommée chitine. Cetle substance est blanche; clle ne contient pas d'azote; elle se dissout dans l'acide nitrique à chaud sans y jaunir; elle brûle sans se boursoufler, et donne un charbon qui conserve la forme de l'organe soumis à la combustion. Cette substance a été trouvée aussi dans l'enveloppe des crustacés. On l'obtient en plongeant la partie dans une dissolution de potasse et en faisant chauffer cette dissolution; la potasse dissout les matières animales et met à nu la chitine. Mémoires de la socićté d'histoire naturelle de Paris, t. I, part. 1, pag. 29. (Notc d. s tmulucteurs.) 


\section{1) ANATONIE COMPARÉE.}

cés à l'intérieur, constituent des systèmes parliculiers, les systèmes cartilaginenx et osseü. De l'ensemble de ces diverses parties dures résulle le squelette; entouré des parties molles, principalement des muscles, il est appelé squetette intéricur; on le nomme extériezur lorsqu'il leur sert denveloppe. Dans ces deux conditions, le squelefle représente très-généralement la forme de :uut le corps, mème après la destruction de toutes les autres parties. Celte règle s’applique également aux cas où il n'atteint que des dimensions froportionnellement fort restreintes.

A ces systèmes qui constituent presque en enlierl'appareil passif de la locnmotion se joignent des parties plus molles que les os et les cartilages. Ces parties sont de deux sortes; on les lésigne sous le nom générique de ligamens, 'quoique à l'état parfait elles présentent entre elles des différences remarquables.

Ces deux sortes de partiés appartiennent it d'autres systèmes que l'on rencontre aussi dans des appareils différens.

Les plus solides, qui sont principalement destinées à maintenir l'union des organes durs ntre eux et avec les muscles, appartiennent au système fibreux qui revêtles os, dont il constitue le périoste, et les cartilages, dont il forme le périchondre. Ici le systeme fibreux a aussi pour usage de soutenir les ramifications des vaisseaux qui pénètrent ¿ l'intérieur des os. 
Les ligamens de la seconde sorte sont moins en rapport avec la connexion qu'avec la mobilité des parties dures entre elles; ce sont les ligamens synoviaux, ou capsules synoviales; ils appartiennent au système séreux, forment des sacs sans ouverture au dehors, à parois minces, étendus d'un os ou d'un cartilage à l'autre, tapissent les faces opposées de ces parties, et sécrètent une humeur onctueuse, transparente, visqueuse, la synovie.

$$
\text { \$. } 3 .
$$

Les procédés suivant lesquels sont unies entre elles les différentes portions du squelette sont très-diversifiés; mais toutes ces variétés peuvent être ramenées à deux modes d'union principaux. Ou les surfaces opposées sont unies entre elles par une substance intermédiaire étendue de l'une à l'autre, et dont la consistance tient le milieu entre la dureté et la mollesse, ou le contact est opéré entre ces surfaces par une membrane qui naît du pourtour de chacune d'elles, et qui leur permet de se toucher et de glisser l'une sur l'autre.

Dans le premier cas, le moyen d'union consiste en une substance fibreuse, ou fibro-cartilagineuse, ou cartilagineuse; dans le second cas, c'est une membrane synoviale que renforcent des portions isolées et intermédiaires de tissu fibreux et cartilagineux, qui, dans le plus grand 


\section{n'ANATOMIB COMPAILÉE.}

nombre des jointures, n'existent qu'à l'extérieur, qui quelquefois sont situées à l'intérieur des capsules elles-mêmes.

On conçoit que dans les modes de connexion oì les surfaces ne sont unies entre elles qu'à leur pourtour, et sont libres dans le reste de leur étendue, la mobilité doit être beaucoup plus considérable, tout étant égal d'ailleurs, que Jors de la disposition contraire. On désigne ces deux états de connexion opposés sous le nom d'union mobile ou articulation proprement dite, et d'union sans mozvement. Ces deux sortes d'articulations offrent plusieurs différences sous le rapport de l'étendue et de la solidité.

La plus solide est l'articulation immobile, lorsque les surfaces correspondantes sont inégales et opposent réciproquement des enfoncemens à des éminences qui s'engrènent mutue!lement, et que la substance intermédiaire n'offre qu'une épaisseur proportionnellement peu prononcée. Cette disposition reçoit le nom de sulure. Les sutures varient entre elles, et présentent des degrés très-remarquables, qui dépendent du volume, du nombre, de la complication des inégalités formant l'engrenage que constitue cette sorte d'articulation.

Lorsque les surfaces correspondantes ne sont que peu ou point inégales, lorsque la substance qui les unit est en outre large et molle, on donne à cette disposition le nom d'harmonie. 
La présence de ligamens fibreux ou de cartilages intérieurs, fait de cette articulation, considérée sous le double rapport du mode d'union et du degré de solidité, le passage à l'articulation mobile.

L'articulation mobile présente en effet réunies les deux espèces de connexions. Que l'on en suppose les cartilages intermédiaires suudés aux deux os, les ligamens intermédiaires étendus jusqu'à former des expansions qui se touchent, elle représentera une véritable harmonie.

Les surfaces destinées à glisser les unes sur les antres sont toujours lisses, souvent encroûtées de cartilage. Cette disposition est commune chez les animaux supérieurs. Ordinairement il n'y a que deux os articulés ensemble; quelquefois on en rencontre un plus graud nombre. Les surfaces planes offrent le moins de mobilité; l'étendue des mouvemens y décroît en raison de la roideur plus considérable et du développement. moindre des capsules ligamenteuses. Les os ar.ticulés entre eux n'exécutent alors, en effet, qu'un seul mouvement : il consiste dans un glissement qui a lieu de haut en bas et de bas en haut, entre les surfaces articulaires.

Ciest dans cette sorte d'articulation, nommées roides à cause du peu d'étendue des mouvemens, que l'on trouve le plus souvent des ligameus intermédiaires. On la remarque surtout entre de petits os. 
Les autres articulations permettent, soit la flexion et l'extension, soit la torsion, ou l'une et l'autre à la fois.

Dans l'un et l'autre cas, ce sont des surfaces arrondies qui sont en rapport. Mais lorsque les mouvemens se bornent à la flexion et à l'extension, c'est-à-dire daus l'articulation en ginglyme, en charnière ou en genou, les surfaces articulaires ne forment que de petits segmens de sphère auxquels correspondent des enfoncemens, et sont interrompues ou bornées en outre par des éminences ou des cavités qui existent à différens poinls, et empèchent, à lles degrés variables, le mouvement dans une autre direclion que celle de la longueur.

I,es articulations de torsion varient également sous le rapport du degré; mais on voit ici toujours des éminences rondes, simples, s'articuler avec des cavités analogues.

Le moindre degré de mobilité caractérise l'articulation trochoïde (rotatio), dans laquelle un os, etc., représente l'axe autour duquel s'en meut un autre, tous denx ne se tonchant que par des éminences et des enfoncemens proportionnellement peu prononcés. Souvent ce sont deux os, notamment des os longs, qui sont unis entre eux ì leurs deux extrémités par des surfaces sphérö̈des. Cette disposition n'existe pas toujours. Lorsque cela n'a pas lieu, l'axe est enfermé par cles ligimens forts, qui forment un 
cercle complet en s'écendant d'une partie de l'os mobile à l'aulre. Il résulte re cette disposition que, dans celte articulation, la torsion n'est possible que dans un plan.

On peut considérer comme une modification de cette articulation, ou bien comme une espéce d'articulation intermédiaire à la précédente et à celle qui suit, l'articulation en anneau, qui se rencontre chez quelques poissons, et dans laquelle deux os s'engrènent par des crochets ou par des anneaux complets.

Un rapprcchement de cette disposition nous est offert dans l'articulation trochoïde, lorsque les ligamens qui entourent l'axe formé par un os unique vienuent à s'ossifier, et forment ainsi un cercle osseux parfait. J'ai fait celte observation sur le squelette de l'hippopotame du cabinet d'histoire naturelle de Paris; en étudiant l'articulation de l'atlas avec l'axis, on voit dans cette pièce que le ligament transverse qui passe derrière l'apophyse odontoïde de l'axis, est ossifié.

I.e mouvement est beaucoup plus libre, lors que les surfaces qui se touchent représentent les segmens d'une sphère plus considérable, et constituent une portion comparativement plus petite des os que l'articulation unit. La conséquence de cette disposition est une rotation libre dans tous les sens, et d'autant plus étendue, que la cavité destinée à recevoir la tête sphéroïde offre moins de profondeur et de surface. Lorsque la 
cavité articulaire est grande en proportion et entoure toute la tête, l'articulation reçoit le nom d'énarthrose; petite et aplatie. elle forme l'articulation la plus libre, l'arthrodie.

Quand on compare les divers animaux sous le rapport de ces articulations, la seule observation générale qu’il soit possible de faire, c'est que les mêmes parties dures s'articulent entre elles dans des êtres différens d'une manière extraordinairement variée; diversité extrême qui dépend de ce que l'un présente souvent la plus grande mobilité sur un point donné, tandis que chez l'autre on y rencontre une suture ou une harmonie.

$$
\text { S. } 4 \text {. }
$$

Des systèmes que nous venons de considérer, le musculaire est le plus important; c'est à lui qu'est inhérente lafaculté locomotive. Cependant on peut en faire précéder la description de ce!le des parties dures; je crois même cet ordre plus convenable. Nous ne traiterons donc des muscles qu'après avoir étudié les organes passifs de la locomotion. 


\section{CHAPITRE II.}

DESCRIPTION SPÉCIAIE DU SQUELETTE DANS LES DIFFÉRENTES CLASSES "N NIMAUX.

PREMIERE SECTION.

SQUELETTE DES ZOOPHYTES.

S. 5.

La disposilion la plus simple du squelefle se renconire daus les zoophyles, où il recoit le nom de polypier ou corail.

La situation du corail l'a fait distinguer en extérieur et intérieur; dans le premier cas, il est creux : dans le second, plein; c'est sur cela qu'est fondée la division des polypes en corticiféres et en axif'eres. Il est digne de remarque que les polypes des zoophytes intérieurs, à tige creuse, ont une forme moins parfaite que ceux dont le tronc est solide, et quile revètent à l'extérieur; nouvelle confirmation du principe général que les animaux pourvus d'un'squelette extérieur, sont moins bien crganisés que ceux dont le squelette est sifué profondément. 11 est vraisemblable que la situation relative du squelelle el de la substance molle, est au fond la 
nème dans les uns que dans les autres, et ne differe que sous le rapport que nous venons d'indiquer (1).

Lorsque le squelette est intérieur, il occupe sans doute constamment l'axé du tout, que le volume en soit considérable ou non.

11 en est ainsi, du moins dans les genres pennatille et vérétille, aussi bien que dans la gorgone verruqueuse (gorgonia verrucosa), espèce à liquelle se rapportent toutes celles chez lesquelles les parties dures sont prédominantes.

Le volume proporionnel du squelette varie considérablement. Dans plusieurs genres, la †ige solide. soit extérieure, soit intérieure, forme la partie la plus considérable de toute la masse, et représente, par conséquent, irès-exactement tout l'animal. Nous en trouvons des exemples parmi les polypes corticifères, surtout chez les tubipores, parmi les polypes axiferes dans les

(1) Cette remarque de M. Meckel est confirmée par les observations de MM. Quoy et Gainard, naturalistes de l'expédıtion commandée par le capitaine Freycinet. IIs ont constaté que les tuyaux parallèles du lithophyte connu sons le nom d'orgue (tubipora musica), et dont la couleur est d'un si bean rouge, étaient enveloppés d'une couche membraneuse d'un vert très-brillant, continue au reste de l'animal. Cet être, qui est une hydre, est lui-même renfermé dans le tube où il peut s'enfoncer à volonté, et d'où il peut faire sortir quand il lui convient ses huit tentacules. Voyage autour du monde, sur l'Uranie et la Physicienne, partie zoologique, 182\%. (Nole des traducteurs.) 
genres corail noir (untipalhes), isis, madrépore, millépore.

Chez d'autres, le squelette est, au contraire, extrèmement petit, relativement à la substance molle; cela se voit dans les pennatules et les vérétilles. Chez les premières, tout l'animal, par sa forme arborescente extérieure, ressemblè beaucoup de genres dont le polypier est considérable; chez les secondes, tout le corps, ainsi que la tige intérieure, représente un cylindre simple. J'organisation de ces dernières forme le passage aux espèces dans lesquelles la substance dure manque tout-à-fait, comme, par exemple, chez les alcyons, et ceux-ci conduisent aux polypes simples, vivant isolément.

Le polypier disparaît-il et revient-il périodiquement chez certaines espèces où il est trèspetit? On est fondé à l'admeltre, si on s'en rapporte à l'autorité d'un grand naturaliste (1), qui dit que le polypier manque chez le veretillum cynomorium. Il est cependant plus vraisemblable qu'il a échappé à l'iuvestigation de cet observateur, soit par accident, soit à cause de sa ténuité; je l'ai en effet toujours trouvé, quoique de volume très-différent, dans un très-grand nombre d'individus que jai examinés avec soin. Il faut toutefois ajouter que tous ces individus furent pris à la mème époque, et que cette époque était le milieu de l'été; il serait par conséquent

(1) Pallas, miscellanea zonlogicn, 1. 17?. 
D'ANATOME COMPARÉE.

possible qu'ii manquât dans d'autres saisons.

Sa consistance et partant sa composition varient. Chez les cératophytes il est corné; il est pierreux chez les lithophytes.

La substance animale, plus molle, qu'il loge dans son intérieur, est de nature gélatineuse; l'écorce dure est composée, soit uniquement, soit en majeure partie, de carbonate de chaux : dans le dernier cas, il y a une petite proportion de phosphate de chaux ( 1 ).

Sa forme extérieure est extraordinairement váriée; tout ce qu'on en peut dire, c'est qu'elle est généralement celle de tout le zoophyte, et détermine cette dernière. Cette observation s'applique surtont aux espèces oì celte partie est considérable relativement à la partie molle, moins à celles où le polypier est en proportion d'un petit volume. Ainsi dans les pennatules il ne forme qu'une tige sans ramification, quoique l'ensemble présente la forme d'une plume. Il est également fort simple dans le genre vérétille. Mais dans l'un et l'autre il coïncide, par son amincissement aux deux extrémités, avec la forme du tout. Il est même digne de remarque que dans les pennatules, nù l'ensemble rappelle encore la structure ramifiée d'autres espèces, on le trouve beaucoup plus long et plus gros que dans les véréillles, où cette structure n'existe

(1) Hatchett, Expér. chim. sur les zoophyles, ctc . Philos. transaclions. 
plus. Outre l'axe contral, les pennalules ont encore d'autres parties dures. Celles-ci constituent la base des ailes, et sont des épines alongées, droites, minces, apointies, disposées, les unes à côté des autres en forme d'éventail, depuis la racine jusqu'au bord de chaque aile, entre les lames cutanées de celles-ci, et qui dépassent leur bord libre. Ces épines sont coinposées de plusieurs fibres plus petites, juxła-posées; mais elles ne sont pas unies entre elles. Chacune d'elles correspond à deux paires de polypes, dont l'une est inférieure et l'autre supérieure; et les ovaires, ainsi que les vaisseaux, s'étendent tout le long entre elles, depuis le bord jusque vers la racine de l'aile.

Les rayons des différentes ailes ne communiquent nullement entre eux, quoiqu'ils soient bien rapprochés les uns des autres à la racine; ils ne sont unis que par le parenchyme mou. Ils sunt encore moins en connexion avec l'axe.

Cette disposition forme évidemment le passage des vérétilles, ete., qui n'ont qu'un axe, aux zoophytes, dont le polypier se ranific d'une manière non-interrompue.

D'autre part, elle se ratlache aux alcyons, qui ne présentent aucune trace de substance calcaire intérieure, mais chez lesquels il existe une croûte calcaire extérieure, qui diminue d'épaisseur et de dureté de la racine vers les extrémités des branches. 
Lia surface extérieure du polypier est ou lisse, comme chez les pennatules et les vérétilles, ou parcourue par un nombre variable de sillons longitudinaux, comme dans le corail du commerce '(corrallium), 'etć.

11 ne présente très-généralement point de traces d'articulation, mais forme un tout continu que n'interrompt aucune solution. Une exception est fournie, parmi les espèces où le polypier est fort développé en proportion de la substance molle, par l'isis et les polypes qui s'en rapprochent; ces êtres offrent un exemple de structure articulée digne d'être remarqué, à cause de l'analogie qu'elle présente avec les squelettes des animaux supérieurs. Leur polypier est formé de portions alternativement cornées et calcaires. La consistance de ces deux substances présente, dains la même espèce, les mêmes gradations. Ainsi, là où les porlions calcaires sont très-dures, les portions intermédiaires sont cornées, tandis que lorsque les premières ont une structure moins compacte, les secondes sont d'une consistance spongieuse.

Parni les espèces elles-mêmes où la substance molle prédomine beaucoup sur la substance dure, ou du moins parmi celles qu'une structure moins dendroïde et une longueur plus considérable du tronc en rapprochent, les encrines ont des parties dirres articulées.

31. 
La structure intime de cette partio du polypro est simple.

Elle esi siluće, du moins chez les pronalulies et les vérélilles, dans une enveloppe propre, mince, solide, lisse, quil l'entoure sans lui adlúrer. Cette enveloppe, qui est attachéc d'une manière lâche à la masse molle circonvoisine, peut en quelque sorte ètre comparée au périoste des animaux supérieurs.

Dans le genre pennatule, colle poche, en raison du développement beauconp plus cousidérable de la lige, est hien plus distincte que dans le genre vérétille.

Dans l'un et l'autre on voit des faisceaux qui partent de la face intérieure de la substance molle, et se fixent à cette enveloppe dans une direction oblique du sommet a la base du tronc; (n peut comparer ces faisceaux aux muscles des animaux supérienrs.

Je ne sais si cette enveloppe existe également chez d'autres zoophytes, surlout chez ceux oi le troncest en proportion plus volumineux. Elle manque positivement daus la gorgone verruquense. Le trouc n'y est pas mème uni à la partic molle par une substance intermédiaire, analogue au tissu muqueux, de sorte quen faisant une incision, on sépare très-facilement les deux substances de l'animal.

I a tige elle-même est en général parfailenrent homogime; quelques gemres la présentent co- 
pendant à divers degrés de complication. Nous avons déjà indiqué sa structure articulée chez certaines espèces; ce phénomène se voit encore chez. d'autres. Chez les gorgones, elle est composée d'une partie extérieure plus molle, et d'une partie intérieure plus dure occupant l'axe, et qui est toujours beaucoup moins considérable que l'antre. Ces deux parties sont surtout trèsdistinctes dans la gorgone verruqueuse (1). L'extérieure est brune, cornée, se compose de plusieurs couches concentriques, l'une dans l'autre, qui se confondent en se continuant; l'intérieure est blanche, lisse, cylindrique, et séparée sans transition de l'extérieure; elle est plus dure et beaucoup moins développée que celle-ci.

Daus la pennatule grise ( pennatula grisea), la structure du tronc est encore plus compliquée. Sa surface extérieure est formée par une couche blanc-jaunàtre, d'une minceur proportionnelle. Après colle-ci on en trouve une seconde, grise, un peu plus épaisse, mais beancoup plus dure; enfu une troisieme plus épaisse encore, semblable a la premiere sous le rapport de la couleur et de la consistance, et enveloppant un rylindre minec, dur, situé daus l'axe du tronc.

I.es différentes portions de la lige des isis ar-

(1) Ta gorgone vermouluc (gorog. vermiculuta) présente aussi un exemple tris-fraplant de la disproportion des deux substances.

(Note des tradurteurs.) 
ticulés ne présentent pas des démarcations également tranchées.

Lorsque les deux substances du tronc sont solides et dures, on les trouve exactement séparées; dans le cas contraire, elles se confondent en quelque sorte; la plus molle des deux substances contient des filamens de la portion dure, et péuètre elle mème dans les divisions de celle-ci.

I.es gorgones et les pennatules me semblent faire le passage des autres genres aux isis; c'est en effet chez elles que commerice la distinction des deux̃ substances; mais celles-ci s'étendent encore comme un tout continu à travers tout le squelette, ou du moins à travers sa plus grande partie.

Le mode de développement du squelette des polypes est peu conruu.

On peut admettre, par analogie, qu'il se forme postéricurement à la substance molle.

Des individus trè-jeunes de la gorgone verruqueuse, ayant la forme de petits boutons rouges, et dont les plus parfaits avaient déjà les huit bras dentelés, ne présentèrent en effet à Cavolini ( 1 ) aucune trace de squelette intérieur, quoique la substance corticale contînt déjà de la

(1) Memorie per servire alla storia naturale dei polipi marini. Napoli, 1785, p. 133.

Le fait consigné dans la note de la page 15 sur le tubipora musica confirme encore l'idée que la substance molle est la watrice de la substance dure, et par conséquent la précide.

(Note des traducteurs.) 
clalux corm rne chez les individus adultes. J'oblins les mòmes résultats dans mes recherches sur cet inimal. Avec ce phénomène coïncide la faculté que possède la substance corticale, de continuer à vivre pendant un laps de lemps assez considérable, a près avoir été isolée du tronc, sans que celle circonstance soit suivie de la reproduction nécessaire du polypier, fait d'autant plus remarquable, que l'on trouve peu de temps aprés la séparation les traces d'un nouveau squelette sous la substance molle ou corticale enlevée.

DEUXIEMME SECTION.

SQUELETTE DES ÉCHINODERMES.

$$
\text { S. } 6 .
$$

L'étude du polypier conduit à celle du squelelle dres échinodermes. lues polypes à polypier articulé me semblent surtout offrir la transition aus échinodermes, qui, à leur tour, forment le passage aux animaux arliculés.

$$
\text { S. } 7 \text {. }
$$

L.e squelette des échinodermes est très-généralement articulé, à l'exception de celuide quelques érolinides. Il se compose de pièces tout-àfait pleines, ayant une structure absolument hromogene, saus offrir de traces de tissu fibreux ou cellulaire. 
Il est formé principalement de carbonate de chaux et dinne petite proportion de phosphate calcaire, ce qui avait déjà élé indiqué par Hatchefl (1). M. Tiedemann (2) ne signale que d'une maniere générale la prisence de ces deux subslances. Les recherches de M. Stolize confirment celles de Matchett. Suivant lui, il existe dans les oursins beancoup plus de phosphate de chaux que dans les astéries.

Le mode de développement de ces parties est peu connu.

Ce quil y a de certain, c'est que le nombre des différentes parties qui composent lo squelette de cos animaux augmente avec l'âge; on les trouve constamment moius nombreuses chez les jeunes astéries et oursins de la mème espece, que chez les adultes.

La supposition que la substance de ce squelette pourrait être formée dans un organe particulier (3) a élé émise. Ce que l’on a considéré comme étant cet organe, est une petite surf́ace arrondie, située entre deux rayons ì la face dorsale du disque des astéries communes, surface garnie de petites éminences, et d'où naîl, nour se porter en bas et en dedans, un canal contourné, rempli d'une substance grumeleuse, dure.

(1) Hatchetl, sur le test des testacés. Philos. trans.

(2) Ueber den Isan der Rochrenholothurie, elc 1:16. (Sur la structure de l'holothurie tubuleuse.)

(3) Tiedemann, loco cilato. 
1) ANATOMLE COMPAREE.

()n a cité à l’appui de cutte opinion:

1\%. Jinsertion de ce canal calculifere dans un systime vasculaire qui se ramifie daus les différens rayons;

2". I á concordance de composition entre la substance contenue dans le canal calculifére et le squelette;

5o. Labsence de vaisseaux aux vertèbres, de sorte qu'il semble que celles-ci n'ont pas d'autres organes de nutrition, et qu'il fallail par conséquent que la chaux contenue dans le système vasculaire transsudât à travers celui-ci.

Sans nier absolument l'exactitude de cette opinion, on peut néañmoins faire la remarque :

$1^{\circ}$. Qu'il est en général plus vraisemblable que les portions du lest se forment comme les autres parties. aux lieux mèmes qu'elles orcupent. On. pourrait citer comme exem ple analogue les cuncrélions calculeuses dites yeux d'écrevisses; mais il ne faut pas oublier que celles-ci se forment au moyen du nouveau test, lors de la mue, randis que chez les échinodermes il n'y a pas de changement semblable, du moins d'apres ce qu'on en sait jusqu'à ce jour.

$2^{\circ}$. Les faits cités ne sont pas tout-à-fait conchans, puisque le premier peut aussibien auforiser à ìgarder le canal calculifere comme un organe excréteur; que le second, ou peut donner lieu ì la même opinion, ou nindique pas néressairement une relation de cause it effel; que 
le troisième ne milite nullement en faveur de la prétendue source de nutrition des parties calcaires, car si la masse pierreuse peut se produire dans une partie de la peau, cela doit aussi bien être possible partout où elle se trouve.

\section{Ajoutez à cela :}

$3^{\circ}$. L'absence de cet organe che z les ophiures, et très-vraisemblablement aussi chez les ou:sins.

La circonstance que le canal calculifere a ses racines dans le système vasculaire, et se dirige de eelui-ci vers la peau de l'animal, joinle aux argumens ci-dessus énoncés, et à la grande quantité de subsiance solide chez les astéries et lesoursins: me fait croire, ce me semble, avec plus de vraisemblance, que ce canal est un organe excréteur. Avec cela s'accorde très-biein l'absence de l'organe à calculs dans les oursins, où le test calcaire a atteint un développement beaucoup plus considérable. Comme la présence d'un organe sécrétant de l'acide urique avait été démontrée, il n'y a pas long-temps, chez beaucoup d'animaux, il n'était pas sans intérêt de chercher si la substance du canal calculifère ne contiendrait pas d'acide urique. M. Stoltze fit l'analyse de ces parties avec beaucoup de soin, mais il n'y trouva que de la malière animale, beaucoup de carbonate de chaux et une petite quantité de phosphate de clraux.

Le squelelte le plus composé est celui des 
stellérides, le plus simple celui des holothuries; celui des échinides leur est intermédiaire.

1. Stellérides.

\$. 8.

Chez les astéries proprement dites, nous noterons en particulier l'astérie orangée (asteria aurantiaca): chaque rayon se compose d'une quartité très-considérable de pièces ossiformes, qui se succèdent de la base au sommet du rayon, et se répètent exactement sous le rapport de la position et de la forme. Le volume seul cesse d'ètre le même, et diminue insensiblement de dedans en dehors. Ces pièces se correspondent anssi parfaitement d'un côté à l'autre dans le mème rayon, et sont unies entre elles d'une manière mobile. Les pièces des deux côtés de chaque rayon réunies, constituent une gouttière dont la convexité est tournée en haut et la concavité en bas. Les deux moitiés latérales se réunissent sous un angle obtus, et forment par leur face supérieure le plancher de la cavité viscérale du rayon.

Celie cavité est fermée supérieurement par la peau qui est solide et dure, ćtendue d'un côté à l'autre, de sorte que chaque partie de la goutlière dont le mode de formation vient d'être indiqué est transformée cu un anucau aplati. articulé à sa partie inférieure, el tre présentant 
atucune trace de Jrisure ì sa partic supérieure.

Ces pièces solides sont tapissées en grande partic a leur face interne par me membrance fibreuse blanche, épaisse, très-résistante, qui se porle de lá au dos de l'animal, et représente le. derme.

Quand on examine de plus près la formation de ce squelette, on trouve les particularités suivantes :

A côté del'axe du rayon existe la pièce: la plus considrable, que l'on peut nommer lapièceprin. cipale ou le corps. Sa forme es! celle d'un carre longr; sa plus grande longueur s'élend de l'axe en dehors vers le bord latéral. Elle est considérablement plus haute qu'épaisse, et diminue en même temps d'épaisseur à partir de l'axe. Elle a sa face interne large, très-rugueuse, tournée vers la pièce correspondante et de mème nom; au point de jonction des deux picces, existent. des filores musculaires, transverses, qui les unissent en haut; en bas ce sont des fibres tcndincuses, blanches, roides, courtes et dirigres dans le même sens. Vers la base et le sommet du rayou, celte extrémité interne des pièces principales présente une surface carrée, moins ruyucuse, par laquelle elle s'unit aux deux pièces voisines et de mème nom, an moyen d'une substance plus molle, d'un rouge-brun, incontestatb!ement musculcuse.

Du milien de la face antericure de la biece 
sóve une saillie triangulaire se portant cu devant vers le hord inférieur; plus en dehors et en haut, il en part une plus considérable de la face postérieure, se portant en arrière et s'élendiant jusquà l'extrémilé externe.

Par ces deux saillies les pièces de même non se touchent sans cependant s'unir.

La partie interne du bord inférieur est libre, l'externe s'appuie sur une pièce inférieure qui va être décrite.

Le bord supérieur est encore plus libre; il offre seulement à son extrémité exteruc, au devant de la saillie postérieure dont il a été fait mention, sur une surface rugueuse, peu étendue, une très-petite pièse alongée, plongée touta frit au milieu d'une substance rendineuse et. élroitement unie à ce bord, d’où elle se porte d̀ une autre pièce latérale considérable.

Cette pièce principale n'est pas, comme les intres, revètue à sa face interne de substance cutanée tendineuse, elle est au contraire entièrement libre.

Entre les moitićs internes de deux de ces pièces du mème côté, il existe une lacune considérable, dans laquelle est située la partie moyenne de la vésicule du pied.

A l'endroit de la ligne médiane, où les pièces qui se correspondent latiralement se renconIreat, clles recouvrent le vaisseau el te cordon nerveux du rayou. 
Outre la pièce principale qui vient d'ère décrite, il tn existe encore quatre autres de chaque côté.

La première de celles - ci (la seconde en commençant à compter par la pièce principale), qui est la plus petite, est la pièce alongée dont il a déjà été question; elle est situẻe entre la première et la troisième, comme une espèce de poutre transversale. On peut l'appeler pièce transversale intermédiaire.

La troisième, qui est la seconde par le volume, peut être nommée la pièce latérale inférieure. Elle a la forme d'un carré long, est convexe en dehors, concave en dedans; elle est plus épaisse que la promière pièce dans la direction de la base au sommet du rayon, de sorte qu'elle correspond toujours à une pièce principale et à la moitié d'une autre de ces pièces, ou à deux de ces parties; elle devient insensiblement plus mince de haut en bas.

Elle nes'articule pas avec la pièce principale, mais touche par sa partie moyenue à la seconde pièce, ct par sa moilić inférieure ì la quatrième. Elle est unie à cette dernière de manière à en recevoir une entière à sa partie moyenue, et une moilić à son extrémité antérieure et postérieure, re sorte qu'ici deux pièces latérales inféricures voisines se réunissent pour recevoir une pièce latérale supérieure. Vers la base et le sommet du rayon elle s'articule mobilement, au moyen 
le fibres ligamenteuses transverses, avec la pièce antérieure et postérieure du même nom.

Ia surface extérieure de cette pièce est hérissée d'un grand nombre de petites apophyses qui ont la forme d'écailles. Ces éminences sont plus considérables vers son bord postérieur, où elles se transforment en une série simple de piquans mousses, alongés, un peu àplatis, entièrement mobiles, dirigés vers le sommet du rayon, se succédant de haut en bas, et diminuant de volume dans la mème direction. Ces piquans sont ordinairement au nombre de cinq. Les deux plus supérieurs, surtout le premier de tous, ont des tètes très-arrondies, au moyen desquelles ils reposent sur de petits tubercules concaves de la pièce latérale inférieure. Ises fares articulaires des piquans moyens sont à peu près planes; celles des deux piquans inférieurs offrent une disposilion contraire à celle des piquans supérieurs. Ils ne sont unis à la pièce latérale inférieure qu'aú moyen d'une substance fibreuse, blanche, disposée seulement autour deleur base, qui est rugueuse.

La quatrième pièce peut être désignée sous le nom do pièce latérale supérieure. Elle ressemble i la précédente, mais elle a une forme plus carrée et plus d'épaisseur; en s'y réunissant elle constitue le bord latéral de la cavité viscérale, s'articule avec elle inférieurement, et n'est unie avec les pièces voisines du même nom que 
vers la base et le sommet rlu rayon; enfin elle olfre à sa surface des saillies semblables, mais moins grandes el moins nombreuses; une ou trois seulement de ces saillies sont mobiles.

La cinquiène pièce est l'inféricure. Elle est carrée, un peu convexe vers la base, concave vers le sommet du rayon, et se trouve entre lat piéce principale et la pièce latérale inférieure; elle leur est unie par des fibres tendineuscs.

Elle cst mie par des fabres musculaircs aux pièces antérieure et postérieure de mème nom qu'elle,

Sa facc inféricure qui est libre, est recowverte d'une grande quantité de piquaus mobiles, alongós, aplatis, qui augmentent considérablement de volume de dehors en dedans, mais qui n’égalent pas les épines de la pièce laterale inférieure.

La peat qui ferme la cavité viscérale de chaque rayon el cn forme la paroi stpéricure, se compose :

$1^{\circ}$. 1)'une conche intéricure tendineuse, qui se continue sur la face interne des pieces qui viennent d'ètre décrites, al l'exception de la première et de la cinquième;

$2^{\circ}$. De tubercules trè-rapprochés les uns des autres, variant considérablement suivant les espees, sous le ripport du volume et de la forme, qui rendent cette partic fort ruguense, et qui scmblent avoir de lanalogie avec les piquans 
mohiles qui hérisseut les côtés et la fare inférieure, mais qui se confondent plus que ceux-ci avec la hase qui leur sert de support.

Linnion de ces différentes fièces entre elles, outre qu'elle est opérée pandes connexions musculaires, l'est en grande partie par des proddolious d e nature fibreuse qui s'étendent entre les deux faces opposées; elles jouissent par cónsé(fuent de peu de mobilité les unes sur les autres. l.es piquans, au contraire, qui arment plusieurs d'entre elles sont articulés d'une manière trèsmobile, an moyen de ligamens capsulaires lâches.

Chaque portion de rayon, en considérant les rleux moiliés latérales, se compose, abstraction faite des piquans mobiles:

$1 \%$ De deux pièces principales,

2 . De deux pièces transverses.

$5^{\circ}$. Enfin de deux pièces inférieures.

A cela se joint de chaque côté :

$1^{\circ}$. Une moitié de pièce latérale supérieure,

$2^{\circ}$. Et une moitié de pièce latérale inférieure.

Il y a par conséquent à peu près huit pièces prour former une portion vertébrale.

Chaque rayon se compose d'environ quatrevingts de ces portions, et contient par conséquent de 6 fo a jo pièces osseuses isolées. Tout le corps est douc formé de 5200 à 500 pièces propres. quoiqu'il n'y eu ait pas despéciales dans ledisque.

Cies pieces varient. de la hase all sommet du 
rayon; elles deviennent en général plus petites vers l'extrémité libre, mais elles présentent la même distribution.

Il est, en outre, plusieurs pièces latérales de même nom et du mème côté, qui se confondent vers l'extrémité du rayon. Celte partie de l'animal se termine enfin de façon que la peau supérieure ou dorsale intermédiaire disparaît, et que les dernières pièces latérales supérieures se courbent en haut et se soudent dans la ligne médiane.

Quant aux bases des rayons, elles offrent surtout les modifications suivantes:

$\mathbf{I}^{\circ}$. Les pièces principales deviennent plus courtes de l'axe vers les bords latéraux, plus longues de haut en bas, et plus étroites de dehors en dedans;

$2^{\circ}$. Les apophyses dont elles sont garnies en avant et en arriere sont plus imparfaites;

$3^{\circ}$. Elles se rapprochent davantage les unes des autres;

$4^{\circ}$. La pièce la plus interne s'épaissit subitement de dehors en dedans, et parvient à surpasser au moins trois fois les pièces précédentes dans le sens de cette dimension;

$5^{\circ}$. Les pièces latérales supérieures, à compter de la dixième ou onzième portion vertébrale, s'éloignent de l'axe en se dirigeant plus vers le côlé, vers les rayons voisins, et augmentent, dans le même rapport, de hauteur et d'étroitesse. 
Aux cinq ou six portions les plus internes, les pièces latérales supérieures manquent, et la dernière portion s'unit, par des fibres tendineuses, avec celle de même nom du rayon voisin.

$6^{\circ}$. Les pièces latérales inférieures se conservent plus long-temps. A partir de la dixième ou onzième portion vertébrale, elles se dirigent aussi sur le còté. Dans la région de la septième portion, la pièce latérale inférieure se porte tellement en haut, qu'elle se détache de la pièce principale, et vient à ètre placée entre les pièces |rincipales voisines et la pièce latérale supérieure. Les trois ou quatre pièces placées le plus en dedans, qui se trouvent jusque vers la troisième portion, diminuent promptement de volume, s'aplatissent considérablement d'un côté à l'autre, sont situées de dehors en dedans dans l'angle de deux rayons, et se joignent exactement aux pièces de même nóm du rayon voisin.

$7^{\circ}$. Les pièces transversales semblent manquer à partir de la cinquième portion; cette absence n'est cependant qu'apparente; elle est la conséquence de ce que les vertèbres les plus internes les ont refoulées et poussées en dedans.

Les quatre pièces principales situées le plus en dedans, semblent remplacées par une pièce considérable, unique, située en avant et un peu sur. le côté de la première pièce principale. En effet, tout-à-fait au-dessous de la base des deux rayons 
on trouve entre les vertèbres les plus internes, une grosse pièce mobile, en forme d'un Y, qui peut ètre considérée sans contredit comme une réunion des quatre pièces principales les plus internes. On pourrait, à la vérité, la regarder comme un analogue des pièces latérales inférieures également les plus internes; mais l'opinion que j’ai énoncée me paraît la plus juste, $1^{\circ}$ parce que les pièces transversales les plus iuterues manquent tout-à-fait; $2^{\circ}$ et que cette pièce en forme d'Y n'atteint pas la surface, et ne supporte point d'épine mobile.

$8_{0}$. Lespièces inférieures deviennent très-étroites à partir de la cinquième division; elles changent leur direction, jusqu'alors transversale, en une oblique, de sorte qu'elles forment de dehors en dedans avec l'axe du rayon un angle très-oblique. La dernière s'applique dans toute sa longueur contre la pièce du même nom du rayon voisin.

On voit très-distinctement, d'après ce qui vient d'être dit, le mode suivant lequel s'élargissent les rayons à leur base, et se forme une cavité centrale, discoïde, ample; en effet, les pièces principales se contractent vers l'axe, les pièces latérales inférieures les plus internes, les pièces inférieures et les transversales, changent en parlie de direction, en partie s'éloignent de leurs rayons principaux pour se porter en dedans et se souder entre elles, tandis que les pièces latérales supérieures s'éloignent de l'axe, se diri- 
D'ANATOMIE COMHARÉ.

geant vers la circonférence, pour disparaître enfin tout-à-fait aux portions les plus internes.

\section{\$. 9 .}

La structure des ophiures est plus simple, mais elle est essentiellement la même. Chez l'ophiura lacertosa, chacun des rayons, formant un cône très.alongé, est composé à toute sa surface extérieure de plaques dures, unies par un contact très-étroit. Les supérieures forment le plus souvent une rangée double, quelquefois cependant il n'y en a qu'une, quelquefois trois. Elles correspondent sans doute à la peau dorsale des astéries. Vis-à-vis de ces plaques, c'està-dire à la face inférieure, est située une série plus étroite, ordinairement unique, de semblables plaques, que l'on peut considérer comme correspondantes aux pièces inférieures des astéries, qui se seraient portées plus en dedans, et soudées au milieu. Ces deux espèces de plaques sont privées de saillies; mais de chaque côté il y a une autre série de plaques, garnies à leur bord postérieur d'épines courtes, de même longueur, dirigées en arrière; elles offrent, en outre, à leur moitié inférieure une crête calcaire, étroite, saillante en dedans. Ce sont incontestablement les analogues des pièces latérales supérieures et inférieures des astéries. Ces quatre rangées de plaques entourent très-étroitement une série 
d'autres plaques en même nombre, discö̈des, de plus en plus étroites de la base au sommet du rayon, qui se terminent en haut et en bas, à côté de la ligne médiane, par deux pointes mousses, et forment dans leur partie moyenne une saillie considérable en devant et en arrière, prononcée particulièrement dans ce dernier sens, où elles sont par conséquent aussi beaucoup plus épaisses qu'ailleurs. Elles représentent évidemment les pièces principales des astéries, unies à leurs petites pièces transversales; elles ne se distinguent même des premières que par une hauteur plus considérable, leur forme arrondie, la soudure des deux pièces latérales dans la ligne médiane, et lear grand volume relativement aux autres parties, enfin, parce qu'elles occupent presque toute la cavité du rayon, le canal intestinal et les ovaires ne s'étendant pas ici dans cette cavité comme chez les astéries.

Les crêtes calcaires des pièces latérales se placent entre les pièces précédentes.

A la face dorsale du disque central, qui est proportionnellement beaucoup plus considérable chez les ophiures, on voit disparaitre à l'extérieur beaucoup plus tôt qu'à la face abdominale toute trace des plaques du rayon. A cellelà, on n'en voit plus rien au-delà du pourtour du disque; à celle-ci, au contraire, les plaques inférieures et les latérales s'étendent jusqu'à l'orifice qu'elles entourent. Quand on ouvre le disque 
ì sa pirtie supérieure, on trouve cependant les pièces discoïdes situées au côté intérieur de la face inféricure , et correspondant aux pièces principales des astéries, s'étendre aussi loin en avaut que là. I a plus interne de ces pièces est beaucoup plus haute et plus épaisse que les autres; elle est divisée en deux moitiés latérales égales, qui, réunies, forment un arc dont la convexité est tournée err dehors, et supporte plusieurs rangées d'épines courtes, situées les unes au-dessus des autres. Les arcs des différens rayons se réunissent sous des angles aigus, rentrans en dedans; la saillie qui en résulte supporte des épines plates, plus grandes, placées les unes an-dessus des autres.

En haut, les moitiés latérales des arcs des rayons juxıa-posés sont en outre retenues ensemble par deuz plaques transverses.

A la circonférence extérieure du disque on trouve immédiatement au-dessus de chaque rayon une plaque calcaire triangulaire, après laquelle vient une crête alongée, étroite, occupant la moitié du disque. Cette plaque ne représente pas, comme on pourrait le croire, les pièces latérales soullées entre elles, puisque celles-ci existent séparées, et s'étendent presque jus. qu'à la circonférence intérieure du disque, à côté de l'ouverture buccale.

Dars d'autres espèces, notamment chez l'ophiurr syuamalu et ciliaris, $1^{\circ}$ les epines des pieces latérales sont beaucoup plus grandes et 
plus mobiles; $2^{\circ}$ le disque est plus dur; $3^{\circ}$ on voit aussi à l'extérieur de leur face dorsale des stries calcaires, qui correspondent à l'axe des rayons, s'étendre jusqu'au milieu; $4^{\circ}$ ces stries et d'autres situées entre elles, qui convergent vers le centre du test dorsal où elles se réunissent, supportent des épines mobiles, mais plus petites.

L'ophiura texturata offre une organisation intermédiaire aux deux précédentes; il est digne de remarque que même les pieds de ces animaux présentent une semblable strie intermédiaire (1).

Les ophiures conduisent, sans contredit, aux eur'yales et aux comatules. Les premières, quand on fait exception de l'absence des épines et de la division multiple des bras, présentent à peine quelque différence; les comatules en offrent au contraire de fort considérables, qui sont très-remarquables à cause du passage qu'elles forment aux échinides.

L'animal est formé, $1^{\circ}$ d'un disque, $2^{\circ}$ de cinq à dix rayons inférieurs, externes, plus grands, $3^{\circ}$ d'un nombre plus considérable de rayons supérieurs, internes, plus petits. Ces deux ordres

(1) Chez l'ophiura texturata il y a décidement de petits pieds aux rayons, quoiqu'ils doivent manquer chez l'ophiura à courtes épines. Voy. Lamarck, anim. sans vertèbres, II, 541 . Chez l'ophiura squamata et ciliaris, les pieds sont beancoup plus longs, et ressemblent parfaitement à ceux des oursins. 
de rayons se portent en haut, les externes en dedans, les internes en dehors, de manière à se rencontrer au milieu de la face supérieure du tout, en face du disque. Si on se figure ces rayons déployés et réunis, on a un oursin ordinaire. Tous ces organes sont articulés; les plus grands supportent deux rangées latérales, alternes, de rayons articulés plus petits, qui ressemblent aux rayons internes, mais ne sont pas aussi développés que ceux-ci.

Le disque plus grand à la face abdominale, présente ensuite un orifice non-seulement buccal, mais aussi un anal. L'ouverture buccale n'occupe pas exactement le centre. L'anus représente le plus souvent une saillie en forme de crête, située également un peu vers la circonférence du disque; l'existence de cette crête n'est cependant pas constante, il n'en reste souvent qu'un petit orifice (1).

2. Échinides.

$$
\text { S. } 10 .
$$

La forme totale des échinides, et la disposition du test qui la détermine, établissent, il est vrai, au premier coup d'cil, une différence bien

(i) Voyez ce que j’ai dit sur les caractères remarquables qui distinguent ce genre d'avec les antres stellérides, au vol. 8, cah. 3 de mes Archives de physiologie, 1823. 
tranchée entre la conformation de ces animaux et l'organisation qui vient d'ètre décrite : cependant il est facile de les ramener l'une à l'autre. même sans tenir compte des formations intermédiaires qui les rattachent (1).

\section{\$. I1.}

Tous les échinides offrent la disposition suivante. $1^{\circ}$ Le test entier, plus ou moins arrondi, se compose de deux ordres de divisions; chaque ordre en renferme cinq qui sont égales, ou du moins très-semblables les unes aux autres, et qui alternent avec celles de l'autre ordre.

Leur forme est en général alongée, mais la longueur en est variable; on peut les considérer chacune comme formée de deux triangles adossés par leurs bases.

$2^{\circ}$. L'extrémité supérieure de ces divisions occupe toujours le milieu de la moitié supérieure de l'animal; elles s'étendent de là vers la plus grande circonférence, au point où la face supérieure se continue avec l'inférieure.

$3^{\circ}$. Toutes ces parties, et conséquemment presque toute la surface supérieure du test, offrent une quantité très-considérable de tubercules arrondis, représentant des boutons fort convexes, de volumes divers. Ces tubercules sont

(1) Voy. vol. 1, p. 125 de cet ouvrage. 


\section{D'ANATOMIE COMPARÉE.}

entourés d'un sillon plus ou moins considérable, et se composent eux-mêmes, du moins le plus souvent, d'une base plus large et d'une extrémité globuleuse plus saillante. Ces petits boutons supportent des piquans, grands et petits, sans nulle cavité intérieure, dont le volume et la forme présentent souvent les plus grandes différences chez le même animal aussi bien que dans les différentes espèces, et qui sont unis aux tubercules par une articulation très-mobile.

4'. Les deux ordres de divisions dont il vient d'ètre question se distinguent l'un de l'autre sous plus d'un rapport.

a. Par leur largeur. Ainsi les unes sont toujours un peu plus larges que les autres; souvent même cette différence est fort prononcée.

b. Par leur structure. Toutes ont des tubercules armés d'épines, mais les plus étroites présentent constamment, soit des tubercules et des épines d'une dimension beaucoup moindre, soit plusieurs rangées d'orifices arrondis assez réguliers et très-serrés, se dirigeant de leur base vers leur sommet. Ces orifices sont en général plus petits que les tubercules, et ordinairement disposés le long du bord latéral de ces portions du test. Ces rangées sont les ambulacres, et les plaques qui en sont garnies peuvent ètre nommées plaques ambulacraires.

c. Les divisions largeses s'étendent toujours, sans interruption sur tout le test, et se ren- 
contrent les unes les autres à l'extrémité buccale; - les plus étroites, percées d'orifices, se terminent, au contraire, souvent beaucoup plus tôt, c’est-à-dire déjà bien haut à la face supérieure.

\section{S. 12.}

Les différentes espèces d'échinides sont loin de rentrer exactement dans les conditions du type dont je viens d'esquisser l'idée gérérale.

L'organisation la plus régulière se rencontre chez les oursins proprement dits.

L'excès de largeur des divisions les plus considérables sur les autres n'est pas exagéré. II existe tout au plus une différence de moitié. Elles portent huit à dix rangées de gros tubercules trèssaillans, destinés à recevoir de grands piquans. Les bases des gros tubercules sont entourées d'une quantité considérable de tubercules plus petits. De ces dernières rangées, il n'y en a que deux qui s'étendent de l'orifice supérieur à l'inférieur; ce sont les troisièmes à compter du bord externe.

Les petites divisions ont deux rangées de gros tubercules, entre lesquelles on voit deux rangées plus courtes, hérissées d'éminences moins élevées. Les petits tubercules offrent un poli luisant.

Les ambulacres forment sur chaque côté des petites pièces, plusieurs bandes sinueuses de 
trous, quelquefois au nombre de quatre et de six, qui sont éloignées de celles de l'autre côté par les rangées des éminences, à une distance dont la largeur est de beaucoup supérieure à leur largeur propre. Ces séries s'étendent sans interruption de l'orifice anal à l'ouverture buccale.

Les deux ordres de pièces dont il vient d'être parlé, les grandes et les petites, sont très-distinctementformées de plusieurs lames pentagonales et hexagonales transverses, larges, mais peu hautes, légèrement convexes en dehors, concaves en dedans. La face externe de chacune d'elles est armée de dix à douze éminences en forme de tubercules; l'interne est unie; les bords sont inégaux, ils présentent des dents et des creux qui sont disposés verticalement sur chaque face. Les lames ne sont articulées entre elles qu'au moyen de ces inégalités qui s'engrènent mutuellement; leur union est très-solide, quoique je n'aie pas pu découvrir d'autre moyen de connexion. Cet engrenage des lames au moyen des lignes dentelées, a fait dire qu'elles avaient beaucoup de ressemblance avec les sutures du crâne de l'homme ( I); cependant cette analogie est très-éloignée, puisque les dents de cette suture sont, $1^{\circ}$ toujours de même longueur, $2^{\circ}$ simples, $3^{\circ}$ en ligne droite et tranchantes.

Les plaques diminuent considérablement de

(r) Tiedemann, 1. c, p. 86. 
largeur, moins de hauteur, depuis le milieu de la périphérie, vers les ouvertures buccale et anale. Celles des grandes divisions sont plus hautes et pourtant moins nombreuses que les plaques des petites; il en résulte que, tandis que l'espace compris entre la bouche et l'anus est rempli par vingt et quelques de ces plaques, il y en a ici environ trente pour occuper le même espace.

Toutes s'engrènent de telle sorte, qu'à deux de l'une de ces rangées il en correspond une de l'autre série.

Chaque division se partage en deux moitiés parfaitement symétriques, de façon que tout le test est formé par vingt rangées de plaques, dont dix grandes et dix petites.

A l'extrémité inférieure ou buccale, toutes les divisions forment des saillies plus ou moins considérables, qui partent, sous un angle droit, de la plus interne de chaque série, et se dirigent en dedans. Cette saillie manque, ou est du moins très-petile à la partie interne et moyenne des petites divisions; elle est, au contraire, trèslongue sur la partie externe qui renferme les ambulacres. Ces parties très-alongées se rencontrent versl'endroit de la circonférence où la saillie manque; il résulte de cette disposition cinq dents très-proćminentes. Entre ces dents et le test extérieur, on trouve un orifice arrondi, qui correspond à la ligne médiane des plaques ambula- 
D'ANATOMIE COMPARIE.

craires. Les saillies des larges divisions sont beaucoup plus déprimées que celles des étroites.

Ces deux sortes de saillies semblent se distinguer en outre, du moins fréquemment, par le nombre des parties qui les forment. La saillie qui correspond aux deux moitiés latérales d'une grande division est unique; les saillies plus prononcées des petites divisions peuvent être, au contraire, séparées en deux moitiés latérales. C'est du moins ce que j'ai trouvé dans toutes mes recherches.

A côté des saillies des petites divisions, on voit, près de chaque bord latéral, dans les grandes divisions, de chaque côté, une incisure plus ou moins profonde, que l'on peut voir aussi à l'extérieur, et qui conduit à ces saillies.

Au côté supérieur opposé existe une plaque pentagonale simpleà l'extrémité de chacune des divisions grandes et petites. Celles de ces pièces qui correspondent aux grandes divisions, ont deux ou trois fois plus d'étendue que celles qui limitent les petites plaques. L'angle moyen, plus grand, est tourné en haut, le bord inférieur, concave, est dirigé en dedans. Elles renferment une ouverture considérable par laquelle l'uvaire s'ouvre en dehors; on peut par conséquent les désigner sous le nom de plaques ovariules (1). Les petites plaques sont également

(1) M. de Blainville les nomme plaques génitales.

(Note clu iraducteur.) 
munies d'un trou, mais beaucoup plus petit; il appartient au syst ème vasculaire. Toutes les grandes plaques, mais non toutes les petites, s'étendent jusqu'au bord interne du cercle formé par ces dix plaques. Une des plaques génitales est ordinairement déux à trois fois plus grande, en même tems plus rugueuse à cause d'un grand nombre de petites saillies, et enfin plus convexe que les quatre autres. Cette disposition représente, sans contredit, le disque arrondi des astéries, qui est en rapport avec le canal calculifère. Le cercle entouré de toutes ces plaques est rempli par un nombre plus ou moins considérable d'autres plus petites, qui ne sont pas, comme les autres, unies immobilement, mais qui jouent les unes sur les autres, à l'aide d'une peau plus molle qui les unit. Elles entourent l'orifice étroit de l'anus qu'il est rare de voir exactement au milieu.

Les épines des oursins poprement dits ne sont pas très-longues et épaisses, et ne varient pas beaucoup sous le rapport du volume.

$$
\text { S. } 13 .
$$

Les autres échinides sphéroïdes offrent en général la même organisation; mais le genre cidarite s'en éloigne, $1^{\circ}$ par le volume extraordinaire et le nombre en proportion moindre des épines principales; $2^{\circ}$ par la diminution considérable 
D'ANATOMIE COMPARÉs.

du nombre et l'ampliation des pièces des grandes divisions, disposition en rapport avec la première, en ce qu'à chaque piquant principal il ne correspond qu'une plaque; $5^{\circ}$ par un volume beaucoup moindre des petites plaques ambulacraires et par une position plus droite de leurs orifices; $4^{\circ}$ par la circonstance que la saillie ou apophyse interne réfléchie de la grande division ne se courbe pas par dessus l'ambulacre, d'où résulte une lacune qui n'est pas fermée en dedans. Les saillies épineuses des divisionslarges sont beaucoup plus élevées que chez les oursins; celles des divisions étroites n'existent presque pas. Les premières peuvent en outre être partagées en deux moitiés latérales, du moins chez le cidarite hystrix; leur séparation est indiquée déjà par un raphé profond qui part de leur bord interne; $5^{\circ}$ une circonstance surtout digne de remarque est la disposition des tubercules qui supportent les piquans principaux, et partant le mode d'articulation de ces piquans avec les pièces du test. Les tubercules sont perforés dans toute leur hauteur au centre, et contiennent ici un filament qui en sort et s'attache au milieu de la face inférieure de l'épine. Comme je n'avais que des individus secs, je n'ai malheureusement pas pu constater si ce filament est un ligament ou un muscle. M. Lamarck (1) le prend pour un muscle, sans déter-

(1) Hist. nal. des anim. sans vertèbres, t. III. 1. 53. 
miner les motifs de son opinion. Son assertion que le piquant est également perforé est certainement inexacte, du moins chez le cidarite hystrix, où il est, proportionnellement à son volume, à peine aussicreusé qu'une épine ordinaire $; 6^{\circ}$ les cinq plaques génitales ont toutes le même volume et la même disposition; aucune d'elles ne se distingue par une structure granuleuse. Ce fait est curieux en lui-même, et en outre parce que, réuni ̀̀ lá considération du développement considérable des piquans, il concourt à confirmer l'opinion énoncée plus haut, que l'organe calculifère est un organe excréteur.

$$
\text { S. } 14 .
$$

On trouve des différences plus considérables chez les espèces alongées et aplaties, où l'orifice anal n'est pas opposé à l'ouverture buccale, mais où il est placé plus ou moins près de la circonférence et surtout à une extrémité de l'axe, longitudinal; la bouche occupant au contraire le milieu de la face inférieure, ou bien s'étant portée dans l'axe longitudinal vers un point de la circonférence opposé à l'anus.

On voit très - généralement dans ces espèces que, $1^{\circ}$ leur test n'est pas bien distinctement composé de pièces isolées, grandes et petites, au point que souvent on n'en remarque aucune trace; 


\section{n'ANATOMIF. COMPARÉT.}

$2^{\circ}$. Les ambulacres sont incomplets; ils partent en effet d'un point de la face supérieure et convexe; ce point se trouve silué au milieu de l'axe longitudinal, ou vers la circonférence du tesl, mais il est toujours indiqué par les orifices des ovaires; de là les ambulacres s'étendent. avec des interruptions, jusque vers l'orifice buccal opposé au point de départ.

On rencontre dans ces espèces des différences graduelles, remarquables; ainsi quelquefois, comme par exemple chez les spatangue, les ambulacres n'existent complèternent que sur une partie de la face supérieure du corps, puis manquent dans une étendue plus ou moins considérable de la face inférieure, ensuite reparaissent daus la région de l'ouverture buccale. Mais celte partie inférieure differe de la supérieure $: 1^{\circ}$ en ce que chaque ambulacre n'est formé que de deux rangées d'ouvertures, tandis qu'i la face supérieure il y en a quatre; $2^{\circ}$ en ce que les ouvertures, dans chaque rangée inférieure, sont, ou plus petiles, ou beaucoup plus éloignées les unes des autres, que dans les supérieures; $3^{\circ}$ en ce que les rangées elles-mèmes sont beaucoup plus courtes et en même tems plus étroites à la première qu'à la seconde de ces régions. Il résulte de ces dispositions qu'un ambulacre supérieur, qui tombe dans l'axe longitudinal et se dirige en face de l'orifice buccal, est trés-souvent plus étroit quc les autres, et que II. 
l'étroitesse et la petitesse des orifices qu'il présente, ainsi que la risposition de ces trous on deux rangées, le rendent tout-à-fait semblable à ceux de la face inférieure.

Chez d'autres, comme chez les clypéastres. il n'existe point de traces d'ambulacres inférieurs; mais les supérieurs s'étendent un peu plus loin. et même le cinquième ambulacre, qui cst impair, est aussi complet que les autres.

Ici, il y a encore bien moins de traces d'articulation que dans la disposition précédente. A cette différence il s'en ajoute d'autres très - remarquables dans la structure intérieure, qui s'éloigne beaucoup de celle des spatangues et des autres genres.

En effet, la cavité viscérale des clypéastres est partagée par des cloisons comme pierreuses, qui s'étendent en général de la face supérieure à l'inférieure, mais qui sont interrompues par places. Ces cloisons naissent à l'ouverture buccale, où elles commencent avec la saillie interne dont il a été question dans la description générale; mais elles sont plus déprimées à leur origine que cette saillie. En cet endroit elles sont loin d'atteindre la face supérieure, elles restent au contraire déprimées jusque vers le milieu de la distance quiles sépare de l'ouverture buccale et vers la circonference, mais alors elles se prolongent tout a coup. el arrivent promptement à la face supérieurc. lilles forment intériemrement cinq divisions 
triangulaires, in terrom pues sur plusieurs points, qui correspondent exactement aux ambulacres visibles à la face extérieure, de sorte qu'elles se dirigent le long du bord externe d'une division formée par deux ambulacres.

Dans la premiere modification des oursins aplatis il n'y a pas de trace de cette disposition à la face interne; mais à l'externe on voit à la surface supéricure, un sillon concave en dehors, convexe en dedans, se dirigeant du sommet d'un ambulacre à l'autre, et formant un carré assez régulier. Ce sillon exisie aussi à la face inférieure du test, mais il est ici plus large, plus superficiel et plưs faiblement indiqué.

C'est sans doute à la forme alongée, peut-être aussi à l'absence d'articulation qu'il faut rattacher le nombre moindre d'orifices ovariaux qui n'existent qu'au nombre de quatre chez les spatangues. Ces orifices sont disposés par paires; l'une d'elles, tournéevers l'vuverture anale, est toujour's plus grande que l'autre. Les dimensions en sont quelquefois triples:

$$
\text { \$. } 15 .
$$

Nous avons déjá fait remarquer plus haut que le test des échinides pouvait être ramené au même type que celui des slellérides. L'exposition que nous venons de faire contient non-seulement de nouvelles preuves à l'appui de celle proposition, mais en donne méme une expli- 
cation plus détaillée. Lat division interne des clypéastres est surtout importante.

Les pièces du test, chez les échinides, correspondent aux rayons des stellérides de manière à ce que deux ambulacres placés l'un à côté de l'autre, réunis à la moilié de l'une des grandes pièces qui leur correspondent à chacun, représentent un rayon.

Cela est moins prononcé chez les oursins proprement dits; chez les ciclarites la disposition est déja plus distincte parce que les plaques am. bulacraires sont plus étroites et les ambulacres de la mème pièce plus rapprochés; enfiu, parec que les pièces les plus considérables sont plus larges. Celle disposition est surtout distincte dans les espèces aplaties et principalement dans celles que parıgent des divisions internes, chez lesquelles la périphérie extérieure est plus ou moins concave précisément à l'endroit où deux divisious se touchent à l'intérieur. Partout les orifices des ovaires tombent exactement entre deux moitiés des grandes divisions.

Les petites plaques, ou les plaques ambulicraires des échinides, correspondent incontestablement aux pièces intérieures, ou principales des portions en lesquelles on partage les rayous des astéries; tandis que les grandes divisions des premiers correspondent aux pièces inférieures et aux deux pièces latérales do ces mêmes astéries. Cola résulle aussi bien du vo- 
li A

lunc que de la posilion et de la forme des pieces comparées ensemble daus les différentes espèces.

Cette analogie est confirmée en outre par la circonstance que, chez les échinides dont le squelette est aplati, oblong, les plaques ambulacraires font en dedans une saillie en forme de toit, plus ou moins distincte, souvent extraordinairement prononcée, comme cela a lieu che\% les astéries, pour les pièces principales des diflérentes portions des rayons. On trouve mème quelquefois ici, à la partie interne des ambula. cres, un grand nombre de saillies transversales quileur correspondent, el qui, quoiquen cofondues, laissent néanmoins des lacunes plus ou moins considérables entre elles, et rappellent distinctement les pièces principales des astéries.

3. Holothuries (1).

$$
\text { S. } 16 \text {. }
$$

Le squelelte des holothuries, comparé à celrii des échinodermes considérés jusqu’ici, est extraordinairement dégradé; il a presque entièrement disparu. On ne trouve, en effet, choz ces animaux qu'un anneau mince, très-étroit en proportion de la longueur de l'animal, formé de dix pièces calcaires molles, qu'unit entre elles, d'une manière mobilt, une substance Gibreuse, lâche.

(1) Jees holothuries sont comprises dans les fistulides de 3. de Lamarck.

(Nole des traducteurs:) 
Le sens dans lequel toutes ces pièces sont le plus étroites est de dehors en dedans; celui de leur plus grande largeur est d'un côté à l'autre. Elles ne sont pas beaucoup moins larges d'avant en arrière que de dehors en dedans, et offrent un bord postérieur, cor.cave, creusé d'une seule échancrure, et un antérieur, dentelé, apointi, alternativement concave et convexe. L'ensemqui résulte de ces pièces prend la forme d'une ble couronne peuélevée, ayant ses rayonstournés en devant.

En considérant ces dix pièces de plus près, on s'aperçoit qu'elies forment deux ordres dont les pièces respectives alternent les unes à l'égard des autres.

Les grandes pièces présentent en devant deux dents latérales, entre lesquelles se trouve un enfoncement moyen; les petites pièces ont, au contraire, deux enfoncemens latéraux et une dent moyenne. A la partie externe des deux dents latérales, qui terminent les grandes pièces, s'attachent les deux trousseaux des muscles longitudinaux qui occupent toute la longueur de l'animal; tandis que toutes ces dents envoient de leur face interne de nombreuses fibres tendineuses qui se rendent au commencement du canal intestinal. Après ces pièces, il n'y a pas. d'autre partie dure dans tout le corps. 


$$
\text { S. } 17
$$

Les opinions sont parlagées sur ce que doivent représenter les parties des holothuries qui vienuent d'être décrites.

Bohadsch (I), qui du reste na pas fait de distinction entre les grandes tet les petites pièces, et donne par conséquent l'orifice comme u'étant formé que de cinq portions, les regarde comme des dents.

M. Cuvier, an contraire, qui purte exactement leur nombre à dix, sans cependant indiquer la différence qui subsiste entre ces deux ordres de pièces, se déclare contre l'opinion qui en veut faire des organes masticaleurs : fondé sur ce que, quoique revêtues de la membranc interne de la bouche, elles ne renferment pas de dents, il établit qu'elles servent seulement de points d'attache aux muscles et aux tentacules (2).

1. Tiedemann, en considérant qu'elles servent d'insertion aux muscles longitudinaux, les regarde également comme un rudiment de squelette (5). Sans contredit, l'opinion de MM. Guvier ct Tiedemann est la plus exacte; on voit, en

(1) Reschreibung einiger minder bekannten Secthicre. 5. S1. (Bescription de quelques animaux marins peu connus.)

(2) Leecons al'Anculomic compuréc, vol. III, p. 336.

(3) L., C., 5. 27. 
effet, par la pusition, la direction, lat forme et la connexion de ces parties avec les voisines, qu'elles sont les analogues des pièces antérieures, que nous avous vues se réfléchir au test des oursins.

\section{TROISIĖME SECTION.}

\section{SQUELETTE DES ANNELIDES (I).}

$$
\text { S. } 18 .
$$

Dans les annelides, qui viennent après les échinodermes, l'appareil de la locomotion consiste sou.

(1) M. Savigny admet dans les annelides les particularités suivantes. Il reconnait une téte à celles que l'on appelle NÉrńı́ć Es. Celte portion consisle en un petit renflement antérieur et supérieur, sans articulation mubile. Il élablit que le corps de plusieurs d'entre elles, qui est formé d'une série successive d'anneaux, porte à chaque anneau une paire de pieds. Les mêmes néréldéEs et les serpulées en fournissent des exemples. Les pieds des nćréid ḱes se subdivisent en deux rames, l'une supérieure ou dorsale, l'autre infírieure on ventralc. La dernière est la plus saillante. Les pieds des SERpuléxs se composent aussi de deux parlies, dont l'une, propreà l'action de la nage, répond à la rame dorsale des NÉRĹíćes; et l'autre, beaucoup moins propre à l'action de nager qu'à l'action de s'accrocher et de se fixer, répond à leur lame ventrale. Les deux rames sont presque constamment unies dans les serpulérs; leurs soies servent à les différencier. On distingue dans la rame le cirre et la soie. La première paì'e de pieds, et une, ou deux, ou même trois des suivantes, dans les NéRÉldéEs, manquent entièrement de soies et ne conservent que leurs cirres, qui d'ordinaire acquièrent plus de développement. M. Savigny les nomme 
vent uniquement en parlies musculeuses, molles. De ce nombre sont, par exemple, les espèces dugenredessangsues, etc. Chez d'autres, on peut dire chez la plupart, il existe en outre des parties dures, alongées, purement extérieures, des cirres tentaculaires. La dernière paire de pieds de ces animaux constitue, par une transformation analogue, les styles ou longs filets qui accompagnent l'anus et terminent ordinairement le corps. Ces filets, suivant la remarque de $\mathbf{M}$. Savigny, se présentent dans beaucoup d'insectes apiropodes et hexapodes. Certaines autres parties des pieds serublent parfois privées de cirres; c'est sur les espèces où celte absence a lieu que se manifeste la présence des élytres et écailles dorsales, appendices propres à la seule famille des aphrodites. Le mème auteur ajoute que les élytres on ailes des insectes hexapodes sont attachées au deuxième et a troisième segment du corps, quoiqu'il y ait de petites familles où le premier segment porte deux élytres, tandis que le second en est dẻpourv. Il arrive aussi quelquefois que dans les SERPULÉES la première paire de pieds, et une, ou deux, ou même trois des suivantes, affectent des formes anomales qui ne paraissent pas convenir au mouvement progressif, et qui, jointes au volume des segmens antérieurs, donnent à ces segmens réunis l'aspect d'une tête.

Les cirres sont des filels tubuleux, sub -articulés, communément rétractiles, fort analogues aux antennes. Ce sont les antennes du corps; les cirres des rames dorsales, ou cirres supérirurs, sont constamment plus longs que les cirres inféricurs. Les cirres manquent en tout ou en partie dans les SERPCLÉEs. Lorsqu'ils existent, on n'en trouve qu'un à chaque frifd, c'est ordinairement le cirre supérieur. (Description de l'Erypte, Hisl. nat., t. 1, 3 liv., $2^{c}$ sect. , $3^{\mathrm{e}}$ part., AnELIDEs, Minoires parliculiers, par Jules-Cisar Savigny. ( Note des traducteurs.) 
soies (1), qui ont pour caractère général d'être attachées aux parois latérales du corps par plusieurs faisceaux, et de nètre pas articulćes.

(1) M. Savigny partage les soies en soies it crochets (LNciculi), en soies subulées (SETr subutate), et en soies à paletles (seta spatelluia).

Les soies à crochets sont de petites lames, minces, transparentes, comprimées latéralement, courtes ou peu longues, exactement alignéès, très-serrćes les unes contre les autres, et découpées sous leur sommet en plusieurs dents aiçuës à rrochets qui sont d'autant plus longues qu'elles se rapprochent davantage de la base de la soie. Rarement elles sont à un seul crochet. Ces soies, disposées sur deux rangs, occupent le bord saillant d'un feuillet ou d'un mamelon transverse qui réunit les muscles destinés à les recevoir, et dans l'épaisseur duquel elles peuvent se relirer. Quoiqu'elles occupent généralement la place de la rame ventrale, elles peuvent prendre, soit à tous les pieds, soit seulement sur un certain nombre, la place de lá rame dorsale. Ces soies restent contenues dans l'épaisseur de la peau; elles sont particulières aux SERPuLÉES.

Les soies subulées doivent elles-mêmes être distinguées en soies proprement dites et en acicules.

Les soies proprement dites ( festuce) sont cylindriques, prismatiques ou aplatics, droites ou légèrement courbées, et presqque toujours rétrécies sensiblement de la base au sommet; vers ce sommet quelques-unes ont une pelite dent, et paraissent fourchues : d'autres sont légèrement dilatées et garnies d'aspérités. Il y en a mème qui ont la pointe réfléchie, ou courbée, ou torse, surmontées d'une arête ou d'une petite lame mobile; toutefois la plupart l'ont droite et aiguë. Il est rare que leur intérieur soit fistuleux; presque loules sont solides, fermes et roides; cependant certains genres cir portent qui sont fines et flexibles comme des cheveux. Les soics 
Outre les soies, il y a dans quelques genres, comme, par exemple, dans le genre aphrodite,

sululces traversent les fibres de la peau et pénètrent avec leurs fourreaux dans l'intérieur $d u$ corps où sont fixés les muscỉes destinés à les mouvoir. Elles sont, dans les NérélDÉEs, grêles, nombreuses, rassemblées par rangs complexes, ou par faisceaux qui ont chacnn leur gaine propre, et sortent des côtés ou du sommet de chaque rame. La rame ventrale n'a communément qu'un seul de ces rangs ou de ces faisceaux; la rame dorsale en a souvent deux, et quelquefois davantage. Dans les serpulÉEs on les trouve souvent clistribuées dans plusieurs gaînes qui toutefois se réunissént en un seul faisceau, constamment dépourvu d'acicules. Ce faisceau constitue ordinairement la rame dorsale, et c'est la seule partie du pied à laquelle le nom de rane convienne exactement. Les soies subulées sont sujettes à manquer dans la partie postérieure du corps des serporées. Elles y sont quelquefois remplacées par la troisième sorte de soies, les soies à palettes (Spatellula). C'est une troisième sorte de soies dont le bout est aplati horizontalement et arrondi en spatule.

Les acicules sont des soies plus grosses que les autres, coniques, droites, aiguës, conterlues dans un fourreau dont l'orifice particulier se reconnaît à la saillie. Les acicules se reconnaissent encore par leur couleur brune, noire, ou différente de celle des autres soies auxquelles ils sont associés. Quelques genres des NÉRÉIDÉES en manquent; et quand ils existent on en trouve rarement plus d'un à chaque rame et à chaque faisceau principal. Celuide la rame ventrale est constamment le plus fort; on nele trouve que dans les NÉrÉInÉES.

Il existe dans les échiures (ordre des courbicrnées) deux sries rapprochées et crochues, placées au-devant du corps et répondant a peu près au quatrieme anneau. Les lombrics n'offrent au mème point qu'un renflement des anneaux 
à la face supérieure du corps, des plangres qui semblent correspondre aux arceaux supérieur's des insectes, des arachnides et des crustacés, ainsi qu'aux coquilles des mollusques. Ces plaques ne sont pas en rapport immédiat avec les soies dont il est ici question.

C'est le squelette des aphrodites qui offre la disposition la plus parlaite, propre aux animaux de celle classe; je le choisis, comme exemple. pour l'exposition du développement le plus parfait de ce type, que l'on voil surtout trés-bien dans l'aphrodite aculeata.

\section{S. 19.}

Ici les plaques, an nombre d'environ quinze de chaque côté, sont siluées dans la cavité respiratoire, immédiatement au-dessous du tégumont feutré du dos, auquel elles n'adhèrent nullement. La cavité respiratoire est formée par cette enveloppe et la membrane sous-jacente commune qui entoure les organes digestifs. Les plaques représentent des disques arrondis, minces, mais d'une étendue considérahle, qui se recouvrent réciproquement des deux còtés et d'avant en arrière. Leur face inférieure est appliquée,

numériquement les mîmes. J.es HrunINéEs sont tout-à-fait nues. Voyez la partie d'histoire naturelle de la description de l'Ĺrypte, I. I, et les Mémoires particuliers de M. JulesCésar Savigny.

(Note des traducteurs.) 


$$
\text { D'ANATOMIE COMPARÉE. }
$$

dans sa moitié externe, au moyen d'une bande transversale étroite qui est à égale distance de leur hord antérieur et postérieur, sur l'expansion membraneuse commune qui enveloppe les oryanes digestifs; c'est là leur unique attache.

Dans d'autres espéces, par exemple dans l'aphrorlite squammata, ces écailles sont parfaitement libres.

Elles forment en partie la face supérieure du corps, en partie servent de protection aux organes de la respiration situés sous elles.

Les organes principalement passifs sont des soies ou piquans noirs et roides.

Chez l'aphrodite aculeata, les soies sont disposées dans toute la longueur du corps, trèspres les unes des autres, par environ trentecinq rangées transversales de chaque côté, qui occupent toute la hauteur des faces latérales. Elles diminuent considérablement de nombre, le volume et de dureté, à partir de la partie moyenne du corps vers ses extrémités an térieure et postérieure, de sorte que les postérieures sont surtout très-petites.

Les inférieures se distinguent des supérieures en re qui elles sont placées au sommet d'une éminence conoïde, fort saillante, qui se termine ordinairement par trois petits mamelons. Chacun de ces mamclons supporte quelques soies 'fui se succerlent d'avant en arrière, et forment dela sorte trois rangées situées les unes au-dessus 
des autres, dont la plusinférieure se compose de quatre à cinq soies, et chacune des supérieures de deux, mais qui sont toujours beaucoup plus fortes que les premières.

Les soies supérieures forment une série, et ne sont pas supportées par des tubercules. Elles sont en général plus grandes el plus fortes que les inférieures. Elles sont environ au nombre de quatorze dans la rangée transversale qui occupe la région moyenne. Si on compte seulement seize soies pour chacune des trente-cinq rangées transversales, on obtient pour chaque côté de lanimal, environ six cents de ces soies.

A la moitié inférieure de la portion supérieure de chaque rangée transversale, existent, trèsrapprocliés des soies, des poils réfléchissant l'éclat des couleurs méralliques, phus longs et plus nombreux, mais aussi plus faibles que les soies. Entre ces poils et les soies il se trouve un tissu comme feutré, qui retient les soies à leur partie inférieure, et revèt tout le dos de l'animal; ce feutre ne s'éiend pas jusqu'aux soies pédiculées les plus inférieures.

Les soies inférieures jouissent, soit par l'absence de ce tissu, soit par leur position sur un cône tronqué et saillant, de mouvemens plus libres que les soies supérieures qui, retenues par la substance feutrée, forment, réunies aux poils qui sont très-serrés, une série de rames.

Toutes s'élargissent un peu du sommet à la 
Dinatonie comparé.

base, mais sans se renfler en bulbe. Leur partie inférieure est cependant beaucoup plus molle que le reste; elle offre aussi un éclat doré, landis que toute la longueur de la soie est noire. Lil partie inférieure des différens faisceaux est renfermée dans une gaîne commune, qui est. un repli de la peau extérieure qui sert d'enveloppe aux viscères. Les soies sortent de ce kyste chacune par son ouverture propre, aux bords de laquelle elles sont intimement unies.

Dans les autres genres, les piquans sont en général beaucoup plus courts, et forment des rangées transversales moius composées.

Ils occupent ordinairement toute la longueur du corps; d'autres fois, au contraire, comme chez l'arénicole lombricoüde, ils n'existent que sur ses deux tiers antérieurs.

Cliez ceux des entozoés qui appartiennent à cetle classe, les piquans que les autres genres ont sur les parties latérales du corps, se sont poriés ì l'extrémilé antérieure, où ils forment des couronnes de crochets simples ou multiples, placés au pourtour de l'ouverture buccale. 


\section{QUATRIEME SECTION.}

SQUELETTE DFS INSECTES, DHS ARACINIJES

ET DES CRUSTACÉS. (1)

\section{S. 20.}

Le squelette des insectes, des arachnides et des crustacés, est si bien formé d'après un même

(1) Ce squelette a été étudié dans ses rapports généraux et dans ses détails par M. Victor Audouin. Il a fait connaître le nombre des pièces qui entraient dans sa composition, et après leur avoir imposé des noms, il les a snivies dans chaque classe et dans chaque ordre, à travers les modifications nombreuses de formes et d'usages qn'elles subissent. Le résultat général de l'étude comparative qu'il en a faite est que : $1^{\circ}$ le squelette des animaux articulés est formé d'un nombre détcrminé de pièces distinctes ou soudées intimement entre elles; $2^{\circ}$ que dans plusieurs cas les unes diminuent, disparaissent réellement, tandis que les autres prennent un développement excessif'; $5^{\circ}$ que l'accroissement d'une pice semble exercer sur les picices voisines une sorte d’influence qui explique toutes les lifférences qu'on remarque entre les individus de chaque ordre, de chaque famille, de chaque genre.

Le plus grand état de simplicité dusqueletle se rencontre dans les annelites, dans certains insectes myriapodes (les scolopéndres, lesiules), dans les chenilles, et en général dans toutes les larves d'insectes. Denx deini-segmens unis à chaque extrémité composent chez ces êtres un anneau complet; ces anneaux, dont l'organisation est très-simple, se repètent ainsi depuis la tête jusqu’a l'extrémité du corps, on en d'antres termes, ils sont tous sensiblement semblables 
Iype, que si le reste de l'organisation de ces anientre eux. Dans d'autre cas, l'arceau se fracture; il s'isolede nouvelles parties plus ou moins distinctes. C'est au thorax des espèces qui sont à la fois pourvues de pieds et d'ailes, que la plus grande complication se manifeste. Tel est le thorax de la plupart des insectes hexapodes. M. Audouin reconnait trois anneaux au thorax, le premier ou le plus voisin de la tête est le prothorax, le second le mésothorax, et le troisième le métathorax. Chacun de ces anneaux est essentiellement composé des mêmes pic̀ces; nous allons les étudier dans le mésothorax. Cet anneau porte la première paire d'ailes et la seconde paire de pattes; il peut être partagé en quatre régions : une inférieure, deux latérales et une supérieure. Celle-ci constitue le dos, et les trois autres concourent à formec la poitrine.

Ũne seule pièce occupe la région inférieure, c'est le sternum; les régions latérales, ou les flancs, se divisent en trois pièces principales, qui sont l'épisternum, l'épimère et le paraptère. La région supérieure, qui a reçu le nom de tergum, est composée de quatre pièces nommées prcescutum, sculum, scutellum, post-scutellum.

Le sternum est une pièce, commune à tous les insectes, plus ou moins développée, souvent distincte, souvent aussi intimement soudée aux pièces voisines avec lesquelles elle se. confond.

Des trois pièces ordinairement latérales qui forment les flancs, la première, qui estl'épisternum, s'appnie sur le slernum et s'étend jusqu'a la partie supérieure; la seconde, ou l'épimére, situće en arrière de la précćdente, se soude avec elle, remonic aussi jusqu'i la partie supcérieure, et repose dans certains cas sur le sicrum. Mais ce qui lin caractérise essentiellement, c'est que toujours elle est cn rapport avec la hanche et forme quelguefois la circonférence du trou qui la reroit; clle s'articule avec elle an moyen d'une petite pièce appelée trochantin. Lat troisième pièce concourant à former

II. 


\section{maux n'établissait pas entre eux des différences}

les flancs est le paraptère; c'est une pièce fort petite, rarement visible, en contact avec l'épisternum et avec l'aile; elle est toujours appuyće sur l'épisternum, quelquefois prolongée le long de son bord antérieur; d'autres fois elle est libre, passe au-ilevant de l'aile, et mếne accidentellement se trouve placée au dessus.

De la jonction du sternum et des flancs résulte la poitrine. Fin faisant choix de certains insectes tels que le dytique, le hanneton et la plnpart des coléoptères, on reconnaîtra facilement dans le mésothorax les pièces qui viennent d'ètre signalées comme formant la poitrine.

Dans l'intérieur da thorax, la face supérieure du sternum donne naissance à une pièce que $\mathbf{M}$. Audouin nomme entothorax. C'est la pièce en $\mathbf{Y}$ de M. Cuvier; il sert à protéger le cordon mélullaire et donne insertion à des muscles.

L'épisternum à son bord antéricur, quelquefois le sternum, et même la partie supérieure du corps, présentent une ouverture stigmatique entourée d'une petite pièce souvent cornéc; cette pièce est le péritrème. On la retrouve ordinairement au pourtour de tous les stigmates.

Des quatre pièces qui composent le tergum, la plus antérieure, le prescutum, et la dernière, le post-scutellum, sont presque toujours cachées dans l'intérieur du thorax; le sculellum, ou la troisième pièce, connu sous le nom d'écusson, a seul été décrit à cause de sa grandeur. On n'a jamais rien dit du sculum ou de la seconde pièce.

Le prosculum est quelquefois assez grand; le scutum sarticule avec les ailes, il est souvent très-développée. Le post-scutellum est quelquefois libre, quelquefois confondu avec le scutellum. On relrouvera facilement toutes ces pieces du dos en etudiant le mésothorax d'un papillon quelconque.

Le prothorax et le métathorax sont formés des mêmes pières que le inésothorax.

On trouve dans l'intérieur du thorax des sortes de lames 
cornées qui naissent toujours le long de la soudure de deux pièces entre elles, ou des deux portions paires de la même pièce réunies sur la ligne moyenne; M. Audouin les nomrne APODE்uES : apodèmes d'insertion celles qui donnent attache à des muscles; apodèmes articulaires celles qui s'observent au sommet des pièces de la soudure desquelles elles résultent, et qui servent ordinairement a l'articulation des ailes. Il existe encore d'autres pelites pièces au dedans du thorax qui ont quelque analogie avec les apodèmes d'insertion, avec la différence qu'elles ne naissent pas de la soudure de deux pièces et sont autant de parties indépendantes et libres, ce sont des Éprdènes. Ces petites lames mobiles donnent tantôt insertion aux muscles et représentent alors autant de petits tendons: elles ont reçu dans ce cas le nom d'épidémes d'insertion; tantôt elles existent à la base des ailes et représentent autant de petits osselets qui facilitent leurs mouvemens : on les nomme alors épidèmes d'articulation.

Tel est le summum de complication d'un anneau du thorax. La simplicité des autres anneaux du corps s'opère par la réduction et la sondure de ces pièces. Toules les différences entre les portions diverses du corps du même individu, et entre des animaux d'ordres différens, résultent de modifications survenues par suite de l'absence ou de la présence de ces parties, et des changemens opérés dans leur forme ou dans leur développement; changemens qui en en trainent dans les usages. Un anneau de la téte ne diffère par conséquent d'un anneau du thorax que par la soudure de ses pièces; on y retrouve des pièces analogues. L'entothorax lui-mème existe distinctement, M. Audouin l'a nommé alors enlocéphale. Enfin on observe dans les articulations des màchoires une disposition analogue à celle que l'on rencontre darisl'articulation des pieds avec les segmens du thorax.

I.es anneaux de l'abdomen présentent, beaucoup de sim. plicité; ils sont distincts entre eux et généralement composés 
des sous-ordres d'une seule et mème classe (1). Leur squelette est en même temips beaucoup plus parfait que le squelette des vers et des mollusques, sans que cela soit une raison de placer les animaux qui nous occupent en ce moments au-dessus des mollusques. Leur squelette est en effet surtout un squelette extérieur qui disparaît insensiblement dans les mollusques, et qui est

de deux arceaux qu'aucune pièce ne subdivise, parce qu'il n'y a plus d'appendices aussi développés que les aîles et les pattes qui s'y insèrent. Les mots thorax, téte, abdomen, seront définis plus loin, selon le sens rigoureux qu'y attache M. Audouin. Nous engageons nos lectenrs à consulter, pour de plus amples détails, le beau rapport que MI. Cuvier a fait à l'académie des sciences dans la séance du 19 février 1821 , et le travail de l'auteur inséré dans les Annales des sciences naturelles, recueil très-intéressant rédigé par MIM. Audouin, Brongniart et Dumas.

(Note des traducteurs.)

(1) Il est bien vrai de dire que le squelctte des animaux articulés est formé sur un mème plan; mais il présente dans chaque classe des différences qu'on ne saurait méconnaître, et qui ont été très-bien indiqquées par M. Audouin.

Dans tous les insectes (les myriapodes exceptés) trois anneaux du corps sont dévcloppés outre mesure et constituent le thorax. Dans les arac'inides, ce thorax est formé par quatre anneaux. Dans la plupart des crustacés, on cn compte cincq sur lesquels l'accroissement s'est opréré. Beaucoup d'autres différences se voient encore dans la structure de la têtc, dans les proportions de l'abdomen, et surtout dans le développement relatif des diverses pièces qui constituent le thorax. (Voye la note qui précérle ci celles insérées ainx pages suivantes sur les crustacís et sur les arachnitles.

(rote des traducteurso) 


\section{V'ANTOMIE COMPARÉ.}

remplacé par un système osseux indépendant, séparé du squelette extérieur.

$$
\text { S. } 21 \text {. }
$$

Chez tous les.animaux que nous allons examiner, les parties dures sont ordinairement beaucoup plus nombreuses, plus grandes et plus compliquées que chez les vers; elles enveloppent très-généralement le reste du corps; la peau de ces ètres s'endurcit et forme un test composé de plusieurs portions; disposition dont on trouve déjà un indice dans les écailles dorsales des aphrodites. Quelquefois ses parties dures ne se bornent pas à constituer une enveloppe extérieure, elles se continuent aussi en dedans, entre les organes entourés par la portion dure extérieure, et forment un second squelette intérieur, plus ou moins parfait ( $I$ ).

$$
\text { S. } 22 \text {. }
$$

De mème que tout le corps de ces animaux est composé de plusieurs portions arrondies ou

(1) Ce squelette intérieur dont parle M. Meckel est essentiellement formé par les cntothorax et par les prolongemens nommés apodémes, qui naissent sur les lignes de soudare des différentes picces du squelette extérieure entre elles. (Voyer la note de la page 64.)

(Note des traducteurs.) 
segmens qui se succèdent d'avant en arrière, de même le squelette se compose de plusieurs anneaux. Ceux-ci ou sont fermés complètement, à l'exception de leurs extrémilés antérieure et postérieure, ou bien offrent une moilié supérieure et une inférieure unies entre elles par la peau non durcie. Cette peau restée molle unit entre eux les anneaux qui se succèdent d'avant en arrière.

La portion la plus antérieure de ces anneaux; ou la tête, est communément fermée complètement, si on en excepte son ouverture antérieure ou buccale, et la postérieure ou l'ouverture qui communique avec le tronc: on peut dire la même chose de la seconde série d'anneaux chez la plupart desinsectes; mais ces anneaux, chezles crustacés, sont en général composés de deux arceaux séparés; tandis que la troisième série d'anneaux qui constituent la queue, est formée en général d'anneaux qui sont complets chez les derniers, et n'offrent aucontraire qu'une moitié supérieure et une inférieure chez les insectes. Chez les arachnides, du moins chez les scorpions, tous les anneaux antérieurs se partagent en un arceau supérieur et en un inférieur; les anneaux postérieurs ou de la queue sont au contraire complets, et le dernier anneau abdominal offre la première disposition dans sa partie antérieure, qui est la plus grande, et la seconde daus sa partie postérieure. 


$$
\text { S. } 25 .
$$

A ces anneaux s'ajoutent très-généralement des parties alongées, plus ou moins articulées, et différentes, en outre, sous le rapport du degré et du mode de composition; ces parties sont creuses comme tout le squelette, disposées par paires à la face inférieure du coŕps; elles s'étendent dans la presque totalité de sa longueur, comme plusieurs insectes et la plupart des crustacés en offrent des exemples. 11 résulte de celle disposition que chaque anneau en porte une ou deux paires; mais le plus ordinairement ce.pendant ces parties sont bornées aux portions antérieures du corps, comme l'exemple en est fourni par la plupart des insectes et des arachnides. Toutes sont des organes de locomotion, et quelles que soient leurs différences, le plus souvent elles sont essentiellement frormées d'aprèsun type commun. Celles de ces parties quise trouvent à la tèle sont ou des organes des sens, ou des instrumens de manducation; celles de la poitrine sont des pieds. Le passage des pieds aux organes de manducation et réciproquement, est si insensible, surtout chez les crustacés, qu'il est difficile d'étallir une différence précise entre ces organes, et que souvent les mâchoires d'une espéce ont la plus grande analogie avec les pieds de l'autre. (1)

(1) Si l'on s'en était tenu à comparer les formes el les usa- 


\section{$\$ 24$.}

Les subdivisions des pieds ou pattes, qui sont les seules de ces parties que je considérerai ici, sont: $x^{0}$ la hanche; $2^{\circ}$ la cuisse; $5^{\circ}$ la jambe; $4^{\circ}$ le pied proprement dit ou le tarse. Ce dernier est composé lui-mème de plusieurs parties successives, propres, quoique petites.

La hanche est placée le plus souvent verticalement en travers, et est ordinairement ouverte dans une étendue plus considérable à son extrémité supérieure qu'à son inférieure. Elle est une des portions les moins longues, souvent la plus courte de tout le pied; elle est sitnéc dans une ouverture de la pièce thoracique (I), et se meut

ges pour établir l'analogie des parties, on ne fút pas arrivé à celte conséquence. C'est ici que la loi des connexions est. presque seule applicable. A l'aide de ce principe, M. Savigny a établi la ressemblance dont il est question; en employant ce même guide, M. Audouin a pu compter les anneaux qui composent la tête des insectes. Par l'étude de la bouche d'un iule, on voit que la première patte est déjà refoulée vers le menton. Dans la scolopendre, les deux premières paires de pattes font partie de la bouche, etc.

(Note des traducteurs.)

(1) C'est surrout avec l'épimère que la hanche s'articule, et 1 articulation a lieu au moyen d'une très-petite pièce découverte par M. Audouin et qu'il a nummé trochantin. (F oyez la nole de la page 64 .)

( Note des traducteurse) 
D'AYATOML COMPARÉE.

davant en arriere. Son ouverture supérieure est simple et droite, horizontale; l'inférieure, ordinairement située un peu plus en dedans, présente la plupart du temps une saillie antèrieure et une postérieure, plus ou moins développées par lesquelles elle embrasse l'extrémité interne de la cuisse.

La cuisse est̂ communément plus longue et plus mince. Sun extrémité interne est arrondie, et offre, à sa face antérieure et postérieure, un enfoncement dans lequel les saillies de la hanche s'engrènent exactement, de manière à rendre impossible le mouvement en devant et en arrière, en sorte que le monvement est borné à lia flexion et à l'extension. En haut et en dehors, sn devant et en dedans, on voit au-dessus de cet enfoncement une petite saillie qui consolide encore davantage l'articulation.

L'ouverture externe est composée, du moins souvent, de deux parties unies entre elles sous un angle droit, dont l'externe affecte une position verticale, l'inférieure étant disposée horizontalernent.

La jambe est également longue. L'extrémité supérieure en est arrondie en tête, qui s'enfonce dans l'extrémité externe de la cuisse; il y a souvent ì la base de la tête, en avant et en arriere, une petite saillie qui concourt à cousolider l'articulation. Lu bas on trouve, 
chez les coléoptères, une petite lame propre, isolće, qui s'engage dans la portion verticale de l'ouverture externe de la jambe. Il n'existe donc également ici que des mouvemens de flexion et d'extension.

L'extrémité inférieure a une ouverture arrondie, simple, ordinairement entourée de saillies en devant et en arrière qui horuent considérablement tous les mouvemens autres que la flexion et l'extension.

Le tarse, communément plus mince que les autres portions de l'appendice locomoteur, est formé de plusieursarticles qui se dilatent plusou moins à l'une ou à l'autre de leurs extrémités, mais principalement à l'extrémité inférieure ou externe. Ils ont à cette dernière une saillie antérieure et une postérieure, ce qui fait de l'articulation un ginglyme. Le dernier article est ordinairement plus consistant que les autres, et forme un ou plusieurs crochets juxtaposés d'avant en arrière, qui dans le dernier cas peuvent être rapprochés et éloignés les uns des autres, et sont en outre capables d'exécuter des mouvemens de flexion et d'extension.

A ces organes de locomotion, situés à la face inférieure du corps, s'ajoutent ehez plusieurs insectes, et de tous les animaux articulés seulement chez ceux-ci, des ailes disposées également par paires, mais à la face supérieure du corps. Nous considérerons ces parties plus loin, 
puisqu'on ne les rencontre que chez les insectes parfaits (1).

$$
S .25 .
$$

Le squelette des animaux articulés se conpose, à des degrés variables, de plusieurs couches superposées qui sont les différentes couches de la pcau; c'est par cette raison que nous en parlerons seulement, lorsque nous traiterons de cet appareil.

Le tissu en est ordinairement homogène, sans disposition fibreuse distincte.

Il est fréquemment garni, sur des points trèsvariables, de prolongemens qui ressemblent à des poils et des plumes.

La consistance en augmente avec l'âge. Le squelette extérieur offre souvent des couleurs très - variées, tandis que l'intérieur est unicolore. (2)

(I) On a réuni, sous le nom générique d'appendices, les mâchoires, les mandibules, les antennes, les pattes, les ailes, les filets qui terminent l'abdomen, etc. Toutes ces parties ont cela de commun qu'elles sont accessoires aux anneaux dont la succession forme, à partir de l'ouverture buccale, lout l'animal. Les travaux de MM. Savigny, et Audouin prouvent combien cette pensée est philosophique.

(Note des traducteurs.)

(2) Nons avons dit que M. Meckel donnait le nom de squelette intérieur à toutes les saillies ou prolorgemens que l'on r)bserve dans l'intérieur du corps. Parmi ces sortes d'apophyses, les plus importantes sont les entothorax; les autres 


\section{$\$ 26$.}

La composition chimique des parties solides et dures, n'est pas la mème chez tous les animaux qui nous occupent en ce moment.

Le test des insectes et des arachnides, du moins des scorpions, est entièrement formé d'albumine concrétée, suivant les expériences de Hatchett ( $\mathrm{r}$ ).

La substance qui donne au test des crustacés sa solidité est du carbonate et du phosphate de chaux. Le premier de ces sels l'emporte de beaucoup en quantité sur le second.

$$
\text { S. } 27
$$

Quoique le squelette de plusieurs insectes

consistent en des espèces de lames ou d'épines qui naissent de la sondure des différentes pièces entre elles, et anxquelles M. Audouin a donné le nom général d'apodèmes. (Voyez la note de la page 64.)

(Note des traducteurs.)

(1) Expér. chimiques sur les zoophytes, avec quelques remarques sur les principes constituans des membranes, etc. Voy. Philos. transactions. - Mérat-Guillot, Annal. de chimie, t. XXXIV, p. 7. - Chevreul, dans le Mémoire de Geoffroy sur le thorax des animaux articulés en général et des insectes en prarticulier, inséré dans les Annales genérales des sciences physiques, t. VII, [. 1. 
soit le plus complet par le développement et le nombre des pièces qui le composent, c'est néanmoins dans cetle classe que l'on trouve les formes les plus imparfaites; on peut donc, mème sous le rapport du squelette, considérer les trois classes des insectes, des arachnides et des crustacés, dans le même ordre que leur assigne d'ailleurs le reste de leur organisation.

\section{SQUELETTE DES INSECTES.}

A. Conditions générales.

$$
\text { S. } 2 S \text {. }
$$

Le squeletle le plus simple d'un insectc nous est offert par ceu: qui ne subissent pas de métamorphose. Ia structure de celui des autres est plus compliquée sous tous les autres rapports, principalement celui des insectes ailés, dont le corps est loin d'ètre formé d'un aussi grand nombre de segmens, mais se compose, en revanche, de parties très-dissimilaires, plus compliquées et plus parfaites, en sorte que l'on peut admettre que les matériaux qui ont été employés, chez les premiers, à former des parties semblables, se repétant un certain nombre de fois, ont servi à produire des parties plus variées chez les seconds. 


$$
\text { \&. } 29
$$

Voici à peu près les conditions les plus générales de la disposition de ce squelette:

$1^{\circ}$. Tout le squelette de la plupart des insectes, principalement de ceux à mélamorphoses, se partage, comme le corps dont il détermine la forme extérieure, en deux divisions principales: le tronc et la tête, dont chacune peut être subdivisée en deux portions, le tronc en thorax et abdoinen, la téte en crâne et face.

$2^{\circ}$. Le thorax ( 1 ) ou le corselet est le siége des organes de la locomotion. Les pieds sont disposés à sa face inférieure, tandis que les ailes en occupent le plus souvent les faces supérieure et latérales.

3". Le thorax est composé de plusieurs segmens, notamment de trois, qui se succédent d'avant en arrière en forme d'anneaux, ouverts à leurs extrémités antérieure et postérieure, et habituellement fermés dans le reste de leur étendue, à l'exception de quelques lacunes destinées à l'insertion des pieds.

(1) Le mot thorax a été substitué par M. Latreille à la dénomination vague de tronc, par laquelle on entendait aussi toutes les parties comprises entre la tètre et l'abłomen. Ia capacité du prothorax a reçu de $\mathbf{H}$. Audonin le nom de tron pharyngien, celui du mésothorax de trou cesophagien, celui du métathorax de trou stomacal. (Note des traducteurs. 
1)e ces trois sections successives, la première, le prothorax, est communément la mieux séparée des autres (du mésothorax et du métathorax) ( 1 ) et de la tète; les deux derrières forment plus constamment ensemble un anneau unique, mais beaucoup plus grand.

$4^{\circ}$. De la partie inférieure de la surface intérieure de ces portions du thorax, on voit naitre, le plus souvent sur la ligne médiane, des apophyses simples, ou paires, qui sont disposées symétriquement à égale distance de celte ligne, et dirigees d'ordinaire plus ou moins en dehors, où elles se terminent, tantòt en restant libres, tantôt en s'articulant avec d'autres apophyses qui leur correspondent, et qui sont situées à la face interne des parois latérales. Ces prolongemens servent surtout d'attaches aux muscles des pieds et des ailes, et forment en outre des moyens protecteurs destinés à abriter le cordon nerveux central. Leur nombre, déterminé par celui des annearix et des pieds, est toujours de trois paires, qui se suivent d'avant en arricre. Les apophyses internes peuvent ètre dé-

(1) Ces distinctions ont été établies d'une manière très-rigoureuse par M. Audouin, et c'est sur elles que repose toute étude philosophique du thorax. Quand on veut prendre une idée précise de cette partie du squelette, il faut nécessaireincont étudier successivement chacun des anneaux, sans quoi il régnerait une très-grande confusion dans la descriplion rgu'on en ferait, et leur étude comparative serait impossible. J'observation montre qu'ils sont, quant à leur compo- 
signées, à cause de leur forme, sous le nom de branches furculaires (furcula, rami furculares). les externes sous celui d'apophyses latérules (processus laterales.) ( 1 ).

Toutes font partie du squelctte intírieur des insectes.

$5^{\circ}$. La portion antérieure du thorax, la plus petite, supporte les pieds de devant; la postérieure, beaucoup plus grande, supporte les deux paires de pieds postérieures et les ailes (\%).

Liées au développement d'ordinaire plus considérable des paires le pieds postérieures et à la présence des ailes, les apophyses internes ou branches furculaires de la portion posterieure du thorax sont communément beaucoup plus développées que celles de la portion antérieure.

On a proposé de donner le nom d'entoster-

sition, une répétition les uns des autres : seulement les pièces sont plus ou moins distinctes, plus ou moins développées. De là le choix que l'on doit faire de tel anneau de préférence à tel autre pour en étudier les parties conshituantes.

\section{(Note des traducteurs.)}

(1) Ces pièces sont les unes des cntoihorax et les autres des apodèmes.

(Note des traducteurs.)

(2) Cette portion postérieure n'csl pas formée d'un seul segment; elle est composée du mésothorax et du métathorax, chacun porte une paire de paltes et une paire d'ailes dans les insectes à quatre ailes. Tous le répétons, l'étude générale du thorax est impraticable si on 11 a pas toujours présente à l'esprit sa division cn trois anmeaux.

(Note des traducteurs.) 
num à la paire postérieure des apophyses furculaires, à cause de leurs branches considérables ( $I$ ).

M. Audouin appelle entothorax toutes les partıes moyennes inférieures, et apodèmes les apophyses latérales (2).

6. Les conditions les plus générales qu'offrent les pieds ont été indiquées plus haut.

$7^{\circ}$. Les caractères généraux des ailes, mais qui sont sujets à un nombre variable d'exceptions, sont : une attache mobile à la face supérieure du corps, aux limites de celle-ci et de la face latérale; leur division en deux paires. l'une antérieure, l'autre postérieure, entièrement séparées l'une de l'autre; une forme alongée; un aplatissement considérable du haut en bas, de façon qu'elles forment des lames minces

(2) Chabrier, sur le vol des insectes. Journal de phrsique, t. XCI, p. r99.

(2) M. Meckel ne paraît pas avoir connules recherches importantes de M. Audouinsur le squelette des insectes; il semble avoir pris ces indications dans l'ouvrage de M. Chabrier, qui s'est mépris souvent sur la synonymie, et dont le travail n'é tant pas comparatif ne peut être d'aucun secours pour l'étude de l'anatomie générale. L'entothorax de M. Audouin est cette piece trés-reconnaissable ordinairement en forme d'Y, que l'on trouve a l'intéricur de chaque anneau du thorax, et qui nait au-dessus du sternum; les apodemes sont des pièces accessoires fort différentes. (Voyez les notes précédentes et les Annales des sciences naturelles, tome I er et suivans.)

( Note des traducteurs.)

II. 
plus ou moins triangulaires; une composition consistant en deux substances, l'une mince, molle et membraneuse (I), et l'autre plusépaisse, solide et dure. C'est la première de ces substances qui prédomine très-généralement; elle consiste en plusieurs pièces creuses, mobilement unies entre elles : celle qui occupe la base se compose de pièces plus petites, arrondies, tandis que les autres pièces, répandues sur le reste de l'aile, sont formées de rayons minces, mais très-alon. gés, et l'emportant en masse et en numbre sur la première. Les plus longs et les plus forts de ces rayons sont situés le long de l'aile, le plus souvent à son bord antérieur; les plus courts et les plus faibles se dirigent de la base vers le bord postérieur, qui n'est pas, comme l'antérieur, renforcé par des stries cornées, mais qui n'est que membraneux (2). L'existence de ces deux

(1) La substance membraneuse est elle-même, suivant M. Jurine et M. $\Lambda$ udouin, constamment composée de deux feriillets superposés; ces feuillets constituent à eux seuls, dans quelques cas rares, l'aile tout entière: le plus souvent ils occupent des intervalles limités par des lignes ou nervures formées de substance dure. (Note des traducteurs.)

(2) Ces lignes de substance cornce sont contenues entre les deux feuillets membraneux et présentent deux faces; la supérieure, qui est très-cornée et souvent arrondic, adhère intimement a la membrane. Leur face inféricure, plus plane, d'une consistance moindre, peut en ètre isolée plus facilement. Ces filets sont autant de tubes dont la coupe transversale est ovalaire. (Voyez Jurine.) (Note des traducteurs.) 
substances différentes permet très-généralement à l'aile de se plisser, à l'état de repos, en long et mème en travers, et de s'épanouir dans l'exercice de la locomotion ( 1 ).

(1) On a distingué à l'aile-une base et un sommet, plusieurs bords et plusieurs angles, dénommés d'après leur rapport avec la ligne médiane du corps de l'insecte; les nervures ont été également désignées, suivant ce qu'elles rappelaient d'analogue avec la disposition des animaux supérieurs, nervure radiale, nervures cubitale et brachiale. Les espaces membraneux ont été appelés cellules : cellules radiales, cellules cubilales, suivant leur rapport de position. Des osselets existent à la base de l'aile; M. Andouin les a tous compris sous le nom général d'épidèmes articulaires. M. Jurine a dénommé ainsi ceux qui entourent l'articulation de l'aile antérieure avec l'arceau supérieur du mésothorax : petit radial, grand radial, petit cubital, grand cubital, naviculaire, petit huméral, grand huméral; il en a trouvé cinq a l'articulation de l'aile postérieure a vec le métathorax, et leur a imposéles noms d'ćchancré, de scutellaire, de diadémal, de fourchu, de massue; ces pièces du métathorax, dont deux ont disparu, sont la répérition, suivant M. Audouin, de celles du mésothorax. L'analogic des ailes et des pattes proposée déjà par M. de Latreille a été confirmée et élablie sur des raisonnemens très-so!ides par M. Audouin. Les considérations sur lesquelles il s'appuie sont les suivantes : elles découlent de la manière dont il envisage un anneau du corps qu'il considère formé de deux demi-arceaux joints le plus souvent par leurs extrémités. Ces demi-arceaux sont composés de la même manière, le supérieur, du tergum et de deux épisternum, un de chaque côté; l'inférieur, du sternum sur la ligne moyenne et de deux épimères; les ailes s'articulent avec le tergum et avec les deux autres pièces de l'arceau supérieur, comme les pattes s'articu. lent avec le sternum et les deux pièces de l'arcean inféricur.

LI.

6. 


\section{\$. 3o.}

L'abdomen (1) est plus grand, mais moins parfait que le thorax. Il n'offre ni pieds ni ailes chez la plupart des insectes, et n'est par conséquent pas en rapport avec la locomotion; sa principale destination est d'envelopper les organes de la digestion et de la génération. Il se compose de pièces supérieures qui, le plus com-

Ces appendices deviennent tellement comparables parla forme, qu'il est des animaux, les blattes, par exemple, où les filets terminaux de l'abdomen, les uns supérieurs ou représentant les ailes, les autres inférieurs ou analogues aux pattes, ne sauraient être distingués que par leur position. De mème que les appendices inférieurs se modifient dans les diverses régions du corps, à la tête, au thorax et à l'abdomen; de mème les supérieurs affectent des variétés de formes très-nombreuses. Ainsi ce sont des antennes à la tête, des ailes modifiées en élytres, en balancier, en cuilleron, au thorax; des filets à l'abdomen, qui, en se réunissant, constituent l'étui de la tarière ou de l'aiguillon. Les ailes n'ont donc pas une existence plus anomale que les pattes. (Voyez Audouin, Dictionnaire classique d'histoire naturelle, t. I, art. Aile.)

\section{( Nole des traducteurs.)}

(1) L'abdomen, suivant M. Audouin, est cette partie du corps faisant suite au thorax, composée d'un nombre quelconque d'anneaux constamment dépourvus d'appendices articulés, essentiellement locomoteurs, n'en présentant tout au plus que des vestiges. Ces anneaux sont des cylindres creux, fort courts, rentrant, dans certains cas, les uns dans les autres, comme les tubes d'une lunelte, tantôt constitués par deux demi-cylindres qui s'abouchent ordinairement pât les deux bords de leurs sections. Les arceaux inférieurs pré- 
munément, sont minces, ou quelquefois consistent en peau molle, et d'inférieures plus solides et plus dures, qui sont unies mobilement entre elles par une bande plus ou moins large de peau. Ces arceaux supérieurs et inférieurs of freut rarement des apophysès analogues à celles toujours distinctes du thorax; quelquefois cependant il y en a aux demi-anneaux inférieurs, mais elles sont peu prononcées et à peine saillantes.

$$
\text { S. } 31
$$

La partie postérieure de la tête, ou le crâne, est arrondie; mais elle ne l'est pas également dans tous les insectes; cette portion est en général plus petite que la partie antérieure, qui est principalement composée de la réunion des organes de la mastication. Le crâne porte plusieurs organes des sens, surlout les yeux et les antennes; il renferme dans son intériecir les ganglions antérieurs du cordon nerveux central, les muscles des organes masticateurs, et le commencement de l'œ. sophage. Il est ouvert en avant et en arrière, forme un anneau complet, quoique constitué par

sentent intérieurement, dans quelques insectes, des éminences qui ne sont autres que les analogues des entocéphales et des entothorax; M. Audouin les nomme entogastres. Le nombre d'anneaux qui forment l'abdomen dans chaque ordre, dans chaqque famille, etc., ses usages et ses rapports avec les organes qu'il constitue, son mode d'insertion, varient beaucoup. (Voyer. l'article abdomen du Dictionnaire classique d'histoire naturclle.)

(Note des traducleurs.) 
plusieurs lames, et s'articule mobilement avec le premier anneau du thorax (1).

De sa face inférieure et interne partent le plus souvent d'avant en arrière, deux apophyses (pro cessus), une de chaque côté, qui se dirigent en haut. Tantôt ces deux pièces ne s'atteignent pas; tantôt elles s'unissent entre elles et partagent la cavité crânienne en une moitié supérieure, ordinairement plus ample, et en une moitié infé. rieure plus petite (2). L'oesophage se trouve constamment dans la moilié supérieure (3).

$$
\text { S. } 32 .
$$

Nous allons faire conmaître mintenant les particularités qu'offre le squelette des insectes, dans les differens ordres de cette classe.

1. INSECTES A MÉtTaMORPHOSES.

A. Diptères.

$$
\$ .33 .
$$

Chez les diptères, les trois anneaux antérieurs

(1) La tête est forméc de plusicurs anneanx soudés enscmble. M. Audouin en a déterminé le nombre en étudiant celui des appendices; mais il parait que son travail sur cette partie du squelette est encore inédit. Nous n'en parlons que d'après les leçons que ce jeune professeur donne chaque année au jardin du Roi, en remplacement de M. de Lamarck.

(Note des traducteurs.)

(2) Cette pièce n'est autre chose que l'entocéphale décrit par M. Audouin.

(Note des traducteurs.)

(3) L'orifice antérienr de la tête a été désigné sous le num de buccal; le postérieur sous celui d'occipital. (N.destr.) 
1) A АTOMH: COMPARÉE.

du tronc, ceux du thorax, sont confondus en une seule pièce; les appuis intérieurs des mus. cles sont petits, minces et fribles (1).

Les différentes portions qui constituent les pieds des diptères sont longues et minces, à l'exception des hanches qui sont courtes, libres, et font une saillie verticale à la face inférieure du corps.

A la face inférieure d'un ou de plusieurs ar-ticles du rarse, dans le premièr cas à celle du dernier, existent des lames conformées comme une cuiller, creuses inférieurement, quelquefois clentelées à leur pourtour, qui peuvent ètre mues volontairement, et servent à l'animal à sattacher aux corps; il se soutient au moyen des ventouses qu'elles forment, surtout lorsqu'il marche contre la gravité de son propre corps, la tête en bas et les pieds en haut ${ }^{2}$ :

Les diptères n'ont que les ailes antérieures.

Elles sont alongćes, minces, présentant beaucoup de nervures dans le seris de leur direction longitudinale; mais ces nervures sont faibles.

(1) Malgré cette confusion des pièces lu thorax, on peut tris-bien reconnaitre les trois anneaux qui le forment, et surtout étudier le mésothorax, dont la partie supérieure est trés-développée, ct dont les pièces sont assez distinctes.

(Nole des traducteurs.)

(2) E. Home, Farther observations on the feet of animals, whose progressive motion can be carried on against gravily. Philos. trans., 1816, p. 523. 
La place des ailes inférieures est occupée par un petit disque, simple, plus consistant, que l'on nomme le cuilleron, et qui est situé au-dessous et derrière les ailes. Plus bas et plus en arrière existe une petite pièce alongée, claviforme. c'està-dire renflée vers son extrémité libre; c'est le balancier, qui appartient sans doute aussi à la paire des ailes postérieures.

Les anneaux abdominaux sont peu mobiles les uns sur les autres, davant en arrière; dans plusieurs espèces les supérieurs et les inférieurs sont soudés ensemble.

Je n'ai pas pu découvrir de division en moitié supérieure et en moitié inférieure, ni dans l'intérieur cie la tête qui est arrondie, ni dans le trou occipital.

Le corps des laryes des diptères se compose d'anneaux très-mous, moins distinctement séparés; l'extrémité postérieure en est mousse, l'antérieure pointue.

On rencontre surtout à son extrémité postérieure une couronve de soies mousses.

Je n'ai pas pu trouver de trace de squelette intérieur chez ces animaux.

B. Hémiptères.

$$
\text { \$. } 34 \text {. }
$$

Parmi les homoptères ou hémiptères à ailes égales, du moins dans le genre tettigone (tetti- 
gonia), l'anneau thoracique antérieur ou le prothorax est complet, très-large à sa face supérieure, extrèmement étroit à l'inférieure, surtout vers sa partie moyenne. Il offre inférieurement de chaque côté l'ouverture destinée à recevoir la hanche, qui est ici complètement fermée. Non loin de la ligne médiane, il naît de chaque côté de la paroi inférieure un crochet court, déprimé, fortement dirigé en-dehors. Un autre semblable, beaucoup plus long, naît un peu plus en devant de la paroi latérale, se dirige en arrière et en dedans, par-dessus l'ouverture de la hanche, et s'applique contre le premier.

L'anneau postérieur, beaucoup plus considérable, est partagé en une moitié antérieure beaucoup plus grande et en une postérieure beaucoup plus petite, par une lame triangulaire transversale, qu'une ouverture étroite interrompt au milieu dans les deux tiers inférieurs de sa longueur. Cette lame détache, près de l'extrémité inférieure de cette fente verticale, un appendice presque horizontal qui se dirige en devant; sur les parties latérales elle ne s'étend pas jusqu'à la paroi inférieure; il en résulte ici une lacune transversale.

On rencontre au milieu de la face inférieure lappareil de charpente ordinaire. Il est peu élevé, mais compliqué. En arrière se dirigent deux apophyses larges, sur lesquelles s'appuient les appendices horizontaux de la lame posté- 
rieure. En devant on voit naitre: $1^{\circ}$ deux apophysés courtes, dirigées l'arrière en avant; $2^{\circ}$ deux autres latérales plus longues, qui vont se rendre à une autre apophyse plus courte, mais plus grosse, qui naît de la paroi latérale.

Au bord postérieur de la face tournée en bas, existe entre les deux ouvertures de la hanche une apophyse furculaire, simple, plus petite. Celle-ci, ainsi que toute la partie de la pièce postérieure du thorax à laquelle elle correspond, est située derrière la paroi transversale que j'ai décrite ( $r$ ).

Les pieds de l'insecte parfait sont courts et faibles. Cela s'applique surtout au tarse qui est trìs-mince. Chez la larve au contraire, les pieds sont proportionnellement considérables, principalement la première paire qui est très-longue, volumineuse, et propre à creuser la terre à l'aide du grand développement qu'a pris le tarse.

Les ailes sont consistantes, fortes, volumineuses, et offrent un grand nombre de nervures.

Les anneaux abdominaux supérieurs et inférieurs sont consistans et durs, entièrement ou presque entièrement soudés ensemble, en haut et en bas. lls ont les uns et les autres, à leur

(1) C'est surtout dans les cigales qu'il est curieux d'éludier le thorax. M. Audouin a montré que l'orgnane du chant propre au mâle était composé par les uềmes pic̀ces qui existent dans les autres insectes, mais développées differemment.

(Note des traducteurs.) 


$$
\text { D'ANATOMIE COMPARÉE. }
$$

bord antérieur, de petits appendices qui, sur les anneaux supérieurs, sont plus rapprochés dela ligne médiane. Le plus antérieur des anneaux inférieurs supporte, une pièce cornée, formée de chaque còté d'une branche interne, inférieure, large et horizontale, et d'une externe, mince, unie à la première sous un angle obtus.

L'ouverture postérieure de la tête est très-large, et n'est fermée inférieurement que par une strie cornée, transverse, extrêmement mince, de sorte que la tête est presque entièrement ouverte à sa partie inférieure. Je n'ai rien pu découvrir des parties intérieures qui existent chez d'autres insectes.

$$
\text { \$. } 35 .
$$

Les hétéropières ou hémiptères à ailes inégales, ont une structure encore plus simple.

Chez le nèpe gris (nepa cinerea) le premier anneau thoracique est large, surtout à sa partie supérieure; il s'avance un peu par son bord postérieur sur l'anneau moyen. Le second et le troisième soudés ensemble, ne sont pas beaucoup plus grands que le premier ( 1 ).

Le squeletle intériez'r du thorax est très-im-

(1) Ce qui distingue surtout les hémiptères de cette section, c'est le développement excessif de l'écusson du mésothorax. Cette particularité est très-sensible dans les pentatomes, et surtout dans le genre sculcllaire.

(Nole des traducteurs.) 
parlait; il se compose d'éminences inférieures peu considérables, très-éloignées d'un côté à l'autre, et n'atteignant pas les parties latérales.

Les pieds sont imparfaits; le tarse, qui est mince, n'a en effet qu'un seul article outre le crochet qui est simple. Il est, après la hanche, la portion la plus courte du pied. Les hanches congénères des côlés opposés sont très-éloignées; elles sont arrondies et très-courles.

Les pieds de derrière sont les plus longs; les moyens sont à beaucoup près les plus courts; les antérieurs sont les plus forts. Les hanches sont longues, s'appuient sur l'extrémité antérieure de la face inférieure du premier anneau thoracique, et sont dirigées tout droit en devant. Ie reste du pied est de même tourné en avant autour de son axe; la cuisse est très-longue et volumineuse, sillonnée à sa face autérieure de manière à recevoir la jambe. Celle-ci est également considérable. Le tarse est un simple crochet tourné en devant.

Les ailes antérieures ne sont guère plus longues que les postérieures, mais elles sont considérablement plus fortes et plus épaisses, surtout dans leur partie antérieure.Ellesnese plissent pas.

Les anneaux de l'abdomen, qui est très-aplati, ne sont pas mobiles; ils ne peuvent pas s'engager les uns dans les autres.

La téte est très-petite, étranglée à sa partie postérieure. Je n'ai pas trouvé sa cavité partagcéc en une moitié supéricure et en une inférieure. 
C. Lépidoptères (1).

$$
\text { S. } 36 \text {. }
$$

Le segment antérieur du thorax, ou prothorax, est très petit, étroit et court; il se divise dans toutesa longueur en deux moitiés latérales qui ne sont pas fermées supérieurement, mais qui forment un anneau ouvert.

La face inférieure de ce segment est la plus llarge; elle offre, à sa partie postérieure, deux ouvertures disposées l'une au-dessus de l'autre, et destinées à la première paire de hanches; elles: ne sont séparées que par une strie transversale étroite. De cette strie s'élève, des deux còtés, une paire d'apophyses latérales, déprimées, qui n'atteignent pas la paroi latérale; entre ces deux apophyses on en trouve une autre moyenne, plus longue, dirigée en arrière, qui s'unit au moyen d'une articulation mobile à une apophyse semblable, née de la paroi inférieure du segment qui vient après.

La portion postérieure du thorax (2) est fer-

( I) L'ordre des lépidoptères se distingue essentiellement par l'accroissement excessif du mésothorax; au contraire le métathorax est très-peu développé, et le prothorax encore moins. Le mésothorax offre à sa partie supérieure quatrc piéces très-distinctes, désignées par M. Audouin sous les nors de prosculum, sculum, sculellum et post-scutellum. (Yoyez la note p.64de ce volume.)

( Note des traducteurs.)

(2) Celte portion ne comprend réellement que le mésotho- 
mée d'une manière imparfaite par deux lames qui descendent de la paroi supérieure; l'une est en devant, l'autre en arrière. La postérieure est plus longue que l'an térieure, et chacune est formée de deux moitiés latérales fendues dans presque toute leur longueur. De la face inférieure du segment naissent deux apophyses furculaires, larges, ayant à peu près la même hauteur; l'antérieure correspond à la seconde, la postérieure à la troisième paire de hanches. L'apophyse antérieure, qui occupe la région moyenne du tout, se prolonge en' une crête disposée sur le milieu, tranchante, qui se termine au bord antérieur; la postérieure occupe à elle seule presque toute la longueur de la pièce qui lui correspond. La première, réunie à une autre apopḩyse que fournit la face latérale, forme un trou transversal considérable; la seconde reste isolée.

Cette portion du corps se partage en deux parties : de ces deux parties, l'antérieure (1), plus grande, correspond à la paire de pieds moyens et à la paire d'ailes antérieures; la postérieure (2), beaucoup plus petile, est en rapport avec les parties postérieures de même nom. Cette disposilion est prononcée surtout à la face inférieure.

$\operatorname{rax}$. Qui constitue presque à lui seul le thorax des papillons.

(Note des traducteurs.)

(2) C'est le mésothorrix et le métathorax réunis.

( Note des traducteurs.)

(3) Le métathorax. 


$$
\text { DANATOML COMPAREE. }
$$

Les pieds sont faibles, les hanches longues et verticales. Le tarse articulé forme la portion la plus longue de lappendice.

Chez płissieurs espèces les pieds les plus antérieurs stizit très-rabougris.

Les ailes sont membraneuses, parcourues par de fortes lignes cornées; leurs deux faces sont garnies de petites plumes diversement colorées, le plus souvent serrées. Ces plumes donnent aux ailes leur couleur.

Les ailes postérieures sont communément plus courtes que les antérieures.

La disposition remarquable que présente le mode d'union à l'aide duquel les ailes des papilions crépusculaires et nocturnes sont si exactement fixées l'une à l'autre, a déjà été indiquée au premier volume ( 1 ).

En avant, en dedans et en haut de la paire d'ailes antérieure, on trouve une petite écaille dite épaulette; cette écaille est arrondie, convexe, très-velue, surtout en arrière (2).

Les anneaux de l'abdomen sont formés d'un arcean supérieur et d'un arceau inférieur. Tous ont à peu près une consistance égale; l'inter-

(1) Vol. I, p. 3o6, 3o7.

(2) Cetle pièce, qui ici est libre dans presque tout son coniour, se retrouve dans les antres ordies d'insectes, mais sruuléc et confondue avec les autres pièces du thorax. C'est le paraptère de M. Andouin.

(Note des traducteurs.) 
valle qui les sépare est occupé par une bande de peau étroite. Les bords postérieurs des anneaux placés en avant recouvrent un peu ceux qui les suivent.

La tête est toujours très-petite; l'ouverture occipitale, qui est proportionnellement considérable, est partagée en deux moitiés par une petite strie cornée, dirigée transversalement. La moitié supérieure est un peu plus grande que l'inférieure.

A l'état de larves, les lépidoptères n'ont des parties dures qu'à la tête et aux pieds.

Les pieds sont de deux sortes : les pieds vrais et les pieds faux. Les premiers, qui dans la presque totalité de ces êtres sont au nombre de trois paires, sont fixés aux anneaux antérieurs; les derniers s'articulent avec les anneaux postérieurs; il en résulte que la dernière paire des pieds vrais est séparée des autres par plusieurs anneaux.

Les pieds vrais ont beaucoup d'analogie avec les pieds des insectes myriapodes; comme ceux de ces derniers, ils sont courts, courbes et terminés par un crochet simple. Plusieurs portions cornées qui se succèdent, les composent.

Il existe ordinairement cinq paires de pieds faux, dont la structure diffère d'une manière très. remarquable de celle dẹ pieds vrais. Ils sont, pour la plupart, plus courts, mais plus larges, et garnis, à leur extrémité libre, d'une quantité 
Ii.ANATOMIR COMPAREE.

considérable de crochets cornés; ces crochets sont de deux ordres, distincts seulement par leur inégalité de développement, et disposés d'une manière alternative. ils sont profondément engagés dans un disque formé par les tégumens généraux; l'extrémité inférieure en est recourbée et cachée daus ce disque; il en résulle une insertion très-solide. La disposition et le nombre de ces crochets varient aux deux pieds d'une mème paire et à ceux des différentes paires dans un animal donné. Les pieds correspondans de diversindividus de la mème espèce, étudiés aux différentes périodes de la vie, présentent également des différences sous ce double rapport. On peut dire en général que la quantité en augmenle considérablement-avec l'âge; celle augmentation peut mème en doubler le nombre. Les quatre paires antérieures de pieds n'offrent point entre elles de différences constantes qui sojent disnes de remarque; elles se distinguent seulement de la dernière ou cinquième paire, du moins chez beaucoup de chenilles : I par le nonsbre bien plus considérable de crochets; $2^{n}$ par l'élat plus ou moins complet des anneaux quils forment; entiers̀ dans les premiers, réduils à un seul arceau dans les seconds.

$\mathrm{Ni}$ !e pied vrai, ni le faux ne correspondent à des parties de peau endurcie particulières; nous rencontrerons plus loin celte disposition dans les larves des coléoptères.

II. 
La tête de ces animaux est en général fort dure. Elle est oblongue et disposée de manière à présenter sa plus grande longueur de haut en bas, et sa moindre étendue d'avant en arrière. L'intérieur de cette partie ne m'a offert aucune trace de division en deux moitiés, l'une supérieure et l'autre inférieure.

D. Hyménoptères ( 1 .

$$
\$ .37 .
$$

Chez les hyménoptères l'anneau le plus antérieur du thorax est partagé en denx moitiés latérales dans tonte sa longueur. L'extrémité postérieure seule ne l'est pas; ces deux moitiés sont distantes l'une de l'autre en haut; en bas elles sont rapprochées, mais ne se confondent que postérieurement. De cette partie postérieure naît une apophyse furculaire, d'une hauteur égale à celle du demi-anneau qui est lui-mème pen élevé et s’enfonce sous la tète; elle atteint la paroi latérale de cet arceau, sans s'unir à elle.

Il u'y a ni ouverlure, ni échancrure propre à recevoir la paire de hanches la plus antérieure; la hanche ne pénètre pas dans l'anneau, elle est seulement située tout près et derrière lui. Ses muscles sont placés dans la lacune que lais-

(1) C'est encore le mésothorax qui a pris iri un plus grand développement que le prothorax, et qui constitue presque lui seul le thorax de l'insecte. (Note destraducteurs.) 

sent entre elles la paroi latérale et la fourche.

La portion postérieure du thorax est presque cutièrement fermée en arrière; ce qui dépend de ce que les parois supérieures et latérales se replient en bas et en dedans.

Lintérieur de la cavité présente, à peu près vers le milieu de la paroi inférieure, une apophyse moyenne, très-peu saillante, quise divise bientòt en deux branches latérales (1) C Celles-ci, à peu de distance de leur origine, rencontrent dans la ligne médiane une pièce transversale qui les unit; elles forment ainsi une ouverture centrale, considérable, contenant un ganglion du cordon médullaire. De la partie supérienre de cette apophyse, il s'en détache une autre transversale, mince et alongée, dirigée en dehors et en haut, qui s'applique contre la paroi latérale. Un peu au-dessus et derrière cette apophyse, la paroi latérale fournit une lame alongcée, considérable, qui s’applique contre la face interne de sa parlie postérieure : la paroi est par conséquent doublée en cet cndroit, disposition qui coincide sans doute avec les engainemens des anneaux abdominaux.

L'apophyse moyenne produit en devant, au dessous de l'ouverture, nne crête qui se replie supérieurement, et forme aiusi un demi-canal transversal et superficiel.

(1) C'est l'entothorax du second anneau du thorax.

(Note des traducteurs.)

3.

7. 
Les deux ouvertures, complètes pour les seconde et troisième paires de hanches, sont situées l'une en avant, l'autre en arriére de l'apophyse furculaire.

Les pieds sont ordinairement alongés et considérables, le volume en aurmente de la paire antérieure à la postérieure. Les tarses, torjours articulés, forment souvent la plus longue portion de la troisieme paire. Les hanches sont alongées, mais plus larges et plus courtes que les autres parties; elles sont placées verticalement sur la face inférieure du corps, et sont entièrement ou presque entierement libres.

Les ailes antérieures sont beaucoup plus longues que les postérieures. Les unes et les autres sont membraneuses, minces, parcourues par des nervures et très-alongées. Les antérieures sont plissées longitudinalement chez les diploplères. La moitié interne du bord qui regarde en arrière forme, chez un très-grand nombre d'espèces, une saillie recourbée en has et en dedans, qui s'adapte à une autre éminence placée en face sur le bord interne supérieur; par cet ajustement, les deux ailes sont retenues ensemble et forment une aile unique plus forte, disposition qui rappelle celle que nous avons si. gnalée chez les papillons nocturnes et crépusculaires.

En avant et au-dessus de la racine de la paire d'ailes antérieure, on tronve sonvent, à la face 
supérieure du corps, comme chez les lépidoptères, une petite écaille cornée dite écaille de l'épaule ou épaulette (1).

i’abclomen est formé de lames dures, supérieures et inférieures. Elles sont larges et peuvent rentrer très-profondément les unes dans les autres, au point même que les antérieures re couvrent presque entièrement les postérieures. Les supérieures dépassent également un peu les inférieures sur les parties latérales; les unes et les autres offrent ici de petites apophyses qui s'élèvent de leur bord antérieur.

Louverture arrondie qu'offre la tête pour le passage de l'osophage et du cordon médullaire est petite. Immédiatement en arant de sa partie inférieure, on trouve un anneau trèsmince, mais fort étendu, qui envoie de chaque còté, en devant et en dehors, un prolongement considérable, conslituant un pont qui s'applique sur la partic inférieure de la circonférence du crâne, et s'étend jusque vers l'extrémité antérieure des yeux.

Le corps des larves des hyménoptères est formé d'anneaux entièrement membraneux. La tite seule fait exception. On voit sur les parties latérales des cinq ou six anneaux moyens, au dessrutis des stigmales, cinq ou six paires de pieds qui ont la forme d'autant de pointes mousses et courtes.

1) Le paraptire de M. Audonin. (Note des traduct.) 
La tête est très-petite, arrondie, cornée. Je n'ai pas trouvé le trou occipital partagé en parties supérieure et inférieure.

\section{E. Névroptères (1).}

\section{§. 38 .}

Chez les névroptères, le premier anneau du thorax est séparé des autres; mais il est propor. tionnellement très-petit, et fort étroit à sa partie inférieure. On trouve ici, à l’intérieur, une pelite pièce furculaire qui n'atteint pas les parois latérales, qui complète la circonférence de l'anneau et s'unit à ses parties supérieure, et latérales au moyen d'une articulation mobile.

La pièce postérieure (2), beaucoup plus considérable, porte inférieurement, dans la ligne médiane, une saillie longitudinale simple, peu élevéc, et sur les còtés une crète antérieure et postérieure. Cés productions sont toutes deux tries-faibles.

Les pieds sont petits; les ailes grandes, mem.

(1) Le prothorax est ici peu développé; les deux anneaux qui suivent, le mésothorax et le métathorax, ont un trèsgrand développement; ce sont les flanes qui ont pris surtout une très-grande élenduc. (Note des traducteurs.)

(2) Il est presque inutile de répéter que cette pièce postérieure du thorax correspond au mésothorax et au métathorax de M. Audouin, qu'il est toujours essentiel de distinguer.

(Nole des traducteurs.) 
J'ANATOMIK COMPARÉE.

braneuses et minces, à nervures rétiformes, et ne se plissent pas.

Les anneaux abdominaux superieurs sont considérablement plus larges que les inférieurs, sur lesquels ils s'appliquent de dehors en dedans. Les premiers ont partout une largeur égale; les derniers sont plus larges à leur par. tie antérieure qu'à la postérieure qui est la plus grinde. Plusieurs apophysés latérales naissent de leur partie antérieure. La tête est aplatie d'avant en arrière. Le crâne et le trou occipital sont partagés en une moitié supérieure et en une inférieure par une barre transversale de matière cornée. La structure des lurves est très-analogue à celle de l'animal parfait, quand on fait exception des ailes; l'abdomen en est seulement plus court et plus large. Les ailes sont plissées, mais parfaitement développées et renlermées dans des gaînes dures et très-courles.

Les pieds sont proportionnellement plus longs que chez l'insecte parfait.

\section{F, Orthoptères (1).}

$$
\text { S. } 39 \text {. }
$$

Les orthoptères offrent plusieurs différences dignes de remarque; ainsi le genre de vie et le

(1) J.e prothorax des orthoptires est souvent très-développé à sa parlie supérieure. Daris le genre telrix, cette partie supéricure se prolonge jusqu'a l'extrémité du ventre. 
mode de locomotion de quelques-uns, notamment de la courtilière commune (gryllotalpa), s'éloignent beaucoup de ce qui existe dans la plupart des autres. Ces différences se rencontrent surtout au thorax.

En général, mais plus particulièrement dans les grillons et les locustes, la paroi inférieure de l'anneau le plus antéricur du thorax offre de chaque côté, à peu près vers le milieu de sa longueur, une barre transversale, mince, qui se dirige en dehors et en haut, s'élargit vers la paroi latérale et se convertit en une lame ransversale, large, qui se soude à cette paroi. L'extrémité supérieure donne naissance ellemème à une crète saillante qui règne dans toute la hauteur de la paroi latérale. La partie postérieure de la paroi inféricure est une lame particulière, séparée, qui, réunie à la partie antérieure, forme la lacune destinée à recevoir la hanche la plus antérieure, et porte une crête alongée à sa face supérieure et interne.

Une disposition fort analogue est offerite par ces parties chez les truxales.

Chez les courtilières ou taupes-grillons, le squelette interieur du premier anneau thoracique est extrèmement fort, sans doule plus que chez tout

Le mésothorax et le métathorax sont également développés, et le principal accroissement s'est porté sur les pièces des flancs (l'épisternum et l'épimère), qui sont ici très-distinctes.

(Note des traducteurs.) 
autre insecte; développement qui est en rapport avec le volume et la fonction des pieds de devant. Les apophises de cet anneau, qui lorsque cette disposition n'existe pas sont simples, minces et faibles, occupent ici toute la longueur et presque toute la hauteur du segment, puisqu'au milieu elles s'étendent jusqu'à la paroi supérieure avec laquelle elles se confondent.

Les deux apophyses postérieures sont situées très-près l'une de l'autre, entre les extrémités également postérieures des deux ouvertures deslinées à la première paire de hanches, et qui appartiennent uniquement au premier anneau du thorax.

Il en raît d'abord, en haut et en dehors, une saillie considérable qui occupe à peu près la hauteur de loute la pièce thoracique. Puis ces pièces ossenses se dirigent en devant et en dehors, et se terminent par deux prolongemens. L'apophyse qui occupe le milieu, beaucoup plus développée, se porte en dedans et en íaut, se réunit à celle du còté opposé sur la ligne médiane; enfin, la haule crête longitudinale qui en résulte s'unit à la face interne de la partie supérieure.

L'apophyse du devant se dirige plus en dehors ct se termine, en s'élargissant, à l'extrémité antérieure de cette même partie.

Les ouvertures destinées à recevoir la première paire de hanches nesont séparées l'une de l'autre que par une piece longitudinale très-mince. 
Dans les mantes le squelette intérieur du premier segment thoracique est très-faible, quoique la première paire de pieds soit très-développée; mais il faut dire aussi que le développement se fait d'après un autre type. Le squelette intérieur se compose ici seulement de deux plaques triangulaires, partout séparées l'une de l'autre, et renforcées par une lame cornée vers le bord postérieur. Ces deux plaques sont situées dans la partie antérieure de la première pièce thoracique qui est très-alongée; elles s'étendent d'avant en arrière. Ces parties sont dans ces animaux proportionnellement plus fortes que chez les locustes et les grillons.

La portion postérieure du thurax offre dislinctement une tendance à se partager en une moitié postérieure et en une antérieure. La paroi inférieure présente, à des distances à peu près égales du bord antérieur et du bord postćrieur et les unes des autres, deux apophyses furculaires, séparées, peu élevées, qui s'épanouissent, s'excavent en haut et s'ajustent avec des apophyses de la paroi latérale dirigées au-devant d'elles, mais sans s'y unir. Ces apophyses de la paroi latérale produisent des crêtes longitudinales qui vont en haut se terminer à la racine des ailes. Entre ces apophyses, la lame supérieure envoie en bas, de chaque còté, une lamé transverse, mince, occupant à peu près un tiers de la hauteur de tout l'anneau, cette lame est, sur la li- 
gne médiane, en contact avec celle du côté opposé.

Les apophyses inférieures et latérales, dont nous avons déjà parlé, et qui, en s'unissant entre elles et avec la partie voisine de la circonférence des parois latérales et inférieures, forment des trous ronds, partagent d'avant en arrière l'ouverture destinée à recevoir la seconde et la troisième paire de hanches.

Chez le taupe-grillon, ces parties, et principalement les apophyses inférieures, sont plus fortes et plus élevées que chez les autres orthoptères; il nait de l'antérieure, en arrière et en haut, un fort prolongement moyen, qui s'avance jusque dans l'intervalle qui sépare les deux apophyses postérieures.

Dans les mantes et les truxales, au contraire, elles sont à peu près aussi faibles que chez les autres orthoptères. Chez les derniers elles ne s'alteignent pas dans la ligne médiane.

Les pieds et les ailes offrent des différences très-considérables. Les premiers sont cependant en général longs et gréles; ceux de la partie posıérieure du corps sont plus volumineux que les auires, dont la longueur est à peu près égale. Cette disposition est commune à tous ces insectes; mais elle se rencontre principalement chez les grillons, les locustes et les truxales, où les paires postéricures servent ì exćcuter le saut. Les mantes sront ceux de ces animaux qui ont les pierls les moirs inégaux. Les postérieurs sont peu 
développés dans ces ètres. Il en est de même chez le taupe-grillon, où les pieds postérieurs, plus longs que les antérieurs, sont pourtant beaucoup plus faibles que les premiers.

Les hanches sont très-généralement courtes et arrondies; la cuisse et la jambe sont longues; le pied est court.

Les mantes et la courtilière commune offrent des dispositions particulières, surtout à la première paire de pieds.

Chez les mantes, les hanches sont beauroup plus longues que chez les autres orthoptères, les pieds de derrière sont beaucoup plus faibles, les deux paires antérieures les dépassent de beaucoup. La première, presque aussi longue que la troisième, est de beaucoup la plus forte. La hanche surtout est très-longue, elle dépasse beaucoup celle des autres paires. La cuisse n'est guère plus longue, mais elle est plus large et plus plate. La jambe, beaucoup plus courte, se termine en arriere par un crochet pointu, fortement recourbé et dirigé en haut, qui s'étend presque tout le long de la moitié supérieure du tarse; celui ci est très-mince, et son premier article, très-considérable, est aussi long que tous les autres ensemble.

La paire de pieds antérieure est un long crochet à l'aide duquel l'animal saisit sa proie; elle sert peu à la marche.

Chez le taupe-grillon, tous les pieds sont très- 
épais, et proportionnellement courts ; il faut en excepterlat première paire. Cette paire, qui sertà l'animal à creuser, forme une pelle très-large, comprimée latéralement, et composée de plusieurs parties, qui sont toutes, à l'exception du tarse, à peu près de la même hauteur et d'une longueur égale.

La hanche, qui est forte, représente un anneau large, mais court, ayani en devant, à sa face externe, un enfoncement qui reçoil l'extrémité postérieure de la cuisse, sans qu'il y ait articulation.

Elle porte, à son exirémité antérieure, une pièce triangulaire, alongée, qui se termine en devant par un crocbet pointu, dirigé en haut. Cette pièce est mobile dans ces animaux; on la rencontre aussi chez les autres insectes, ou elle est habituellement soudée à la hanche et ne forme point de saillie.

La cuisse est la portion la plus considérable de l'appendice; elle est dirigće en haut. La jambe se porté en bas et en avant; elle se termine inférieurement par un bord tranchant, large, garni de quatre dentelures longues, dirigées en bas. Elle est triangulaire et beaucoup plus large en bas qu'en haul. Cette portion est reuforcée par le premier article court et large du tarse, qui, repuis le milieu de sa face externe est appliqué sur son quart anléricur et inférieur. Cet article est triangulaire et terminé par deux fortus pointes 
dirigées en avant. Les autres articles sont presque nuls et sans utilité.

Les ailes sont, dans la plupart des animaux dont il s'agit, longues, mais faibles et minces, les nervures en étant peu solides et peu résistantes. Ia longueur en est ordinairement égale; les postérieures sont, au contraire, plus larges que les antérieures. Chez le taupe-grillon, les postérieures sont surtout beaucoup plus longues que les antérieures.

Les lames inférieures de l'abdomen sont en général plus minces que ne le sont celles placées en hant, qui elles-mèmes offrent peu d'épaisseur et de consistance.

De l'extrémité antérieure du bord latéral et de l'extrémité externe du bord antérieur des lanıes situées inférieurement, on voit naître une apophyse mince, mais étendue, qui sc partage en une pointe antérieure et en une postérieure; ces pointes se prolongent dans la cavité de l'abdomen; elles sont sans doute les analogues de celles qui existent plus généralement dans le thorax, mais elles ont une texture bcaucoup plus délicate que celles--ci, quoique leur largeur soit plus considérable.

Dans la tète, la cloison qui résulte de la rénnion des deux apophyses inférieures est trèsconsidérable, forte et longue; clle occupe tonte la longueur du crâne; mais je ny trouve au- 
D'ANATOMIE COMPARÉE.

cinne Irace d'un anneau supérieur particulier destiné au cordon médullaire.

Chez les mantes cette cloison est courte et large, confurmément à la disposition de la tête. Tout le premier ganglion du cordon médullaire abdominal est situé sous cette pièce transversàle.

\section{G. Coléoptères (1).}

$$
\text { S. } 40 .
$$

Chez les coléoptères, notamment le cerf-volant (lucanus cervus), l'orycte nasicorne (geotrupes nasicornis), le hanneton commun (melolonthu vilguris), les dytiques (dytiscus marginalis et latissimus), l'hydrophile (hydrophilus piceus), le blaps géant (blaps gigas), l’anneau antérieur du thorax est formé d'une pièce dont la paroi inférieure offre antérieurement et postérieurement un talus à sa face intérieure, qui s'enforice ou sous la tète, ou sous le second anneau du tho-

(1) Les coléoptères sont remarquables par le développement du prothorax et du métathorax. Le mésolhorax, ou l'anneau intermédiaire, cst beaucoup moins développé; il supporte les ćlytres, dont l'action, dans le vol, n'est pas trés.jprononcée. C'est dans les coléoptères qu'on peut retrouver facilement les pièces conslituant la poitrine, et que M. Audonin a désignées sous les noms de sternum, d'épio sternum, d'épimére et de paraplère. Les copris, le bousier sarré, par exemple, offrent toutes ces partics tris-développées.

(Note des traducteurs.) 
rax. Des deux côtés, à la partie inférieure de la périphérie de l'anneau, mais un peu plus en arrière qu'en devant, on voit une ouverture con. sidérable, fermée de toutes parts, destinće à loger la hanche de la premiere paire de pieds. Cette cavité est tantôt simple, comme chez les lucanes, tantôt partagée par une barre transversale en une partie antéricure plus grande et en unepostérieure plus petite, comme chez les blaps. Immédiatement en dedans de celte ouverture, on voit naître du bord antérieur de l'enfoncement destinéà la portion postérieure du thorax, une petite apophyse verticale de chaque côté : ces deux apophyses ne sont unies nulle part et se présentent partout distantes de la ligne médiane, dans une étendue proportionnellement considérable.

La portion postérieure du thorax ( 1 ) est formée de plusieurs pièces que l'on peut séparer les unes des autres, notamment d'une inférieure et de trois supérieures.

La pièce inférieure est perforée en devant, des deux côtés, pour la hanche de la seconde paire de pieds, postérieurement pour celle de la troisième paire.

Sa face inférieure fournit, en haut et en dehors, plusieurs apophyses inégalemenl déve-

(I) Celte portion postérieure serait plus facile à étudicr dans le détail de ses pièces si on la divisait préliminairement en deux anneaux. En effet, elle est composée du mésothorax et du métathorax.

(Note des traducteurs.) 
loppées chez tous les coléoptères. Le cerf-volunt offre une disposition des plus compliquées. La paire d'apophyses antérieure, rélativement beaucoup plus petite, est fort éloignée de la ligne médiane; elle monte, large d'abord, de la partie interne du pourtour de la lacune destinée à la hanche antérieure, produit erısuite au dessus et en avan t de cette cavité, une apophyse transverse mince qui se dirige en dehors et s'applique presque à la paroi latérale, de sorte qu'il reste un grand espace carré entre ces parties.

Un peu plus en avant, on voit se détacher de la paroi latérale un autre prolongement mince, plus court, qui, après s'être dirigé en dedans et en arrière, se termine librement.

Une seconde apophyse, beaucoup plus volumineuse, naît du milieu dı bord postérieur, entre les extrémités internes des lacunes destinées aux deux dernières hanches. Elle se porte loin en haut et en avant, et envoie du milieu cle sa longueur, en haut et en dehors, une forte branche de chaque côté. On voit également naître en bas, de la ligne médiane, et en avant de cette dernière apophyse, une forte crête montante. La partie antérieure de l'apophyse et de ses deux branches la térales est creuse.

Des pieces supérieures la plus grande, parallèle à l'inférieure, est située le plus en arrière. Elle porte à sa face interne deux crêtes en forme d'X. 
Le petit bouclier triangulaire, situé devan! cette partie, présente une forte crète longiludinale à sa face interne.

Au dessous et derrière ce petil bouclier existe une petite lame carrée, mince, descendant verticaleinent du bord antérieur de la pièce postérieureet supérieure: celte lame rélrécit en devant l'entrée de la portion postérieure du thorax.

Les dytiques offrent une disposition ana!ogue. L'apophyse postérieure, siluée inférieurement . est en proportion mème beaucoup plus forte; elle est très-large et profondément excavée, sur. tout dans sa partie postérieure moyenne el dans ses deux parties latérales. I.es apophyses anlérieures vont jusquà la face latérale et se confondent sur la ligne médiane, de sorte qu'elles forment un anneau considérable avec la face inférieure.

Chez les hydrophiles, le métathorax est semblable, mais un peu plus faible. Les apophyses antérieures alteignent les parois latérales et forment également un anncau très-ouvert avec la paroi inférieure (1).

L'urganisation est au contraire très-imparfaite chez d'autres coléoptères, particulièrement chez

(1) Ces apophyses qu'on remarque à l'intérieur du thorax ne sont antre chose que les entothorax de M. Audouin, ou les pièces en forme d'Y de M. Cuvier; il en exisie une à chaque anneau du thorax sur la ligne moyenne du sternum.

(Note des traductcurs.) 


$$
\text { "ANATOMH: COMPARÉ }
$$

ceux qui ne volent pas, comme les blaps. Toutes les picces sont ici soudées ensemble; tout le thorax ne forme presque qu'un arceau; disposilion qui résulie de ce que l'anneau n'est fermé à la partie supérieure que par une petite parlie, correspondant presque uniquement au petit houclier. Le reste de l'espace est rempli en haut par une membrarre molle et mince, l'aualogue de lit lame posı́érieure, mais qui u'esı pas endurcie. Lapuphyse pustérieure inferieure se parlage aursitòt en deux branches longues et minces, dirigées en bas et en dehors, et s'étendant jusqu'à la lame supérieure. Les apophyses antérieures sont larges et se ríunissent en forme de pont, mais nuffent point de branches laterales.

La disposilion des carabes et des genres voisins est très-analogue.

Les pieds présentent beauconp de variétés en rapport avec le genre de vie de l'animal. Cependaut on remarque très-génćralement:

$1^{\circ}$. Qu'ils angmentent de longueur de la premiere à la troisième paire;

$2^{Q}$. Que la cuisse et la jambe sont les portions les plus longues;

3". (Que les tarses représentent la partie la plus mince, composé de trois à cinq articles, dont le rle rnicres' armé de deux crochels juxlaposés.

Les coléppteres qui courent vice ont tous les pieds lon $\leq$, grètes, très-alongés et arrondis; les harıches sont courtes et sphériques. Plus ils cou-

8. 
rent vîte, plus le tarse est long. Cela existe chez les carabes et genres voisins, dans les blaps, etc. Parmi les coléoptères nageurs, les hanches sont également conrtes et arrondieș chez les dyliques; chez les hydrophiles, au contraire, elles sont larges et longues. Les autres parties du pied sont plus ou moins alongées et aplaties dans ces deux genres. Cela se voit surtout à la dernière paire de pieds chez les dytiques, et à la seconde chez les hydrophiles. Deux aiguillons mobiles, qui se trouvent très-généralement à l'extrémité inférieure de la jambe, sont ici surtout forts et longs. Les tarses de ces paires de pieds sont garnis, des deux côtés, de cirres serrés.

Chez les dytiques la hanche de la troisième paire est soudée à la face inférieure du corps.

Il est digne de remarque, que c'est précisément chez les coléoptères aquatiques que la paire de pieds antérieure, qui ne dévie pas du type ordinaire, offre souvent des différences sexuelles, puisque le tarse du mâle a un appareil propre à retenir la femelle; appareil que l'on peut comparer au pouce des grenouilles qui se gonfle à l'époque des amours.

Chez les hydrophiles, où cette différence est moins marquée, elle consiste en un prolongement du cinquième article, transformé en une lame triangulaire, considérable, tournée en devant.

Chez les drtiques, les trois premiers articles 


$$
\text { I'ANATOMHé comparée. }
$$

sont très-larges et forment ensemble une espèce d'assiette ronde, concave à sa face inférieure, et garnie en outre de quelques ventouses considérables et d'une grande quantité d'autres plus petites.

Chez les pétalocères les pieds sont courts en proportion, les hanches très-larges, grandes, plates et susceptibles d'un mouvement unique. savoir, d'avant en arrière autour de leur axe; les cuisses et les jambes sont le plus souvent plates, surtout les premières; les tarses sont faibles.

Chez les coléoptères qui vivent sous terre, et que ce mode d'existence oblige à creuser à des profondeurs variables, la jambe du premier appendice locomoteur surtout est large, plate, garnie à son bord antérieur et externe de dentelures plus ou moins longues. Le tarse manque à cette paire dans plusieur's genres; la pointe.inférieure de la jambe est au contraire ici, et aux autres paires de pieds, très-longue, forte, solide et quelquefois simple.

Quant aux ailes, les antérieures sont consistantes, dures et cornées : on les a nommées pour cela élytres, parce qu'elles recouvrent les ailes proprement dites. Elles consistent très-généralement en deux lames: l'ure externe, cornée, épaisse; l'autre interne, membraneuse, beaucoup plus mince; toutes deux sont ordinairement intimement unies entre elles; mais quel- 
quefois elles sont moins serrées l'une contre l'autre, surtout en avant, disposition qui permet à l'air de pénétrer darrs l'intervalle qui les sépare, comme, par exemple, chez les hydrophiles.

Chez plusieurs insectes qui ne rolent pas, par exemple, le genre blaps et plusieurs especes de carabes, surlout chez le premier, les deux élytres s'appliquent si exactement l'une contre lautre dans la ligne médiane, qu'elles sembleut soudlés; cette disposition n'est toulefois qu'apparente; car on pent les scparer saus solution de coutimuté, mòme chez les blaps. Elles sont ordinairement presqueaussi longues que l'abdomen; chez les brachéfyeres; elles sont beancoup plus courles. Une saillie siluée ì l'extrémité antérieure de leur bord postérieur, el qui est d'autant plus longrue que l'insecte vole plus parfilitement, sert à les unir à l'extrémité anterieure du bord latéral du bonclier. A còlé de celte saillic on voit en outre quelques pertites pirces cornćes, articulés d'une maniere mobile arec le bouclier. Celle de ces pieces qui est postérieure et interne se transforme, chez quelques-mins, particulièrement chez les dytiques et les hydrophiles, en une lamelle membraneuse, mince, siluée à l’origine de l'élyıre, qui sert peut-ètre à recevoir de l'air introduit ainsi entre ellemême et l'élytre.

Les ailes postérieures ou ailes proprement 
D'ANATOMIE: COMPARÉ.

dites, sont urdinairement plus longues et plus larges que les antérieures on élyıres; elles se plissenl par conséquent au moins une fois en travers, el mème à des degrés variables dans le sens de la lingueur; elles sont formées du reste d'apres le type ordinaire.

Chez quelques-uns, par exemple, les blaps, etc., elles manquent tout-i-fait; chez d'autres, comme la plupart des espèces de carabes, elles sont extremement petites, et la longueur daus ce cas l'emporte iellement encore sur la largeur et le volume, qu'elles sont presque filiformes et qu'il est très-facile de les perdre de vue.

L'abłomen est formé d'environ cinq plaques à la partie inférieure, et de six à la supérieure.

Les inférieures sont plus minces, et non-senlement beaucoup plus solides et plus dures. mais en mème temps bien moins mobiles. Cette remarque sapplique surıout aux plaques antérieures, qui, dans quelques espèces, par exemple, chez les blaps, se soudent et n'en forment qu'une, qurique lon distingue bieu les limites de séparation. La plaque la plus antérieure présente, en avant el de cluaque côté, un enfoncement qui contribue à la formation de la fosse coxale, lestinće à loger la troisieme paire de pieds.

Les plaques supérieures sont des membranes minces et molles, surtout chez les insectes aprteres, et chez les blaps, dont les élytres, plus ou 
moins soudées entre elles, recouvrent constamment l'abdomen.

La dernière des plaques supérieures et des inférieures est beaucoup plus mobile et beaucoup plus petite que celles qui la précèdent; aucune dimension intermédiaire ne conduit à cette disproportion extrème. C'est entre ces deux plaques que s'ouvrent en dehors les organes de la digestion et de la génération.

La tête présente quelquefois, chez les géotrupes, par exemple, au bas de sa partie postérieure, une saillie fort convexe qui correspond à un enfoncement semblable de la pièce antérieure de la poitrine; elle est ordinairement plus faible, ou manque tout-à-fait; la tète ne représente alors qu'un anneau simple.

Chez quelques-uns, par exemple, dans les blaps, les carabes et les méloës, on voit se diriger de la face inférieure, mais en avant seulement, deux saillies d'une hạuteur peu sonsidérable, entièrement séparées ou qui ne sont unies que par une membrane mince; elles forment une gouttière.

Chez d'autres, comme l'orycte nasicorne (geotrupes nasicornis), le cerf-volant (lucanus cervus), le dylique bordé (dytiscus marginalus), l'organisation de la tète est plus compliquée. Non-seulement les deux saillies, racourcies dans la même proportion d'avant en arrière, sc réunissent dans la ligue médiaue, d'oủ résulte 
un canal court; mais on voit en outre, à leur partie supérieure, un denxième anneau, plus petit, tout-à-fait fermé, qui embrasse l'origine du cordon nerveux central, situé derrière l'encephale.

L'organisation des hydrophiles est intermédiaire aux deux précédentes. On voit monter de la face inférieure deux lames parallèles, dirigées d'avant en arrière, et donnant naissance par leur bord supérieur, vers le milieu de leur longueur, à une production cornée, très-mince, qui s'élève en forme de pont au dessus d'elles, et qui est leur seul moyen d'union.

Les larves des coléoptères se distinguent des insectes parfaits par la disposition des parties dures comme par toutes les autres. J'ai choisi, pour mes recherches, la larve du cerf-volant, parce qu'elle est grande et qu'on peut se. la procurer facilement. La peau du corps est en géréral molle, mais beaucoup moins à la face supérieure qu'à l'inférieure. Lєs seules parties qui se distinguent par une dureté considérable sont: $1^{\circ}$ quelques points à l'extrémité an térieure du corps, immédiatement derrière la tète, qui sont la plupart en rapport très-intime avec l'insertion des pieds; $2^{\circ}$ les pieds; 30 la téte; $4^{\circ}$ les stigmates.

On voit immédiatement derrière la tête :

$x^{\circ}$. De chaque côté du corps, au dessus de la premiere paire de pieds et du premier stigmate, 
une plaque brunâtre, ayant la forme d'un carré long, qui se continue immédiatement dans le reste de la peau par toute sa circonfisence.

$2^{\circ}$. Plus bas, exactement au dessus des pieds, on trouve quatre peliles bandes cornées, dures, brunâlres, un peu saillantes, situées immédiatenient les unes derrière les aulres. Isa premirre a la forme diun carre long et vient après la tèle, avec la circonférence inféricure de laquelle elle s'articule par son extromité antérieure. Derrière elle et au dessus de la jremicre paire de pleds, on troure la scconde qui est un peu plus étroile. Les deux autres, siluées audessus des deuxième et lroisième paires de pieds, sont encore plus petites et plus minces; elles sont en avant plus larges queu arriere. : es trois plaques postérieures ont en outre une branche antérieure, transverse, dirigée du dehors en dedans.

'Iel est l'aspect extérieur de ces parties; si on les examıne à l'intérieur, on trouve une disposition bien plus compliquée.

La premiere plaque porte, le long el au dessous de son hord supérieur, une saillie peu élevée; son bord postérieur, au contraire, se replie en dedans en formant une saillie semblable, mais beaucoup plus forte.

Les trois plaques suivantes, beaucmup plus driprimées, saillent un peu en dedans dans toulé leur hauteur. La branche iransverse antérieure 


$$
\text { D'A NTOMIE COMPARE. }
$$

est beancoup plus haute que la postérieure; elle est blanche et dure à l'intérieur. Outre cette branche Iransversale antérieure, visible même alextérieur, il en existe une seconde, postérieure, de mème hauleur, qui s'unit en dehors à la précédente et ì la branche longitudinale, sous un angle aigu", de façon que les deux branches transverses forment un $\wedge$ silué immédialement au dessus de la première portion des pieds.

Les trois paires de pieds sont très-pelites, et leurs parties cornées plus molles que les pièces qui viemnent d’èıre décriles.

De leurs cinq portions alongées, la première estla plus longue. après elle vient l'avant-derniere, consuite la troisieme. La cinquieme est la plus courle, elle forme un ongle simple, légèrement recuurbé.

La premiere, ou la banche, offre une large échancrure, en haut à sa partie postérieure, en bas à l'antérieure; disposition qui permet une flexion et une extension considéribles entre elle el le corps diun côté, et eutre elle eı la cuisse de l'autre còte; ces mouvemens sont beaucoup nusius prononcés aux autres portions du membre.

Le crâne, arrondi, est aplati d'avant en arriere; il offre une ouverture circulaire trèsgrande, qui en occupe presque toute la face postérieure.Cette ouverture n'est fermée, en bas, 
que par un pont étroit, mais épais; c'est à ce pont et à toute la circonférence de la face postérieure que s'altache le tégument cutaré du corps. L'espace compris entre cette insertion et le pourtour de l'ouverture est rugueux, il sert à l'atlache des muscles de la tète.

L'osophageseul sort par le grand trou occipital; les deux cordons médullaires qui unissent entre eux l'encéphale et la moëlle spinale sortent, au contraire, de la cavité du crâne, audessous de la partie inférieure de la circonférence du trou occipital. Ils ne sont pas contenus ici dans un anneau; mais il y a de chaque còté une petite saillie dirigée en dehors qui les sépare des parties voisines. Les deux saillies forment un $\wedge$, dont le sommet est tourné en haut; cette disposition a quelque analogie avec l'arc d'une verièbre. En bas on trouve immédiatement la peau.

Ainsi que les pièces du tronc, la tête, brune à l'extérieur, est blanche dans son intérieur.

Je ne parlerai pas ici des cercles cornés des stigmates; il en sera question lorsque nous exposerons l'appareil de la respiration.

2. Insectes sans métamorphose.

$$
\text { S. } 41 .
$$

Je ne considérerai ici que les myriapodes. Ceux-ci ont le squelette beaucoup plus simple 
1)'ANATOMIE COMPARÉE.

que les insectes considérés jusqu’ici; ils forment par là le passage aux arachnides.

Tout le corps se compose, dans cette famille, d'un nombre toujours très-considérable d'anneaux ayant à peu présle même volume, dont les antérieurs recouvrent un peu les postérieurs. Chezles iules tous les anneaux sont complets, à l'exception des plus antérieurs, et portent deux paires de pieds. Parmi les premiers anneaux, celui qui vient immédiatement après la tête est plus large d'avant en arrière que tous les autres; mais il n'existe que dans sa moitié supérieure. Il conlient cependant, dans sa cavité, deux plaques alongées, disposées d'avant en arrière; chacune de ces plaques supporte une paire de pieds. L'anneau suivant est complet, mais beaucoup plus large dans sa moitié supérieure, qui est plus grande que la moitié inférieure. Il présente un sillon transverse; ce mode de conformation est transitoire et conduit aux autres anneaux non sillonnés qui ont une longueur à peu près égale rans toute l'étendue de leur circuil. Il ne supporte que deux paires de pieds. Il en résulte qu'à sa partie inférieure il ne forme qu'un anneau simple.

Tous les pieds sont situés immédiatement en avant du bord postérieur de chaque anneau, exactement ì côlé de la ligne médiane, et les deux paires de chaque cercle sont à côté l'urie de l'autre. 
Ils sont petits, minces, convexes en dehors. concaves en dedans, et composés de six à sept articles simples devenant insensiblement plus longs; le dernier de ces articles est un crochet simple.

Les anneaux des scolopendres sont moins nombreux et distinctement divisés en deux moiliés: l'une supérienre el l'aulre infépieure, dont le seul mode d'union est une peau plus molle. Entreces deux moitiés sortent les pieds des deux côtés; de sorte que les paires correspoudantes sont ici très-éloignées l'une de l'autre. Leur structure ressemble du reste à celle des pieds des iules, à cela près qu'ils sont plus volumineux et quil n'y a pour chaque arliculation du corps qu'une paire qui correspond au milieu de celte articulation. La dernière el l'avant-dernière paires, la premiere de ces deux paires surlout, salongent considérablement, et se tournem en arrière, de manière à devenir parallèles l'une à côté de l'autre.

La téte, chez les iules et les scolopendres, est formee de pliques supérieures et inférieures.

Il n'y a dans aucun de ces animax dapophyses inlérieures partant des pièces du test; c'est pourquoi tous les viscères sont situés dans uue seule et même cavité (1).

(1) Chez ces animaux, le développement des anneaux a été uniforme; chafue segment est pourvu dappendices à l'arceau inférieur uniquement. Il résulte de la préspnce de 
II. SQUELETTE IES ARACHNIDES.

$$
\text { S. } 42 \text {. }
$$

Les squeletles extérieur et intérieur des arachnides sont beaucoup plus imparfaits qu'un ne les reucontre daus la plupart des invect $s$. Dans l'extérieur, la disposılion articulée est beancoup moins développeje; l'intérieur est beaucoup plus petil et moins compliqué.

A. Arachnides proprement dites.

$$
\text { S. } 43 .
$$

Les araignées proprement dites offreut, sous l'un etl'autre rapport, l'organisation la plusimparfaite. La peau peu consistante de l'aldomen, qui est oblong, n'offre point d'articulation à sa face superieure; quelquefois seulerrent il y a une légere disposition arliculée à sà face inférieure, comme, par exemple, chez la mygale aviculaire.

ces appendices à chaque ar:neau dı corps, que les Myriaponesniont pas d'abdomen, et qu'ils sont seulement composés d'un thorax, d'apuès la définition d $\mathrm{M}$. Audonin; ;en animaux arliculés, dépourvus n'ilpendices, présentent letype 10 l'uniformité la plus complète, et en même temps de la plus graude simplicité (les appendices de la bouche exceplés). Pour avoir i'idée de la plus grande complication, on peut supposer un animal entiérement formá de segrmens pourvus chacun d'une paire de pieds et d'une paire d'ailes.

(Note des traducteurs.) 
L'extrémilé postérieure scule de cette portion du corps est quelquefois munie de paires d'appendices oblongs et articulés.

Le corselel (1) se compose d'un test supérieur et d'un test inférieur. Le supérieur est plus grand que l'aulre; il est ordinairement un peu étranglé à sa partie antérieure nù il correspondà la tète, et supporte, ou mieux recouvre les yeux. Sa partie postérieure présente, au milieu de la surface intérieure, une forte saillie d'où partent, vers chaque côté, quatre fortes apophyses, dont l'antérieure et la postérieure sont les plus fai-

(1) Le thorax des arachnides est beaucoup plus simple que celui des insectes, quoiqu'il soit composé d'un plus grand nombre d'anneaux. En effet, abstraction faite de la tête, qui est confondue avec lui, on lui compte quatre anneaux, c'est-à-dire un nombre égal à celui des quatre paires de pattes. Ces anneaux se composent d'une pièce inférieure. le sternum, et de pièces latérales ou flancs. Les quatre sternums sont exactement sondés entre eux, et forment un petit plastron entre les pattes. Les flancs sont aussi réunis entre eux, mais on apercoit les lignes de soudures; ils constituent le dos de l'animal. La partie supérieure, ou le tergum des insectes, manque ici complètement. IN. A udonin a démontré ce fait. On peut se figurer ce qui a lieu ici en comparant cette organisation à celle d'un crustacé décapede brachyure auquel on aurait enlevé la carapace. Dans l'araignée les flancs obliques en dedans et en haut sont arrivés au point de contact; ils se touchent tous par leur sommet. Dans le crabe les flancs, qui sont aussi obliques en dedans et en haut, ne sont point assez prolongés pour se toucher; il existe entre eux un intervalle.

(Note des traducteurs.) 
bles, qui sont toutes des traces d'insertions musculaires.

Le test inférieur correspond seulement à la partiepostérieure du test située supérieurement. 11 est droit, et du milieu de son bord postérieur s'élève une petite apophyse qui bientôt après se divise, de chaque côté, en une branche semiannulaire, longue, mais étroite, qui se dirige en devant immédiatement sous les parois latérales du test supérieur, et sert d'attache aux muscles des hanches, an. dessus desquelles le test est situé. Cette apophyse forme la paroi latérale de la poitrine qui est très-déprimée.

Il existe en général quatre paires de pieds qui ont à peu près la même grandeur et la mème structure. Ils sont silués immédiaternent les uns derrière les autres, entre les deux tests du bouclier thoracique, et sont libres dans toutes leurs pariies. La premiere portion en est alongée, la seconde beaucoup plus courte. La troi. sième, bien plus longue que la précédente, est suivie d'une portion très-courte; la cinquième est la plus longue, mais plus grêle que la troisieme. La sixième et la septième diminuent brus. quement de longueur. La dernière est formée par plusieurs petites pièces dures, placées les unes au-dessus des autres.

Les petits articles intermédiaires ne semblent pas exister dans toutes les espèces.

La première portion ne correspond Ires-vraiII. 
semblablement pas à la hanche des insectes, mais représente les parties latérales du thorax. Cela est très-distinct chez les scorpions, comme on le verra par leur description.

Je ne trouve aucune trarce de squelette intérieur chez les araignées, à moins qu'on ne veuille regarder comme telle une partie tendineuse, épaisse, solide, très-fortement développée chez les grandes araignées, particulièrement chez la mrgale aviculaire; partie qui est située sous la plaque supérieure dı bouclier thoracique, qui supporte, à ses faces supérieure et inférieure, deux rangées latérales d'apophyses verticales, et à chaque bord une rangée simple d'apophyses transverses, prolongées dans l'intervalle qui sépare les muscles des pieds.

Mąis cette analogie a contre elle la position de cette partic au-dessus du canal intestinal, la présence simultanée de cette partie et du squelette intérieur ordinaire, chezles scorpions, et en général le peu de ressemblance qui existe entre cette partie et le squelette intérieur des autres animaux articulés.

\section{B. Scorpionides.}

\section{S. 44.}

Les scorpionides ont un squelette extérieur et un squelelte intérieur beaucoup plus composés; mais, conformément au type général des arach- 
nides, la tète et la poitrine sont encore confondues. Ces deux cavités réunies constituent une portion du corps beaucoup plus petite relativement que chez les araignées ordinaires.

On ne trouve au dos qu'une plaque carrée, dure, s'élargissant d'avant en arrière, qui porte plusieurs points oculaires au milieu et sur les parties latérales; ceux de ces points qui occupent le milieu sont situés plus en avant que ceux qui sont sur les côtés. Sous l'extrémité antérieure de cette plaque existent en haut les mâchoires supérieures, en bas les mâchoires inférieures très-développées, et les parties buccales en général.

La paroi inférieure est beaucoup plus compliquée. Elle est composée de trois paires de pièces diversement disposées, qui se succèdent immédiatement et se rejoignent tout-à-fait ou presque tout-à-fait dans la ligne médiane. Les extrémités externes et ouvertes de ces pièces supportent les hanches des pieds qui leur sont lâchement unies par une peau molle.

La jièce antérieure est de forme rectangulaire, sa plus grande longueur est d'avant en arrière; il en résulte que la cavité est située en dehors. Elle supporte la première paire de pieds, qui sont séparés l'un de l'autre par une saillie triangulaire, alongée de la seconde pièce qui se prorte en avant et se place entre eux.

I a seconde pièce est plus grande, triangu- 
laire; sa dimension transversale est la plus longue. Les deux pointes de cette pièce qui portent la seconde paire de pieds se rencontrent dans la ligne médiane.

La troisième pièce est encore plus grande et plus alongée. Elle est également triangulaire. Les pointes internes se rejoignent presque sur la ligne médiane, elles ne sont séparées que par une petite plaque triangulaire. Elle correspond aux troisième et quatrième paires de pieds par deux ouvertures articulaires tout-à-fait séparées, dont la postérieure est située beaucoup plus en dehors que l'antérieure.

De la face supérieure interne de ces pièces naissent des saillies qui divisent imparfaitement la cavité de la poitrine d'avant en arrière. Les denx saillies antérieures sont des plaques hautes, molles, membraniformes, verticales, triangulaires, situées sur le bord postérieur de la première et de la seconde pièce. La saillie de la troisième pièce est moins haute et beaucoup plus dure et plus solide, du moins chez le scorpion roussâtre (scorpio occitanus), et n'est en rapport avec la périphérie extérieure qu'à ses extrémités externe et interne. Llle nait de l'extrémité interne de la dernière pièce, et se dirige, sous forme d'un pont mince, de dedans en delors et d'avant en arrière, pour se fixer entre les ouvertures destinées à recevoir les troisième et quattrième paires de pieds. 
Les pieds qui sont longs, mais faibles, augmentent considérablement de volume d'avant en arrière, au point que la dernière paire est deux fois plus longue quela première; ils forment des fractions d'arcs dont la convexité est diriçée en arrière, de sorte que leurs extrérnités externes s'éiendent très en avant; ils sont composés chacun de sept portions. La première, ou la hanche, entièrement libre et s'articulant avec la partie externe des pièces décrites, est courte, épaisse et arrondie. La seconde portion est la plus longue, mais elle est aplatie de dehors en dedans. 1.a troisième est plus courte, mais aussi plus large et plus épaisse. Les quatrième el cinquième sont à peu près de la mème grandeur, mais beaucoup plus pelites que la troisième. La sixième est inférieure en volume aux deux précédentes; la septième, formée de deux crochets latéraux, est la plus petite.

L'abdomen se divise en deux sections: une antérieure, plus courte, mais beaucoup plus large, et une postérieure, plus longue, mais considérablement plus étroite; cette deruière est la queue.

La section antérieure ne se compose que de quatorze demi-anneaux, sept en haut et sept en bas, unis entre eux par une membrane intermédiaire, mince. Quoique le nombre des demi-anneáux inférieurs semble moindre, je crois néanmoins qu'il est également de sept. En haut, il 
ne saurail y avoir le moindre doute sur leur nombre; on en voit distinctement sept qui s'élargissent d'avant en arrière. En bas, on n'en aperçoit d'abord que cinq, qui correspondent aux cinq derniers anneaux de la série supérieure, et qui sont un peu plus larges; mais on en trouve réellement, en avant du premier de ces cinq anneaux inférieurs, encore deux autres, quoique beaucoup plus petits, qui s'appliquent contre la petite plaque triangulaire que nous avons décrite avec le thorax. Le postérieur de ces deux petils arceanx est carré et plus petit que l'antérieur, qu'une incisure longitudinale, passant par sa ligne médiane, partage en deux plaques triangulaires, juxtaposées. C'est entre ces trois plaques que se Irouve l'orifice de l'organe générateur. Liarceau postérieur supporte des deux côtés un corps plat, alongé, également dur, puisqu'il est corné, que l'on peut en quelque sorte comparer à un peigne à courtes dents. Ce corps se compose de trois portions qui se suivent d'avant en arrière. La plus antérieure est formée, de la racine à la pointe, de trois articles, dont le plus interne est le plus long et le plus fort. La seconde portion ou série contient environ une vingtaine de petits disques; la troisième se compose d'une quantité, très-considérable chez certaines espèces, de lamelles alongées, dirigées en arrière, qui représentent les dents du peigne, sont séparées dans 
tonte leur longueur, mais sont très-rapprochées les unes des autres.

Un célèbre naturaliste a pris ces parties pour des organes de respiration (1). Il y a déjá quelque temps que j'ai prouvé l'inexactitude de cette opinion en démontrant les véritables organes respiratoires de ces animaux (2). Comme elles se trouvent au voisinage de l'orifice de l'appareil générateur, je les regardais alors comme des parties externes de la génération (3); opinion qui est peut-être confirmée par la circonstance dont j’ai fait la découverte plus tard, que Irès-constamment ces parties sont beaucoup plus volumineuses chez les mâles que chez les femelles, du moins dans le scorpion d'Europe (scorpio europoius).

Celte différence de volume dans les deux sexes ne suffit pas pour prouver directement que ces parties appartiennent à la génération. L'opinion la moins sujette à erreur consiste peut-être à considérer ces parties comme des rudimens de pieds, ce qui n'exclut pas la pre. mière opinion; elles représenteraient alors les faux pieds des crustacés. Cet excès de volume qu'elles offrent chez les mâles est digne de

(1) Dnméril ; Zoologie analytique, p. 290.

(2) Beitræge zur vergleichenden und menschlichen Anatomie, T. I, p. 109, I 16.

(3) Ibid. 
remarque, parce que le développerneut de la première paire de faux pieds est également si considérable dans beaucoup de crustacés qu'on les regarde comme des pénis.

La queue est formée de six articulations creuses, parfaites et oblongues, qui augmentent en longueur d'avant en arrière jusqu'à l'avantdernière qui est la plus longue. Toutes se prolongent à leurs extrémités antérieure et postérieure en une saillie, et sont lâchement unies entre elles par une peau intermédiaire qui est molle; mode d'union d'où résulte qu'elles ne se recouvrent pas mutuellement et peuvent s'appliquer les unes sur les autres dans tous les sens. L'orifice anal est placé entre la dernière et l'avant-dernière articulation. La dernière articulation renferme l'organe venimeux, et se termine par un stylet fin, convexe en haut.

\section{IIJ. SQuelette ves crustacés.}

$$
\text { \$. } 45 \text {. }
$$

Les crustacés sont sans contredit ceux de tous les animaux articulés extérieurement qui ont le squelette le plus compliqué (1).

(1) C'est-d̀-dire, selon nous et M. Audouin, celui qui est formé par un plus grand nombre d'anneaux; mais ces anneaux, étudiés séparément, sont beaucoup plus simples que ceux des insectes. Abstraction faite de la carapace, chaque anneau du thorax se compose d'un sternum et des flancs. 


\section{D'ANATOMIE COMPAREE.}

L'écrevissé de rivière ordinaire, dont je me sers de type pour celte classe, offre la disposition suivante :

Toutes ces parties sont soudées entre elles, mais on peut les distinguer facilement en comptant le nombre de paires de pattes. Il donne toujours le nombre des anneaux du thorax.

La complication apparente du squelettc des crustacés provient de la multiplicité de lames qui partagent en cellules la cavité de leur thorax. La disposition de ces lames est presque inextricable tant qu'on ne les rapporte pas aux pièces dont elles sont les analogues; rapport qu'on ne saurait élablir qu'en ayant égard à leurs connexions essentielles. Guidé par ces vues philosophiques, M. Audouin a reconnu dans ces lames les apodèmes des insectes prolongés et èlargis. En effet tontes ces cloisons naissent de la soudure des sternums et des flancs entre eux, et devieunent, en se joignant, les parois d'un grand nombre de cellules. La position relative de ces séries de cellules les unes à l'égard des autres, est différente dans les brachyures et dans les macroures. Celles des flancs sont superposées et forment deux étages dans le maja, qui appartient au premier de ces ordres. L'étage inférieur a pour base le sternum; l'élage supérieur correspond à la voûte des flancs qui sont obliques, et qui font partie d'un thorax large et arrondi. Dans le homard, crustacé de l'ordre des macroures, les loges qui limitent les lames sont verticales et disposées sur un mẻme plan; ici le thorax est étroit et alongé. Les cellules sternales du maja forment une rangée de chaque còté du corps; celles du homard sont réunies entre elles au. dessus du sternum qui les sépare inférieurement. Les cellules des flancs sont supérieures dans le premier, externes dans le second. Les cellules supéricures et inférieures communiquent entre elles chezle maja; cette communication est le résultat de l'absence de voûtes pour les derniers et de planchers de la part des cellules des flancs; on nomme ces lácuncs trous 
Tout le squelette, ainsi que le corps en général, peut ètre partagé en une moitié antérieure et en une postérieure; la première com-

inter-cloisonnaires; ces trous sont disposés à la circonférence du thorax où la lacune existe uniquement; chaque cellule des divers segmens a ainsi extérieurement une ouverture commune qui reçoit la patte correspondante; elle s'ouvre d'autre part par un orifice particulier dans l'intérieur du thorax, à l'opposé de l'ouverture précédemment indiquée. Dans le homard, les cellules des flancs ne communiquent point entre elles; les cellules sternales s'ourrent les unes dans les autres par une espèce de fente ovalaire qui occupe la ligne médiane; ainsi réunies elles constituent un canal longitudinal qui communique avec les cellules des flancs par les trous inter-cloisonnaires.

Dans les crustacés décapodes, le squelette, abstraction faite de la tête et de l'abdomen qui sont fort rudimentaires, est composé de huit segmens qui supportent les cinq paires de paltes ambulatoires et les trois paires de pieds-mâchoires. Les trois premiers segmens sont rudimentaires. Les cinq suivans ont un très-grand développement. Dans le maja, de l'ordre des brachiures, les sternum, soudés entre eux, forment un large plastron qui occupe la face inférieuredu thorax; les pièces des flancs constituent deux espèces de boucliers sur les parties latérales. Dans le homard, ordre des macroures, il n'existe plus de plastron proprement dit; tous les sternum, soudés bout à bout, constituent une espèce de crête médiane placée entre la base des pattes qui sont rapprochées au point de se toucher. (Voyez les recherches anatomiques sur le thorax des animaux articulés par M. Audouin, et postérieurement le travail fait en commun avec M. Milne Edwards sur la circulation dans les crustacés. (Annales des sciences naturelles, t. XI.)

(Note des traducteurs.) 
prend la tête et le thorax, la seconde la queue.

l.e thorax forme la plus grande partie de la premiére moitié; il renferme les organes respiratoires et générateurs en entier, et en outre les pièces les plus importantes des appareils di. gestif et circulatoire; il porte en mème temps les cinq paires de pieds vrais ou ambulatoires. Comme la tête et le thorax ne peuvent guère ètre séparés l'un de l'autre, il vaut mieux les décrire comme ne faisant qu'une seule portion du corps, qui se partage en une moitié supérieure et en une inférieure.

Isa première est simple et forme un bouclier oblong, convexe, ouvert à ses parties antérieure, postérieure et supérieure; ce bouclier recouvre la moitié inférieure et les organes de la partie supérieure et externe, dont il vient d'élre question, et les dépasse en devant; là cette partie se termine en deux pointes et est elle-même partagée par un enfoncement qui se dirige d'un bord latéral à l'autre, en deux portions, une antérieure plıs petite, et une postérieure plus grande. A la racine de l'apophyse à deux pointes, on voit une lame verticale, mince, faire saillie en bas.

Immédiatement après, cette partie s'étrangle considérablement; il ne reste alors qu'une apophyse étroite, moyenne, formée de deux prolongemens qui se réunissent sous un angle droit; avec cette apophyse s'articule d'une manière peu 
mobile une lame triangulaire, large. La partie supérieure de la lame perpendiculaire dont il a été question, présente une ouverture transversale par laquelle sortent les yeux.

I.a moitié inférieure a une structure beaucoup plus compliquée. Elle forme pour la plupart un demi-canal scaphoïde, convexe à sa face inférieure, concave à la supérieure, que l'on peut diviser en une partie moyenne et deux latérales, qui, quoique solidement unies entre elles, peuvent être séparées.

La partie moyenne s'élargit d'avant en arrière et constitue une goutlière en forme de toit. Sur ses côtés s'élèvent verticalement plusieurs a pophyses, élroites dans leur partie inférieure, mais s'élargissant à la supérieure tellement en avant, en arrière, en dehors et en dedans, qu'elles ont la forme d'une plaque. Celte partie supérieure est garnie de bords tranchans et considérablement dentelés; l'iuférieure a des bords unis.

Les parties latérales forment, dans leur portion externe, une membrane élevée, verlicale, continue, pourvue d'un bord supérieur convexe, et que consolident des espèces de cylindres ossiformes, durs, qui sont surtoùt consistans, larges et épais dans leur partie inférieure. En bas, ces cylindres s'épanouissent en plaques qui se joignent entre elles.

La partie moyenne de chaque plaque est sur- 
D'ANATOMIE COMPARÉ.

montée en dedans par une saillie transverse considérable, formant avec elle un angle droit; saillie qui se bifurque à peu de distance en une branche antérieure et en une branche postérieure.

L'antéricure, plus considérable, se partage en deux, une supérieure et une inférieure; la branche postérieure est simple, plus petite, et correspond seulement à la division supérieure de la branche antérieure.

lies saillies internes de la partie latérale sont situées de manière à occuper l'espace qui existe entre les apophyses montantes de la partie moyenne, dont elles sont pourtant distantes de toute la largeur-qui sépare la partie latérale de la moyenne.

Les différentes divisions des parties moyenne et latérales s'unissent très-étroitement entre elles sur plusieurs points.

Celles de la partie moyenne s'appliquent toutes les unes contre les autres dans leur portion moyenne et inférieure, et se confondent en ce point en un tout, à l'exception de la plus antérieure et de la plus postérieure.

Les lames supérieures des apophyses de mème nom des deux côtés se recourbent fortement les unes vers les autres, à tel point qu'en cet endroit, ces apophyses sont le plus rapprochées les unes des autres. Les plus antérieures s'alteignent et forment une véritable su- 
ture; les autres sont unies seulement par une membrane, et il y a d'autant plus d'espace entre elles qu'elles sont plus postérieures. Les apophyses d'un mème côté se recourbent aussi un peil les unes vers les autres par leurs plaques supérieures; mais il n'y a que la première et la seconde qui soient en contact.

La partie moyenne s'unit avec la latérale par trois apophyses verticales. Les branches antérieures de l'apophyse interne de chaque section latérale s'appliquent en haut contre l'apophyse verticale la plus voisine de la pièce moyenne; les postérieures s'appnient contre celles qui en sont le plus rapprochées.

Les pièces latérales se rejoignent ici en même temps par leurs branches antérieures et postérieures.

De cette disposition résultent, en procédant de dedans en dehors, et en passant de chaque point à celui qui est à côté :

$I^{\circ}$ Un enfoncement longitudinal simple, moyen, entre les corps et les apophyses verticales de la pièce moyenne; enfoncement qui loge le cordon rachidien;

$2^{\circ}$ Une série interne;

$3^{\circ}$ Une série externe de canaux arrondis, très-larges, qui se suivent d'avant en arrière et sont dirigés de haut en bas.

Les canaux internes sont situés immédiatement au-dessus de l'enfoncement longitudin?! 


$$
\text { D'ANATOMIE COMPARÉE. }
$$

moyen, entre les apophyses verticales qui sont placées au milieu, et les branches des apophyses internes des pièces latérales. Ils s'ouvrent supérieurement, en dedans, dans l'enfoncement longitudinal dont il vient d'être question; inférieurement ils sont fermés par l'enfoncement plus court de la pièce moyenne.

Les canaux externes se trouvent entre les apophyses internes et les plaques situées sur le còté de la pièce latérale. Ils sont entièrement fermés partout.

Ces deux séries sont séparées l'une de l'autre par les branches inférieures et postérieures des apophyses internes des pièces latérales.

On trouve, en outre, entre ces deux séries de canaux, de chaque côté, une ligne de petites ouvertures verticales, dirigées un peu obliquement, de dehors en dedans et d'arrière en avant, entre les apophyses verticales de la pièce moyenne et les hranches antérieures et postérieures correspondantes des apophyses latérales; d'où résulte une communication entre la série interne des canaux et entre les canaux interne et externe les plus voisins.

Ces canaux se confondent du reste dans la majeure partie de leur trajet inférieur, parce que les branches des apophyses latérales sont étroites.

Ces canaux reçoivent les muscles de la première portion des pieds et ceux des mâchoires; la 
série externe correspond au muscle extenseur, et l'interne au fléchisseur.

\section{S. 46 .}

Le thorax porte les pieds proprement dits ou pieds antérieurs, qui, ainsi que tout le squelette, sont des pièces testacées, creuses, composées de plusieurs portions qui se succèdent de la base au sommet, dont le nombre n'est pas le même dans tous les pieds, et qui varie de six à huit.

La première portion, la hanche, est courte, épaisse, irrégulièrement quadrilatère, toujours unie à la partie latérale de la face inférieure du thorax, de manière à correspondre à l'un des canaux verticaux précédemment décrits. Elle est attachée au pourtour de ce canal par une membrane lâche, mais ne se meut qu'en avant et en arrière, et pas mème fort librement dans cette dernière direction; bornée dans ses mouvemens par les canaux qui la reçoivent. Elle est considérablement plus haute dans la partie postérieure de sa circonférence que dans l'antérieure.

La seconde portion est beaucoup plus étroite, mais plus alongée, triangulaire, plus déprimée supérieurement qu'inférieurement. Elle ne forme une pièce particulière qu'aux quatre pieds postérieurs; au premier pied, elle est confondue 
avec la troisieme piéce. Elle est unie à la hanche, à la partie inférieure et supérieure de la circonférence de son extrémité postérieure, au moyen d'une membrane lâche, et sur les parties latérales par une membrane roide, qui ne permet par conséquent qu'un mouvement d'élévation et d'abaissement, on de flexion et d'extension sur la hanche.

La troisième pórtion du membre est plate, alongée, plus volumineuse que la précédente. Son extrémile postérieure est coupée en biseau, l’antérieure à pic. Elle ne se meut qu'en dehors et en dedaus, en avant et en arrière.

La quatrième portion est encore plụs considérable; elle est la plus longue de toutes, quand on fait abstraction du pied le plus antérieur. Son bord postérieur est droit. Il en est de même de la partie supérieure de la circonférence de son extrémité antérieure; l'inférieure présente au contraire une profonde échancrure en arrière; il en résulte une flexion et une extension trèsconsidérables entre cet article et l'article suivant, tandis que ses mouvemens sur la pièce précérlente ne peuvent se faire que davant en arriere et de dedans en dehors.

Iá cinquieme portion est plus courte. Son exlrémité postérieure forme supérieurement, au milieu et sur les parties latérales, une forte saillie; inférieurement elle est coupée droit. Antérieurement elle est échancrée à la partie II. 
externe et interne, tandis qu'en haut et en bas elle forme une saillie. Par celle disposition les mouvemens de flexion et d'extension sont presque entièrement nuls entre celte piéce et la sixieme; mais le mouvement latéral est très-libre.

Ia sixième pièce présente postéricurement deux saillies, l'une externe et l'autre interne, qui se terminent par un bord concave ayant en haut et en bas un pelit condyle par lequel chacune d'elles correspond ì la cinquieme portion. In avant, cette pièce se termine constamment par un bord droit.

Les trois premiers pieds se distinguent des autres par l'existence d'une pointe qui termine cette pièce antérieurement, au-dessus de l'endroit oi s'insère le dernier article; pointe qui est presque aussi longue que celui-ci et qui forme pince avec lui.

Gette sixième pièce est, ainsi que la quatrième, partout la plus longue; au premier pied, elle est tellement développée en lont sens qu'elle est à elle seule plus volumineuse que le reste du pied.

La septieme pièce est en général pelite, apointie, et surtout plus mince que les autres. Elle n'est très-longue qu'au premier pied, mais aussi plus mince que les autres. Elle se fléchit et s'étend librement sur la pièce précédente.

Par suite cie la disposition de ces différentes faces articulaires, le pied peut, au moyen de la 


\section{D'ANATOME COMPARÉE.}

hanche, se mouvoir en avant et en arrière sur le thorax; mais ce mouvement n'est pas trèslibre.

Le reste du pied se fléchit et s'étend au contraire très-librement sur la hanche.

Les troisième et quatrième pièces ne jouissent que du mouvement transversal; la cinquième s'étend et se fléchit très-aisément; la sixième se meut assez librement en dehors et en dedans; la septième a des mouvemens de flexion trèslibres.

\section{S. 47 .}

La partie la plus antérieure, représentant la tète, a déjà été décrite en partie plus haut, en parlant de l'extrémité antérieure de la pièce supérieure de la portion céphalo-thoracique.

Outre les parties déjà indiquées, on trouve. ici différens organes des sens et les mâchoires.

Les premiers remplissent de chaque côté l'es pace compris entre la lame supérieure et l'inférieure. On trouve d'arrière en avant et de dehors en dedans trois pièces mobilement unies entre elles par de la peau molle.

La plis postérieurc est une lame quadrilatère, transverse, qui se termine par une saillie mamelonée où se trouve contenu l'organe auditif.

Apres celte pièce viennent deux autres pières testacées, creuses, siluées l'une au dessus de l'autre, dont l'externe est beaucoup plus 11. 
volumineuse que l'interne. La première supporte une apophyse pyramidale, courte; la seconde porte une longue antenne. Au point situé le plus en dedaus el en devant, on trouve un cylindre alongé qui supporte les antennes internes, plus petites que les externes.

\section{\$. 48 .}

La portion postéricure du corps, ou la queuse, se compose: $1^{\circ}$ de six anneaux qui se succèrlent d'avant en arrière, et $2^{\circ}$ de plusieurs appendices.

Les anneaux sont tous docaucoup plus larges ( au moins six fois), beaucoup plus corivexes et plus durs dans leur moitié supérieure qu'a l'inférieure; la première de ces moities est colorée en brun, la seconde en blanc. Ces anneaux se touchent par conséquent à leur moitié supérieure; ils sont au contraire à l'inférieure éloignés l'un de l'autre et unis seulement par de la peau plus molle; ils peuvent, en outre, se glisser les uns sous les autres, de maniere à ce que la partie antérieure de l'un s'enfonce sous la partie postérieure de celui qui le précède, et que les anneatux les plus postérieurs ne sortent tonl-à-fait des plus antérieurs que lors de la courbure la plus furte de la queue, ou les portions inférieures des anneaux sunt rapprochées au contact. Cette série d'anneaux est termınée par deux plaques qui se suivent et ne correspondent qu'à la moitié supéricure des anneaux, mais ne 


$$
\text { DaNATOME COMPARÉ. }
$$

sont pas aussi larges et sont bien moins convexes que ceux-ci.

Chacun des six anneaux antérieurs porte de chaque còté, au commencement de l'arceau inférieur, un appendice alongé, articulé mobilement; c'est un faux pied. La paire la plus antérieure de ces faux pieds est simple et forme un demi-canal concave en dedans; les autres sont plus composés, ils sont formés de trois pièces, d'une supérieure, pièce basilaire, arliculée avec la portion inférieure de l'anneau, et de denx inférieures fixées à l'extrémité inférieure de celle dernière, pièces appendiciales, dont l'externe est entière; l'interue étant artisulée et velue à son extrémité inférieure qui est libre.

I.e septième anneau est dépourvu de ces faux pieds, qui sont remplacés àsa partie postérieure et aux lamesterminales: $1^{\circ}$ par une pièce testacée, creuse, triangulaire; $2^{\circ}$ par deux lames, l'une externe et l'autre interne, supportées par cette pièce. L.a première est inférieure et se partage, comme la lame terminale supérieure, eu deux autres qui peuvent se glisser inférieurement dans cette mème lame terminale située supérieurement.

$$
\text { §. } 49 \text {. }
$$

Les différences que présente le squelette des crustacés à la surface externe du corps, faisant. 
l'objet de la zoologie, je ne considérerai ici surtout que quelques-unes des variétés qu'offre le squelette intérieur.

La disposition qui vient d'être décrite chez l'écrevisse fluviatile (cancer astacus) tient, sous plusieurs rapports, le milieu entre les deux extrêmes, et a pu par conséquent ètre choisie avec avantage pour représenter le type de cette classe.

Un de ces extrêmes est formé par les lécapodes brachyures (1).

Ces animaux ont le thorax en général trèsgrand et très-large en proportion de sa longueur; il en résulte que le squelette intérieur est ou plus solide, ou beaucoup plus ramassé d'avant en arrière, et plus large que celui de l'écrevisse de rivière.

Ce squelette est formé de deux portions. La postérieure, plus considérable, constitue un canal moyen, déprimé, court et étroit, d'où partent des deux côtés quatre lames verticales, minces et hautes. De ces lames, les antérieures sout dirigées en avant, les deux moyennes en droite ligne et les postérieures en arrière; cette disposition a quelque analogie avec une étoile. Les ouvertures triangulaires laissées entre ces lames reçoivent les quatre paires de pieds postérieurs. La cavité moyenne est parfaitement

(1) Voyez la note de la page 136 de ce volume. 


$$
\text { D’avatume companée. }
$$

fermée par la réunion, de ces lames, mais elle est loin de s'élendre tout-à-fait jusqu'à l'extrémité postérieure du thorax.

La portion antérieure, plus petite, a une forme semblable, et est constituée également par quatre parois transversales de chaque côté. Il n'y a que les deux postérieures qui se rencontrent en haut dans la ligne médiane, et qui, en se réunissant, forment une ouverture que ferme exactement une éminence prolongée d'où s'élève, en outre, une saillie moyenne, dirigée en arrière. Les lames antérieures, beaucoup moins élevées, natleignent pas la ligne médiane; elles ne s'étendent pas nón plus jusqu'à la lame postérieure.

Entre la division antérieure et postérieure existe un grand espace triangulaire, tout-d̀-fait ouvert en haut; c'est une dilatation de la cavité moyenue.

Les anneaux de la queue sont plus plass, la peau de la face inférieure est plus dure; les moitiés inférieures des anneaux sont proportionnellement plus considérables, et il existe inférieurement. entre les grands anneaux dés arceaux intermédiaires, d'un petit diamètre.

Les hermites (pagurus) offrent un rapprochement avec cette disposition du thorax.

Outre que les deux premières apophyses vêrticales, situćes au devant de la premiere paire de pieds, se réunissent dans la ligne médiane, réu- 
nion qui a également lieu chez les écrevisses proprement dites, la seconde et la troisième paire d'apophyses, non-seulement s'atteignent dans la ligne médiane, ce qui produit un canal complet, mais encore sont unies a leur prartie supérieure, entre elles et à la première, par une bande étroite, dirigée d'avaut en arriere; d'où résulte la fermeture du canal dans toute sa longueur. Les apophyses postérieures, au contraire, ne s'atteignent pas dans la ligne médiane.

On connaît le développement imparfait cle leur queue. Les anneaux n'y sont pas parfaits, mais ils y existent d'une maniere distiucte dans leur partie supérieure qui est partout la plus forte; ces portions d'anneaux sont en outre beaucoup plus minces et séparées par des interstices membraneux plus considérables que chez d'autres crustacés. I.es écailles terminales, ainsi que la partie antérieure des autres pièces, offrent une consistance égale à celle que l'on rencontre ailleurs.

\section{S. 50 .}

Chez les scyllares le thorax présente une disposition contraire.

Les apophyses verticales internes et latirales externes existent; elles ont entre elles les mèmes rapports que dans le plus grand nombre des crustacés; mais il n’y a que la paire la plus antérieure, située contre les premiers picds, qui, par sa réunion dans la ligne médiane, forme un an- 
neau; les autres divergent très-fortement en haut et en dehors, et n'envoient point de lames saillantes en dedans; raison pour laquelle le demicanal moyen est très large, peu haul, et sans trace de voùte qui le recouvre. Les parois latérales et verticales de la totalité du thorax sont au contraire plus épaisses et plus dures que je ne les ai vues partout ailleurs. Elles sont, ainsi que les portions moyennes, tout-à-fait confondues en un tout, conformément au type des astacoïles.

\section{S. 51 .}

Celte disposition forme le passage à celle quoffrent plusieurs décapodes, par exemple les penées (penceus), de plus, les stomapodes ou mantes de mer, notamment les squilles, c'està-dire, vraisemblablement tous ceux qui ont la queue fort développée, ou les macroures (1).

Les saillies moyennes, verticales et latérales, existent; mais elles sont peu élevées, minces et trì-éloignées les unes des autres, tant celles qui diffèrent par la position que celles de même nom; les internes sont à une grande distance de la ligne médiane, en sorte que les ouvertures el les canaux ne sont entourés que de bords irés-faibles et pas tout-à-fait fermés. Ces apophyses internes ne s'atteignent pas dans la ligne médiane.

(1) Voyer la note de la prage 136 de ce volumc. 
Dans tous ces geures, les anneaux qui forment la partie inférieure du thorax sont entièrement séparés les uns des autres : chez les squilles la partie superieure, oule bouclier, présente mème cette division. Les parois latérales sont faibles et en grande partie membraneuses.

Au développement plus considérable de la queue, chez les squilles, se rattache la présence de faibles apophyses dans la portion inférieure de la plupart des anneaux antérieurs de la queue, mais qui, comme celles des anneaux du thorax, sont très-éloignées les umes des autres et nioffrent aucune tendance à se confondre ní d'un côté à l'autre, ni d'avant en arrière.

Les pieds offrent des différences extrèmement considérables sous les rapports de la forme, du volume et même de la position.

Chez plusieurs déca podes brachyures, les pieds, surtout la dernière paire, sont considérablement aplatis de haut en bas; ils sont destinés à la natation. Cette disposition est surtout marquée aux deux dernières portions de la paire postérieure, tandis quaux pieds antérieurs la dernière division est étroite el apointie.

(ivelques décapodes brachyures ont les deux dernières paires de pieds sur le dos.

Chez les squilles, les cinq pieds ordinaires ou thoraciques, surtout ceux de la seconde paire, sont d'une longueur considérable et comprimés latéralement dans le sens longitudinal. Ceux de 
l'avant-dernière paire sont les plus larges; leur bord autérieur forme une gouttière dans laquelle s'implante le dernier pied, qui forme un crochet pointu. Les trois premiers pieds de la queue sont minces, alongés et formés de quatre articles, doni le troisieme porte un crochet mince, mais également dur. Les picds des six derniers segmens de la queue sont plus courts, mais beaucoup plus lärges et plais. Une pièce basilaire, large, supporte deux lames, une exierne et une interne, larges, minces, arrondies, formées, latéralement et de la base au sommet, de plusieurs parties plus ou moins distinctes. Aux cinq premières paires de ces pièces, on voit à la partie interne de la base de la lame externe, une branchie considérable. 11 n'y en a pas à la sixième paire, qui est beaucoup plus, développée, principalement dans sa lame externe, plus distinctement divisée dans le sens longiturinal, et dont la pièce basilaire commune est fort alongée.

\section{S. 52 .}

Parmi les autres crustacés, les amphipodes et isopodes de M. de Latreille ont la tête beancoup) plus distinctement séparée du reste du corps que les autres; chez les entomostracés (entomostracia Müll., branchiopoda Latr.), elle est confondue avec le thorax.

Il résulte de cetle disposition que le reste du 
corps, dans les deux premiers ordres, se partage en un nombre considérable d'anneaux ou mieux de demi-anneaux; car la partie supérieure et postérieure du corps est seule divisée de cette manière; l'inférieure étant membraneuse, beaucoup plus mince et continue. ILe corps des branchiopodes constitue au contraire bien plus exactement une masse unique, qu'il existe ou non un grand bouclier dorsal.

Je n'ai trouvé dans ces animaux aucune trace de squelette intéricur, du moins chez les cymothoés, les talitres et les apus. Il existe peut-ître ('hez les limules, que je n'ai pas pu me procurer.

Les espèces de ces trois ordres de crustacés offrent aussi de grandes différences sous le rapport des pieds. La plupart de ceux-ci sont composés de plusieurs parties : chez les amphipodes ils forment une pince anterieurement, tandis que chez les isopodes et les branchiopodes ils se terminent ordinairement par un crochet simple, mais souvent très-fort. Plusieurs des derniers, comme les apus, etc., possèdent des pieds en forme de rames, qui sont, comme par exemple chez les limules et caliges, compliqués de demianneaux, et chez les argules mèrie de ventouses, placées au devant des pieds comme des rames. La plupart des pieds de ce dernier ordire sont munis de branchies. 


\section{GHAPITRE V.}

SQUELETTE DES CIRRIPIDES.

\section{S. 53.}

Les cirripèdes, par la disposition de leurs parties solides, conduisent des crustacés aux mollusques.

Le test ou le squelette extérieur se compose, chez les lépas aussi bien que chez les balanices, de deux paires de valves trianguiaires, alongées, faiblement convexes en dehors, situées dans la peau molle qui les unit entre elles d'une manière mobile; les sommets de ces valves sont tournés en haut, et la supérieure est plus grande que l'inférieure.

Chez les lépas ces pièces sont unies d'une manière moins étroite; la supérieure est encore considérablement plus petite que l'inférieure. Dans quelques espèces, par exemple le lepas coriacea, elles sont exiraordinairement petites en proportion du reste de la peau et du volume de tout l'animal.

Dans les lépas, il existe en outre une petite piece particulière, moyenne, scaphoïde, qui s'étend, à la face dorsale, entre les deux paires de valves presque jusqu'à l'extrémité supérieure de tout l'ensemble. 
Cette valve impaire manque dans les balanites, du moins je ne l'y ai pas trouvée; mais à sa place il existe un second test beaucoup plus épais, qui enveloppe tout l'animal. Celui-ci se compose de quatre pièces triangulaires, qui, du moins dans plusieurs espèces, se replient sur elles-mèmes lans leur partie inférieure, sous un augle droit. Elles sont exactement appliquées les unes contre les autres dans tout leur trajet, se confondent mème ensemble dans leur partie inférieure; tandis que dans leur partie supérieure, plus considérable, elles sont unies d'une manière presque aussi intime par une suture formée de saillies transversales, très. rapprochées les unes des autres. De là résulte une espèce de coupe, ouverte en haut par un orifice étroit, close en bas par un fond; coupe dans laquelle l'animal, libre supérieurement, vit attaché inférieurement au moyen de muscles.

La transition qui couduit à cette organisation me semble offerte par quelques lépas, par exemple le lépas pollicipes, où la valve supérieure et intérieure est en proportion plus considérable qu'il n'est habituel de la voir; l'inférieure se partageant en une pièce antérieure plus considérable et en une grande quantité d'autres plus petites, qui, si elles étaient portées en dehors et élevées davantage, représenteraient l'organisation des balanites.

Outre cet appareil testacé, on trouve, à la par- 


\section{D'ANATOMIE COMPARÉE.}

tie antérieure de la face inférieure du corps, de chaque còté, six paires de cirres mobiles se succédant de très-près d'avant en arrière et trèsvoisins de cenx du còté opposé. Les deux cirres de chaque paire sont disposés très-près l'wn de l'autre, de dedans en dehors, sur un long pédicule charnu. Ils sont comme cornés, creux, distinctement articulés, et se terminent par une pointe très-alongée. Ieurs bords antérieur et postérieur sont munis de soies délicates, non articulées; celles de ces soies qui sont situées au bord postérieur et convexe, sont beaucoup plus petites, en moindre nombre, et n'existent qu'i l'extrémité antérieure de chaque article; les antérieures sont beaucoup plus longues et occupent tonte la partie antérieure de la circonfé. rence du cirre, quoiqu'elles forment aussi, pour chaque article, un faisceau composé d'environ douze soies inégalement développées. Les cirres deviennent plus grêles d'arrière en avant; de sorte que la première paire présente à peinc le quart du volume de la dernière. Elle est en mème temps beaucoup moins distinctement articulée, plus molle et d'une coloration plus claire que les autres, chez lesquelles le nombre des articles diminue également d'une manière insensible.

Un fait digne d'intérêt est que, chez les lépas, cespartiesscient proportionuellement beaucoup plus développées que chez les balanites; il en re- 
sulte un antagonisme entre le développement des valves de ceux-ci, qui ressemblent à des co. quilles de mollusques, et ces parties des lépas qui correspondent aux pieds des crustacés. Les cirres des balanices sont en effet beaucoup plus petits, plus minces, plus mous, moins distinctement articulés et plus faiblement ciliés. La première paire seule fait ici une exception; du moins l'un des cirres est, chez les balanites, en proportion considérablement plus long que chez les lépas. Le cirre externe, qui est plus court, offre en outre cela de particulier, que ses articles portent, à leur bord interne, une apophyse considérable, sur laquelle sont implantés les cils ou soies. 


\section{CHAPITRE VI.}

\section{SQUELETTE DES MOLLUSQUES.}

\section{\$. 54 .}

Si on sépare les céphalopodes des mollusques (1), on peut dire que ces derniers n'ont très-généralement qu'un squelette extérieur formé par l'endurcissement de la peau et connu sous le nom de coquille (2), qui offre les plus grandes variétés sous le rapport de la présence, du volume, de la forme, du nombre el de la composition chimique des pièces qui le composent.

Cette coquille, si elle existe, est formée de plusieurs lames superposées, se formant peu à peu de dehors en dedans, qui s'appliquent les unes

\section{(1) Voyez vol. I, 1. 97 et 193.}

(2) La coquille est un produit sécrété. Ce sont de petits pores placés dans le rebord libre du manteau qui opèrent cette sécrétion. La matière produite est déposée dans une partie de l'épaisseur de la peau, an-dessous de l'épiderme, le pllus communément entre le réseau vasculaire et le pigmentum de cette enveloppe, quelquefois dans l'épaisseur du derme lui-mème. Elle ne corsiste pas en un endurcissement de la peáu par un arnas de molécules calcaires déposées dans les mailles d'un tissu cellulaire, mais bien en un dépôt de malière mucoso-crélacée. Ducrotay de Blainville, Manuel de malacologie et de conchyliologic. Paris, 1825 , pages 86 et suivantes.

II. 
contre les autres daus toute leur étendue, ou qui restent plus ou moins sćparcées vers leur bord libre. Je développement de ces lames n'est pas uniforme, mais il se fatit a différentes époques de l'année (1). Lille offre communément un haut

(1) Ces couches superposées sont suriout évidentes dans les Huîrnes. On a donnéle nom de stmecture feuilletéc a la dis position offerte par la cuquille de ces animanx. Les bords de's lames quise voient à la face externe de la coquille cons li uent ce qu'on nomme les stries d'accroissement. La structure forillticic devient surlout évidente après que ces coifuilles, formées de celte manière, ont été exposées à la chaleur et à la longue action de l'air, a cause de la destruction de la matière muqueuse qui liait non-seulement les molícules de chaque lame, mais encore les couches superposées.

La structure des peignes et des patelers differe de celle deruicre; les couches composantes y sont mieux licies, les molécules calcaires y sont plus rapprochées; ces corquilles peuvent être cliauffées fortement sans se déliter.

Les Jamronneaux présentent une antre disposition : les molécules calcaires, en formant des lames composantes, se correspondent et se placent au-dessus les unes des autres dans toutes les couches qui composent la coquille. Cette siructure est la structure fibreuse.

Dans quelques cocpuilles les denx structures alternent; une partiede leur épaisseur est simplement ferilletíe, lantre est fibreuse; c'est une structure fibro-Irincelleuse. Les coquilles nacrées se rapprochent beuncoup des dernières; la partie nacrée semble être toujours lamelleuse, et l'autre fibreuse et plus ou moins oblique.

Il existe encore une autre sorte de structure: c'est la simicture vitreuse; elle est produite a l'eporue ou la cocpuille est parvenue an degré de grandenr dont elle est susceprible; le 
degré de consistance, qui provient d'une grande quantité de carbonate de chanx, quelquefois mòlé

derme paraît sécréter alor's une plus grande quantitć de matiere calcaire et moins de matière muqueuse; les molécules sont plus serrées, plus entassées et plus uniformément disposées; les frottimens du manteau achèvent avec l'âge de polir la surface du dépôt.

On trouve des exemples de la structure vitreusc particulierement a louverture de la coquille dans les casuues, les puncelatiss, elc. C"est cetle maticre qui remplit les trous dont cette coquille peut étre percéc par accident; c'est de cette matière qu'est formée la partie postérieure de la spire des coquilles turriculées, ce qui force l'animal à l'abandonncr. La substance calcaire de structure vitreuse est très-cassante, et dans toutes les directions; cela permet d'expliquer la ciécollation de la spire dans plusieurs mollusques céphalés.

Les couches composantes sont rarement colorćes; la coquille est le plus ordinairement blanche; une coquille completcment dermale n'est pas colorće, parce que le pigmentum est restí à la partie de la peau qui la recouvre.

La coloration des coquilles peut donc être due au pigmentum; teile est la coloration de leur surface externe: elle peut en outre dépendre de l'arrangement mécanique des molícules calcaires; tello est la coloration irisée, comme le prouvent les expériences de 11 . Brewster; elle est quelquefuis, a la surface interne de certains bivalves, le résultat d'une imprégnation (fui s'étend pert à peu en surface et en profonleur, et rue produit une humenr formée par quelrue organe de l'animal qui la teint de cette manière.

Les borls du mantran fonrnissent seuls la premiere de ces colorations, celcest párliculierement lorsquel'animal est jeune et cu’il est expres: a linfluence de la lumiere, que les couleurs sont plus varićes et plus intenses.

lifaumur a fait a ce sujel des expériences; cette coloration

11. 
d'une petire portion le phosphate. Ce carbonate est uni à la subslance albuminiforme ani-

est superficielle et se détruit par la chaleur. Dans les PORCEratines elle est plus profonde. Bruguieri a expliqué ce fait. Lorsique l'animal est adulte, les bords découpés du manteau. pourvus l'espaces fournis de pigmentum, déposent, avec la malière éburnée qui épaissit la coquille, une nouvelle sorte de matière colorée qui est disposée jar taches, parce que les espaces ne tombent pas justement sur les lieux de premier dépôt.

L'ordre dans lequel se fait le dépôt de couches successives qui forment la coquille est tel, que la moins étendue et la première formée est aussi la plus externe.

La forme de la coquille est entièrement subordonnée aux formes mêmes du mantean; $€$ tle est découpée et prolongée en épines creuses, si le manteau est lui-même découpé et présente des lanières; cesse-t-il d'offrir à certains âges ces lanières $\mathrm{ft}$ ces découpures, la coquille s'accommode encore à sa forme, et sa nouvelle disposition en reproduit le dernier changement.

L'accroissement dé certaines coquilles est uniforme; celui d'un certain nombre d'autres est sujet à des intermittences. Dans les premières les stries d'accroissement se recouvrent d'une manière presque insensible: dans les secondes, des intervalles macrués entre les couches diverses, ou des bour. relets saillans indiquent ces intermittences. Au terme de l'accroissement, la coquille présente un bourrelet; si la matière vitreuse qui le forme conlinue is se déposer, l'ouverture peut en être fort diminuce el être garnie d'un péristôme continu. Les dents qui modifient l'orifice d'une coquille univalve sont produites par les cannelures du inanteau.

Les coquilles présentent des échancrures, des sinus et des impressions qui sont le produit de la pression de quelqu: organe, et souvent des prolongemens du manleau ou des 
male, qui constitue à elle seule la coquille beaucoup plus molle qui est propre à certains molLusques.

On peut, en traitant les coquilles avec des acides étendus, en précipiter le sel, de sorte qu'il ne reste que la matière animale conservant la forme de la coquille. Les coquilles molles se trouvent chez plusieurs gastéropodes, par exemple dans les aplysies et les pleurobranches, où elle est réduite à n'être qu'une petite lame mince, cachée en totalité ou en majeure partie dans la peau du dos. Lienveloppe des acéphales nus, quoique bien plus élendue, doit sans contredit èłre rangée ici; elle est à la coquille des acéphales testacés ce que le squelette des poissons cartilagineux est à celui des poissons osseux. Il est digne de remarque qu'elle u'offre point de traces de divisions analogues à celles des coquilles; on voit également la tête des poissons cartilaginemx se distinguer de celle des poissons

insertions musculaires. La coquille est d'abord membraneuse, elle ne se solidifie que graduellement. Les élémens calcaires en augmentent avec l'âge. Par les progrès de cette circonstance, les tubercules et les piquans que présentaient la coquille cessent d'ètre tubuleux, ils s'usent et s'effacent; les sinus s'agrandissent, les couleurs se ternissent, la coquille se: convre de dépôt crétacé, la capacité en diminue, les univalves se cloisonnent. Quand la mort de l'animal est arrivéc, ia substance solicle se cristallise, elle devient très-friable. 1) ucr. de blainv., Manuel de malac., p. 86 et suivantes.

(Nole des traducleurs.) 
osseux par le tres-petit nomlire de pièces dont elle est composée.

La coquille est liće aux autres parties par les mêmes rapports que cenx qui unissent les autres organes entre cux; on peut démontrer qu'elle reçoit des vaisscaux d'autres régions du corps.

La présence de piéces calcaires, grandes et petites, qui, daus quelques espèces, par exemple, chez l'arion de M. de Férussac, constilucut la coquille, n'est pas un fail contradictoire à la dépendance mutuelle de cetle partie de l'animal et des autres organes; puisque ces pieces sont unies entre elles par du tissu muqueux.

On peut élever contre l'opision d'apres laquelle la substance de la coquille serail forméc dans un organe particulicr, le sac calcuire, les mêmes objections qui ont été fatites plus haut (1), au sujet de la formation du test des échinodermes.

Les mollusques qui viennentimmédiatement après les cirripèdes sont, à l'exception des oscabrions, les mollusques multivalves, dirisiou la plus inférieure desacéphales, dans laquelle on comprend avec raismu les conchifires crassipèdes de M. Lamarck.

On trouve chez les tare's (terecto), a l'extrémité antérieure et a la postéricure du corps qui est très-alongé, deux petites coquilles laté-

(1) Pag. 22-23. 


\section{D'ANATOMIE COMPARÉ.}

rales symétriques, qui sout par conséquent séparées l'une de l'autre de toute la longueur de l'animal. Les pièces principales, siluées à l'extrénité buccale, sont les plus considérables; la furme en est très-convexe; leur plus grande largeur' s'étend de la face dorsale à la face abdominale; elles sont plus élevées à la première qu’à la seconde, et offrent à la partie postérieure de la face interne plusieurs saillies servant de points d'attache aux muscles.

Les valves situées à l'extrémité opposée et qui entourent les deux tubes par lesquels le corps de l'animal se termine, sont aplaties, oblongues; leur plus grande étroitesse existe à la base, par laquelle elles sont fixées dans le manteau.

Je doute que le tube, ouvert aux deux extrémités, qui renferme le corps de l'animal, lui soit aussi étranger qu'on l'admet communément. Malheureusement je n'ai eu occasion d'examiner que des individus sortis du tube; mais j’ai trouvé souvent quelques pièces de ce tube encore attachées à l'extrémité postérieure de l'animal et trés-intimement unies avec le manteau. Joignez it cela que, dans les genres voisins, aspergillum et clavagrella, les valves sont fixées dans le tuhe.

Il est assez vraiscmblitble que les cing valves des lepas, ainsi que la coquille externe et le., quatre valves intérieures des lalcnites corres. pondent aux quatro valves of au tube des tarets.

Chro les pholadnines la paire de valves, qui est 
la seule existante chez les autres bivalves (1) a pris sur les pièces accessoires un développement proportionnellement plus considérable; celles-ci existent, mais ne se trouvent pas toujours à la même place.

(1) On a séparé des bivalves les tarets et genres voisins; ils font partie des tulivalves. Le nombre des pièces qui entrent dans la composition des coquilles non tubuleuses a servi à les partager en coquilles : $1^{\circ}$ univalves, qui sont formées d'une seule pièce; $2^{\circ}$ subbivalves, dans lesquelles on rencontre une pièce principale et une pièce accessoire; $3^{\circ} \mathrm{bi}$ valves; $4^{\circ}$ tubivalves, dans lacomposition desquelles entrent deux pièces principales et une pièce accessoire; $5^{\circ}$ multivalves, que constitue un grand nombre de pièces.

Les multivalves sont de trois sortes: $1^{\circ}$ les sériales, qui sont composées de plusieurs pièces transversales, imbriquées comme dans les oscabrions; $2^{\circ}$ celles qui sont formées de cinq valves ou plus, symétriquement rangées à droite et à gauche et quelquefois même placées en écailles et réunies entre elles au moyen de la peau comme dans les $\Delta$ Matiges; $3^{0}$ enfin celles qui sont disposcés d'une manière circulaire, comme dans les baLAres et genres voisins. Ce sont les subcoronales de M. de Lamarck.

Les tubiralves ont les deux valves des bivalves proprement dites, mais sont entourées d'une troisième pièce en forme de tube, les tanets, les pholades, les fistulanes, etc.

Les bivalves ont deux pièces appliquées sur les côtés de l'animal, et dans un rapport plus ou moins étroit avec lui.

Les subbivalves ont aussi deux pièces; mais l'une d'elles est analogue à la coquille univalve; l'autre est une pièce accessoire : ce sont des univalves operculées.

La forme de la pièce unique qui forme les coquilles univ'alves est très-variable. De Blainville, ouvrage déja cité.

( Note des traducteurs.) 
Il n'entre pas dans mon plan de décrire plus longuement les coquilles des bivalves, je ferai remarquer seulement ce qui suit (1):

I L'extrémité supérieure de la face interne. de ces coquilles présente très-généralement, sur le bord supérieur et au dessous, plusieurs. saillies et enfoncemens : ces parties, variablement prononcées, s'engrènent et forment la

(I) On a placé diversement la coquille bivalve pour en détcrminer les différentes régions. Réaumur, qui a été imité par Müller, Dacosta, Draparnaud, M. Cuvier, etc., et qui a été également suivi par M. Meckel, a supposé qu'elle reposait sur sa tranche, le ligament qui unit les deux valves étant placé en haut, comme si l'animal eût marché devant l'observateur. Dans cette direction, le ligament est. en arrière du côté de ce dernier, et la partie plus ou moins saillante, vers laquelle convergent les bords, est placée en avant.

On a distingué à la coquille ainsi posée un diamètre vertical que M. de Blainville nomme sa hauteur. Ce diamètre cst étendu du ligament à la tranche. Sa longueur est constituée par le diamètre opposé à celui-ci. L'extrémité antérieure de ce diamètre est désignée par l'appellation d'ouverture buccale; la postérieure est anale.

Le bord ou se trouve le ligament et qui correspond au dos de l'animal a été nommé bord dorsal; il présente en avant le somnet ou les sommets; celui qui forme la tranche est appelé bord abdomial. I a circonférence de la coquille est constituée par tous ces bords (margines). Le limbe est compris entre les bords. L'une des valves est gauche, l'autre droite. Linnée, H. II Lamarck et Bosc ont considéré la coquille dans le sens inverse par rapport au bord dorsal et abdominal; mais les còtés restent les mêmes. (De Blainville.)

(Note des traducteurs.) 
charnière ( cardo). Celle-ci offre des degrés de composition très-variés. Dans quelques genres, par exemple le genre acarde ( acardo), il n'en existe pas la moindre trace; l'huître (ostrea) n'offre qu'un léger enfoncement dans chaque valve. On trouve au-dessus de la charnière, à l'extérieur de la cavité de la coquille, un ligament très-élastique, composé en partie de substance fibreuse, en partie de substance musculaire; cette production étendue d'une valve à l'autre agit par son élasticité en sens contraire de l'action contractile des muscles qui rapprochent les valves.

$2^{\circ}$ Les valves sont très-généralement symétriques sous tous les rapports; cette règle souffre des exceptions. Il est des animaux dans lesquels ces pièces de la coquille offrent des dissemblances, soit de volume, soit de forme, soit de coloration.

$3^{\circ}$ Les valves sont ou toutes deux libres, ou l'une d'elles est altachée au sol ou à d'autres corps. Aver cette circonstance coïncide généralement le défaut de symétrie le plus prononcé.

Il n'est pas non plus rare de voir les valves de mollusques bivalves, parfaitement libres, offrir elles-mêmes un haut degré d'asymétrie. La plus remarquable, quand on fait abstraction du volume et qu'on n'a égard qu'à la forme, est sans doute offerte par les térébratules.

Dans ces ètres, non-seulement l'une des valves est plus convexe que l'autre, matis elle la de- 
fuisse mème considćrablement au point de leur union par me apophyse mousse un peu recourbéc. Elle est en ontre, au milieu de cette partie, perforée par une grinde ouverture arrondie. De chaque còlé de cette ouverture et de la base de la valve nait une apophyse coracoïde considérable qui s'engrène au moyen d'un enfoncement correspondant avec la valve opposée; la face interne de cette dernière est surtout remarquable par la dent qui la surmonte en dedans; dent qui ne se borne pas, comme de coutume, à la région du bord supérieur de la valve, mais qui s'avance jusqu'au-delà du milieu de sa face interne. Ia parie supérieure, qui en est la plus considérable, présente la forme d'une losange; elle est creusée de trois enfoncemens, dont l'un, beaucoup plus grand que les deux autres, est situé au milieu. Ces enfoncemens reçoivent les deux dents latérales (1) de la valve supérieure. Du milieu du bord inférieur de cette partie, on voit une saillie étroite, mais élevée, se diriger exactement dans le sens de la ligne módiane de la face interne vers le bord inféricur; elle se termine un peu au dessous du milieu de la face interne; en avant de sa termillaison, immédiatement sous son bord in-

(1) Los ćmineroes que présente la charnière sont appelées drenl. Les principales qui sont sur le sommet sont les den!s cardinales. Les dents bacérales occupent les còtés du somzris:1,

(Note des troducterers.) 
terne et libre, on en voit naître, en avant et en arrière, deux apophyses latérales, transverses, d'un volume assez notable; disposition qui rappelle incontestablement le squelette intérieur de la plupart des animaux articulés.

L'organisation des coquilles des bivalves semble se lier par plusieurs voies transitoires à celle des céphalophores. $1^{\circ}$ Ces passages sont principalement fondés sur le manque de symétrie offert par les céphalophores, chez lesquèls une des valves disparaît insensiblement.

Ce rapprochement rend très-digne d'intérêt l'existence d'un opercule ( I ) à la face inférieure du pied de plusieurs gastéropodes. Cet opercule, formé de plusieurs couches et coloré de la même manière que la coquille, semble être, quoique imparfaitcment, une répétilion de celle-ci; placé à l'orifice de la coquille, il en clot l'ouverture quand le pied est rentré, et se comporte comme on voi t une valve se comporter à l'égard de l'autre chez les mollusques bivalves (2).

$2^{\circ}$ Un autre passagge est établi, si je ne me

(1) Liopercule est une production de la pean du pied. Il varic beaucoup en volume et en forme. Les variations de formes sont liées aux diversités de configuration de l'ouverture. Sa composition est égaleınent variable; on doit le distinguer de l'épipluragme, pièce indépendante de la cuquille.

(Note des traducteurs.)

(2) Cette analogie de l'opercule avec une valve, avancec d'aboud par Adanson, est contestéc par M. de Blainville, qui 
trompe, des bivalves symélriques par les genres fissurelle, émarginule, patelle, pleurobranche et halyoticte, aux autres genres. Supposez deux valves symétriques soudées ensemble dans la ligne médiane, qu'il reste une lacune au milieu ou sur un point de la circonférence, vous aurez la coquille des fissurelles ou émarginules; si ces lacunes disparaissent, ce sera la coquille des $p a$ telles, qui n'est pas encore contournée. Chez les halyotides la coquille se contourne; mais elle est très-plate et ne présente que des comrbures indistinctes. Plusieurs coquilles courbées et contournées, mais symétriques, nous citerons en particulier le capulus de Montf., forment des transilions insensibles entre les coquilles plates ou les patelles proprement dites et les halyotides qui se distinguent par leur peu de symétrie.

L 'exactitude de celte manière de voir est confirmée évidemment par les traces nombreuses dorganisation transitoire que présentent les genres que nous venons de mentionner dans toute leur structure intérieure.

$\bar{j}^{\circ}$ Les oscabrions (1) paraissent être le chaî-

se fonde sur ce que les deux valves d'une coquille bivalve correspondent chacune à un côté de l'animal; tandis que dans les subbivalves la coquille correspend constamment a la face drorsale et l'opercule au pied. (Voyez Manuel de malacologic, P. 115.)

(Note des tratucteur.s.)

(3) Les coquilles de ces êtres sont des multivalves.

( Note des traducteurs:) 
non intermédiaircqui lic les tarets, les pholariaires, etc., aux gastéroporles. I'cut-circe le point d’intersection de cette transition avec la précédente est-il offert par le gemre patclle, dont on peut concevoir aussi bien la coquille comme résultant de la soudure des valves, que las oscabrions ( chitons) présentent disposéc's dans un ordre successif d'avant cu arrière, que de la disparition de toute trace de scivation ente les denx moitiés latérales.

Iues valves des oscalurions, qui sont tres-généralement au nombre le huil, se recouvrent les unes les autres d'avant en arrière, et sont fixées lâchement à des éminences transverses du mauleau, formées de muscles et de tendons qui leur eorrespondent.

İa plus antérieure et la plus postérieure des valves sont arromlies vers l'extrémité correspondante du corps; les autres ont un bord antéricur et un hord posterien droils. Cics valves terminales sout plus larges d'avant en arrèere, plus étroiles d'un côté à l’autre que les valres intermédiaires. Toutes les valves, à l'excepion des premières, portent de chaque cúce de leur bord antérieur, une apophyse peu large, mais assez longue, dirigée de dehors cu dedans el de bas en hatut; cetle apophyse s'applique sous la portion musculaire qui précéde immódiatement la valve.

Immédiatement au-dessus de ces valves prin- 
cipales, il existe ordinairement, sur toute la périphéric du manteau, une multilude de petiles pièces testacées, oblongues, très-rapprochées les unes des autres.

Il est plus rare de trouver en ces endroits des faisceáux isolés de soies dures.

Arec le genre halyotide commence la disposition contournée de la coquille des univalves (1). Nais la coquille de ces auimaux est cncore trèsalongée, déprimée, plate et extrêmement peu contournée. On y remarque seulement: $1^{\circ}$ is lextrémité postérieure de la face externe de la

(I) On distingue aux coquilles univalves : $I^{0}$ un sommet qui est le point d'origine; $2^{\circ}$ une base qui en est le terme; $3^{\circ}$ un corps compris entre ces deux points cxtrêmes. On place l'animal d'une coquille univalve daus les mìmes rapports que celui d'une coquille bivalve. Il en résulte, en admettant la méthode adoptée prar Réaumur, MH. Cuvier, de Blainville, etc., que le sommet est en haut et en arricre, l'ouverture ou la base en avant et en bas; qu'il existe deux côtés à la coquille, l'un droit et l'autre gauche, et que l'un des bords de l'ouverture est antéricur et l'autre postérieut.

Le corps de cerlaines univalves est sans excavation; il est tubuleux dans quelrues autres et très-conlourné. Cet enroulement du corps de la corquille constilue la disposition des corkleürles ou spirivalues. Généralement les lours de spire se sont opérés du sommet à la base en procédant de grauche a droile : celles-ci sont les spirales normales; d'antres s'enromlent en sens contraire. La cavité de la coquille est uniloculaire, monothalame, ou multilocalaive, polylialame, cliambrie, cellulée et nème cloisonnép, eic. (De Blairville, Mrunuel de malacolorgie. Paris, 1825.)

(Note des traducteurs.) 
coquille, surtont vers son bord droit, un tour de spire court et peu élevé, dirigé en haut et à gauche, mais distinct du reste de la surface de la coquille; $2^{\circ}$ à la face interne, un enfoncement contourné, correspondant au tour de la spire; cet enfoncement est imparfaitement séparé du reste de la cavité fort aplatie de la coquille, par une saillie droite, un peu concave à gauche, qui s'étend depuis la pointe de la coquille jusque vers l'extrémité postérieure du bord gauche; $5^{\circ}$ une différence considérable entre le bord droit et le bord gauche; le premier étant plus petit, plus droit, simple, tranchant et tourné en dehors; le second commencant à la saillie dont il vient d'ètre question, étant plus long, bien plus convexe et proéminant en dedans dans presque toute sa loagueur, à l'exception de sa partic la plus antérieure; $\mathbf{t}^{\circ}$ enfiu la série longitudinale d'orifices le long du bord. Ces orifices augrmentent de capacité d'arrière en avant, deviennent plus nombreux et plus considérables avec l’âge; mais les postérieurs s'oblitèrent à mesure qu'il s'en forme de nouveaux à la partie antérieure.

Si d'une part la convexité plus considérable, si la disposition distinctement spiroïde, mais encore symétrique de la coquille du capulus mine des patelles aux halyotides; si la convexité plus profonde, si un contournement plus prononcé de la coquille, et une saillie plus 
forte du bord sauche de cetle pièce calcaire, daus le genre stomale, conduisent des halyolides aux autres univalves; il existe d'autre part d'autres genres voisins, dont la forme extérieure établit un rapport plus étroit avec les patelles, que la disposition intérieure de leur coquille rapproche davantage des univalves plus fortement contournés; chez ces genres, en effet, la cavité de la coquille esı b́eancoup mieux séparée par une saillie que chez les halyotides et les stomates. De ce nombre est le genre calyptrée, dont la coquille est conoïle, pointue, courte, presque pas contournée, surmontée à sa face interne et à son sommet par une saillie verticale, peu élevée, concave, et nes'étendant pasjusqu'au bord de l'orifice. La mème disposition s'observe dans les genres navicelle et crépidule, dont la coquille est alongée et présente surtout dans le dernier une forte saillie horizontale dirigée de la partie postérieure de la circonférence en avant. Les crépidules sont incontestablement le plus rapprochées des autres univalves, puisque les tours de spire de leurs coquilles sont toujours dirigés à droite. et par conséquent ne sont pas symétriques, tandis que les coquilles des autres offrent uns symétrie parfaite ou presque parfaite.

Une autre disposition curieuse des navicelles consiste dans l'existence diune pièce testacée, située dans l'intéricur du rlos quiforme le passage aux oscabrions. 
Chez les antres univalves la coquille devient plus convexe. Le bord droit se comporte comme chez les halyotides et genres voisins, mais le gauche se recourbe plus fortement en dedans; disposition dont nous avous déjà signalé les indices. Ce bord se renfle d'une manière variable vers la droite, et forme ainsi une coquille intérieure plus composée dans la coquille primitivement extérieure qui recouvre la première, Cette coquille intérieure, née d'un développement considérable du bord gauche de la coquille principale, forme ordinairement un grand nombre de tours de spire qui sont dirigés de l'extrémité postérieure à l'antérieure; de ces tours de spires, les internes et les postérieurs deviennent insensiblement plus petits; ils se contournent de droite à gauche autour d'un axe central, renflé, souvent crenx. qui s'étend depuis l'extrémité antérieure de l'urifice de la coquille et du point de jonction du bord droit avec le gauche jusqu'au sommet ou à l'extré mité postérieure de la coquille. Cet axe, la columelle, a la forme d'une tige solide, et représente la moitié gauche et la plus petile du bord de ce côté qui se divise en deux branches d'avant en arrière.

Au point de jonction des bords gauche et droit, on trouve à gauche, chez beaucoup d'univalves, un demi-canal d'une longueur variable; ce n'est souvent, dans un grand nombre. qu'une simple échancrure; cette ou verture reçois 


$$
\text { n'ANATOMIE COMPARÉE. }
$$

un prolongement correspondant de la cavilé respiratoire de l'animal.

Ce n'est pas ici le lieu d'indiquer les différences presque infinies qu'offre la forme de la coquille.

J'ai déjà parlé de l'opercule (1), que l'on rencontre dans plusieurs gastéropodes.

(1) Voyez p. 172 


\section{GHAPITRE VII.}

SQUELETTE DES CÉPHALOPODES.

\section{\$. 55 .}

Les céphalopodes, si généralement admirables par toute leur structure, représentent d'une manière très-remarquable, dans la disposition de leurs parties dures et de tout leur appareil de locomotion, des réunions de formes séparées. ailleurs; car un très-grand nombre d'entre cux possèdent non-seulement une coquille, dont l'état de perfectionnement est variable, mais peut-être présentent-ils plus généralement encore un rudiment de squelette intérieur; squelette qui est cartilagineux, et qui existe à divers degrés de perfection. Ajoutons que ces êtres portent en outre, au pourtour de la bouche, des bras ou pieds munis de suçoirs. Plusieurs espèces sont mèmes pourvues de pointes disposées en crochets.

$$
\text { S. } 56 .
$$

La coquille (1), qui ne manque qu’aux octopo. des, est ou externe ou interne. La première disposition est beaucoup plus rare, n'existe peut-ètre que daus l'argonaule, et pas mème encore cer-

(L) La coquille des céphlalopodes est inoperculce et polythalame.

(Note des traducteurs.) 
lainement dans ce genre (1). Cette coquille, soit intérieure, soit externe, est symétrique et le plus souvent spiroïde, comme dans les univalves. La coquille spirale intérieure de la plupart des animaux de cette classe, nous nommons en particulier les nautiles et genres voisins, est en grande partie fossile; elle offre, en outre, cette particularité, qu'elle est formée de plusieurs loges disposées successivement d'avant en arrière, et séparées les unes des autres par des cloisons verticales, qui sont rraversées, dans beaucoup d'espèces, par un tube ou interrompli ou continu, destiné à les unir entre elles, et renfermant peut-être, dans les nautiles, un ligament; ce ligament étendu dans toute la longueur de la coquille allacherait celle-ci au reste du corps de l'animal. Ce caractère devient au reste trés-invraisemblable par l'absence du ligament précité chez les spirules, où la coquille contournée est située dans l'intérieur du dos.

Daus les genres sciche et calmar, la coquilie n'offre pas de iraces distinctes de circonvolution; on la reconnaît surtout dans le premier. Elle est située, chez l'un et l'autre, dans une cavité particulière de la face dorsale, à laquelle rien ne l'unit, rt rout la paroi inférieure, mince, la sépare de la cavité viscérale soüs-ja-

(1) Leach, Observations on the genus Ocyl hoë. Philosoph. trans., 18 I6, p. 29.3.-Say, on the gemus Ocythoë. Ib. 1819 , p. $10 \%$ 
cente; elle est ainsi partagée, par la ligne médiane, en deux moitiés exactement égales. Elle est fort alongée, surtout dans les calmars, où sa largeur est à sa longueur dans le rapport de $\mathbf{b}$ 6 ou de 1 à 7 ; tandis que, chez les seiches, ce rapport n'est que de 1 à 3 ou de 1 à 4 .

C'est dans le milieu qu'elle est le plus épaisse, surtout chez les seiches; elle est plus mince sur les bords et à la partie postérieure.

Dans le genre calmar, elle est en général beaucoup plus mince et plus étroile que dans le genre seiche. Dansle premier, le quart antérieur en devient subitement très-étroit; dansle second, au contraire, cette partie n'est guère plusétroite que la postérieure. Daus l'un et l'autre elle est convexe à sa face supérieure, et concave à l'inférieure, du moins en partie. Cette disposition règne dans tonte la longueur de la coquille chez. les calmars, de façon qu'elle est ici composée de deux mitiés latérales réunies en forme de toit. Chez les seiches elle est convexe en bas dans sa moitié antérieure, plus grande, surtout au milieu et un peu en avant, où elle est le plus épaisse. En arrière, elle est au contraire concave à cette même face inférieure. Il existe en même temps, à cette face, chez les seiches, près de l'extrémité postérieure, un trou borgne dirigé vers cette extrémité, et à la face supérieure une pointe recourbée en bas qui dépasse le bord postérieur. 
La coquille consiste, dans l'un et l'autre genre, en une substance cornée, verdâtre. Dans les calmars, elle est formée uniquement de cette substance; dans les seiches on n'en trouve, même dans les individus d'une grande taille, qu'une couche mince. Cette couche existe $: 1^{\circ}$ à la circonférence où elle a environ un quart de ligne de hanteur, sur trois ou quatre lignes de largeur; $2^{\circ}$ au dernier dixième de la face dorsale, où elle est un peu plus épaisse et adhère d'une maniére plus lâche à la croûte calcaire qui lui est sous.jacente.

A cette substance cornée s'ajoute, chez les seiches, qui par cela se rapprochent davantage des céphalopodes, considérés précédemment, une substance dure, mais lâche et friable, en proportion beaucoup plus considérable que la substance cornée, et constituant presque seule toute la masse de la coquille qui lui doit sa dureté (1). Des éminences et des impressions rendent rugueuse la face supérieure, surtout dans sa partie postérieure. La moitié postérieure de sa face inférieure est, au contraire, rendue inégale par un grand nombre de lignes convexes en avant, qui, se suivant de très-près et devenant plus grandes d'avant en arrière, lui donnent un aspect feuilleté. La moitié antérieure de la face supérieure offrè la même dispo-

(1) John, Analyse chimique de l'os des seiches dans Meckel's Archiv. Bd., t. IV, p. 43 I. 
sition, mais d'une manière moins prononcée.

Cette portion calcaire de la coquille se compose diun nombre considérable de petites lames très-minces, descendant obliquement de haut en bas et d'avant en arrière, el qui en occupent, non toute la longueur, mais toute la largeur. Ces lamelles sout séparées les unes des autres par de petits espaces rćgalièrement disposés, beaucoup plus larges, et offrant eutre eux à peu près la mème étendue. Ces petits espaces sont remplis d'une multitude innombrable de petites colonnes étroiles, très-rapprochées et placées parallèlement sur les lamelles; elles sont d'une cousistance plus lâche que celles-ci. Au milieu, point où la coquille est le plus épaisse, ces lames existent conséquemment en plus grand nombre: on en compte jusqu’à près de cinquante.

\section{S. 57.}

Il existe en outre, chez les céphaloporles, un squeletie intérieur distinct de la cuquille, et composé des principales divisions du squelette des animaux supérieurs, le tronc, la tète et les membres.

Ce n'est pas le prétendu os des sciches que nous venons de décrire, ou l'épée cles culmar's, qui est la trace du rachis, comme l'ont admis quélques anteurs et parliculierement Spix (1).

(1) Cephalogenesis, p. 33: 
Les véritables rudimens de la colonne vertébrale sont représentés par quelques cartilages situés en avantde ces parties, enlre elles et le cartilage crinien. Cependant ces parlies n'existent pas chez tous les céphalopodes. Ainsi je n’ai pas pu les trouver chez les octopodes, quoique j'en aie examiné aveo soin plusieurs individus de différentes lailles. Elles existent, au contraire, dans les seiches et les calmars, du moins dans la seiche officinale et chez le calmar commun, le grand calmar (loligo sagittata).

Il serait curieux dẹ rechercher s'il existe un squelette cartilagineux intérieur et quel est son degré de peffectionnemerit chez les genres aryonaute et nautile, dont la coquille est considérablement développée. Comme ce squelette est très-prononcé chez les seiches, chez lesquelles la coquille quoique intérieure, est fort considérable, tandis que chez les octopodes, où il n'existe point de coquille, il est au minimum du développement, on ne saurait en tirer la conséquence qu'il existe une coquille volumineuse au défaut d'un squelette intérieur. Une telle conclusion serait d'autant plus erronée que le squelette intéritur a été trouvé généralement chez toutes les espéces examinées jusqu'a ce jour.

Lescartilages du troncesont situés à la face dorsale du cou, immédialement sous la peau, qui est tres-mince en cet endroit et qui leur arlhere intimement; ils sont entierement libres daus toute la 
ligne médiane. La paroi dorsale de l'abdomen et la partie solide qu'elle contient dépassent et recouvrent tellement ces cartilages qu'on ne les voit pas ou presque pas, si on ne soulève et rejette en arrière ces parties. Mais ils sont séparés tout-à-fait du crâne et de la coquille elle-même, auxquels ils ne sont unis que par la peau et des muscles longitudinaux, élendus du sac abdominal au crâne, et entre lesquels ils sont fixés en partie.

Ils sont minces en proportion de leurs autres dimensions; c'est au milieu de leur longueur qu'ils offrent le plus d'épaisseur. Leur forme présente de remarquable, dans les seiches et dans les calmars, un bord antérieur, convexe, se terminant en pointe au milieu. On trouve toujours, en outre, dans l'un et l'autre genre, au moins deux de ces cartilages, un supérceur et un inférieur. Le supérieur, plus mince, forme la partie antérieure de la gaine membraneuse qui enveloppe la coquille; l'inférieur en est entièrement séparé dans la membrane qui recouvre les viscères derrière la tête; il est renflé tout le long de sa ligne médiane, où il présente une saillie parcourue à son tour par un eufoncement régnant aussi dans le milieu de toute sa longueur.

Outre ces proprićtés générales, le rudiment du rachis en offre d'autres qui sont particulicres aux différens genres.

Il est à l'état le plus imparfait chez les seiches. 
Chez ces ètres, il n'existe que deux cartilages, couverts de la peau, qui sont très-minces, puisque chez des individus longs de sept ponces, depuis la bouche jusqu'à la partie postérieure ducorps, ils ont à peine un quart de ligne d'épaisseur. Ils sont absolument droits; les saillies longitudinales en sont peu élevées; la gouttière qui régne dans la ligne médiane du cartilage inférieur est large; la saillie mème, en devenant graduellement plus plate, disparaît presque entièrement au commencement du dernier tiers de la longueur du cartilage. Ils ont tous les deux une forme semi-lunaire, la pointe moyenne du bord antérieur étant trèsobtuse et leur bord postéricur peu concave. Il en résulte qu'en les mesurant le long de leur ligne médiane, on les trouve beaucoup moins longs que larges, quoique la distance d'une des deux pointes latérales postérieures à la pointe moyenne du bord antérieur soit un peu plus considérable que d'une pointe latérale à l'autre.

Le cartilage supéricur est un peu plus large, mais plus court que linférieur; il offre, daus toute sa face inférieure, une forte saillie longitudinale.

Chez les calmars le cartilage inférieur est beaucoup plus épais; rhez un calmar lonğ de cinq ponces, il a, en effet, an milieu et vers les bords latéraux, une ligne d'épaisscur, et dans le reste de son élendue rette épaisseur n'est pas au-dessous d'une demi-ligne. 
Sa forme est celle d'une losange; il est circonscrit par deux bords antérieurs et deux postéricurs, sans courbure, qui se rencontrent sur les còtés, sous des angles presque droits, et en avant el en arrière sous des angles très-aigus.

La saillie longitudinale moycune en est trèsprononcée et s'étend dans toute la longueur du cartilage. Elle est ici fort concave à sa face inférieure; la gouttière supérieure de la saillie est au contraire très-étroite et aplatic. Les parties latérales sont droites.

La longueur mesuréc dans la ligne médiane estè la largeur comme 3 à 2.

Les ailes latérales du cartilage sont entièrement couvertes de substance musculaire; il n'y a de libre que la crète longitudinale moyenne.

Indépendamment de ce cartilage principal on en trouve un autre chez les calmars; celui-ci est beaucoup plus pelit, mais plus large, également rhomboïde, et garni à sa face supérieure d'une saillie longitudinale moyenne; il est situé au devant du premier, en partie recouvert par sa pointe antérieure, mais il ne lui est uni qu'au moyen de la peau et des muscles.

Par opposition à ce développement considérable des cartilages inférieurs, le supérieur est peu développé, très-alongé, fort mince dans toutes les directions, presque uniquement sensible par sa saillie longitndinale, inférieure, et à peine distinct de la peau qui l'enveloppe. 
Ia position de ces cartilages, leurs rapports avec les parties voisines et le mode de développement du rachis dans la série animale et dans le mème organisme, me déterminent à considérer ces pièces cartilagineuses comme le rudiment de la colonne vertébrale.

Mais cette mème position, jointe à leur forme, rend vraisemblable qu'ils ne correspondent pas à des vertèbres entières; qu'ils ne représentent que l'arc de ces os; le volume du cartilage principal et le mode de formation, que présentent les corps des vertèbres dans les animaux plus élevés, ne militent pas moins en faveur de l'opinion qui les fait considérer comme les analogues des arcs de plusieurs vertèbres réunis. La remarque faite par M. Cuvier (1), que certains cartilages qui renforcent le dos des octopodes ne peuvent pas être conçus comme des vertèbres n'est pas contraire à notre opinion; les cartilages dont il est ici question correspondant à des parties d'organisation très-différentes.

Les parties, au contraire, mentionnées cidessı:s, et qui sont considérées par quelques auteurs comme le rachis de ces animaux, correspondent clairement à la coquille des mollusques, et en particulier à celle des gastéropodes; cela nous parait du moins résulter très-positivement

(1) Mémoire sur les poulpes et leur anatomie, p. 42, dans les Mémoires pour servir ic l'histoire et ic l'analomie des mollusques. Paris. Mèm. I. 
et de leur connexion lâche avec les parties molles qui les enveloppent, et de leur structure stratifiée, enfin de leur composition.

Il est véritablement digne de remarque de trouver ainsi à la fois, chez plusieurs céphalopodes, un squelette intérieur et une coquille ou squelette extérieur, formant en général d'une manière si distincte la transition des animaux invertébrés aux vertébrés, et constituant en effet une division intermédiaire à ces deux grandes divisions du règne animal (1).

Un autre fait non moins curieux est la série queforment les différens genres de céphalopodes, sous le rapport du degré de développement de ces parties. Les nctopodes n'ont ni coquilles, ni rachis. On ignore, autant que je sache, comment se comportent à cet égard les argonautes dont la coquille est très-développée. Chez les seiches, où la coquille est imparfaite, on voit apparaître deux lames encore très-minces, comme premier rudiment de la colonne vertébrale, et chez les calmars dont la coquille est encore moins parfaite, le rudiment du rachis a pris un développement plus considérable; il est non-seulement plus épais et plus étendu, mais en avant de la pièce principale qui existe aussi chez les seiches, il s'en est formé une autre beaucoup plus petite, analogue à ce que l'on ren-

(1) Voyez vol. I, p. 97 et 195 . 
contre chez la plupart des poissons, où la première vertèbre cervicale est petite et imparfaite; tandis que chez beaucoup d'autres la vertèbre suivante, formée de la réunion de plusieurs au. tres soudées ensemble, se distingue par son volume considérable.

\section{S. 58.}

Plusieurs céphalopodes, principalement les seiches et les calmars, ont encore d'autres os du tronc, outre les rudimens du rachis.

Ces os sont situés à la face inférieure du corps, et contenus en partie dans la substance de la lame inférieure du manteau, à laquelle ils sont unis intimement, et au dessus du niveau de laquelle ils forment une saillie qui s'avance dans la cavité des branchies. On en trouve toujours un de chaque côté. Leur plus grande longueur est d'avant en arrière, leur petite étendue d'un côté à l'autre.

Ils sont beaucoup plus développés chez les seiches que chez les calmars; ils y sont mieux séparés des parties environnantes; et plus en rapport avec la forme de tout le corps, ils sont, chez les premiers, arrondis et épais, chez les seconds alongés et minces. Chez ceux-ci, ils s'étendent jusqu'au bord antérieur de la paroi inférieure du manteau; chez les autres, où ils se portent en général plus en arrière, ils ne l'atteiguent pas dans toute leur longueur. 
Je n'ai pas pu trouver de trace de ces cartilages chez les octopodes.

A l'opposite de ces pièces on trouve, des deux côtés, à la base de l’infundibulum, dans un prolongement latéral, valvuliforme de celui-ci, d'autres cartilages qui correspondent parfaitement aux premiers par la position, la forme et la direction; très-concaves chez les seiches, plus aplatis chez les calmars, ils sont reçus exactement par les pièces que nous venons de décrire, et forment avec elles la clôture assez complète de la partie antérieure de la cavité viscérale.

On trouve bien la valvule latérale chez les octopodes, mais aucune trace de ces cartilages. Ces cartilages sont-ils les vestiges de certaines parties du squelette des animaux supérieurs; et quelles sont ces parties? cette question est difficile à résoudre. Il est vraisemblable qu'ils correspondent à la série des os situés vis-à-vis du rachis chez les vertébrés.

$$
\text { S. } 59 \cdot
$$

Outre les parties décrites qui représentent les cartilages des os du tronc des vertébrés, il existe chez les seiches, les calmars et les o.topodes, des cartilages qui sont tres-vraisemblablement les analogues des partics solides des membes des animaux supérieurs.

Ils sont situćs sur le côté du corps, très cxac- 
tement plongés dans la substance musculaire, surtout à leur face supérieure; leur forme est toujours alongée et apointie aux deux extrémités.

Dans. les seiches et les calmars, ils sont plats, plus épais à leur bord interne qu'à l'externe, et descendent le long de toute la base des nageoires aux muscles desquelles ils donnent insertion. En dedans, ils sont séparés de la face latérale du corps par une lacune considérable, tapissée d'une membrane propre, entière.

Chez les seiches, où les nageoires sont plus longues, ils offrent également beaucoup plus de longueur que chez les calmars. Dans les premieres, ils correspondent à toute la longueur du tronc; dans les seconds, ils se bornent à sa plus grande partie qui est postérieure.

Dans les octopodes ils n'existent qu'à l'état rudimentaire; disposition conforme au développement imparfait de tout le squelette; ils sont beaucoup plus courts, oblongs, un peu apointis en avant et en arrière, ne correspondent environ qu'au troisième quart de la longueur du tronc, et ne sont séparés en dedans de la substance musculaire par aucun intervalle.

Cee sont là les parties dont M. Cuvier ( $\mathrm{r}$ ) dit; avec raison, qu'on ne peut pas les considérer comme des vestiges de rachis; mais cela n'in-

(1) Voyez l'ouvrage cité a la page $\mathbf{1 8 9}$

II. 
firme en rien l'exactitude de l'opinion qu'une foule d'organes des céphalopodes peuvent être ramenés à des organes analogues des vertébrés, et que les céplialopodes présentent confondus les caractères des invertébrés et ceux des animaux à vertèbres.

\section{S. 60 .}

Enfin les céphalopodes, nous cilerons les seiches, les calmars et les octopodes, possèdent du moins un cartilage céphalique distinct et très-développé, dont voici les traits généraux.

Il est situé derrière la masse de la bouche, profondément caché dans la substance musculaire; il est plus large que long, fort concave en devant, convexe en arrière, et se compose d'une partie moyenne, déprimée, et de deux parties latérales. La partie moyenne est percée inférieurement par une ouverture ronde, considérable, destinée surtout à laisser passer l'œsophage et à recevoir le cordon médullaire; en haut, elle présente une forle concavité pour contenir l'encéphale : il en résulte que cette partie renferme une cavité spacieuse, spéciale, dont la paroi postérieure forme une protubérance moyenne, propre, qui n'est pas aussi saillante en arrière que les partics latérales. La circonférence supérieure de l'anneau est un peu obliquement dirigée de haut en bas et d'avant en arrière; l'inférieure, moins concave, est située transver- 
salement. Celle.ci contient la partie inférieure du collier médullaire avec l'organe auditif, et est percée pour le passage du corłon de la moelle abdominale.

Les parties latérales sont plus plates; leur paroi interne, ascendante, les sépare un peu, quoique imparfaitement, de toute la hauteur de la partie moyenne. Elles renferment l'neil et le nerf optique qui y pénètre à la sortie de l'anneau. Elles portent, à l'extrémité antérieure et interne de leur bord inférieur, une lame mince, dirigée de dedans en dehors, qui les complète en quelque sorte en avant, où elle s'applique au devant de l'œil et se perd dans la peau.

Les différens genres offrent quelques déviatious de celte disposition générale.

Chez les seiches et les calmars, le cartilage céphalique est beaucoup plus solidé et plus épais que chez les nctopodes; chez les calmars, il est presque le plus épais. Il est en outre, chez les derniers de ces animaux, le plus convexe; si convexité est au contraire la moindre chez l'octopode. La lame antérieure est de plus, dans les seiches et les calmars, une pièce propre qui ne s'articule qu'avec le cartilage principal, et est beaucoup plus longue en dehors, mais aussi moins élevée que chez les octopodes, où elle est haute, arrondie, et seulement une apophyse née du cartilage principal. Enfin, chez les octopodes, l'ouverture destinée au nerf op- 
tique est oblitérée en avant et ne forme qu'une échancrure, tandis que chez les autres c'est un véritable trou.

\section{S. 61 .}

Outre ces cartilages céphaliques, qui appartiennent sans doute à tous les céphalopodes, il existe chez les seiches, et autant que s'étendent mes recherches ce fait est absolument propre à ces animaux, un cartilage impair, volumineux. Ce carti lage est situé transversalement au-dessous de la masse buccale, à peu de distance en avant du cartilage céphalique qui vient d'être décrit et auquel il n'est uni que par de la substance musculaire. C'est un demi-anneau très-imparfait, puisqu'il est un peu convexe en dehors et concave en dedans. Il envoie de son bord antérieur, qui est concave, trois apophyses; il n'en naît qu'une, moyenne, de son bord postérieur qui est convexe.

Ce cartilage, dont il n'existe point de traces dans les autres genres, et qui constitue un point d'appui pour les bras, est assez considérable; puisque chez une seiche longue de sept pouces, il a près d'un pouce de long sur deux à quatre lignes de largeur d'avant en arrière, et d'épaisseur de dehors en dedans. 


\section{S. 62.}

Les parties le plus généralement répandues du squelette cartilagineux intérieur des céphalopodes sont par conséquent:

$1^{\circ}$ Les cartilages céphaliques.

$2^{\circ}$ Les cartilages des membres.

11 faut y joindre, chez les seiches et les calmars, les deux cartilages dorsaux; de ces cartilages, l'inférieur se multiplie d'avant en arrière chez les calmars; dans l'un et l'autre genre, le cartilage céphalique antérieur se sépare également du cartilage principal; les cartilages des membres deviennent plus grands aussi et plus libres, surtout chez les seiches. Enfin celles-ci portent un carlilage propre, situé en avant des cartilages céphaliques, au pourtour de la masse buccale et de la base des bras.

C'est donc chez les seiches que l'appareil cartilagineux et la coquille intérieure sont le plus parfaits; tandis que chez les octopodes ils offrent aux deux appareils le plus d'imperfection. Ce système n'est composé que de trois pièces distinctes dans les derniers; il est formé de douze chez les seiches et les calmars.

\section{S. 63.}

Il résulte de ce qui vient d'être exposé que 
les céphalopodes sont très-généralement pourvus d'une coquille et d'un squelette cartilagineux. On rencontre plus rarement, chez ces animaux, des parties dures; ces parties correspondent très-vraisemblablement aux organes locomoteurs, soyeux, de plusieurs vers et aux pattes des animaux articulés. Cette disposition, que jai eu occasion de voir au musée de Hunter, à Londres, a été décrite par Rellonius (1), Rabricius (2), Molina (3), Berget Lichtenstein (4).

D’après les faits comnus jusqu'à ce jour, ces parties n'existent qu'aux deux bras les plus longs, où elles remplacent les cupules, en forme de ventouse, dont elles ne sont sans doute qu'une modification. Ces cupules n'ont póur appui principal qu'un anneau corné, dentelé, et les crochets ne sont que ces anueaux prolongés. Ils alternent comme les ventouses, sont supportés par un pédicule charnu, et entourés presque jusqu'au sommet d'une gaîne membraneuse, qui est une transformation des anneaux membraneux de la cupule. Le nombre et le volume de ces crochets varient suivant les espèces.

(1) Bellonius, de Aquatilibus, p. 340 .

(2) C. Fabricii Fauna Gronland. , p. 559 .

(3) Molina, Saggio sulla stor. del Chili, p. 199.

(4) Lichtenstein, dans l'Isis d'Oken, 1818, p. 1491. Onychoteuthis. Seiches à griffes, planche 19. 


\section{GHAPITRE VIII.}

SQUELETTE DES VERTÉBRÉS OU SYSTÈME OSSEUX.

$$
\text { S. } 64 .
$$

Le squelette intérieur des céphalopodes conduit à celui des autres animaux supérieurs : il peut en effet ètre considéré, à juste titre, comme le rudiment immédiat du squelette des vertébrés.

Ce squel:tte iniérieur, ou le système osseux proprement dit. se distingue de l'extérieur, de la coquille ou du test, par la position, la forme ex'érieure, le tissu et la composition. L'exposé de ses caractères généraux renfermera en même temps le plus grand nombre de ces différences. Il est, dans les diverses classes des vertébrés, à tous égards, beaucoup plus disposé d’après le même plan, que ne le sont les parties solides des animaux que nous avons considérés jusqu“ici. Il est par conséquent facile et convenablo d'en réunir les propriétés générales et particulières dans un chapitre spécial, avant de tracer la disposition qu'il offre dans les différentes classes. 
I. CARA CTÈRES GÉNŔRAUX DU SYSTÈME OSSEUX.

Les conditions gérérales que présente le système osseux sont surtout les suivantes:

\section{Position.}

La plupart des os sont situés profondément entre les muscles qui les entourent, et sont séparés de la peau par ces mêmes muscles, ainsi que par les vaisseaux et les nerfs qui se rendent à ceux-ci et à l'organe cutané. Les muscles s'attachent par conséquent, au moyen de leurs tendons, à la périphérie des os. Il est rare que des pièces osseuses, propres, de formes diverses, soient situées librement dars la peau; cependant les poissons offrent assez souvent cette disposition, et l'on peut en quelque sorte considérer comme un rapprochement vers cette disposition, la tendauce que manifestent certains de leurs os à se porter en dehors, surtout au crâne et aux membres antérieurs; il en est ainsi d'autres os, principalement des côtes, chez les chéloniens.

\section{-2. Forme extérieure.}

Les caractères les plus généraux de la forme extérieure des os sont :

$1^{\circ}$ Un certain degré de lisse de la face exté- 
rieure dans la majeure partie de son étendue. $2^{\circ}$ La présence de plusieurs inégalités, souvent considérables, que n'exclue pas cette disposition lisse, mais qui offrent elles-mêmes une surface unie, et dont les contours suivent. fréquemment des courbes diverses.

Cies inégalités affectent plusieurs formes: $\mathrm{d}^{2} \mathrm{a}$ bord celles d'éminences, de saillies, d'apophyses, qui ont surtout une domble destination. Elles sont le plus communément en rapport avec l'attache des muscles; elles servent à en renforcer l'action, en agrandissant l'angle sous lequel ils s'insèrent et en augmentant eu outre la surface d'attache.

D’autres éminences servent à unir les os entre eux.

Ces deux espèces d'éminences se distinguent entre elles : les dernières sont encroûtées de cartilage; les premières en sont dépourvues. La plupart des éminences qui servent à l'attache des muscles sont rugueuses à des degrés différens.

Les éminences articulaires sont presque toujours lisses, mais quelquefois elles offrent aussi des inégalités très-considérables, dans les cas où elles concourent à former une articulation so. lide, qui en général est immobile.

Les os offrent, en outre, des enfoncemens, des cavilés, des sillons, des fosses, des gouttières, situés en partie isolément, en partie sur 
les apophyses dont il vient d'ètre question, et qui servent de point d'attache aux muscles. assureut la position des tendons, protègent les vaisseaux et les nerfs, et reçoivent des saillies osseuses.

Après ces enfoncemens viennent des ouvertures. des trous, des conduits, des canaux.

Quelquefois une gouttiere est convertie en une ouverture, parce qu'il se dépose de la substance osseuse dans la partie ordinairement libre de sa circonférence, qui n'est formée que par du tissu cellulaire ou de la substance fibreuse. Il existe aussi des ouvertures propres, conslantes, qui, lorsqu'elles ne sont pas en rapport a vec la nutrition des os, sont, d'une manière plus ou moins passagère ou permanente, formées par plusieurs os. Ces onvertures. ou sont placées à l'entotir de grandes ou petites portions des systèmes circulatoire et nerveux, ou contribuent à diminuer le poirls du système osseux, ce qui rent spécifiquement plus léger le reste du corps, ou bien enfin donnent passage aux vaisseaux nourriciers des os et aux vaisseaux sécréteurs de la substance fluide qu'ils renferment.

On peut ciler pour exemples de la première espèce, le grand trou occipital, le trou moyen des vertèbres, la plupart des grandes ouvertures latérales de celles-ci et des os du crâne ; une ouverlure de la seconde espéce est le trou ovale du bassin. 
Les ouvertures qui donnent passage aux vaisseaux nourriciers et sécréteurs de la substance médullaire, se divisent très-généralement en grandes et en petites; celles ci existent presque partout en très-grande quantité; celles-là sont beaucoup plus rares et ne se trouvent que sur des points déterminés.

Tous les os ont un rapport général de configuration extérieure, en ce sens qu'ils forment, ce qui n'est pas également distinct dans tous, soit des anneaux entiers, qui sont des vertébres, soit des parties d'anneaux, qui, par leur forme convexe et leur tendance à se souder entre elles, indiquent manifestement leur origine. Quoique ces purtions d'anneaux soient parfois sensibleblement droites, on peut néanmoins démontrer leur nature primitive en étudiant leur mode de formation.

\section{Forme intérieure ou texture.}

Les os sont formés très-généralement de deux substatices, dont l'externe, la substance compacte, est beaucoup plus consistante, plus dure et plus dense que l'interne, la substance sponzieuse. Mais elle ne different pas essentiellement entre tlles, par plusieurs raisons : $1^{\circ}$ Elles passent insensiblement l'une dans l'autre; $2^{\circ}$ trèscommunément l'une d'elles se développe sur les divers points d'un même os, ou sur des os

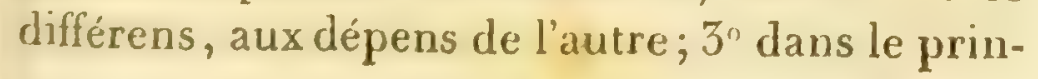


cipe, toute la substance de l'os possède les propriétés qui n'appartiennent plus tard qu'd la sul). stance intérieure; $4^{\circ}$ leur composition est absolument la mème; $5^{\circ}$ enfin, la substance externe peut se transformer morbidement en substance interne et réciproquement.

La substance spongieuse, qui est lâche, formée plus ou moins distinctement de lames et de filamens, de configuration variable, constitue, dans l'intérieur des os, une cavité diversement composée, remplie d'une humeur qui contient assez généralement de la graisse, la moelle.

Dans la plus grande partie de leur surface extérieure, les os sont étroitement enveloppés d'une membrane fibreuse, le périoste; les vaisseaux s'y ramifient avant de pénétrer dans l'os. Cette membrane ne manque qu'aux faces osseuses tournées l'une vers l'autre, elle passe ici par dessus l'espace intermédiaire et s'épaissit ordinairement par places pour former les ligamens fibreux.

Il y a d'autres vaisseaux qui entrent dans les os pour s'y ramifier, ou en sortent, comme il a été indiqué.

\section{Composition.}

Les os se composent de deux substances : l'une d'elles est spéciale, propre, animale, gélatineuse, et l'autre dure, formée elle-mème de plusieurs combinaisons terreuses, surtout de plos- 
phate de chaux. La première sert de liaison à la seconde, qui est généralement plus abondante; cependant l'os dépouillé de sa substance lerreuse n'en conserve pas moins toute sa forme. Le phosphate de chaux n'est pas l'unique sel qui entre dans la composition des os; il y en a encore. d'autres, mais dont les proportions sont beaucoup moindres.

\section{Propriétés physiques.}

Les os sont en général d'une couleur blanchejaunâtre.

Quoique variant pour l'élasticité, ils possèdent toujours un haut degré de consistance et de dureté.

\section{Propriétés vitales.}

Les os se distinguent d'un très-grand nombre de parties organiques : $1^{\circ}$ par la lenteur et la simplicité de leurs phénomènes vitaux, analogues à ceux des végétaux; $2^{\circ}$ et, en conformité avec ce dernier rapprochement, par l'énergie et la perfection de leur faculté régénératrice, qui est plus développée dans ce tissu que dans toutes les autres parties de l'organisme.

$$
\text { S. } 65 \text {. }
$$

Les caractères qui viennent d'être indiqués appartiennent à tous les os en particulier. L'en- 
semble du système se montre comme un tout continu, non interrompu, mais qui varie dans ses degrés de connexion, comme il a été indiqué plus haut (1); on peut le nommer sque. lette intérieur ou osseux. On peut y tracer une première coupe, et le diviser en une partic moyenne ou centrale et en deux latérales. La première se compose : ${ }^{\circ}$ des os du trone, du rachis et des os accessoires qui lui sont unis, des côtes et du sternum; $2^{\circ}$ du crâne et de quelques os de la face.

Les secondes sont formées des os des membres et de quelques os latéraus de la têle. La partie centrale est formée, en majeure partie, d'anneaux qui se succèdent d'avant en arrière et qui sont unis en un tout; ces anneaux constituent les vertèbres; elle est en outre formée de parties dont il est aisé de reconnaître la plupart pour des fractions et des répétitions de ces vertèbres. La partie centrale se partage elle-même en deux moitiés latérales qui sont le plus souvent parfaitement symétriques.

Comme les os de la tête, quoiqu'ils soient des répétitions des os du tronc, en sont fort distincts, ainsi que de ceux des membres, que du reste ceux-ci le plus souvent répètent manifestement les parties latérales des vertèbres et ont des rapports variés d'action et de connexion

(1) Voyez le $\$ \cdot 3$. 
D'ANATOMIE Comparée.

soit directs, soit indirects, avec la colonne verlébrale, j’ai cru devoir, pour l'étude spéciale du squelette dans les différentes classes, faire suivre la description des os du tronc par celle des os des membres et terminer par celle de la têle.

II. Caractères particuliers ou différences

DU SYSTÈME OSSEUX.

$$
\text { S. } 66 \text {. }
$$

Les caractères que nous venons d'indiquer, et qui constituent autant de points de contact, tant pour toutes les parties du squelette intérieur du mème animal que pour le squelette de tous les animaux, ne sont pas les seuls dignes de remarque.

Le système osseux offre encore, soit dans le mème organisme, considéré à la même époque et à des phases différentes, soit entre des organismes variés, des différences relatives à la région du corps où les os sont situés, à la période de la vie où on les observe et aux classes dans llesquelles on les étudie.

A. DIFfÉnENCES RELATIVES A LA RÉGION.

$$
\text { \$. } 67 .
$$

J es différences relatives à la région lu corps permettent de déterminer quatre modes de configuration, et de reconnaitre : $1^{\circ}$ des os courts 
ou arrondis; $2^{n}$ des os larges ou plats; $3^{\circ}$ des os alongés ou cylindriques; $4^{\circ}$ enfin des os mixtes qui se rapprochent plus ou moins de la forme des autres os, surtout des deux premières espèces.

Les os courts ou arrondis ont un diamètre à peu près égal dans toutes leurs dimensions; les os plats sont très-généralement minces, mais à peu près aussi larges que longs; les os alongés sont minces et étroits relativement à leur longueur. Les os courts sont souvent fort irréguliers. Les os plats sont communément plus réguliers, triangulaires, ou carrés, ordinairement convexes à leur face externe, concaves à l'interne. Les os longs sont plus droits et renflés habituellement aux deux extrémités, qui sont encroûtées de cartilage, parce que c'est surtout aux extrémités qu'ils s'articulent. d'une manière mobile avec d'autres os. Les os cylindriques sont les plus grands. Les courts sont les plus petits. Les premiers constituent d'ordinaire la plus grande masse du squelette des régions qu'ils concourent à former.

Les os longs sont composés, comme les os courts et plats, de deux substances, dont l'une est interne, l'autre externe; mais ils different des derniers par l'absence de la substance interne qui manque dans une partie considérable de leur longueur, surtout dans leur partie moyenne, et par l'existence d'un organe médul- 
laire particulier qui remplit la cavité résultant de l'absence de la substance interne. Cet organe médullaire est une membrane délicate, dont le produit est ici plus graisseux que dans les autres os. Dans les os courts et dans les os plats, le tissu làche, ou le diploë, existe dans toute leur étendue.

Il faut cependant ajouter qu'à peu d'exceptions près la plupart des os sont mixtes, en ce sens qu'ils offrent quelque chose de la composition de ceux que la totalité de leur mode de configuration a fait rapporter à des os d'un autre ordre. Ainsi certaines de leurs fractions appartiennent constamment à des os différens. D'autre part, ces fractious sont primitivement séparées les unes des autres, et ne se soudent entre elles en un tout que par suite de progrès insensibles. Les vertèbres, par exemple, que l'on cite ordinairement comme des os courts, ne le sont que sous le rapport de leur corps; elles sont, au contraire, des os plats ou longs quand on a égard. $\therefore$ leurs autres parties. Dans les os cylindriques, le corps, ou la diaphyse, est seul alongé, les extrémités renflées sont courtes et épaisses.

Les os courts constituent surtout les portions du corps ou il a fallu un haut degré de solidité jointe a quelque mobilité. On les trouve généralement à la base du carpe et du tarse. Les corps des vertèbres forment d'une manière analogue la partie la plus solide de la colonne i1. 
vertébrale. C'est pour cela que ces os sont d'ordinaire très-solidement articulés entre eux au moyen d'une harmonie qui embrasse la totalité ou une partie de leur étendue. On voit en outre se développer, dans les tendons des muscles, des os qui jouissent des propriétés appartenant aux os courts; ils servent, soit à donner à ces muscles plus de solidité, soit à leur fournir une base d'insertion plus élargie.

Les os plats, seuls ou réunis à des os courts et mixtes, forment principalement des cavités qui renferment des organes importans. 1ls sont communément unis aux os voisins pardes sutures encore plus solides que ne le sont les modes d'union qui joignent les os courts. Leurs bords sont par conséquent variables, inégaux et dentelés à des degrés différens, et ont, surtout au crâne, une grande tendance à se souder entre eux pour former une cavité.

Les os longs se rencontrent dans les portions du corps qui exécutent les morvemens les plus étendus et les plus libres; on les trouve dans les membres.

Le passage des os longs aux os plats est formé par les côtes, qui constituent en général la plus graude partie de la cavité thoracique.

B. DIFFÉRENCES QUI DÉPENDENT DES AGES.

$$
\text { S. } 68 \text {. }
$$

Les différences que présentent les os aux diverses phases de la vie sont extrêmement cu- 


\section{D'ATATOMIE COMPARÉE.}

rieuses, soit par elles-mêmes, soit parce qu'elles coïncident avec des différences de classes qui persistent.

Dans le principe on trouve àda place de l'os une substance qui en diffère totalement : cette substance, qui est un cartilage, quoique présentant la forme de l'os qu'il précède, s'en distingue par une structure entièrement ou presque entièrement homogène, par la solidité, l'absence oula proportion très-minime de principes terreux , c'est-à-dire par plus de mullesse et d'élasticité, enfin par une couleur d'un blanc-bleuâtre.

Iرorsque des apophyses et des prolongemens se développent à la surface et principalement à la partie moyenne de cette substance primitive (1). on trouve très-communément sur plusieurs points, et surtout à son intérieur, une substance osseuse qui est partout entonrée de cartilage; alors seulement les vaisseaux commencent à charrier du sang rouge.

Dans la plupart des animaux, ces noyaux partiels d'ossification se réunissent en un seul os, suivant un temps qui varie; souvent ces noyaux d'ossification qui se réunissent en un tout sont très-nombreux, comme, par exempie, chez les oiseaux, quelquefois aussi chez les mammifères, où presque tous les os du crâne et un grand nombre de ceux de la face se soudent en-

(1) Dutrochet, Mémoire sur l'ostéogénie. Journal de Physique, to LXXXXV, p. 161 . 
semble. Ordinairement il se forme d'abord quelques os du tronc, après eux vienuent plusieurs os de la tète, enfin ceux des membres. Leur perfectionnement suit à peu près le mème ordre. Souvent, dans les longs os par exemple, la partie moyenne, qui est la plus considérable, se forme la première; lans d'autres, au contraire, dans les vertèbres et les os du crâne, par exemple, les parties latérales se développent avant celles qui occupent le centre.

Primitivement il n'existait que des cariilages mous à la place des os; ceux-ci sont, par la même raison, d'abord plus arrondis et contiennent proportionnellement plus de substance molle, animale, que de substance terreuse. Plus tard celle-ci augmente; ils deviennent plus consistans, plus durs, en même temps plưs anguleux, et augmentent de volume dans toutes les directions. Plus tard encore, particulierement à l'âge avancé, leur volume, surtout dans le seus de l'épaisseur, diminue dans diverses proportions : ils devienment souvent beaucoup plus mous qu'ils ne le sont dans les premiers temps de la vie.

D'après Ilatchett et Ifome, il faut compter une certaine proportion de graisse pour la formation des os. Leur opinion est fondée sur ce que les .ufs des animaux dépourvus d'os n'ont pas de graisse dans leur jaune, et que dans le cas ou cette graisse manque dans le jaune de l'neulf d'a- 
minaux pourvus d'os. patr exemple dans les larres de grenonilles, il se forme dans leur abdomen, vers le temps de l'ostéogenèse, une masse de graisse qui disparait insensiblement à mesure que lesos se développent (i). Cetle observation ne suffit cependant pas pour rien ôter a la justesse de la remarque faite par M. Prout (2): "Que, dans l’état actuel de nos connaissances, rien n’autorise à admettre que la graisse déposée dans ces parties se transforme réellement en la substance terreuse des os. „Il est très-vraisemblable que ce tissu adipeux n'est ici, comme partout ou on le rencontre, en général qu'un dépôt de matieres nutritives; et on concevrait tout au plus, d'après les conjectures de M. Prout (3), que le jaune prît part à la formation des os, au moyen du phosphore qu'il contient en grande, proportion. Cet élément disparaît en effet pèndant l'incubation, et se transforme en acide phosphorique qui entre dans la composition des os.

\section{DLFFÉRENCES DE CLASSLS.}

$$
\text { 6. } 69 \text {. }
$$

Les principales différences que présenle le (1) On the formation of fat in the intestins of the tadpole and of the use of the yelk in the formation of the embryo in the egg. Phil. Trans. 1816 , p. 301.

(2) Some experiments on the changes which take place in the fixed principles of the egg during incubation. Phit. Trans. 1822 , p. 399.

(3) Ibid. 
squelette extérieur dans les divers animaux parvenus ঐ̀ l'état parfait, sont les suivantes.

\section{r. Forme extérieure.}

Telle est l'extrême diversité offerte par les os du même animal, que l'on conçoit â priori que les différences présentées, sous le rapport de la forme extérieure des os, par les grandes et petiles divisions introduites dans le règne animal, sont insuffisantes pour servir à établir avec certitude des caractères de classes et d'ordres fondés sur l'ensemble du squelette. La comparaison des os du même animal fournit un caractère de classe assez gériéral : ils sont d'autant plus semblables lés uns aux autres que l'organisme entier est moins élevé dans la série. Ainsi les différentes vertèbres et les côtes se ressemblent beaucoup plus entre elles chez les poissons et les ophidiens, que chez les animaux qui leur sont supérieurs. Les os postérieurs du crâne de ces mêmes ètres ont beaucoup plus d'analogie avec les vertèbres qu'ils n'en offrent dans les vertébrés placés au dessus; enfin les membres de plusieurs poissons, ainsi que les pièces des mâchoires des ophidiens et des batraciens, ressemblent tout-àfait à des côtes.

Ces faits sont fondés sur la loi : que les différens os sont formés d'après un type primitif, qu'ils peuvent être ramenés, soit à̀ des vertèbres entières, soit à des parties de vertèbres, et que c'est dans sa forme la plus simple et la moins 
parfaite, et à une époque diversement rapprochée de sa première apparition dans la série animale, que tout os représente le mieux ce type primordial.

Les seuls cas où l'on puisse emprunter des caractères au squelette entier pour différencier des collecions plus ou moins grandes de la série animale, sont ceux où le système osseux présente exceptionnellement des particularités irès-saillantes, en rapport avec le genre de vie des animaux, et spécialement avec leur mode de locomotion. Un exemple remarquable de cette circonstance nous est fourni par les oiseaux, qui, outre les enfoncemens et ouvertures que l'on rencontre dans les os des autres animaux, y offrent une espèce particulière d'ouvertures qui établissent la communication entre la cavité des os et les organes respiratoires, et chez lesquels les voies qui laissent entrer l'air dans le corps, souvrent dans les os par des conduits d'une étendue variable.

Les principaux points de vue sous lesquels on peut considérer cette disposition sont :

$1^{\circ}$ La position;

$2^{\circ} \mathrm{La}$ forme;

$3^{\circ}$ Le volume;

$4^{\circ}$ Le nombre de ces ouvertures;

$5^{\circ}$ La situation relative de l'organe respiratoire avec lequel elles communiquent;

$6^{\circ}$ La proportion des os, ainsi en rapport avee 
l'air ; tous ou quelques-uns pouvant offrir cette eondition.

$7^{\circ}$ Le nombre des oiseaux qui présentent cette disposition. A ce sujet on peut poser les ques. tions suivantes: Tous la présentent-ils? existe-til des différences à cet égard? quelles sont-elles?

$8^{\circ}$ Linfluence des phases diverses de la vie; ce qui amène à rechercher si cette influence produit des différences et quelles elles sont.

1. Posilion. I es ouvertures aérifóres sont toujours cachées; elles occupent la face intérieure des os; aux os longs elles sont presque toujours situées à l'une des extrémités, et plus particulièrement à l'extrémité la plus rapprochée du centre.

2. Forme. La forme en est constamment arrondie; les bords qui les entourent sont lisses et ronds; la direction en est souvent oblique, de sorte qu'il en résulte un conduit court qui établit la communication avec l'intérieur de l'os; ce conduit ne s'y ouvre quelquefois pas immédiatement par tout son diametre, mais il forme une fosse dont le fond est percé de plusieurs petits orifices.

3. Volume. L'étendue des onvertures et celle des cellules auxquelles elles conduisent varient; il existe en général entre elles un rapport direct.

4. Nombre. Quelques os, particulièrement fes vertèbres, les côtes et leurs appendices, le 
sternum lui-mème, sont percés communément de plusieurs ouvertures. La plupart des autres us n'en présentent qu'une seule.

5 . Le plus grand nombre des os, et particulièrement du tronc et des membres, communiquent arec les poumons au moyen de canaux qui partent de ces organes; les autres, c'est-àdire ceux de la tête, s'ouvrent immédiatement dans la cavité buccale à l'aide d'un canal qui est commun à la bouche, à la cavité du tympan et à la trompe gutturale; chez quelques oiseaux, principalement les hibous, le rapport de ces derniers os avec l'organe respiratoire, est aussi élabli par l'intermédiaire du nez.

6. Ces ouvertures n'existent pas dans tous les os. Ceux qui en sont privés généralement sont les os de l'avant-bras et de la main, de la jambe et du pied, les os zygomaliques et les sus-orbitaires, l'os hyoïde et le cercle osseux qui entoure les yeux. Parmi les os aérifères mêmes, il n'y eu a pas un seul qui ne soit solide chez quelque oiseau, tandis qu'il est creux chez les autres. J'humérus et la mâchoire inférieure perdent rarement leur caractère d'os aériens; ceux qui le perdent le plus souvent sont les apophyses ptérygoïdes.

On truuve chez quelques oiseaux, entre les os parfaitement aéritères, dénués de moelle, et les us qui en sont pourvus, des os intermédiaires qui, dans d'autres espèces, sont parfai- 
tement aériens. La plupart de ces os intermédiaires contiennent de la moelle, et possèdent en outre une petite cellule aérienne qui s'ouvre par un orifice étroit.

Indépendamment de ces différences qui ont lieu entre les divers os du même oiseau, il y en a d'autres qui dépendent des individus, des genres et des périodes de la vie.

Les différences individuelles se rencontrent surtout dans les os de petit volume qui sortent plus souvent que les autres du rang d'os aériens, par exemple l'os communiquant, qui tantôt n'est plein que d'un côté, tantôt l'est également de tous deux, qui parfois est tont-à-fait creux.

7. Les différences génériques sont très-considérables. Chez plusieurs oiseaux de haut vol, d'un grand volume, tels que les pélicans, les fous, les cigngnes, tous les os qui ont été indiqués comme aéricns communiquent avec l'organe respiratoire; il en est presque de même chez les aigles et les vautour's, où il n'y a exception que pour les os communiquans.

Chez les grèbes (podiceps), les hirondelles de mer (sterna), les foulques (fulica), quelques os de la tête présentent seuls une cavité qui n'en occupe même qu'une fort petite étendue.

Du reste, il y a souvent des variétés, à cet égard, dans des genres et espèces très rapprochés.

8. Les différences de développement de ces os sont très-remarquables. 


\section{I) ANATOMIE COMPAREE.}

Il est constant que les os ne deviennent aériféres qu'après la naissance; tous ces os, sans exception, contiennent d'abord de la moelle comme les autres. Ce n'est que peu à peu que cette substance disparaît ; elle commence constamment à s'effacer à parlir de l'ouverture de communication. Le développement complet de la cellule aérienne n'a lieu ordinairement qu’à l'époque du développement parfait des parties génitales, les fonctions respiratoire et génératrice, ainsi que leur's organes respectifs, atteignant partout en mème temps leurs derniers perfectionnemens. C'est également à l'approche de la puberté que les vices organiques du cour et du poumon, susceptibles de troubler l'intégrité de la respiration, exercent l'influence la plus fâcheuse sur l'organisme; ces altérations peuvent même devenir un obstacle aux conditions qui caractérisent cette époque de la vie..

\section{S. 70 .}

Le squelette considéré dans son ensemble fournit difficilement des différences assez générales pour bien caractériser des groupes entiers d'animaux; il n'en est pas ainsi des modifications que subit un os donné dans la série des vertébres; elles suffisent pour rendre facile la détermination de la division à laquelle l'animal dont cet os est tiré ap partient. Le même os est, à la vérité, formé partout d'après un type com- 
mun; mais les changemens que ce tyje épronve sous l'influence de plus d'une cause sont tels, qu'il est difficile de le reconnaître, même pour l'oil le plus exercé.

Les causes de ces modifications peuvent itre ramenćes surtout à deux : la première semble avoir son siége dans les os mèmes; la seconde dans d'autres parties. Un exemple de la première espèce nous est fourni dans la disposition des vert èbres, des côtes et du sternum, chez les chéloniens; nous en croyons voir un de la seconde espèce dans les formes très-variées que présentent les os du crâne, considérés, soit c'n particulier, soit en général; variétés en rapport avec la configuration et le volume de l'encéphale, avec la disposition des muscles masticateurs, et qui ne sont qu'une suite des modifications qu'offrent ces parties elles-mêmes. Il ne serait pas exact cependant de dire d'une manière absolue, que la cause des différcnces de forme qui se remarquent dans les os réside en eux-mêmes; ces différences peuvent ètre détcrminées partout par la condition de configuration d'un autre système; ainsi les systèmes nerveux et musculaire n'y sauraient jamais ètre considérés comme étrangers. On voit, en effet, chez les chéloniens, les muscles du tronc se déyrader dans le même rapport que les os de cette partie changent de forme et prennent plus de volume et d'extension. Jiapparition tardive du 
systeme osseux dans la série animale et dans les embryons, la masse peu considérable des os comparée à celle du système musculaire, donnent de nouvelles forces i cette conjecture. Admettons donc cette dépendance, mais sans nier que les différens vices de conformation originelle, et les déviations de forme et de tissu qu'of. frent les os après la naissance, permettent de cruire qu'ils renferment primitivement en eux la cause de plusieurs modifications.

Les différences de forme des os sont, du reste, tellement considérables, qu'un seul et même os appartenant à des animaux divers, est à peine reconnaissable, surtout quand on le vuit hors de ses rapports naturels. Ainsi les côles des poissons sonten général très-minces, putites, extrèmement alongées, et.proportionnellement très. éloignées les unes des autres. Chez les chéloniens, elles sont, an contraire, très-généralement des os d'un grand volume, larges, entourés de bords dentelés que des articulations immobiles unis-sent enire eux, it que rles sutures solides fixent aur vertèbres et au sternum. Le sternum offre des différences au moins aussi saillantes. Chez le. viseaux, il est presque saus exception formé d'une seule pic̀ce, très-long, fort convexe, large, garni au milieu de sa face antérieure d'une crete longitudinale, fort saillante en avant et en haut; chez les autres animaux, il est le plus souvent aplati, étroit, composé de 
plusieurs pièces impaires qui se succèdent d'avant en arrière; chèz les chéloniens et animaux voisins, il est également plat, mais proportion. nellement plus grand encore que chez les oiseaux, et formé de plusieurs pièces, en partic paires, en partie impaires.

Les os du crâue des mammifères, surtout de ceux qui sont au haut de l'échelle, sont fort convexes; ceux des animaux inférieurs sont planes et aplatis.

Les os des membres offrent aussi les différences de forme les plus extrêmes; le rapport de leurs dimensions est très-variable; souvent ils sont percés d'ouvertures considérables et destinées à des usages importans, comme, par exemple, au condyle interne de l'humérus; ces ouvertures 'manquent dans d'autres animaux; d'autres fois la forme des surfaces articulaires et des apophyses diffère considérablement dans diverses espèces.

2. Volume.

Les différences de volume des os peuvent donner lieu à diverses considérations. On peut demander : $1^{\circ}$ si certains os ne varient pas de préférence sous ce rapport. Cette question peut être décidée affirmativement. Les vertèbres sont de tous les os ceux qui offrent le moins de diffé. rences dans le volume; les os des membres, surtont ceux de la main et du pied, sont les plus 
variables sous ce rapport. Entre ces deux exirèmes sont placés les os de la tête et les côtes.

Une telle diversité de volume dans les os des membres dépend évidemment de ce qu'en général les membres dont les os forment la base, offrent des variétés extrêmes qui influent sur le mode de locomotion.

$2^{\circ}$ Le rapport du système osseux avec les autres organes offre des différences très-considéirables. Ainsi, par exemple, dans les chéloniens, il atteint un développement relatif tel, que dans une tortue grecque, qui pesait une livre neuf onces, je trouvai le squelette du poids de dix onces. Le squelette qui reste dans les dimensions les plus restreintes, du moins sous le rapport de la masse, est en général celui des oiseaux et des poissons.

\section{Nombre.}

Après l'étude des différences de la configuration et du volume des os vient naturellement celle de leur nombre.

En général les vertébrés inférieurs possèdent le plus grand nombre d'os séparés. La preuve en est facile à donner; il suffit de comparer, en effet, le nombre des os de la tête d'un poisson ou d'un reptile avec celui des os d'un mammifère. On obtient le même résultat en rapprochant les os du tronc de ces animaux, les os des membres 
des poissons et des sauriens. Cette particularité dépend de deux causes.

D'un côté on voit, à l'état de séparation, chez. les animaux inférieure, des pièces osseuses, qui, chez les animaux supérieurs, se soudent entre elles; d'un autre còté, il y a plusieurs os, chez les premiers, qui se répètent réellement un plus grand nombre de fois que che\% les derniers. Liexemple de la première de ces circonstances nous est surtout fourni par les os du crâne et de la face, celui de la seconde par ceux du tronc et des membres. Ajoutons quil est possible cependant. et même très-vraisemblable, qu'il n'y ait ì ces deux états qu'un principe commun, et qu'ils rentrent l'un et l'autre dans le premier. L'exces de nombre des vertèbres des ophidiens et des poissons pent dépendre de lampliation des disques osseux antérieur et postéricur du corps de ces os; la présence chez beaucoup de poissons de plusieurs rangées d'apophyses épineuses supérieures et inférieures, peut provenir du développement en os propresque subissent les points d'ossification qui existentau sommet des apophyses épineuses de plusieurs mammilères. La rangée de côtes supérieures de beaucoup de poissons peut résulter également de l'ampliation et de l'état de séparation du point d'ossification particulier que l'on rencontre souvent au sommet des apophyses iransverses des verièbres. Quelques-uns, si ce 
n'est pas tous, se présemtent disposés successivement de la base au sommet; les nageoires des poissons, surtout des chondroptérygiens, sout sans doute les apoplyyses des vertèbres grossies et non soudées au corps. Le nombre considérable des rayons des nageoires euxmêmes, qui présentent la même disposition, s'explique peut-ètre de la même manière, quand on se rappelle que l'os métacarpien et le métalarsien des ruminans se compose primilivement de deux moitiés latérales entièrement séparées, qui ne se confondent en une seule piece que par la disparition insensible, mais totale, de leur paroi interne.

Quelque explication que l'on donne de son origine, celte augmentation du nombre des os est très-fréquemment accompagnée d'un déveIoppement imparfait de tout le squeletle ( 1 ). Il

(1) C'est cet état particulier de séparation ou de fusion des pièces osseuses, observé comparativement dans le squelette des espèces inférieures et dans celui des animaux élevés, à des âges divers et dans des étals variẻs d'anomalies, qui a permis à M. Geoffroy-Saint-Hilaire d'établir qu'un certain nombre d'élémens organiques concourait à la formation des parties analogues, que ces élémens organiques étaient dans une dépendance réciproque telle qu'ils restaient fondamentalcment et invariablement joints à certains d'entre eux, même dans l'èlat le plus rudimentaire, quoirqu'ils fussent susceptibles de quelques relalirns accidentelles avec certains autres, dans les conditions d'un développement différent; c'est celte analyse naturelle des piéces constitutives que $\mathbf{M}$. Geoffroy-Saint-Hilaire a décou. 
semble qu'un accroissement sur un point r'a jamais lieu qu'aux dépens d'un développement supprimé ou diminué à des degrés variables sur un autre.

Ainsi, chez les ophidiens, les sauriens et les poissons, le nombre variablement augmenté des vertèbres et de leurs os accessoires, qui sont multiples chez les derniers, coïncide avec l'absence ou la brièveté des membres en général; et dans les sauriens et les poissons, le développement plus considérable de la partie qui correspond à verte et dontil s'en faitl'historien, en imposant des désignations fixes et particulières à ces matériaux qu'il a poursuivis de modifications en modifications et d'une manière graduelle, après les avoir primitivement étudiés au maximum de leur développement. Ces observations lui ont donné la clef de différences insolubles sans les principes dont elle lui ont fait reconnaitre l'existence; tel est le principe rles affinite's de ces élémens entre eux, tel est celui de l'invariabilité des connexions. Il doit à ces recherches également d'avoir approfondi la loi suivant laquelle s'effectuent ces oppositions de développement dont parle ici M. Meckel, et que M. Geoffroy a rendues par l'expression de balancement des organes. $\mathbf{\Lambda}$ l'aide de la connaissance des matériaux constitutifs, des lois qui régissent lcur rapport de développement de connexion ou leurs afiinités, toutes les différences deviennent les modifications d'une même constitution primitive, et les analogies sont irrésistiblement établies. Les faits particuliers qui servent de base à ces considérations fourniront l'occasion, dans le cours de cet onvrage, d'exposer les prenves alléguées par M. Geoffroy-Saint-Hilaire en faveur de son système. L'analyse des pièces vertébrales, sternales, hyoïlales et scanılaires sera donnée d'après $\mathbf{M}$. Geoffroy quand il s'agira du rachis, de la lête, etc.

(Note des traducteurs.) 


\section{D'ANATOME COMPARÉE.}

la main et au pied, est dans un rapport direct avec l'absence ou la petitesse des fractions supérieures des membres; application de cette loi générale qui régit les phénomènes vitaux, et d'après laquelle l'accroissement de l'action vitale sur un point dépend d'une diminution sur un autre, ou la détermine.

\section{Texture.}

Sans parler du remplacement entier ou presque entier des os par des cartilages chez les céphalopodes, ainsi que chez un grand nombre de poissons, on peut dire que les véritables os offrent, sous le rapport de la texture, plus ou moins de différences dans les diverses divisions établies parmi les animaux.

Dans la plupart des os des oiseaux, surtout dans les os longs, aérifères ou non, le tissu spongieux est en général plus rare, plus grossier, conséquemment plus lâche; il en résulte que la cavité médullaire est proportionnellement beaucoup plus grande que dans les os des autres animaux; la substance compacte ou corticale est au contraire plus solide. Dans les os de la tête, c'est le diploé qui est fort développé et qui l'emporte de beaucoup sur la substance compacte. Cela existe surtout chez les oiseaux de proie nocturnes. Les os longs de quelques reptiles, spécialement des chéloniens, n'ont au contraire point de carité médullaire; il n’en existe pas de trace dans 
les os même les plus longs, comme dans l'humérus et le fémur: ces os n'offrent partout que dn tissu spongieux. Leurs côtes, très-fortement dé. veloppées, ont la même structure; celles des ophidiens sont tout-à-fait creuses el sans tissu spongieux.

Les os des poissons ont en général, à l'extérieur, une structure rayonnée, lâche, qui rappelle celle des os du foetus des mammiferes; ils n'ont pas de cavité médullaire, ou elle n'est du moins pas considérable; mais on y peut discerner le diploé, distinct de la substance compacte ou corticale, que l'homogénéité et l'absence de la disposition fibrilaire font également recunnaître. Chez certains mammifères il y a des os qui dévieut de la structure générale. Ainsi, par exemple, l'humérus et les os de l'avant-bras des cétacés, du moins dans le dauphin ordinaire (delphinus delphis, et dans le marsouin commun (phocaena), n'ont absolument point de cavité médullaire; ils sont tout-à-fait remplis de substance spongieuse solide et à petites aréoles. I.es os de ces animaux sont toujours à leur face externe, ainsi que dans leur sulsstance corticale en général, plus làches que chez les autres mammifères; c'est pourquoi il y a moins de différence entre leurs deux substances.

\section{Consistance.}

La consistance des os n'offre pas de différen- 
ces bien considérables, mais les os des oiseaux me semblent les plus cassans. Les os sont naturellement d'autant plus flexibles et plus mous que la proportion de la substance calcaire y est moindre.

\section{Composition.}

Les os des différéntes classes de vertébrés se distinguent particulièrement les uns des autres par le rapport de la substance animale avec les principes terreux. La première est plus aquense chez les vertébrés inférieurs, sirtnut les poissons; mais quoique après la dessiccation le rapport entre la substance animale et les élémens terreux y soit plus rapproché des conditions qui sout propres aux vertébrés supérieurs, elle y est toujours plus abondante que chez les derniers. Le squelette des poissons cartilagineux n'est pas uniquement formé de cartilages, quoique les os de leur tête, surtout chez la raie lisse, ne renferment qu'une très-pelite proportion de prineipes terreux (1).

L'analyse chimique y démontre en effet du sulfate, de l'hydrochlorate et du carbonate de soude, du phosphate de chaux, de la magnésie et du fer, du sulfale de chaux, etc. (2).

(1) Hatchett, sur le test des crusiacés et les os. Dans les Philos. trans. 1799.

(2) Chevreul, Annal. de mus., vol. XVIII, p. 150. 
Le squelette des poissons osseux contient toujours du phosphate de chaux en quantité, un peu de carbonate de chaux, et peut-être aussi, mais moins généralement, du phosphate de magnésie.

Il serait à désirer que l'on possédât plus d'analyses chimiques des os des reptiles.

Les différences considérables que présente le développement proportionnel de leur squelette rendent cette question intéressante. On sait cependant que les os de la vipère contiennent beaucoup de phosphate et peu de carbonate de chaux.

Chez les oiseaux, du moins chez les plus élevés, il y a une portion beaucoup plus considérable de sels terreux, surtout du phosphate de chaux, très-peu de carbonate, et une petite quantité de phosphate de magnésie.

Des analyses récentes (1) ont dérnontré aussi, dans les os de plusieurs mammiféres, du fluate de chaux, qui manque peut-être à d'autres animaux, particulièrement aux poissons, du moins si l'on en juge par la seule donnée que nous en. ayons.

\section{Coloration.}

Les différences de coloration des os sont ex-

(i) Analyse chimique des os de poissons, par Duménil, dans Trommsdorf's Journal für die Pharmacie. Bd. 4 , St, : S. 278 . 
trèmement insignifiantes; elles n'ont surtout aucun rapport avec les classes. Les plus saillantes sobservent dans certains genres, ou mème dans quelques espèces de diverses classes; de ce nomire est, par exemple, la couleur verte des os de l'orphue (esox bellone); et leur couleur noiritre, propre à plusieurs variétés des gallinacés.

8. Modes d'articulation.

L'articulation des os offre surtout des différences frappantes : il y a beaucoup d'os qui présentent tous les modes d'articulation possibles. Ainsi, par exemple, la partie antérieure et interne de la mâchoire supérieure, l’os intermaxillaire, est, chez la plupart des vertébrés, non-seulement séparé du reste, mais il s'articule mème avec l'os maxillaire supérieur proprement dit, d'une manière mobile chez la plupart des poissons et ophidiens; chez l'homme il y est soudé déjà long-temps avant la naissance, et chez beaucoup de mammifères cette soudure u'a lieu qu'arec l'âge. L'occipital est également uni par une articulation mobile avec le reste du crâne chez plusieurs sauriens, tandis que chezles autres animaux cette union a lieu par dessutures. Chez les mammifères la jonction des vertèbres entre elles, au moyen d'une substance fibrocartilagineuse qui occupe la majeure partic de leurs faces ct bords en regard, est fonl peu mo- 
bile; chez les oiseaux, l'articulation des vertibres cervicales contient un cartilage intermédiaire libre; chez les ophidiens ce carrilage n'existe pas, et dans toutes ces articulations, où, chez les mammifères, la connexion est opérée par des ligamens fibreux, il existe des ligamens synoviaux. Chez les chéloniens, au contraire, non-seulement les arcs des vertèbres, mais encore leurs apophyses épineuses, sont partout unies d'une manière tout-à-fait immobile par des sutures profondes. Un exemple encore plus frappant de ces variétés nous est offert par les côtes; chez les animaux verlébrés autres que les chéloniens, les côtes ne s'atteignent d'avant cu arrière que daus des cas exceptionnels, encore n'est-ce que daus de très-petites étendues; elles sont articulées en outre d'une manière plus ou moins mobile au moyen des ligamens synoviaux avec les vertèbres et le sternum; chez les chéloniens, elles sont soudées d’une manière imrnobile par des sutures, tant entre elles qu'avec les vertèbres et le sternum. Les membres offrent des différences encore plus grandes, s'il est possible, par le lieu aussi bien que par le mode de l'articulation. Chez la plupart des mammifères qui n'out point de clavicules, ou seulement des rudimens de clavicules, les membres antérieurs ne sont unis an reste du squelette que par des muscles, nulle part par des os et des ligamens; chez d'autres, cette con- 
D'ANATOMIF COMPARÉE.

nexion est opérée par une clavicule; chez les oiseaux et les saurieus, par une seconde clavicule, qui n'est autre que l'apophyse coracoïde grossie. I.es omoplates, libres vers la face dorsale du corps chez la plupart des vertébrés, s'appliquent, chez les poissons, aver. Jeurs extrémités tournées vers celte face, contre le crâne ơu le rachis; en revanche elles ne sont pas attachées à un sternum. Les os du bras et de l'avant-bras, articulés mobilement chez les autres animaux, sont, chez eux, communément confondus en un tout. Les membres postérieurs présentent des différences peut-être plus considérables. Chez les oiseaux et les mammifères, leurs premières fraclions, les os coxaux, sont articulées, on peut dire immobilement, avec l'extrémité inférieure de la colonne vertébrale. Chez les mammifères et les reptiles pourvus de membres parfaits, et chez les poissons eux-mêmes, ces os, dits de la hanche, salteignent en mème temps sur un ou deux points dans la ligne médiane; ils sōnt dans cés mèmes points séparés chez les oiseaux, à peu d'exceptions près.

Chez beaucoup de reptiles ils ne sont, au contraire, unis au rachis que très-lâchement; leur jonction qui est mobile, a lieu par des ligamens capsulaires; chez les poissons, les muscles sont les moyens d'union.

Le fémur est uni, chez la plupart des vertébrés, a l'os coxal par un ligament intéricur, le 
ligament rond, qui manque cependant chez quelques-uns, par exemple chez lorang-outang, l'aï ou le paresseux à trois doigts, et chez les chéloniens.

Chez les oiseaux le péroné monte jusqu'au fémur; chez les mammifères le tibia seul est en contact avec l'os de la cuisse.

Les différences considérables dans la disposition des facettes articulaires des os du carpe et des orteils, déterminent les degrés très-divers de mobilité de ces fractions des membres.

\section{Différences périodaires.}

Outre ces différences qui se rencontrent à l'élat parfait, le système osseux présente, dans les divers animaux, d'autres différences trèsconsidérables qui dépendent des phases de la vie, et qu'il n'est pas facile de ramener à des lois générales.

A. Les plus frappantes et les plus générales de ces différences se rapportent au temps de l'apparition de certains os et parties d'os, et in celui de la soudure de ces dernières en un seul tout.

La circonstance que, chez les vertéłsrés inférieurs, plusieurs pièces osseuses, qui se confondent en un seul os chez les hauts vertébrés, restent séparées pendant toute la durée de lit vie, pourrait faire présumer que la rapidité du 
développement des os est en rapport direct de la perfection de l'organisation. Mais cette conjecture est contredite par l'expérience. Ainsi, par exemple, chez le porc, on trouve le corps, les graudes ailes et les moitiés latérales de la moitié antérieure du sphénoïde, soudés ensemble vers le milieu de la vie intra-utérine. Vers la mème époque existe déjà, chez cet animal et chez le lapin, le corps de la première vertèbre cervicale. Chez le chien il existe aussi déjà quelque temps avant la naissance. Chez le foetus à terme de la brebis et du cabiai (hydrochaerus), les arcs des vertèbres sont déjà confondus en un seul os. Ils offrent de même, à cette période, déjà tous les noyaux osseux dont se forment insensiblement les os cylindriques.

Chez le chien et chez l'homme les os longs n'offrent, à la naissance, qu'un noyau osseux, et les diverses portions du sphénoïde sont encore plus ou moins séparées les unes des autres.

Il se présente ici une question: Existe-t-il réellement entre les divers animaux une si grande variété dans la rapidité du développement des os? Il serait fort possible que l'état embryonique, chez quelques-uns, surtout chez ceux dont le système osseux se développe en apparence plus tardivement, constituât une portion plus courte de toute la vie que chez d'autres. Par conséquent, si on faisait abstraction de la différence entre cet état de l'embryon et celui de 
l'animal formé, différence qui ne doit évidemment pas être considérée sous le rapport du développement de ces organes, la période de l'origine et du développement des os serait la mème pour tous les animaux; mais cela n'a pas lieu en effet. Ainsi, chez l'homme, le point d'ossification du corps de la première vertébre cervicale se manifeste dans le sixième mois après la naissance; chez le chien, avec le deuxième, chez la brebis, le porc, le lapin, avec le troisième mois de la vie embryonique, et enfin chez le cabiai, au milieu de la seconde senaine après la conception. Si on admet pour toute la durée de la vie de l'homme tout au plus 70 ans, 15 pour celle du chien, 14 pour celle de la brebis, 20 pour celle du porc, 9 pour celle du lapin, 6 pour celle du cabiai, il en résulte, entre le temps de l'origine de cet os et la durée de la vie de l'animal, le rapport suivant:

Chez l'homme celui de $1:: 46$.

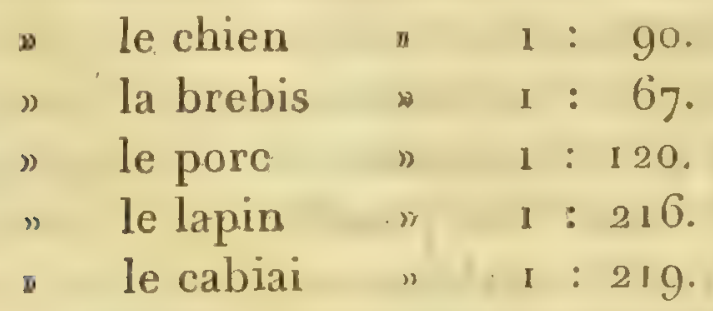

Le rapport dans le développement des autres os offre des différences très-analogues. La conséquence immédiate et la plus générale de ces exemples est que l'ostéogénic s'opère d'autant plus rapiclement que l'animal est plus fécond, 
DANATONIE CuMPARE.

puisque les animaux chez qui elle est la plus prompte alleignent l'âge adulte le plus vite, conçoivent le plus souvent pendant la même période, et apportent au monde le plus grand nombre de petits. Le rapport entre le degré de l'intelligence et la rapidité du développement des os marche en parlie de front avec la faculté génératrice, quoique cela n'ait pas lieu, par exemple, chez la brebis, chez laquelle la formation est plus lente que chez le chien.

() n peut en outre établir en général, d'après les recherches connues jusqu'à ce jour, que l'ostéogénie est lente chez l'homme.

Quoiqu'il $y$ ait des os, chez les animaux, par exemple le sphénoïde, qui restent séparés en plusieurs piéces pendant toute la durée de la vie, il est d'observation que la soudure de ceux qui se soudent habituellement 'hex eux s'opere communément plus tôt que chez l'homme. Une différence plus spéciale entre l'homme et les animaux semble consister en ce que, chez ces derniers, les portions d'un même os, placées bout à bout, ne se soudent pas pendant toute la durée de la vie; tandis que les parties latérales de même nom se soudent plus vite, soit entre elles, soit avec un os impair moyen. Ainsi le sphénoïde antérieur et le postérieur restent constamment séparés chez la plupart des mammiféres; les maxillaires supérieurs et l'os inter maxillaire ne se sourdent également qu'ì 
l’âge le plus avancé; on voit au contraire les parties latérales des sphénoïdes antérieur et postérieur se souder respectivement entre elles bien plus tôt que chez l'homme. Cette différence semble dépendre de trois causes: $1^{\circ}$ de la ressemblance entre les os du crâne et les vertèbres, plus grande chez les animaux que chez l'homme; ce qui tient à ce que la ressemblance des différentes parties augmente en général en descendant dans la série animale. Les portious antérieure et postérieure du sphénoïde des animaux, considérées chacune à part, ressemblent en effet plus à une vertèbre que ne le fait le sphénoïde de l'homme, formé de ces deux portions. $2^{\circ} \mathrm{On}$ peut considérer comme la seconde cause, la loi d'après laquelle se développent les os de la tête et même en général tous les os dans la série animale. Ce développement se fait évidemment dans le sens de la longueur. De mème qu'il n'existe d'abord que la colonne ver. tébrale, de mème c'est d'arrière en avant que se forment inseusiblement d'abord les os de la tête situés dans la ligne médiane, auxquels s'ad. joignent seulement plus tard les os latéraux. Les os les premiers formés restent d'autant plus séparés que l'animal est plus inférieur et se soudent d'autant plus vite que les os latéraux se développent davantage. $3^{\circ}$ La troisième cause consiste dans la différence de volume. Ainsi le sphénoïde antérieur est, relativement au postérienr, 
beancoup plus volumineux chez les animaux que chez l'homme; sourent même il a plus de volume absolu; les ailes latérales du dernier sont surtout beaucoup plus petites. L'os intermaxillaire est aussi, relativement à l'os susmaxillaire, beaucoup plus considérable. De là la tendance qu'il offre à se développer en un os propre et à ne pas se réunir avec l'os auquel on le trouve annexé chez l'homme.

B. Les différences dans le mode de développement des os en parliculier et de tout le système osseux en général sont beaucoup plus rares.

Il se présente trois questions sur le premier rapport:

$I^{\circ}$ Chez tous les animaux, le même os est-il formé du mème nombre de pièces?

$2^{\circ}$ Chez tous, ces pièces se soudent-elles entre elles dans le mème ordre, ou cette circonstance In'a-t-elle pas lieu?

$3^{\circ}$ La disposition et la position relative de ces Ipièces osseuses sont-elles partout les mêmes?

Quant à la seconde condition, il s'agit de -savoir si les différens os présentent, chez tous les animaux, le même rapport réciproque, eu égard à l'épeque de leur apparition, ou si chez quelques-uns les mêmes os se manifestent plus tòt que d'autres; si chez d'autres, tels os se montrent et se soudent plus tôt, et tels autres plus tard.

dutant que je puis conclure d'un nombre 
considérable de recherches faites ì cet égard, les différences, dans le sicond cas, sont bien moindres que dans le premier, sans doute parce que les différences qui intéressent les fonctions ou la manière d'être d'un organe constituent une plus grande déviation du type général que celles qui n'ont trait qu'au nombre des pieces qui entrent dans sa composition. On en trouve toutefois plusieurs exemples.

Ainsi, sous le premier rapport, aucun des mammifères, hormis l'homme, ne présente, it la septième vertèbre, de noyau osseux particu. lier, entourant en avant le trou vasculaire. quoique ce trou existe constamment; chez quelques animaux, et particulièrement les ruminans, du moins la brebis, il y a un point d'ossification propre pour l'apophyse épineuse de plusieurs vertèbres moyennes, surtout de celles à longues épines; ce-noyau osseux manque aux autres.

L'occipital se forme, chez la plupart des mammifères, d'un nombre plus considérable de pièces que cela n'a lieu chez l'homme et le porc, du moins dans un grand nombre de cas.

Chez les oiseaux, la mâchoire inferieure est composée, de chaque côté, d'un nombre considérable de pièces osseuses; il n'en existe qu'une seule chez les mammifères.

Les exemples de la seconde espèce sont plus rares; cependant nous en voyons un dans le mode d'ossification du sphénoide. Chez l'homme 
la lame interne des apophyses ptérigoïdes se soude bien plus tôt à la grande aile que celle-ci ne s'unit au corps du sphénoïde. Le contraire a lieu chez divers animaux, particulièrement chez le chien, la brebis, le porc; phénomène extrêmement curieux, puisque la longue durée de la séparation de cette pièce osseuse d'avec la grande aile rappelle la séparation constante de cet os chez les oiseaux, les reptiles et les poissons.

L'occipital offre des phénomènes analogues. Chez l'homme ses pièces latérales et supérieures se soudent entre elles plus tôt qu'avec le corps del'os; chez les ruminans et le porc, au contraire, les pièces latérales s'unissent au corps bien plus tôt qu'avec la partie supérieure ou occipitale proprement dite. Les chiens offrent quelque chose d'analogue, puisque la pièce la plus supérieure de la portion occipitale reste séparée encore fort long-temps après que toutes les autres sont soudées ensemble.

La disposition et la situation des pièces osseuses isolées est en général la même; mais il y a aussi des exceptions à cette règle. Ainsi, par exemple, la partie moyenne de la mâchoire inférieure des oiseaux, qui comprend la partie antérieure et en même temps la plus considérable de chaque moitié, est formée, d'un os impair, quoique chacune des moitiés se compose d'un grand nombre de pièces osseuses; chez les 11. 
mammifères, les deux moitiés latérales de la mâchoire inférieure sont, au contraire, primikivement séparées dans toute leur longueur, et ne se soudent que dans la ligne médiane.

Un exemple à l'appui de la proposition que j'émels sur le développement des différens os du mème squelette, qui ne se fait pas partout exactement dans le même rapport, nous est fourni par la mâchoire inférieure: chez l'homme, les deux moitiés se soudent d'extrêmement bonne heure, beaucoup plus tôt que les pièces diverses de la plupart des os; chez le plus grand nombre des animaux elles restent, au contraire, séparées bien plus long-temps que dans les autres os. 


\title{
GHAPITRE IX.
}

\section{SQUELETTE DES POISSONS.}

\author{
A. OS DU TRONC.
}

a. Colonne vertébrale (1).

1. Poissons cartilagineux.

$$
\text { S. } 69 \text {. }
$$

\section{Les premières traces de l'arc des vertèbres se} :sont rencontrées chez les céphalopodes. La co-

(1) Neuf élémens composent, suivant M. Geoffroy-SaintHilaire, une vertèbre parvenue à son maximum de développement. Le volume proportionnel, l'état d'ussification plus ou moins avancé, la condition de séparation ou de soudure, quelques jeur dans les rapports secondaires de position, quelques accidens de forme, la présenc ou l'absence de ces mêmes pièces constituent toutes les différences qui caractérisent la série vertébrale dans les diverses espèces d'animaux observées aux âges successifs deleurvie; et sil'on étend cette considération, sil'on ad’met qu'un certain nombre d'élémens distncts se retrouvent également dans la composilion des membres, des systèmes hyoïdanx, sternaux, etc., si les mèmes variations de condition dans ces matériaux primitifs donnent aussi la clef des modifications subies par les diverses parties du squelette; la connaissance première de ces faits très-simples suffira pour comprendre les innombrables différences que présentent tous les états variés de l'ensemblc du systeme osseux. Par ce système, dont les fonctions sont dans une si étroite dépendance avec les autres parties de l'économie, on peut avoir des données sur ces parties ellesmèmes; ainsi les rapports les plus intimes unissent le sys- 
lonne vertébrale des cyclostomes, qui font partie des poissons cartilagineux, présente des perfectionnemens nouveaux: ou l'arc des vertèbres s'y développe davantage, ou ces os offrent de plus un corps.

Je n'ai pas eu occasion d'examiner les ammo. coetes et les myxinés. M. Cuvier dit que les premiers ont constamment un rachis membraneux (1). Voici la disposition qque j'ai trouvée chez les pétromyzons.

tème osseux anx vaisseaux et aux nerfs dónt il est toujours le moyen de protection; ainsi il n'est qu'une portion du système dermoïqne qui en est la matrice. L'étude des pièces osseuses peut donc conduire aux connaissances les plus profondes des phénomènes de l'organisation. L'analyse des divers élémens osseux présente dans les poissons cartilagineux des difficultés qui ne s'offrent pas ailleurs. L'état cartilagineux ne permet pas de circonscrire chaque élément isolé. Il n'en est pas autrement du larynx humain; pris à un âge trèsjeune encore, cet organe n'est formé que d'une seule pièce; alors c'est une seule membrane dans l'épaisseur de laquelle il se manifeste plustard des noyaux plus consistans qui s'isolent et finissent par présenter la multiplicité des pièces qui constituc l'état adulte de cette cavité. Les poissons cartilagineux sont arrêtés à ce premier degré de développement; ce n'est que dans les poissons osseux que l'on voit prononcer les traits de démarcation propres à déterminer chaque élément spécial; aussi est-ce une des espèces que comprend ce dernier groupe dont M. Geoffroy a fait choix pour établir ses considérations sur la vertèbre, qui seront rappelées aux poissons osseux.

( Note des traducteurs.)

(1) Sur la composition de la máchoire supérieure des poissons, Mémoires du muséum d'histoire naturelle, t. I. 
l) ANATOMIE COMPARḰ.

La partie du rachis qui représente la série des corps des vertèbres constitue deux cônes adossés par leur base; l'ensemble qu'ils forment est très-alongé, s'étend dans toute la longueur du poisson, et se termine à la tête qu'il en faut excepter; l'extrémité antérieure représente un sommet mousse, qui s'appuie sur le crâne, et se continue en effet immédiatement avec lui; l'extrémité postérieure, beaucoup plus apointie et plus mince, forme en outre l'extrémité de tout le corps. Ce rachis est composé de deux parties, l'une externe et l'autre interne, qui diffèrent sous le rapport de leur consistance.

La partie extérieure est creuse, fibro-cartilagineuse, très-élastique et formée de parois qui sont très-minces, si on a égard à la capacité qu'elles renfermeut ; chez un petromyzon marinus, long de plus de deux pieds, elles ont à peine un cinquième de ligne d'épaisseur. Elle forme un tout parfaitement homogène.

La forme en est généralement circulaire; les faces externe et interne en sont unies, elles adhèrent intimement aux parties voisines. Cependant quand on examine de plus près ces deux faces, on voit qu'un très-grand nombre d'incisures circulaires extrêmement rapprochées les unes des autres, en rendent la superficie inégale, re quifait paraître cette partie externe composée d'un nombre extraordinaire d'anneaux disposés dans un ordre successif d'avant en arrière. 
A la région inférieure de la circonférence du canal que forme cette partie extérieure, existe - des deux côtés une strie saillante, courte, triangulaire, plus consistante, plus jaunâtre; cette strie, dont le sommet libre est tourné en dehors, lui adhère assez intimement, mais peut en être séparée sans qu'il en résulte de lésion ni pour elle, ni pour la partie qui lui sert de support. Les deux rangées de stries sont unies et tenues entre elles par une membrane aponévrotique, blanchâtre, mince, mais solide, qui s'attache aussi supérieurement sur les côtés du double cône cartilagineux; cette membrane est fermée au dessus de la portion de la colonne qui représente les arcs des vertèbres, portion dont elle constitue, en effet, toute la parties upérieure.

La partie intérieure est pleine. En avant, le cône cartilagineux se termine insensiblement par une pointe mousse; il pénètre de quelques lignes dans le crâne et occupe à peu près le dixième postérieur de toute la longueur de la tête. Sur le vingtième antérieur de toute la longueur du rachis, l'enveloppe aponévrotique devient à la fois plus épaisse et plus facile à séparer exactement; les pièces latérales deviennent plus dures, réellement osseuses, et sont inférieurement plus rapprochées vers la ligne. médiane, jusqu'à ce qu'elles s'y confondent au crâne. Dans la partie postérieure du rachis, elles se portent également en bas; plus antérieurement, 


$$
\text { 1) ANATOMIE COMPARÉE. }
$$

tlles sont dirigées en dehors; enfin, tout à fait en arrière, elles s'atteignent dans la ligne médiane.

La substance contenue dans ce canal est demifluide; elle ressemble à de la gélatine coagulée; elle est partout de même consistance; sà couleur est le blanc-jaunâtre; elle se divise trèsfacilement en quatre cordons, deux supérieurs et deux inférieurs : les uns et les autres, placés sur les côtés, se rencontrent dans la ligne médiane sans laisser de lacune entre eux. Chacun de ces cordons semble composé d'un nombre infini de lamelles triangulaires, extrêmement minces, qui se succèdent de très-près d'avant en arrière, et sont unies entre elles par du tissu cellulaire serré. Cette substance remplit exactement le canal; aussi est-il difficile de l'en séparer sur quelques points, et paraît-elle par conséquent ne faire qu'un avec lui.Il semble du moins qu'il faille lui attribuer l'aspect sillonné de ses parois. Mais elle n'est pas plus entourée d'une membrane propre, qu'elle ne renferme une cavité et un fluide dans son intérieur.

I.es conditions générales de la disposition de la partie inférieure du rachis ont déjà été indiquées par MM. Duméril (1), Home (2)et Carus(3).

(1) Sur les foissons cyclostomes. Paris, r 8 ro.

(2) Lect. on comp. anatomy. Lond., 1814, p. 88.

(3) Sur quelques particularités de la lamproie. Meckel's Archiv. Bd., 2. S. 600 . 
M. Duméril décrit la substance aponévrotique, la plus extérieure, comme ronde, molle et plus dure à certaines périodes de l'année; remarque qu'il ne m’a pas été possible de confirmer jusqu'ici.

D'après Home, la structure de cette partie dans les lamproies de mer (petromyzon) coinciderait exactement avec celle des esturgeons (accipenser). Cette opinion renferme plusieurs inexactitudes, puisque Home décrit mal cette disposition chez l'esturgeon, et que ces deux genres offrent, à cet égard, de grandes différences. Carus n'est pas plus exact quand il dit " que la cavité du canal cartilagineux disparaît - vers le milieu du corps; que, par conséquent, - la portion postérieure de la colonne verté- brale n'est formée que d'un cylindre cartila" gineux très-élastique, rempli intérieurement "d'une substance cartilagineuse plus molle." Je trouve, en effet, dans toute la longueur du corps absolunient la mème disposition de la substance contenue, et le même rapport de cette substance avec le cylindre qui la contient.

Cette différence de la partie intérieure aux diverses régions de la colonne vertébrale n'a donc pas lieu dans la nature.

Aucun des auteurs qui m'ont précédé n'a, au contraire, indiqué, à ce que je sache, les rudimens distincts des apophyses transverses; on ne connaissait pas mieux le passage de la por- 
D'ANATOME COMPARÉE.

tion inférieure du rachis dans la base du crâne.

La partie de la colonne qui correspond aux arcs est plus petite que celle qui représente les corps des vertèbres, et forme une gouttière triangulaire, dont la base est dirigée en bas, le sommet en haut. Cette gouttière est presque entièrement aponévrotique dans sa partie supérieure, et se compose de deux conduits superposés. Le plus inférieur de ces conduits entoure la moelleépinière, et forme, avec la partie moyenne et supérieure du corps cartilagineux des vertèbres, le canal triangulaire déprimé de haut en bas, qui est destiné à loger la moelle. Les parois de ce conduit sont aussi composées extérieurement d'une substance cartilagineuse, en dedaus d'une substance aponévrotique, blanchâtre, substances qui se continuent, sans interruption, avec celle des parois $\mathrm{du}$ cartilage qui correspond au corps des vertèbres. Il existe, en outre, des deux côtés une série de pièces triangulaires, concaves à leurs faces supérieure et inférieure, alongées, beaucoup plus dures, assises sur la face externe de cette substance cartilagineuse, unies entre elles par cette substance et dépassant ce canal en haut. Ces pièces concourent ainsi à la formation du canal qui est situé au-dessus. Ces pièces cartilagineuses ne sont unies partout dans la ligne médiane que par de la substance fibro-cartilagineuse, et ne se confondent nulle part en une seule. 
Le canal supérieur est triangulaire, apointi en haut; il est formé inférieurement, des deux cótés, par la partie supérieure de la série de pièces cartilagineuses mentionnées, au milieu par la substance fibro-cartilagineuse qui unit ces pièces, aux parties latérales et supérieure uniquement par l'enveloppe tendineuse qui entoure extérieurement le corps et la partie inférieure.

Il recèle une masse molle, colorée en rougebrun dans la partie moyenne, qui est la plus considérable du rachis, où il est beaucoup plus ample que le canal rachidien.

On voit naître de son sommet et se continuer, dans la ligne médiane, jusqu'à la peau, dans la majeure partie du corps, une membrane tendineuse, mince, qui partage le corps de l'animal dans toute sa longueur en deux parties latérales, égales.

La partie inférieure de la colonne vertébrale, celle qui correspond aux corps des vertèbres, aussi bien que la supérieure, correspondant aux arcs, n'offre pas les mêmes conditions dans toute la longueur du rachis. Vorci les plus dignes de remarque.

Toute la partie qui représente les arcs est peu élevee, mais large dans la portion la plus antérieure de la colonne vertébrale; les pièces osseuses dures convergent pen, et sont par.conséquent unies par une masse fibro-cartilagineuse, large; la cavité supérieure située au-dessus de 


\section{1) ANATOMIE COMPARÉE.}

la moelle spinale est à peine indiquée; elle est remplie de substance fibro-cartilagineuse; il existe à peine une trace de la cloison fibreuse. Vers la portion moyenne de la colonne rachidienne, cetle cavité et cette cloison se développent de plus en plus; la cavité se remplit d'une masse plus molle; les pièces cartilagineuses se tournent un peu les unes vers les autres, mais il reste encore beaucoup de distance entre elles. Dans la moitié postérieure, la partie supérieure des arcs offre surtout une modification remarquable. A l'endroit qui correspond à la large nageoire du dos, c'est-à-dire dans presque toute la moitié postérieure, cette cloison aponévrotique se transforme en une multitude de rayons cartilagineux, très-oblongs, devenant insensiblement plus minces, qui, se succédant immédiatement les uns aux autres, sont cachés d'abord, quelques-uns seulement, surtout en avant, dans la partie charnue par toute leur moitié inférieure, mais qui s'étendent partout jusqu'au bord des nageoires dorsales qu'ils concourent à consolider. La plupart d'entre eux, principalement ceux qui existent dans la nageoire postérieure, se bifurquent à peu près vers le commencement de leur tiers supérieur; il en résulte un rayon antérieur et un postérieur. Le premier se partage quelquefois à son tour en une moitié antérieure et en une postérieure; mais jamais on ne les trouse composés de plu- 
sieurs pièces dans le sens de la longueur. Le nombre de ces rayons surpasse considérablement celui des moitiés d'arcs; très-serrés et très-étroits, ils leur sont dans le rapport de 4 à 1. On voit en même temps disparaître la cavité supérieure; le rudiment qui en subsiste se remplit de substance fibro-cartilagineuse; d'où il suit que les rayons cartilagineux semblent, du moins en partie, être formés aux dépens de la substance contenue dans cette cavité. Les moitiés d'arcs qui se succèdent bout à bout sont plus fortement dirigées les unes vers les autres dans la ligne médiane; elles ne sont jamais soudées, mais on les trouve partout unies entre elles par une couche proportionnellement large de substance ligamenteuse.

$$
\text { S. } 70 .
$$

La disposition du cylindre sous-jacent à la moelle spinale, et le mode de passage des vertèbres dans le crâne, placent les esturgeons après les cyclostomes. Mais les chimères et le polyodon-feuille (squalus spatule) sont encore plus rapprochés par la disposition du rachis entier. Aussi ces animaux seront-ils les premiers dont nous allons traiter.

Dans la chimère arctique le corps est formé d'un cylindre cartilagineux, d'une largeur égale dans sa moitié antérieure, et qui se rétrécit ensuite peu à peu jusqu’à un degré remarquable; il est 
absolumentcreux jusqu'al'extrémité dela queue; son calibre, chez un individu de 2 pieds 6 pouces, offre euviron 3 lignes à l'end̆roit de sa plus grande largeur, l'épaisseur de ses parois ayant un peu plus d'une demi-ligne. Les trois cinquièmes antérieurs, à l'exception du premier Itrentième, sont formés par une quantité extraordinaire d'anneaux cartilagineux complets, très- rapprochés les uns des autres et unis entre eux I par du tissu fibreux d'une manière tellement étroite qu'ils ne se manifestent que sous la forme de légères saillies. Chez l'individu précité, il y a environ quarante de ces anneaux dans l'étendue d'un pouce; ils n'ont pas tout à fait un tiers de ligne de largeur. Ces petites saillies manquent dans les deux cinquièmes postérieurs, et le cylindre qui constitue le filament caudal a la forme d'un carré alongé, comprimé d'un côté à l'autre, et considérablement plus élevé de haut en bas.

Ces anneaux manquent dans la partie la plus antérieure, qui est un peu plus étroite ; le cylindre semble par conséquent formé ici d'un cartilage simple : il en résulte cette particularité remarquable, que l'extrémité -antérieure ressemble à la postérieure.

Le cylindre est ici fermé par une lame cartilagineuse mince, qui opère l'articulation avec le crâne; elle sera décrite plus tard, en traitant des raies et des squales.

Dans l'intérieur de ce cylindre creux, on en 
trouve un second, beaucoup plus étroit, membraneux, fibreux, étroitement attaché à celui qui le contient. J'examine ce cylindre sur un squelette que j'ai préparé il y a quelques années et que j'ai fait amollir très-facilement; je n'y trouve aucune trace de division; il est vraisemblable qu'il ne contenait, comme chez les cyclostomes, qu'une substance gélatineuse sans discontinuité.

Le cylindre cartilagineux présente en haut et en bas, des deux côtés, une saillie plus molle, plus membraneuse et plus mince; cette saillie devient d'autant plus molle qu'elle s'en éloigne davantage: elles peuvent ètre séparées toutes deux du cylindre, sans lésion pour les anneaux. Les inférieures représentent de courtes apophyses transverses, et forment les parois d'un demi-canal large, peu élevé; les supérieures sont les arcs, unis supérieurement dans la ligne médiane, et formant, avec le cylindre, le canal destinéà la moelle spinale.

On ne distingue pas bien sur ces saillies une division en plusieurs pièces qui succèdent d'avant en arrière, comme sur le cylindre qui représente les corps des vertèbres.

En avant, où les anueaux manquent, cette partie qui représente les arcs est plus intimement unie avec le corps, elle est ici en mème temps plus élevée et se continue, en outre, en une plaque carrée, considérable, haute, aplatie 
d'un còté à l'autre, qui s'étend en arrière encore un peu plus loin que la partie antérieure du cylindre qui est dépourvue d'anneaux. Le bord supérieur de cette plaque est semi-lunaire, fort concave; en avant aussi bien qu'en arrière, mais surtout ici où elle est plus élevée, la plaque s'élargit des deux côtés, les prolongemens qu'elle four* nit sont analogues à des ailes; elle se termine supérieurement par deux pointes latérales, recourbées en arrière; les pointes antérieures, plus petites, sont entièrement libres, les postérieures reçoivent entre elles les nageoires dorsales antérieures qui s'y articulent par ginglyme,

De la partie inférieure de l'aile postérieure qui est transverse, naît un pont cartilagineux, mince et court, qui se joint à l'arc suivant.

La nageoire dorsale antérieure se compose d'une épine osseuse, aigue, très-forte, longue, convexe en avant, et d'une plaque triangulaire, plus mince, large d'avant en arrière, comprimée d'un côté à l'autre, qui est située derrière l'épine et forme la base de la partie postérieure de la nageoire.

La base de la nageoire dorsale postérieure, qui est beaucoup plus longue, mais bién moins élevée et occupe la majeure partie du corps, est formée par des rayons cartilagineux, simples, alongés, éloignés les uns des autres de toute leur largeur, occupant la ligne médiane, et en rap- 
port avec les arcs par une bande membraneuse qui est située plus haut.

La nageoire elle-même se compose seulement d'un bien plus grand nombre de rayons membraneux très-rapprochés les uns des autres.

Chrez le polyodon feuille, la formation est plus parfaite, il y a un même nombre de corps et d'arcs des vertèbres (1).

$$
\$ \cdot 71 .
$$

Cette organisation de la colonne vertébrale semble être suivie de celle des esturgeonis; ils ont également un canal cartilagineux, parcourant toute la longueur du tronc, situé sous la moellespinale et rempli d'une masse gélatineuse et molle; mais déjà cette partie offre des traces d'un développement plus élevé, sa paroi est, relativement à la cavité qu'elle renferme, au moins deux fois aussi épaisse que dans les lamproies. Cee canal est extraordinairement extensible; chez de jeunes individus, du moins, on peut sans le casser lui donner, par la traction, trois fois la longueur du tronc; quand je n'employais pas cette extension en longueur, je ne pouvais pas remarquer quatre rangées de stries qui s'unissaient en arrière sous un angle aigu; mais en employant cet artifice on les voit paraître; elles

(1) Cuvier, sur la composition de la máchoire supérieure des poissons. Mémoires du muséum d’histoire naturelle. 
résultent d'une modification subie par dea stries circulaires qui existent en effet et qui se convertissent en celles dont il s'agit. La masse gélatineuse contient dans son intérieur, pas touta-fait dans l'axe, mais un peu plus bas, un canal rond entouré d'une gaîne particulière, résultant d'un épaississement et d'un endurcissement de la substance gélatineuse; ce canal renferme un fluide ténu.

D'après Home (I), ce canal doit être composé d'une manière très-remarquable; il formerait, suivant cet auteur, une série de cavités rhomboïdes, correspondant au nombre des anneaux cartilagineux dont il va être question, et communiquant entre elles par des orifices étroits; mais je n'ai pas pu trouver cette disposition : elle a également échappé à Baer (2); et je regarde par couséquent, avec cet anatomiste, les dilatations observées par Home comme accidentelles et dues à des ruptures.

La colonne vertébrale offre encore d'autres perfectionnemens dans sa disposition : ils consistent en partie dans l'ampliation et dans le développement plus complet de la masse qui représente les arcs des vertèbres, en partie dans la formation de pièces cartilagineuses à la périphérie du cylindre que nous avons décrit.

(1) Lectures on comparative anatomy. T. I, p. 87-88.

(2) Baer dans son second rapport sur les travaux anatomiques de l'Université de Knnigshberg, 1819, p. 18.

II. 
On observe, en, effet, sur les parties latérales, et sous la face abodominale de ce cylindre, un nombre considérable de cartilages carrés, minces, convexes à la face externe, concaves à l’interme, plus longs d'avant en arriere que de haut eu bas. Dans la moitié antérieure de la colonne vertébrale, leurs bords inférieurs ne s'inclinent les uns vers les autres que sur la ligne médiane, et sont unis par une membraue mince; daus la moilić postérieure, au contraire, ils se confondent!toul-à-fait,

Immédiatement sous leur bord antérieur, on voit naîlre du plus petit nombre des veriebres antérieures, c'est-i-dire dienviron quarante d'entre elles, une apophyse transverse, courte en proportion, dont le sommet est uni à la côte par un ligament.

Leur face interne donne naissance, un peu au-dessous de son milieu et dans toute sa longueur, à une apophyse plus courte, mais plus large, qui, dirigée en dedans et réunie à la partie inférieure du cartilage, forme un demi-canal dont le bord interue euvoie une membrane minçe qui unit ices apophyses des deux côtés. Ces apophyses réunies, la membrane qui en remplit l'iutervalle et la partie inférieure du cartilage, constituent un canal en partie membraneux, en partie cartilagineux; ce canal qui existe dans la portion inférieure de la colomne vertébralè loge Taorte. 
D'ANATOME COMPARÉE.

Indépendamment de ces pièces latérales et inférieures, il y en a quisont latérales, moyennes et supérieures; elles recouvrent le cylindre cartilagineux d'en haut et forment la partie qui correspond aux arcs.

Ces pièces supérieures et latérales ont la forme d'un triangle alongé; elles se rétrécissent de bas en haut, devienuent convexes en dehors, et forment, en se réunissant des deux côtés, deux conduits superposés.

Le supérieur, qui renferme une substance ligamenteuse, est alongé, plus profond et plus étroit que l'inférieur et n'est qu'un demi-canal, parce que les sommets des pièces latérales des deux còtés ne se rencontrent pas dans la ligne médiane; mais une partie de la base de l'os supérieur, moyen, s'interpose entre elles et complète ainsi le canal. D'après d'autres recherches (1), les deux côtés des pièces latérales supérieures formeraient à eux seuls ce canal supérieur; mars je puis assurer que la disposition que j’ai indiquée est la véritable.

La paroi inf́érieure de ce canal n'est au contraire formée que par une saillie interne des deux pieces latérales, qui ne se soudent cependant pas ici, mais qui sont séparées par une couche étroite de substance ligamenteuse.

Cette saillie forme en même temps la paroi supérieure du canal inférieur.

(1) Baer, li. d. ; p irg. 
En bas, vers le cylindre cartilagineux, les pièces latérales et supérieures se tournent également les unes vers les autres, dans la direction de la ligne médiane; mais elles ne sont jamais unies en cet eudroit que par une membrane mince, assez large en proportion. De cette disposition résulte un canal régnant dans toute la longueur du rachis, formé uniquement par les arcs des vertèbres et contenant la moelle-épinière. La membrane mince qui unit inférieurement les pièces latérales et supérieures permet de distinguer, à travers, l'éclat de ce cordon médullaire; ni le cylindre, niles pièces cartilagineuses inférieures qui le recouvrent, ne prennent pas part à la formation de ce canal.

Le sommet des pièces latérales et supérieures est surmonté d'autres pièces cartilagineuses, propres, alongées, rétrécies de bas en haut, comprimées d'un côté à l'autre et très-rapprochées les unes des autres|; ces pièces sont dirigées également de bas en haut et d'avant en arrière; elles complètent, comme il a déjà été dit, la paroi qui regarde en haut du canal supérieur; ce sont les apophyses épineuses.

Ces diverses pièces ne sont pas toutes en contact. Les apophyses épineuses sont appliquées immédiatement sur le sommet des moitiés d'arcs; les pièces qui se succèdent d'avant en arrière sont très-rapprochées; les pièces des arcs s'atteignent presque au-dessus du cordon rachi- 
D'ATATOMIE COMPARÉ.

261

flien; mais les pièces latérales et inférieures sont séparées des arcs par une lacune qui varie en largeur.

$$
\text { 5. } 72 .
$$

La colonne vertébrale de l'esturgeon offre du reste, dans ses différentes régions, les variétés suivantes.

Les deux portions principales dont elle est formée, la partie inférieure ou les corps, et la partie supérieure ou les arcs réunis aux apophyses épineuses, ne sont pas également rapprochées l'une de l'autre. L'intervalle qui les sépare, considéré d'une manière relative ou absolue, croît dans la région postérieure, et la bande membraneuse qui les unit augmente proportionnellement de largeur à mesure qu'on l'examine plus près de l'extrémité caudale. La plus grande distance absolue que l'on remarque entre elles existe dans les deux quarts moyens de la longueur de la colonne. Elle est, à cette partie de la longueur, de trois lignes dans des esturgeons longs de seize pouces, tandis qu'elle est presque nulle en avant où les corps et les arcs se touchent pour ainsi dire.

La forne de la colonne vertébrale varie également.

Nous avons déjà fait remarquer plus haut que les quarante premières vertèbres environ sont munies de chaque côté d'une courte apo- 
physe transverse, que les corps des vertèbres antérieures ne se réunissent pas en bas dans la li ñne médiane, tandis que cette réunion a lieı entre les vẹtèbres postéricures.

Les corps des vertèbres antérieures ne se joignent toutefois entre eux que fort au-delà du point où les a pophyses transverses sont disparues.

A cette disposition coïncident la compression latérale du corps des vertébres à mesure qu'elles sont plus postérieures, leur configuration en dos d'âne et leur conversion graduelle en apophyses épineuses inférieures.

Il existe dans la nageoire anale neuf rayons qui se succèdent immédiatement d'avant en arrière, sont entièrement séparés des arcs des vertèbres et formés de trois articles disposés bout à bout de dedans en dehors. De ces articles l'interne est le plus grand, le moyen est plus petit de moitie et l'externe plus petit encore. Ils ne forment que la base de la nageoire, car celle-ci se compose de deux lames cutanées, dures, élroitement appliquées l'une contre l'autre, consistant en un nombre beaucoup plus considérable d'articulations bien plus petites.

La nageoire caudale est beaucoup plus simple; les apophyses épineuses inférieures senles s'y prolongent considérablement surtout en avant; elles en occupent la majeure partie de la longueur. Le sommet des sept apophyses épinẹcuses placées après la première, supporte de 
n'ANATOME COMPARtí.

plus que les autres un deuxième noyau, peu élevé, ayant la forme d'un carré arioudi. Vers lextrémité de la quene ces épines deviennent très-minces, courtes, et semblent se confondre en arrière.

Les corps des sept premières vertèbres, la première exceptée, offrent une disposition trèsremarquable. Ils sont partagés en une partie interne plus volumineuse et en une externe beaucoup plus petite, l'une et l'autre unies seulement par une membrane plus lâche. Les dernières supportent les sept côtes antérieures. Les internes se soudent ensemble en une pièce. Au niveau des sixième, septième et huitième arcs, chacune de ces pièces reste séparée de l'arc auquel elle correspond; en avant elles se soudent au contraire à ces parties. Il en résulte que, comme ceux-ci se soudent égralement, la vertèbre qui en est formée est forí longue.

Elle se continue, sans interruption, arec les os de la base du crâne; on n'eu peut pas indiquer les limites.

Le canal qui loge le cylindre cartilagineux devient insensiblement plus petit et se continue presqu’à travers tout le quart postérieur de la base du crâne, jusqu'au-dessus du premier arc branchial. I a base du crâne ne devient solide qu'à compter du puint où elle recoit ce canal.

Sous la partie interne, entre elle et l'externe. cst situće, entourée d'une membrane làche, 
l'extrémité postérieure d'une lame osseuse, iongue, mince et étroite, qui se continue, sans interruption, sous la portion interne de la base du crâne.

Les pièces supérieures des vertèbres offrent aussi plus ou moins de variétés dans les différentes régions de la colonne vertébrale.

Les arcs ont à peu près une hauteur égale dans la portion moyenne du rachis qui en est la plus considérable; en avant ils sont plus hauts, en arrière bien plus déprimés.

Les quatre arcs antérieurs sont tout-à-fait confondusdans toute leur hauteur, et l'on ne reconnaît les limites des différentes vertèbres que par les paires de nerfs spinaux qui sortent du canal rachidien.

Les quatre arcs les plus antérieurs se confondeut insensiblement avec les portions latérales et supérieure de l'occipital.

Les apophrses épineuses existent dans la plus grande partie de la colonne, à l'exception des trois ou quatre premières vertèbres, où elles manquent absolument. La portion postérieure de la nageoire dorsale antérieure, qui fait plus de la moitié de la longueur du corps de jeunes esturgeons longs de seize pouces, ne m'en offre non plus aucune trace; je n'en trouve pas plus dans l'espace compris entre celle-ci et la nageoire dorsale postérieure ou caudale. Ces mèmes apophyses subissent, dans la portion antérieure de 
la première de ces nageoires, une dépression qui devient, par degrés insensibles, fort considérable; mais elles se trouvent au-delà des six vertèbres les plus antérieures.

Dans la nageoire dorsale antérieure, elles sont remplacées en grand nombre par des noyaux cartilagineux, propres, qui constituent la base de la nageoire et deviennent, comme elle, plus petits d'avant en arrière. A l'exception du rayon le plus antérieur de la nageoire, ces noyaux forment trois rangées, placées les unes au-dessus des autres, dont les deux inférieures sont alongées; la supérieure est arrondie, elle est trèsdéprimée, au moins cinq fois plus que la série moyenne, qui offre en hauteur à peu près la moitié de la rangée inférieure. La division supérieure manque au premier rayon; l'inférieure y est au contraire plus de deux fois plus haute que les apophyses épineuses ordinaires. En avant les rayons sont appliqués sur les arcs au moyen des petites apophyses épineuses; en arrière cette application est immédiate. Ces séries de cartilages sont à moitié plongées entre les muscles, à moitié libres, mais ne forment qu'une trèspetite partie de la nageoire. Celle-ci est formée le plus souvent de deux lames de peau trèssolides, unies par du tissu cellulaire, divisées de la même manière de haut en bas, mais composées de rayons bien plus fins, très-rapprochés, par conséquent plus nombreux, dont cha- 
cun est formé lui-même d'un noinbre considérable d'articulations.

Outre les différences de forme indiquées, les vertèbres de l'esturgeon présentent aussi des différences de composition et de texture assez uotables. Le cylindre inférieur, rempli de substance gélatineuse, est partout fibro-cartilagineux.

Les lames qui l'entourent latéralement et inférieurement sont aussi cartilagineuses; il n'y a qu'environ dix vertèbres, celles comprises entre la quinzième et la vingt-quatrième, dont les apophrses transverses qui sont courtes, soient tout-à-fait osseuses.

Une structure entièrement osseuse se remarque beaucoup plus généralement dans les arcs. du moins à leur partie supérieure et dans les apophyses épineuses du haut. Cette disposition ne cesse que derrière le premier tiers des nageoires dorsales.

Les deux séries inférieures des rayons composant les nageoires $\mathrm{du}$ dos et de l'anus sont aussi tout-à-fait osseuses. On remarque la mème structure dans les rayons qui forment la série simple dont est composée la nageoire caudale, du moins dans la moitié antérieure des parties supérieure et inférieure de cette nageoire. 


\section{\$. $7^{3}$}

La colonne vertébrale des raies et des squales se distingue de celle des poissons que nous venons de considérer, par la disposition de la substance gélatiniforme contenue dans son intérieur: celle-ci est divisée, par les corps des vertèbres, dont le volume a proportionnellement augmenté, en autant de fois deux cônes arlossés par le sommet qu'il y a de vertèbres; chaque vertèbre est un peu plus longue que haute et large, elle est très-concave dans ses parties antérieure et postérieure. Ces deux concavités se contractant de dehors en dedans, se rencontrent presque dans le milieu; il en résulte que la série des cônes doubles, gélatineux, forme à peu près encore un tout continu. Cependant les vertèbres ne sont jamais percées au milieu, les doubles cônes sont par conséquent séparés chaque fois entre deux vertèbres.

A l'endroit où les faces latérales des corps des vertèbres se continuent avec la face inférieure, les corps, surtout la plupart des antérieurs, se prolongent à des longueurs variables et constituent une apophyse comprimée de haut en bas: ce prolongement, du moins chez les squales et les raies, supporte des os transverses, alongés, mobiles sur lui; ce sont les côtes.

J’airemarqué sur la colonne vertébrale de l'ai- 
guillat (squalus acanthias), une différence de développement très-digne de remarque. Chez lui les vertèbres sont tout-ł̀-fait creuses à l'état de foetus et forment des anneaux qui ont partout la même largeur. Cette organisation que je trouve constante chez des foetus qui ont environ un pied de long les rapproche par conséquent, tant qu'ils se trouvent à cette période, des chimères, des polyodons, des esturgeons, etc. Je n'en trouve plus de traces chez un individu long de deux pieds, chez lequel les corps des vertèbres ont tout-à-fait la structure de ceux des poissons cartilagineux supérieurs. Peut-être tous les poissons cartilagine ux offrent-ils transitoirement cette disposition, et leur organisation se perfectionne-elle plus tard par la formation insensible d'anneaux de substance solide qui s'appliquent de dehors en dedans contre la face interne des anneaux des corps des vertèbres, se développent de leurs deux extrémités vers la partie moyenne et se soudent entre eux et avec la face interne de l'anneau, tandis que la substance contenue dans le double cône que forme la réunion de deux vertèbres reste au contraire plus molle.

$$
\text { S. } 74 .
$$

La partie de la colonne vertébrale qui correspond aux arcs n'est pas exactement formée suivant le même type chez les raies et les squales. Ces deux genres s'accordent en ce que cetle 

partie est composée, chez eux, de pièces plates, séparées des corps, inclinées les unes vers les autres supérieurement et unies entre elles; mais le nombre et le rapport de ces pièces avec les corps varient dans chacun d'entre eux. Chez les raies, dont les corps des vertèbres sont beaucoup plus petits et plus nombreux, chacun ne correspond qu'à un arc; dans les squales, ils sont en rapport avec un arc et demi: l'un est un peu plus grand et s'applique sur le milieu de chaque corps de vertèbre; l'autre est plus étroit, mais non plus déprimé, et il est situé entre le premier et entre chaque couple de vertèbres. Ces arcs se correspondent par la forme : réunis entre eux et au corps des vertèbres, ils constituent un seul tout dont les parties sont jointes par de la substance molle.

La différence qui existe entre les squales et les raies est compensée parce qu'il existe chez les raies, au-dessus des arcs, dans la ligne médiane, une seconde série de pièces osseuses, intercalées de la même manière que chez les squales, entre les pièces situées sur le milieu des corps. La plupart de ces pièces osseuses sont larges et peu hautes; quelquefois, cependant, elles sont minces et élevées, par exemple, chez l'aigle de mer (raja aquila): on peut se convaincre de l'exactitude de celte assertion, en considérant surtout la partie moyenne et postérieure de la colonne vertébrale, où ces pièces 
osseuses, saillantes, s'arrangent en dos d'âue et se prolongent considérablement en bas et des deux côtés, jusqu'à descendre aussi bas que les autres plaques, au niveau des vertèbres, comme chez les squales:

Les raies offrent par conséquent le développernent dislinct d'une disposition qui. n'est qu'indiquée chez les squales, c'est-i-dire qu'elles présentent une série d'os particuliers, différens des veriébres, situés au-dessus de lá colonne vertébrale primitive, et qui se développent en général d'une manière encore beaucoup plus parfaite chez les poissons osseux. Celte porion de la seconde série d'os qui, chez les raies, s’élève au-dessus des autres, est remplacée trèsvraisemblablement par un grand nombre de nageoires dorsales; la nageoire dorsale la plus antérieure est en effet formée par plusieurs séries superposées d'os longitudinaux, la postérieure par une série unique: le nombre et le yolume de ces os correspondent à peu près aux parties saillantes de la seconde série des raies.

Les deux moitićs latérales de tous les arcs ne sont pas soudées daus la ligne médiane, mais seulement unites par de la substince cartilagrineuse, ce qui est suriout très-distinct chez les squales. La partie postérieure du rachis, qui est la plus considérable, se distingue en outre de l'antérieure par la présence d'un prolongement moyen, comprimé d'un còté à lautre; ce pro- 
longement bifurqué dans sa moitié supérieure est formé de deux racines latérales que sépare l'une de l'autre une lacune large, d'où résulte un canal dirigé sous les arcs des vertèbres. Ces apophyses peuvent être considérées comme des arcs inférieurs des vertèbres, qui se forment parce que les apophyses transverses se tournent en bas et én dedans et se réunissent dans la ligne médiane en apophyses épineuses inférieures; ces apophyses s'alongent considérablement à l'extrémité de la colonne vertébrale chez les squales, les torpilles ou raies électriques, mais non chez les raies proprement dites; elles s'aplatissent dans le mème rapport par la soudure de leurs deux moitiés latérales qui commence depris la racine, de sorte que la cavité y disparaîl.

Les anges (squalus squatina) offrent plusieurs particularités dignes de remarque: les arcs des vertèbres sont plus courts que chez d'autres squales et se rapprochent par là de la disposition qu'offrent les raies; ils sont en outre, surtout les antérieurs, très-larges, concaves à leur face inférieure, et beaucoup moins renflés aux deux extrémités, et par conséquent plus plats sur les còtés.

Les rayons des nageoires dorsales et anales sont composés, chez les squales en général, de trois séries de forles pièces cartilagineuses, alongées, dont la piece moyenne est considéra- 
blement plus longue que l'inférieure et que la su. périeure; le nombre de ces rayons est pour l'ordinaire de la moitié plus considérable que celui des corps des vertèbres auxquels ils correspondent; il égale celui des apophyses épineuses, et est double de celui des corps; ordinairement aussi ils ne correspondent qu'à la base de la nageoire.

A près la troisième série de cartilages, vient, entre les deux lames de la peau, un nombre infini de petites stries cartilagineuses, minces et longues.

Le squalus squatina fait également exception à cette règle : $1^{\circ}$ il existe dans cette espèce, au devant de la première nageoire dorsale, dans une étendue trois fois plus longue que sa base, et au devant de la seconde, dans une étendue un peu moins considérable, une série simple de rayons alongés; $2^{\circ}$ un bien moindre nombre de corps de vertèbres leur correspond, puisqu'il y a en avant 17 à 18 corps et seulement 10 à 11 rayons, tandis qu'en arrière il y a 5 rayons et 8 à 10 corps; $3^{\circ}$ la nageoire ne contient que deux séries de rayons, qui sont toutes deux larges d'avant en arrière et peu élevées, et dont linférieure se compose de deux rayons trèslarges, tandis que la supérieure est formée de quatre ou cinq rayons plus étroits.

Les nageoires des raies sont beaucoup plus étroites que celles des squales et se composent 
d'un nombre moindre de rayons; mais chacun de ces rayons est le plus communément composé d'un nombre plus considérable d'articulations que chez les squales.

Les torpilles sont, aussi sous ce rapport, formées d'après le type des squales.

$$
\text { S. } 75 \text {. }
$$

La partie antérieure de la colonne vertébrale offre chez les raies une disposition digne de remarque, qui ne se représente pas dans les squales, du moins dans toutes les espèces; la condition la plus essentielle de cette disposition consiste dans la soudure de plusieurs vertèbres pour n'en former qu'une seule :il en est ainsi déjà chez les chimères et les esturgeons.

A peu près à compter de l'endroit qui correspond aux membres antérieurs, la colonne vertébrále grossit considérablement dans toutes ses parties, et les vertèbres se confondent en une masse, que l'on ne reconnaît pour être formée de plusieurs vertébres que par deux séries d'onvertures superposées, à travers lesquelles sortent les deux racines des nerfs spinaux. Les corps aussi bien que les arcs sont exactement sourlés ensemble; les apophyses transverses des corps forment, de chaque côté, aussi une lame qui devient sensiblement plus large des extrémités antérioure et postérieure vers le milieu dela colonne; les parties antérieure et moyenne, II. 
les plus cunsidérables et les plus larges de cette lame, sont un peu recourbées en hant, à cause des branchies.

Cette lame recourbée offre toujours son étendue la plus large au milieu de sa longueur; c'est en ce point qu'elle prend brusquement une autre direction, de manière à se réfléchir en dehors, en faisant un angle presque droit avec: la partie postérieure; la courbure de la partic antérieure est au contraire une pente insensible. Elle forme par conséquent un triangle dont le bord postérieur est droit, tandis que l'antérieur descend obliquement de haut en bas, de dehors en dedans et d'arrière en avant. Chez les torpilles, cette lame est beaucoup plus développée que chez les raies proprement dites. Ainsi, chez une torpille brune( $T$. Galvani), longue d'un pied trois pouces, je lui trouve neuf lignes dans sa plus grande hauteur, en tout quatorze lignes de long, et chez un raja oxyrynchus de mème taille, je ne lui trouve que neuf lignes de long sur trois de hauteur. Chez les torpilles, elle déborde par conséquent le reste de la colonne vertébrale dans une étendue considérable en lehors, ce qui a lieu beaucoup moins chez les raies.

Son sommet externe, qui est libre, est en outre, dans les torpilles, situé beaucoup plus haut que le bord libre des apophyses épincuses; aette disposition n'a pas lieu daus les raies. 


$$
\text { D'AVATOML: COMPABE. }
$$

yuoique cetle lame s'y recourbe fortement el forme un demi-canal que l'on ne retrouve pas dans la torpille.

En firisint sécher cette lame, séparée des forts muscles qui la recouvrent, elle se recourbe trèsfacilement en haut et en dedans, se porte à la rencontre de l'autre, de sorte qu'elles finissent par se toucher chez les torpilles; circonstance qui pourrait faire donner des idées inexactes de la position et de la forme de cette partie.

Les arcs et les corps, séparés dans les autres vertèbres, sont également unis : en un mot, la partie antérieure de la colonne vertébrale forme un cartilage unique; ce cartilage, formé par la réunion de plusieurs vertèbres confondues, est absolument creux dans son intérieur; mais cette cavité est d'une autre nature que celle du cartilage vertébral des lamproies, des chimères et des esturgeons, car elle n'est pas due à une dilatation du corps, mais bien de l'arc, accompagnée d'un amincissement considérable du corys, aux dépens de la dimension en largeur; elle se continue par conséquent en avant dans la cavité crânienne, en arrière dans le canal vertébral, tandis que chez les premiers de ces poissons, ces cavités sont parfátement séparées l'une de l'autre.

Mais c'est précisément cette différence qut rend cetle disposition très-digne de remarque, parce qu'elle démontre des degrés fort divers 
dune mème organisation. Chez les lamprotes ha substance aponévrotique, représentant les corps, ne forme encore dans toute la longueur du rachis qu'une masse homogène indistincle des apophyses transverses; chez les chimères il se manifeste une disposition articulée, imparfaite, dans la plus grande partie de lit longueur de la colonne; ce n'est qu'en avant que les pièces sont encore confondues.

- Le rachis de l'esturgeon présente des traces d'articulation beaucoup plus évidentes, quoique les parties antérieures y soient confondues encore; mais on recomiait, d̀ cet état mème, une tendance à la séparation manifeste, surtout aux apophyses épineuses. Dans les deux genres précédens le corps des vertèbres est encore crenx, il est au contraire plein dans les raies.

Cette partie la plus antérieure de la colonne, formée de vertèbres soudées, n'offre cependant pas exactement les mêmes caractères daus toutes les raies. Nous venons de parler de la différence présentée par la longueur et la hauteur de la lame que forment les apophyses transverses soudées, et qui monte obliquement en dehors. Remarquons en outre que le rapport de la longueur de cette partie, comparée à celle de toute la colonne vertébrale, n’est pas partout la mème. Ainsi je le trouve chez la raie blanche (raja batis) et la raie bordée (raja rostellain) exprimé par $1: 5$; il est le mème chez la tor- 
pille brune (torpedo Galvani), quand on y comprend, comme dans les deux espèces précédentment cilées, toutes les sertèbres dont les apophrse's transverse's sont soudées; chez la raie bouclée (raju clavala) et chez les rhinobates, ce rapport est de $1: 6$; chez la raie lentillat (raja oxy'ynchus) et la raie Cuvier (r. Cuvierii), seulement comme $1: 7$, chez la pastenague commune (raja pastinaca), comme 1: 20; variété qui semble se rattacher à la différence qu'offre la Iongueur de la queue.

Les vertèbres sondées ne sont pas non plus loutes confondues au même degré.

Chez la torpille brune (torpedo Galsani), on distingue très-bien dans la moitié postérieure de la partie soudée, qui est un peu plus grande que l'antérieure, onze vertèbres cervicales, dont le corps et les apophyses épineuses sont tout-àfait séparés par des incisures et unies senlement par leurs apophyses transverses: le nombre en est de douze dans le raja rhinobates; chez la raie lentillat, il n'y en a que deux qui forment le dernier dixième de celte partie. Chez la raie blanche, le sixième postérieur est formé de cinq vertèbres très-petites de cette espèce.

Le nombre des vertèbres qui composent celle portion varie également. Pour déterminer com. bien de vertèbres concourent à la former, on a, outre les limites de séparation, la ressrource de compter les trous intervertébraux. 
qui marquent la sortie des nerfs. In faisant usage de ce moyen on trouve que cette partie antérieure de la colonne vertébrale serait com. posée, chez la raie blanche, de trente et quelques vertèbres, chez la torpille, de vingt-deux, et chez la raie lentillat, de vingt. Il résulte du moins de ces exemples, que l'étendue proportionelle occupée par les vertèbres confondues n'est pas en rapport nécessaire avec leur nombre; cette étendue est en effet à peu près la même chez la raie blanche et la torpille, où le nombre des vertèbres soudées est fort différent. Chez la raie lentillat, "la longueur proportionelle est au contraire beaucoup plus petite que chez la torpille, tandis que le nombre des vertebres soudées est à peu près le mème.

On voit un rapprochement de cette organisation chez les anges (squalus squatina). Les quatre premières vertèbres deviennent insensiblement plus larges que les autres; cet excès de dimension est tel, que la largeur de la plus antérieure est presque doublée si on la compare à celles qui suivent la quatrième; celles-ci, à l'exception des vertèbres de l'extrémité, offrent, comme on le voit ordinairement, peu de différence à cet égard. Il faut ajouter que les rudimens de côtes, séparés jusqu'alors, se soudent au corps de la vertèbre, depuis la quatrième jusqu'à la première, et se dirigent plus latéralement. 


\section{\$. 76.}

Les chimères, les raics, les torpilles et les squales, diffèrent très-fortement des lamproies et des esturgeons, par le mode d'articulation de la colonne vertébrale avec le crâne. Loin que chez les premiers le rachis soit continu au crâne et s'y réunisse pour ne former qu'une seule pièce comme chez les seconds, ces deux parties sont parfaitement séparées, et le mode d'rnion qui les rapproche leur permet une mobilité variable. Cette articulation mobile consiste, chez tous ces genres, en plusieurs points de contact tout-à-fait éloignés les uns des autres. Cette disposition a aussi été indiquée exactement en général, par M. Cuvier, à l'égard des raies et des squales, lorsqu'il dit que, chez ces animaux, la tête s'articule avec le rachis par deux condyles (1); mais il n'a pas cité les différences remarquables que présentent ces deux tribus; il s'est en partie trop avancé, du moins relativement aux raies, en ajoutant que cette articulation n'est pas très-mobile.

Après les poissons qui viennent d'être considérés viennent les squales, qui ont le moins de mobilité dans cette articulation.

Les corps des autres vertibres s'unissent entre? eux au milieu par de la substance cartilagineusc;

(1) Anat. comp., i. I, p. 232. 
celui de la vertèbre antérieure s'unit, au moyen d'une semblable substance, avec le corps de la vertèbre crânienne, au dessous du trou occipital. Ces deux cartilages, semblables aux portions de deux vertèbres opposées l'une à l'autre, sont très-concaves et forment un cône double. A côté et au dessus de cette articulation trèssolide on rencontre un second mode d'union, opéré par un ligament circulaire, court, s'étenlant de toul l'arc de la première vertèbre au pourtour du trou occipital.

Ces moyens de jonction sont renforcés par une. troisième el une quatrième connexions latérales. Il existe, en effet, de chaque côté de la face antérieure de l'apophyse transverse de la première vertèbre, un léger enfoncement arrondi, dirigé d'avant en arrière et de dedans en dehors, qui correspond à une éminence semblable, saillante sur le côté de la facette articulaire moyenne de l'occiput. Ces parlies sont entièrement séparées l'une de l'autre et de la facette articulaire dont il vient d'être question; elles sont retenues ensemble par des ligamens capsulaires, courts et roides.

L'arc qui correspond au, trou occipital est le premier qui soit intercalé entre ceux qui sout soudés avec les vertẻbres; il est par conséquent situé un peu en avant de la première.

Chez les chimères et les raies l'articulation est beaucoup plus libre; dans les unes et les autres 
la connexion inférieure, opérée par de la substance ligamenteuse, est remplacée par une capsule articulaire. Chez les chimères la partie du rachis qui représente le corps des vertèbres se termine par une surface carrée, convexe d'un còté à l'autre, concave de hant en bas, qui correspond à une autre surface large, concave d'un cóté à l'autre et de haut en bas. Au dessus de ces surfaces on trouve le large trou occipito-rachidien; au dess'us et au dessous d'elles, à la partie inférieure du pourtour de cette ouverture, et de chaque côté, on remarque une facette articulaire particulière, entourée d'une capsule; ces deux facettes sont légèrement convexes à la première vertébre cervicale, concaves sur l'occipital, et beaucoup plus hautes que larges sur les deux os.

Chez les raies on observe: $1^{n}$ que la facette articulaire moyenne, considérablement renflée, s'avance un peu dans la cavité du crâne; $2^{\circ}$ que les deux facettes articulaires latérales, également beaucoup plus élargies et séparées, comme chez les chimères, sont situées beancoup plus sur le còté; $3^{\circ}$ que celles de la première vertèbre alıssi bien que de l'occipital sont très-convexes; $4^{\circ}$ qu'il se trouve entre toutes deux un cartilage articulaire intermédiaire, très-arrondi.

C'est du moins la disposition que je trouve chez les torpilles, les raies lentillat et blanche. Chez l'aigle de mer (raja aquila) le mouvement 
est plus horné et fort semblable à celui des squales; toutes les facettes y sont, en effet, moius élevées, et les cartilages inter-articulaires manquent, quoique l'amphiarthrose soit remplacée par une diarthrose.

Il existe toutefois chez les raies, en haut ef en bas, une lacune constante entre le trou occipital et l'arc des vertèbres; chez les squales, an contraire, l'arc est appliqué immédiatement contre ce trou.

2. Poissons osseux (1).

$$
\text { S. } 57 .
$$

Les vertèbres des poissons osseux ont en géréral la méme forme que celles des cartilagineux

(I) Ce sont les poissons osseux qui offrent les álémens vertébraux séparés de la manière la plus distincte. C'est chez ces êtres que ceux de ces matériaux qui se correpondent s'observent plus scublables entre eux et qu'il est plus facile de suivre les changemens graduels, survenus dans leurs rapports de développement et d'arrangement relatifs, qui donnent licu à toutes les modifications de conformation dont les vertèbres préscntent les exemples. M. (ieoffroySaint - Hilaire, en étudiant les poissons osseux, y a trouvé un terme de comparaison qui lui a permis de concevoir et d'expliquer comment avait été modifié le type unique de l'organisation animale. Placés entre les insectes et les hauts vertébrés, ils lui ont fourni le lien de transition qui unit ces grandes coupes du rigne aniuial; ce sont les découvertes qu'il a failes en observant les poissons osseux qui ont fait connaître à ce profond anaiomiste quelle ćtait la 
supérieurs, mais leurs apophyses sont plus par. jaites. Les vertèbres antérieures sont générale-

condition essentielle du passage qui ramène à une communauté de type des organisations si diverses. Il a isolé nettement chez ces animaux : $1^{\circ}$ une pièce centrale, un noyau vertébral, qu'il a désigné sous le nom de cyctéaL, c'est le corps de la vertèbre. Ce noyau tubulaire est membraneux dans les premiers temps de la formation, il se remplit ensuite de couclies concentriques dans l'âge plus avancé des poissons, reste plus ou moins largement perforé, après avoir subi par le retrait des fluides qu'il contenait une sorte d'éiranglement moyen, qui donne lieu à la disposition de deux cônes adossés par le sommet. Chez les insectes et dans la salamandre mẻme', la cavité intérieure dn cycléal ne se remplit pas; dans les premiers, elle contient tous les ortranes importans à la vie, le tissu épidermique s'y applique immédiatement à la surface extérieure, comme il s'applique dans les tortues sur celle de la carapace. M. GeoffroySaint-Hilaire signale aussi : $2^{\circ}$ deux pièces destinées à coîffer le canal médullaire, et il donne à chacune d'elles le nom de PB́RIAL; ces osselets, dont l'affinité nécessaire est le cycléal et la connexion indispensable le canal médullaire, éprouvent des modifications en rapport avec les conditions de la jortion du sytème nerveux à laquelle ils servent d'étui; plus écartés, plus alongés, lorsque l'organe contenu est plus développé, et réciproquement plus restreints dans leurs dizensions, plus accollés, même confondus, lorsque la partic ‘u'ils protégent est très-peu volumineuse. Aux périaulx s'a. joulent: 5" de chaque côté une pièce qui, si les parties nerveuses deviennent tellement volumineuse que les périaux ne suffisent plus à les contenir, peut être employée avec ces osselels à former la capacité qui les doit renfermer; cette juiece est l'Éplar.

Les deux ḱpaux, dans le cas où les périan: sont plus que: 
ment un peu plus courtes que les autres, et deviennent plus étroites d'avant en arrière. Ja dernic̀re parvient surtout, dans le plus grand nombre des cas et d'une manière brusque, ‘̀ une dimensiou considérable de haut en bas, en s'amincissant dans la mème proportion, et en prenant la forme d'un triangle dont le sonmet est tourné en avant, vers l'avant-dernière verièbre, et la base en arrière. Le bord postérieur de cette vertèbre supporte la nageoire caudale. Elle est simple chez quelques poissons, par exenple chez la baudroye commune (lophius piscatorius ); chez d'autres elle est partagée en

suffisans, deviennent des pièces additionnelles qui s'y ajoutent et qui sont susceptibles de degrés divers de développernent. En se plaçant bout à bout, les épiaux fournissent des rayons natatoires. Opposés aux périaux, el comme ceux-ci liés par une affinité nécessaire avec le cycléal, mais dans le sens opposé, on rencontre : $4^{\circ}$ les deux pièces désignées sous le nom de paracux; celles-ci sont dans les mêmes nécessités de connexion avec le système circulatoire, ce mot étant pris dans le sens le plus étendu, que le sont les périaux avec le système nerveux. Elles subissent les mèmes accidens de dévelóppement et de rapport, l'une relativement à l'autre, que les périaux. entre eux, accidens qui coïncident également avec les dispositions corrélatives de l'appareil circulatoire. A ces piẻces se joignent : $5^{0}$ les cataud, pièces correspondantes aux épiaux; ces osselets sont pour les périaux. ce que sont les épiaux relalivęment aux périaux. (Voyez les Considérations générales sur les vertèbres, par M. GcoffroySaint-Hilaire; deuxième collection des Annales du Muséum, tome 9).

(Note des traducteurs.) 
deux pièces, par exemple, chez les saluth ( $s$ lurus glunis), en quatre chez les balistes, et en un bien plus grand nombre chez les moles (tetroclon mola), où l'on trouve jusqu'à neuf pièces superposées. La pièce interne représente le corps, les pièces supérieure et inférieure correspondent aux apophyses épineuses supérieures et inférieures. Cette verlèbre porte constamment sur son bord postérieur, qui est convexe, une quantité considérable de rayons, longs ou courts, qui se succèdent de haut en bas et forment la nageoire caudale, mais dont les antérieurs, ceux de la partie supérieure aussi bien que de l'inférieure, correspondent généralement aux apophyses épineuses de vertèbres coccygiennes qui précèdent.

Il y a cependant quelques exceptions à celte disposition de la dernière vertèbre caudale, surtout chez les poissons très-alongés. Ainsi, particulièrement chez le saluth, cette vertèbre n'est guère plus haute que la précédente. Chez le ruhan (cepola tocnia) elle a bien la forme indiquée, mais elle n'y est pas plus haute que les autres ou du moins, elle ne les dépasse que de fort peu.

Chez les murènes et les murénophis les vertébres deviennent, sans interruption, de plus en plus courtes, moins élevées, pliss étroites et plus imparfaites, sans que la dernière change de forme, comme celí a encore lieu chez le ruban. 
Chez les fistulaires on trouve une disposition remarquable : les vertèbres y deviennent dc plus en plus petites, et se prolongent aut-delà de la nageoire caudale en un filament long et très-mince, mais qui ne semble présenter la séparation propre aux vertèbres imparfaites qu'ì son commencement.

\section{S. 78.}

Les corps des vertèbres des poissons osseux portent très-généralement: $1^{\circ}$ une paire d'apophyses articulaires ou obliques antérieures, $2^{0}$ une paire de semblables apophyses postérieures; $3^{\circ}$ une apophyse épineuse supérieure; $4^{\circ}$ des apophyses épineuses inférieures, et $5^{\prime \prime}$ des apophyses transverses. Ces deux dernières sortes d'apophyses se rencontrent moins généralement. que les premières.

Lcurs vertèbres sont unies entre elles par les corps, qui sont cartilagineux comme ils le sont dams les poissons chondroptérygiens, et en outre par les apophyses obliques. Les apophyses énineuses, plus rarement les apophyses transverses. se soudent également quelquefois d'une manière plus ou moins parfaite. I a première vertèbre s'articule comme dans les poissons cartilagineux: $1^{\circ}$ par son corps au moyen d'une ampliarthrose opérée par de la subsiance fibro-cartilagineuse, absolument à la manière des autres verlèbres, avec une apophyse impairc également fort con- 
cave ; $2^{\circ}$ plus haut et en avant très-ordinairement par de fortes apophyses articulaires inférieures ivec la partie de l'occipital qui représente l'arc de cette vertèbre (cette disposition est si générale que je la regarde comme une règle constante); $5^{0}$ la portion squameuse ou occipitille proprement dite de l'occipital, s'applique, en outre, très-fréquemment par son bord postérieur contre le bord antérieur de l'apophyse ćpineuse inférieure de la première vertèbre. Par ces différentes connexions la réunion de la tète avec la première vertèbre cervicale devient encore plus solide qu'on ne le dit communément. La disposition qui vient d'être décrite rapprocho de très-près l'articulation occipito-atloïdienre de celle que nous avons rapportée plus haut chez les squales (1).

Les vertèbres peuvent ètre partagées de lá manière la plus générale, en vertèbres abdominales et en vertèbres coccygiennes: les premières viennent immédiatement après la tête, et portent les apophyses transverses et les côtes, lorsque ces os existent; mais elles ne souliennent pas d'apophyses épineuses inférieures. Les vertebres coccygiennes au contraire sont ordinairement dépourvues d'apophyses transverses et de côtes, mais elles servent de base à des apophyses épineuses inférieures.

Le norrbre en est très-considérable chez les

(1) Voyc\% plus haut, p. 279. 
poissons en général, quoique che\% certains d'entre eux, tels que les télrodons et les balistes, il y en ait fort peu.

$$
\text { S. } 79 .
$$

Le corps des vertébres des poissons osseux offre en général un rapport de conformation avec celui des poissons cartilagineux parfaits; comme chez les premiers il est composé de deux cônes creux tournés l'un vers l'autre, et qui se rencontrent au milieu par leurs sommets, où souvent ils ne sont séparés que par une membrane mince, qui quelquefois est même perforée (1). Le plus ordinairement ils ne sont pas

(1) Cette phrase semble ne porter que sur une circonstance peu importante. L'auteur de la découverte dèla perforation du centre de toute vertèbre dans les poissons, M. Geoffroy-Saint-Hilaire est d'un avis bien différent dans ses Mémoires sur les insectes apiropodes (Journal compl., vol. VI, p. 149.) Cet anatomiste y parle pour la première fois du trou existant au fond des corps vertébraux, trou qui s'obstrue dans les poissons qui prennent de l'âge, ce qui n'arrive paś chez tous. C'est le fait qui caractérise au plus haut degré l'organisation vertébrale des animaux supérieurs, et qui n'existe en minimum chez les poissons que comme rappelant cet état de chose. Cependant une rouvelle organisation s'ensuit chez ces derniers, on y observe l'arrangement de disques distincts et entrecoupés, comme dans une pile galvanique par une substance qui n'est pas fibrense, et d'une demi-consistance dans l'intérieur des cônes, de telle sorte que cet axe général traverse les corps vertébraux, et établit des rapports entre les parties de chaque anneau.

( Note des traducteurs.) 
n'ANATOMYE COMPARÉ.

tres-longs d'avant en arrière; mais il y a des exceptions à cette régle, par exemple, aux vertèbres postérieures du ruban et de l'ombrine barbue (grmnetrus cepedianus), ainsi qu'à celles des syngnathes, où ils ont une forme très-alongée. Il n'existe toutefois nulle concordance nécessaire entre la forme des corps des vertèbres et celle de tout le corps de l'animal, comme on pourrait le croire d'aprés ces exemples; car chez les anguilles (murana), et chez les murènes proprement dites (murcenophis), poissons trèsalongés, les corps des vertèbres sont très-courts et d'une hauteur proportionnellement assez considérable.

Une saillie longitudinale, transversale, tantôt plus faible, tantòt plus marquée, située au dessus de l'apophyse transverse dont elle diffère entièrement, partage le plus souvent, d'une manière plus ou moins distincte, les corps des vertèbres des poissons en une moitié supérieure et en une inférieure très-concave; disposition surtout très-prononcée chez plusieurs silures, ensuite chez les anarrhiques (anarrhichas) et les sargues (sargus).

Chez quelques silures (le harmouth-hale), cette organisation est surtout remarquable, parce que, sur la plupart des vertèbres postérieures, les concavités des deux côtés se confondent plus uu moins parfaitement dans la ligne médiane, en s'ouvrant l'une dans l'autre, à travers la iI. 
vertèbre, ct que cette ouverture partage plus ou moins complètement le corps en trois pièces superposées. Cetle disposition est remarquable surtout en ce qu'elle semble indiquer déja, dars les autres vertèbres, la forme en éventail percé à jour, qui est parliculière à la dernière.

Chez le vomer Brorvnii, il existe, depruis la hritième jusqu’à la seizième vertèbre, une semblable ouverture dans la moitié inférieure du corps et à la racine de l'apophyse épineuse inférieure.

\section{S. 80 .}

Les apophyses abliques ou articulaires, se Irouvent, de chaque còté de la face supérieure du corps et de la racine de l'apophyse épineuse supérieure ou de l'arc, exactement à l'extrémité antérieure et postérieure du corps; ce sont ordinairement les plus petiles des apophyses, elles ont une forme triangulaire et mue direction portée en haut et un peu en dehors.

Les antérieures sont plus longues, elles sont situées plus en dedans et sont par conséquent recouvertes en dehors par les apophyses articulaires, postérieures, de la vertebre qui est placée immédiatement au devant; ou si cela n’a pas lieu à cause de la briéveté des apoplıyses arliculaires postérienres, ces deruieres ne reconvrent pas les antcrieures, mais elles leur sont directement contiguës. 
Les antérieures semblent exister presque constamment; chez les syngnathes, elles manquent cependant ainsi que les postérieures, ou sont du moins peu prononcées. Les postérieures manquent chez le congr', tandis que les antérieures y sont très-developpées.

\section{S. $8 \mathrm{r}$.}

Les apophyses transwerses des vertèbres des poissons sont, sous tous les rapports, sujettes à un très-grand nombre de variétés.

Sous le rapport de leur présence, ce sont, sars contredit, les parties des vertèbres qui manquent le plus souvent; elles manquent surtout chez les tétrorlons, en particulier chez les tétrodon mola, hispidus, fahaca, testudinarius, les diodons, les coffres (ostracion), la baudroye commune (lophius piscatorius), les chironectes, le batrachoïle de Surinam (batrachus surinamensis), le poisson lune (zeus luna), les callionymes, les serrasalmes, le coltus insi. diator.

Les vertèbres de ces poissons offrent évidemment lá forme la plus imparfaite; elles ont, à la vérité, très-généralement des apophyses obliques plus ou moins distinctes, et des apophyses épirenses supérieures et inférieures (ces dernières n'existent cependant qu'aux vertèbres postérieures); mais on n'y aperçoit, pour trace d'aprophyses transverses, que des crêtes transver- 
sales, larges, qui sont surtout distinctes aux vertèbres postérieures.

Cette organisation n'est pas proportionnellemeut fréquente; on ne la rencontre pas du moins ordinairement, car un nombre très-supérieur des poissons osscux possèdent des apophyses transverses qui naissent généralement des faces latérales des corps des verlèbres.

Elles manquent ordinairement aux vertèbres les plus antérieures, sont courtes aux premières où elles se manifestent, augmentent de volume peu à peu et diminuent ensuite de nouveau aux vertèbres postérieures; elles ne sont, en outre, presque jamais droites, mais elles sont plus ou moins dirigées de haut eu bas et de dedans en dehors.

Le volume, la forme, la direction, le nombre même de ces apophyses varient extraordinairement.

Le summum du développement de ces apophyses se voit chez les silures, particulièrement dans quelques espèces : je citerai le $s$. gourgar carasch, où je les trouve longues de près d'un pouce chez un individu qui n'a pas un pied de long. Il en est de même chez les gades, surtout l'égrefin ( $g$. agrefinus), et le merlus ordinaire (merlucius). Chez l'égrefin, elles sont surtout très-longues, chez le neerlus ordinaire, clles présentent aussi une largeur très-grande, une convexité très-prononcée en haut, une disposilion concave en bas et une extrime minceur; 
ce qui leur donne une forme connidle; elles sont peut-ètre le plus considérables chez l'anableps tetrophthalmus.

La forme en est très-variable.

La plupart sont triangulaires, alongées . apointies de dedans en dehors, renflées à leur bord postérieur, plus minces dans leur partie antérieure. Il en résulte que leur face supérieure forme une gouttière : quelquefois elles sont droites, d'autres fois convexes supérieurement, concaves inférieurement, quelquefois comprimées d'avant en arrière, tantôt de haut en bas. La dernière disposition est la plus ordinaire.

La direction de ces apophyses présente aussi des différences : chez la plupart des silures, ainsi que chez les anableps, elles sont, par exception à la règle, entièrement ou presque entièrement droites; chez d'autres poissons, surtout les blennies, les anarrhiques, les labres, les gobies, les scares, les spares, elles sont au contraire très-fortement tournées en bas. Cette disposition est digne de remarque, parce que, comme le démontre l'étude des apophyses épi. neuses inférieures, celles-ci résultent de la réunion et du prolongement des apophyses transverses.

L'organisation d'une nouvelle espèce trèsvoisine duscarus croicensis (1) est surtout remarquable sous ce rapport. Les apophyses transver-

(1) Blocl's Fische, Taf. 221. 
ses de la cinquième vertèbre s'y réunissent, non loin de leur origine, dans la ligne médiane, d'où résulte une ouverıure située sous le corps de la vertèbre; cette capacilé est fermée inférieurement par une bande osseuse, large, s'éten. dant transversalement d'un côté à l'autre. Après s'être réunies pour former cette ouverture, les apophyses transverses s'écartent de nouveau et se dirigent en bas. L'ouverture et le pont augmentent considérablement de dimension d'avant en arrière, de sorte qu'à la dixième vertèbre, qui est en mème temps la première vertèbre coccygienne, il existe au dessous de la capacité supérieure ordinaire une autre ouverture placée plus bas, et sous les apophyses transverses, une apophyse épineuse inférieure, longue, formant le bord antérieur de la queue.

La même organisation s'observe anx dernières vertèbres dorsales de la daurade ordinaire ( sparus aurata) et du sparus virginicus: chez le calastomus teres, le silurus niger, le s. felis, le machoïran ( s.mystus) et le s. clarias, cette disposition existe aux deux dernières vertèbres dorsales; chez les plagusies (plagusia), c'est aux cinq; le saluth (silurus anguillaris) la présente aux six, et chez le silurus basre, elle se reproduit même aux sept dernières de ces vortèbres; elle est indiquée sur quelques-unes des vertébres qui précèdent, par une petite saillie interne située de chaque côté. 
D A INATOME COMPARÉ

29,5

La disposition la plus remarquable est celle qui existe chez les asprèdes (silurus aspredu). Toutes les vertèbres, à l'exception des dernieres coccygiennes, purtent des apophyses transverses ordinaires, pas très-longues, mais tout-à-fait droites. L'on trouve, en outre, sur toutes les vertèbres qui succèdent aux vertèbres réunies, à compter de la première, un canal complet cheminant au dessous du corps des vertèbres, et se contiumant avec le demi-canal des vertebres les plus antérieures, soudées entre elles: canal dont la parvi inférieure est large en proportion et porte la côte sur ses deux bords. Les apophyses transverses sont donc ici partagées en une partie transversale et une descendanie, unies dés deux còtés dlans la ligne médiane.

Dans quelques poissons, par exemple, dans le vomer Brownii, et vraisemblablement dans plusieurs espéces très-plates, les apophyses transterses des dernieres vertebres dorsales, constituent, en se soudant, une semblable apophyse moyenne, supportant la côte à son extrémité inférieure, et munie d'une ouverture transversale qui passe en devant dans l'ouverture longitudinale qui vient d'être décrite.

Toutes les apophyses transverses ne présentent pas cette conformation; on ne la retrouve pas dans les dorées (zeus) et les pleuronectes; elie n'existe pas non plus dans les chétodons. 'Tous ces poissous ont les apophyses transver- 
ses des vertèbres dorsales entièrement séparées.

Les apophyses transverses des poissons, sont ordinairement simples, quelquefois bifurquées; cette bifurcation peut avoir lieu de deux manières, soit d'avant en arrière, soit de haut en bas. On les trouve bifurquées d'avant en arrière chez le serpent de mer (murcena serpens). Les quatre-vingt-une premières sont solides, larges, aplaties d'avant en arrière, triangulaires, et naissent presque de toute la longueur du corps des vertèbres. A compter de la quatre-vingt-unième, elles se divisent en une moitié postérieure, plus large, et en une antérieure, plus étroite, qui ont leurs extrémités libres inclinées l'une vers l'autre, et dont enfin les antérieures disparaissent, mais seulement aux dernières vertèbres coccygiennes, tandis que les postérieures restent jusqu'à la fin. Les vertèbres sont en outre munies, à compter de la quatre-vingt-unième, d'apophyses épineuses, inférieures, larges, mais courtes.

Chez les congres (murcena conger) et la murène commune (murcenophis helena), les àpophyses transverses se partagent en une pointe supérieure et en une inférieure. De la seconde jusqu'à la cinquième vertèbre des congres, il y a une apophyse inférieure, large, occupant toute la longueur de la vertèbre et une apophyse étroite et pointue, mais longue. Les apophyses transverses des quarante vertèbres 

qui suivent sont simples; sur la plupart des auires, a l'exception des quarante dernières, l'apophyse transverse est bifurquée en une pointe supérieure et en une inférieure. Cette dernière est bien plus grande que l'autre; à compter de la dixième de ces vertèbres, elle se réfléchit en dedans pour la formation de l'apophyse épineuse inférieure, et se soude à celle du côté opposé. Cette pointe inférieure persiste sur toutes les vertèbres coccygiennes, tandis que la supérieure disparaît aux dernières vertèbres.

Il existe une semblable organisation chez la muréne commune. A l'exception des quatorze premières vertèbres, chaque apophyse transverse porte au-dessus de sa pointe externe, un petit condyle supérieur, qui en est séparé par une incisure. Ce petit condyle s'alonge peu à peu considérablement, de sorte qu'il en résulte une apophyse transverse supérieure et une inférieure. En outre, les apophyses transverses inférieures se tournent en bas et se confondent au milieu, à compter de la soixante-douzième, pour former des apophyses épineuses inférieures. Les apophyses transverses supérieures ne disparaissent complètement que sur les vingt dernieres vertèbres.

Chez le serpent de mer, on voit des apophyses épineuses inférieures se détacher de l'endroit où les apophyses transverses commencent à se partager dans la direction longitudinale; cet animal 
at par conséquent en même ternps l'organisation des murènes, etc.

Les apoplyyses iransverses cessent commu. nément à la queue; elles y sout remplacées par les apophyses épineuses inférieures. II n'y a quiun petit nombre de poissons qui fas sent exception à cette règle. De ce nombre sunt surtout plusieurs pleuronectes, par exemple, le turbot ( $p /$ maximus) et la plie fraiche $(p)$ platessa), surtout les $p l$. rhombus et rhombö̈des, auxquels il faut ajouter l'acanthure (theutis hepatus), l'hirondelle de mer (trigla volitans), le congre, la murene commune. Cies apophyses qui chez les pleuronectes se trouvent à l'extrémité antérieure đu corps des vertèbres et diminuent considérablement de volume d'avant en arricre, constituent plus ou moins distinctement les parties supérieures les apophyses transverses antérieures; les parties inférieures de celles-ci s'agrandissent et se dirigent en bas pour former les apophyses épincuses inférieures. Chez la barbue ( $p l$. rhombus), cette séparation se voit déjì a la dernière vertébre abtominale et à la première coccygienne, dans lesquelles les parlies supérieure et inférienre ne sont unies entre elles que par une lame osseuse, mince. A compter de la première vertèbre coccygienne la partic supérieure se porte davantage en haut et en arrière, affectant souvent une direction transversale, tandis que l'inférieure, se sou- 
dant à celle du côté opposé, descend dircetement en bas et se place daus la ligne médiane.

Chez les acanthures (theutis) le rebord latéral ordinaire devient une crète large.

Chez l'hirondelle de mer il se détache, supérieurement et latéralement, de toutes les vertèbres (dont il faut excepter la seconde cervicale et les trois dernières coccygiennes), des apophyses supérieures, longues, triangulaires. Ces apophyses sont toutes très-aiguës et dirigées cn haut et en avant ; la première fait seule exception. ()n voit en outre, à compter de la sixième verlèbre coccygienne, de semblables apophyses inférieures naître du bord iuférieur de la face latérale et se diriger en bas.

Les premières semblent correspondire aux côtes supérieures, les dernièresaux inférieures qu'elles remplacent en effet.

J'ai déjà décrit leur disposition chez les ruguilles et les murènes proprement dites.

\section{S. 82 .}

I.es apophyses épineuses, supérieures et inférieures, sont en général beaucoup plus longues chez les poissons osseux que chez les cartilagineux. Elles sont surtout fort développées , en particulier les inférieures, aux vertèbres postéricures du tetrodron mola, du romer birownii, des chélodons, des pleuronectes, des spares; 
chez ces poissons, elles sont en grande partie cause de la hauteur du corps, et leur nombre comme celui des vertèbres est, en général, remplacé par la longueur. Cependant la hauteur du corps de la plupart de ces poissons ne dépend pas uniquement de ces apophyses, mais elle résulte en outre plus ou moins de l'existence de rayons accessoires supérieurs et inférieurs; leur longueur est toutefois ordinairement en rapport direct avec la hauteur du corps des poissons; il en résulte qu'elles sont très-considérables chez les poissons élevés, et beaucoup plus petites chez ceux dont le corp" est déprimé. Ces apophyses sont au contraire trèscourtes chez les anguilles et les murénophis. Elles manquent tout-à-fait aux quatre vertbères antérieures des moles (tctrodon mola). Chez le congre, les trcize premières de la face supérieure ont, à l'exception des deux premières, la même largeur dans toute leur élendue, de manière qu'elles se touchent dans toute leur hauteur et forment, en se réunissant, une espèce de toît long, non interrompu; organisation indiquée chez les gades, les uranoscopes, les balistes et plus encore chez les silures.

Les apophyses épineuses, supérieures, forment très-généralement un triangle reposant sur la face supérieure des corps des vertèbres par deux côtés qui ne tardent pas à se réunir dans la ligne médiane. Ce triangle est dirigé de bas en 
baut et d'avant en arrière, et sa partie supérieure, qui est la plus grande, est simple.

La plupart des apophyses épineuses supérieures sont arrondies et alongées. Cela existe dans les genres saumon, serrasalme, brochet (esox), exocet (exocotus), hareng (clupea), chez la plupart des silures, des carpes (cyprinus), des anableps, des loches (cobitis), et chez le plus grand nombre des gades, des pleuronectes, des labres, des blennies, des anarrhiques, des rubans (cepola), des rascasses (scorpana), des surmulets (mullis), des uranoscopes, des sphyrènes, des spares, des soldado (holocentrus), des pagres, des sciènes (sciaena), des perches, des trigles, des batrachoïdes (batrachus), des dorées (zeus), des cheilodiptères, des coryphènes, des scombres. Chez plusieurs poissons, coutes ou quelques-unes de ces apophyses sont plus ou moins larges d'avant en arrière, soit dans toute leur longueur, soit dans leur partie inférieure; lorsque quelques-unes seulement offrent cette forme, ce sont le plus souvent les antérieures. Parmi les poissous cités il y a dans les brochets, les silures, les gades, les cyprins, les uranoscopes, un nombre variable de ces apophyses antérieures qui ont une largeur plus ou moins considérable. Chez le polypterus bichir toutes sont assez larges dans toute leur hauteur; chez les tétrodons, les diodons ei les bralistes, elles ont toutes une largeur considé- 
rable dans cette direction. La même chose a lieu chez les acanthures (theutis), les scares, les chétodons. Elles ont ici plus souvent à leur bord autérieur un renflement de volume variable, de sorte qu'elles sont en même temps, en cet endroit, assez larges d'un côté à l'autre. Dans les acanthures, les scares, les chetodon arcuatus et bicornis, les balistes, les tétrodons, surtout le tetrodon hispidus et les coffres, plusieurs de ces apophyses antérieures se confondent, surtout à leur extrémité inférieure, et constituent par là une cloison osseuse, continue entre la moitié droite et la moitié gauche du corps.

Les apophyses épineuses, supérieures, formeıt ordinairement, par une disposition semblable, dẹs rayons simples, situés dans la ligne mé* diane et résultant de la réunion de deux moitiés latérales. Cependant quelques silures font exception à cette règle. Ainsi chez le silurus niger la pointe des sept premiers rayons est bifurquée en deux moitiés latérales; elle l'est également chez le harmouth-hale, depuis le second jusqu'au septième rayon. La mème disposition est offerte par les seconde, troisième, quatrième et cinquième des vertèbres qui succèdent aux grosses vertẻbres antérieures, soudées entre elles, chez le cloras carinatus, le silure bagre. Dans ces dernières, celte organisation est due on au moins se raltache a l'existence des 
petites épines accessoires qui se placent entre les deux moitiés latérales.

Chez le salmo saurus cette bifurcation existe depuis la seizième jusqu'à la vingt-cinquième vertèbre. dux vertèbres moyennes les pointes sont tout-à-fait partigées en deux moitiés; aux vertèbres antérieures et postérieures, elles se coufondent d'abord pour s'écarter ensuite au bont supérieur.

Chez l'espadon ( xiphias gladius) la bifurcation a lieu de la première vertèbre à la troisième.

Cet écartement des épines supérieures est encore plus pronouté chez le tetrodon fahaca. Aux six premières vertèbres dorsales, c'est-àdire à presque toutes, ces épines larges, triangulaires, à pointe mousse, s'élèvent de la base de l'arc. séparées entièrement daus toute leur hanteur, de toule la largeur de la vertèbre. Elles sont parallèl's aux vertèbres antérieures, convergentes vers les postérieures jusqu’à ce quielles se confondent dans la septième.

Dans plusieurs espèces de diodons la déviation de la regle est encore plus grande. Les larges épines des dix premières vertèbres dorsales s'écartent fortement l'une de l'autre, à partir du cócé de la base de l'arc, se dirigent en arrice et forment jor là une goutlière ouverte en hant. Ciette goullière est formée, aux deux premierces vertelores, par larc osseux, qui est 
mince, aux autres seulement par une mem. brane ou un cartilage, qui recouvré supérieurement le canal où est logé le cordon rachidien. Ce sont là réellement les épines; il existe en effet indépendamment d'elles des apophyses transverses, distinctes, aux quatre dernières de ces vertèbres. Cette organisation est évidemment très-digne de remarque, parce qu'elle offre la plus grande analogie avec la fissure irrégulière des vertèbres connue sous le nom de spina bifida.

Chez le tetrodon fahaca le canal destiné à contenir la moelle-épinière est partout fermé par une forte lame osseuse; par conséquent la disposition s'éloigne moins de la règle. La déviation est encore moins considérable chez le tetrodon hispidus; la disposition est bien essentiellement la même, mais la fente ne s'étend que jusqu'à la quatrième vertèbré, et les moitiés des épines sont plus rapprochées l'une de l'autre.

Chez le tetrodon testudinarius, les larges apophyses épineuses des trois premières vertèbres montent verticalement, de sorte que chez un sujet de huit pouces de long, il y a près de six lignes de distance entre ces deux prolongemens. La partie antérieure de la quatrième apophyse offre de légères traces de cette disposition.

Cette organisation n'existe pas chez les coffres (ostracion), du moins chez l'ostracion triqueler et cubicus; mais les arcs portent, chez ces pois- 
sons, des épines supérieures, aiguës, peu élevées, en forme de crêle. Les apophyses épineuses des syngnalles sont bifurquées d'une autre manière. C'est dans le sens d'avanì en arrière que la fissure est dirigée. Leurs moitiés sont formées de cinq à six petites racines trèsminces, montant presque verticalement et se réunissant presque toujours en une lame longue, moyenne, simple, mais peu élevée.

Cette lame, au-dessous de la nageoire dorsale, s'y modifie de deux manières; d'une part elle prend une hauteur beaucoup plus considérable; de l'autre, elle se divise d'avant en arrière, en trois, quatre ou cinq parties distinctes qui supportent les rayons des nageoires.

\section{\$. 83.}

On rencontre chez la plupart des poissons osseux la seconde série d'épines, qui existe chez. plusieurs de ceux dont le squelette est formé de cartilages; elle y règne dans une étendue qui varie, souvent elle se retrouve dans toute la longueur de la colonne vertébrale, entre la partie supérieure de la première série, mais remplissant bien moins exactement la lacune; elle laisse mème un espace fermé par une expansion membraneuse.

Ces épines accessoires, situées à la région stipéricure, sont daus le rapport le plus intime II. 
avec les nageoires dorsales. Ces dernières s* prolongent-clles sur une grande parlie du rachis, les premières sont nombreuses et occupent toute l'élendue de la longueur presque entière de cette région. Elles manquent presque tout-à-fait lorsque les nageoires dorsales sont petites. Le plus grand nombre de vertèbres en est privé dans la baudroye commune, les diodons, les tetrodon hispidus et fahaca, la plupart des silures, des anableps, des brochets, des harengs, dans l'elops suurus, chez plusieurs espèces de cyprins, par exemple l'ablette (c. alburnus), le cyprius nasus. On ne les rencontre, dans tous ces poissons, qu’à la petite étendue qui correspond à la petite nageoire dorsale.

Chez d'autres, par exemple, le salmo salar. le salmone (s. ville), la truite (s. fario), la truite saumonée (s. trutta); le characin nefasch (s.ctgyp. tius), l'ombre commun (s. thymallus), la raie du Nil (s. niloticus), la marène (s. marena), l'éperlan (s. eperlanus), chez le serrasalme citharine (serrasalmus citharinus), le myletes rhomboidens, les cyprins, du moins dans le plus grand nombre des espèces de ce genre, les épines accessoires existent daus la moitié antérieure de la colonne vertébrale, à compter de la tète jusqu ì l'extrémité postérieure de la nageoire dorsale.

Elles existent, au contraire, dans toute l'étendue du rachis, chez un très-grand nombre de 
I'ANATOME COMYARÉ.

yenres el d'espèces très-voisins de ceux oir elles manquent, ainsi, par exempke, chez le chironectes lievigatus, le batrachus surinamensis, le tetrodon mola. Elles se trouvent en général dans toute ou presque toute l'étendue de la colonne vertébrale chez les polyptères, les gades, les pleuronectes, les phycides ( $p$ hycis), les blennies (blennius), les anarrhiques, les labres, les rubans, les murènes, les lutjans, les jarretières (lepidopus), les ceintures (trichiurus), les spares, les sargues, les soldado (holocentrus), les scares, les acanthures (theutis), les muges ( mugil), les ténianotes, les scorpènes (scorpona), les surmulets (mullus), les pagres (pagrus), les sciènes ( scirena), les vives (trachinus), les trigles (trigla ), les perches ( perca), les urinoscopes, les donzelles (ophidium), les coryphenes (coryphena), les vomers, les pomacanthes, les chétodons, les dorées (zeus).

Il est digne de remarque cependant que dans les espèces mêmes où elles semblent, au premier aperçu, occuper tsute la longueur de la colonne vertébrale, elles manquent, presque sans exception, sur quelques unes des dernières vertèbres coscygiennes.

Le genre gade en est dépourvu communément à plusieurs des premières vertèbres cervicales; chez les pleuronectes et genres voisins, au contraire, elies s'avancent dans une grande étendue sur le crâne; il y en a plus de douze 
qui deviennent insensiblement presque paral. lèles à sa face supérieure sur laquelle elles sont appliquées immédiatement et qui atteignent jusqu'à la face.

Le nombre des épines accessoires est, en général, en rapport avec l'étendue qu'elles occupent sur la colonne vertébrale et avec le nombre des vertèbres qui composent celle-ci, chacune d'elles correspondant à l'un de ces os; cependant il y a des exceptions à cette règle, quoiqu'en petit nombre. Quelquefois la série des épines accessoires existe sur une grande partie de la colonne vertébrale, mais le nombre en est proportionnellement pelit, puisque chaque vertèbre n'a pas son épine accessoire correspondante. Ainsi, chez le bichir (polypterus niloticus) il n'y en a que vingt-six pour soixantehuit vertèbres, parce qu'elles ne commencent à paraître qu'à partir de la sixième vertébre, et que l'absence d'un plus ou moins grand nombre d'entre elles interrompt la continuité jusqu'aux dernières qui se manifestent constamment. Chez les pleuronectes et dans les genres voisins, au contraire, le nombre en surpasse extraordinairement celui des vertèbres. On trouve dans ces poissons, très-généralement: $1^{\circ}$ deux épines accessoires pour chaque apophyse épineuse, une antérieure et une postérieure, qui, par leurs extrémités inférieures, entourent la pointe de l'apophyse; $2^{\circ}$ il restc 
D'ANATOME COMPARLE.

lans la moitié postérieure du rachis, une épine propre, libre à son extrémité inférieure, entre l'épine accessoire, antérieure et postérieure, de chaque couple de vertebres qui se suivent. Chez le flétan (pl.hippoglossus), trois de ces épines accessoires sont situées entre les deux avant-dernières vertèbres qui portent des nageoires. Ces rayons intermédiaires semblent appartenir surtout aux grands pleuronectes. Je les trouve du moins principalenent chez le turbot ( pl. maximus) et le flétan; on les rencontre moins dans les pelites espèces, il n'en existe quelquefois pas du tout dans quelques espèces. Dans d'autres, comme chez le turbot ( $p l$. maximus), elles s'étendent jusque dans la moitié antérieure du rachis, où les vertèbres n'ont de rayons accessoires que par places, disposition qui se reproduit aussi dans la plupart des autres espèces.

Les rayons accessoires dans les balistes, les chétodons, les vomers, leș dorées, les acanthures, les spares, le brama raji, les coryiphènes, les sciènes, les perches, reproduisent exactement les mêmes conditions. Dans tous ces poissons, à l'exception des balistes, ils n'existent cependant que dans la moitié postérieure de la colonne vertébrale, et leur simplicité, dans la partie antérieure, est compensée par leur largeur plus considérable. Chez les balistes, tous sont si larges qu'ils se confondent entr. cux et aver: les apophyses épineuses supé- 
rieures, de manière à former une cloison osseuse. Ces mêmes balistes portent, dans la moitié postérieure de la colonne rachidienne, entre chaque couple de vertèbres, deux épines accessoires, outre les deux épines appartenant à chaque vertèbre, si toutefois on peut se servir du nombre des crêtes transversales comme d'un moyen de détermination.

L'augmentation du nombre des rayons accessoires est, comme leur dimension, dans le rapport le plus intime avec l'augmentation et la diminution numérique des rayons des nageoires.

Cette organisation coincide avec la hauteur considérable du corps, et correspond, sans contredit, à l'augmentation des nageoires que l'on remarque chez les raies et les squales.

Le nombre des épines accessoires supérieures surpasse en outre celui des vertèbres chez les pleuronectes et dans les genres voisins; cette circonstance dépend de ce qu'elles se développent au crâne jusque vers l'extrémité antérieure de la tête. Ce mode de formation, auquel les rayons antérieurs des nageoires participent également, offre quelques différences assez intéressantes, tant sous le rapport de son extension que sous celui de sá disposition.

Sous le premierrapport, quelques poissons, par exemple, les pleuronectes solea, linguatula (1),

(1) Ces deux espèces ne constitıent gue l'espèce des soles de M. Cuvier. 
microchirus, heterochirus, l'achirus fisciatus, les plagiusies, l'emportent considérablement sur les autres; ces épines accessoires existent chez. eux jusqu'à l'extrémité antérieure de la tête; chez d'autres, au contraire, par exemple chez le pleuronectes rhomboideus, elles ne vont que jusqu'au devant des orbites.

Quant à leur disposition, on peut remarquer qu'elles sont formées le plus sonvent sur le type des apophyses épineuses. Mais il y a aussi des différences sur ce point. Chez quelques-uns, par exemple, chez le pleuronectes macrolepidolus, ces épines crâniennes ont la même direction que les apophyses épineuses des vertèbres et ne sont pas sensiblement plus rapprochées les unes des autres; chez d'autres, on peut dire chez la plupart, mais particulièrement chez les achires, elles se rapprochent insensiblemeut d'arrière en avant et prennent une direction plus oblique, qui devient à la fin presque horizontale; de sorte qu'elles sont appliquées sur le crâne. Les plus inférieures et les plus antérieures se prolongent en outre considérablement. Elles forment voûte en haut et se recourbent en bas. Les rayons des nageoires conservent avec cela le plus souvent leur direction, leur. forme et leur volume ordinaires.

Cette disposition conduit à l'organisation des plrıgusies, ou les épines situées au devant de l'orbite se confondent en deux pièces: l'une de ces 
pièces est postérieure, supérieure, plus considérable, triangulaire, alongée, a la pointe tournée en arrière; ses fibres représentent par leur direction les rayons des achires, etc.; l'autre, alongée, placée immédiatement en avant de la première, descend vericalement de haut en bas jusqu'au devant du maxillaire supérieur. A la partie antérieure de cette dernière on trouve en outre une pièce membraneuse, pectiniforme, beaucoup plus volumineuse, convexe en avant, qui se porte en bas et se termine à la même hauteur que la seconde pièce.

Cette disposition est extrêmement intéressante : elle concourt à établir l'analogie des os crâniens avec les vertébres; les rayons accessoires sont placés en effet sur les crêtes pariétale et occipitale, de la mème manière que ceux du rachis sont situés sur les apophyses épineuses supérieures.

La longueur des rayons accessoires est fort généralement en rapport direct avec le développement du corps des poissons dans la direction de la face dorsale is l'abdominale; elle est par conséquent très-considérable chez les poissons aplatis, beaucoup moins chez les autres.

Ils sont très-généralemeut plus courts que les apophyses épineuses supérieures; cependant il y a quelques poissons, par exemple les saumons, le spare on le brama raji, le poisson. lune, plusieurs espèces de silures, où ils sont, 
du moins en partie, plus longs que ces mêmes apopliyses.

Cies rayons accessoires ne s'étendent communément qu'à l'extrémité supérieure des apophyses épineuses auxquelles ils correspondent; dans certaines espèces, au contraire, les silures par exemple, dont les apophyses épineuses sont bifurquées à cet effet, ils descendentbeaucoup plus bas. Chez les mêmes poissons, ces rayons sont, à la partie postérieure du corps, séparés par un large intervalle des sommets qui terminent les apophyses épineuses.

Les rayons accessoires sunt en général plus ou moins obliquement dirigés de bas en haut, et d'avant en arrière; et cette direction est d'aulant plus oblique qu'on les examine plus postérieurement. Chez les pleuronectes, au contraire, ceux du devant sont non-seulement droits, mais même obliquement tournés de bas en haut et d'arrière en avant, en sorte qu'ils sont situés presque horizontalement. Celte direction se reproduit chez quelques autres poissons à corps élevé; mais elle est moins prononcée.

Ils sont généralement alongés. Chez les saımons, plus encore chez les gades, les anarrhiques, les blennies, le callionymus lyra, ils sont communément minces, mème plus minces que les apophyses épineuses. Celte disposition est surtout remarquahle chez la lingue ( 5 inclus molua), et chez le merhus ordinidre (s. me'r- 
lucius ), à cause de la largeur considérable des apophyses épineuses; mais ils sont très-souvent beaucoup plus forts et plus larges d'un côté it l'autre ou d'avant en arrière. Ils se dilatent surtout dans la première direction; et ils offrent en outre très-généralement une saillic latérale, transverse, aiguë, soit à leur portion moyenne, soit à leur partie postérieure. Cette organisation existe surtout chez les balistes, chez le raji du Nil ( salmo niloticus), le salmo dentex, le serrasalme citharine, le lack (elops saurus), l'esox lucius, les cyprins, chez plusieurs silures, principalement aux épines accessoires qui sont en outre grandes et épaisses; clle existe aussi chez les sargues, les ténianotes. les scorpènes, les priacanthes, en partie chez les perches, les sciènes, les trigles, les dorées (zeus), les scares, les acanthures (theutis), les chétodons. Dans les quatre derniers genres les épines accessoires sont assez épaisses pour s'alteindre d’avant en arrière, et se réunir en une lame osseuse plus ou moins continue. Chez les harengs ( clupea), leur plus grande largeur est d'un côté à l'autre.

Chez quelques-uns, surtout chez les balistes, chacune des pièces osseuses, formant celte seconte série daus sa partie postérieure, qui est la plus considérable, sout soudées en devant et confondues en cicux grandes pièces: l'une de ces pièces, beancoup plus grande que 
l'autre, s'étend de l'occipital en irrière; la seconde, plus petite, descend obliquement de haut en bas et d'avant en arrière, entre l'extréwité postérieure de la première pièce et les apophyses épineuses des quatrième et cinquième vertèbres. Une organisation rapprochée de ce que nous venons de dire est celle des trigles, ou ces rayons s'élaryissent en avant et en arrière, de l'extrémité supérieure jusque'sur le côté, et forment de larges lames, imbriquées. Les exemples en sont fournis particulièrement par le perlon ( $t r$. hirundo), le rouget (trig. cuculus), le gronau (tr. lyra), le trigla aspera, moins chez les $t$. carolina et volitans. Le loricaria cataphracta offre la même disposition que les trigles; et dans ce genre ceux des boucliers transverses formés de celte manière, qui sont placés en de'vant, se soudent sur les parties latérales avec les apophyses transverses accessoires tournées en haut. Ce n'est que sur cette seconde série que sont posés les rayons des nageoires dorsales, qui se terminent en pointe et qui sont toujours simples, quoique la longueur en soit très-variable.

$$
\text { S. } 84 \text {. }
$$

La plupart des poissons osseux possèdent, à la face inférieure du corps d'un plus ou moins grand nombre de vortèbres, des apophyses qui peuvent ètre nommées apophyses épincuses in- 
féricures. Elles forment avec les supérieures un angle plus ou moirs aigu, et sont dirigées, avec des degrés d'inclinaison variés, de haut en bas et d'avant en arrière.

On les trouve le plus généralement aux vertèbres coccrgiennes; elles forment une partie plus ou moins considérable de la hauteur que présente la moitié inférieure de la queue; mais on en rencontre souvent déjà des indices aux vertèbres abduminales, surtout aux dernières.

Comme elles n'existent ordinairement qu'à la queue, elles sont moins générales que les supérieures; le nombre en est déterminé le plus communément par la longueur de cette partie.

Elles ont, chez quelques poissons, la même forme et la même longneur que les apophyses épineuses supérieures; chez les autres elles sont, tantôt un peu plus longues, tantôt plus courtes. Cette différence est cependant d'ordinaire peu considérable. Elles sont on droites, ou légèrement convexes en devant, concaves en arrière.

La plupart sont alongées et simples; elles naissent par deux racines, et présentent par conséquent à leur extrémité supérieure une ouverture transverse pour le passage des troncs vasculaires. Dans la majeure partie de leur longueur, ces deux moitiés latérales sont réunies en une épine unique.

Celles du milieu sont ordinairement plus longues que les antérienres et que les postérieures. 
L'épaisseur de la plupart des antérieures dépasse celle des autres quelquefois à un haut. degré; elles sont bifurquées inférieurement. Cette disposition est présentée surtout par celles qui arment les vertèbres caudales et supportent des còtes, comme cela se voit, par exemple, dans les genres saumon et scombre, chez plusieurs pleuronectes et dans le vomer Brorvnii. Chez la sole (pleuronectessolea,) les apophyses épineuses inférieures des vertèbres abdominales sont trèsalongées; la longueur s'en accroît même considérablement d'avant en arrière. Chez le pleuronecte rhomboideus elles ne sont pas bien hauıs, mais très-larges dans le sens antéro-postérieur; il en résulte qu'elles se touchent presque les unes les autres; elles sont d'ailleurs trèsminces d'un còté à l'autre.

Les apophyses épineuses, inférieures, portent quelquefois, comme les supérieures, particulièrement en haut, des crètes longitudinales plus ou moins larges, qui sont beaucoup plus minces d'un côté à l'autre que les épines proprement dites, et qui n'existent que dans une étendue variable. Chez les acanthures et les scares, ces crêtes parient seulement du bord postérieur de l'épine; chez plusieurs chétodons, par exemple le chcetodor arcuatus et le cornutus, elles partent aussi du bord antérieur.

Plusieurs des apophyses épineuses, inférieures, surtout des verlèbres caudales, se souden! 
entre elles chez les scares et les chétodons; les acanthures font exception: mais le nombre de ces apophyses, inférieures, soudées n'y est jamais aussi considérable que celui des supérieures.

Les poissons, chez lesquels elles existent déjá aux vertèbres abdominales, sont les laudroyes (où leur présence est d'autant plus remarquable que le développement du reste des vertèbres est fort imparfait ), les chironectes, les saumons, les serrasalmes, les harengs, les mormyres, quelques pleuronectes, par exemple le $p l$. rhomboideus, la sole, le $p l$. macrolepidotus, le pl. heterochirus, le pl.microchirus, en outre les plagusies, l'achirus macrolepidotus, l'achirus heterochirus, l'acl. microchirus. Elles manquent, au contraire, sur ces mèmes vertèlıres, ou présentent du moins une tout autre forme dans la plupart des autres pleuronectes. Elles existent trèsgénéralement dans les genres scare, spare, sargue, boops, rascasse (scorpcena), ainsi que chez les perches et les muges.

Elles sont longues et situées fort en avant sur tout chez le sparus raji, les romers, le poisson de Saint-Pierre(zeus faber), les chcilodiptères, le dophin (coryphona hippurus), les scombres.

Ces apophyses ne doivent pas être considérées comme des parties propres. Elles ne sont, en effet, que les apophyses transverses inférieures des corps des vertébres qui supportent les cotes du bas: ou seulement la portion infé- 


\section{D'ANATOMIE COMPARÉE.}

rieure de ces apophyses, cxistant ou seulcs on rćunies à ces còles, qui se confondent entre elles dans la ligne médiane et forment un anneau armé d'une épine.

Cie rapprochensent résulte des considérations suivantes:

$I^{\circ}$ Ces apophyses transverses se continuent, sans interruption, avec les apophyses épineuses inférieures; cette continuité s'opère de la manière suivante : elles se prolongent, se portent l'une vers l'autre en bas, se soudent avec les còtes, se confondent daus la ligne inédiane, et y constituent un anneau armé d'une épine. Un exemple manifeste de ce passage se rencontre surtout chez les gades, principalement chez le gadus barbatus, où la première de ces apophyses épineuses inférieures forme un anneau énorme avec une épine à peine sensible; sur cet anneau et sur le second, on reconnait encore très-distinctement l'endroit de soudure entre lapophyse transverse et la côte. La plupart des gades offrent des organisations semblables, mais une qui est surtout digne de remarque est celle du merlan noir ( g. carbonarius), où les apophyses transverses de la première vertèbre qui est munie d'une apophyse épineuse inférieure, se prolongent toul-à-coup considérablement, se recourbent latéralement. l'une vers l'autre, mais ne s'atteignent pas encore. 
$2^{\circ}$ Les apophyses épineuses inférieures, qui existent aux vertèbres abdominales chez les pois. sons dont il vient d'être fait mention, portent souvent les côtes inférieures à leur sommet. II en est ainsi surtout chez plusieurs des pleuro. nectes précités, dans les genres scombre, spare. vomer, dorée, coryphène, moringre, saumon, scare, rascasse.

On ne peut pas objecter, comme contraire it cette manière de voir, la présence d'apophyses transverses avec les apophyses épineuses inférieures que j’ai considérées plus haut, car précisément ces apophyses transverses sont, ou des parties propres, ou des développemens plus considérables de la crête moyenne du corps des vertèbres, ou enfin seulement la partie supérieure des apophyses transverses ordinaires, qui se continuent avec les apophyses épineuses inférieures.

Outre ces apophyses épineuses inférieures qui existent surtout dans la région postérieure du corps, il y en a quelquefois aussi à l'extrémilé antérieure de la colonne vertébrale (1).

(1) La jeune plie, ou carrelet (pleur. rhombeus), a servi d'exemple à M. Groffroy-Saint-Hilaire pour la détermination des pièces constitutives de la vertébre. C'est en ítudiant nn tronçon osseux, emprunté au rachis de cet animal qu'il a pu saisir avec une évidence incontestable la correspondance exacte, existant entre les pièces qui occupent la région dorsale du cycléal ct celles qui sont situćrs à sa région 
Ainsi les corps des quatorze premières verlèbres de la murène commune portent des apophyses épineusés inférieures, larges, réunies en une crète occupant toute la longueur des corps; elles sont beaucoup plus considérables que les

ópposée. Dans ce poisson, les vertèbres coccygiennes présentent du còté du canal médullaire une disposition qui se reproduit avec une fidélité parfaite du côté du système sanguin. Deux pièces alongées s'élèvent des parties latérales du cycléal, se rapprochent, convergent vers la ligne médiane de cet élément, et s'y soudent pour n'en former qu'une seale, séparable en deux moitiés, qui est elle-même prolongée ei grêle. Ces pièces laissent, entre leurs racines, le point où elles se réunissent et le cycléal, un espace occupé, d'un côté, par le système nerveux, et qui, de l'autre, contient le foyer circulatoire. Ces pièces sont au dos les périaux, dans le sens opposé les paraaux, ou apophyses épineuses supérieures et inférieures. La pièce composée, qui résulte de l'union des périaux d'une part et celle qui de l'autre part est produite par la sondure des paraaux, est surmontée par deux autres pièces, qui, ajoutées bout à bout, forment les nageoires dorsales et abdominales, enfin les rayons accessoires, pièces identiquement pareilles, et qui employées à la protection. du système sanguin, sont désignées par $\mathbf{M}$. Geoffroy sous le nom de cataaux, et sons celui d'épiaux, si elles servent au système nerveux. I.'exigence d'une plus grande capacité se fait-elle davantage sentir? cet ajustement bout à bout disparait: plus dapophyses épineuses, plus de rayons accessoires, mais des apophyses transverses, des côtes, des pièces pariétales, frontales, etc. (Voyez la note de la page 282 de ce volume, voyez plus loin Cr.are, etc., et GeoffroySaint-Hilaire, Annales du Muséum, ventèвne.)

\section{(Note des traducteurs.)}


supérieures, deviennent insensiblement plus petites d'avant en arriere, et se confondent peu à peu avec les apophyses transverses, au point que la dernière se bifurque et se dirige en de. hors; mais elles ne contiennent point de cavité.

Il n'existe aucune trace de cette disposition chez les anguilles, du moins chez le congre.

$$
\text { S. } 85 \text {. }
$$

Les apophyses épineuses inférieures des poissons sont, comme les supérieures, très-généralement accompagnées d'épines accessoires, qui, par leur présence, leur nombre, leur forme et leur volume, représentent ordinairement celles de la face supérieure.

Mais il arrive souvent que la concordance n'est pas entièrement exacte. Ainsi, par exemple, les inférieures sont très-fortement développées, sous le rapport du volume et du nombre, dans toute la longueur considérable de la queue des silures, où l'on ne trouve aucune trace des supérieures.

Elles existent, au contraire, communément en nombre moindre que les supérieures; ce qui dépend de ce qu'elles ne font toujours que correspondre aux apophyses épineuses inférieures.

Elles sont pour la plupart arrondies et alongées; mais, chez certains poissons, surtout chez les poissons plats et ceux qui ont le corps 
I) NATOME COMPARÉE.

élevé, une étendue plus ou moins grande de leur portion moyenne se prolonge et se dilate en crètes disposées dans le sens transversal et dans la direction antéro-postérieure; il en résulte une sorte de croix et le contact des crêtes antérieures avec les postérieures dans une partie plus ou moins considérable de leur longueur. Chez les pleuronectes, cette disposition nexiste que dans une très-petite partie inférieure, et seulement chez les espèces qui ont le corps haut et non alongé; elle règue, au contraire, dans toute la longueur des apophyses épineuses inférieures, chez les dorées, les scares, les acanthures, les vomers, le chatodon arcuatus, les balistes, les coryphènes, où ces crêtes s'atteignent réciproquement. C'est chez les balistes et les chétodons que le développement des crêtes transversales est surtout le plus considérable.

Chez la plupart des poissons, les épines accessoires, inférieures et supérieures, sont parlout séparées entierement les unes des antres et des apophyses épineuses proprement dites; les antérieures dépassent, en avant et tout-ifait librement, les apophyses épineuses inférieures auxyuelles elles correspondent. Dans un nombre de poissons qui est proportionnellement petit, surtout chez ceux qui ont le corps haut, les épines accessoires unféricures situées en avant s'applicjuent très-étroitement contre ies apophyses épineuses inférieures. I a disposi. 
tion qu'offrent les pleuronectes, les chélodons, les dorées, les vomers, les balisles, c'est-à-dire, à ce qu'il paraît, presque tous les poissons ou il existe une connexion aussi étroite, consiste en ce que l'épine accessoire inférieure, qui est la plus antérieure, l'emporte de beaucoup en force, en longueur, en épaisseur et en largeur sur les autres, s'élève, en s'apointissant de bas en haut, presque jusqu'au corps de la première vertèbre coccygienue, en avant de l'apophyse épineuse inférieure de cette vertèbre, dont la forme est le le plus souvent un peu concave en avant et convexe en arrière, sa longueur et sa largeur étant également assez considérables. Ces deux apophyses se réunissent étroilement d'àvant en arrière, dans la majeure partie de leur longueur, pour former un rayon très-fort, qui ferme postérieurement la cavité abdominale, et augmente considérablement la solidité de la queue.

Il est quelques variélés de cette disposition. Chez le vomer Brorwnii et les pleuronectes, ce rayon n'est formé, comme nous l'avons indiqué, que par ces deux pièces. Dans la sole ces deux pièces sont, relativement aux autres, trèscourtes et minces, et ne se touchent que dans une petite étendue. Chez les plagusies, il n'existe pas même un semblable rayon antérieur plus développé, mais les épines accessoires inférieures et antérieures, qui sont les plus longues et qui présentent une concavité en avant, s'ap- 
pliquent seulement, de haut en bas, les unes contre les autres, de manière à pouvoir être séparées; clles s'élèvent ainsi, réunies en faisceau supérieurement, jusqu'à l'extrémité inférieure de l'apophyse épineuse de la première vertèbre caudale, avec laquelle elles ne sont unies que d'une manière lâche. La solidité est au conrraire beaucoup plus considérable chez les $b a$ listes et les chétodons, où la dernière vertèbre abdominale s'applique en haut et en avant contre le rayon dont il est question, au moyen d'une apophyse inférieure, forte; quoique beaucoup moins longue. Chez le pourc (balistes capriscus), chez les balistes monoceros, ater, et aculeatus, il est éloigné supérieurement de la seconde apophyse inférieure et de la première épine accessoire par une lacune considérable; il y est, au contraire, attaché dans toute sa longueur chez les $b$. diemensis, oxyrhynchus, verrucosus, ainsi que chez les chélodons. Chez le poisson Saint-Pierre l'apophyse épineuse de la seconde vertèbre coccygienne s'applique même contre ce rayon.

Chez les trois dernières espèces de balisies, lapophyse épineuse inférieure de la dernière vertêbre abdominale s'élargit brusquement ì son extrémité supérieure, et d'un côté à l'autre, en une espèce de bouclier considérable, dont les autres poissous n'offrent proint de trace. La premiere épine accessoire inférieure correspond 
le plus souvent, d'une maniere plus ou moins distincte, à plusieurs rayons de la nageoire anale, et n'est sans doute qu'un composé de plusieurs rayons accessoires. Elle porte inférieurement, chez le chatodon arcuatus, deux crètes transversales, larges, placées l'une après l'autre, tandis que les autres n'en cnt qu'une.

Dans cette disposition, les épines accessoires inférieures qui suivent immédiatement la première, sont ordinairement plus ou moins rudimentaires; celle-ci est, au contraire, trés-développée. Cela se voit surtont chez les pleuronectes à corps court et élevé, par exemple, chez le flétan ( $p l$. hippoglossus), le turbot ( $p l$. maximus), la limande ( $p l$. limanda), elc.; moius chez la sole; ici plusieurs rayons accessoires se sont soudés en un seul qui est volumineux; rien de pareil n'existe chez les balistes et les chétodons, chez lesquels le rayon accessoire suivant n'est guère plus petit que le premier, et où tous diminuent de volume d'avant en arrière.

\section{\$. 86 .}

Aux épines el épines accessoires des vertèbres de poissons se rattachent les rayons des nageoires. Toutes les nageoires, les dorsales, les caudales et les anales, renferment, à quelques exceptions près, par exemple, le saluth ( silurus glanis), des rayons disposés successivement 
d'avant en arrière, qui en forment la base osseuse, et s'écartent plus ou moins à leur base pour constituer deux apophyses latérales, courtes, par lesquelles ces rayons sont appliqués sur les extrémilés supérieures de l'épine accessoire qui leur correspond, et avec laquelle ils sont unis d'une manière mobile.

Leur longueur correspond très-généralement à celle de la nageoire ou de la partie de la nageoire où ils se trouvent.

Tous ou du moins la plupart d'entre eux sont bifurqués d'avant en arrière, vers leur extrémité libre, á des profondeurs variables, ce qui augmente la base de sustentation pour la nageoire qui s'épanouit vers le bord libre; mais ils sont simples, du moins en avant, chez plusieurs poissons, par exemple dans les genres scombre, dorée, chétodon, scare.

Chez plusieurs silures, par exemple le doras carinatus, le s. seren, s. carasch, s. bagre, s. abusari, le second rayon de la nageoire dorsale antérieure est très-grand, apointi, plus ou moins séparé du reste de la nageoire, qu'il dépasse; il est fortement dentelé à son bord antérieur, quelquefois aussi à son bord postérieur.

Chez les balistes le premier rayon seulement, ou le premier et le second, même le troisième, sont ainsi séparés des autres nageoires et formés de la même manière. Le premier et le second sont très-rapprochés dans le dernier cas, 
le troisième étant isolé. Le premier est toujours le plus long, le troisième le plus court. Tous sont des armes puissantes. La description détaillée de cette disposition sera faite un peu plus loin.

$$
\text { \$. } 87
$$

Outre les conditions générales que nous venons de considérer, certains groupes de poissons en offrent encore de particulières.

Il faut ranger ici d'abord la réunion de plusieurs vertèbres ou parties de vertèbres entre? elles.

La tendance des vertèbres de la partie antérieure du rachis à se confondre en un seul os, très-générale chez les poissons cartilagineux, se montre beaucoup plus rarement chez les poissons osseux. On voit un indice de cette disposition dans l'organisation de la seconde vertèbre cervicale chez les carpes. Elle est heaucoup plus volumineuse que les autres et munie, de chaque côté, de deux apophyses transverses; l'une est postérieure, descendante, plus longue, l'autre est antérieure, ascendante, plus courte. Mais cette disposition est beaucoup plus prononcée chez les silures. La première vertèbre cervicale est petite, n'est presque formée que de son corps qui est mince, et ne se montre presque que comme un fibro-cartilage ossifié; mais la seconde est au moins six fois plus grande qu'elle et les 
D ANATOMIE COMPARÉ.

suivantes, plus de trois fois plus volumineuse que les plus grandes des autres, et non-seulement beaucoup plus longue, mais aussi beaucoup plus large, quoique ses apophyses épineuses soient plus courtes. Elle porte, chez le silure commun, deux apophyses transverses de chaque còté, une antérieure et une postérieure, qui se réunissent à leur base. J'antérieure, beaucoup plus volumineuse, est partagée en deux pointes; celle du devant, plus forte et plus large, s'applique contre l'os de l'épaule par une facette articulaire, encroûtée de cartilage; la postérieure est libre. L'apophyse épineuse est de inème partagée en deux moiliés, dont l'antérieure, bien plus"grande, dépasse de beaucoup la première vertèbre cervicale et s'articule étroitement avéc la crête occipilale. La largeur de ses apophyses transverses, qui excèdent de beaucoup celles des autres vertèbres, est un indice de l'organisation costale qui commence à la troisième vertèbre, quoiqu'elle ne se manifeste ici encore que dans un état extrêmement rudimentaire. Quand on considère cette vertèbre d'avant en arrière, on la voit très-distinctement formée de deux.

Cette particularité est encore plus développée dans quelques autres espèces de silures que dans le saluil. Chez celui-ci et chez le sharmuth (s. anguillaris) la vertèbre en question est, en tffel. la plus petite, vient ensuite le schilbé 
(s. mystus), puis le doras, le s. liagre et felis, l'asprède. Dans la dernière espèce j'ai trouvé cette vertèbre d'un pouce de long et de large dans un individu qui n'avait guère plus d'un pied. Plus cette vertèbre est développée, plus elle contribue par ses apophyses épineuses a appuyer la nageoire dorsale, et se montre par conséquent formée d'un plus grand nombre de vertèbres.

Quant' à la forme, il est permis de dire également que la vertèbre grossie a toujours la forme d'une vertèbre composée. Il existe du moins deux apophyses transverses, une antérieure et une postérieure, dont la premıère est communément plus développée et bifurquée plus ou moins profondément en deux moitiés, l'une antírieure et l'autre postérieure. Chez le piemodes auratus, la portion transverse de la verlèbre, qui est très-longue, ne forme qu'une écaille large et simple, qui a quelque analogie avec la carapace des chéloniens. Le nombre des apophyses épineuses correspond ordinairement à celui des apophyses transverses. Au moindre degré de développement la forme de cette vertèbre ne présenté pas d'autre particularité; mais dans d'autres espèces, surtout chez le s. seren. le s. clarias, le doras, un large bouclier osseux s'applique. sur les apophyses épineuses jusqu'au premier rayon de la nageoire dorsale, unissant ainsi entre eux le crâne et la vertebre. 
Chez d'autres cet os est plus étroit, fendu latéralement à sa partie postérieure. Une disposition remarquable est celle qui se voit chez le $\therefore$ bagre. Il part des deux côtés et de toute la hauteur de la première apophyse épineuse, qui est tres-large, une lame transversale également irès-large, qui s'étend jusqu'à l'os qui recouvre les apophyses transverses; cette lame est l'indice du prolongement du crâne en arrière. Un semblable indice se voit dans l'articulation des sommets des apophyses transverses avec les parties latérales de l'occipital, articulation qui esi constanle. On trouve également, chez beau. coup de silures, le sommet de l'apophyse épineuse antérieure articulé par suture avec l'occipital. Dans plusieurs espèces, par exemple, les doras, le s. bagre, il naît de l'extrémité postérieure et externe du pariétal un os propre yui aboutit à celte vertèbre, et entre ces trois (s) on trouve une ouverture qui rappelle celle qui existe chez la plupart des reptiles entre le pariétal et l'occipital. Chez plusieurs silures la premiere vertebre manque comme os propre, et son articulation avec l'occipital est dentelée, cest-id-dire operée par suture. Chez le s. niger, l'arc de cette vertèbre est éģalement uni à celui re la vertibre suirante par une suture à dentelures aiguës.

Des rapprochemens vers ces organisations se vient dans le serrasalme citharine et le chu- 
racin nefasch, où la première vertèbre porte une apophyse épineuse supérieure, très-large d'avant en arrière, qui dépasse le corps surtout en devant, et qui chez le characin nefasch est unie par une suture avec l'os interpariétal.

Chezles fistulaires, la première vertèbre, qui est très-alongée, est également formée de plusieurs pièces unies entre elles par harmonie. Chez le $f$ tabacaria, cette vertèbre constitue à peu près un neuvième de toute la colonne rachidienne; elle est un peu plus courte chez le $f$. chinensis (1).

La seconde vertèbre cervicale est grossie d'une autre manière chez la loche d’étang (cobitis fossilis). Le corps se gonfle des deux côtés en bas et en arrière, el constitue un renflement fort considérable, arrondi, lâche, à parois minces, dont les deux moitiés sont séparées à l'extérieur, inférieurement et postérieurement, par une incisure superficielle, tandis qu'elles forment un tout continu daus la ligne médiane. Ce renflement contient la vessie natatoire.

\section{88.}

Cette région antérieure du rachis est encore plus compliquée, shez un certain nornbre de poissons, par la présence de plusieurs os, pro-

(1) Voyez le squelette du premier, décrit et fiçuré par Rosenthal dans ses Tables ichlingotomiques, I, a, 1. Jo . pl. IX. 
porlionnellement très-petits et très-délicats, si on les compare aux autres os de cette région.

Ces us n'ont été démontrés jusqu'ici que chez les carpes, les silures et les loches d'étang; je n'ai pas pu les trouver dans quelques genres voisins. Je citerai, par exemple, l'exucet (exocoetus exsiliens), et le céphalo (mugilcephalus), sans parler des autres genres où ils manquent, d'après les recherches de M. le professeur Weber.

Ces os sont ordinairement au nombre de trois, situés tout à côté de la première et de la seconde vertèbre, entre elles et l'occipital; ils se succèdent d'avant en arrière, unis entre eux par des articulations mobiles.

Celui qui est postérieur et qui occupe le plan inférieur, dépasse de beaucoup en volume les deux autres; il est alongé, plus ou moins apointi à ses extrémités antérieure et postérieure, recourbé et surmonté d'une apophyse supérieure qui s'élève un peu en avant du point moyen desalongueur. Celui de ces os qui le suit en avant, le moyen, est ordiriairement le plus petit; il est mince, alongé, simple chez le silure et la loche d'étang; il est le plus composé chez la carpe, où il se bifurque à son extrémité postérieure, et donne naissance. à une apophyse supérieure et à une apophyse inférieure.

Le plus antérieur et le plus supérieur de ces us est formé, chez la loche d'étang, par une pe- 
tite lame légèrement concave. Chez la carpe, cette lame plus concave se termine supérieurement 'et inférieurement par deux apophyses courtes; chez le silure, on trouve derrière elle un condyle arrondi, au moyen duquel cet os est mobilement articulé sur la première vertèbre, eı d'où part une forte apophyse qui se dirige en haut.

La lamelle qui caractérise cet os forme une ouverture appartenant à l'organe auditif; l'os le plus postérieur est, au contraire, placé sur la vessie natatoire.

Frappé du rapport qui unit ces os à l'organe auditif, M. Webèr (1) les a considérés comme appartenans à cet organe, et les a comparés aux osselets de l'ouie des mammifères; le postérieur correspond, selon cette manière de voir, au marteau, le moyen à l'enclume, l'antérieur à l'étrier.

La position et les connexions de ces os militent, en effet, fortement en faveur de cette opinion. On ne saurait objecter contre ce rapprochement l'imperfection relative qu'offrent, sous le point de vue du nombre et lu développement, les osselets de l'ouie chez des animaux placés au-dessus des poissons, tels que les reptiles et les oiseaux, quoique cette analogie rapproche les premiers de ces ètres beatl-

(1) De aure et aulitu hominis et animalium, $p .1$. Ine "aure animalium aquatilium, 1820. 
D'ANATOMIE COMPARÉE.

coup plus près des mammifères, et que le développement uniforme des autres parties, tant internes qu'externes de l'organe auditif, forme une série presque parfaite.

Rosenthal, qui précédemment a figuré quelques-uns de ces os (1), mais qui n'a pas aperçu l'os moyen, donna au postérieur le nom d'os en forme de sabre ou d'apophyse ptérygoïde de la seconde vertèbre (2), et désigna lantérieur sous le nom de petite écaille osseuse unie avec la première vertèbre (j), sans rien dire sur leur nature (4).

Je reviendrai à ces os en traitant de l'organe auditif, attendu que, quelque soit leur usage, c'est avec cet organe qu'ils ont le plus de rapport.

$$
\text { \$. } 89 \text {. }
$$

Il est plusieurs poissons chez lesquels les verlèbres sont plus composées encore; de nouvelles apophyses s'ajoutent à celles qui existent

(1) Ichthyotom. Tafeln. H. 1, r812. H. 2, 18,6, Taf. I, fig. 3-6. Taf. 9 , fig. 3.

(2) H. I, S. 8, H. 2, S. 29.

(3) H. 1, S. 8.

(4) Ce sont les pièces de l'opercule qui ont fourni à M. Cenffroy-Saint-Hilaire les analogues des os de la caisse du tympar. (Philos. anal., t. I. Paris 1818.-Il sera ques. tion phus an long de cette opinion a-propos de l'appareil ie l'onie.

(Note des traslucteurs.) 
déjà. Il faut ranger ici surtout plusieurs silures, et particulièrement l'espèce harmouth-hale (silurus, seu heterobranchus bidorsalis ).

A compter de la cinquième vertìbre, toutes les apophyses ont, à leur base, une éminence tournée en bas, qui s'alonge d'une manière insensible et proportionnelle dans le sens antéropostérieur; en partant du sixième de ces os, on trouve inférieurement, à l'extrémité postérieure de la face latérale du corps, une saillie semblable; ces apophyses se touchent réciproquement; disposition qui augmente naturellement la solidité de la connexion.

Une disposition analogue, mais beaucoup moins développée, se trouve chez le sharmuth ( silurus anguillaris), et le silur'us niger.

Le scomber albacore présente une organisation qui est encore plus compliquée. Outre les cûtes courtes, latérales et supérieures, appliquées immédiatement sur le corps des verlèbres et dirigées en arrière, il porte au corps de toutes les vertèbres, les antérieures exceptées, des apophyses antérieures et postérieures qui se dirigent en bas. Celles des vertèbres antérieures sont séparées; celles des postérieures sont, à une hauteur considérable, appliquées très-étroitement les unes contre les autres, et ne s'écartent latéralement que plus loin. Les apophyses postérieures du quart antérieur de la colonne vertébrale ne sont que des pointes. 
D'ANATOME COMPARÉE.

comme chez les silures, entièrement séparées des apophyses antérieures de la même vertèbre et de la vertèbre suivante, quoiqu'elles descen-' dent immédiatement au devant des dernières. Mais à partir de ce point, elles s'alongent tont à coup considérablement, et se bifurquent à leur extrémité inférieure en deux pointes, dirigées en dehors et en devant, qui s'appliquent contre de semblables pointes, fournies par l'apophyse anterieure de la mème vertèbre.

Les apophyses antérieures sont simples dans le sixième antérieur de la colonne, mais se bifurquent au-delà en s'alongeant; des deux pointes qui en résultent, l'antérieure est plus courte, et la postérieure est plus longue; la dernière se confond, comme il a été dit, avec l'apophyse postérieure, se prolonge ensuite en bas et en arrière, porte une côte antérieurement, et reste ici d'abord séparée de celle de même nom du côté opposé, puis se réunit à elle, tandis que dans la queue elle s'y soude aussilôt et forme avec elle l'apopbyse épineuse inférieure. La pointe antérieure, plus courte, est libre inférieurement dans les vertèbres antérieures et postérieures; aux vertébres moyennes elle s'applique contre la pointe postérieure de la vertèbre qui précede immédiatement. 


\section{\$. 90 .}

On trouve des particularités, chez quelques poissons, surtout aux apophyses épineuses supérieures et inférieures et à leurs développemens, les épines accessoires et les rayons des nageoires.

Les plus remarquables de ces particularités sont les suivantes:

Chez une espèce de chétodon (chcetodon arthriticus) (1), il existe des renflemens arrondis, spongieux, mous, très-volumineux, remplis d'une moelle huileuse : $1^{\circ}$ à l'extrémité supérieure de la première épine accessoire supérieure; $2^{\circ}$ sur quatre des épines supérieures de la moitié postérieure, à peu près vers le milieu de leur longueur, mais un peu plus haut, de sorte qu'il y a chaque fois entre deux épines de cette espèce une épine ordinaire; 30 à l'extrémité inférieure de la première épine inférieure qui est très-longue; et au milieu d'une des dernières, laquelle correspond à la dernière des épines supérieures ainsi conformées. Les renflemens supérieurs et inférieurs, placés postérieurement, règnent environ sur la moitié de la longueur de toute l'épine; le renflement infé-

(1) Descr. of a species of chaetodon, called by the Malays, ecan bonna. 13y M. W. Bell, etc., at Bencoolen. (Philosoph. iransact. $179^{3}, \mathrm{P} \cdot 1, \mathrm{P} \cdot 7$, tab. 6.) 


\section{D'ANATOMIE COMPARÉE.}

rieur et antérienr n'en occupe que le quart. L'olservateur dit expressément qu'ils existent sur tousles poissons de cette espèce et coustammentaux mèmes os. On ignore l'influence qu'ils exercent sur le genre de vie de l'animal; peutcitre est-elle nulle; du moins elle ne peut être considérable sur le mode de locomotion lent, qui est propre à plusieurs de ces poissons dont le squelette n'offre pas cette déviation. On peut admettre que le surcroît de poids qui en résulte pour le corps de l'auimal est compensé par le volume considérable de la vessie natatoire.

Une autre particularité de ces parties, c'est le développement de plusieurs rayons des na= geoires en pointes isolées, plus ou moins aiguës, dentelées, souvent assez longues. Ces pointes occupent communément le dos, où elles sont le plus fortes et le plus nombreuses, mais on en Irouve aussi à la face abdominale. Elles sont situées en général au devant des nageoires dorsale et anale, sont nues dans leur partie supérieure; quoique dans un grand nombre de cas, si ce n'est dans tous, la peau subsiste et leur forme une gaine molle et lâche qui les entoure. Elles sont en outre généralement beaucoup plus mobiles que les rayons des nageoires et peuvent se cacher, par un côté, d'une manière plus ou moins complete, dans une gouttiere variablement profonde, formée par la peau, les os et les muscles voisins, et de l'autre, se dresser et 
prendre une direction tout-à-fait ou presque tout-ì-fait verticale. On en tronve des exemples avec différentes modifications chez les centronotes, les balistes, les ericius.

Chez les centronotes, il existe entre la nageoire dorsale et la tête, une série de pointes de cette espèce, dont le nombre varie suivant les espèces. Chez les $c$. lyzan (Risso) il y en a sept. La première est tout-à-fait immobile, horizontale, immédiatement appliquée sur le bord supérieur du dos, et porte sa pointe directement tournée en devant. Les sept postérieures sont mobiles, mais leur mobilité diminue considérablement d'avant en arrière. Elles peuvent se lever plus ou moins verticalement et tourner leur sommet en arrière; les antérieures peuvent même se cacher entièrement ainsi dans une gouttière.

Le même poisson poṣsède, au devant de la nageoire anale, deux pointes situées l'une après l'autre, qui peuvent se dresser en bas presque tout-à-fait verticalement, et se cacher en arrière dans une semblable gouttière.

Les balistes offrent une disposition intéressante à plusieurs égards. Comme il a déjà été dit (1), la portion la plus antérieure des épines accessoires dorsales qui suit immédiatement la tèle est transformée en deux os. Le postérieur, arrondi, alongé, beaucoup plus petit, est dirigé

(1) Voyez pag. $31 / 4$ de ce voinme. 
I ANatomil Comparie.

d'arrière en avant et de bas en haut et rencontre ici l'extrémité postérieure de l'os supérieur. Celui-ci, qui touche en avant à l'occipital, est alongé et scaphoïde, la concavité profonde qu'il forme est tournée en haut et la partie convexe et longue, en bas. L'extrémité antérieure est un peu relevée au dessus dù reste de l'os, et porte: $1^{\circ}$ deux éminences articulaires, latérales, arrondies, considérables; $2^{\circ}$ en dedans et au devant de ces èminences, deux autres petites saillies qui passent en arrière dans une éminence arrondie, moyenne, située entre les premières. L'épine antérieure qui est la plus volumineuse, est articulée avec les dernières.

Immédiatement en arrière de ce point; l'organisatıon de cette pièce est encore plus compliquée. Du milieu de sa face supérieure qui estconcave s'élève : $1^{\circ}$ une éminence longitudinale, considérable; $2^{0}$ les deux lames latérales sont percées par une ouverture alongée, encore plus considérable, qu'une forte barre partage en une moitié antérieure plus petite et en une postérieure plus grande.

Cet os supporte trois épines, unies entre elles à leur partie inférieure par de la peau molle, libres de toute autre arhérence; la plus antérieure est beaucoup plus grande que les deux autres qui ont à peu près la même hauteur. Partagées à leur base en deux apophyses articulaires latérales, elles sont toutes un peu con- 
vexes en avant, concaves en arrière, apointies; l'antérieure est tapissée à sa face antérieure d'une peau dure, très-rugueuse et dentée, et à sa face postérieure d'une peau molle et délicate, qui enveloppe les deux autres sur toute leur périphérie. La première et la seconde se distinguent, en outre, d'une manière remạquable, l'une de l'autre et de la troisième. La première présente. en effet, au dessus et en arrière des courtes apophyses articulaires, de chaque côté, une forlc saillie longitudinale, postérieure, interne; entre ces saillies existe dans le milieu une gouttière longitudinale, considérable; la seconde épine se termiue inférieurement par deux prolongemens latéraux fort longs. qui sortent par la moitié postérieure des ouvertures dont nous avons parlé, et qui sont situés sur les côtés die l'os principal. Ces deux prolongemens sunissent ici étroitement, mais mobilement avec les faces latérales. Immédiatement au dessus de ce point l'épine se renfle en un condyle arrondi, convexe en avant, élevé au milieu surtout dans le sens longitudinal, qui s'engrène par cetle éminence longitudinale avec la gouttière postérienre de la première épine. Inférieurement ce condyle, surtout par son éminence articulaire, est fortement apoiuti en avant, et concave en arrière:

Ces deux épines sont situées immédiatement l'une à la suite de l'autre; la troisième en est 
fort éloignée; elle est située presqu'à l'extrémité postérieure de l'os principal.

Elles sont étroitement unies les unes arx autres, un peu au dessus de leur extrémité inférieure, par un fort ligament fibreux, qui s'étend de la face postérieure de l'épine antérieure à la plus postérieure; il résulte de cette disposition qu'elles doivent nécessairement s'élever et s“incliner en arrière d'un mouvement simultané. Liépine antérieure retenue immédiatement en position verticale par les éminences latérales, internes et antérieures de l’os principal, ne saurait être mue en avant, et ne peut, à cause de la saillie moyenne dans laquelle passent ces éminences, être inclinée sur le còté. C'est surtout la seconde épine qui assure la solidité de toutes trois; lorsqu'elle est dressée verticalement elle glisse en devant avec son condyle inférieur par dessus la seconde émi. nence, moyenne, de l'os principal sur laquelle elle se meut, et s'engage dans un enfoncement corısirlérable, situé entre cette éminence è la première. Elle est encore plus fixée dans cette position parles éminences postérieures et internes de la première épine qui viennent d'en haut se presser contre sa base. On presse par conséquent en vain la première épine sur la seconde, ni l'une ni l'autre ne cède; au contraire la seconde en devient plus solide dans sa position; ce n'est qu'en tirant en arrière l'épine 
postérieure que l'on peut faire baisser les antérieures pour les faire entrer dans la gouttiere formée parl'os principal; elles sont situées ainsi tout-a-fait horizontalement et presque entièrement couvertes sur le côté.

L'organisation des pomocentres et des balistes se trouve réunie d'une manière extrêmement compliquée dans un poisson décrit par plusieurs naturalistes, mais surtout avec soin par Tilesius (1) qui le vit en vie. A la place des nageoires dorsales, il existe dans cet animal trois épines longues et trois autres plus courtes qui s'entrecroisent lorsqu'elles sont érigées. Elles sont atlachées à leur base par un fort tendon dans le profond sillon dorsal où elles peuvent s'abaisser et se cacher; mais quand elles s'élevent, elles s'entrecroisent avec tant de force qu'il est impossible de les faire fléchir en arrière. Chacune de leurs articulations est rectangulaire, et présente une échancrure dans laquelle s'engrène une saillie du sillou dorsal; cet engrenage empêche que l'épine ne puisse être forcée en arrière, la rend immobile, jusqu'à ce que le tendon la retire de la saillie tranchante.

Cette description n'est pas tout-à-fait satisfai-

(1) Ericius cataphractus mihi; der spanische Reuterfisch; matskasaoibo Japon.; monocentris carinata Bloch ; Gasterosteus Japon. Houttuyn sciæna cataphracta Thunberg. Münchner Denkschr. 1811, 1812, p. 77. (Voyez les Mémoires de l'Académie des sciences de Munich de 1811 et 1812 .) 


$$
\text { D'ANATOMIE COMPARÉ. }
$$

sante, car elle ne délermine pas exactement la forme des différentes épines, ni la disposition des muscles, ni leurs rapports entre eux; cependant les figures font voir d'une manière dislincte la composition précédemment indiquée.

$$
\text { b. Côtes. : }
$$

$$
\text { S. } 9 r \text {. }
$$

Les poissons sont très-généralement pourvus de côtes; celles-ci sont des os alongés, situés sur les faces latérales du corps des vertèbres, le plus souvent des seules vertèbres abdominales, ordinairement depuis la première jusqu'a la dernière; ces os s'articulent mobilement avec les vertèbres et sont libres à l'autre extrémité. On peut les considérer en général comme des répétitions et des prolongemens des apophyses transverses et les étudier successivement, cornme les vertèbres, dans les poissons cartilagineux et dans les poissons osseux.

1. Poissons cartilagineux.

$$
\text { S. } 92 .
$$

J'ai déjà dit précédemment (1) que, coutrai-

(1) Dans ma trad. allem. des Leçons d'anatome comparée de 1. Cuvier, vol. I, p. 494. 
rement à l'assertion émise par Vicq d'Azyr (1) et M. Cuvier (2), plusieurs poissons cartilagineux, en particulier les raies, les squales et les esiurgeons possèdent des côtes; il est cependant vrai qu'elles manquent à plusieurs, savoir aux chimères, aux lamproies, sans doute aussi aux genres voisins; je n'en ai pas pu trouver de trace chez l'aigle de mer (raja aquila).

Chezles raies et les squales, qui en sont pourvus, ainsi que chez l'esturgeon, les côtes sont appuyées sur dés a pophyses transverses, courtes, Iriangulaires, apointies, qui se trouvent situées constamment à l'extrémité inférieure de la face latérale des corps des vertèbres.

Chez l'esturgeon et la torpille, elles sont proportionnellement plus longues que dans les autres poissons cartilagineux; chez les raies elles sont en géuéral plus petites que chez les squales. Parmi ces derniers je trouve que c'est l'ange (sq. squatina) qui a les plus longues.

Chez l'esturgeon elles sont droites et disposécs obliquement de haut en bas et de dedans en dehors; la direction en est transversale chez les raies et les squales.

Il n'y a jamais plus d'une rangée de chaque côté. Elles sont de même beaucoup plus petites que chez la plupart des poissons osseux.

(1) Mémoires des savans étrangers, to VII , p. 23.

(2) Cuvier, Anatomie comparée, t. I, pag. 213. 
D'ANATOMIE COMPARÉ.

a. Poissons osseux.

$$
\text { S. } 93 .
$$

11 existe des côtes chez le plus grand nombre des poissons osseux.

Les principales conditions qu'elles présentent sous le rapport de la présence, de la disposition, du nombre, de la position, de la connexion avec lesvertébres, de la direction, de la forme et du volume, sout les suivantes:

$1^{0}$ Présence. Elles existent très-généralement; quelques poissons font seulement exception, par exemple les tétrodlons, les diodons, les coffres, les baudroyes, les chironectes, les syngnathes, les espadons, les fistulaires. Lorsqu'elles existent, elles se montrent presque toujours déjà à compter de la première vertèbre, ou commencent du moins déjà à quelque distance de l'extrémité antérieure de la colonne rachidienne.

$2^{\circ}$ Disposition. Toutes les côtes n'ont pas la même disposition; on peut les distinguer surtout en deux espèces ou ordres, en côtes supéricures et en côles inférieures. L'ordre des inférieures est plus général que le supérieur, ordinairement plus développé et existe souvent sans le supérieur, tandis que celui-ci n'existc jamais sans l'autre. (On trouvẹ réunies ces deux espèces de cưtes dans les genres saumon, brochet, mor- 
miye, hareng, carpe, pleuronecte, gade, labre, blennie, ruban, anarrhique, sargue, rascasse, ténianote, mulle, farlouse, pagre, spare, scare, sciène, perche, vive(trachinus), uranoscope, trigle, brama raji, acanthure (theutis), chélodon, scombre, coryphène; en"général chez la plupart des poissons osseux. Chez quelques-uns, nous citerons spécialement le genre hareng: il y a encore, outre les côtes supérieures ordinaires, une troisième rangée au dessus de celle-ci; elle est séparée des autres de toute la hauteur du corps des vertèbres. Cette organisation est vraisemblablement indiquée chez beaucoup de poissons, par exemple chez les brochets uu ésoces, par une bifurcation profonde de l'extrémité in terne des côtes supérieures.

$3^{\circ}$ Nombre. Le nombre des côtes est en général déterminé par celui des vertèbres abdominales, les vertèbres coccygiennes étant sans côtes. Quelques poissons font cependant exception a cette règle; ils ont leurs vertèbres caudales garnies de côtes. Ce sont constamment, dans ce cas, des côtes supérieures, courtes, parce que les inférieures se soudent entre elles et avec les apophyses transverses pour former les épines inférieures. Cette organisation se trouve surtout chez le lump de nos mers (cyclopterus lumpus), le sargue ordinaire, le pagre ordinaire, les coryphines, le chatodon arcuatus, et les scombres. Chez les 6combres ces côtes existent sur la plupart des 
vertèbres coccygiennes; chez les autres seulement sur un petit nombre des premières. Chez la bonite rayée (sc. pelamys) elles se confondent aux dernières vertèbres caudales et forment une large crète transversale qui se soude avec les verièbres.

$4^{\circ}$ Position et connexion. Les côtes supérieures sont toujours situées sur la partie latérale du corps des vertèbres; les inférieures correspondent aux mêmes régions latérales, ou en partie à ces régions, en partie au dessous, ou enfin tout-à-fait au dessous des vertèbres. La dernière disposition a lieu quand elles sont attachées ic la face inférieure des vertèbres, la première quand elles le sont sur les parties latérales.

Elles sont situées, soit immédiatement sur le corps, soit sur des apophyses de cette partie des vertèbres. Le premier mode de connexion est ordinairement celui des côtes supérieures, le second celui des inférieures. Cependant les côtes supérieures ne sont unies quelquefois aux vertébres que par des filamens tendineux, longs et minces, et semblent par conséquent être seulement plongées dans la partie charnue. La description des vertèbres a fait connaître chez quels poissons les eôtes inférieures sont siluées sur des apophyses transverses.

Chez les saumons, les mormyres, les harengs, le brama raji, les coryphènes, les scombres, les côtes supérieures sonł situées sur les parties la- 
térales du corps des vertèbres, à plus ou moins de distance de la racine des apophyses épineuses supérieures, quelquefois presque jusqu'auprès des inférieures, et conséquemment à une hauteur, qui n'est pas la même dans tous, au dessus des côtés inférieures. Chez le polypterus bichir, il existe d'autres côtes qui correspondent aux côtes supérieures ordinaires; ces côtes occupent la partie antérienre du corps des vertèbres, c'està-dire la partie postérieure de la face inférieure des apophyses transverses; elles sont situées partout au devant des côtes inférieures; dans aucune région elles ne sont au dessus.

Chez les pleuronectes, les gades, les anarrhiques, les labres, les spares, les scares, les ténicnotes, les vives, les sciènes, elles sont situées à peu de distance des còtes inférieures sur les apo. physes transverses.

Chez plusieurs espèces de gades, de labres, de perches, de chétodons, les côtes supérieures occupent la partie postérieure de l'extrémité supérieure des côtes inférieures, surtout de celles du devant. Quelquefois les còtes accessoires an. térieures s'attachent aux inférieures, et les postérietires tout à côté d'elles aux apophysestransverses. On en a un exemple dans le genre rascasse.

Les côtes inférieures sont beaucoup plus or dinairement siruées sur les parties latérales, comme cela résulte en partie déjà de la remar- 
D'ANATOMIF, COMPakéE.

que faite ci-dessus que les apophyses transverses ont ordinairement une direction plus latérale. Lorsque celles-ci sont dirigées en bas, les côtes en question se trouvent tout-ì-fait sous la vertèbre. Cela s'applique aussi aux côles inférieures qui sont appliquées immédiatement à la face inférieure des vertèbres sans apophyses transverses.

Les scombres et les coryphènes offrent surtout cette disposition.

Elles sont situées plus latéralement, au contraire, chez la plupart des autres poissons.

$5^{\circ}$ Forme et direction. Les côtes inférieures des poissons osseux sont communément plus ou moins convexes en dehors, concaves en dedans, renflées en une petite tête à leur extrémité supérieure, d'où elles s'amincissent insensiblement jusqu'à leur extrémité inférieure, qui dégénère en une pointe. Elles sont ordinairement alongées et arrondies, et descendent de haut en bas et en mème temps un peu en arrière.

On les trouve convexes dans le sens que nous avons indiqué, par exemple dans les genres saumon, serrasalme, carpe, brochet, mormyre, 'xocet, hareng, gade, pleuronecte, blennie, labre, sciène, perche, muge, dorée.

Chez d'autres poissuns et en particulier les balistes, les loricaires, le plus souvent aussi chez les silures, les uranoscopes, les trigles et les bairaclius, elles son! au contraire convexes en 
avant, concaves en arrière, et sont situées horizontalement, ou seulement un peu obliquement tournées en bas.

Elles sont planes chez un petit nombre, par exemple dans le polypterus nilolicus.

Quelquefois elles ne sont pas alongées et arrondies, mais elles sont, surtout supérieurement, beaucoup plus larges de dehors en dedans que d'avant en arrière, direction suivant laquelle elles sont fort minces et comprimées. Les saumons, les serrasalmes, les mormyres, en quelque sorte aussi les brochets, les loches, les anableps offrent cette disposition. On la retrouve aux deux antérieures chez le pimelodus biscutatus, à toutes ou au moins à la plupart de ces côtes chez les carpes, les labres, et p articulièrement le $l$. livens, la vicelle ( $l$. vetula), le l. obscurus, chez les soldado (holocentrus), les sargues, les pagres, les spares, les sciènes, les perches, les muges, les acanthures, les scares, les chétodons, les coryphènes, les scombres, les exocets. Leur bord externe est à la fois plus épais que le reste, de manière qu'elles sont concaves en dedans.

On ne les trouve pas, autant qu'il m'a été permis de m'en assurer, larges d'avant en arrière, et comprimées de dehors en dedans.

$6^{\circ}$ Volume. La plupart des côles inféricures sont longues, larges et plus ou moins épaisses, principalement chez les serrasalmes, les cyprins, les labres, les sargues, les pagres, les spares: 


\section{D'ANATOITE COMPARÉE.}

les mornyres, les sciènes, les perches, les scombres, les chétodons, chez l'opah (zeus luna), les acanthures; très-courtes chez les pleuronectes, les polyptères, la plupart des silures, des anguilles, des rubans, des anarrhiques, des $c y$ cloptères.

Elles ont une longueur considérable, mais une ténuité extrème, chez les harengs; chez les balistes elles sont courtes, mais épaisses.

Elles ont une longueur et épaisseur moyennes chez les autres.

Les cótes supérieures n'ont pas toujours le mème rapport avec les inférieures. Elles sont généralement plus petites et plus minces; cependant la différence n'est pas bien considérable chez quelques-uns, particulièrement chez les harengs, les exocets, les brochets ou ésoces. les pleuronectes, les gades (surtont chez reuxci), les labres, les anarrliques, les vives, les brama raji, les scombres.

La différence est au contraire très-grande chez les spares et les genres qui en ont été séparés, chez les perches, les acanthures, les chécoclons, les coryphènes, les centronotes.

Une exception digne de remarque se rencontre che'z le trenianolus tuberculosus, où les côtes supérieures ne sont pas aussi longues, mais plus épaisses que les inférieures. Chez le polypterus niloticus, les côtes supérieures, placées en avant, sont beaucoup plus putites et plus minces 
que les inférieures; les postérieures sont plus grandes, quoique plus minces.

Elles sont ordinairement droites et dirigées en dehors, en arrière et en haut; mais chez le trenianotus tuberculosus elles sont assez fortement convexes à leur face inférieure, concaves à la supérieure. Chez plusieurs poissons, par exemple, "chez les labres, dans un certain nombre de spares, de centronotes, de scombres, de coryphènes, elles sont convexes en dehors.

Chez le polypterus bichir, les antérieures sont plus petites, droites; les postérieures convexes.

Chez plusieurs poissons, par exemple chez les balistes, le polypterus niloticus, les cyprins, les brochcts, les labres, toutes sont tout-à-fait ou presque tout-à-fait semblables.

Elles offrent, au contraire, chez le plus grand nombre de poissons des différences plus ou moins frappantes. La première ou les deux premières sont ordinairement ires-petites, ensuite elles grossissent subitement à compter de la seconde ou troisième vertèbre, et. augmentent d'abord insensiblement de volume d'avant en arrière, puis diminuent de même peu à peu, de sorte que les premières et les dernières sont les plus courtes. Les postérieures deviennent en même temps plus minces.

Chez quelques poissons, par exemple le salmo nilotic:s, le myleces rhomboidalis, les cyprins, la première des lougues còtes est de beaucoup 


\section{D'ANATOMIE COMPARÉE.}

plus large, en même temps plus droite que les autres. Chez les cyprins, la différence cst moins saillante à cause de la largeur des autres côtes.

Cela a lieu pour les deux premières côtes chez le silurus schal carasch.

Chez les gades, les quatre à cinq premières còtes sont appliquées immédiatement, les autres au moyen d'apophyses transverses, sur les parties latérales des corpss des vertèbres, et sont plus courtes, mais beaucoup plus épaisses que les postérieures. Celles-ci sont à la fois tout-à-fait ou presque tout-à-fait droites, les premières sont courbes.

La mème différence de connexion nous est présentée par d'autres poissons encore, par exemple, par les trigles et les scombres; il u'y a que cette distinction à remarquer, c'est que, chez les trigles, les premières côtes sont situées très-haut comme chez les garles, tandis que chez les scombres elles se trouvent très-rapprochées de la face inférieure du corps des vertèbres.

Les huit premières de l'opah sont beaucoup plus courtes que les moyennes.

\section{c. Sternum.}

\section{S. 94 .}

Le sternum ou les sternums, quand ils exisitent chez les vertébrés, sont généralement des 
os carrés, très-simples, le plus souvent minces, d'une forme alongée et aplatie, situés à la face abdominale du corps, à l'opposite de la colonne vertébrale à laquelle ils sont unis par les côtes. Jeur étendue varie considérablement. Ordinairement ils correspondent seulement à la cavité thoracique; mais il n'est pas bien rare de les voir descendre davantage et fermer la cavité abdominale à sa partie inférieure.

Il n'y a guère d'os sur la détermination desquels il règne autant d'opinions contradictoires que sur le sternum des poissons. On n'a gratifié de celte dénomination pas moins de quatre os différens. Duverney (I) voulait que le sternum füt représenté par de petits os, situés solus les ares des branchies, et non, comme le prélend M. Geoffroy, les arcs branchiaux eux-mêmes; le sternum fut pour Gouan (2) un os impair, situé d'avant en arrière, entre la tête et la clavicule, sur la lignne médiane; Vicq-d’Azyr (5) considéra comme tel les clavicules et les omoplates; M. Cuvier (4) une série de pièces osseuses que l'on rencontre chez quelques poissons, et qui occupent le milieu de la face inférieure de l'abdo-

(1) Mémoires sur la circulation du sang des poissons, etc. Mém. de l’Acad. des sciences, 1 or , $\mathrm{p}, 296$

(2) Hist. piscum. Argentor, , 1750, p. 64.

(3) Prenier mémoire, p. s. à l'anatomie des poissons. Dans les Mémoires présentés, etc., t. VII , I. 24 .

(4) Lecons d'anatomie comparce, vol. I, P. 21/. 
men, vir elles se succèllont dans le sens antéropostérieur. M. Geoffroy( I) voit chezles poissons cartilagineux l'analogue du sternum daus un large cartilage, placé entre les branchies; chez les poissons osseux, il signale comme tel, outre la pièce indiquée par Gouan, et qui est pour lui la portion moyenne, les deux branches latérales de l'os hyoïde, et les rayons branchiostèges situés sur ces dernières sont pour lui les côtes sternales (2).

(1) Troisième mémoire sur les poissons, où l'on traite de leur sternum, etc. Annal. du Phusceum d'hist. natur., t. X, p. 87. - Phil. anat., t. I, p. 5 g.

(2) Le sens du mot sternum a étć généralisé par M. Geoffroy-Saint-Hilaire. Cet anatomiste en a fait un nom collectif qu'il applique à un ensemble de pièces, formant la partie inférieure du thorax des ventébnés. Cet appareil sternal, qui appartient nécessairement à la poitrine, sert à en aider le mécanisme ou en protéger les viscères.

Chacune de ces pièces est déterminée sous les rapports de ses fonctions et présente un caractère propre. Elles sont jartagćes en deux ordres : dans le premier sont compris les os sternaux proprement dits; dans le second sont renfermées les cốtes sternales. Les os sternaux sont au nombre de neuf; les cótes sternales ne sont pas en nombre fixe.

Dans les animaux qui ont la poitrine alongée, les os sternaux sont disposés bout à bout; ils s'accouplent, se rangent sur un plan transversal, se doublent ou s'écartent selon les modifications imprimées à la forme de la poitrine. Un seul, qui est impair, conserve sa position centrale.

11. Ceoffroy leur a assignć des noms particuliers. 11 appelle ḱpistervaux les plus antéricurs, entosterana celui qui 


\section{De toutes ces opinions, celle de M. Cuvier} me paraît seule la véritable. Celle de M. Geoffroy

occupe Ie centre, Hyosternaux les annexes látérales et antérieures, Hyposteknatex les annexes latérales postérieures, XIPHISTEnNuUx les os postérieurs et médians; son terme de comparaison ayant été choisi dans le cas oủ la joitrine est aussi large et aussi ample que possible.

L'appareil sternal a des caractìres propres dans chaque classe. Dans les poissons osseux, il est retenu dans des limites fort resserrées; il est composé d'un ḱpISTERNä à deux têtes et puis des annexes: les HYosternaux et les HYPOSTRRNAUX; L'EnTOSTERNAL n'y domine pas. Les cinq pièces précitées n'éprouvent alors aucun obstacle à leur développement; elles croissent jusqu'à ce qu'elles s'atteignent et s'appliquentaux hyoüdes. L'HYosternat et l'ḱpIsternal sont tellement séparés qu'on les croirait étrangers l'un à l'autre. Ce sontle Hyosternadx qui supportent les côtes sternales que M. Geoftroy signale dans les rayons branchiostiges. Ainsi loin de refuser un appareil sternal aux puissons, M. Geoffroy l'analyse et le suit dans loutes ses variétés de disposition. (Voyez Philos. anat., t. I, P. 162 , et t. II, P. 200 ).

Les pièces sternales réunies aux pieces entrant dans la composition de la bouche, du larynx, etc., etc., sont les seules que M. Robineau Desvoidy admette dans les crustacés, les arachnides et les insectes. Il considère ces animaux comme vertébrés, mais avec cette différence, par rapport aux animaux supéricurs, que ceux-ci ont le chapelet des vertèbres dorsales prédominant, landis que chez les crustacés, les arachnides et les insectes il n'existe que la sćrie des pièces abdominales dont il assimile les groupes successifs aux appareils osseux qui forment le rachis des rertébrés et qu'il appelle vertèbres; après avoir étendu la définition de cette expression, comme il sera dit plus tard, il a 
est bien exposće, et a beaucoup pour elle au premier abord; mais elle est renversée par la remarque que la large pièce cartilagineuse des

constaté neuf élémens à ces vertèbres de la série inférieưre qui prédomine exclusivement chez les crustacés, les arachnides, et les insectes.

Chacun des élémens de ces appareils osseux, successifs, a reçu un nom particulier. Il appelle вAsIaL la pièce centrale de chaque vertébre. Pour se faire une idée du basiax, on peut supposer un animal dont chaque division ne serait protégée en dessous que par une seule pièce; cette pièce serait le вasıa. Il nornme costaux denx pièces qui se développent ordinairement sur le dessus et sur les côtés du corps de l'animal et naissent de la face supérieure du basial. Ces pièces représentent vraiment les côtes sternales. Ces cosTAUx peuvent se sonder par leur extrémité ou leur bord correspondant et former ainsi le tergum ou l'anneau dorsal. Leur usage est de constituer un appareil de protection aux organes digestifs et aux nerfs.

Lne autre paire se développe de la face inférieure du BasiaL: ceux-ci sont les pozergaux. Ces pièces mettent l'animal dans les rapports les plus grands et les plus variés avec le monde extérieur; elles constituent ses organes de préhension, de mastication, renferment les organes des sens, de copulation, et portent souvent ceux de respiration. Deux autres paires ont encorécété admises : les artroméraux et les arthrocéraux. Toules c'eux waissent dans la plupart des cas du rolergal de chaque cùlé. Jes arturocérauy se développent ordinairement en las; ils fournissent le plus communément les organes de la locomotion terrestre, aquatique, aérienne. Celte paire se brise en plusicurs fragmens qui forment ce qu'on appelle les élytrr's, les ailes, les nageoires, les cuisses, les jambes, les larese et eouvent l:es liver's instrumens de leurs arts. Elle peut 
poissons cartilagineux, bien qu'assimilée exactement par lui à la pièce osseuse que Gouan prenait pour le steruum, n'est pourtant pas une pièce sternale, mais correspond très-vraisemblablement à l'os hyoïde moyen, postérieur des oiseaux. quoique cet os soit beaucoup plus petit en proportion. Un examen plus approfondi qui sera fait, en traitant de l'os hyoïde et de l'organe respiratoire, démontrera que toutes les pièces osseuses, considérées par M. Geoffroy comme appartenant au sternum, ne font absolument partie que de l'os hyoïde, considérablemient développé chez les poissons. Une circonstance qui milite surtout contre l'opinion de ce dernier naturaliste, et qui peut être présentée ici par anticipation, c'est que l'os qu'il prend pourle sternum est situé, chez les raies et les squales, non au dessous et en avant du cour, mais au dessus de

être modifiće en organe de protection de l'antenne, devenir rudimentaire et disparaitre.

La seconde paire ou les ARthrocéraux, qui nait ordinairement sur le dessus des porkrgaux, consiste en un double appendice articulé qui s'étend sur le monde extérieur; elle forme les palpes, les antennes, les balanciers et souvent une partie des ailes; elle exerce le tact, l'odoration, le goùt; son développement est en rapport inverse avec celui des ARTHROméraUX. Recherches sur l'organisation vertébrale des crustacés, des arachnides et des insectes, par J.-B. Robineau Desvoidy. Un vol. Paris, 1828.

( Note des traducteurs.) 
D'ANATOMIE COMPARÉ.

ce viscère; disposition qui serait contraire à toute analogie ( 1 ).

Daprès uotre manière de voir, la plupart des poissons sont privés de sternum. Chez ceux qui en sont pourvus, il est constitué le plus

(1) Ce n'est pas une opinion qu'a émise M. Geoffroy-SaintHilaire, c'est un fait qu'il a énoncé. Une première inspection lui avait fait prendre, par une véritable inspiration irréfléchie, cette pièce carlilagineuse pour l'entosternal. Il abandonna cette idée après un examen plus attentif; mais la doctrine qu'il combat, c'est que les poissons soient sans sternum. On répugne à croire qu'il y ait défaut d'un appareil sternal parmi ces animaux; $1^{\circ}$ Quand on voit que l'appareil hyoïdien est complet; $2^{\circ}$ que les rayons bra nchiostèges restent sans analogues; $5^{\circ}$ qu'un grand mouvement a porté la poitrine vers la tête, et que dans ce refoulement en avant les pièces sternales ont dủ être écrasées, prrssées, rejetées sur les côtés; $4^{\circ}$ qu'elles ont dû suivre les branchies au service desquelles elles sont dévolues; $5^{\circ}$ que la multiplicité des pièces sternales suffit à expliquer les modifications nombreuses qu'a éprouvées cet appareil, en concevant des changemens de proportions et de rapport entre elles; $6^{\circ}$ que les connexions y sont favorables; $7^{\circ}$ qu'enfin une semblable exception au plan général qu'a suivi la nature dans la formation des vertébrés, serait un argument qui ne tendrait rien moins qu'à infirmer la loi d'unité de type reconnue par M. Meckel dans son premier volume.

Comment admeltre en effet que la nature, astreinte à une certaine règle pour dix-neuf vingtièmes de ses ouvrages, quitterait pour le dernier vingtième sa voie adoptée? Il y aurait là une contradiction unique qui s'évanouit si l'on admet les fails démontrés par M. Gcoffroy-Saint-Hilaire.

(Note des traducteurs.) 
souvent par un nombre variable d'os en forme de $\mathrm{V}$, dont le sommet est dirigé en bas, et la base en haut, qui s'imbriquent d'avant en arrière, et s'étendent, immédiatement sous la peau, depuis la nageoire anale jusqu'au point de réunion des deux os de l'épaule. Leur série n'est pas interrompue par les os pelviens, quelques-uns d'entre eux s'appliquent mème autour de ceux-ci. Le degré du développement de ces os varie; ils sont peu considérables chez le poisson Saint-Pierre; chez l'alose, au contraire, ils se terminent par une grande pointe, nonseulement en avant, mais particulièrement en haut sur les parties latérales. Chez l'anchois (chupea utherinoides), ces pièces sterinales sont en proportion encore plus considérahles que chez l'alose. Celles du milieu ont, chez un grand individu, six lignes de haut, et toutes ont une largeur de deux lignes et demie. Dans ce poisson on ne peut pas méconnaitre la nature de ces os; les apophyses supérieures et latérales de ces pièces s'étendent en effet jusquiaux côtes abdominales, et s'appliquent contre elles par une partie considérable de leur longueur : il en résulte que ces os semblent représenter le sternum et les cartilages des còtes. On ne doit pas s'étonner de ce que ces os s'étendent dans toute la longueur de l'abdomen, puisque le crucodile et plusieurs autres subriens offrent quclque chose de très analogue; on peut dire par con- 
sćquent que la partie postérieure du sternum, laquelie, chez les crocodiles, ne s'est pas développée autant que l'antérieure, a pris ici tout ou presque tout son accroissement, tandis que l'antérieure ne s'est pas formée.

Les balistes offrent une forme moins parfaite à cet égard; je crois du moins que l'on peut considérer, avec quelque raison, comme sternum abdominal, un os simple qui s'applique, par son extrémité antérieure, à la face interne des deux principaux os inférieurs des membres antérieurs, et qui, comprimé latéralement et offrant un bord supérieur convexe et un inférieur concave, s'étend en arrière à la face inférieure de l'abdomen. Chez le balistes vetula cet os est très-simple, comme il vient d'être dit; chez le b. biaculeatus il est, en proportion, beaucoup plus grand et plus large, et se termine par deux pointes supérieurement et vers sa portion moyenne, où les pointes se présentent sur son burd inférieur : il s'applique par les pointes antérieures contre les clavicules; immédiatement en arrière des postérieures, on trouve les deux longues épines que l'on peut considérer comme les rudimens des membres postérieurs.

B. OS DES MEMBRES.

La plupart des poissons possèdent les deux paires de membres des animaux vertébrés. Les 
os ou cartilages de ces partics forment plusieurs fractions qui, de la ligne médiane et de l'intérieur du corps, se dirigent vers la périphérie.

Afin de pouvoir indiquer convenablement les particularités que présentent les os des n^embres dans les poissons, nous croyons devoir, comme nous l'avons fait pour la colonne vertébrale, considérer ces parties d'abord dans les poissons cartilagineux; nous les éludierons ensuite d'une manière spéciale dans les poissons osseux. Nous séparons cette étude, parce que ces deux ordres offrent des différences Irès-considérables, que les individus de chaque groupe se rapprochent au contraire, sous ce rapport, par des ressemblances fondamentales. La mème raison nous conduit à décrire les membres antérieurs et les postérieurs isolément.

a. OS DES MEMBRES ANTÉREURS.

1. Poissons cartilagineux.

$$
\text { S. } 9^{5} \text {. }
$$

Les raies, les squales et les chimères, avec les genres qui en ont été séparés, offrent beaucoup plus de particularités et de caractères com. muns que les estrngeons. On sait que le squelette des premiers présente une disposition particuliere, taudis que les esturgeons, non-seu- 
lement forment le passage aux poissons osseux, mais sont mème, sous plusieurs rapports, organisés plutòt d'après le type de ceux-ci que d'après celui dont les cartilagineux offrent l'exemple.

Les membres antérieurs des genres précités se composent surtout de deux portions principales.

La première est formée d'os alongés et plats; elle constitue un anneau ou ceinture plus ou moins complète; ce cercle entoure constamment le corps à sa face inférieure, et quand il est parfait, il le recouvre même à sa face supérieure.

La seconde portion, articulée avec la précédente à l'endroit où elle se replie de la partie latérale en bas, consiste en deux fractions, dont la première, composée d'os larges et plats, sem. blables, mais plus ou moins triangulaires, constitue la base de la nageoire proprement dite.

La seconde fraction de la deuxième portion du membre est formée de plusieurs séries successives de cartilages alongés ou de rayons des nageoires.

Láa première portion qui occupe la région de l'épaulc consiste très-généralement en deux cartilages; l'un de ces cartilages est supérieur, étroit, communément plus petit; l'inlérieur est beaucoup plus volumineux. Le premier est droit, le second est plus ou moins recourbé. Ce der- 
nıer est formé d'une partie laiérale supérieure, et d'une partie latérale inférieure, dont la dernière se soude, dansla ligne médiane, à la partie de même nom, située au côté opposé. A l'endroit où il se recourbe, ce cartilage offre toujours sa plus grande largeur d'avant en arric̀re; la surface en est garnie de saillies convexes de différens volumes, dont la hauteur la plus considérable est dans la direction de haut en bas; avec ces saillies sarticule la portion suivante; cette articulation permet à cette portion des mouvemens libres surtout dans le sens vertical, de haut en bas.

Ces caractères généraux des os de cette portion offrent en partie, dans différens genres, et même dans diverses espèces du même genre, des variétés fort notables; ces variétés se rapportent à la connexion, au nombre, à la forme et au volume.

1. Connexion. En ce qui concerne cette condition, on oberve la différence remarquable que chez les squales; sans exception, même chez l'ange (squalus squatina), ainsi que chez les chimères et les torpilles, la pièce supérieure n'est pas unie avec le rachis ou le crâne; chez les raies, au contraire, elle est articulée solidement, par harmonie, avecl'extrémité postérieure de la première portion du rachis, formée de l'assemblage de plusieurs vcrtèbres; chez les esturgeons, elle s'applique même contre l'occipital, 
à la manière que l'on observe dans les poissons osseux.

Les différences graduelles qui s'observent sous ce rapport sont curieuses.

La connexion la plus solide se rencontre chez quelques raies, par exemple dans l'aigle de mer. Dans cette espèce la pièce supérieure est appliquée, par son bord supérieur et interne, contre la ligne des apophyses épineuses, et par son bord inférieur qui est plus étroit, mais correspondant toujours à la moitié postérieure du superieur, contre la ligne des apophyses transverses. Celte partie inférieure manque dans d'autres raies, par exemple, la rnic blanche et la raie lentillat; elle y est remplacée par un fort ligament, de sorte que le cartilage s'applique seulement contre la ligne des apophyses épineuses.

Chez les torpilles la pièce supérieure ne s'attache absolument pas à la colónne rachidienne, mais se confond dans la ligne médiane avec sa congérière du côté opposé; il en résulte un anneau étroit, tout-à-fait mobile, qui n'est nulle part en connexion avec la colonne vertébrale.

Chez les squales enfin les parties supérieures de même nom, des deux côtés, sont entièrement séparées l'une de l'autre et du rachis.

La connexion des deux pièces inférieures dans la ligne médiane n'offre pas moins de différences, quriqu'elles soient moins saillantes. 
Chez les raies, les chimères et l'ange, ces deux pièces se réunissent par une articulation immobile en un tour unique, et offrent leur plus grande épaisseur en cet endroit; chez plusieurs squales, je citerai spécialement le griset ( $s q$. griseus) et le marteau ( $s . z$ gcena), ainsi que chez les torpilles, les pièces en question sont le plus minces à l'endroit de leur réurion, et jouissent par conséquent de quelque mobilité. Chez les torpilles cette surface de contact est, conformément à la forme générale du corps, étroite d'avant en arrière, et très-large d'un còté à l'autre; la disposition contraire a lieu chez les squales.

$2^{\prime \prime}$. Nombre. Le nombre des pièces qui composent cette portion du membre antérieur n'a pas plus de fixité. Il est une loi générale à laquelle je ne connais pas d'exception, c'est qu'elles ne sont jamais moins de deux. Un examen superficiel pourrait faire croire que chez l'ange la pièce supérieure manque; mais elle existe constamment, elle n'est que très-petite et avortée. Il y en a au contraire trois dans les torpilles: $1^{\circ}$ une petitc pièce alongće, la plus interne, contiguë à celle du côté opposé : $2^{\circ}$ une autre plus large et plus de deux fois plus longue, située entre la précédente et la deuxième pièce ordinaire;

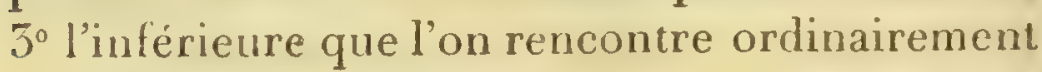
et qui est volumineuse. Cette différence est incontestablement en rapport avec l'ampliation considérable de l'arc de ces poissons. 
D'ANATOMIE COMPAREE.

$5^{\circ}$. Forme. Les deux pièces, l'inférieure surtout, varient aussi sous ce rapport.

La pièce supérieure est fort alongée, apointie en haut chez les chimires et le plus grand nombre de squales, mais principalement chez les premières; elle est au contraire carrée chez les raies, particulièrement les raies communes, tandis qu'elle est plus prolongée dans le sens de la largeur chez les torpilles.

L'intérieure, qui est la plus simple chez les squales, offre à l'endroit où elle se recourbe deux faceltes articulaires convexes; l'une de ces facettes est supérieure et antérieure, l'autre est inférieure et postérieure; toutes deux sont séparées par une ouverture assez peu considérable.

Cette ouverture manque chez les chiméres oit l'on rencontre les deux facettes articulaires; en revanche la partie inférieure de la pièce inférieure y est beaucoup plus large que la supérieure, dont elle se distingue en outre par plusieurs éminences et enfoncemens.

Chez les torpilles et les raies cette pièce s'étend surtout l'avant en arrière à l'endroit où elle se recourbe; plus loin elle se rétrécit de nouveau. L'ouverture qui existe chez les squales s'agrandit en outre considérablement et se multiplie. On trouve en effet chez les torpilles et les raies, indépendamrient de l'ouverture postérieure, plus petite; qui s'est ici considérablement étendue, une autre ouverture antérieure, plus 
grande, correspondant à la moitié antérieure de la pièce. Cette dernière ouverture est surtout grande chez les torpilles; la partie du cartilage qui est située au devant est transformée en un pont etroit. Chez la raie lentillat on trouve encore en outre, en dedans et en bas, près l'ouverlure postérieure, une seconde ouverture qui est pareillement considérable. Chez, l'aigle de mer il y a même une ouverture inférieure et antérieure à côté de la supérieure, le bord csterne qui manque chez d'autres raies se complétant dans cette espèce. On en rencontre même une cinquième qui est antérieure, supérieur: et externe; celle-ci est formée aux dépens du bord externe dont les parties supérieure et externe donnent naissance à un pout osseux particulier.

Il existe de plus, chez les raies et les torpilles, un troisième condyle, moyen, assez considérable.

Toutes ces différences sont en rapport direct avec le différent degré de développement de la nageoire.

$4^{\circ}$. Volume. C'est chez les torpilles que cette portion des membres est la plus grande; viennent ensuite les raies, puis les chimères, en dernier lieu les squales.

La seconde portion est toujours située au bord interne de la nageoire dont elle forme $l_{i 1}$ base.

Elle est toujours unie par des faceltes articu- 
linires, concaves, avec la partie articulaire de la première portion; les différences que présentent ces faceltes correspondent exactement à celles de celte portion qui ont été décrites.

Cette surface est toujours la plus étroite de la seconde portion, qui, à partir de ce point, s'élargit tonjours en arrière, et plus ou moins aussi en devant chez l'ange, les torpilles et les raies.

Elle est la plus petite chez les chimères, et se compose de trois pièces, parmi lesquelles celle du milieu est de beaucoup la plus grande et la plus alongée. La postérieure de ces pièces, qui est la plus petite, est située sur les premières, la premiere et la seconde sont au contraire en rapjiort de contiguité avec la partie articulaire de la première portion du membre.

Chez les squales la pièce antérieure se divise en deux autres plus petites, ayant à peu près le même volume. La seconde de celles-ci se grossit considérablement chez l'arge, et augmente surtout en largeur. La troisième petite pièce, postérieure, que nous avons mentionnée, manque ici.

Chez les raies et les torpilles, le nombre de ces pièces augmente encore plus considérablement; elles deviennent en outre plus longues, moins plates, et se renflent surtout à lear bord interne, en haut et en bas, de manière à former "ne crête qui agrandit beaucoup la surface d'aitache pour les muscles. Taudis que dans les précédentes espèces elles ne s'étendent quien 
arriere, elles se dirigent ici en avant, où elles sont toujours plus nombreuses et plus volumineuses qu'en arrière. Cela est surtout très-frappant chez les torpilles, chez lesquelles on trouve, en avant, environ dix de ces pièces très-considérables; il y en a à peine cinq en arrière et un beaucoup plus grand nombre de petites. Ordinairement il y a quelques petites pièces moyennes qui passent immédiatement en dehors dans des rayons de nageoires, tandis que la plupart de ceux-ci sont simplement appliqués sur le bord externe de ces bases cartilagineuses. Cette disposition est surtout distincte chez la raie lentillat, où il existe un grand cartilage de cette espèce, ayant la forme d'un carré long, qui se contimue avec huit rayons de nageoires et qui est suivi de plusieurs autres plus petits correspoudans it un ou deux rayons de nageoires. Chez l'aigle de me" il existe, entre le premier et le dernier cartilnge, une série d'autres carlilages plus petits, mais qui ne se continuent pàs immédiatement avec les rayons. Le nombre de ces carlilages basiques est très-variable et ne dépend nullement de l'étendue de la nageoire. Chez l'aigle de mo', il n'y a le plus souvent qu'un cartilage très-volumineux qui correspond à la moitié antérieure et postérieure de la nageoire; chez la raie blanche, la raic lentillat et les corpilles, il y en a plusieurs qui diminuent considérablement de volume de la base au sommet. 
D'ANATOMLE COMPAREE.

Chez les iorpilles, la pointe antérieure de celte série de cartilages serencontre presque avec une stric cartilagineuse, alongée, tournée en dehors, qui vient de l'extrémité antérieure de la tète.

Chez l'aigle de mer également, il y a un cartilage carré, large et fort, qui, venant de l'extrémité antérieure de la tête, va au devant de r'ette série de cartilages et s'applique à sa face interne, en s'unissant avec elle d'une manière intime. Mais la série de cartilages et toute la nageoire se portent encore plus en avant et en dedans, de manière à former la partie la plus antérieure de tout le corps, et à s'atteindre presque dans la ligne médiane.

La seconde fraction de cette portion de l'ap-pendice locomoteur, vers laquelle la fraction qui vient d'ètre considérée forme en partie, du moins par ses derniers articles, une transition insensible, est composée d'un nombre variable de rayons fort alongés, un peu renflés aux deux extrémités, unis entre eux et avec les bords latéraux des pièces de la première fraction, d'une manière peu mobile et par amphiarthrose.

Ils sont généralement d'autaut plus multipliés qu'ils sont moins volumineux, et que la nageoire est. plus large et plus fractionnée; doù il suit riécessairement que les plus grandes nageoires sont anssi les plus mobiles, à cause de l'augincutation du nombre des pièces articulées. C'est 
à la partie moyenne, qui est à la fois la plus large de la nageoire qu'on en compte davantage; ils diminuent de nombre, mais moins de volume, vers l'extrémité. Les raies offrent les plus grands développemens. J'en trouve plus de vingt rangées daus la région moyenue de la nageoire de la raie Llunche, même trente et quelques chez l'aigl' de mer' et la raic lentillat, chez les torpilles seulement dix à douze, chez le griset ( squalus griseus), l'aiguillat (acanthias), la petite roussette (catulus), le marteau (zygana), trois à quatre, et seulement trois chez l'ange ( $s q$. squatina.)

Le nombre des rangées que forment les rayons varie de l'extrémité antérieure des nageoires à leur extrémité postérieure. Il y en a communément de quatre-vingts à cent chez les raies, de cinquante à soixante chez les torpilles, vingt et quelques chez les squales et les chimères.

Quand on les considère d'avant en arrière, on trouve ordinairement que ceux du milieu sont les plus longs.

Ceux de la partie externe sont ordinairement aussi plus petits que les internes. Cette circonstance a lieu surtout pour les postérieurs, que l'on rencontre quelquefois, comme chez les chimères et la petite roussette (squalus calulus), subitement beaucoup plus petits et à la fois larges et discoïdes.

Chez les raies, les dẹniers articles diminuent 
V'a Natome COMPané.

aussi peu à peu de la moitié; mais cela n'est pas général parmi elles. Ainsi, par exemple, chez le griset ( $s q$. griseus), l'article moyen est beaucoup plus court que l'externe et l'interne, quiont tous deux à peu près le même volume.

Le marteau ( $s q . z y$ grana) présente une organisation encore plus digne de remarque. Il y a trois articles, dont le plus externe, par ses rayons antérieurs, est huit ou dix fois plus grand que le second, qui est lui-même trois fois plus petit que le premier. Cutte troisième série se termine par une pointe mince, et diminue brusquement de volume, à compter du milieu de la nageoire.

D'après mes recherches, les rayons des nageoires ne forment généralement qu'une couche simple, située entre les muscles supérieurs et inférieurs; le nombre des séries qui se succèdent d'avant en arrière est, au contraire, très-généraiement doublé chez les raies et les corpilles, par la bifurcation de la plupart des rayons externes. Ordinairement la bifurcation commence seulement au milieu d'un rayon, et à chacune de ses moitiés correspond une paire d'autres rayons. (?) uelquefois cela a lieu sans bifurcatiou préalable d'un rayon simple. Chez les corpilles et la raic lentillat, cette organisation commence au tiers exierne de la nageoire; chez l'aigle de mer, à peine au dernier dixième.

Le's syuales offrent quelques indices de cette 
disposition dans la bifurcation de l'extrémité libre des rayons externes.

Quelquefois, par exemple chez les chimères, la série miyenne est considérablement plus longue que les autres.

On voit par conséquent par là qu'il y a une différence très-considérable dans le rapport de chaque article; ce fait est curieux, soit par luimême, soit à cause des différences analogues qui existent entre les parties correspondantes des membres des autres classes d'animaux dans leurs divers ordres.

A compter du troisième article, la nageoire est ordinairement appuyée et consolidée par un très-grand nombre de stries cornées, fines, pointues, que l'on peut comparer évidemment avec les ongles. Elles sont surtout développées cher les chimères et les squales, beaucoup moins chez les raies; celte inégalité de développement dépend de ce que chez celles-ci les rayons des nageoires s'étendent dans toute la largeur des nageoires, tandis que chezles squales il y a toujours une portion considérable de la partie externe des nageoires qui n'est pas occupée par les rayons.

La forme et le volume des nageoires sont du ressort de la zoologie; cependant nous devons en indiquer ici la disposition chez l'aigle de mer'; cette disposition ne pouvant pas ètre reconnue sans en examiner la structure intérieure. 
La nageoire est ordinairement le plus large dans sa partie moyenne; elle diminue plus ou moins à partir de ce point, mais uniformément et sans interruption vers les deux extrémités. L'aigle de mer fait exception à cette règle d'une manière digne de remarque. Les deux tiers postérieurs de la nageoire ont la structure ordinaire, seulement elle est plus courte d'avant en arrière qu'on ne la rencontre communément. Mais un peu en avant du premier tiers, les rayons disparaissent tout ̀̀ coup et presque entièrement; au lieu d'être transverses comme jusqu'à ce point, ils prennent une direction exacte d'arrière en avant; vers l'extrémité antérieure, ils se dirigent même peu à peu de dehors en dedans et d'arrière en avant : il en résulte que les extrémités antérieures convergent fortement et parviennent presque à se toucher. En arrière, il n'y a qu'une série simple qui devient insensiblement quadruple antérieurement.

Chez le céphaloptère (raja cephaloptera), il $y$ a à peu près la même disposition, à cela près que les nageoires thoraciques se portent plus en avant et ne convergent pas, mais s'écartent au contraire fortement l'une de l'autre. 


\section{S. 96 .}

L'esturgeon fait, comme nous l'avons déjà dit(1), le passage des poissons cartilagineux aux osseux.

La portion supérieure ou antérieure des membres forme à peu près un demi-cercle, ayant sa convexité tournée en arrière et sa concavité en avant. Elle s'articule d'une manière peu mobile par son extrémité supérieure, qui se termine en pointe mousse, de côté et d'eı haut, à la partie postérieure de la tête, et se compose de trois pièces qui se succèdent de haut en bas.

La plus supérieure est très-simple; c'est une plaque alongée, arrondie, cachée dans son tiers supérieur sous la partie céphalique avec laquelle. elle s'articule; elle est libre et tont-i-fait osseuse et rugueuse dans ses deux tiers inférieurs.

La seconde, qui est moyenne, est beaucoup plus composée et la plus grande de toutes.

Elle forme une pyramide creuse, dont la base est en bas, et le sommet en haut.Les parois inférieure et externe sont beaucoup plus larges que l'interne. Elles sont en outre osseuses; la derniere est cartilagineuse. La paroi interne s'articule avec l'exicrne et l'inférieure par deux

(1) Pag. 252 de ce volume. 
D'ANATOMLE COMPARÉE.

saillies cartilagineuses parfaitement séparées l'une de l'autre. Cies saillies parlagent l'espace, qui existe entre les trois parois, en trois conduits ou lacunes qui se dirigent d'avant en arrière; lintervalle moyen est bien plus grand que les autres, l'interne ou inférieur est le plus petit.

Le sommet de la pyramide, dans la moitié supérieure de sa hauteur, glisse au dessous de la moitié interne de la première pièce.

La troisième pièce, placée au devant de la seconde, est un peu plus petite, et consiste en deux lames, dont l'une est horizontale et inférieure, et l'autre supérieure et externe, plus grande; toutes deux sont unies entre elles sous un angle aigu, et articulées arec les parois inférieure et externe de la seconde pièce par ine suture presque immobile; leurs extrémités antér.eures, attachées l'une à l'autre aumoyeu de da peau, se meuvent librement. Il existe en outre en cet endroit, sur la partie inierne de l'extrémité antérieure de celle troisième pièce, une lame osseuse, carrée, très-petite.

La seconde portion, la nageoire proprement dite, est alongce, triangulaire, et se compose de leux parties, l'une antérieure, plus pelite, et l'autre posterieure, bien plus considérable.

Iíd partie antérieure a la forme d'un carré ir régulier; sa plus grande largeur est d'un côté à l'autre; en arriere elle est limitée par un bord 
convexe, en avant par un bord concave. Elle est appliquée par ce dernier bord, qui est le plus épais, à sa partie externe, sur la région moyenue de la pyramide formée par la seconde pièce de la portion précédente; il en résulte que la lacune moyenne reste libre.

Elle est formée de plusieures pièces cartilagineuses, ou rayons très-alongés. La première série en comprend six. Ie plus externe est court, épais, triangulaire; les internes sont plus longs et plus grêles. En avant des cinquième et sixième rayons, qui sont les plus internes, il en existe un autre, arrondi, très-petit.

La seconde série est formée de pièces cartilagineuses plus petites et plus arrondies; elle est unique dans sa moitié externe, double et même triple dans sa moilié interne.

La partie antérieure de cette série, qui l'emporte de beaucoup en développement, est revêtue d'une membrane molle.

La partie postérieure se compose d'environ quarante rayons plus durs, cornés, très-serrés les uns contre les autres, mais unis entre eux par une membrane interunédiaire plus molle. Le premier ou le plus externe de ces rayons, qui correspond seul à la première pièce cartilagineuse de la partie antérieure, a beaucoup plus d'épaisseur que les autres; il présente la forme d'un carré alongé. Ceux du milieu sont les plus longs; les internes sunt les plus courts 
et les plus fins. 'Tous, à l'exception du plus externe, sont en apparence divisés par des articles nombreux, alongés, qui ne sont indistincts qu’à la base, et qui sont environ au nombre de vingt sur les rayons les plus longs. Le premier excepté, tous se divisent en une couche supérieure et une inférieure, unies étroitement entre elles par un tissu cellulaire dense.

\section{Poissons ossenx.}

$$
\text { S. } 97 \text {. }
$$

Chez les poissons osseux le squelette des membres antérieurs est en général très-composé. Ces membres présentent toutefois des degrés très-différens de développement. I.e degré le plus inférieur, qui se rattache à l'entière absence de ces parties chez les cyclostômes, parmi les poissons cartilagineux, est offert par les murènes proprement dites, les unibranchapertures, en partie aussi par les anguilles. Quant au perfectionnement du squelette chez les poissons osseux, il est digne de remarque qu'ils ont toujours des membres antérieurs, quoique l'on trouve des exemples du défaut de ces appendices chez les poissons cartilagineux dont it vient d'être question.

Chez les murénophis on voit surtont distinctement que les membres ne sont essentiel- 
lement que des développemens ultérieurs des côtes. Ces poissons offrent, de chaque côté du corps, derrière les branchies et la place occupée ordinairement par les membres, plus développés chez les autres poissons, un seul os mince, concave en avant, convexe en arrière, entouré partout seulement de peau et de muscles, sans connexion aucune avec les autres os du corps, pas même avec l'os congénère du côté opposé, quoique lé' extrémités de ces deux os soient très-près l'une de l'autre. La disposition de cet os est exactement la même que celle des arêtes qui sont plongées uniquement dans les parties charnues, et qui y forment une ou plusieurs séries des deux côtés de la colonne vertébrale, chez la plupart des poissons, et particulièrement dans la murène commune (murcenophis helena).

Chez les unibranchapertures il n'y a de mème qu'un seul os, disposé de la mème manière, mais beaucoup plus gros que chez la murène commune.

A celte organisation se rattache celle du serpent de mer (murcena serpens). On y trouve aussi ce petit os, unique, convexe; mais il y est tellement rudimentaire, que sur un squelette long de huit pieds, il m'a présenté à peine une demi-ligne d'épaisseur. Mais ce n'est pas le seul, il y a encore d'autres os à la périphérie qui sont beaucoup plus développés. 


$$
\text { D'ANATOMIE COMHARÉ. }
$$

Ce poisson forme par ces derniers os la transition à la disposition ordinaire..

$$
\text { S. } 9^{8}
$$

La disposition commune des membres chez les poissons osseux est, dans les points essentiels, la mème que chez les cartilagineux.

Les membres antérieurs sont également formés ici de trois portions; l'une est supérieure ou antérieure, la seconde moyenne, et la troisième est inférieure ou postérieure.

La portion moyenne est communément beaucoup plus petite que les deux autres, disposition qui est sans doute la plus générale.

La troisième est ordinairement la plus grande.

La portion moyenne est très-généralement articulée d'une manière mobile, par l'intermédiaire de la première, avec la région supérieure, postérieure et latérale du crâne; sa disposition se distingue par là de celle que nous avons vue dans la plupart des poissons cartilagineux, les esturgeons exceptés.

La premiere portion forme, comme chez les cartilagineux, un demi-cercle composé de plusieurs pièces osseuses qui se succèdent de haut en bas et qui sont unies entre elles d'une manièré peu mobile, mais toujours plus mobile que chez les premiers. A l'extrémité inférieure de ce 
demi-cercle, les os les plus longs de la première portion se réunissent des deux côtés, dans la ligne médiane, par une articulation ordinairement mobile; ce qui constitue une seconde différence entre la plupart des poissons osseux et des poissons cartilagineux.

La troisieme portion, ou la nageoire propre-ment dite, est en outre moins horizontale, mais plus verticale.

\section{\$. 99.}

La portion antérieure peut être partagée en deux fractions : une supérieure et une inférieure.

La fraction supérieure, que l'on peut nommer l'épaule, est ordinairement plus petite que l'inférieure, mais se compose très-généralement de plusieurs pièces osseuses. La plus constante de ces pièce:; est alongée, aplatie, et recouvre, par une partie plus ou moins considérable de sa moitié inférieure, la face externe de l'os de la seconde portion. Cet os est très-ordinairement suivi, en haut et en avant, d'un ou de plusieurs autres qui l'unissentau crâne. Ils sont toujours articulés avec cet os et avec le crâne d'une manière variablement mobile ; circonstance qui me fait croire qu'ils ne font pas partie des os du crâne, mais de ceux des membres.

Les principales conditions et modifications 
D'A NATOMIE COMPARÉE.

particulières que présente l'épaule sont les suivantes.

Sous le rapport de la connexion de cette partie avec les autres os, les murénes se distinguent des aulres poissons; chez elles, l'épaule nes'unit ni au crâne, ni à la colonne vertébrale; mais on la rencontre, à quelque distance du crâne, plongée librement dans les chairs.

Lorsque cette connexion a lieu, il existe trèsgéuéralement, mais non nécessairement et toujours, un second os supérieur, et les différences graduelles que l'on observe à cet égard sont fort dignes de remarque.

Chez les murènes, oú il n'existe point de connexion, cet os se termine, supérieurement et inférieurement, par une pointe mousse et simple.

Chez les baudroies, du moins la baudroie. commune, ainsi que les chironectes, il s'articule avec le crânc, mais se prolonge en haut également et se termine par une pointe monsse et unique.

L'organisation est plus composée dans les silures. Il n'y existe également qu'un os, du moins chez le saluth (silurus glanis); mais il est plus lürge, et se compose le quatre branches: deux de ces dents sont supérieures et deux sont inférieu. res; elles se rencoutrent dans une petite partie moyenne et Iransverse. De ces branches, la supérieure, interne el transversale, est la jlus grande; l'inféricure, qui est extcrne et verticale, 
est la plus petite. Par les branches supérieures qui forment ensemble un arc considérable, l'os s'applique inférieurement contre le corps de l'occipital, supérieurement contre le temporal et au dessus de cet os; par les deux branches inférieures il embrasse, de dehors et de dedans. l'extrémité supérieure de l'os de la seconde portion.

II représente distinctement, dans sa partie supérieure, l’os le plus supérieur de l'épaule, lequel existe communément; sa partie inférieure correspond à l'os inférieur de l'épaule; disposition qui indique par conséquent déjà la séparation de cette partie en ses deux os ordinaires.

L'os le plus supérieur, se manifestant après l'inférieur, offre toujours une structure un peu plus compliquée. Il est recourbé et se bifurque plus ou moins baut en deux prolongemens; l'un esi supérieur et se continue dans la direction de la pièce du bas, l'autre est inférieur, plus court, très-souvent faiblement développé, saillant en dedans plus ou moins transversalement. Le prolongement supérieur glisse d'arrière en avant et de dehors en dedans, par dessus la face supérieure du crâne; l'inférieur s'applique contre la face latérale du corps de l'occipital, ou du moins se rapproche de cet os.

Il existe, en outre, en avant et au dessus de cet os, d'un à trois autres os; il en résulte que, 
D'ANATOMIE COMPARÉE.

dans le dernier cas, les os de cette région s'élèvent au nombre de cinq, nombre qu'ils ne dépassent jamais autant que je sache.

$$
\text { S. } 100 .
$$

Tous ces os présentent encore d'autres différences. Celles qui sont relatives au volume seront étudiées plus loin; ici nous ne parlerons que de la forme.

L'os inférieur a très-généralement la forme simple décrite plus haut. On le trouve dans les genres carpe, brochet, exocet, gade, trigle, baudroye, chironecte, pleuronecte, saumon, tétrodon, brame, spare, perche, sciène, gobie, mulle, scombre, muge, ophidie, blennie, vive, cotte, labre, sphyrène; il ne varie que dans le rapport qui existe entre sa longueur et sa largeur.

Quelquefois cette disposition est plus compliquée; ainsi, chez l'uranoscopus scaber, où cet os est également très-large et peu haut, cette complication a lieu de la manière suivante : $1^{\circ}$ son bord postérieur se prolonge en une pointe saillante, aiguë; $2^{\circ}$ sa face externe est partagée par une forte saillie, longitudinale, en une moitié postérieure, plus grande, et en une antérieure, heaucoup plus petite.

L'os le plus inférieur, lorsqu'il s'unit à un os 25. 
supérieur, est terminé, chez plusieurs poissons, par une pointe simplequi s'unit avec ce dernier par de la substance fibreuse; il est seulement un peu plus mince dans une étendue variable, con. cave et rugueux à l'extérieur. Cela s'observe, par exemple, dans les genres carpe, brochel, hareng, gade.

Il y a plus de complication dans d'autres cas, par exemple chez les trigles et les labres. Lios se termine par deux pointes, dont l'une externe et l'autre interne; l'extrémité inférieure de l'os supérieur s'applique contre la dernière. Cette disposition offre aussi des variétés. La condition que l'on observe chez les uranoscopes se rattiache à la disposition la plus simple. La région interne de cet os se renfle chez ces arimaux et constitue une facette articulaire, considérable, transverse, descendant obliquement; la région externe est au contraire plus déprimée; elle est dirigée aussi obliquement en bas, et forme un enfoncement inégal, au devant duquel existe une éminence transverse, considérable. Toute cette surface est fort large dans le sens transversal.

Ia région interne s'élève un peu plus quњ l'externe et forme un condyle encroûté de car.. tilage; l'cxterne présente une cavité articulairc, égaltement encroîtée de cartilage; des éminences et des entoncemens sur l'os supéricir correspondent à ces saillies et à ces cavités. 
Les muuvemens sont par conséquent ici plus libres.

Chez quelques poissons l'ns supérieur est plus simple quien général, par l'absence du prolongement inférieur et interne Cela se voit, par. exemple, ch'z les çprins. L'os, très-petit, forme ici seulement un triangle alongé, légèrement recourbé, qui s'apointit considérablement de bas en haut. Il a la même disposition chez les ¿xocets, à cela près qu'il est aplati supérieurement au lieu d'ètre apointi.

D'autres, par exemple, les ésoces, les scorpènes, les trigles, funt le passage à l'organisatiou commune. L'os dans sa moitié postérieure et inférieure s'élargit insensiblement en devant et se prolonge en une seconde apophyse descendante, légèrement recourbée, qui est large et haute chez les esoces, épaisse et dépriméc chez les scorpènes. Dans les trigles, tout l'os est triangulaire, convexe en dehors, concave en dedans, en devant beaucoup plus large, postérieurement prolongé en une pointe aiguë.

Chez le saumon il s'en détache inférieurement une petite saillie distincte.

Chez d'autres poissons, particulièrement les mugres, les brames, les spares, les sciènes, les labres, les harengs, les gobies, le côté inférieur du triangle est très-long, mais il est toujours plus court que le supérieur.

Dans les uranoscopes, ou tout l'os est fort 
large et court, on trouve la branche inférieure aussi grande et beaucoup plus épaisse que la supérieure.

Ordinairement l'os est entièrement caché sous la peau; chez les trigles son extrémité postéricure forme une saillie fort pointue, elle constitue la plus supérieure des épines qui existent aux membres antérieurs.

\section{S. 101 .}

Les parties osseuses qui font suite à l'os dont il vient d'être question, sont toujours situées un peu en dehors et en haut.

Chez les scorpènes, les uranoscopes, le mullus barbatus, les labres, les pleuronectes, il n'existe qu'un seul os; quelques spares, par exemple le $s p$. aurata, en ont deux; d'autres en possèdent trois, comme le sparus macrophthalmus, le muge céphale.

Leur disposition varie.

Chez les uranoscopes, les scorpènes, les mulles, les labres, l'os unique est intercalé étroitement entre les os supérieurs ordinaires et les os correspondans du crâne; il est peu mobile.

Dans les deux premiers genres, il est quadrilatère, aplati; dans les deux autres, il se montre alongé, un peu recourbé.

La disposition n'est pas la même dans tous 
1) ANATOME COMPARÉ

les pleuronectes. Ainsi, chez le pleuronecle plalessı, par exemple, il est situé comme chez los poissons précédens, il est simple et a la forme d'un carré long; chez la sole, au contraire, il offre une disposition très-particulière. Il est, comme de coutume, placé au devant de l'os supérieur généralement existant; mais il sort de la rangée des autrés os, de manière à ce qu'il est situé très-superficiellement, et peut, par conséquent, échapper très-facilement à l'attention de l'observateur. Il a la forme d'un Y et s'unit làchement par sa base avec les muscles sousjacens, ses deux branches étant fixées intimement à la peau de cette région.

Chez les spares les deux ou trois os les plus supérieurs forment un pont étroit quise porte de bas en haut et d'arrière en avant. Chez le sparus aurala ce pont est formé d'une pièce inférieure, large, et d'une supérieure, étroite. Chez le sp. macrophthalmus la pièce supérieure se divise en trois, qui diminuent de volume de bas en haut. Il existe toujours un espace triangulaire, considérable, sntre cette série d'os et la face supérieure ordinaire du crâne.

Ces pièces supérieures ne me paraissent êłre que le prolongement supérieur, alongé et articulé, de l'os supérieur de l'épaule qui existe ordinairement. Elles ont en effet absolument la mème direction, et chez plusieurs poissons, par exemple les grobir's et les clupées, le prolon- 
gement supérieur, simple, de cel os est aussi d'une longrueur considérable.

Chez le muge céphale les trois os les plus supérieurs, comme l'os simple qui leur correspond chez la sole, représentent un Y. Le prolongement postérienr, os simple et-alongé, rencontre l'os supérieur, qui existe ordinairement, vers l'endroit où celui.cise bifurque. Le prolongement antérieur qui suit ce dernier se partage aussi en deux branches: l'une inférieure, horizontale, et l'autre supéricure, verticale, plus petite. La première est contiguë, au dessus de l'opercule, à la paroi supérieure du crâue, ou elle se continue avec la paroi latérale; la seconde est contiguẻ au troisième os, qai est le plus petil, qui affecte la même direction, et s'applique par l'extrémité qui le termine supérieurement, immédiatement en avant du prolongement d'eo haut de l'os supérieur ordinaire, au hord postérieur de la face supérieure du crâne.

Il reste également une lacune considérable entre ces os et la face supérieure du crâne qui est située au dessous. Ces os sont, surtout dans le genre muge, une imilation et un perfec* tionnement ultérieur de l'os le plus supérieur qu'il est ordinaire de rencuntrer dans cette région.

Dans les harengs on remarque une organisation intermédiaire aux deux extrêmes de développement de cet os. Il existe en effet daus ces 
D'ANATOML COMPARÉE.

animaux deux os à l'endroit où existent les trois os des muges; le premier est formé des deux os infirieurs des muges, le supérieur reste isole.

\section{\$. 102 .}

I a fraction inférieure de la porlion antérieure de ce membre, est, sous le rapport du volume des pièces qui le composent, de tous les os des membres antérieurs le plus considérable; il surpasse toujours en grandeur la portion supérieure de la portion antérieure, et peut être nommé la clavicule. Elle est très-généralement composée de deux os, l'un antérieur et externe, plus volumineux; l'autre postérieur et interne, plus petit.

Le premier forme un aro dont la convexité est dirigée en arrière; il est aplati de dehors en dedans, ordinairement plus ou moins convexe en avant et en dehors, et variablement concave en arrière, de sorte que cette surface qui est étroite forme une gouttière longitudinale; en haut il est cuntigu à l'omoplate, dont il est recouvert lans une partie plus ou moins grande de sa moilié supérieure; en bas il se rencontre en avant, dans la ligne, médiane avec son congénère, en arrière et en dedans, avec des os de la portion suivante. Cette pièce peut être désignée sous le nom de clnvicule antérieure ou exlerne.

Le serond os est alongé, presque droit, com. 
munément apointi à sa partie inférieure, et des. cend d'un point plus ou moins élevé de la face interne du premier auquel il est uni lâchement. On peut lui donner le nom de clavicule postérieure ou interne.

Ces deux os offrent plusieurs variétés, dont quelques-unes sont assez considérables.

\section{S. 103.}

C'est chez les murènes que la clavicule antérieure offre la conformation la plus simple.

Elle y est très - alongée et étroite, aplatie de dehors en dedans dans sa moitié supérieure, prismatique dans l'inférieure, de façon que la face externe et inférieure est plane, tandis que l'interne est légèrement concave.

On observe la même disposition chez les silures; avec cette différence qu'au lieu de se terminer supérieurement par une pointe, comme chez les anguilles, elle se bifurque à son extrémité en deux pointes, dout l'antérieure est plus longue et plus mince que la postérieure.

Cet os n'est aussi simple que dans son quart postérieur qui est le plus élevé; dans le reste de son étendue, qui est plus horizontale, il est assez concave à sa face interne; cette concavité provient de ce que le bord supérieur forme une saillie assez considérable en dedans. Il présente en outre, à sa face interne, à l'endroit de jonction de son quart postérieur avec le reste, 
une cavité articulaire de forme auriculaire consilérable: il offre aussi des inégalités à cette mime face interne, qui résultent de trois facettes lamineuses, inégales, par lesquelles il s'articule avec l'os de la portion suivante. La première de ces facettes est longue, occupe la moité interne, la plus grande, de la saillie dont j'ai parlé ; la seconde est beaucoup plus petile, également située an bord supérieur au point de jonction du quart supérieur de l'os avec sa partie antérieure; la troisième ressemble à la second̉e elle se trouve vis-à-vis d'elle sur le bord inférieur.

Chez la plupart des autres poissons, l'os en question est encore plus ou moins creusé en gouttière; son bord supérieur se recourbe plus fortement en dedans et en bas. Cette courbure est très-prononcée surtout chez le muge céphale, l'exoccetus exsiliens.

Il se développe en mème temps sur différentes parties de son étendue, des crêtes longitudinales plus ou moins saillantes.

La première de ces lignes est externe. La paroi externe, précédemment simple, se transforme en une face supérieure et externe; toutes deux se réunissent sous un angle obtus on droit. Chez les labres, cette saillie est trèsfaible; elle est plus marquée chez les muges, les spares, mais bien plus forte chez la carpe.

Une seconde crête, qui est interne, se voit 
sur les limites entre les faces externe et interne. Elle n'est que peu développée dans les carpes; elle l'est beaucoup plus chez les muges, où elle commence par un crochet très-saillant; ensuite elle disparaît presque entièrement pour reparaître plus prononcée vers l'extrémité inférieure de l'os.

C'est surtout chez le poisson lune que cette crête interne est prononcée; elle s'étend dans toute la longueur de l'os, et se replie en dedans, à son origine, sous la forme d'une apophyse large.

Cette organisation offre chez beaucoup de poissons un autre mode de complication; il y a des dents d'une longueur variable, souvent trèsaiguës, qui partent du bord postérieur. Cela se voit, par exemple, dans les trigles, les zées, les scorpènes, les uranoscopes.

Plusieurs silures et genres voisins offrent, à cet égard, une organisation surtout digne de remarque.

$1^{\circ}$ La clavicule est très-large; $2^{\circ}$ elle s'articule avec celle du côté opposé, dans toute la largeur du bord interne; $3^{\circ}$ elle délache de son bord postérieur une apophyse transverse, considérable, qui se porte en ligne directe et verticale à la rencontre de l'autre, avec laquelle elle s'articule, par son extrémité inférieure, au moyen d'une suture dentelée, mais ne l'atteint pas supérieurement. De là résulte, entre son 
bord concave et le commencement de la colonne vertébrale, uné ouverture étroite par laquelle passe l'osophage. Par suite de cette disposition, les cavilés thoracique et abdominale sont séparées l'une de l'autre par une véritable cloison osseuse.

Le silurus senen et le silurus clarias sont les espèces qui m’ont le mieux présenté cette disposilion.

La clavicule du loricaria cataphrıcta est également munie d'une apophyse verticale aussi forte; mais elle n'atteint pas tout-à-fait celle du côté opposé.

$$
\text { S. } 104 .
$$

Le second os de cette portion du membre, la clavicule postérieure ou interne, existe un peu moins généralement que le précédent.

D'après M. Geoffroy-Saint-Hilaire, il manque dans les blennies, l'uranoscopus scaber, le ruban, l'echeneis remora, les gasierostées, le gobius niger, le mullus barbatus, l'anarrhichas lupus et les murènes (1).

Cela est vrai pour plusieurs d'entre ces poissons, notamment pour les genres uranoscope, gobie, échénéide, murène, anarrhique. Mais il existe chez le surmullet (mullus), le blennius

(1) Second mémoiresur les poissons. Annales du Muséum, t. IX, p. 4 is. 
pholis et le ruban. Je l'ai même trouvé fort considérable dans ce dernier.

D'un autre côté, il manque réellement à plu. sieurs poissons auxquels il est attribué par M. Geoffroy, ou du moins auxquels ce naturàliste ne le refuse pas (1).

La première assertion s'applique aux silures,

(1) C'est à M. Geoffroy-Saint-Hilaire qu'ont été dues les premières études analogiques faites sur l'os que M. Meckel désigne ici sous le nom de clavicule postérieure, et que l'anatomiste français avait appelé clavicule coracoüde. Ce furent même là les premiers pas de M. Geoffroy dans la carrière qu'il a depuis si agrandie. Les principes qui l'ont conduit à ses découvertes ultérieures n'étaient encore que soupçonnées. Imbu, comme tous les anatomistes de cette époque, de cette idée préconçue, liéritée d'Aristote et mal définie, dont il restait à prouver la vérité, que less animaux appartenaient à un même plan général, comme eux il n'avait encore chcrché que des différences, et il n'osait que soulever encore, sans guide et sans règle, le voile qui couvraịt les ressemblances dont les rapports n'étaient pas une évidence tout-à-fait visuelle. Il a donc pu arriver que M. Geoffroy ait alors annoncé quelques faits négatifs, quelques nonexistences, que des recherches faites après aient découverts; mais il n'est aucun fait positif sur lequel on puisse prouver une erreur. Jusqu'à plus ample information, force était à M. Geoffroy de ne pas admettre ce qui n'était pas trouvé, quoiqu'il y eut li un fait de moins en faveur des analogies dont M. Geoffroy et l'école allemande ont défini depuis les lois; nouvelle direction donnée aux esprits qui ne pouvait être que le fruit d'une longue accumulation de travaux, mais qui doit élever l'anatomie comparée au rang des sciences parfaites.

(Note des traducteurs.) 
D'ANATOMIE COMPARÉE.

du moins au silurus glanis; il est même vraisemblable qu'il manque aussi aux autres, attendu que, comme on verra plus loin, l'os, que M. Geoffroy considère comme la clavicule postérieure, correspond fort vraisemblablement à un autre os de la portion suivante du membre.

Un exemple à l'appui de ma seconde assertion, in est fourni par l'exocretus exsiliens, chez lequel je n'ai pas pu trouver de trace de cet os, malgré les recherches les plus exactes. Il est possible qu'ici l'os ne se soit pas formé à cause du développement fort considérable de toutes les autres parties du membre antérieur.

Les autres variétés que présente cet os sont relatives au nombre, au volume, à la forme, à la direction et à la connexion.

Ordinairement il est simple, parfois double. Cette dernière disposition est offerte, par exemple, par le muge cephale, les labres, les scorpènes, les spares. L’os supérieur est alors communément plus large que l'inférieur. Ils descendent ordinairement tous deux en affectant la mème direction; mais chez le tétrodon tesiudinarius, le supérieur se porte en dehors; l'inférieur, qui est ici, contrairement à la règle, plus fort, se dirige en arrière.

L’inférieur repose ordinairement sur l'extrémité inférieure de l'os supérieur; chez le tétrodon cela n'a pas licu, il est plus rapproché de son extrémilé supérieure. 
Une question s'élève ici : l'os supérieur est-il réellement une partie de la clavicule interne? n'est-il pas une partie appartenante à la portion suivante du membre? Cette dernière opinion est d'autant plus fondée que la première pièce de la portion suivante manque dans les cas où l'os inférieur de la clavicule interne semble se parlager en deux.

Dans cette disposition l'os inférieur, qui, d'après ce qui vient d'ètre dit, constitue peutêtre à lui seul la clavicule interne, est le plus souvent petit, mais ordinairement d'une longueur considérable. Cela est vrai surtout pour les genres zéé, scare, tétrodon, muge, baliste et centrisques.

Il est dans la plupart de ces poissons trèsalongé, triangulaire, apointi, quelquefois aplati de dehors en dedans. Telle est sa disposition chez les labres, les spares, les scorpènes.

Chez le tetrodon testudinarius, il est très-large et plat dans sa inoitié postérieure et inférieure; en avant il est an contraire mince et étroit.

Ordinairement il se porte directement en bas et un peu en dedans, mais quelquefois il est tourné fortèment en arrière, comme, par exemple, dans le muge céphale.

Chez le tétroclon il est également fortement tourné en arrière, uni d'une manière lảche, et par une petite éteudue de son extrémité supé- 
rieure, avec la face interne de la clavicule antérieure.

Lorsque l'os le plus supérieur de la portion suivante, se prolonge en apparence, d'après la conjecture que nous venons d'émettre, pour former la moitié supérieure de la clavicule interne ou postérieure, celle-ci est appliquée sur l'extrémité inférieure 'de la clavicule externe, parce que l'os en question s'interpose entre les deux clavicules.

Chez plusieurs poissons, par exemple le zeus s. argyreiosus vomer, les os de même nom des deux côtés se confondent à leur extrémité inférienre dans la ligne médiane.

Chez d'autres, par exemple les siganes, ils s’appuient avec leur extrémité inférieure sur les épines coccygiennes autérieures et inférieures.

Chez d'autres encore, surtout le muge céplale, et quelques espèces de chétodons, ils s'unissent, par leur extrémité postérieure et inférieure, avec la région postérieure des os pelviens, et supportent par conséquent les membres postérieurs; mode d'articulation particulier, dont M. Geoffroy s'est servi pour former un ordre particulier de poissons (1).

Chez quelques poissons, et particulièrement chez la baudroie, cet os est très-mince et situé superficiellement. Il se dirige en arrière, et entre

(1) Annales du Muséum, 1. c., p. 416. 
dans la paroi postérieure de la membrane branchiale qu'il renforce dans cette partie, descendant jusqu'à l'ouverture de la branchie.

D'après M. Geoffroy, cet os se transformerait, chez les silures épineux, d'une manière extrêmement remarquable, et deviendrait l'épine la plus supérieure, libre, de la nageoire pectorale (I). Cependant les argumens allégués en faveur de cette opinion ne me paraissent pas tout-à-fait convaincans. Sans doute l'épine supérieure, la plus forte, s'articule, dans ces espèces, avec la clavicule externe; mais évidemment cela prouve seulement que cet os peut, sans contredit à cause de son grand volume, quelquefois sauter la portion située entre la clavicule et la nageoire; anomalie d'articulation qui se rencontre souvent, comme on sait, sur d'autres os.

Le second argument allégué par M. Geoffroy est fondé sur ce que, chez les silures non épineux, par exemple le silurus electricus, la clavicule postérieure se trouve à sa place accontnmée, sous la forme d'un os mince, sourlé à d'autres os par ses deux extrémités : cette preuve n'est pas plus convaincante que la première, car cet os existe aussi chez d'autres silures, nommément chez le saluth (silurus glanis), où le premier rayon de la nageoire présente cette métamorphose; cet os n'est autre chose qu'une

(1) Ibid., p. 426 
D'ANATOMIE COMPARÉE.

pièce ordinairement existante à la seconde portion du membre; celle qui quelquefois s'alonge pour supporter la clavicule interne, et qui s'articule alors moins exactement avec la clavicule et les autres os de cette portion.

D'après ces considérations, l'opinion de 1I. Geoffroy ne me paraît pas conforme à la uature.

\section{S. 105.}

La seconde portion des membres antérieurs, ou portion moyenne qui succède à la première en arrière et en dedans, est composée, chez la plupart des poissons osseux, d'os courts et plats, qui reposent sur la elavicule externe. L'un d'eux est ordinairement plus long que les autres, tandis que ceux ci ont à peu près la même longueur. Ils sont le plus souvent au nombre de trois, plus rarement au nombre de deux ou d'un: En arrière ils sont contigus à la première fraction de la troisième portion du membre.

Les murénes, les lophies et les silures, du moins le murcenc conger et anguilla, ainsi que la baudroie (lophius piscatorius) et le saluth ( silurus glanis), offrent diversement des organisations moins parfaites que celles qui, par suite de modifications différentes dans chaque espèce, conduisent à la disposition ordinaire, qui est la ulus compliquée. 
Chez les murènes, cette portion du membre se compose seulement de deux os aplatis de dehors en dedans, se succédant de haut en bas, situés sur les limites de l'omoplate et de la clavicule, de manière à ce que l'os supérieur corresponde à la première, l'inférieur à l'extrémité supérieure de la seconde.

Tous deux sont un peu convexes en dehors, concaves en dedans. Le supérieur est près de deux fois plus volumineux que l'inférieur, un peu plus élevé de haut en bas que d'avant cn arrière, supérieurement apointi, convexe en devant, inférieurement échancré; il eu résulte une petite lacune entre lui et l'os suivant.

Celui-ci a sa plus grande dimension daus la direction d'avant en arrière; il est convexe en haut, échancré en bas, terminé par une pointe mousse en avant et en arrière.

Chez la baudroie il existe à la face interne de la clavicule externe, précisément au point de réunion de la branche supérieure avec l'inférieure, un os très-court qui se divise en deux moitiés. La postérieure, beaucoup plus considérable que l'autre, est alongée, tout-à-fait cartilagineuse dans sa parlie postérieure, qui se termine par une surface articulaire, arrondie. fort convexe. Elle est percée, en ontre, an conmencement de cette surface, vers le milieu de sa hauteur, dine ouverture qui descend verticalement. La moitić antérieure est un stylet mince, 
D'a NATOMIE COMPARÉE.

descendant obliquement d'avant en arrière et de dehors en dedans.

Ces deux moitiés sont contiguës à la clavicule: la postérieure par toute sa face interne, qui est larğe; l'antérieure par sa pointe; d'où résulte, entre les deux os, une ouverture triangulaire considérable, formée en dehors par la clavicule, en dedans par le stylet.

Suivant M. Geoffroy-Saint-Hilaire (I), cet os, $1^{\circ}$ se souderait, avec l'âge, à la clavicule; 2 " il y en aurait en outre deux autres appartenant a cette portion du membre. Mais je ne crois ni lune ni l'autre de ces deux assertions. En effet, sur les sujets les plus volumineux il m'a été facile de disjoindre les deux premiers os par la macération, et les seconds me semblent devoir ètre, avec plus de raison, comparés à des os de la portion suivante.

Les silures n'ont également qu'un os pour cette portion du membre; mais il est beancoup plus compliqué que les deux os des murènes, et que l'os simple de la baudroie, avec lequel il offre du reste une plus grande analogie; il représente, en effet, déjà les trois os ordinaires des autres poissons osseux.

Il est très-long, considérablement rétréci d'arrière en avant, puisquil commence par une

(1) Premer mémoire sur les poissons, 1. c., p. $\tilde{3} 6 \mathrm{~g}$. 
base courte, mais large et élevée, et qu'il se termine par une pointe très-alongée.

Sa partie postérieure se compose principalement d'une branche supérieure, verticale, et d'une inférieure, horizontale. La première est plus longue, aplatie, s'applique contre la seconde des surfaces rugueuses décrites ci-dessus (1), et plus haut contre la partie inférieure de la face interne de la branche ascendante de la clavicule. Elle renferme infériéurement une ouverture ronde, considérable.

La branche horizontale, inférieure, qui est plus petite, est également percée, à sa base, d'une ouverture, mais qui est moins large; elle s'applique contre la troisième des faces articulaires de la clavicule que nous avons décrites plus haut (2).

La branche supérieure, qui est ascendante, détache vers le milieu de sa face interne une apophyse mince, qui descend obliquement en dedans, et qui, vis-à-vis de la base de l'os, rentre dans sa face interne.

Cette portion du membre présente ainsi trois lacunes, dont la plus considérable est celle qui vient d'être décrite.

L'apophyse antérieure, simple, plus longue, mais plus déprimée, s'applique, dans la majeure

(1) Voyez pag. $591-595$.

(2) Mêmes prages. 


$$
\text { D'ANATOMIB COMPARE. }
$$

partie de sa longueur, contre la première des surfaces articulaires de la clavicule décrites plus haut (1): il en résulte entre ce point et la partie postérieure une lacune alongée et considérable.

Otez par la pensée, à l'os décrit ci-dessus, sa pièce longue, et ôtez de mème antérieurement le pont à la partie supérieure de la branche verticale, vous aurez exactement la disposition qui existe dans les baudroies. .

Je n'ai pas pu résoudre la question de savoir si, chez des individus très-jeunes, cet os, fort compliqué, est formé de plusieurs pièces unies entre elles par une substance cartilagineuse; cependant cela ne me paraît pas vraisemblable, si on fait abstraction des premières périodes du développement; car, chez un silure long de dix pouces, je n'ai pas pu découvrir de traces de séparation. Lit disposition ne différait ici de celle qui vient d'être décrite que par la circonstance que la seconde ouverture horizontale n'était pas fermée en avant.

(1) Voyez pag. 594. 


\section{S. 106.}

Les trois os qui se rencontrent chez la plupart des poissons osseux, représentent les parties de l'os unique qui vient d'être décrit che\% les silures; mais ils semblent rester séparés pendant toute la durée de la vie; je les ai du moins trouvésabsolument disposés de la même manic̀re chez les sujets les plus volumineux et chez les plus petits.

Le plus long de ces trois os correspond à la partie antérieure et styloïde de l'os unique. Il est plus large supérieurement, plus ou moins apointi dans sa moitié inferieure qui est la plus longue, et il s'applique, avec son bord externe supérieur, contre le bord postérieur de la lame interne de la clavicule extcrne; de telle sorte qu'il reste une ouverture d'une étendue variable entre ses extrémités supérieure et inférieure et celte clavicule.

Cet os est souvent contigu, par son extrémité postérieure, aux deux petits os postérieurs et supérieurs, qui, situés l'un à côté de l'autre et l'un sur l'auire, touchent en dehors également à la face interne de la clavicule, et représentent la base de l'os simple des silures. Il est attaché en outre, dans une petite étendue de son bord postérieur et en haut, aux os inférieurs de la portion suivante. 
L'os le plus supérieur est alongé, plus ou moins recourbé en dedans, plus développé supérieurement qu'inférieurement, et articulé ici avec la partie supérieure du grand os antérieur. Il s'avance communément le plus en dedans, de manière à sortir à des distances variables de la rangée des deux autres, qui sont davantage disposés sur un plan.

Le second des os supérieurs, qui est le moyen, est toujours plus ou moins quadrilatère; il forme un anneau percé d'une ouverture arrondie vers son bord antérieur, et est situé en haut, entre les clavicules externe et interne, et l'os antérieur, qui est le plus grand, et auquel il est contigu par son bord inférieur.

A son bord postérieur il s'unit à l'os supérieur de la première fraction de la portion suivante, quelquefois même avec les rayons les plus supérieurs des nageoires; c'est en effet le point où celles-ci s'attachent particulièrement à cette fraction.

Ces trois os offrent des variélés assez considérables à plusieurs égards.

Dans plusieurs genres, par exemple les carpes et les saumons, ils offrent la plus grande res. semblance avec la disposition que l'on rencontre dans les silures; en effet, ils sont : $1^{\circ}$ arrondis, cylindriques, courts, recourbés; $2^{\circ}$ ils sinfléchissent en dedans à une assez grande distance 
de la clavicule externe; il en résulte des lacunes considérables entre eux et celle-('i; $3^{\circ}$ tous sont unis par du cartilage, quoiqu'ils puissent être séparés; $4^{\circ}$ l'os antérieur, le plus volumineux, est uni par une suture à la clavicule dans presque toute sa longueur, et même dans sa partie postérieure.

Au contraire, dans beaucoup de poissons, peut-être dans la plupart: $\mathrm{I}^{\circ}$ ces os sont beau-

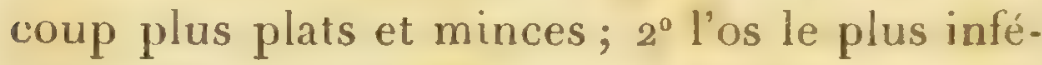
rieur n'est appliqué contre la clavicule que supérieurement et souvent dans une étendue trèscourte; $3 \cdot$ le plus supérieur est beaucoup plus alongé, plat, très-peu courbe, a beaucoup' muins de connexion avec les autres, de sorte qu'eu haut il n'est uni que lâchement à la clavicule, et n'est attaché en bas que faiblement à l'os le plus inférieur; $4^{\circ}$ tous les os sont situés beaucoup plus en arrière quàà côté de la clavicule; il en résulte une surface plate, plus simple; $5^{\circ}$ l'os le plus supérieur supporte la clavicule interne à son extrémité inférieure.

Cette disposition, du moins relativement aux quatre premières circonstances ci-dessus indiquées et à la forme de ces os en général, est offerte spécialement par les spares, les brames, les scorpènes, les muges, les centronotes, l'esox. belone, les sphyrènes, les gades, les mulles, les trigles.

La troisience ef la cinquième proposition que 
je viens d'établir sur les conditions qu'offre l'os le plus supérieur, et avec lesquelles on peut comparer ce qui a été dit plus haut (1) à l'occasion du nombre des pièces de la clavicule interne, devraient ètre énoncées de la manière suivante, si mon opinion sur l'identité de cet os avec la clavicule interne ne se confirmait pas.

"L'os le plus supérieur manque forl souvent; mais alors la clavicule interne se divise ordinairement en une moilié supérieure, plus grande, et en une inférieure, plus petite.

Mais de ces hypothèses la dernière me parait la moins vraisemblable, parce que d'après elle le nombre des différentes pièces osseuses de cette région restant le même, l'une disparaîtrait tout-à-fait tandis qu'une autre se partagerait en deux; dans la première supposition, au contraire, il n'y a point de disparition de pièce; mais l'une est refoulée par l'autre, de telle façon qiie celle-ci descend de son point d'attache ordinaire. et va s'altacher à la pointe intérieure de celle qu'elle a déplacée.

Mais dans l'une et l'autre hypothèse, le plus supérieur ou le plus interne des trois os de la seconde portion des membres, serait toujours sujet au plus grand nombre de déviations, puisque, dans la première, il changerait Je plus suaverut et le plus complètement de forme, de 
position et de connerion, et que, dans la seconde, il manquerait très-fréquemment, tandis que les deux autres sont, it tous égards, beaucoup plus constans.

Cela s'applique principalement au second os, qui est situé au milieu, et qui a la forme d'un anneau.

Ciet anneau, modifié à sa partie postérieure en une face articulaire très-convexe pour s'unir à la portion qui vient après, forme la fraction postérieure de la seconde portion du membre de la baudroie commune que nous avons décrite plus haut ( 1 ); il n'y a, au contraire, nulle trace de fraction supérieure, à moins qu'on n'admette qu'elle se porte en arrière, pour constituer l'os qui a également été décrit (2); cas dans lequel ce poisson n'aurait point de clavicule interne; ce qui ne serait pas tout-àfait impossible, par la raison qu'elle manquè̀ plusieurs autres poissons (3).

Ce qui vient d'ètre dit au sujet du second os, prouve en outre que la détermination des os qui viennent après, donnée par M. Geoffroy, est inexacte: l'un de ceux, en effet, dont il les considère comme une modification, existe à la fois

(I) Voyez pag. 404-405.

(2) Voyez pag. $401-402$.

(3) Voyez pag. 897 
D ANATOMIE COMPARÉE.

avec ces os. Il est conformé en tout suivant le type ordinaire lles poissons, et est articulé avec les autres de la manière accoutumée.

Peut-ètre est-il mime possible de démontrer le plus supérieur de ces os chez les lophies.

J'ai réussi, en effet, par la macération, à séparer sans difticulté la pièce annulaire de l'os, en apparence commun de la seconde portion, en une pièce externe, plus grande, et en une interne plus petite. La première se confond, du moins autani que j’ai pu le constater jusqu'ả ce jour, avec l'os le plus antérieur; elle représenterait par conséquent ce dernier et l'os moyen ou annulaire. La piece interne, qui a la forme d'un anneau ouvert en avant, pourrait ètre considérée comme l’os le plus supérieur qui aurait glissé contre la face interne de l'os moyen. Il est positif que cette pièce existe isolée chez la baudroie commune; mais je n'ose décider si tle est réellement ce que je dis, ou si elle n'est pas la pièce moyenne. On peut alléguer en faveur de la première opinion, qu'indépendamment de cette pièce il y a la pièce annulaire, et que la pièce supérieure ne forme jamais, je crois, d'annean. La seconde opinion pourrait s'étayer de ce que la pièce séparable forme la fraction interne de la face articulaire pour la portion suivante; disposition dont je ne conuais jas d'autre exemple.

Dans la premiere supposition, la longue 
pièce, se portant à la superficie du corps, serait la clavicule interne; daus la seconde, la question sur la détermination de cet os serait indécise; mais, dans l'une et l'autre de ces suppositions, l'opinion de M. Cieoffroy est toujours erronée.

Nous croyons devoir réserver la comparai. son des deux premières portions du membre chez les poissons cartilagineux et les poissons ossenx, ainsi que la désignation plus exacte de ces parties, pour le moment où nous décrirons leurs analogues chez les vertébrés supérieurs.

\section{S. 107.}

Isa troisième portion du membre antérieur des poissons osseux se divise, comme chez les poissons cartilagineux, en deux fractions, la base out le carpe, et le's doigts ou orteils qui se subdivisent, à leur tour, en leurs phalanges. L'une et l'autre fraction est composée de plusieurs os se succédant de haut en bas, situés dans un plan, et se touchant les uns les autres.

\section{S. 108.}

Le carpe est beaucoup moins développé que la fraction digitale, et se compose d'un nombre d'os bien moindre qu'elle. Ceux-ci sont à la fois 
beaucoup plus larges, plus plats et renflés aux deux extrémités.

Cette partie de la nageoire est formée communément de trois à quatre os. La dernière condition est beaucoup plus fréquente que l'autre.

Une exception à cette règle se présente dans le genre lophie, où il n'y a que deux os à cette partie. Cette partıcularité, jointe à la longueur et à l'étroitesse de ces os, circonstances toutes deux évidemment fort accidentelles et nullement essentielles, a été cause que $\mathbf{M}$. Greoffroy a considéré ces os comme représentant l'avant-bras des animaux supérieurs; erreur qui est réfutée, si je ne me trompe, de la manière la plus concluante :

$1^{\circ}$ Par la présence des os auxquels les assimile M. Geoffroy, dans la seconde portion dû membre antérieur ( 1 ).

$2^{\circ}$ Par ce qu'a de contradictoire avec la disposition de tous les autres poissons, l'absence des os carpiens dans les lophies, qui résulterait de ladoption de cette analogie.

$3^{\circ}$ Par la présence de trois os dans cette fraction chez les chironectes, qui offrent la même conformation que les lophies dans la seconde fraction, et le mème défaut d'auires os à la base de la nageoire.

Parmi les différentes espèces de lophies que

(1) Yoyez pag. $40 \%$ 
j'ai pu comparer entre elles, j’ai trouvé que les deux os dont il s'agit sont les plus petits chez la baudroie commune, et les plus longs chez le lophius punctatus.

Les os même que M. Geoffroy considère comme des os de l'avant-bras chez le bichir (polypterus niloticus), me semblent être des os du carpe. Ils sont tous deux fort alongés, l'inférieur presque deux fois plus long que le supérieur. Ils s'écartent postérieurement l’un de l'autre, et dans l'intervalle il existe un os arrondi, plus court et aplati.

On ne trouve done ici et chez les chironectes que trois os. Lesbatrachoüdes s'accordent, avecles poissons qui viennent d'ètre décrits, par le développement considérable de cette région et la forme fort alongée de chaque os en particulier; mais ils ont eux-mèmes un os de plus que de coutume; ils en ont cinq.

Ces os divergent d'avant en arrière et deviennent, comme à l'ordinaire, plus longs de haut en bas. Les quatre supérieurs sont étroits; l'inférieur est fort large. Postérieurement ils ont une grande largeur, beaucoup plus considérable que chez les polyptères.

Les os du carpe augimentent très-généralement de volume de haut en bas; l'inférieur est communément plus grand et augmente d'une manière brusque. Il en résulte que les inférieurs correspondent à un nombre beaucoup plus 
D'ANATOMIE COMPARÉE.

considérable de rayons de nageoires que les supérieurs.

Cette disposition réfute aussi l'opinion de MI. Geoffroy, sur la détermination des os de cette région chez les lophies et les polyptères; car l'os inférieur a pour le moins dix fois plus de masse que le supérieur, et supporte environ trente rayons; le supérieur n'en a que trois.

D'autres différences portent sur la présence, la furme, le rapport réciproque et la siluation de ces os, et en somme sur la disposition de toute cette région, qui est en général déterminée pat. celle de ces os.

Il paraît qu'ils manquent fort rarement; du moins l'unique exemple de cetle espice qui me soit connu, est fourui par l'exocotus exsiliens, chez lequel leur absence dépend vraisemblablement du grand développement de la fraction qui vient ensuite.

Comme la face articulaire des rayons de la nageoire est ici formée de la même manière que chez les autres poissons, il est possible que les os du carpe soient confondus avec ceux de la fraction placée au devant d'eux. Cette soudurè est d'autant plus vraisemblable que ces derniers ne font qu'un avec la clavicule externe, et que tous les rayons de la nageoire s'articulent avec cet os.

Sous le rapport de la forme, ils sont prismatiques ou oblongs chez les balistes, les murènes, 
les silures, les cyprins, les saumons, les brames, les ésoces, les soles, le corb (scicena umbra), les perches, la sargue ordinaire, le maquereau (scomber scombrus), les spares, le dophin, le poisson Saint-Pierre, les labres, principalement chez le remora (echeneis remora), la mole (tetrodon mola), les baudroies. Ils se montrent larges et aplatis dans les scorpènes, l'uranoscopus scaber, les pleuronectes, le boulereau (gobius niger), le coffre (ostracion trigonus), les callionymes, les ophidies, surtout très-distinctement chez le trigla gumardus, le malarmal (trigla cataphracta) L., la vive ordinaire ( $t$ achinus draco), où ils forment un carré presque régulier.

Les splyyrènes et les gades établissent la transition entre ces formations.

Ces os sont situés le plus souvent les uns audessus des autres; chez la baudroie commune, ils sont au contraire l'un à cóté de l'autre, de façon que le petit se trouve situé très en delıors, le grand tout-à-fait en dedans.

\section{\$. 109 .}

La fraction digitale de la troisième portion du membre thoracique est toujours une des plus considérables de tout le membre, et l'emporte surtout constamment de beaucoup sur la fiaction carpienne. Elle est plus ou moins alongée. 
Ia description de sa forme et du nombre des rayons qui la composent, etc., appartient à la zoologie. Ici nous ne devous exposer que la forme des rayons particuliers, telle que la dissection nous la démontre.

Quoiqu'ils ne forment, au premier aperçu, qu'une série simple, on trouve néanmoins, en examinant attentivement, que chaque rayon peut ètre divisé, d'une manière plus ou moins parfaite, en un externe et un interne, qui sont seulement unis très-étroitement ensemble.

Ils sont en outre diversement divisés de haut en bas, dans une étendue variable de leur partie postérieure, vers leur bord libre.

Mais en revanche ils ne sont pas partagés dans le sens de la longueur, cornme les rayons de plusieurs poissons cartilagineux, en plusieurs articles séparés, quoique cette conformation soit indiquée très-généralement par des renflemens et des rétrécissemens alternatifs.

Les exceptions d̀ celte règle sont extrêmement rares. Je n'en connais qu'un seul exemple fourni par le genre polypière. Dans le squelette qui se trouve au Muséum de Paris, on voit du cûlé gauche, après les os du carpe, dix-neuf os alongés, disposés en rayons, augmentant de volume de haut en bas, auxquels succèdent les rayons ordinaires qui sont plus longs. Il est tres-digne de remarque qu'au côté droit il ne se tronve à leur place que cinq os plus larges, 
dont la largeur s'accroît considérablement de haut en bas. Cette disposition rappelle virement la conformation des batrachoides décrite plus haut, mais chez lesquels les cinq os larges sont suivis immédiatement des rayons ordinaires.

Les polyptères auraient par conséquent la nageoire divisée, comme est la main des animaux supérieurs, en carpe, métacarpe et doigts.

Le rayon le plus supérieur est communément un peu plus épais que les autres; sonvent l'épaisseur en est beaucoup plus considérable. Ce rayon est moins distinctement formé de deux couches. Chez plusieurs poissons il se prolonge beaucoup, est dentelé et sert par conséquent d'arme efficace.

Les rayons sont toujours très-alongés et deviennent insensiblement plus grêles de la base au sommet.

A leur base les rayons des deux rangées se terminent par une apophyse ayant sa face antérieure tournée vers la première fraction de la nageoire; celle des rayons de la rangée externe descend obliquement en dehors, celle de l'interne descend en dedans. Ces dieux rangées forment ensemble une face articulaire, triangulaire, concave, par laquelle elles embrassent, plus haut ou plus bas, le bord postérieur du carpe.

De cette manière, la majeure partie de ces rayons s'articule avec les os du carpe, et tous 
réunis constituent un bord antérieur, égal; mais souvent le rayon supérieur s'avance en avant sur les autres, les dépasse et ne s'articule pas avec les os du carpe, mais avec l'os annulaire de la seconde portion du membre, et même avec la clavicule externe.

Les carpes et les anguilles offrent des exemples de la première dispositiou; les silures en présentent pour la seconde.

Cetle connexion a fait admettre à M. Geoffroy, comme nous l'avons déjà dit (1), que ce rayon n'est pas un rayon de nageoire ordinaire, mais la clavicule interne qui aurait été refoulée au dehors. Mais outre les argumens déjà précédemment allégués contre l'opinion de M. Geoffroy, elle devient manifestement invraisemblable par la remarque que les carpes et les anguilles offrent le commencement de cette organisation, dont celle des silures n'est qu'un degré supérieur, bien qu'elles possèdent très-distinctement une clavicule interne, ordinaire. Chez les silures, l'extension de l'articulation avec la clavicule externe est sans doute en rapport avec le volume considérable du premier rayon de la nageoire.

Les trigles offrent une disposition particutiere des nageoires. Ce sont trois rayons, séparés des autres, qui ne sont unis ni avec ceux-ci ni entre eux-mémes. Ils occupent la fraction la 
plus inférieure, sont situés sur le troisième et le plus inférieur des os carpiens, et ont nonseulement plus d'épaisseur que les autres rayons unis entre eux, quoiqu'ils soient plus longs, mais ils sont encore : $1^{\circ}$ distinctement formés d'articles unis seulement par des couches de cartilage; $2^{\circ}$ non divisés de haut en bas vers leur extrémité libre; $3^{\circ}$ beaucoup plus susceptibles que les autres d'être séparés en deux couches longitudinales juxtaposées.

\section{S. 110 .}

Les différentes portions du membre anlérieur sont loin d'offrir partout les mèmes rapports. Cette remarque ne s'applique pas seulement aux os des portions mèmes, mais encore à ceux des diverses fractions de ces portions. Cependant les différences qui en résultent ne peuvent être bien saisies qu'à l'aide d'un tableau qui les renferme toutes, et auquel je renvoie le lecteur d'autant plus volontiers qu'il contiendra encore plusieurs autres conditions des os des membres anlérieurs, qui n'ont pas pu être détaillées ici et qui ne sout pourtant pas sans importance, soit par elles-mèmes déjà, soit par rapport à la détermination de ces os. 
b. Os des membres postérieurs.

$$
\text { S. } 111 .
$$

Les os des nuembres postérieurs des poissons, surtout ceux des portions extérieures, sont, en géuéral, formés d'après le même type que ceux des membres antérieurs; mais ils en diffèrent presque toujours ainsi que l'ensemble des poslérieurs eux-mêmes, par des conditions contraires au type ordinaire des vertèbres, et qui consiste en un volumé beaucoup plus petit et un développement beaucoup moindre, sous le rapport du nombre des pièces.

Je ne connais d'autre exception à cette loi que celle fournie par le platycéphale (cottus insidiator ); chez lequel les membres postérieurs l'emportent sur les antérieurs, dans toutes leurs portions, tant sous le rapport de la masse que sous celui de l'étendue.

Les nageoires offrent, il est vrai, le rapport crlinaire, puisqu'elles sont formées d'un nombre moindre de rayons; mais elles sont plus longues, et les rayons en sont plus épais qu'aux membres antérieurs. Rien de semblable n'existe dans les autres espèces de ce genre, du moins dans le meunier (cottus gobio) et le scorpion de mer (c.scorpius). Lorsque, comme chez plusieurs trigles, exocets, et chez les poissons vo. 
lans en général, les rnembres antéricurs grossissent souvent fort consilérablement, les postérieurs ne dépassent pourtant point ou que peu la mesure ordinaire. 11 n'est également pas rare de les voir manquer dans cette classe de vertébrés, è jamais, du moins autant que je puis savoir, la portion interne n'existe quand l'externe ou la nageoire abdominale manque, comme cela se voit quelquefois lors de l'absence de la nageoire thoracique.

Tandis que chez la plupart des poissons les membres antérieurs sont unis, soit au rachis, soit au crâne, les postérieurs ne sont jamais en connexion immédiate avec le reste du squelette, mais seulement d'une manière médiate, ou à l'aide de muscles, ou enfin au moyen des os des membres antérieurs.

Dans les gastérostées, du moins dans le gasterosteus aculeutus, il existe cependant, à cet égard, un rapprochement vers le morle d'articulation des membres antérieurs des poissons, ou des nembres postérieurs des autres vertébrés: une apophyse verticale, externe, existant ordinairement sur la premiere portion du membre, d'une hauteur et d'une largeur considérable, monte fort haut, un peu au-devant du milieu de la portion, à la face externe des còtes de cette région, sur lesquelles elle est appliquée immédiatement, et ne se termine que vis-à-vis de la colonne vertébrale. 
11 n'y a point de trace de cette disposition dans. le gastré (gusterosteus spinachia). Je n'ai pas trouvé davantage chez l'anableps tetrophthalmus, l'aiguillon decrit par M. Cuvier (1), qui se détache du hord externe et se replie dans la direction des còtes, el qui pourrait rappeler cette même organisation. Le sujet que j'ai examiné était pourtant frais, volumineux et bien conservé; d'où je conclus qu'il y a eu peut-être quelque méprise.

Les poissons se distinguent en outre des autres animaux, sous le rapport de la position de ces nageoires; elles ne se trouvent pas partout derrière les nageoires thoraciques et à une distance considérable, puisque dans certains poissons elles sont situées immédiatement audessous d'elles, et chez d'autres même au-devant d'elles. Les premiers ont été nommés poissons thoraciques, les seconds jugulaires; ceux où les nageoires postérieures se trouvent derrière les antérieures sont connus sous le nom d'abdaininaux. De ces trois ordres, le dernier est incontestablement le plus parfait, et celui des jugulaires le plus imparfait, non-seulement parce que celui-ci s'éloigne le plus du type des vertébrés supérieurs, mais parce qu'il n'y a le plus souvent qu'une paire de membres, ce qui les rapproche par conséquent des apodes, qui n'ont

(1) Anatomie comparéc, t. I, p. $40 \%$. 
point de membres postérieurs. Chez les poissons jugulaires et thoraciques, les nageoires abdominales s'unissent ordinairement aux parties inférieure et antérieure de la face interne des clavicules externes. Chez quelques-uns, par exemple, chez l'uranoscopus scaber', elles y sont complètement soudées; disposition qui, des divers degrés de l'imperfection que sont susceptibles d'offrir les nagenires abdominales comparées, sous le rapport de la position et de la connexion, avec les nageoires thoraciques, est sans contredit le plus inférieur. Dans d'autres poissons, au contraire, par exemple, les muges, les scares, elles ne sont pas unies avec ces os; mais cette union a lieu à l'aide d'un ligament avec le sommet des clavicules internes. Cette derniere conformation est évidenment un degré intermédiaire à celle des poissons thoraciques et abdominaux.

Le membre abdominal des poissons peut aussi être divisé facilement en une portion intéricure et en une exlérieure; la première de ces portions peut êlre nommée portion coxalc ou hanche, ou bien, portion pelvienne ou bassin, et l'autre nageoire abdominale. 
a. Poissons cartilagineux.

$$
\text { S, } 112
$$

1. Portion coxale ou hanche.

La hanche des poissons cartilagineux n'est pas partout formée d'après le même type. Chez les raies et les squales, elle constitue une sorte de barre transversale qui est considérablement plus large d'un côté à l'autre que d'avant en arrière, a sa plus grande minceur de haut en bas, et se termine à ses deux extrémités, en avant et en arrière, par une apophyse, dont l'antérieure est tournée en avant et en bas, la postérieure en haut et en arrière. Chez les chimères, au contraire, les deux moitiés du bassin ne sont pas unies entre elles; elles consistent en deux pièces cartilagineuses, séparées, plates, minces, courbes, ayant la convexité dirigée en dehors, et la concavité en dedans.

$$
\text { S. } 113 .
$$

2. Nageoire.

La seconde portion, la nageoire abdominale de's poissons cartilagineux, particulièrement des raies, se compose, comme la nageoire thoracique, de deux parties: " d'une série de pièces 
osseuscs, fort alongées, plates, quadrilatères, se suivant d'avant en arrière, et dimimuant de volume dans le mème ordre, dont la première s'articule avec le bassin au point de réunion de sa prartie transverse avec ses parties latérales; $2^{*}$ de quelques séries de pièces minces, plates, alongées, diminuant aussi beaucoup de volume vers le bord libre, où elles sont bifurquées; les antérieures sont beaucoup plus développées que les postérieures, et s'attachent immédiatement à l'extrémité externe de l'os pelvien.

Chez les chiméres, la place des plaques cartilagineuses, à la base de la nageoire abdominale, est occupée par une lame unique, d'une largeur considérable, qui n'est suivie que d'une série de lames longitudinales, le reste de la nageoire étant membranéux.

La première partie peut être nommée la racine de la nageoire abdominale, le tarse; la seconde constituerait le métatarse et les orteils.

\section{S $11: 1$.}

I a plupart des poissons cartilagineux offrent une différence sexuelle, remarquable, dans le développement des membres postérieurs; ces membres sont beaucoup plus grands chez les mâles que chez les femelles; disposition qui se rattache à la présence d'une glande considérable chez les prenicrs. Coest pour cette raison que je 


$$
\text { D'ANATOMHE COMPARÉ. }
$$

ne décrirai cette particularité des os qu'en traitant des glandes en question.

$$
\text { S. } 115 .
$$

Les esturgeons forment également le passage des poissons cartilagineux aux poissons osseux par la disposition de leurs membres postérieurs. Ils ont : $1^{\circ}$ une petite hanche, triangulaire, ayant son sommet tourné en avant, laquelle est séparée de celle du côté opposé de presque toute la largeur de la face inférieure du corps; $2^{\circ} \operatorname{sept}$ rayons cartilagineux, ayant à peu près une largeur égale, appuyés sur le bord postérieur de la hanche, descendant de dehors en dedans, et d'avant en arrière; $5^{\circ}$ approchant trente rayons de nageoire beaucoup plus longs, formant une couche supérieure et une inférieure, qui ne contiennent pas de traces de cartilages.

La séparátion des deux moitiés du bassin est évidemment une analogie avec les poissons osseux; la présence de cartilages tarsiens représente la disposition des poissons cartilagineux. Il est curieux en outre; sous plusieurs rapports, que les moitiés du bassin soient extrêmement imparfaites, petites en proportion, et considéra. blement éloignées l'une de l'autre. 


\section{b. Poissons ossern.}

\section{S. 116.}

x. Hanche.

La hanche des poissons osseux est formée ordinairement, de chaque côté, d'un os triangulaire, situé horizontalement, ou obliquement dirigé de dedans en dehors, qui a sa base tournée vers celui de l'autre côté, et sa pointe regardant en dehors. A son bord externe, il devient toujours plus large. Quoique cet élargissement ne se manifeste pas au même point de la longueur dans toutes les espèces, il occupe constamment la région moyenne ou l'intervalle qui sépare cette région moyenne de l'extrémité postérieure. Ainsi élargi, il constitue une surface articulaire entièrement ou en majeure partie convexe en dehors, qui supporte la nageoire abdominale.

Abstraction faite des différences de position précitées, la connexion et la forme de la hanche. présentent plusieurs modifications.

En général, les os coxaux sont unis entre eux par leurs bords internes; cependant on voit une exception à cette règle dans les cxocets, les ésoces, du moins dans le brochet (esox lucius), l'orphie (esox belone), le scombresoce camporien (esox brasiliensis), les polvptères, l'elops 
saumus . l'anableps tetrophthalmus, les saumons, le silure noir, où ces bords sont seulement tournés l'un vers l'autre.

Les conformations transitoires, qui conduisent à cette séparation totale, sont celles où la connexion des bords internes est interrompue dans une étendue plus ou muins considérable. On observe cela dans la baudroie commune, le mormyre salheych (mormyrus labiatus), la herse commune (kaunum), le ban (crprinö̈des), le pimelode scheinan (silurus clarias), le docmac, le bagre, la carpe ordinaire, l'alose, où ils ne sont unis que dans leur plus petite partie postérieure. tandis qu'ils sont fort éloignés l'un de l'autre dans toute leur longueur. Cette union des deux iparties latérales s'étend un peu plus loin dans leur moitié postérieure, chez le muge céphale, lle thon (scomber thynnus). Le passage de ceuxci est formé par le platycéphale (cottus insidiator), chez lequel elles sont fort convexes en dehors, de sorte qu'il se trouve entre elles une ouverture très-large. En arrière et encore plus -en avant, ils ne sont unis que dans une trèspetite étendue.

On les trouve entièrement soudés chez la vive ordinaire (trachinus draco), l'uranoscopus scaber, le poisson Saint-Pierre, les labres, les acanthinions, le surmulet (mullus surmuletr.s), les clupées, les sciènes, les perches, plusieurs silures, par exemple, le silurus senen, 
le doras carinatus. Chez les labres, les scorpenes, et chez tous ceux où la soudure n'est que partielle, les bords externes sont soudés ensemble; chez les acanthinions, le poisson aintPierre, l'union a lieu entre les faces internes ou inférieures.

Dans les trigles, le saluth, les gades, les chabots, ils sont unis dans leur moitié postérieure, et dans une petite étendue de l'antérieure; mais dans la majeure partie de la dernière, l'excavation notable du bord interne produit un intervalle considérable.

Chez le scorpoena horrida, il existe entre ces deux bords une petite lacune moyenne, alongée, à peine sensible.

La forme la plus simple de la hanche cousiste en l'existence d'une plaque triangulaire, le plus souvent alongée, apointie en avant. Mais cette forme est sujette à un grand nombre de modifications.

Chez les castagnoles (brama raji), les deux tiers antéricurs forment une épine extraordinairement longue, très-étroice par rapport au tiers postérieur, qui est haut et aplati d'un còté à l'autre.

Chez le sharmuth (silurus anguilluris), ce sont, au contraire, des plaques courtes, presque carrées.

Chez les trigles, la moitié antérieure est formće de chaque côté d'une partie externe, in- 
1) ANATOMLE COMPARÉE.

férieure, verticale, plus petite, et d'une interne, supérieure, horizontale, plus grande, qui sont unies ensemble sous un angle droit, et du point de jonction desquelles il s'élève une crète en haut, en sorte que la lame transverse, supérieure, touche à peu près au milieu de la lame externe et verticale. De l'extrémité antérieure de la jonction de la moitié postérieure, on voit saillir en avant et en bas une épine courbe, considérable, que l'on trouve aussi chez d'autres, par exemple, chez le surmulet (mullus surmuletus), le muge céphale (mugil cephalus).

Chez l'uranoscopus scaber, la vive ordinaire, (trachinus draco), le thon (scomber thynnus), il existe une semblable épine dans la même région; mais toutes deux sont fort éloignées l'une de l'autre, tandis que chez le premier de ces poissons elles sont unies et situées dans la ligne médiane.

La division de la région antérieure de la partie interne des os coxaux en deux pointes, que l'on troure chez les silures, l'exoccetus exsiliens. les cyprins, paraît ètre l'indice d'une semblable épine.

Chez plusieurs poissons, par exemple, les trigles, les mulles, les scombres, les scorpènes, les dorées, les acanthinions, ils se prolongent, au contraire, a leur extrémité postérieure, en une épine plus ou moins longue, qui, dans ces genres, se confond avec celle du côté opposé.

II 
Dans les cyprins, il y a une épine analogue; chacune d'elles est très-ćloignéc de celle du còté opposé.

La conformation la plus particulière est celle que présente l'exocotus exsiliens, chez lequel chaque hanche est formée de deux moitiés, dont l'une est antérieure, horizontale, triangulaire, plus large, et l'autre postérieure, verticale, étroite, mais seulement un peu plus courte, tournée en dehors et en haut vers la face dorsale; ces deux moitiés se réunissent sous un angle droit au point d'insertion des nageoires abdominales. La moitié antérieure est, du reste, dans la majeure partie antérieure de sa longueur, partagée par une lacune considérable en une portion interne, plus large et plus mince, et en une externe, plus étroite, qui est cylindrique.

Chez le trigla volitans, la hanche est fort developpée et garnie d'un grand nombre d'apophyses. Elle consiste en une partie interne et une externe. La première se dirige obliquement de dehors en dedans et de bas en haut, et est soudée dans toute sa longueur avec la partie de même non du côté antérieur. En arrière elle se prolonge en deux apophyses latérales et en une moyemne, impaire. De la partie externe de la hanche on voit se détacher en avant et latéralement une apophyse longue et mince qui s'unit au milieu du bord antérieur de la clavicule. 
3. Nageoires.

$$
\$ 117 .
$$

Chez les poissons osseux la structure des nageoires est moins parfaite que chez les cartilagineux; elles ne consistent qu'en une série de rayons longitudinaux, divisés à leur extrémité interne en une branche supérieure et en une inférieure, et s'imbriquant d'avant en arrière; il en résulte une fosse profonde, dirigée d'avant en arrière, qui entoure l'éminence ara ticulaire des os pelviens.

Les poissons osseux sont privés par conséquent, en général, des portions du membre situées entre la hanche et les orteils. Chez les polypières, la conformation est plus parfaite. Après le bassin vient une série de quatre os fort alongés, dont l'interne est plus épais et plus long que les trois externes, et séparé de ceux-ci, qui sont immédiatement appliqués les uns contre les autres.

C. OS DE LA TÊTE.

$$
\text { S. } 118 .
$$

Afin d'éviter les répétitions et les détails trop minutieux, nous croyons devoir faire précéder l'étude du crâne des poissons par un exposé géréral de la disposition du crâne chez les animaux vertébrés en général. 
Il est toujours formé de deux parties principales, savoir: d'une partie postérieure et supérieure, le crâne, et d'une inférieure et antérieure, la face. Le crâne constituè une cavité qui contient dans son intérieur l'encéphale et renferme des deux côlés, en avant et en bas, soit seul, soit conjointement arec des os de la face, les organes de l'ouie, de la vue et de l'olfaction : la face est aussi le siége des organes de ces mêmes sens, et en outre de l'urgane de la gustation; elle se compose principalement des os qui entourent la cavité buccale, et qui servent médiatement et immédiatement à la mastication.

Quelque variables que soient la forme de la tète entière, le rapport de ses différentes parties et l'état des os qui la composent, on peut néanmoins, outre les deux grandes divisions qui viennent d'être établies, déterminer dans chacune d'elles certaines régions, le plus ordinairement certains os, qui se répètent dans les divers animaux, quant à leurs conditions les plus essentielles.

On trouve ainsi au cräne, en le considérant d'arrière en avant:

1. L'os basilaire. Il formela région postérieure, inférieure et moyenne du crâne, et s'articule avec la première vertèbre du tronc. Il constitue tonjours un anneau, et a très-distinciement une. forme de vertèbre (1). Cette disposition existe

(1) L'idće de rencontrer une organisation vertébrale, 
surtout à sa partic postérieure, qui est lat porlion ociprilale; à sa partie antérieure, quiest la $\mu$ or-

dans les os de la tête,", parait 'être néédans l'esprit de plusicurs anatomistes a fois; le mode ${ }^{\mathrm{s}} \mathrm{d}^{\mathrm{r}}$ investigation de l'cpoque et l'état de la science avaient amené celte proposition a maturite.

On attribue a M. OKen d'avoir énoncé le premier, en 1807 , quelques idées sur le rapport qu'il aurait trouvé exister entre la conformation des vertebres qui terminent le rachis đu côté de la tête, chez-les poissons, et la disposition des pièces osseuses commençant, au mème point, la cavilé du cràne. Ces premières données seraient imprimées dans un écrit intitulé : Ueber die Bedeutung der:Schadelknochen; Jena, 1807.

La même pensée avait cependant déjà été indiquée, en 1805, par M. Burdin. On lit dans son ouvrage: "Dans l'appareil osseux, on voit la variété de formes que présente le squelette, depuis celui qui consiste seulement en une série de vertébres semblables, dont la téte paraîl n'étre qu'une plus compliquée, jusqu'à celui qui se compose d'une colonne vertébrale, de la tête, de la poitrine et des membres." (Btrdix, Cours d'études médicales, ᄂ. I, p. xvi. Paris 1803.)

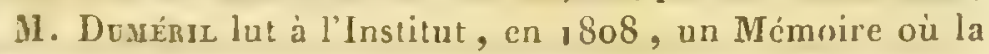
même comparaison fondamentale est également élablie. Cet anatomiste, qui, suivant ce qu'en rapporte M. GeorfroxSairt-Hinaire (Annales des Sciences naturelles, t. III, 1. $1 ; z$ ), indiqua une partie de l'analogie de composition reconnuc depuis par un certain nombre d'anatomistes entre la tête et !e rachis, idée des plus hardies pour le moment, se réduit toutefois dans ses conclusions imprimées, à ćlablir que c'est seulement sous le rapport des mouvemens de l'ensemble qu'il considère la téle comme une vertébre très-développée.

Il admet, dans ce travail que le trop, occipital est la continnation du canal rachidien; qu'une ressemblance parfaite existe entre l'apopliyse basilaire jointe au corps du spleć- 
tion sphénö̈dale, cette ressemblance est beaucoup moins parfaite et ne devient distincte qu'à

noïde et le corps d'une vertibre; que les facettes articulaires sont représentées par le condyle simple ou double; les apophyses épincuses et les lames, par la protubérance occipitale, la crête qui la continue en arrière, les irrégularités latérales, et les espaces compris entre ces parties; enfin. les apophyses transuerses par les protubérances masioides. M. Duмḱrıl ajoute plus spécialement au sujet des poissons osseux : "La téte n"est pas plus mobile sur l’échine que les autres vertèbres ne le sont entre elles; aussi ces animaux. $n^{\prime}$ ont-ils pas de condyles au crîne, mais une cavilé conique située au dessous du trou occipital, et articulée absolument comme les vertebres qui suivent." (Mag. encyclop., 6, 3,

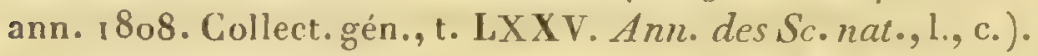

Dès l'année 1814, M. De Buarnvilie, dans le cours qu'il fit au jardin du Roi, en remplacement de M. Cuvier, avait professé que quatre vertèbres entraient dans la composition du crẩne. Les considérations qui ont amené M. DE Blainville à établir ces démarcations, entre les pièces ossenses de la tête, sont dues à l'impulsion donnée par $\mathbf{M}$. le docteur GaLL aux recherches sur l'organisation de l'encéphale. C'est comme enveloppe superficielle de la substance nerveuse, la dure-mère formant elle-même une enveloppe plus profonde, que M. DE Buainvicte les a considérées. Cet anatomiste à fondé l'existence de verièbres céphaliques, aunombre de quatre, sur la détermination d'un nombre égal de ganglions nerveux avec appareil extérieur, c'est-a-dire servant d'origine, camme ceux que présente à la moelle la naissance de chaque nerf rachidien, aux nerfs qui sortent du cerveau, et dont la plupart lui présentent des racines antérieures et des racines postérieures analogues à celles des nerfs spinaux.

Les limites que cet anatomiste a trácées aux vertèbres de la tête. sont les suivantes :

lin procédant d'avant en arrière, la première est formée 
l'aide d'autres os qui ferment l'anneau supérieurement.

par le vorner et les os propres du nez; elle correspond au bulbe olfactif ou ganglion d'origine des nerfs de l'olfaction.

La seconde se compose du sphénoüde antérieur et des frontaux: ; elle est destinée au ganglion optique (couche du mème nom), qui fournit origine aux nerfs optiques, ct à la quatrieme paire (analogues des branches postéricures des ne:f's spinaux), et aux troisième et sixième paires (analogues des branches antérieures).

La troisième est constituée par le sphénoïde postéricus et les pariétaux; elle est dévolue au ganglion nerveux qui doune naissance au nerf trifacial, ganglion qui forme la pyramide antérieure.

La quatrième est produite par l'occipital; elle correspond au corps olivaire et au ruban gris, qui donnent naissance an nerf acoustique (branches postérieures), et aux nerf's pneumo-gastrique, glosso-pharyngien et grand hypoglosse (branches antćrieures ).

Une partie des idées de M. de Blainville furent publiées en $18: 6$ dans les Mémoires de la Société philomatique; les limites qu'il admet sont imp rimées dans l'article MА мMIғÈnEs du Nouv. dict. d'hist. natur., de Déterville. Paris 1818.

Ces idées reçurent une extension extraordinaire dans l'ouvrage de M. SpIx, partisan extrème de la doctrine des homologistes allemands, c'est-àt-dire des analomistes qui veulent retrouver, à travers les différences de conformation des diverses parties du même être, les répétitions des mêmes conditions de disposition et de structure. i1. SPIX considere l'animal comme forme de deux tronçons, dont l'un est ramassé, c'est la téte; l'autre est plus distendu, ciest le tronc. Il retrouve dans la tête les analogues des verlibres rachidiennes; la mâchoire supérieure lui représente les membres supéricurs; les inférieurs sont reproduits par la mâchoire d'en bas. Le cràne est divisé, par M. Spıx, en 
2. L'os temporal qui est double et situé en dehors du précédent. Il représente la partie posté-

trois vertèbres qu'il nomme : occipilale nu cranique, pariétale ou thoracique, frontale ou abdominale. La seconde dénomination rappelle l’idée des régions du corps auxquelles il les compare (Cephalogenesis, 18,5). Ces recherches rentrent dans un système général de la nalure qui trouve d'ardens prosélytes en Allemagne, et qui consiste à considérer un plan unique dans loutes les productions de la nature. L'animal est la répétition de certaines porlions de lui-même, qui en répètent de plus petiles. Le globe représente en grand un animal; l'univers représente le globe, elc.

M. Cuvier, en 1817 , dans les considérations générales sur les mammifères (Règne animal, t. $\mathrm{I}^{\mathrm{ez}}, \mathrm{p} .73$ ), subdivise leur cráne en trois ceintures formées: l'antérieure, par les deux frontaux et l'ethmö̈le; l'intermédiaire, par les pariétaux et le sphénöide; la postérienre, par l'occipital; os anxquels s'ajoutent les temporaux intercalés entre l'occipital, les pariétaux et le sphénoïde.

En 1820 et $182 \mathbf{I}, \mathbf{M}$. OK $\mathbf{k N}$, développant ses premières idées, reconnaît à la tête qualre vertèbres; trois au crâne, et une a la face. Il les limite comme M. de Buainvilue. Chacune de ces vertèbres est destinée à un appareil de sens. A cause de cette destination, M. Orin les désigne sous le nom de verièbres auriculaire, linguale, oculaire et nasale. (1sis, $n^{n} 6$, année 1820 , pag. 1552. Esquisse d'un système d'anatomie, de plyssiologie, etc. , pag.41. Paris, Béchet).

D'autres auteurs ont encore traité des mêmes considérations; on peut lire à ce sujet Urric, Opuscule in- $4^{\circ}$, savoir: Annotationes quadam de significatione ossiun capitis; Berolini apnd Dümmlerum; Bojards, dans plusieurs cahiers de l'Isis; année 1818 , p. 498 et 1819 : Carus, daus son Lehrbuch der Zootomie; Leipzig, 18 i 8, in-8。. Ces citations sont empruntées à M. Geoffhor-SalNr-Hilarre.

La connaissance des élémens dont se compose chaque. 
rieure et inférieure des parois latérales, avec la parlie externe de la face inférieure du crâne, et

vertebre rachidienne, a conduit M. Geoffroy-Santi-HILaInE à rechercher si les mêmes élémens ne se rencontreraient pas dans les pièces osseuses de la tête. Le premier fait qu'a obtenn M. Geofroor, en interrogeant les époques foeiales de l'espèce humaine, et l'état adulte des animaux daris lesquels les pièces de cet appareil ossenx restent au plus grand élat de séparation, consiste dans lear existence au nombre de 63. Ce résultat, divisć par le chiffre 9 qui représente la quantilé des élémens d'une vertèbre, dorne $\eta$ pour le nombre des vertèbres composant la lête. Le second résul. tat vers lequel cet anatomiste a dirigé ses investigations, a été la détermination analogue de chacun des élémens vertébraux de la tête, rapporté à chaque élément des vertèbres dı rachis ; cette recherche l'a amené à considérer en procédant, d’avant en arrière, la première vertèbre qu'il appelle haAIALE comme formée : 10 par les carlilages du nez, qui en constituent le corpss, dans lequel il a reconnu l'élément analngue à celui qu'il a nommé cycléal; il a désigné ce corps sous le nom de prótosphÉNaL (la terminaison sphénal étant adoptée pour lous les corps des vertèbres céphaliques); $2^{\circ}$ par les cornets inférieurs du nez, cenx-ci sont les analogues des périaux; chacun de ces cornels est appelé nнINoPHYSAL; $5^{\circ}$ par les cornets supérieurs qui sont les épiaux de

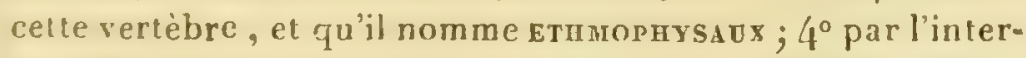
maxillaire de chaque côté; cet os, l'analogue du paraal, est désigné sous le nom d'ADNaSal; $5^{\circ}$ par la portion dentaire du maxillaire de chaque côté, rapporté an cataal, nommé

\section{A DDExtal.}

La denxieme vertèbre ou la vertèbre Nazare, a pour $c y$ cléal la lame ethmoïdale, désignée par le mot de rHinospuéral; pour périaux. de chaque côté, l'os unguis appelé a acryar ; pour épial, l'os du nez appelé nasal; pour paraul, la portion palatine du maxillaire, nonmée adovstal; 
forme, d'une manière plus ou moins distincte, une moilié latérale de vertèbre.

pour cataal le vomer, qui a reçu le nom de vomḱrat.

La troisième vertèbre, ou oculankr, a pour élémens : ro (sur le milieu), comme cycléal, le corps de l'ethmoïde qui constitue l'ethMospnéNal; $2^{\circ}$ ( sur chaque côté), commé périal, le cartilage tarse ou palpébral; $3^{\circ}$ comme épial, le coronal ou fnontal; $4^{\circ}$ comme paraal, la prortion orbitaire du maxillaire; $5^{\circ}$ comme cataal, le palatin ou PALATAL.

La quatrième vertèbre, ou cÉRÉBRALe, a : $1^{\circ}$ (sur le milieu), pour cycléal, le premier corps du sphénoïde; ce corps est l'entosphésad; $2^{\circ}$ ( sur les parties latérales), pour périal, chaque petite ailc ou ingrassial; $5^{\circ}$ pour épial, chaque grande aile ou ptéréAx; $4^{\circ}$ pour paraal, l'os zygomatique ou JUGAL; $5^{\circ}$ pour calaal, l'appophyse ptérygoïde interne ou HÉBISSÉAL.

La cinquième vertèbre, ou la evanRIJUnaLe, est formée sur le milieu : $1^{0} \mathrm{du}$ deuxieme corps du sphénóide, analogue du cycléal, et désignć sous le nom d'Hyposphéral: (sur les côtés) $2^{0}$ de chaque os des tempes, analogue du périal, et nommé temporal; $5^{\circ}$ du pariétal, analogue de l'épial, ct qui conserve le nom de pariécs ; fo dela grosse tubérosité du cercle du lympan, analogue du paraAs, et qui est nommé SFRRIAL; $5^{\circ}$ d'une parlie innominée, analogue an catral, et désignée par l'appellation de COTYuÉAL.

La sixième vertébre, ou l'auricularke, comprend: io le premier corps du basilaire, qui en est le cycléal, et qui est appelé otosfá́nal; $2^{\circ}$ (sur les parties latérales), le rocher, ou le RuPÉAL, qui représente le périal; $5^{\circ}$ la première partie de l'occipital, ou l'Inten-parútat qui est l'spial; $4^{\circ}$ le cercle du tympan, denxième partie du préopercule, ou le traparal qui reproduit le paraal; $5^{\circ}$ le marteau (inter-opercule), ou le marxíaz.

La septième vertibre a : $1^{0}$ pour ycléal, le deuxiemi 


\section{D'Anatome Comparét.}

5. L'os pracicial, placé en avant de la partie supérieure de la portion occipitale du basilaire et au-dessus du temporal. Il atteint la ligue médiane, et constitue une lame mince, correspondant à un arc de vertèbre.

1. L'os frontal, qui a beaucoup de rapport par sa position et sá forme, avec l'os précédent, en avant duquel il est situé, mais qui est un peu plus compliqué que lui.

5. L'os elhmoïde, qui devrait plutôt être rangé parmi les os de la face, et qui est placcí au dessous et en avant du frontal.

corps du sphénoïde, ou BASISPHÉNAL; $2^{0}$ pour périal, la troisième partie de l'occipital, ou exoccrpitaI; $3^{\circ}$ pour épial, la deuxième partie de l'occipital, ou suroccirttal; $4^{\circ}$ pour paraal l'étrier, ou sturést; $5^{\circ}$ pour calaal l'enclume, ou ricéaL.

Les pièces de la mâchoire inférieure que M. Geofrnoy arimet au nombre de sept de chaque cóté, sont désignées ainsi qu'il suit dans l'ancienne nomenclature, et en procédant d'avant en arrière: $1^{\circ}$ dentaire; $2^{\circ}$ operculaire; $3^{\circ}$ supplémentaire; $4^{\circ}$ coronoüdien; 5o angulaire; $6^{\circ}$ articulaire ; $7^{\circ}$ subangulaire. M. Gropfnox propose d'y substituer les noms suivans en procédant dans le même ordre : 10 subdental; $2^{\circ}$ subvoméral; 30 subpalalal; $4^{\circ}$ subhérisséal; 5 "subcotyléal; $6^{\circ}$ submalléal; $7^{\circ}$ subincéal. Ces pièces sont, suivant cet anatomiste, opposées aux vertèbres céphaliques, comme le sont, aux vertèbres cervicales, les pièces de lappareil hyoïlien, comme l'est le sternum aux vertèbres dorsales, les intersections aponévrotiques anx vertèbres lombaires, elc. (Lisez Ann. des Sc. nat., Géfrrox-SantHitatRz. t. IIL, pag. 173 et 245$)$.

(Note des traducteurs.) 
On rencontre trés-généralement ì la face:

6. L'os maxillaire unféricur, os considérable, en forme d'are, ordinairement armé de dents et articulé mobilement, à son extrémité postérieure, avec le temporal.

7. I'os maxillaire supérieur, en face et andessus du précédent; sa partie antérieure, qui est fort souvent séparée du reste, reçoit le nom d'os intermaxillaire.

8. L’os palatin, situé derrière le maxillaire. supérieur.

9. L'os malaire ou zygomatique, situé à côté et en dehors de l'os maxillaire supérieur.

10. L'os du nez, situé au-dessus du malaire, entre lui et le frontal; il occupe la ligne médiane.

11. L'os lacrymal ou unguis, situé dans la mème région, mais un peu plus en dehors.

12. Le cornet inférieur, en dedans du maxillaire supérieur.

13. Le vomer, os moyen et impair.

$$
\text { S. } 118 \text { bis. }
$$

Les poissons cartilagineux et ossenx se distinguent considérablement les uns des autres par la disposition de leur crâne, de sorte qu'on ne peut guère leur assigner de caractères communs. Parmi les cartilagineux eux-mêmes, les cyclostômes el les plagiostrimes, offrent si peu de concordance qu'on est obligé d'úludier chaque: groupe à part. 


\section{a. poissons cartilaginevi.}

a. Cyclostômes:

$$
\text { S. } 1 \text { I } 9 .
$$

I a tète des crclostômes est fort alongée et se compose de plusieurs pièces successives s'imbriquant en partie les unes les autres d'arrière en avant el de haut en bas.

La plus postérieure, qui est à la fois la plus, grande de ces pièces, présente une forme trèsirrégulière. Elle a la configuration d'un carré long, et est formée d'une moitié postérieure et d'une antérieure, dont la première est fort compliquée. Elle est un peu plus large, mais plus courte que l'antérieure, se montre convexe en arrière, légèrement concave en avant. A son extrémité postérieure elle se renfle des deux còlés en une éminence osseuse, arrondie; ensuite elle se déploie en un large anneau, descendant obliquement en dehors, dont la cavité est remplie par une membrane, et derrière lequel immédiatement descend un crochet légèrement convexe en dehors et aplati d'avant en arrière.

Dans la fosse antérieure, il existe un os transversal, arrondi, creux, qui s'étend presque dans toute la largeur de celte pièce d'un côté à l'autre.

La moilié antérieure de celte pièce forme une 
plaque simple, convexe à sa face supérieure, concave à l'inférieure, terminée par un bord antérieur légèrement concave.

La seconde portion des cartilages céphaliques consiste, en majeure partie, en une lame semblable à celle qui vient d'être décrite; mais elle se termine en avant par un bord légèrement convexe; elle est un peu plus petite que la poslérieure, qui la recouvre dans sa moitié tournée en arrière.

Elle est terminée postérieurement par une pointe mousse, et il nait des extrémités latérales de son bord postérieur une forte apophyse verticale qui se dirige en bas.

Entre cette apciphyse et l'anueau de la première portion, qui est la plus postérieure, on trouve une lame transverse, en forme de pelle, aplatie de haut en bas, mousse en avant et en arrière; elle est unie à ces deux parties au moyen d'un ligament; sa plus grande longueur est d'avant cu arrière; en dehors elle est limitée par un bord convexe, en dedans par un bord concave.

En avant de l'apophyse descendante postérieure, il en existe une autre semblable, mais plus courte, qui est unie lâchement à la face inférieure du deuxième bouclier.

La troisième portion des cartilages céphaliques se compose d'un nombre encore plus considérable de pièces.

La plus antérieure de celles-ci est un auneau 
D'ANATOMIE COMPARÉE.

roud, fort, armé de dents, qui entoure l'onver. ture buccale; il touche par sa partie supérieure au bord antérieur du second bouclier qui le recouvre en partie.

Au point de réunion deses moitiés supérieure et inférieure est appliqué un os fort, triangulaire, alongé, qui leur est uni mobilement au moyen d'un ligament capsulaire; il se dirige en arrière et en bas, et est situé sous les pièces latérales que nous avons décrites en traitant de la seconde portion.

Au milieu de sa moitié inférienre on voit s'articuler un os mince, un peu plus long supérieurement, qui s'élargit en plaque à sa partie antérieure.

Au-dessus de cette pièce, mais aussi sous l'œsophage, on trouve un cartilage beaucoup plus long, aplati d'un côté à l'autre, fort alongé, apointi en arrière, s'étendant jusqu'à l'origine des organes respiratoires.

Ce dernier supporte à son extrémité antérieure un cartilage court qui lui est uni par un ligament; ce cartilage a la furme d'un Y dont les branches sont tournées en haut; il est situé entre les pièces latérales, antérieures et courtes de la seconde portion, et remonte vers la pièce moyenne.

Tous dounerons la désignation de toutes ces pieces lorsque nous considérerons les os de la tète des poissons osseux. 


\section{b. Plagiostómes.}

\section{S. 120.}

Les plagiostômes diffèrent des cyclostômes et entre eux-mêmes; ces différences sont quelquefois très-prononcées. I.es caractères les plus généraux de leur tête sont les suivans.

La partie supérieure et postérieure, qui est la plus grande, consiste en un cartilage creux, quadrilatère, ordinairement peu élevé et plus ou moins alongé, qui contient, $1^{\circ}$ l'encéphale; $2^{\circ}$ les organes de l'ouie, de la vision et de l'olfaction. On y peut distinguer très-géneralement une face postérieure, une supérieure, une inférieure, deux latérales et une antérieure, ou au moins une extrémité antérieure ou un bord antérieur.

La face postérieure de ce cartilage céphalique commun est peu haute, assez verticale; elle contient dans son milieu une ouverture ronde, destinée au passage de la moelle-épinière, et qui conduit dans le canal vertébral : c'est le trou occipital.

A côté et un peu au-dessous de ce trou, on voit, de chaque côté, un condyle qui sert ì l'arti. culation du cartilage céphalique avec les apophyses articulaires les plus antérieures du rachis; au-dessous on trouve, dans la ligne mé- 
1) Anatomie comparí.

diane, une surface de forme variée qui correspond aux corps de la colonne vertébrale.

¿. a fuce supérieure, large, est, dans une étendue plus ou moins considérable de sa partie moyenne, beaucoup plus mince que dans le reste ct que tout le cartilage céphalique en général. Celte partie moyenne peut être lésignée sous le nom de fontanelle.

La face inférieure offre à peu près la même longueur que la supérieure; elle est simple, sans inégalités, plane ou légèrement concave.

Les faces latérales, pour la plupart moins élevées, sont plus composées. Elles présentent postérieurement une saillie plus ou moins forte, apres laquelle vient une cavité orbitaire le plus souvent considérable, ouverte en avant et en arrière, fermée supérieurement et inférieurement par une partie saillante des faces supérieure et inférieure; il en résulte, en cet endroit, un rétrécissement variable de la tête.

En avant de l'orbite, il existe, de chaque côté, une saillie plus faible, creuse et ouverte en bas, la cavilé olfactive qui renferme l'organe de l'odorat.

On trouve fort'généralement, si ce n'est constamment, en avant de la cavité olfactive, un "artilage considérable, alongé, Iransverse, mince et aplati.

De lí, la tète se prolonge en un museau terminal plus fort ou plus faible, plus ou moins 
composé, consistant en une portion moyenne. et deux latérales, formées chacune de pieces cartilagineuses propres.

\section{S. 121.}

La partie inférieure de la tête, qui est aussi la plus petite, est articulée d'une manière mobile avec la précédente, par deux points: $1^{\circ}$ à sa partie postérieure, latérale et inférieure; $2^{0^{*}}$ plus antérieurement.

La première facette articulaire est un enfoncement situé derrière l'orbite; la seconde est placée en arrière, et endehors de la cavilé nasale.

Dans l'enfoncement, on voit très-généralement s'articuler, par engrenage, un os simple: alongé, l'os carré, qui est la partie inférieure et externe du temporal.

Cet os est dirigé en avant et en bas; il présente à son extrémité inférieure et antérieure, en avant, sur une éminence articulaire antérieure, un cartilage très-considérable, garni de dents; ce cartilage se dirige en avant; il est uni le plus souvent d'une manière mobile dans la ligne médiane avec celui du côté opposé, et forme avec lui un arceau convexe en dehors et en avant. Immédiatement en arrière de ce dernier, on voit s'articuler avec le premier un autre cartilage également dirigé en avant, qui appartient aux organes rle la déglutition et de la res- 
D'ANATOMIE COMPAREE.

piration, et que par cette raison je ne décrirai point ici.

Au-dessus du cartilage armé de dents qui vient d'ètre décrit, on en trouve un autre, ordinairement plus petit, mais semblable, qui lui correspond de haut eu bas; il est égnlement garni de dents, qui sont opposées aux premières, et situé sous la partie antérieure de la face inférieure du crâne. Vers le tiers antérieur du bord supérieur de ce cartilage, on voit naître un ligament par lequel il s'attache, d̀ l'endroit déjà indiqué, à la partie supérieure de la tête.

Ces deux cartilages, les plus volumineux de celte région, s'articulent mobilement à leur extrémité extérne et postérieure; cette articulation permet qu'ils s'ćcarient l'uu de l'autre en haut et en bas, dams les mouvemens nécessaires pour ouvrir et fermer la bouche. Ce point de réunion se divise très-généralement en deux moitiés séparées, dont l'interne est formée en haut par une saillie, en bas par un enfoncement, tandis que la moitié externe présente une disposition contraire.

Il s'y ajoute souvent encore quelques cartilages alongés, beaucoup plus petits, qui descendent en se succédant immédiatement à compter du supérieur jusqu'à l'inférieur, et sont unis mobilement entre eux et avec les précédens. Ils sont situés à la face exteine de ces deux cartilages; le superienr et le moyen, si celui-ci 
existe, sont dirigés de hant ẹn bas et d'avant en arrière, l'inférieur se flirige dans le sens contraire, de sorte qu'ils se rencontrent, à peu de distance de l'articulation des deux grands cartilages, sous un angle aigu ou obtus, et peuvent même preudre tous une position parfaitement verticale, si la bouche est largement ouverte.

$$
\text { S. } 122 \text {. }
$$

Les différens genres des plagiostômes offrent, en partie, des modifications fort notables de cette organisation générale de la tête.

La disposition des chimères diffère le plus de celle des autres, et se rapproche au contraire, sous plusieurs rapports, de celle des cyclostômes, comme cela a aussi lieu pour le rachis.

Toute la partie supérieure de la tête est ici très-alongée, haute, aplatie d'un côté à l'autre; elle cousiste essentiellement en une lame inférieure, trausverse, et en une supérieure, verticale, située dans la ligne médiane.

La portion crânienne est très-petite; elle porte au milieu de sa face supérieure une forte crête longitudinale, verticale. La paroi postérieure descend obliquement en bas et en arrière. Le trou occipital a la forme dune feuille de tréfle; les condyles sont fort alongés, aplatis d'un côté à l'autre; la partie de l'articulation occipitale qui correspond au corps de la ver- 
1'A NATOMIF COMPARÉE.

ièbre forme un enfoncement transversal. La face inférieure est fort concave. Les faces latérales se rencontrent dans la ligne médiane; elles ne sont séparées que par une cloison membraneuse, mince. La face inférieure de l'orbile est très-large; la supérieure est étroite, surtout au milieu; en avant on voit une lame longitudinale, considérable, se détacher transversalement de la lame moyenne et se recourber en dehors; l'orbite est entièrement ouvert à la partie antérieure. La partie située au-devant des orbites forme aussi un toit escarpé, dont l'extrémité antérieure et inférieure est formée par les fosses nasales, situées très-près l'une de l'autre. De leur angle interne s'élève un petit cartilage mince, en forme d'Y, dont le manche ou le corps est tourné en haut.

La supérieure des deux lames dentifères est soudée immobilement à la portion postérieure ct supérieure de la têie; l'inférieure qui est haute, mais étroite, s'articule aussi d'une manière immédiate avec la face inférieure de la tête.

Les cartilages accessoires des denx arcades dentaires sont assez développés; ils touchent par leur extrémité supérieure aux bouts des branches du carilage en forme d'Y.

$$
\text { S. I } 23 .
$$

Les squales at les raies se ressemblent par 
l'aplatissement du crâne; par l'écartement assez considérable des fosses nasales, entre lesquelles la paroi antérienre de la portion supérieure de la tête n'est fermée que par une membrane mince; par la présence de la fontanelle et du cartilage qui supporte l'arcade dentaire inférieure; mais ces poissons diffèrent par les conditions suivantes.

Chez les squales, la partie moyenne de la surface articulaire qui unit l'occiput et le rachis est un enfoncement arrondi, correspondant exactement aux faces antérieure et postérieure du corps des vertèbres; chez les raies, c'est au contraire un bord mince et concave; les condyles sont sitnés transversalement chez les premiers, verticalement chez les dernières. I a tite cntière est plus alongè chez lés raies; clle est à la fois moins convexe que chez les squales. Les orbites de ceux-ci sont, de tons còtés, beaucoup plus couverts que chez les raies. En avant, la paroi supérieure de l'orbite est percée d'une ouverture qui est surtout fort considérable chez l'ange.

La fontanellé est beaucoup moins distincte dans les squales que dans les ruies, à cause de l'épaisseur considérable des parois du cràne. Cette lacune est surtout considérabte chez l'aigle - cie mer et la pastenague comimune (r.pastinach), où elle occupe presque tonte la longueur de la paroi supérieure du crâne; elle est beaucoup 
D'ANATOMIE COMPARÉE.

moindre chez l'oxyrynque et la raie blanche.

La partie antérieure de la portion supérieure de la lète est beaucoup plus développée chez les raies que chez les squales. Elle l'est au moindre degré chez l'ange ( $s q$. squatina), où le carlilage céphalique est fortement tiré en travers dans la région de la cavilé olfactive, la saillie moyenne élant très-peu considérable et même concave au centre. Cette cavité est formée, chez la plupart des squales, de trois cartilages alongés dont un moyen et deux latéraux, qui se rencontrent dans la ligne médiane. Chez les torpilles il n'y a que les deux cartilages latéraux qui se dirigent parallelement. en avant à peu de distance l'un de l'autre. Chez la plupart des raies, ces cartilages se réunissent et forment une pointe plus ou moins longus. Cependant chez l'aigle de mer (r.aquila) cette partie est plus petite que mème chez plusieurs squales.

Les pièces accessoires des cartilages dentifères existent chez les squales, et non, comme il pa... raît, chez les raies. Elles. offrent égalenient des variétés. Ainsi il y en a trois chez le griset et l'ange, seulement deux chez la petite roussette ( $s q$. catulus); on les rencontre au summum de développement chez l'ange, et au minimum chez la petite rousselte, où la pièce moyenne, à en juger par la longueur considérable des deux piéces existantes, semble partagée entre la supérieure et l'inférieure. 
Dans les raies je n'ai pas trouvé de trace de ces pièces, nême sur de grands individus.

I ces raies et les torpille's possèdent, en revanche, de chaque côté un cartilage propre, antérieur, alongé, fort développé surtout chez ces dernières, qui est posé sur l'extrémité externe de la cavité olfactive, et se tourne directement en dehors pour s'unir, d'une manière mobile, aver la région antérieure de la racine de la nageoire.

Chez l'aigle de mer ce cartilage n'est que très petit, mais large, disposition en rapport avec la largeur considérable de la tête.

La forme la plus particulière de la tête se rencontre dans les aygènes. Toute la partie supérieure de cet appareil représente une croix; les parties latérales se distendent de chaque côté, à partir de la cavité olfactive, en formant un angle droit, et constituent un tube large et plat, à l'extrémité externe duquel se trouve inférieurement l'orbite, qui est superficiel et dans lequel chemine le nerf optique.

Vers le milieu de son bord postérieur, on voit se délacher une branche mince qui se porte en dehors et en arrière, et qui à sou extrémité externe se réfléchit de nouveau en avant vers la branche drincipale. La partie antérieure de la branche longitudinale est formée par la pièce cartilagineuse moyenne, et les latérales qui sont situées en avant; ces pièces, d'ordinaire peu dévelop- 


\section{D'ANATOMIE COMPARÉ.}

pées chez les squales, sont ici fort considérables. Les picees latérales, parallèles à la moyenne qui est plus forte, se porient de la base des branches latérales directement en avant, el toutes trois s’attachent avec leur extrémité antérieure, à une grande lame transversalement plus large que le crâne proprement dit. Cette lame n'offre rien de remarquable.

$$
\text { S. } 123 \text { bis. }
$$

L'organisation des esturgeons forme encore en cela le passage des poissons cartilagineux aux poissons osseux.

La forme de toute la tête est fort alongée, triangulaire; all-delà même de l'ouverture buccale siluée un peu en arrière du milieu de sa face inférieure, elle se termine par une longue pointe. La base du tout est cartilagineuse, et sur ce cartilage sont posées des écailles osseuses, qui représentent d'une' manière plus ou moins distincte chacun des os des poissons osseux.

Le cartilage céphalique supérieur se continue en arrière avec le rachis, qu'il déborde un peu supérieurement. A peu de distance en avant de l'extrémité postérieure, la face supérieure descend sans interruption de haut en bas et d'arrière en avant, et présente une ouverture qui conduit à la cavité du crâne. Les faces latérales s'inclinent dans leur moitié postérieure; leur direction est oblique de haut en bas et de 
dehors en dedans; elles affectent un sens opposé dans la moitié antérieure, et se continuent insensiblement avec la face supérieure.

Les orbites sont de faibles enfoncemens fermés en avant par une saillie cartilagineuse qui forme ici le commencement de la muitié antérieure du cartilage céphalique. Ils sont entièrement ouverts en bas et en arrière, et leur circonférence est formée ici et en haut par mu seul os, composé de trois branches qui se réunissent sous des angles droits.

Très-près de l'orbite est située la petite cavité olfactive qui est arrondie.

La face inférienre est très-étroite, surtont dans sa moitié postérieure; elle descend obliquement d'arrière en avant dans cette moitié, et monte au contraire dans sa moitić antérienre: il en résulte que toutes deux se rencontrent sous un angle obtus. Elle offre dans sa partie antérieure une forte saillie, longitudinale, moyenne, et denx sillons considérables qui se dirigent à còté d'elle et disparaissent iusensiblement en avant.

La portion céphalique inférienre, unie d'une manière mobile avec la supérieure, est plus compliquẻe que chez les plagiostomes.

Le stylet cartilagineux qui s'articule avec lat face latérale du crâne existe également ici, mais ii se compose de deux pières cartilagineuses, dout l'une est supérieure, plus grande, et s'élargit 
D'ANATOMLE COMPARÉE.

considérablement de haut en bas, et dont l'antre, inférieure, de moilié plus petite, est dirigée en avant et en bas; elles sont unies par une articulation mobile. A ces cartilages se joint une écaille osseuse, considérable, large, se rétrécissant de haut en bas, qui est située derrière la pièce supérieure, et s’articule avec le bord externe de la face supérieure du crâne.

La pièce stylö̈de inférieure s'articule à son extrémité supérieure avec l'os hyoïde, et touche par l'antérieule, à la mâchoire inférieure qui est alongée; elle mesure par conséquent la distance qui sépare ces deux os.

Elle est en outre contiguë, par son extrémilé antérieure et plus en avant qu'en dehors, conjointement avec la mâchoire inférieure, à deux pièces sus-maxillaires : l'une de ces pièces est antérieure, étroite, osseuse, et l'autre est postérieure, large, cartilagrineuse, bcaucoup plus grande; clles s'uwissent mobilement en avant. entre elles. et dans la ligne médiane, avec leur congénère du côté opposé. Le bord postérieur des deux grandes pièces supporte une plaque rhomboïdale, large; de l'exirémité externe de la piece antérieure, osseuse, on voit se détacher un us styloilde, mince et court, qui se dirige en haut et en dedans.

Cies quatre piéces sont situées en face de la mâchoire inférieure qui est beaucoup plus pelite; elles sont libres sous la face inférieude du 
crâne et forment une plaque large. Vraisemblablement la pièce moyenne, qui est la plus grande, représente le grand os sus-maxillaire supérieur, armé de dents, que l'on rencontre dans les plagiostómes, landis que les deux piéces antérieures qui sont petites, osseuses, sont les analogues des petits os accessoires externes des squales; la plaque postérieure, moyenne, semble être une pièce nouvelle qui a été surajoutée.

\section{b. Poissons osseux.}

\section{S. 124.}

La tête osseuse des poissons osseux se distingue d'une manière variée et frappante d'avec les cartilages céphaliques des poissons cartilagineux: $1^{\circ}$ par la forme totale, puisqu'elle n'est jamais aussi aplatie que la tête de la plupart de ces deruiers poissons; $2^{\circ}$ par sa composition, qui ne consiste pas, comme chez les cartilagineux, en un petil nombre de pièces, mais qui résulte toujours d'unie grande multiplicité d'os. Elle l'em. porte mème, par cette circonstance, sur la tète des hauis vertébrés, soit parce qu'elle renferme plusieurs os propres, soit que certains points d'ossification, qui se soudent entre eux pendant la vie chez les animaux supérieurs, restent constamment séparés chez les poissons. La plupart de ces us sont immédiatement contigus les uns aux autres, sans mobilité, et unis par des sutures 
1) ANATOMIF COMPARÉr.

écailleuses; il n'y a que les os antérieurs et inférieurs, entourant et constituant la cavité buccale, qui soient articulés mobilement avec les os supérieurs qui forment le crâne et la partie supérieure et postérieure de la face.

Plusieurs poissons, entre autres le brochet et le saumon, ont une base cartilagineuse très-distincte, conforme au type de l'esturgeon; cette base supporteles pièces osseuses, seules existantes chez la plupart des poissons osseux. Comme les os de la tète des poissons, sont couverts de peu de muscles, et par conséquent situés presque librement sous la peau, la forme extérieure de la tête osseuse est manifeste : nous pourons d'autant plus passer sous silence les différences qui ont lieu à cet égard, que l'histoire des os en particulier nous fournira l'oceasion d'indiquer la part que prend la forme de chaeun d'entre eux à la forme de l'ensemble.

L'ordre le plus convenable à suivre dans cette description nous semble devoir consister à commencer par la partie de la tête qui se continue avec le rachis, à étudier à part chaque os susceptible d'être isolé, et à déterminer en même temps d'avarce quelle collection de pièces osseuses correspond à un os composé chez les animaux supérieurs, particulièrement chez les mammifères, puisque dans la classe des reptiles le rumbre des pièces osseuses séparées est presque aussi considérable que chez les poissons, 
ct que les os du crâne des oiseaux se confondent d'extrêmement bonne heure en une pièce unique.

Nous devrons par conséquent conmencer par déterminer les pièces qui représentent l'os basilaire, et spécialement surtout la portion occipilale de cet os.

1 Portion occipitale de l'os basilaire.

$$
\text { S. } 125 .
$$

I'occipital des poissons se compose de quatre pièces osseuses : deux sont impaires et occupent la ligne moyenne; deux sont paires et latérales.

I. Immédiatement au devant de la premiire vertèbre existe un os dont la plus grande longueur est ordinairement d'avant en arrière, el la minceur la plus considérable de haut en bas; direction dans laquelle il est un peu aplati.

Cet os présente sa plus grande épaisseur en arrière, où l'on rencontre une facelte articulaire, encrouttée de cartilage, de forme arrondie. concave, un peu plus large dans le sens transversal que longitudinalement. Le bord antérieur de cet os forme une large suture.

La face supérieure en est plus ou moins concave d'avant en arrière, à chacun des còtés. Les deux bords latéraux de cette face, qui sont larges, et par lesquels elle se continuc avec la face inféricure, sont également rugueux. Che\% 
1) ANATOMLE COMPARÉ.

flusieurs poissons, par exemple l'anguille (murcena anguilla), il existe, entre les deux concavités latérales, une troisième concavité moyenne, et les bords latéraux ne s'atteignent pas. Chez d'autres, par exemple les congres ( $m$. conger), le saumon (salmo salar), les carpes, il existe à la place de cet enfoncement moyen une forte crête, dont la moitié postérieure présente une disposition ruguease qui se continue avec les bords latéraux. Cette crête est très-prononcée chez quelques poissons, surtout les turbots (pleuronectes maximus), le flet (flesus), le denté (sparus macrophthalmus), et ia daurade ordinaire ('sp. auralus); mais elle y est interrompue dans sa partie inférieure par une grande ouverture qui résulte vraisemblablement du développement considérable qu'a pris l'ostéïde du labyrinthe, situé en cet endroit.

La face inférieure de l'os est convexe d'un côté à l'autre, plane sur les parties latérales; elle offre au milieu plusieurs saillies longitudinales, considérables, très-rapprochées, par lesquelles elle s'engrène dans des enfoncemens correspondans qui sont creusés dans le corps du sphénoïde, intercalé sous l'os qui nous occupe en ce moment.

Chez les carpes, cet os se prolonge considérabiement en arrière et en bas; il forme une apophyse Iriangulaire, qui est beauconp pluss larue daus sa moitié antérieure que dans la pos- 
térieure, qui est en outre fort concave et qui supporte une éminence odontoide large. Dans sa moitié postérieure cette apophyse cst fortement comprimée d'un côlé à l'autre; elle est trèsconcave à sa face supérieure, et ne tient au reste du corps que par deux racinces larges, séparécs l'une de l'autre par une ouverture considérable.

Cet os est evidemment analogue au corps d'une vertébre. Sa position, sa forme, sit ressemblance avec les corps des vertebres, son articulation avec l'allas, la détermination des os du crâne avec lesquels il s'articule, démontrent cetre analogie d'une manière si positive, qu'il n'y a qu'une opinion sur ce qu'il doit représenter, c'est-à-lire le corps de la vertèbre occipitale.

2. A côlé et au-dessus de cet os existent deux autres os d'une forme irrégulière, mais qui sont généralement composés de trois branches réunies au milieu; de ces lranches l'une est externe, une autre c'st aniérieure, une troisième est supérieure. Cihez quelques poissons par exemple les congres, il s'en ajoule une quatrième qui résulle de la bifurcation de l'antérieure, devenue plus large, en deux autres prolongemens, dont l'interne est le plus petit. Inférieurement cet os présente un bord rugueux, par lequel il sapplique contre le bord égalcment rugueux el supérieur de l'os précédent. et qui, chez divers poissons, offre les mêmes différences que le prémier ainsi daus le cas où l’os précédent a un 
DANATOML COMPARE.

bord ruglieux et moyen, la partie postérieure de la rugosité du second os se porte en dedans et en avant, tandis que si c'est la disposition contraire qui se rencontre, il ne s'éloigne que peu de sa direction. Ces dewx os ne s'alteignent dunc pas sur la ligne médiane dans le premier cas, mais bien dans le second; ce qui produit ce résultat trés-remarquable: que chez un grand nombre de poissons, le corps de l'occipital ne prend pas une part immédiate à la formatiou du cràne, dans une grande partie de son étendue postérieure; ses deux parties latérales se coufoudant, comme chez les batraciens, dans la ligne médiane. Clıez quelques-uns qui présentent la première disposition, nous citerons spécialement J'anguille ( murena anguilla), du moins quelquefois, et le poisson Saint-Pierre (zeus fuber), les deux os latéraux se soudent au contraire dans la région supérieure, sur la ligne médiane.

En haut cet os est circonscrit, dans tout son pourtour, par uu bord large et rugueux.

Il a une face intérieure, une antérieure, une postérieure et une inférieure, qui sont toutes concaves, surtout l'antérieure. Ses faces inférieure, intérieure et antérieure font partie de la surface interne de la cavilé crânienne; l'extérieure appartieut à la surface externe du crâne. Ta partic inférieure de cet os est traversée, de dedans en dehors et d'avant en arrière, par un 
canal considérable qui loge le nerf pneumogastrique, ou au moins une partie de ce nerf.

Cet os s'articule constamment par son bord inférieur avec le corps de l'occipital, souvent aussi avec l'os congénère du côté opposé, par la partie externe de son bord supérieur avec l’os mastoïdien situé au dessus de lui, par la partie interne du même bord avec un autre os qui sera décrit plus loin. Cette pièce de l'occipital offre plusieurs variétés de formes dignes de remarque.

La moindre de ces modifications est le développement de son extrémité supérieure en une espèce de dent; ce dont on trouve des exemples dans les spares, les orphies (esox bellone) et les muges ( mugil). Quelquefois il semble partagé en une moitié supérieure et une inférieure. La supérieure est la pièce ordinaire, qui est ici dentelée; l'inférieure, fixée comme la première sur le corps de l'occipital, est très-petite, alongée, mince, transversale, et présente une ressemblance frappante avec une côte.

Chez les carpes cet os est percé, à la partie interne de sa paroi postérieure, par une ouverture alongée, très-considérable; il forme chez ces êtres et dans ce point un anneau entouré en haut et en dedans seulement par un bord étroit; anneau dont le bord interne se réunit dans la ligne médiane avec celui de l’os du côlé opposé. 
DANATOMIE COMPARÉ.

Cet os est sans contredit analogue à la portion articulaire d'une vertèbre; cela est démontré par la forme, la position et les rapports du trou mentionné, qui donne passage au nerf de la respiration. On peut par conséquent donner à cet os, d'après sa position, le nom de pièce latérale, on latérale inférieure de l'occipital, et d'après sa fonction, celui de partie ou pièce ar.. liculaire de l'occipital.

3. Le troisième os est situé au dessus de la deuxième paire, et entre ou au dessous d'un autre os qui vient en dehors et au dessus, dont je parlerai plus loin, sans décider s'il appartient à l'os temporal ou à l'occipital, ou bien si c'est un os propre. Le troisieme os touche en avant à la parlie postérieure et interne du bord de la clavicule. Il est composé ordinairement d'une moitié supérieure, horizontale, et d'une inférieure, verticale, qui passent l'une dans l'autre sous un angle droit. Sa face postérieure et exterue est surmontée au milieu d'une apophyse plus ou moins prononcée, comprimée d'un côté à l'autre, qui correspond à la crête nccipitale. Chez quelques poissons, par exemple le brochet et les scorpènes, cette apophyse s'élève seulement de la moitié inférieure et verticale de l'os; chez d'autres, par exemple les carpes, les goudes, elle existe déjà à la moitié supérieure. Chez les congres (murcena conger) cet os est tres-petit et mince, sans apophyse. 
M. Cuvier a donné à cet os le nom d'interpariétal, M. Bojanus celui de crête occipitale (crista occipitis). Bien que ce dernier auteur désapprouve, et peut-être à tort, la dénomination donnée par le premier, le nom qu'il lui substitue ne me paraît pas devoir être admis. Supposons que l'os en question corresponde non-seulement à la partie latérale, mass encore à la partie inférieure de la portion squameuse de l'occipital, ce qui pourrait bien être, puisqu'il est presque toujours situé un peu plus bas que le troisième, celui - ci serait évidemment la partie supérieure de la portion squameuse, qui est située en effet plus ou moins entre le pariétal, et qui dans plusieurs mammifères, par exemple le chat, ne se soude véritablement pas avec l'occipital, mais avec le temporal. Le nom choisi par M. Cuvier est par conséquent trèsexact par l'une et l'autre raison, puisque ce nom, sans déterminer de quel os des animaux supérieurs cet os fait partie, n'en indique que la situation. Supposons que l'os que je vais décrire n'appartienne nullement à l'occipital, comme je le présume, la dénomination de portion squameuse de l'occipilal conviendrait mieux que celle de crête occipitale, afin de rappeler son analogie avec l'occipital des animaux supérieurs. Qiroique la portion squameuse de l'occipital soit analogue à l'apophyse épinceuse des vertìhres, on ne peut pas dire que la crète occipritale le soit 
1). INATOMI: COMHЛLEL.

aussi, car celle-ci n'est qu'une partie de ladite portion.

La portion squameuse et surtout sa partie supérieure ou l'os interpariétal, présente chez plusieurs poissons, particulièrement chez les spares, les labres, les bodians, les sphyrènes, les perches, les mules, les muges (mugil) les castaynoles (brama), les scienes, les scombres, les ésoces, les gades, les cyprins, les coryphènes, une crète plus ou moins élevée, souvent trèsprononcée, mince, tranchante, triangulaire, verticale, moyenne, dirigée en haut et en arrière.

Cetle crète est surtout très-haute dans les castagnoles (brama) et les corypliènes, où elle forme la partie postérieure, la plus élevée et la plus longue de la crête moyenne et verticale qui s'élève de la face supérieure du crâne.

Elle est le plus souvent simple; chez quelques-uns, par exemple le bodianus maculatus, elle s'épanouit, au milieu de sa longueur, des deux côtés en deux crêtes transversales. Chez l'anableps tetrophthalmus, elle se termine en arrière par deux longues lames assez larges. Chez l'ornbrine barbue (scicena cirrosa) la partie antérieure de la pièce supérieure de la portion squameuse, pièce supérieure qui est fort grande, n'est pas mince comme de coutume, mais trèséraisse d'un côté à l'autre ei arrondie; la partie postérieure est au contraire mince et tranchante, cornme on la rencontre ordinairement. 
Cette crête manque ou est presque insensible chez d'autres, par exemple le loup ou chat marin (anarrhichas lupus) et l'hirondelle de mer.

Le volume de la portion squameuse varie aussi considérablement. Chez plusieurs poissons, surtout le pimelodes scheilan, elle est petite en proportion et ne s'étend pas fort en avant, de manière que les pariétaux s'arliculent sur la ligne médiane, soit dans la totalité, soit dans la majeure partie de leur longueur.

Chez d'autres au contraire, par exemple le sharmut(silurus anguillaris), les loricaires, le loup marin (anarrlichas lupus), les labres, les sparcs, l'hirondelle de mer, l'ombrine barbue (scicence cirrosa); -cette même portion est tellement développée, que les pariétaux en sont tout-à-fait refoulés sur le côté et sont entièrement séparés.

Quelquefois, surtout chez le saluth (silurus glanis; elle se confond avec les pariétaux ou les anéantit; dans ce cas on n'en trouve point de trace autre que la partie antérieure de la porlion squameuse qui s'étend plus en avant que de coutume.

La partie antérieuré de la portion squameuse, ainsi prolongée, en avant, est fendue sur la ligue médiane, et forme une espèce de fontanelle dont nous parlerons plus loin avec plus de détails.

$$
\text { S. } 126 .
$$

4. Indépendamment de ces os ordinaires, il 
1) ANATOMIE COMPARÉ.

existe, chez plusieurs poissons, deux autres os plus petits, situés en haut et en arrière, entre la pièce supérieure latérale de l'occipital et la pièce osseuse non déterminée dont il vient d'être question. Chez le saumon, ces os sont petits, triangulaires, mais ils font une saillie distincte en arrière.

Peut-ètre ces os correspondent-ils aux os wormiens, qui existent précisément ici entre les os contigus.

2. Portion sphénoïdale de l'os basilaire.

$$
\text { S. } 127 .
$$

1. Dans la direction du corps de l'occipital on trouve en avant un os irès-alongé, légèrement convexe à sa face inférieure, concave à la supérieure. Cet os se termine antérieurement et postérieurement, dans ce dernier sens surtout, par deux branches latérales; dans sa moitié postérieure, du moins au-delà de son milieu, il est considérablement plus large que dans l'antérieure. Sa face inférieure présente très-généralement en avant des sillons longitudinaux; la supérieure est concave chez quelques poissons, par exemple les gades; chez d'autres au contraire elle est convexe, comme chez les murènes.

Postéricurement il s'étend très-loin sous la partie antérieure du corps re l'occipital, ảont il embrasse la branche longitudinale et inférieure 
avecses larges branches latérales; son deuxième tiers supporte, d'avant en arrière, cing os dont trois seulement, peut-ètre seulement deux, appartiennent au sphénoïde chez les animaux élevés; les autres ont reçu une autre détermination.

Cet os correspond également à un corps de vertèbre, et est incontestablement le corps du sphénoïde, dont la forme alongée est en rapport avec la longueur considérable de la moitié antérieure du crâne.

2. La plus postérieure des trois pièces mentionnées est irrégulièrement quadrilatère, légèrement convexe en dehors, un peu concave en dedans; elle correspond à peu près au second cinquième de la longueur du corps du sphénoïde. Sa face interne est partagée par une saillie Jongitudinale en une moitié antérieure et une postérieure; celle-ci reçoit une partie de l'organe auditif, et est ordinairement un peu plus petite que l'autre. La moitié antérieure présente en avant une ourerture considérable pour le passage d'un nerf. Il y a une seconde ouverture de ce genre près du bord postérieur, chez plusieurs poissons, par exemple les carpes.

D’après M. Bojauns, cette lame appartiendrait en toute certitude à l'os sphénoïde, dont elle représenterait la grande pièce latérale, ou sa grande aile; cependant cette détermination ne me parait rien moins que parfaitement certaine, et cela par les raisons suivantes. 


$$
\text { 1) ANATOMIL COMPALEE. }
$$

Cette opinion a contre elle plusieurs considérations.

La première est la position de cet os. En effet, en haut il est contigu seulement à des os qui appartiennent au temporal, en arrière au corps et a la portion articulaire de l'occipital; la grande aile du sphénoïde touche, atu contraire, au pariétal et au frontal, ou au moins à l'un de ces os.

Or les premières conditions sont évidemment celles du rocher des vertébrés supérieurs. L'application de l'os dont il s'agit sur le corps du sphénoïdè, chez les poissons, ne permet de rien. conclure, parce que d'une part les corps du. sphénoide et de l'occipital ne font essentiellement qu'un; que d'autre part cette circonstance est en rapport avec la forme alongée de toute la tête, mais surtout avec la longueur très-considérable et la grande extension du corps du sphénoïde en arrière.

La seconde considération qui s'élève contre la proposition de M. Bojanus est la part que prend cet os à l'organe acoustique.

La troisième est l'étude de la disposition successive d'avant en arrière. Le rapport de cet os avec plusieurs nerfs, existant chez la carpe, éclaire surtout dans cette recherche. Le plus postérieur, qui est tout à la fois le plus gros, est manifestement une partie du nerf respiratoire qui sort entre la portion articulaire de l'occipital et l'os qui vient d'être considéré. Cettc division 
du nerf n'est séparée de l'autre partie que par un pont osseux, mince, existant au bord antérieur de la portion condylö̈lienne, et va se ramifier avec elle à la branchie postérieure.

En avant de cette partie ncrveuse, on voit sortir de l'ouverture postérieure de l'os un nerf plus petit, distribué aux première et seconde branchies et à la partie postérieure de la memisrane palatine. Ce secund cordon nerveux appartient au premier, dont il constitue la partie antérieure.

L'ouverture antérieure donne passage à un troisième nerf ayant à peu près le mème volume, qui se rend à la première branchie et à la membrane du palais. C'est sans contredit la partie antérieure du nerf respiratoire; il correspond vraisemblablcment au nerf glosso-phaigngien des animaux plus élevés.

Enfin au devant de l'os en question, entre lui et celui qui le précède, il se trouve un nerf beaucoup plus considérable, qui se porte à la masse musculaire de la face; ce nerf est évidem. ment le nerf sous-maxillaire.

Or, chezles autres vertébrés, les nerfs pneumugastrique et glosso-pharyngien sortent entre le rocher et l'occipital, le nerf sous-maxillaire par l'extrémité postérieure de la grande aile da sphénoïde, et souvent entre cette aile et le rocher, parce que le trou ovale n'est pas fermé en arrière; la disposition de cet os est donc encore 
1) ANATOMIE COMPARÉE.

la méme que celle du rocher des autres vertébrés.

On pourrait être tenté d'opposer à ces argumens, empruntés à la distribution des nerfs, la remarque de Bojanus, qui établit que le nerf sous-maxillaire entre par le trou dans cet os; mais on a vu qu'elle n'est pas exacte.

D'après tout cela je regarde cet os comme une partie du temporal, et cette partie est le rocher.

$$
\text { S. } 128 .
$$

Le second os, qui vient en avant de ce dernier, est, par opposition, incontestablement une portion du sphénoïde, et, d'après toutes les conditions qu'il présente, la pièce pour laquelle Bojanus a pris l'os dont il vient d'ètre question, c'est-à-dire la grande aile postérieure du sphénoïde; et si l'on a égard à la comparaison qui en a été établie avec des vertèbres, c'est la partie injérieure de l'arc de la seconde vertèbre du crâne.

Cet os est également quadrilatère, un peu convexe en dedans, légèrement concave en dehors; il est appliqué par un prolongement de son bord inférieur sur une petite saillie qui s'élève communément à peu près du milieu de la face inférieure et du bord externe du corps du sphénoide. Postérieurement il touche en outre au bord antérieur de l'os précédent, dont il est cependant séparé inférieurement par une 
grande lacune; plus haut il est contigu par son bord postérieur à la portion écailleuse du temporal; supérieurement au pariélal; en avant, a l'os situé au devant de lui sur le sphénoïde, de sorte qu'il reste inférieurement encore entre eux un espace qui donne passage aux nerfs de l'oeil.

Tout cela rend cet os évidemment analogue à la grande aile du sphénoïde des autres vertébrés. Le nerf sus-maxillaire, il est vrai, ne le traverse pas, mais il se porte en avant à côté de lui, le long de sa face externe. Il est facile d'expliquer cette disposition par l'étroitesse de l'encéphale et par l'exiguité et la forme alongée du crâne du poisson, par suite et en conformité de laquelle la grande aile du sphénoïde se trouve affaissée en dedans. C'est pourquoi il n'a pas non plus d'ouvertures pour le passage des branches du nerf trifacial.

\section{S. 129 .}

3. En avant de cette pièce il en existe ordinairement une troisième, tantôt paire, tantôt impaire, mais communément d'un volume considérable. Elle se compose d'une paroi inférieure horizontale, et de deux parois latérales verticales, s'étend un peu en avant, où clle déborde le corps dusphénoide, dont elle r'atteint pas cependant tout-à-fait l'extrémité inférieure. En arrière elle touche, comme nous l'avons indiqué, à l'aile pustérieure du sphénoidde. en 
n'ANATOMHE COMPARTE.

avant à l'os ethmoïde, en haut au frontal. C'est entre cette pièce et la précédente que le nerf sus-maxillaire et le nerf optique sortent du crâne.

D'après cela cet os est manifestement l'aile antérieure du sphénoüde.

Chez les silures et les saumons, les moitiés latérales de cet os sont unies dans la ligne médiane; chez d'autres, par exemple les carpes, la murène commune (murcenophis helena), elles sont séparées.

M. Bojanus prend cet os pour l'aile postérieure Iu sphénoïde; mais, d'après ce que j’ai dit, il m'est impossible de partager son opinion.

$$
\text { S. } 130 \text {. }
$$

4. Indépendamment des trois pièces du sphénoïde qui viennent d'être considérées, savoir, le corps et les ailes antérieure et postérieure, que l'on peut encore désigner comme les parties inférieures de l'ar'c de la vertèbre ou les portions articulaires, il y en a généralement d'autres de chaque còté, qui sont séparées des pièces précédentes ou ne leur sont unies que par des articulations mobiles. Elles correspondent aussi par leur prosition à des pièces qui, chez les animaux supérieurs, font partie de l'os sphénoïde.

Ce sont les ailes inférieures qui représentent vraisemblablement des apophyses transverses de vertèbres. 
Ces pièces sont communément des os minces, plats, quadrilatères, assez considérables, dirigés de haut en bas et de dehors en dedans, qui sont situćs en face de la région moyenne du corps du sphénoïde, dont ils sont le plus souvent séparés par un intervalle, et qui s'unissent immobilement en avant avec l'os palatin, en arrière avec une pièce latérale descendant du temporal. Chez les anguilles ces os sont fort alongés. Ils manquent à la murène commune (murcenophis helena), dans laquelle ils semblent ètre remplacés par un ligament alongé, s'étendant de la portion latérale du temporal à l'extrémité postérieure de la pièce sus-maxillaire.

\section{Temporal.}

$$
\text { S. } 131 .
$$

Sur le côté de l'os basilaire, et en partie entre ses portions sphénoïdale et occipitale, on truuve les pièces osseuses qui, chez les vertébrés supérieurs, se réunissent insensiblement en un os unique et constituent le temporal. Ces pièces existent toujours de chaquue côté en nombre assez considérable, et se partagent en deux séries, l'une supérieure et l'autre inférieure, qui sont unies entre elles d'une manière mobile, et dont la supérieure concourt à la formation des parois du crâne, l'inférieure descendant, sous la forme d'une lame large, en avant et en dehors, des 
D'ANATONIF COMPARÉE.

branchies à la mâchoire inférieure, avec laquelle elle s'articule par ginglyme.

a. Portion crânienne du temporal.

$$
\text { S. } 132 \text {. }
$$

La portion crànienne du temporal se compose essentiellement d'une pièce inférieure, qui est.le rocher, et dont il a déjà été question plus haut; elle est en outre formée de deux ou trois pièces supérieures qui se suivent d'avant en arrière.

Les conditions essentielles du rocher ont déjà été indiquées. Cet os n'offrant pas de différences dignes de remarque, je passe aussitôt à la description des os qui lui sont supérieurs. Des trois os supérieurs, le moyen et l'antérieur funt certainement partie du temporal; le postérieur, dont il a déjà été question plus haut (I), appartient peut-être au temporal, peut-être à l'occipital, peut-être n'appartient-il même ni à l'un ni à l'autre et forme-t-il un os propre?

1. La forme de cet os est le plus souvent triangulaire. Une de ses faces concourt à former la surface intérieure du crâne; elle est verticale chez quelques poissons, par exemple, la carpe, où l'os est élevé; horizontale chez

(1) Voyez pag. 1,66, 467. 
d'autres, par exemple l'anguille, où l'os est aplati de haut en bas. Il exisle, en outre, trois faces extérieures: une latérale, une postérienre et une supćrieure, qui concourent toutes it la composition de la surface externe du crâne. Les faces supćrieure et postérieure passent l'une dans l'autre sous un angle droit, qui est même aigu chez les congres. Dans le premier cas cet angle s'élève ordinairement vers son milieu, et produit une apophyse considérable.

Cet os offre, à l'angle de réunion qui exisłe entre la face supérieure et l'interne, une facette articulaire rugueuse, par laquelle il est contigu à l'épine, ou crète de l'occipital chez la plupart des poissons, par exemple les cyprins, les ésocies, les gades, les congres; chez d'autres, an contraire, nous noterons en particulier l'anguille vulgaire (murrena anguilla), il se confond, dans la ligne médiane, avec l'os de même nom. Cette soudure me semble résulter du défaut de la portion squameuse de l'occipital, qui manque quelquefois, du moins chez l'anguille. En bas, cet os sarticule avec l'os précédent, en dehors avec le rocher, en avant avec le pariétal.

Il est extrèmement vraisemblalıle que c'est, chez les animaux supérieurs, une partie de la portion squameuse de l'occipital; il correspond alors à une piece osseuse de l'homme, assez souvent séparéc irrégulièrement du reste de la portion squamcuse, et située entre elle, le tem- 
D'A ATOML COMPARÉE.

foral et le paricital (1), pięce qui est sans doute mormale ì une certaine période de la vie embryonique. Si cet os correspond, en effet, à un os anormal de l'homme, la dénomination dos mormien, proposée par M. Bojanus (Isis, 1 $\left.\left.\mathrm{S}_{1 \mathrm{~S}}, 1\right) .502\right)$, est trés-bonne; mais on lui pent objecter, arec raison, que ce n'est pas le seul point sur lequel il se rencontre des os wormiens. Quvique la dénomination d'ocipilal supé-

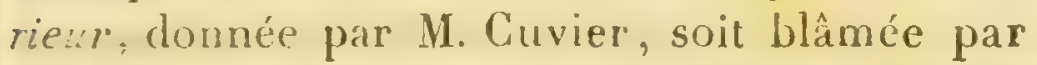
31. Bujanus, je pense que si on y joignait l'épithète de latéral, elle serait préférable à lá première, s'il n'était pas plus utile d'en choisir une autre.

\section{\$. 133.}

2. L'os situé au devant de ce dernier se trouve entre lui, la portion articulaire et la portion épineuse, ou crète de l'occipital, le pariétal, la pièce du temporal qui suit en avant, et le rocher. Il est formé d'une paroi supérieure et d'une inférieure, dont la première est horizontale; la seconde, qui descend plus ou moins obliquement en dedans, a la forme d'un triangle alongé; elle constilue l'extrémité postérieure de la face supérieure et latérale du crâne; elle reçoit dans sa partie interne, oú elle a le plus d'épaisseur, la

(1) J'ai rapproché plusieurs cas de ce genre dans mon Anatomie pathologique, vol. I, et j'en possède un nombre considérable. 
portion supérieure du labyrinthe, et s'articule vers son extrémité antérieure, par sonbord externe, avec la partie postérieure du bord supérieur de l'os carré.

Je regarde cette partie comme la portion mascoïdienne du temporal.

Chez les murènes proprement dites (murrenophis), il existe, au lieu de l'enfoncement articulaire destiné à l'os carré, une suture formée de cavités et d'éminences verticales très-serrées; au-delà de cette suture on rencontre, à la vérité, dans la portion écailleuse, une surface articulaire arrondie; mais il y a une surface semblable qui se retrouve à l'extrémité postérieure de l'aile postérieure du sphénoïde.

Il existe un rapprochement de cette organisation dans les murénes, où l'on voit au milieu rle cet os un petit enfoncement articulaire, rond, largement séparé de celui qui se trouve sur l'os antérieur.

Chez plusieurs poissons, par exemple l'orphie (esox belone), le brochet de mer (sphyrcena spet), le muge céphale (mugil cephalus), l'anableps tetrophthalmus, la face postérieure du crâne se termine supérieurement, des deux côtés, par une plaque alongée qui est formée par cet os ou par celui qui précède. Elle est horizontalement dirigée en arrière, et plus large ici qu'en avant. Elle est très-remarquable chez les splyyrènes, où elle est formée de douze os 


$$
\text { n'ANATOMLE COMPARÉE. }
$$

minces, costiformes, entièrement séparés les uns des autres.

$$
\text { \$. } 134 .
$$

3. L'os supérieur et antérieur est situé entre celui qui vient d'ètre décrit, le rocher, le sphénoïde postérieur, souvent le coronal, le pariétal et l'os malaire. Il fait partie de la région antérieure des parois supérieure et latérale du çàne; il est, dans la plupart des poissons, triangulaire, a pointi en avant, et présente, à la partie externe de sa face inférieure, un enfoncement longitudinal, considérable, qui se continue avec celui de la pièce précédente, et sert à recevoir le bord supérieur de l'opercule.

Cet os est très-petit chez les murènes et les murénophis; il se présente seulement comme un appendice externe et court de l'os précédent, offrant un enfoncement articulaire et arrondi à sa face inférieure, qui ne touche ni au pariétal ni au frontal; ce défaut de connexion est encore augmenté par l'absence de l'os malaire.

Cet os me paraît être l'analogue de la portion écailleuse du temporal.

\section{b. Portion articulaire du temporal.}

$$
\text { ๒. } 135 .
$$

Isa portion articulaire du temporal, à catuse $3 \mathrm{r}$. 
de la forme qu'elle affecte dans cette classe et dans les deux classes suivantes, a reçu le nom d'os carré; cette portion varie plus que la portion crânienne du même os, sous le rapport de la forme, du volume et du nombre des pièces qui la composent.

Elle a cependant toujours, plus ou moins, la forme d'un carré long; elle est plus grande que la portion crânienne, et se compose au moins de deux pièces, une supérieure et une inférieure, unies entre elles par une suture écailleuse.

La disposition la plus simple est offerte par les murènes et genres voisins; il faut seulement remarquer que cette organisation, plus simple, est en effet supérieure à celle qui est plus composée, puisqu'elle rallache ces animaux aux reptiles, chez lesquels cette collection d'os diminue inseusiblement, jusqu'à ce qu'elle finisse par se confondre avec la portion supérieure ou crânienne du temporal. Nous allons donc considérer d'abord les organisations plus compliquées.

Cette portion d'os offre un développement considérable chez la plupart des poissons.

Sous le rapport du volume, ce sont peut-être les syngnathes où elle offre le plus de grosseur. Chez ces poissons la majeure partie de la mâchoire inférieure n'est pas formée par l'os sousmaxillaire proprement dit, mais par la portion qui nous occupe. La pièce la plus supérieure est 
1) ANATOMIE COMYARÉ.

petite, el forme à peine la trentic̀me partie de toute la collection, qui se compose de plusieurs pièces alongées, se recouvrant les unes les autres de dedans en dehors, soit en entier, soit en partie.

Lorsque l'organisation est plus composée, on trouve jusqu'à six piéces, comme, par exemple, chez les carpes et les brachets.

Parmi ces pièces, les deux postérieures et supérieures sont ordinairement les plus fortes. L'une d'elles, plus constante que la seconde, a une forme alongée et présente à son bord supérıeur une surface articulaire, arrondie et longue, par laquelle elle s'articule avec les pièces du temporal qui sont situées au dessus.

Elle offre également à son bord postérieur, non loin de l'extrémité supérieure, une surface articulaire arroudie, convexe, destinée à la pièce supérieure de l'opercule. Elle devient plus mince inférieurement.

En arrière et un peu en dehors d'elle, est située une seconde pièce, également considérable, concave en avant, convexe en arrière, qui se distingue les autres par une série d'ouvertures se succédant régulièrement de haut en bas, et destiuées au passage des canaux mucifères; cette pièce s'unit a l'os précédent et à quelques-unes des pirces inféricures par le moyen d'une suiure.

Apres le promicr os, on reucontec, en bas et 
en avant, un troisième os, styloïde, beaucoup plus petit que celui-ci, sur l'extrémité inférieure duquel il s'appuie.

Entre ces deux os on trouve, du moins chez les carpes, une pièce discoïdle encore plus petite et plus plate.

Au devant de la partie inférieure du premier os et de l'os styloïde, sont situées, l'une au dessus de l'autre, une cinquième et une sixième pièce osseuse; toutes deux sont aplaties; l'inférieure de ces deux pièces présente une surface articulaire, convexe d'avant en arrière, concave de dehors en dedans, par laquelle elle s'articule mobilement avec la pièce de la mâchoire inférieure qui vient après. Ces deux pièces touchent en avant, par le moyen d'une suture, aux pièces qui composent les ailes du sphénoïde.

La même disposition règne chez le saumon, à l'exception du plus petit os, discoïde, qui manque.

Chez ces deiniers poissons et chez les poissons mentionnés précédemment, la pièce styloïde est très-petite; chez la morue, au contraire, qui possède les cinq pièces ordinaires, elle est extrèmement considérable; elle égale en volume les deux pièces inférieures réunies.

Chez les trigles, la pièce la plus posiérieure l'emporte considérablement en développement; elle recouvre extérieurement les trois pièces antérieures, à l'exception de la supérieure qui est 
1)'ANatome CompanéE.

irès-petite, la styloïde étant, au contraire, fort développée. La surface de la pièce supérieure et de la postérieure elle-même, et celle de la plupart des os des régions supérieure et latérale de la tète sont fort rugueuses. La pièce postérieure forme, en outre, avecla supérieure, une partie du pont large qui constitue la face latérale de la tête, comme une couche externe, parallèle à l'interne, qui est représentée par les os ptérygoïdes et palatins. C'est cette disposition qui permet que, chez ces poissons, la pièce en question soit également contiguë au large os malaire qu'elle est loin d'atteindre sans cela.

Chez les silures le nombre de ces pièees est beaucoup moins considérable; ils n'ont que les deux pièces supérieures, postérieures; et l'infé. rieure, qui s'articule avec la mâchoire d'en bas, est ici proportionnellement plus volumineuse qu'ailleurs.

Cette disposition conduil à celle qui nous est offerte par les murènes et genres voisins.

Chez les murènes, la plus inférieure des pièces postérieures existe encore; elle est même fort large. Chez la murène commune (muranophis helena), non-seulement on ne la retrouve plus, mais la pièce inférieure mème est ordinairement petite, de sorte qu'elle semble aussi sur le point de disparâtre. La supérieure, qui est proportionnellement fort volumineuse, présente son apophyse articulaire, très-saillante et des- 
cendant très-bas à cause de l'opercule. Mais ce qu'il y a de plus remarquable sur cette piece, c'est la disposilion du bord supérieur, dont la plus grande partie offre une suture dentelée, interrompue seulement, dans le troisième douzième de l'étendue de ce bord, par un renflement supportant une éminence articulaire, trans. versale et arrondie; il en résulte qu'il n'est que très-peu mobilement uni aux os correspondans du crâne. Celte condition, aussi bien que la disparition de la pièce postéricure et l'extrème petitesse de la pièce inférieure, établit évidemment des rapprochemens remarquables entre l'organisation de ces poissons et celle des reptiles, surtout des salamandies et des ophidiens.

D'après M. Bojanus (1) il n'y aurait, des pièces qui viennent d'ètre représentées comme entrant dans la composition de l'os carré, que la première et l'os styloïde, ou la troisième pièce, qui appartiendraient véritablement à cet os; la seconde, ou la postérieure inférieure apparliendrait à l'opercule, par conséquent à la mâchoire inférieure; une ou les deux pièces antćrieures et inférieures, ou les quatrième et cinquième pièces feraient partie de l'os ptérygoïde. Plusieurs circonstances semblent, à mon avis. militer contre cette opinion en ce qui concerne ha première piece, savoir : l'absence absolue de

(1) Isi., $18,8, p .50 \%$ 


\section{D'ANATOMLE COMPARÉE.}

cancexion de cette pièce avec les os de l'opercule, (condition présentée dans quelques espèces, mais surtout d'une manière évidente par les silures) et l'existence d'une suture qui la fait confondre, au contraire, avec les deux autres os, de manière à former avec eux une mème plaque. Chez les trigles, elle s'unit étroitement, il est vrai, par sa partie postérieure avec l'opercule, et se trouve dans le mème plan que lui; mais en revanche, sa connexion avec les pièces de l'os carré esı beaucoup plus solide ; elle est presque confondue avec l'os styloide, et est exactement unie aux autres pièces qu'elle recouvre. Elle disparait aussi chez la murèue commune (muroenophis helena), et néanmoins l'opercule coïncide par sa forme avec l'us des murènes, qui est encore formé des trois pièces ordinaires de l'opercule.

Que les quatrième et cinquième pièces apparliennent également à la portion tenıporale qui nous occupe ; cela résulte, très-évidemment sans doute, de la circonstance qu'à l'extrémilé inférieure se trouve toujours la surface articulaire destinée à la mâchoire inférieure. Si la pièce rlont il est question était l'os ptérygoïde, celuici se serait intercalé entre la mâchoire inférieure et le temporal; ce dernier n'opérerait plus la connexion entre le crâne et la mâchoire inférieure, conséquence qui est trop contraire à l'aualogiede lous les autres animaux pour qu'elle soit admissible. Mc̀me dans les cas oú cet os se 
partage en deux, je ne regarde pas la pièce supérieure comme une partie de l'os ptérygoïde, parce que celui-ci est représenté par une lame placée au devant de cette pièce.

\section{Pariétal.}

$$
\text { S. } 136 \text {. }
$$

Nous venons de voir comment se comportent les os qui, chez les poissons, représentent l'os temporal des vertébrés plus élevés. A ces pièces et à celles du sphénoïde correspondent, à la face supérieure du crâne, deux os pairs qui, unis aux unes et aux autres, représentent une scconde et une troisième vertèbre cranienne; ces os sont les pariétaux. Chaque pariétal est situé entre la pièce supérieure de l'occipital, la portion écailleuse du temporal et le frontal, et s'unit communément sur la ligne médiane avec l’os de même nom, du côté opposé.

Le pariétal est très-généralement quadrilatère, plat, peu convexe à sa face supérieure, concave à l'inférieure, presque toujours petit par rapport aux autres os. Il est alongé chez le brochet, ainsi que chez les murènes et les murénophis, où il est en même temps apointi en arant; chez le saumon, il est convexe extérieurement. concave intérieurement; chez les carpes, ou il est considérable propurtionnelloment, il reprcsente un carré presque parfait. 


\section{"IANATOME COMPARÉE.}

Chez les poissons pourvus d'une forte crête céphalique et médiane, le pariétal est repoussé sur le côté par la portion squameuse de l'occipital qui forme cette mème crête, de sorte qu'il est entièrement séparé de son congénère. Celte disposition existe, par exemple, chez les coryphènes, les spares, les brames, les zées. Il est en même temps très-petit.

Chez les échénéides, cet os, conformément à la largeur considérable de la tête et à la concavité de sa paroi supérieure, est non-seulement trèsgrand et large, mais il forme aussi un carré presque régulier, et paraît concave à sa face supérieure.

Il manque quelquefois comme os propre; il est alors ou confondu avec la portion supérieure ou moyenne de l'occipital, ou entièrement refoulé par cet os. Il en est ainsi chez les silures, par exemple, où la place de ces trois pièces osseuses est occupée par un seul os, qui par sa partie postérieure correspond à la portion squameuse de l'occipital, et par sa partie antérieure, du moins en grande partie, à l'os pa-, riétal. Il est fendu au milieu dans toute sa moitié antérieure; il en résulie un intervalle étroit, alongé, s'élargissant insensiblement d'arrière en avarıt, qui est une fontanelle postérieure, fermée en avant par la partie postérieure des os frontrux.

La division de cet os dans sa moitié anté- 
rieure, confirme la loi que j’ai tronvée être générale jusqu'ici, savoir que, chez les poissous, les pariétaux ne se réunissent jamais sur la ligne médiane, de manière à former un os impair.

$$
\text { S. } 137 .
$$

Après les os qui viennent d'ètre considérés, il en suit plusieurs antres qui forment la vertèbre céphalique antérieure ou la quatrième, et représentent l'os ethmö̈de et l'os coronal.

5. Os ethmoïde.

$$
\text { S. } 138 .
$$

De ces deux os, l'ethmoüde est situéle plus en bas; il forme la partie antérieure de la cavité crânienne, et reçoit le nerf olfactif qui sort du crâne par cet os. Il est contigu en arrière à l'aile antérieure du sphénoïde; inlérieurement, il repose sur l'extrémité antérieure de la face supérieure du corps du mème os; supérieurement, il supporte l'os frontal.

Il est formé, en général, de trois os; un moyen et deux latéraux.

l'os moyen, qui est impair, consiste le plus souvent en une pièce supérieure et transversale, et en une inférieure, moyenne et verticale; il est concave d'arrière en avant dans la ligne médiane, ou des deux cotés. Jies parties latérales 
forment une lame supérieure et une inférieure, unies en dehors par une paroi verticale, ou un arceau convexe extérieurement, concave en ledans, ou enfin une gouttière. Chez la plupart des poissons, celte gouttière est transforrnée en canal, dans une cerlaine parlie de son étendue, par le moven diune branche ou lame verticale intérieure. Elle est ouverte en dedaus chez les silures.

La pièce moyenne, fendue en avant, se termine très-fréquemment par deux facettes articulaires, latérales, convexes et encroûtées de cartilages, qui portent les os situés au devant et à còté d'eux. l'os maxillaire supérieur et l'os palatin.

Cette disposition se rencontre surtout chez les cyprins.

Chez les poissons qui ont la face on le champfrein fort alongé, comme, par exemple, chez les zées, lesspares, tout l'os ethmoïde ou olfactif est très-aplali, alongé et mince. Lorsque la tête est plate, comme, par exernple, chez les silures, ii est au contraire aplati de haut en bas, et large surtout en avant.

\section{Coronal.}

$$
\text { S. } 139 \text {. }
$$

Ie coronal, d'un développement proportionnel, ordinairement considérable, et dont les di- 
mensions surpassent surtout celles du pariélal, a la forme d'une plaque alongée, s'étrécissant en avant, portant à peu près vers le milieu de sa face inférieure une saillie verticale; il recouvre la partie postérieure de l'os ethmoïde, les ailes supérieure, antérieure et postérieure dı sphénoïde, et la partie antérieure de la portion ficailleuse du temporal; il touche, par son extrémité postérieure, à l'antérieure du pariétal, et par son extrémité antérieure à la postérieur'e de l'os nasal, enfin par son bord interue à son òs congénère du côté opposé.

Chez beaucoup de poissons, les silures, par exemple, les frontaux ne sont pas soudés au milieu dans leur moitié antériéure; il en résulte une fontanelle antérieure, fermée en avant par la pièce moyenne de l'elhmoïde, fendue ellemème au unilieu dans sa partie postérieure. Cette fontanelle antérieure est sé parée de la fontanelle postérieure que nous avons déjà citée, par la moitié postérieure des os frontaux, qui est soudée.

Cet os est surtout irès-développé chẹz les brochets. Il se termine en outre, daus sa moitié antérieure, par une apophyse longue et étroite. Chez les poissons qui ont la crête du crâne forte, cet os, non-seulement touche it la portion squameuse de l'occipital, mais il concourt mème par une forte éminence verticale à former une partie de la crête sincipitale. 
7. Os sus-orbitaire.

$$
5 \cdot 140
$$

Au-dessus de l'œil, et comme complément de la paroi supérieure de l'orbite, il existe, du moins chez plusieurs poissons. surtout chez les curpes, à la partie postérieure du hord externe du frontal, un petit os alongé, aplati, ayant un bord interne, convexe, adhérent, et un externe, concave et libre, qui par sa position et la détermination qu'on lui peut assigner. peut ètre nommé os sus-orbitaire.

Chez l'anableps tetrophthalmus, la paroi supérieure de l'orbite forme une voûte fort convexe; il est vraisemblable qu'il existe chez cet animal un os sus-orbitaire propre, encore plus développé que chez les cyprins.

$$
\text { S. } 141
$$

Les os que nous venons de passer en revue jusqu'ici, correspondent aux os du crâne des animaux supérieurs; ceux que nous allons considérer correspondent aux as de la face. Celleci se partage, comme cela a lieu loujours, en une jartie supérieure qui est la mâchoire supérieure, et en ume inférieure qui est la mâ"hoire inférieure. Les poissons et plusiẹurs au- 
tres repliles, se distinguent des autres vertél)és, principalement des mammiferes, par une articulation plus ou moins mobile de la mâchoire supérienre avec le crâne, en général, et des picces de celte partie entre elles.

$$
\text { S. } 142 .
$$

La partie supérieure de lá face des poissons est formée d'un nombre ordinairement trèsconsidérable de pièces qui ne somt pas partout, faciles à déterminer.

\section{Vomer.}

\section{S. 143.}

En commençant, pour continuer à suivre l'ordre que nous avons adopté jusquici, par la face inférieure ou basilaire de la tète, nous irouvons, immédiatement, au devant du corps du sphénoïle, un os impair, alongé ; cet os est le vomer. 11 est plus ou moins concave ì sa face supérieure, convexe à l'inférieure, mince et plat dais la majeure partie de son étendue pusıérieure; souvent considérablement apointi en arrière, il est ordinairement dans le reste antérieur fortement renflé et étendu en largreur. Sa partie postérieure, qui en est la plus grande, s'enfonce toujours un peu, quelquefois 
D'ANATOME COMPARÉE.

fort loin sous la partie antérieure du corps du sphénoïde.

Chez les murènes et les murénophis, cet os est confondu, à l'état parfait, avec les autres pièces de la mâchoire supérieure; il forme la branche inférieure, postérieure, mince et aiguë de cet os unique. Tant qu'il est séparé, il ne se renfle que fort peu à son extrémité antérieure. Il est large et plat, surtout à sa partie antérieure, chez les poissons à tète large et plate, par exemple chez les silures.

9. Os palatin.

$$
\text { S. 1/4. }
$$

En dehors de cet os, on en rencontre un autre qui, le plus souvent alongé, prend latéralement appui sur l'extrérnité antérieure de l'os précédent, en s'articulant avec lui d'une manière mobile; c'est le palatin. Il est dirigé de dedans en dehors, et d'avant en arrière, et touche ordinairement par son extrémité postérieure à l'os ptérygoïde. Chez les murènes et le murenophis helena, je n'ai pas pu voir cet os d'une manière précise, si on ne veut admettre que, chez les murènes, la partie antérieure de l'os plat et alongé, situé entre la portion articulaire du temporal et le vomer, ne soit l'os palatin. Chez les murénophis, il y a un os plus mince et beaucoup plıs petit, qui de la portion 
articulaire du temporal se dirige en avant; il est beaucoup plus court, n'atteint pas le vomer, et correspond tout au plus à l'os ptérygoïde.

10. Os unguis ou lacrymal.

$$
\text { S. } 145
$$

On trouve en avant, sur la pièce moyenne de l'os ethmoïde, du moins chez plusieurs poissons, particulièrement les carpes, un os arrondi, plat, également articulé mobilement, qui est situé au devant de la cavité olfactive. Cet os correspond, par sa position, à l'os lacrymal des animaux supérieurs. Il supporte l'os palatin à son extrémité externe.

\section{Os nasal.

$$
\text { S. } 140 .
$$

C'est un os alongé, situé au dessus et au devant de la pièce moyenne de l'ethmoïde, avec laquelle il est souvent très-mobilement articulé; il touche par son extrémité antérieure à la région interne de l'os inter-maxillaire, auquel il est uni plus intimement qu'à l'ethmoïde.

Il offre plusieurs variétés.

Chez les cyprins, c'est un os simple, impair, alongé, renflé à ses deux extrémités. Chez 
d'autres, surtout chez ceux dont l'os intermaxillaire est fort développé en haut et en arrière, il est partagé, par les branches supérieures de ce même os, en deux portions déjetées sur les côtés, et qui ne se rejoignent pas mème dans la ligne médiane. Cette organisation existe, par exemple, chez les spares, les gades, les uranoscopes.

Chez les silures, il forme une lame droite, mince, alongée, qui est située sur l'ethmoïde, à la face supérieure de la tête, et est fort éloignée de celle de même nom du côté opposé.

I2. Os maxillaire supérieur.

$$
\text { S. } 147 \text {. }
$$

L'os maxillaire supérieur des poissons osseux est partagé, de chaque côté, au moins en deux pièces, une antérieure et une postérieure, qui se suivent d'arrière en avant, et sont entièrement séparées l'une de l'autre. La postérieure peut être désignée sous le nom de pièce sus maxillaire proprement dite, l'antérieure sous celui de pièce inter-maxillaire. Celle-ci est trèsgénéralement beaucoup plus grande que ne l'est la première et supporte presque toujours des dents qui manquent ì la postérieure. 
a. Pièce sus-maxillaire, ou os maxillaire supéricur postérieur.

$$
\text { S. } 118
$$

La pièce sus-maxillaire est alongée, plate, convexe en arrière, concave en avant, articulée mobilement par des condyles, en haut et en arrière avec la pièce latérale de l'éthmoïde, en baut et en avant avec la branche supérieure du même os, et avec l'extrémité antérieure de l'os nasal. Entre cette pièce et la pièce inter-maxillaire il existe, chez plusieurs poissons, probablement chez tous ceux qui ont l'os inter-maxillaire très-mobile, un cartilage articulaire, triangulaire. Cette disposition est surtout distincte chez les spures. En bas et en dehors, cet os recouvre l'os sous-maxillaire, peu en avant de l'articulation de celui-ci avec la portion articulaire du temporal; il touche ici en même temps à l'extrémité inférieure de la branche inférieure de l'os inter-maxillaire, avec lequel il n'est uni ordinairement, en faisant abstraction de ce point et du point déjà indiqué, que par le moyen de la peau et de la membrane buccale.

Chezle brocket, il est, surtout par rapport à l'os inter-maxillaire qui est ordinairement beatcoup plus considérable, très-grand, alongé, plat, et porte à son extrémité postérieure encore une lame semblable, mais beancoup plus petite, 
qui correspond peut-ère à une partic du palatin qui s'est portée en dehors; mais il correspond aussi, et sans doute plus exactement, à la portion alvéolaire de la mâchoire supérieure.

Chez les silures, cet os est extraordinairement petit, court, épais, et forme la base du filament cartilagineux, long et libre qui en représente peut-être la majeure partie.

Il est très-considérable chez les murénophis, alongé, droit, articulé en avant avec l'os inter-maxillaire, en arrière apointi et garni de dents dans sa moitié antérieure, qui est la plus grande. Chez les murénes, il est plus parfait que chez les murénophis, en ce qu'il est un peu convexe et garni en avant d'une apophyse triangulaire, ascendante, peu élevée. Chez les mu. rènes, il est uni postérieurement par un long et fort ligament, avec l'os inférieur de la portion articulaire du temporal; chez les murénophis, par un ligament plus court, à la mâchoire inférieure, peu en avant de la facelte, où celle-ci reçoit la portion articulaire du temporal, tandis que sans cela il est ordinairement sans connexion avec les deux os.

Il manque, comme os propre, chez les balistes. 
b. Pièce inter-maxillaire, ou os maxillaire supérieur, antérieur.

\section{\$. 149}

La pièce inter-maxillaire, ou l'os maxillaire supéricur cintérieur, forme la partie la plus antérieure de la région supérieure de la face; il est situé entre l'ethmoïde, l'os nasal et le susmaxillaire proprement dit.

Il est formé très-généralement d'une branche supérieure, interne, descendant obliquement de haut en bas et d'arrière en avant, articulée avec celle du côté opposé par une symphyse souvent très-mobile, et d'une branche inférieure, externe, horizontale, descendant de dedans en dehors, et d'arrière en avant, ordinairement armée de dents, qui, par son extrémité postérieure externe, touche à l'extrémité inférieure de l'os sus-maxillaire, et le dépasse un peu en dehors.

Chez les brochets, cet os est petit, uni plus exactement que de coutume avec la pièce susmaxillaire, qui est heaucoup plus considérable que lui. Il ne forme qu'une lame simple, alongée, armée de dents.

Chez les silures, il représente une petite plaque armée de dents, simple, transversale, se rencontrant dans la ligne médiane avec celle du côté opposé, située sous la partie antéricure 
de l'ethmoïle moyen, auquel elle est mnie immobilement, à peu de distance en avant du vomer. dont la partie antérieure, large, armée de dents, présente la même forme.

Il manque, comme os propre, chez les murénophis, du moins dans la vieillesse; il forme un os unique avec le vomer et l'os nasal, peut-être aussi avec le corps de l'ethmoïde. Mais chez les jeunes sujets, cet os unique peut être partagé, en effet, en quatre pièces, savoir : en deux impaires et deux latérales, dont la moyenne et postérieure, qui est la plus petite, représente le vomer; la moyenne antérieure, qui est la plus; grande, représentant l'os nasal ou le corps de l'ethmoïde, les deux pièces latérales, plates et situées sous la pièce moyenne antérieure, représentant les os inter-maxillaires. Ces dernières sont pourvues, à leur partie postérieure, d'un enfoncement articulaire pour recevoir le condyle de la mâchoire supérieure.

Chez les murènes, du moins chez les congres. l'os inter-maxillaire me paraît ètre un os triangulaire, qui est situé à la face externe de la moilié anterieure de la pièce sus-maxillaire, et est considérablement plus haut que celle-ci.

Chez les saumons, cet os est également trèspetit; il ne présente qu'une plaque simple, la branche inférieure. Il est plus grand chez les balistes, mais sa forme est la mème. Les trigles n'offrent qu'une faible trace d'une branche su- 
périeure. Cette branche est aussi très-courte chez les gades, les coryphienes, les scombres, les brames. Elle est un peu plus alongée chez le $m u$ ge céphale, où elle a surtout une largeur et une épaisseur considérables.

Cet os, surtout sa branche supérieure, est d'autant plus développé, que la portion antérieure de la face, articulée mobilement avec le reste de la tête, est plus protractile.

Ainsi, il est très-volumineux chez les spares, les uranoscopes, les cépoles, les chironectes, les lophies, les mules, les vives, les ciorées.

Il est surtout considérable dans ce dernier genre; sa branche supérieure, qui est la plus grande, se termine par deux apophyses, une supérieure et postérieure, plus longue et plus étroite, et une inférieure, antérieure, plus courte, mais beaucoup plus large; la branche inférieure, verticale, est même terminée par trois apophyses, quoique beancoup plus courtes.

L'orgarisation des urunoscopes se rapproche de cette disposition; cependant les apophyses ne sont pas aussi grandes et aussi écartées.

Chez l'orphie (esox belone), la disposition en est tout autre; il se prolonge en avant en un long bec. 
13. Os malaires.

\section{S. 150.}

Il existe très-généralement au desșous de l'oil, entre les os nasaux, les maxillaires supérieurs et la portion écailleuse du temporal, une série d'os plats, formant le bord inférieur de l'orbite qui est convexe supérieurement, concave inférieurement. Les os de cette série diminuent de volume d'avant en arrière, correspondent par leur position aux os malaires des animaux supérieurs, et les représentent du moins par leur partie postérieure. Une semblable division de l'os malaire en une pièce antérieure et en une postérieure au moins, se rencontre même chez l'homme, quoique fort rarement (I).

Cependant il n'y a peut-être que les pièces postérieures qui correspondent à l'os malaire, tandis que les antérieures peuvent représenter rles parties de la mâchoire supérieure des hauts vertébrés, par exemple, l'apophyse nasale ou montante du maxillaire supérieur. Cette supposilion est confirmée assez par la petitesse de l'os décrit comme pièce sus-maxillaire ou os maxillaire supérieur postérieur, aulant que par l'anomalic, assez fréquente chez l'homme, de l'apop'nyse nasale qui est quelquefois entièrement séparée du reste de l'os maxillaire supérieur.

(1) Sandifort, Obs. anal. path., li.1v, p. 134. 
M. Oken regarde ces plaques comme le cerole oculaire des oiseaux qui aurait été jeté en dehors; cependant je ne puis pas partager cette conjecture ingénieuse, par la raison: $1^{\circ}$ que les poissons ne possèdent point de cercle oculaire, mais une forte lame osseuse sous la membrane fibreuse (sclérotique) de l'oil ; $2^{\circ}$ qu'outre les os décrits ils n'ont pas d'autre os malaire; $3^{\circ}$ que les oiseaux en possèdent un à la même place, et qui est primitivement double; et $4^{\circ} \mathrm{enfin}$ que le cercle osseux de l'œil n'existe pas plus chez les poissons pourvus des os qui viennent d'être décrits, que chez ceux qui en sont dépourvus.

Chez les poissons anguilliformes, du moins les murènes et les murénophis, cette série d'os manque ou est extrêmement petite; chez les silures, elle est formée de trois pièces, proportionnellement petites, minces et alongées. Chez les balistes, il n'existe presque qu'une strie cartilagineuse, très-mince; mais dans la généralité des autres genres elle est fort développée, surtout en proportion des pièces sus-maxillaire et inter-maxillaire.

Elle est surtont fort considérable chez les uranoscopes, et plus encore chez les trigles. Les pièces moyenne et antérieure sont surtout grandes, et dépassent considérablement en avant et en haut le maxillaire supérieur, qui est beaucoup plus petit. Dans les spares et les lalires, la pièce antérieure est aussi fort considérable, 
D'ANATOMIE COMPARÉE.

tandis que les postérieures ne sont que petites.

14. Mâchoire inférieure.

$$
\text { S. } 151
$$

Los maxillaire inférieur des poissons osseux est situé au-dessous et derrière les os sus-maxillaire $t$ inter-maxillaire, et la portion articulaire du temporal, avec la partie inférieure de laquelle il s'unit par une surface articulaire transverse, légèrement concave. Il est toujours formé de deux moitiés latérales, convergentes d'arrière en avant, où elles se rencontrent presque toujours sous un angle plus ou moins aigu, et son unies entre elles par une subslance ligamenteuse. Chaque moitié latérale est formée à son tour de plusieurs pièces qui se suivent d'arrière en avant, se couvrent en parlie les unes les autres de dehors en dedans, et s'ernboîtent mème.

I,es données relatives au nombre de ces pièces varient considérablement, aujourd'hui que rlusieurs auteurs comptent, parmi la collection d'os précédemment et régulièrement recornue comme mâchoire inférieure, encore plusieurs pièces beaucoup plus voluminenses (1), qui forment ensemble l'opercule.

(1) Bojanus et Oken, Isis 1818. 
Cela s'applique déjà à la mâchotre inférieure, ainsi nommée ordinairement.

Selon quelques-uns, chaque moitié de la mâchoire inférieure ne se compose souvent que d'une ou de deux, rarement de trois pièces (1). Je crois cependant, d'après mes recherches, qu'il en existe peut-être mème quatre dans la règle.

M. Cuvier cite le polypterus bichir comme l'unique exemple où la moitié en question se compose de trois pièces; mais j'en ai réellement trouvé quatre chez le brochet (esox lucius), l'orphie (esox belone), le brochet de mer, (sphyrcena spet), le saumon (salmo salar), la carpe (cyprinus carpio), l'alose (clupea alosa) le hareng (clupea harengus), la morue ou le cabillaud (gadus morrhua), le muge céphale (mugil cephalus). Parmi ceux auxquels M. Cuvier n'eu reconnaît également qu'une, il y a, outre les saumons, les murènes, surtout les congres et les anguilles qui possèdent évidemment au moins deux de ces pièces. J'en ai trouvé trois chez le poisson Saint-Pierre (zeus faber), l'uranoscopus scaber, le saluth (. silurus glanis), l’anguille (murna anguilla). Le volume des pièces qui composent la mâchoire inférieure diminue en général d'avant en arrière. La pièce antéricure supporte les dents; on la peut appeler à cause de cela piéce dentuire. La

(1) Cuvier, Anat. comp., t, III, p. 16. 


\section{D'ANATOMLE COMPARÉE.}

postérieure ou la moyenne, lorsqu'il existe trois ou quatre pièces, s'applique par son extrémité antérieure, qui est apointie, contre la face interne de la pièce antérieure, ou s'intercale même entre les lames externe et interne de cette pièce, forme avec elle l'apophyse coronoïde, et renferme la surface articulaire par laquelle la mâchoire inférieure s'articule avec l'os carré; on peut par conséquent lui donner le nom de pièce curliculaire. La troisième forme l'extrémité postérieure de la mâchoire inférieure et notamment de son bord inférieur, au-dessous de la face articulaire. Il est digne de remarque qu'elle imite plus ou moins distinctement la forme de la pièce articulaire. Le volume en varie considérablement. Je la trouve extrêmement petite chez les saumons el le brochet de mer (splyrcena spet), un peu plus grande chez le brochet (e. lucius), forl grande chez le poisson Saint-Pierre (zeus faber). On la peut désigner sous le nom de pièce angulaive. La quatrième piècc est située au côté interne de la piéce arliculaire, en avant et au dessous de la surface d'articulation. Elle est alongée et arrondie, appuyée sur une saillie que présente à sa partie interne la mâchoire inférieure plus épaisse en cet endroit, et forme la racine d'un stylet cartilagineux plus ou moins considérable, alongé, arrondi el apointi en avant, qui, conjointement avec l'extremité anlérienre d. In portion arliculaire, sapplique à la 
face interne ou entre les deux lames de la pièce dentaire, et existe aussi chez les reptiles. Cetie pièce est en général la plus petite, et sans contredit celle que M. Cuvier décrit chez le bichir, pour être la troisième qui s'applique contre la face interne de la pièce dentaire, à cela près qu'elle est ici plus ossifiée qu'on ne la rencontre ailleurs. Parmi les poissons à trois pièces sousmaxillaires, les silures et les anguilles ne m'offrent que cette pièce. Elle manque au contraire chez le poisson Saint-Pierre (zeus fruer) et l'uranoscopus scaber, et la troisième pièce est la postérieure. Chez l'orphie (esox belone), je trouve également quatre pièces; mais la disposition est un peu différente. La troisième est proportionnellement plus grande que de coulume; elle est située plus en avant et en dedans, et se partige en une pièce supérieure et en une inférieure. La première de ces pièces est beaucoup plus grande, la seconde forme l'angle. Peut-être la quatrième est-elle seulement descendue plus bas. Il est digne de remarque que le volume de la mâchoire inférieure ne semble pas influer sur le nombre de ses pic̀ces. Chez les carpes, la troisième et la quatrième pièce sont proportionnellement fort considérables. Le long bec inférieur de l'orphie (esox belone) est formé uniquement par la pièce dentaire.

Chez le lépisostée spatule (lepisosteus spalula) la mâchoire inférieure est mème composće de 
D'ANATOME COMPARÉE.

six pièces (1); outre les quatre pièces ordinaires il s'en est formé deux autres par la division des deux plus grandes pièces; ces pièces sont une pièce coronoide formée aux dépens de la portion articulaire, et une pièce antérieure située supérieurement le long de la face interne de la pièce dentaire.

Ces pièces s'adaplant exactement les unes aux autres forment en général une paroi osseuse continue; il existe cepéndant quelquefois, par exemple, chez le poisson Saint-Pierre (zeus faber), en haut, entre la pièce dentaire et la pièce articilaire, un grand espace triangulaire, rempli seulernent par la peau et la membrane buccale.

Dans quelques autres poissons, notamment le lieu ou merlan jaune (gadus pollachius), le merlan commun (g. merlangus), le charbonnier (g. carbonarius), le merlan barbu (g. albidus), il y a des vestiges de cette disposition; mais la lacune est incomparablement plus petite que chez le poisson Saint-Pierre (zeus faber), elle est à peine sensible.

Quelques pleuronectes, surtout la barbue ( $p l$. rhomibis) et le turbot ( $p l$. maximus), tiennent au contraire, sous ce rapport, le milieu entre les gades et les dorées.

(1) Geoffroy, Philos. anat., Paris, 1818, 1. $35, \mathrm{pl} . \mathrm{V}$, fig. $50-5.5$. 
Il faut que cette disposition soit bien rare, puisque je ne l'ai rencontrée que chez les poissons que j'ai mentionnés, bien que j'en aie comparé non-seulement un grand nombre qui m'appartiennent, mais tous ceux qui se trouvent au cabinet de Paris.

Quant au mode de connexion de ces différentes pièces, plusieurs poissons, surtout parmi ceux dont l'os inter-maxillaire est articulé mo-bilement avec les autres os de la tête, se distinguent, non-seulement des autres poissons, mais de tous les autres animaux ì vertèbres, par une mobilité analogue entre la pièce dentaire et la pièce articulaire. La pièce articulaire pénètre bien avec sa pointe antérieure dans la pièce dentaire, mais elle n'y est unie que trèslâchement au moyen d'une substance ligamenteuse. C'est surtout chez les spares, les labres, les scorpènes, les uranoscopes, les trigles, que je me suis convaincu de cette disposition, tant à l'état frais que sur le squelette, où je l'ai reconnue d'abord. Elle est importante, soit parce qu'elle permet d'agrandir l'ouverture buccale un peu plus que de coutume, soit surtout parce qu'elle est en faveur de l'opinion d'après laquelle l'opercule serait la partie postérieure de la mâchoire inférieure, qui aurait été grossie et séparée des autres os, puisqu' ici la partie postérieure de cette mâchoire s'éloigne de l'anterieure beaucoup plus qu'il n'est habituel de le rencontrer. 


$$
\text { D'ANATOME COMPARÉE. }
$$

Cette disposition n'existe pourtant pas chez tous les poissons à museau supérieur, protractile; elle manque aux cyprins et aux dorées.

Après avoir exposé les pièces osseuses qui composent communément la mâchoire proprement dite, nous devons considérer l'opercule sous le point de vue qui, tend à faire voir, en cet os, la partie postérieure de la mâchoire inférieure des animaux élevés: Celte opinion est fondée sur la circonstance que l'opercule, joint à ce que l'on nomme communément la mâchoire inférieure, se compose d'un nombre de pièces osseuses à peu près égal à celui des pièces qui entrent dans la composition de la mâchoire inférieure de plasieurs reptiles, les chéloniens, par exemple. Telle est l'exactitude de ce rapport, que le caractère distinctif de ces deux parties consiste surtout dans l'ampliation affectée par les pièces postérieures, et dans l'écartement qui les sépare les unes des autres. On peut encore citer à l'appui de cette opinion la formation analogue des pièces inférieures du temporal et des pièces du sphénoïde, ainsi que le morcellement du maxillaire supérieur.

Sans méconnaître tout ce qu'il y a d'ingénieus dans cette vue, je crois, pour le présent, ne pas encore devoir l'admettre, et cela par les motifs suivans:

$1^{\circ}$ Il résulte de ce qui précède que, chez le lepisostée (lepisosteus), bien qu'il possède l'oper- 
cule, le nombre et la disposition des pièces osseuses de la mâchoire inférieure proprement dite s'accordent avec ce qu'offre la même partie chez les reptiles mentionués.

$2^{\circ}$ D'autres reptiles, tels que les batraciens et la plupart des ophidiens qui ne possedent point d'opercule, offrent dans chaque moitié de la mâchoire inférieure un nombre de pièces osseuses qui est même moindre que dans la plupart des poissons.

Or, si d'un côté l'opercule peut exister chez les poissons dont la mâchoire proprement dite présente un nombre de pièces osseuses égal à celui qu'offre la même partie chez les reptiles supérieurs; et si d'un autre côté l'absence de l'opercule, chez les reptiles inférieurs, coïncide avec une réduction dans le nombre des pièces de leur mâchoire égale à celle qu'éprouve cet appareil chez la plupart des poissons, il est évident que l'opercule ne doit pas être considéré nécessairement comme une partie dela mâchoire inférieure, et que les os qui le composent peuvent bien ètre des os propres.

$$
\text { S. } 152 \text {. }
$$

15. Forme de la tête des poissons osseux en général.

Quel que soit le nombre des différences que présente le crâne des poissons, même à le considérer dans ses rapports naturels avec les par- 


\section{D'ANATOMIE COMPARÉ.}

lies qui le recouvrent à l'extérieur, néanmoins devons-nous indiquer les conditions les plus générales de la forme de la tête osseuse, ainsi que les régions qui sont en rapport avec des parties dont la position est déterminée par ces régions, et surtout avec les organes de sensations et de locomotion.

La tềte entière est plus ou moins alongée et apointie d'arrière en avant; on y peut distinguer une paroi postérieure, une supérieure, une inférieure et deux latérales. L'extrémité antérieure, située à l'opposite de la paroi postérieure, est percée de l'ouverture buccale.

La paroi postérieure est verticale, peu élevée, en général petite par rapport aux autres; elle offre : ${ }^{\circ}$ les facettes articulaires précédemment décrites; $2^{\circ}$ le trou occipital; $3^{\circ}$ au dessus et près de ce trou, plusieurs enfoncemens et éminences destinés à l'insertion des muscles de la nuque; l'éminence moyenne représente la crète occipitale, qui est souvent considérable.

La face supérieure qui surpasse de beancoup les autres, est communément convexe d'arrière en avant et de la ligne médiane en debors; elle est souvent partagée en deux moitiés par la partie antérieure de la crête occipitale, qui, à cause de sa position dans la région la plus supérieure du crâne, peut ètre nommée créte sincipitale, et acquiert quelquefois une hauteur fort considérable. Cette face est ordinairement plus petite 33. 
que les faces latérales. La partie antérieure de cette face formée par les os ethmoïdes, nasaux et inter-maxillaires, se distingue très-généralement du reste; cette distinction résulte de ce que la région olfactive offre une concavité brusque, plus on moins profonde, qui reçoit les os ethmoïdes et nasaux.

Plusieurs poissons, par exemple les échéneeiddes et les uranoscopes font exception à cette disposition générale; cette face est, chez eux, en partie concave, en partie beaucoup plus large que les faces latérales.

Les faces latérales sont beaucoup plus compliquées que celies qui viennent d'être considérées; elles sont composées entièrement de pièces osseuses unies mobilement avec la face supérieure et qui se suivent d'avant en arrière : Io l'opercule; $2^{0}$ la portion articulaire du temporal, ou l'os carré; $3^{\circ}$ el $4^{2}$ deux parois, dont l'interne est formée principalement par les os ptérygoide et palatin, l'externe par les os malaires.

Les deux faces latérales sont séparées l'une de l'autre par un espace plus ou moins considérable, la fosse temporale, qui est rempli par les muscles élévateurs de la mâchoire inférieure, et qui descenrl obliquement de haut en bas et d'arrière en avant.

Au-dessus de ces faces et au devant de la fosse temporale existe l'orbite, qui commu- 
VinATOMIE COMPARÉ.

nique, par dessus la face inférieure du crâne, avec celıi du côté opposé par une ouverture souvent très-large; il n'est limité inférieurement que dans sa circonférence externe, jamais par une paroi osseuse.

Au devant de cette cavité, et un peu plus en dedaus, on en trouve une autre oblongue ou triangulaire, la cavité olfactive; en bas et un peu au devant de celle ci, on voit une lacune verticale, limitée postérienrement par les os palatin et ptérygoïde, en avaut par l'os maxillaire supérieur; et plus antérieurement existe ordinairement une seconde lacune, plus petite, située entre l'os sus-maxillaire et l'inter-maxillaire.

La face inférieure de la tèle est interrompue par une onverture triangulairè, ayant son sommet tourné en avant, et dans laquelle se trouvent, au-dessous de la face inférieure du crâne, l'os hyoïde, les branchies et les premieres divisions des membres antérieurs. Les bords externes en sont formés par le bord inférieur des deux opercules et des moitiés de la mâchoire inférieure. Beaucoup au-dessus de ces parties, la face inférieure de la tète est partagée en deux moitiés latérales par la paroi inférieure du crâne, qui est très-étroite et qui est formée par le corps de l'os palatin et le vomer; plus au-dessus elle se continue en dehors par de larges lacunes avec l'orbite et la fosse temporale. 
La cavité cränienne qui renferme l'encéphale, est toujours très-petite el d'une forme alongée, disposition en rapport avec le développement imparfait du cerveau. En arrière, elle offre plusieurs éminences el enfoncemens qui, étant en connexité avec l'organe auditif, seront décrits avec celui-ci.

Parmi les ouvertures qui sont en rapport avec le système nerveux, nous avons déjà décrit : $1^{\circ}$ le grand trou occipital; $2^{\circ}$ l'ouverture qui donne passage au nerf pneumo-gastrique (1).

Dans l'os basilaire et le rocher (2), entre celui-ci et la grande aile du sphénoïde, on trouve une fente pour la branche postérieure du nerf trifacial, autrement le trou ovale, qui est partagé quelquefois en une moitié supérieure et en une inférieure; entre la grande et la petite aile du sphénoïde, on voit une autre lacune ordinairement aussi séparée de la première par la partie inférieure et étroite de l'aile du sphénoide, la fente sphénoïdale, par laquelle passe la branche moyenne du nerf trifacial, et une partie au moins de la branche supérieure; plus haut, entre les deux ailes du sphénoïde, existe un trou rond, souvent simple, le trou optique, qui, lorsqu'il est simple, renferme encore la réunion des nerfs optiques; encore plus haut,

(1) Voyez p. 465

(2) Voyez p. 503. 


\section{D'ANATOMIE COMPAREE.}

entre la grande aile du sphénoïde, le frontal et l'orbite, est une petite ouverture par laquelle passe, sous le plancher de l'orbite, une partie de la première branche du nerf trifacial, se portant en avant à la cavité olfactive, dans laquelle elle entre par une fente située entre le frontal et la partie latérale de l'ethmoïde.

En avánt enfin, on trouve, dans l'os ethmoïde latéral, une ouverture simple, ronde, contenant le ganglion du nerf olfactif; c'est le trou du nerf olfactif.

16. Disposition particulière et insolite des os de la tête de quelques poișsons osseux.

\section{S. 153.}

Nous venons d'exposer les conditions ordinaires qu'offre la disposition des os de la tête chez les poissons, avec les principales déviations du type le plus commun. Il en est encore quelques autres que la particularité, et en partie le degré de modifications qu'elles présentent, méritent de faire considérer à part, et qui peuvent être regardées par conséquent, en effet, comme déviations.

$$
\text { S. } 154
$$

Le moindre degré de déviation est présenté 
par la présence d'os mobiles, séparés, situés à la surface du crâne.

Nous en trouvons un exemple dans les lophies et les chironecles. Airsi, la baudroie commune (lophius piscatorius) offre trois pièces osseuses, apointies et fort alongées dans la ligne médiane de la tête. Elles se succèdent d'arrière en avant dans un ordre te!, que la dernière est située immédiatement au devant de l'extrémité postérieure de la tête, et les deux antérieures, qui sont très-serrées l'une contre l'autre, à l'extrémité placée en avant. Elles ne sont pas également grandes; la postérieure est beaucoup plus petite; la plus antérieure un peu plus grande que la seconde.

Toutes reposent sur des os plats, alongés, entourés de forts muscles longitudinaux, qui naissent de l'extrémité postérieure de la face supérieure du crâne.

La postérieure est appliquée seule sur une semblable plaque, très-éloignée de l'antérieure; celle-ci est formée de deux moitiés, l'une antérieure, plus grande, et l'autre postérieure, plus petite; ces deux moitiés sont mobilement unies entre elles; mais la première supporte seule les deux pièces osseuses. La pièce postérieure est située à peu près sur le milieu de sa plaque; la seconde un peu plus avant, la première tout-à-fait à l'extrémité antérieure de la plaque antérieure. Toutes se bifurquent à leur 
D'anatome comparén.

extrémité inférieure, qui est large, en deux moitiés latérales qui, dans les deux pièces antérieures, sont converties en un anmeau à l'aide d'un ligament qui les unit inférieurement; cet anneau est traversé par une petite barre osseuse s'élevant de la ligne moyenne de la face supérieure de la plaque qui sert de support à tout l'os.

A peu de distance de la têle, au dessous de l'apophyse épineuse des seconde et iroisième verlèbres, il existe une plaque absolument analogue, qui supporte deux os semblables, seulement plus petits, situés immédiatement l'un derrière l'autre; elle n’est également fixée à la colonne vertébrale qu'à i’aide de muscles.

Celte circonstance reud déjà très-vraisemblable, que les os dont il vient d'ètre question, ne sont, à la tête, que lés répétitions des épines accessoires et des rayons des nageoires dorsales; opinion qui est corroborée en outre par la nature de ces pièces osseuses, par la difficulté de leur appliquer une autre explication, et par l'organisation des pleuronectes qui a été exposée plus haut.

$$
\text { S. } 155 .
$$

Une particularité de forme bien plus frappante encore, et dont celle qui vient d'ètre décrite est peut-être le premier indice, nous est offerte par la plaque sincipitale et alongée des 
échénéides; à l'aide de cetıe plaque, ces poissons, chez lesquels elle remplit l'office de ventouse, peuvent s'attacher avec force aux corps.

Elle n'est unie que par des muscles et une strie tendineuse, moyenne, à la face supérieure du crâne qui est élargie et un peu déprimée pour la recevoir; cette plaque présente une base osseuse, considérable, qui en détermine entièrement la forme et la grandeur. Son bord postérieur, libre, présente deux saillies transversales, garnies d'un grand nombre de dentelures rugueuses; chacune renferme deux stries osseuses, également transversales. L'extrémité interne des plaques osseuses, inférieures, qui sont plus larges et en général plus grandes que les supérieures, se termine par un pédicule court et étroit qui s'étend jusqu'à la ligne médiane; elles touchent, en outre, aux plaques voisines, et de même nom, par leurs bords antérieur et postérieur. De toute la longueur de leur face supérieure, on voit s'élever une petite crête qui leur est unie mobilement, et qui supporte la seconde plaque, mince, située àu dessous des petites rugosités. Celle-ci, par opposition à la précédente, s'élargit à son extrémité interne, et s'intercale de la sorte entre les pédicules internes et étroits des plaques inférieures. Il existe de plus dans la ligne médiane ver's la face supérieure, entre chaque couple de paires de plaques, des pièces ossenses, styloïdes, alon- 
gées, beaucoup plus petites, qui sont aussi dirigées d'avant en arrière.

Le tout est entouré dans sa partie postérieure, qui est à beaucoup près la plus considérable, d'une lame cartilagineuse étendue, s'élargissant insensiblement d'avant en arrière, qui s'applique contre les bords externes des pièces osseuses inférieures.

Les plaques antérieures et postérieures sont les plus larges et les plus courtes; les premières d'entre elles sont beaucoup plus courtes et plus étroites que les dernières. Les bords supérieurs manquent aux trois dernières plaques; celles des dernières paires situées le plus en dedans, se touchent par leurs bords internes.

Il eșt extrèmement vraisemblable que cet appareil n'est qu'un développement du crâne d'après le type de la colonne vertébrale; et ces pièces osseuses correspondent aux épines accessoires et aux rayons des nageoires.

$$
\text { S. } 156 .
$$

Sous ce point de vue, les modes de conformation qui nous occupent sont surtout intéressans; mais la déviation de forme la plus curieuse et la plus digne de remarque, est le défaut absolu de symétrie dans la disposition de la tète de quelques poissons, principalement des pleuronectes; celte singularité est même très-prouoncée à l'extérieur de ces poissons; ct je crois 
être le premier qui ait décrit en détail cette anomalie en ce qui a trait à leur système osseux (1).

C'est surtout la partie de la tête, la plus rapprochée de la ligne médiane, qui n'est pas sy. métrique dans toute sa longueur; les partics plus latérales s'éloignent beaucoup moins d'une similitude parfaite. Ce défaut de symétrie offre, en outre, des différences graduelles; ainsi, par exemple, chez le turbot, il est beaucoup moindre que chez la plie franche, et bien plus considérable encore chez la sole.

La moitié qui porte les yeux est ordinairement plus grande, et surtout plus large, que la moitié opposée. Elle est, en outre, convexe en dehors; la dernière $\epsilon$ st concave en dedans, et brusquement descendante. La première contient aussi un orbite arrondi, annulaire, ouvert supérieurement et inférieurement, dont le bord externe se porte plus ou moins en dehors, l'interne touchant à la ligne moyenne; cet orbite contient l'oil supérieur.

Conformément à ce qui a été dit plus haut, les us qui offrent le moins de symétrie, sont:

$\mathbf{1}^{\circ}$ L'os basilaire, surtout le corps et la portion squameuse;

(1) Ueber die seitliche Asymetrie im thierischen Kuerper. In meinen anatomisch-physiologischen Untersuchungen. Halle, 1822, p. 271-292. (Sur lc. manque de symétric latérale dans le corps animal. Voyez mes Recherches anatomico-physiologiques.) 
D A NATOMIE COMPARÉE.

525

$2^{\circ}$ Le coronal;

亏ै L'ethmoïde;

$4^{\circ}$ Le vomer;

$5^{\circ}$ Le maxillaire supérieur;

$6^{\circ}$ Le maxillaire inférieur.

Au corps de la portion occipitale, c'est surtout la saillie écailleuse, à la face oculaire, qui est ıplus large que de coutume; la crête médiane imème est tournée du côté opposé.

La portion squameuse est du double plus large au còté oculaire; sa partie transverse, plus llarge, est en même temps plus longue; la crête médiane est dirigée du côté opposé.

Le corps du sphénoïde est fort convexe vers le côté opposé, et est formé ou d'une crête oblique, ou de deux lames, l'une verticale et apparItenant au côté privé d'yeux, l'aùtre plus petite faisant partie du côté où sont les yeux.

Les pariétaux ne diffèrent pas beaucoup entre eux; celui du côté où manquent les yeux est pourtant ordinairement un peu plus grand que l'autre.

La différence la plus saillante est offerte par les os coronaux; elle est même si grande, qu'on ne les reconnaît qu'avec beaucoup de peine pour être des os congénères; s'ils sont sortis de leurs rapports naturels, il est impossible de les reconnaître.

Il existe toutefois entre eux le rapport suivant: l'un et l'autre sont composés d'une partie 
postérieure, plus large, plus épaisse, assez droite, et d'une partie antérieure, beaucoup plus mince, convexe à sa face inférieure, concave à la supérieure; de ces deux parties, la première entre dans la composition de la face supérieure du crâne, la secourle, dans celle de la face inférietire de l'orbite; mais la position et la forme de tous deux manquent également de symétrie.

Sous le rapport de la position, $1^{\circ}$ celui du côté privé d'yeux empiète toujours sur l'autre os par sa partie antérieure, qui est recourbée et dépasse la ligne moyenne; sa partie postérieure reste au contraire du côté qu'il occupe à l'état normal ; $2^{\prime \prime}$ cette partie antérieure de l'os, du côté où les yeux manquent, recouvre la partie correspondante du coronal du côté oculaire, dans une étendue plus ou moins considérable; il en résulte que la partie inférieure et externe de la surface du coronal en est doublée, tandis que derrière l'orbite les os frontaux se rencontrent dans la ligne médiane.

Sous le rapport de la forme, le frontal supérieur, ou celui du côlé oculaire, se distingue principalement de l'autre par un volume plus considérable de sa partie postérieure, qui est carrée. Chez le turbot, la partie antérieure du coronal supérieur, est un peu plus longue et en général pius grande que celle du còté opposé; chez la plie franche, au contraire, cet excès de 


\section{V'ANATOME COMPARÉ.}

dimension est offert par la mème partie du frontal inférieur, et à un degré beaucoup plus considérable.

Les pièces latérales de l'ethmoïde manquent aussi de symétrie; celle du côté oculaire est toujours beaucoup plus grande que l'autre.

La moitié de la pièce moyenne qui appartient au côté oculaire est, au contraire, beaucoup plus petite que l'autre.-

Le vomer est convexe du côté de la face ocu. laire, concave du côté opposé. La courbure a lieu surtout à l'endroit où la partie postérieure, sitnée sous le corps du sphénoïde, se continue avec l'antérieure.

Il est singulier que les os sus-maxillaire et inter-maxillaire du côté gauche soient plus volumineux que du còté droit, sans que cela dépende le moins du monde de la position des yeux.

Cette disposition existe chez le turbot, la plie franche, la sole. Chez la dernière, ces deux os diffèrent plus que tous les autres sous le rapport du volume et de la forme; la pièce inter-maxillaire surtout est trois ou quatre fois plus grande à gauche qu'à droite.

Les pièces sus-maxillaires sont celles qui offrent le moins de différences. Chez le turbot, elles sont presque tout-à-fait symétriques; chez la sole et la plie franche ou carrelet, surtout la première, celles du côté sans yeux sont plus planes que les pièces du côté opposé. 
17. Comparaison de quelques cartilages de la tête des poissons cartilagineux avec des os correspondans chez les poissons osseux.

\section{\$. 157.}

C'est à dessein que nous n'avons pas déterminé plus haut les cartilages mobiles de la face chez les poissons chrtilagineux, afin de chercher leurs analogues seulement lorsque nous aurions décrit les os correspondans chez les poissons osseux.

Nous devons déterminer d'abord les cartilages situés vis à vis de la mâchoire inférieure, et que je n'ai considérés plus haut qu'en général, comme faisant partie de la mâchoire supérieure, dans son sens le plus étendu.

Le grand cartilage, supérieur, mobile et armé de dents, chez les raies et les squales, est considéré ordinairement, à cause de sa position et de sa forme, comme l'os sus-maxillaire; cependant l'explication ingénieuse que nous a dgnnée dernièrement M. Cuvier (1), établit, sinon d'une manière certaine, du moins extrèmement vraisemblable, que ce cartilage est l'os palatin, $1^{\circ}$ puisqu'en dedans de ce cartilage il n'y a point de partie qui puisse être comparée

(1) Mém. du mus. d'hist. nat., vol. I, 1815, p. 103. Sur la composition de la mâchoire supérieure des poissons. 
à cet os; $2^{\circ}$ et que les pièces cartilagineuses (1), situées en dehors dudit os dans la peau de la face, peuvent être considérées comme des pièces du sus-maxillaire et de l'inter-maxillaire.

Chez les chimères, d'après M. Cuvier, ce ne seraient pas mème les os palatins, mais le vomer qui correspondrait de haut en bas à la mâchoire inférieure (2); cependant les argumens cités à l'appui de cette manière de voir, ne me semblent pas tout-à-fait convaincans. La soudure de la pièce supérieure, garnie de dents, avec la face inférieure de la tête, est le fait le plus probant; mais cette soudure pourrait avoir lien aussi pour les os palatins. Aussi serais-je tenté de cousidérer cetle lame comme composée de ces os : je me fonde sur sa largeur, qui est assez prononcée pour que cette lames'étende jusqu'à la circonférence externe du corps, sur sa grandeur, en général, qui est assez considérable pour qu'elle s'étende jusqu'à l'extrémité postérieure de la mâchoire inférieure; enfin sur sa division symétrique en deux moitiés latérales par un sillon. Ajoutons à ces considérations que l'argument emprunté au nombre des pièces labiales ne prouve rien. Chez les chimères, il y en a trois qui, suivant M. Cuvier, correspondent a l'inter-maxillaire, au sus-maxillaire et à l'oss

(1) Voyez plus haut, pages $451 \mathrm{et} 45 \%$

(2) L. c. , p. 127. 
palatin; mais j’ai déjà dit précédemment (1) que chez plusieurs chondroptérygiens, et notamment l'ange, quoique M. Cuvier ne lui accorde que deux de ces pièces, il y a réellement en cet endroit trois pièces cartilagineuses, indépendamment de la disposition ordinaire de la pièce sus-maxillaire.

Il serait possible cependant que l'os palatin fût entièrement chassé par le vomer qui aurait pris un développement plus considérable.

Je ne crois pas non plus pouvoir admettre sans restriction l'opinion de $M$. Cuvier sur les analogues des pièces céphaliques des lamproies (2).

D’après lui, la pièce (3) à dents, simple, annulaire, la plus antérieure, représenterait par sa partie inférieure la mâchoire inférieure, par sa partie supérieure l'os palatin ; l'os styloide, appuyé des deux côtés, correspondrait à l'os carré; la lame la plus antérieure, impaire, située au dessus de l'anneau, devrait ètre considérée comme l'os inter-maxillaire, et celle qui est à côté de lui serait l'os sus-maxillaire. Il me paraît cependant plus exact de regarder:

$I^{\circ}{ }^{\prime}$ 'anneau, uniquement comme les moitiés de la mâchoire inférieure soudées, ou du moins de

(1) Voyez plus haut, pag. 455.

(2) L. c. , p. 128.

(3) Voyez plus haut, p. 446 et 447 . 


$$
\text { D'ANATOMIF COMPAREE. }
$$

considérer la partie supérieure et anférieure pour les os inter-maxillaires, et rion pour les os palatins;

$2^{\text {}}$ Le pédicule latéral comme le maxillaire supérieur;

$3^{\circ}$ La lame située au dessus de l'anneau comme le vomer;

$4^{\circ}$ Les lames latérales comme les os palatins.

Cette opinion est fondée d'un côté sur le rapport de position de ces parties entre elles et avec les autres parties de la tête; de l'autre côté, l'apophyse verticale postérieure du crâne (I) me semble d'autant plus devoir être comparée à l'os carré, qu'elle supporte à son extrémité inférieure un petit cartilage qui pa. raît représenter l'opercule.

Les deux cartilages longitudinaux, moyens et inférieurs des lamproyes (2), font partie de l'appareil hyoïdien. Les parties postérieures de la tête représentent l'occipital, le temporal. l'ethmoïde et le frontal; la lame supérieure et postérieure, qui est la plus grande et qui est située au devant de la cavité olfactive, est sans doute analogue aux cartilages des raies et des squales, réunis en un triangle plus ou moins long; ces deux parties me semblent correspondre aux os propres du nez des poissons osseux.

(1) Voyez plus haut, p. 446.

(2) Voyez plus haut, p. 447. 
Chez l'esturgeon, qui par la disposition des os de la bouche, ainsi que par tout le reste, se rapproche le plus des poissons osseux (1), l'os carré est partagé en deux moitiés, et le nombre des pièces sus-maxillaires est augmenté, puisqu'elles consistent en trois pièces, savoir deux qui sont paires et antérieures, et une impaire postérieure. La plus antérieure des premières est très-vraisemblablement une pièce intermaxillaire; la paire située derrière elle, représente les os palatins. Je regarde la pièce impaire comme le vomer ou les pièces ptérygoïdiennes du sphénoïde. La première opinion devient surtout vraisemblable par le nombre impair de cet os. La seconde manière de voir a en sa faveur en quelque sorte la position de cette pièce en arrière des pièces de l'os palatin; cependant le vomer est aussi situé en partie en arrière de ces pièces.

M. Cuvier, qui ne fait pas mention de la lame postérieure, regarde, ainsi que moi, les pièces postérieures, qui sont les plus grandes, comme des os palatins, celles situées à côlé d'elle comme des os sus-maxillaires, et parle d'os inter-maxillaires situés daus la substance des lèvres; je crois cependant devoir suivre l'opinion que j'ai précédemment citée, parce qu'il y a une troisième paire de cartilages que je n'ai pas

(1) Voyez pag. $457 \mathrm{ct} / 460$. 


$$
\text { 1) ANATOMIE COMPARÉ. }
$$

indiquée plus haut, mais qui se trouve à l'endroit occupé, chez les autres poissons, par les piéces sus-maxillaires. Cette paire est très-petite, alongée ; elle est située en dehors, horizontalement d'avant en arrière, entre l'extrémité inférieure des deux pièces paires, décrites plus haut, et séparée de celle du côté opposé de toute la largeur de ces pièces et de celle de la bouche. Comme la pièce inter-maxillaire est toujours située plus en avant et en dedans que la pièce susmaxillaire, il faut, si je ne me trompe, pour être conséquent, considérer la première de ces pièces comme l'os inter-maxillaire, la seconde comme l'os sus-maxillaire.

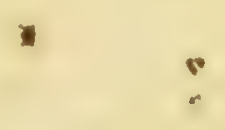




\section{GHAPITRE $\mathbf{X}$.}

\section{SQUELETTE DES REPTILES (1).}

$$
\text { \$. } 158
$$

Les reptiles presentent des différences si extraordinaires, tant sous le rapport de leur

(1) Toutes les parties du système osseux qui se retrouveront plus loin dans les squelettes des autres vertébrés ont été traitées dans les articles précédens à l'occasion des puissons; il faut cependant en excepter l'appareil hyoïdien et toutes les pièces, placées en avant du sternum, que M. de Bu.Aivilute a dósignées sous le nom de pièces médianes inférieures, en prenant pour point de départ, l'axe du canal digestif et en nommant supérieur tout ce qui est au clesswus de cc canal, inférieur tout ce qui est au desšns. Ces pièces ne doivent être décrites par M. MEckei. qu'aux paragraphes qui traiteront des organes de la respiration, de la voix, etc., dont il les considere comme des dépendances plus particulières. Toules les pices médianes inférieures constitucnt, d'après M. de Blalinville, une série que M. Robineau Desvoldy désigne sous le nom de chapelet abdominal, et qu'il regarde, en poursuivant les vues de la philosophie anatomique, comme des élémens de vertébres inférieures. Ces vertébres, plus particulièrement étudiées par M. Robinesu Desvorny dans les animaux inférieurs et spécialement dans les crustacés, les arachnides et les insectes, lui ont fait admettre successivement d'avant en arrière, chez les crustacés, ceux de ces animaux qui offrent le plus de complication: $1^{\circ}$ sept vertèbres intra-buccales formant l'appareil buccal interne, nommées pharyngéale, cricéale, thyréale, arythénéale, et hyoüdionne, en les rapportant aus appareils dont elles rappellent l'existence chez les animanx supérieurs, et servant principalement au jeu de la mastication et de la 
ensemble que sous celui de leur squelette, que dicjil le systéme osseux oblige de diviser cette.

déglutition; $2^{\circ}$ cinq vertébres post-buccales qui consistent dans les cinq paires d'appendices de préhension; $3^{\circ}$ cinq vertebres locomotrices; la première d'elles constitue la pince, les autr.s servent à la progression; $4^{\circ}$ cinq vertèbres abdominales dont l'usage est de porter les oufs ou les organes miles; $5^{\circ}$ une vertèbre natatoire; $6^{\circ}$ une vertibre anale.

La série supérieure ou le chapelet clorsal, l'analogue du rachis et de la boîte crânienne des animaux supérieurs, pré. sente chez ces mêmes crustacés, en procédant également d'avant en arrière, et à partir du mème point: $1^{0}$ une vertèbre labiale; $3^{\circ}$ une vertibre maxillaire; $5^{\circ}$ six vertèles sensoriales qui sont en rapport avec un nombre égal de sens admis par M. Robineau; 40 six vertébres dorsales dont la quantité peut ètre portée jusqu'à treize dans d'autres animaux articulés, (les branchigastres de M. Ronrant ou cruslacés isopodes de M. DE Latreilue), par exemple; 50 une coccy gienne.

Les six sens auxquels M. Roningau rapporte ces vertèbres sensoriales sont: " $1^{0}$ l'organe qui perçoit les molécules

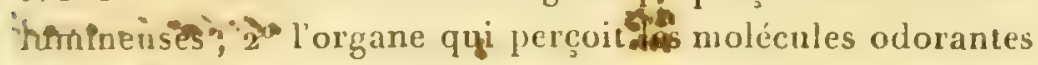
échappêes des corps; $3^{\circ}$ l'organe qui perçoit les vibrations sonores imprimées à l'air; $4^{0}$ l'organe qui perçoit directement la nature chimique des molécules alinentaires; 50 l'organe qui met ur animal, à l'aide d'un son déterminé, en rapport avec un autre animal; $6^{\circ}$ l'organe qui met l'animal supérieur en rapport avec les objels du dehors, à l'aide d'instrumrens'de locomotion qui par la perfection deviennent organes de tact, de toucher.

De lá les six vertèbres de la vuc, de l'olfaction, de l'audition, du gonil, du bruissement et de la mo ilité, ou optique, "lfactive, anditive, gustalc, sonore, motile."

Le nombre at le développement relatifs de crs vertebres 
classe en plusieurs ordres, qui.sont plus tranchés que ceux établis dans les autres classes.

ou de leurs élémens, joints aux usages variés auxquels on rencontre ces élémens de vertèbres employés, établissent parmi les animaux articulés autant de caractères différentiels qui ont permis a M. Rовıneu de changer la classification de ces êlres. Ces différences, d'après l'opinion de l'unité animale, dont M. Roвineau est très-persuadé, ne sont toutefois que des modifications d'un même type, auquel il est même possible de ramener les animaux mollusques et les annelides, en déterminant les caractères propres qui ressortent de l'étude de ees élémens vertébraux dans la constitution de ces êtres.

La possibilité de cette détermination entraîne la nécessité de donner une nouvelle définition du mot vertèbre. Aussi M. Romineav Desvordy, en étendant davantage l'idée qu'a établie a ce sujet M. Geoffrox-Sant-Hilaire, considere-t-il une vertébre comme consistant réellement dans la réunion de divers systèmes qui en font un organe spécial. Chez les animaux pourvus d'un systime solide, elle est formée de neuf pièces élémentaires; l'une des pièces est unique et simple, mais susceptible de se diviser en deux portions. comme on en troive - exempleschez les mollusifues; parm, Jes autres úlémens, quatre sont dans les animaux articulés, simples, ou binaires, ou disposés par paires; quatre autres également binaires sont divisés en plusieurs fractions pour former divers instrumens. $\Lambda$ insi « le système solide, primitif, des animaux se développe tonjours par portions paires ou par couples et ne peut fournir au-delà de cinq paires nour la plus granile perfection de la vertèbre. $\rightarrow$ Les deux paires se peuvent seulement subdiviser. Les usages de la vertèbre sont donc ceux-ci. "Elle enveloppe seulement et protège le corps Ís animaux mollusques; elle protège le corps des animalıx articulés, sert à l'exécution de tous 
Cependant, à les considérer de près, ces différences ne sont pas tellement considérables

leur's actes e! acquiert sur eux sa plus grande perfection, sur. tout pour les organes du mouvement; moins développée sur les animaux sıpérieurs, elle perd ́cón utilité générale à mesure qu'elle sert davantage à recouvrir l'encéphale. nEn réunissant à ces paires ćlémentaires les muscles qui les meuvent et les nerfs qui transmettent la propriété de sentir à tout l'appareil, la vertèbre constitue un ensemble dont on conçoit que la portion solide puisse n'être pas la partie la plus importante, d'où résulte la possibilité de retrouver des traces de la disposition vertébrale chez des êtres dépourvus de squelette comme les sangsues. D'mportantes modifications pourront être également présentées par le siége de la partic dure: elle pourra être le résultat de la sécrélion des muscles, comme M. de Buanvilie le professe pour les os des animaux rcrtébrés, ou clle sera le produit d'une action semblable exccutée par la peau, comme le même professeur admet qu'il arrive pour les animaux inférieurs. Ces deux siéges du système solide on l'absence de ce même système ont tété la base de ces trois divisions du règne en animaux : $1^{0}$ osseux it l'intérieur; $2^{0}$ osseux il l’extéricur; $3^{\circ}$ privés de pièces solides.

Ces appareils vertébraux jouissent d'une sorte de vie à part chez les animaux inférieurs; en se spécialisant el en se centralisant ils perdent de leur existence indépendante, c'est ce qui arrive aux animaux supérieurs chez lesquels les fonclions sont plus particulièrement déterminées et groupées vers un foyer commun. La détermination des vertèbres, qu'il disigne d'après la partie du système nerveux qui leur est spécialement affectée, a conduit M. Roringau à des résultats tout-à-fait neufs. $\Lambda$ insi il regarde le corselet des insectes comme formé par la réunion de plusicurs yerrèbres senso-

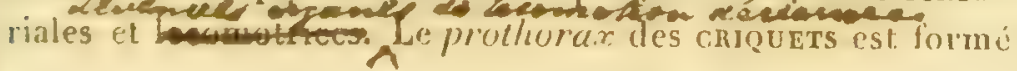


qu'il faille renoncer à la mélhode suivie pour les poissons; on peut par conséquent encore ici suivre chacune des quatre divisions principales du système osseux à travers les quatre ordres principaux de cette classe.

A. OS DU TROKC.

$$
\text { S. } 159 \text {. }
$$

Ce qui a été dit sur les différences considérables de la disposition du squelette, s'applique, à l'exception des membres qui manquent is presque tout l'ordre des ophidiens, principalement aux os du tronc, tant sous le rapport de la forme que du nombre des pièces qui le composent. Comme les différens os du trone sont,

sclon lui par la vertèbre gustale; la vertèbre sonore forme ce qu'on appelle les premiòres ailes; la vertèbre motile donne lieu aux ailes postéricures des insectes et aux balanciers des dipteres en particulier, que cet anatomiste considère comme pourvus d'organes régulateurs du mouvement, comme le sont, dans les vertébrés supérieurs, les organes c'érebelleux. Chezles insectes et chez les articulés en général. la faculté de vouloir et l'instinct d'agir sont renfermés dans l'organe d'exécution. Leurs facultés, centralisées chez les animaux supéricurs, sont disséminées. Leur intelligence n'est done pas une fonction spéciale; résultat que M. Robinesu Desvony généralise. ( Toy. l'ouvrage déja cilé, Recherches sur l'organisation vertébrale des crustacés, elc., $18_{2} 8$, Paris.) Ces vues demandent à être comparées à celles qui ont

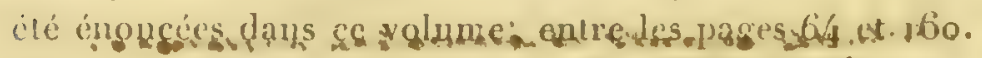

(Noke des tridlitelicurs.) 
D'ANATUMIE COMPARÉE.

dans les divers ordres des reptiles, plus modifiés d'après un type commun que chez les poissons, le mieux que je crois pouvoir faire, c'est de considérer ensemble les os du trone de chaque ordre.

\section{Batraciens.}

$$
\text { \$. } 160 \text {. }
$$

Les os des batraciens offrent dans toutes les parties du tronc, aussi bien que dans tout le squelette, la forme la plus imparfaite, et doivent par conséquent ètre étuliés les premiers.

\section{a. Colonne vertébrale.}

$$
\text { S. } 161 .
$$

Voici les conditions générales qu'offrent les vertèbres dess batraciens.

Elles sont déprimées de haut en bas, pourvues d'apophyses épineuses supérieures, trèscourtes, on en manquent totalement.

Elles ont très-généralement des apophyses transverses, horizontales, plus ou moins courtes, se délachant à peu près du milieu du corps, et de ciraque côté une apophyse articulaire, antérieure et une prostérieure, encroùtée de cartilage, large, mais peu élevée; les antérieures de ces apophyses sappliquent sous les postérieures de la vertèbre qui précède immédiatement. 
1. Batraciens urodèles.

\section{\$. 162}

Les batraciens à queue se distinguent d'une manière très-frappante de ceux qui sont sans quelle.

Chez les premiers, les vertèbres sont toujours, $1^{n}$ beaucoup plus alongées; $2^{\circ}$ plus déprimées et pourvues dapophyses épineuses et transverses beaucoup plus faibles que celles qui leur corresponlent chez les batraciens anoures; $3^{\circ}$ les extrémités antérieure et postérieure des corps de vertèbres sont, d'après le type des poissons, fort concaves et remplies de substance fibro-cartilagineuse; $4^{\circ}$ la queue est formée de vertèbres propres, diminuant considérablement de volume d'avant en arrière, et distinguées des autres par leur brièveté, par leur élat comprimé d'un côté à l'autre, et par la présence d'apophyses épineuses considérables . se réunissant souvent en crête inférieurement; $5^{\circ}$ elles ont, à l'exception des vertèbres candales. presque toutes le même volume, ou à peu prís; $6^{\circ}$ enfin, elles sont considérablement plus nom. breuses.

C'est chez les protées que ces différences sout le plus prononcées; leurs verièbres sont le plus nombreuses et le plus alongeses; en outre 


\section{1) A NTOME COMPALÉ:}

leurs apophyses transverses offrent lá plus grande largeur d'avant en arrière, et les apophyses épineuses, courtes et larges, n'existent qu'à la partie postérieure des vertèbres situées au devant de la queue.

Chez la sirène, les vertèbres sont aussi plus alongées que chez les autres; matis elles sont pourvues d'apophyses transverses plus alongées, plus pointues, situées à l'extrémilé antérieure du corps.

Chez les salamandres et les cécilies, chaque apophyse transverse, courte, des vertèbres sitrées au devant de la queue, est divisée en une moilié supérieure et en nne inférieure.

Chez les salamandres terrestres, ces apophyses sont proportion nellement plus longues que chez les salamandres aquatiques; elles sont simples dans la moitié interne de leur longueur, bifurquées dans l'externe.

Chez les salamandres, mais non chez le protée et la sirène, il y a dans la queue plus de vert tébres que dans la partie antérieure du rachis.

\section{Batraciens inoures.}

$$
\text { \$. } 163 .
$$

Ces batraciens ont un bien moindre nombre de vertèbres, qui, conformément à la forme géviérale du corps, sont considérablement plus 
larges. Les apophyses épineuses sont extrêmement peu prononcées. Elles manquent même touti-fait dans le rana gibbosa. Dans le pipa, le bufo typhonius et la rainette (hyla), principalement chez la dernière, ces apophyses sont au plus grand degré de développement. Mais ce qu'il y a de variable surtout, c'est $: 1^{\circ}$ le rapport des pièces transverses au corps de la vertèbre; et $2^{\circ}$ la disposition de la portion terminale du rachis.

1. Les pièces osseuses transverses sont, dans tous les genres, entièrement soudées avec le corps des verlèbres. Elles se distinguent en outre par leur direction. Les antérieures sont, comme chez tous les batraciens à queue, dirigées en dehors et en arrière; les moyenues sont situées plus transversalement, les postérieures en avant.

Les diverses espèces varient même considérablement entre elles sous le rapport de la disposition de ces pièces. Chez la plupart des grenouilles et des crapauds, chez les premières surlout, elles sont en général plus courtes que chez les pipas. Mais ce développement considérable ne porte ici que sur la seconde et la troisième vertèbre, principalement sur celle-ci; les cinq autres, surtout les quatre dernières, le sont beaucoup moins et sont même absolument plus petites que chez les premières. Isa troisième s'élargit considérablement en dehors, el supporte 
ici un grand cartilage oblong et mobile, beau. coup plus large que la verlèbre elle-mème, et qui présente l'aspect d'un rudiment de côte.

Chez le rana gibbosa, les vertèbres sont à leur minimum de développement, et elles sont au maximum chez le bufo typhonius; elles ont, chez ce dernier, presque toutes un volume égal, à l'exception de la seconde.

La première vertèbre n'a ordinairement point d'apophyses transverses. L'exception faile par le pipa n'est qu'apparente; l'apophyse de celle vertébre appartient évidemment à la seconde vertèbre qui est confondue avec la première.

Les seconde et troisieme apophyses transverses offrent communément la plus grandelargeur; les postérieures sont constamment beaucoup plus étroites que les antérieures; elles sont apointies, tandis que les antérieures s'élargissent plus ou moins vers leur extrémité libre.

2. I.es batraciens sans queve se distinguent de ceux pourvus de queue, par la disposition de la partie terminale de la colonne vertébrale, en ce sens, que toutes les vertèbres situées derrière le sacrum, c'est-à-dire les vertèbres coccygienues, sont soudées ensemble, et for'ment un os creux, à parois très-minces, alongé, comprimé d'un côté à l'autre, plus épais inférieurement, plus étroit supérieurement, qui s'étend presque jusqu'à l'extrémité postérieure les os coxaux; os beaucoup plus mince que les 
autres vertèbres, et pas beaucoup plus court que l'ensemble qu'elles forment.

3. Le sacrum se distingue, dans ces deux orlres, de la mème manière que les autres vertèbres. Chez les batraciens anoures, les trois pièces qui sont séparées dans les batraciens urodèles, sont entièrement soudées ensemble; elles sont en outre plus longues et plus larges, fort renflées en dehors. Chez les gyrenouilles, elles offrent la petitesse proportionnelle la plus extrême. Chezle pipa, elles présentent, à un haut degré, le volume le plus grand, et surtout la longueur et la largeur les plus considérables. Le sacrum du pipa est percé de chaque côté de deux trous successifs pour le passage des nerfs, ce qui est un indice de composition de deux vertèbres. Cet os est absolument simple chez les grenouilles.

Les batriciens anoures se distinguent, en outre, eux-mèmes par le rapport du coccyx avec le sacram; chez les grenouilles et les crapauds, ils sont séparés l'un de l'autre; chez le pipa, ils sont soudés, au contraire, et ne forment qu'un os.

La détermination des os terminaux du rachis des batraciens anoures a été méconnue; le dernier os creux a été pris pour le sacrum, et le sacrum pour la dernière vertèbre; on disait que le coccyx manque (I), et que l'appui de la queue

(1) Cuvier, Leçons d'anatomic comparie, vol. I. 
1) ANATOMIE Comparé.

n'est qu'un cartilage qui disparaît pendant les métamorphoses de l'animal (I). Sans doute le coccyx est purement cartilagineux chez les larves, tandis que les verlèbres sont dejà osseuses; il y parvient même à un volume proportionnellement plus considérable; mais il est réel que cet organe s'ossifie seulement plus tard que les autres os qui se développent beaucoup plus; et que l'os terminal n'est autre chose que cet appui cartilagineux de la queue dans un état de réduction.

3. Différences qui distinguent les vertèbres les plus antérieures des autres.

$$
\text { S. } 164
$$

Les vertèbres du devant se distinguent trèsgénéralement des autres par le défaut d'apophyses transverses. Le pipa semble faire exception en apparence; les apophyses transverses de la première vertébre sont en effet, dans ce genre, beancoup plus petites que les deux suivantes, quoique deux fois plus grandes que les autres, et pour le moins aussi larges que le corps; mais nous avons déjà expliqué ce fait.

Il existe, en outre, plusieurs autres différences fort remarquables qui sont d'autant plus

(1) Froriep, dans une nole à sa Iraduclion de l'anatomie comparće de. Cuvier. 
considérables, que toutes les espèces ne les présentent pas prononcées à égal degré.

La différence la plus frappante est offerte, autant que je sache, par le protée, si toutefois la détermination suivante est fondée.

A l'extrémité postérieure d'une lame triangulaire, longue, mince, apointie en avant, le corps de l'os basilaire, dont le bord postérieur présente au milieu une échancrure, et deux apophyses articulaires transverses, alongées et plates, on voit s'élever verticalement, et un peu en avant, un demi-anneau vertical, étroit, déprimé, qui par la partie inférieure de ses côtés est tout-à-fait soudé à cette lame. Cet anneau est vraisemblablement la première vertèbre cervicale.

Celte opinion est fondée sur les considéralions suivantes:

$1^{\circ}$ La forme de cet anneau en général;

$2^{\circ}$ Sa ressemblance avec la première vertèbre des batraciens anoures, et spécialement des grenouilles, qui est surtout remarquable;

$3^{\circ} \mathrm{La}$ grande ressemblance de la première vertèbre du protée, avec la seconde de la plupart des autres animaux plus élevés, principalement des reptiles supérieurs.

Mais voici des raisons qui militent contre cette manière de voir.

10 Le défaur d'un semblable anneau chez les autres butraciens anoures; 
DANATOMIE COMPARÉE.

$2^{\circ}$ Ia concordance de l'anneau suivant du protée avec le premier des autres batraciens anoures.

Daprès cela, on pourrait être tenté de consinérer cet annean comme une partie de l'occipital.

Dans cette hypothèse, le premier anneau des batraciens urodèles différerait des anneaux suivans, ainsi que du premier anneau des batraciens anoures, par la présence d'une saillie antérieure sur le corps, qui, beaucoup plus étroite et proportionnellement aussi plus courte chez le protée que chez ceux-ci, s'applique en avant et en bas dans la lacune du bord postérieur du corps du basilaire.

Chez les batraciens anoures, le corps du premier anneau est beaucoup plus large qu'aux autres anneaux, surtout dans son extrémité antérieure, où elle est plus de deux fois plus considérable.

Chez les grenouilles, les deux moitiés de l'arc. ne se réunissent pas dans la ligne médiane; disposition que je n'ai pas retrouvée chez les crapauds et le pipa.

La seconde veriébre cervicale n'offre nulle part de grandes différences.

Les autres différences, notamment celles des vertèbres sacrées et coccygiennes, ont déjà été indiquées. 


\section{b. Cỏtes.}

\section{S. 165 .}

Les batraciens urodèles et anoures diffèrent entre eux d'une manière très-frappante, en ce que, très-généralement chez les premiers, les apophyses transverses, du moins plusieurs d'entre elles, surtout de celles de la partie antérieure, supportent des os minces, courts, droits, pointus et mobilement articulés avec elles, tandis que les seconds ne présentent pas ces os.

Ordinairement on considère comme des côles les os mobilement articulés des batraciens urodèles, et les os soudés des batraciens anoures comme des apophyses transverses, en leur refusant les côtes; mais peut-ètre ces deux parties peuventelles recevoir absolument la mème détermination, et les premières et longues apophyses des batraciens anoures sont-elles composées des apophyses courtes et des os accessoires des batraciens urodèles qui sont mobilement articulés avec ces apophyses. Le développement plus considérable des membres chez les batraciens anoures est sans doute cause du non-développement d'une partie de ces apophyses en un os propre. Peut-être aussi les os des batraciens urodèles, considérés comme des côtes, ne sont-ils pas des còtes, mais seulement 
1) ANATOME COMPALÉ.

les os accessoires des apophyses transverses, qui chez ces animaux ne seraient pas soudés avec le reste de la vertèbre, tandis qu'ils le seraient chez les batraciens anoures, qui leur sont supérieurs.

Quoi qu'il en soit, les différens genres de $b a-$ traciens urodèles se distinguent entre eux sous le rapport du nombre et du volume de ces os. Ainsi, chez la sirène (siren), ils n'existent que sur sept verlèbres, depuis la seconde jusqu'à la huitième; chez le protée, seulement sur six, de la cinquième à la dixième; chez les salamandres, ils existent sur toutes les vertèbres situées en avant de la queue, à l'exception de la première.

On remarque, en harmonie avec la différence des apophyses transverses qui se distingue chez les tritons et les salamandres (1), que l'extrémité interne des côtes se bifurque, chez les $s a$ lamandres, plus profondénient en deux têtes, situées l'une au-dessus de l'autre. Chez les mèmes salamandres, l'extrémité externe de la côte la plus antérieure est considérablementélargie, et se termine par un bord arrondi.

Le dernier de ces os costiformes devient, dans les uns et les autres, brusquement beaucoup plus gros que tous les autres, plus long que ceux situés immédiatement en avant, et constitue le sacruin conjointement avec la vertèbre

(1) Voyez plus baut p. 541. 
unique qui le supporte, et qui ne diffère pas du reste des autres vèrtèbres.

Le sacrum supporte l'os coxal, ce qui, joint à la modification précitée de l'os costal, rend plus vraisemblables les présomptions que nous avons émises sur la détermination de ces os chez les batraciens urodèles.

\section{c. Sternum.}

$$
\text { S. } 166 .
$$

Les batraciens possèdent très-généralement un sternum, qui se parlage d'avant en arrière en plusieurs pièces, en partie entièrement séparées les unes des autres par des os intercalés qui proviennent de l'épaule. La présence du sternum est en général admise par les anatomistes; je crois seulement que chez plusieurs de ces animaux le véritable sternum n'a pas été aperçu, ou que d'autres os ont été pris pour lui.

a. Batraciens urodeles.

$$
\text { S. } 167 \text {. }
$$

Jusqu'ici je n'ai pas encore pu trouver positivement le sternum chez le protée; il existe, au contraire, chez les tritons et les salamandres, et même à des degrés de développement très- 


$$
\text { D'. INATOMLE COMPAREE. }
$$

divers, ce qui est fort intéressant à cause de la concordance qu'il présente avec les autres différences graduelles de leur structure.

Le trilon cristatus présente seulement un cartilage alongé, mince, partagé antérieurement en deux branches divergentes, long d'environ quatre lignes, situé dans la ligne médiane, au devant de la symphyse du pubis, et fixé à son extrémité antérieure.

Chez le salamandra atra, ce cartilage est en proportion un peu plus long, surtout plus large, et se termine, à son extrémité antérieure, par deux pointes assez divergentes, dont chacune a environ la moitié de la longueur totale du cartilage.

Il est plus considérable encore chez le salnmandra maculata: les pointes antérieures surtont en sont beaucoup plus développées et aussi longues que le cartilage moyen.

A ce cartilage qui existe partout et qui, quoique situé fort en arrière, est l'analogue du sternum, on voit s'unir chez les salamandres encore deux autres pièces beaucoup plus petites, qui en sont fort éloignées, puisqu'elles se trouvent dans la région des membres antérieurs. Cette disposition paraît, autant que s'étendent mes recherches particulières, au salamandra maculata. Le cartilage dont il est ici question rappelle non-seulement la disposition de plusieurs poissons, mais il est même l'indice du développe- 
ment plus considérable du sternum que l'on remarque chez plusieurs sauriens, et qui est surtout extraordinaire chez les chéloniens.

La plus postérieure de ces pièces, qui est la plus volumineuse, a environ deux lignes en long et en large; elle est partagée à son bord an . térieur en deux lames, l'une supérieure et l'autre inférieure. La gouttière qui en résulte embrasse la partie interne du bord postérieur des deux clavicules.

Uneautre pièce beaucoup plus petite se trouve au devant de ces clavicules dont elle est par conséquent séparée par la pièce qui vient d'être décrite; elle a la forme dune croix, dont les branches transversales sont beaucoup plus considérables que les longiludinales, qui sont trèscourtes et à peine sensibles.

D'après cela, le sternum des batraciens urodèles consiste, lorsqu'il est à son plus haut degré de développement, en trois pieces parfaitement séparées les unes des autres, répandues dans presque toute la longueur du trone, correspondant par conséquent dans les derniers points, de l'espace qu'elles occupent, à la majeure partie du rachis, ce qui est en rapport sans doute avec la forme alongée, et la disposition des os costiformes qui règnent sur toute la longueur du tronc. 


\title{
b. Batraciens anoures.
}

\author{
S. 168 .
}

Les batraciens sans queue offrent aussi des différences considérables.

L'organisation la pỉus simple est cellé offerte par les crapauds, dont le sternum consiste seulement en un cartilage simple, analogue au deuxième os, moyen, dusalamandra maculata; ce cartilage, fixé au même endroit que le premier, savoir à la clavicule postérieure, est alongé, un peu rétréci au milieu, el dilaté dans sa moitié postérieure, où il se convertit en une lame ronde et mince.

Il est, chez les grenouilles, beaucoup plus large dans sa partie située en arrière; et son bord postérieur présente une fente profonde dans sa ligne médiane. Il est en même temps, dans sa partie antérieure qui est alongée, en général plus ossifié que chez les crapauds; postérieurement il se montre cartilagineux.

Indépendamment de ce cartilage, les gre. nouilles en ont encore un autre antérieur, correspondant exactement au cartilage le plus antérieur du salainandramaculata, par la positiou qu'il occupe \& l'extrémité antérieure de la symphyse du pubis, mais qui s'en distingue par sa 
forme trèsalongée, et n'est un peu plus large qu'à sun extrémité antérieure et postérieure.

C'est chez le pipa que le sternum est te plus développé, du moins sous le rapport de l'éten. dué. On y trouve les pièces moyenne et antérieure, dont la première est surtout extraordinairement développée, quoiqu'elles soient cartilagineuses toutes deux.

La première, qui par son extrémité postérieure atteint presque la symphyse du pubis, est une lame quadrilatère, mince, large, qui par ses deux bords antérieurs touche aux bords situés en arrière des deux clavicules postérieures, et a environ un pouce de longueur sur autant de largeur.

La pièce antérieure est beaucoup plus petite; c'est un cartilage semi-lunaire prenant point d'appui sur le bord placé en avant de la clayicule antérieure, ayant sa convexité tournée le mème sens.

D'après quelques auteurs, ce ne seraient pas les seules pièces sternales. Il existe très-généralement, chez les batraciens, à côté de la ligne médiane, le long du bord antérieur de la clavicule ou des clavicules, une lame cartilagineuse considérable, qui, chez les batraciens urodèles et les crapauds, s'avance du côté opposé, de telle façon que celle du côté droit se trouve sous celle du côté gauche, plus près de la superficie; chez le pipa, elle se soude dans la 


$$
\text { D'ANATOMIE COMPARÉ. }
$$

ligne médiane avec celle du côté opposé; elle manque, au contraire, chez les grenouilles, où les clavicules antérieure et postérieure se réunissent.

Cette pièce est considérée, chez les batraciens urodèles, comme le sternum entier; chez les autres, comme une partie du sternum; je crois cependant devoir, avec plus de raison, la regarder seulement comme une partie de la fraction claviculaire de l'épaule, $1^{\circ}$ parce qu'elle a toujours avec celle-ci des rapports plus intimes qu'avec les pièces sternales; et $2^{\circ}$ que de cette manière le chevauchement des pièces de mème nom et des deux côtés l'une sur l'autre, me paraît plus facile à expliquer.

L'ostéologie, aussi bien que la myologie des sauriens supérieurs, et mème des mammifères, offre encore d'autres argumens à l'appui de ma manière de voir; je me réserve de les exposer plus au long en traitant des membres antérieurs.

\section{Ophidiens.}

$$
\text { \$. } 169 \text {. }
$$

Le squelette des ophidiens est le plus simple, non-seulement de ceux des reptiles, mais parmi les squelettes de tous les vertébrés en général, en ce sens que : $1^{\circ}$ il manque, conformément à la disposition du corps entier, de tous ou de la plupart des os des membres; $2^{\circ}$ que 
toutes les vertèbres sont des répétitions presque serviles les unes des autres; $3^{\circ}$ que le sternum leur manque, on peut dire toujours. Néanmoins, j’ai cru devoir considérer les os du tronc des batraciens avant ceux des ofhidiens, parce que: $\mathrm{I}^{\circ}$ ce sont précisément ces os qui sont chez eux, à plus d'un égard, inférieurs à ceux des ophidiens; $2^{\circ}$ que le squelette des chéloniens, auquel conduit celui des batraciens, notamment par le pipa, est aussi plus imparfait que celui des ophidiens, et se rapproche du squelette des vertébrés moins que celui des sauriens, auquel conduisent les ophidiens par des transitions insensibles.

a. Colonne vertébrale.

$$
\text { S. } 170 .
$$

Le rachis des ophidiens se distingue de celui de la plupart des autres reptiles, ainsi que de la plupart des vertébrés, surtout par le grand nombre et l'uniformité des os qui le composent.

Les vertèbres thoraciques et abdominales ont, d̀ l'exception des premières, à peu près le mème volume, et pour la plupart aussi la mème forme; il n'y á què les vertèbres coccygiennes qui différent entre elles, en ce qu'elles diminuent de volume d'une manière insensible, mais ordinairement peu considérable.

Les vertèbres dorsales et lombaires sont plus 
D'ANATOMIF COMPARÉE.

larges, proportionnellement plus courtes que les vertèbres coccygriennes. Leurs apophyses transverses sont souvent beaucoup moins développées en longueur que celles de ces dernières.

Aucun exemple ne saurait, mieux que celui des ophidiens, tirer plus sûrement d'erreur les personnes qui s'attendraient à voir reproduire la forme de tout le corp' dans les parties spéciales qui le constituent. Les vertèbres des ophidiens ue sont en effet nullement fort alongèes. Elles sont, au contraire, du nombre des vertèbres les plus courtes et les plus larges; elles sont notamment bien moins alongées que celles des batraciens urodèles et de beaucoup de sauriens. C'est de là que dépend sans contredit la mobilité extraordinaire et la faculté qu'a le corps de prendre les courbures les plus variées

Celte faculté se rattache à une aurre condi. tion de levir forme. Toutes les surfaces de contact des vertèbres sont très-généralement des facettes articulaires, et l'on trouve par conséquent ordinairement, sur une vertèbre d'ophidien, dix facettes encroûtées de cartilage, qui sont :

1. Deux facettes impaires, au corps de la vertèbre; une antérieure et une postérieure, dont la première est concave, la seconde convexe, et qui s'engrènent l'une avec l'autre. La dernière est distinguée du corps par un petit étranglement ou col; 
$2^{\circ}$ Quatre faceltes paires situées à la base du corps de la vertèbre. Il y en a de chaque côlé, deux inférieures qui sont dirigées en dehors, deux supérieures qui sont dirigées en dedans. Les premières forment des saillies plus prononcées; toutes sont plates et tellement disposées, que les facettes antérieures de la vertèbre qui est en arrière se glissent sous les postérieures de la vertèbre placée en devant. Elles forment la région la plus large de la vertèbre.

Ceste disposition rend possibles les mouvemens multiples et rapides du corps des serpens.

En outre la majeure partie des vertebres d'ophidiens, surtout les vertèbres thoraciques et abdominales, et les plus antérieures des vertèbres coccygiennes, quelquefois même toutes ces verlèbres, offrent, à l'extrémité antérieure de leurs corps, immédiatement sous les facettes articulaires antérieures el externes, des apophyses trunsverses, peu longues, qui sont situées d'ordinaire précisément au dessous des facettes articulaires antérieures, inférieures et latérales.

Jes ophidiens ont très-généralement des apophyses épineuses supérieures qui n'appartiennent nullement aux boas seuls, comme le prétend M. Home ( 1 ).

Elles ne sont jamais fort considérables; le plus souvent elles sont dirigées en droite ligne d'avant en arrière.

(1) Philos. transactions, $\mathbf{1 8 1 2}$, p. 166. 
1)'ANATOMLE COMPARÉE.

Les apophyses épineuses inférieures mêrnè, ne manquent presque jamais ; elles sont ordinairement plus hautes, quoique plus courtes d'avant en arrière que les supérieures, mais elles n'existent pas aussi généralement que les apophyses épineuses supérieures sur toutes les verlebres. Elles sont paur la plupart plus ou moins fortemen t dirigées en arrière. Lorsqu'elles n'occupent que certaines étendues de la colonne vertébrale, elles n'existent qu'à sa partie antérieure et postérieure. Les dernières sont ordimairement les plus longues.

Les épines supérieures et inférieures sont très-comprimées d'un côté à l’antre et minces.:

$$
\text { S. } 171 .
$$

Ces conditions générales offrent dans les différens genres d'ophidiens, surtouı les modifica tions suivantes.

Les verlèbres ont partout à peu près les mêmes dimensions; pourtant les boas me semblent avoir les vertébres les plus courtes et les plus larges.

On rencontre les vertèbres au maximum de développement dans le genre amphisbìne; les iplus petites dans le genre éry $x$.

C'est chez les amphisbènes que les apophyses épinenses supérieures offrent la petitesse la plus extreme. Ce ne sont que de petites crêtes trèspeu prononcées, montant de bas en haut et 
d'avant en arrière, qui s'élèvent peu au dessus des faces latérales de l'ar'c, et qui ne sont distinctes qu'au cinquième antérieur de la colonne vertébrale.

Dans le genre rouleau (tortrix), elles sont un peu plus forles, et règnent dans toute la longueur de la colonne vertébrale.

Elles sont un peu plus fortes encore chez les platures et les éryx; peu élevées aux dernières vertèbres coccygiennes, elles y sont bifurquées en deux moitiés latérales.

Chez les orvets (angunis), les plus antérieures, mais surtout celles des vertebres coccygiennes, sont très-fortes. Elles forment sur ces dernières des épines considérables, presque aussi longues que le corps des vertèbres; ces épines s'élèvent de l'extrémité postérieure du corps, en haut et en arrière.

Elles sont au maximum de longueur dans les genres naja, vipère, couleuvre (coluber, crotale, bongare (peusodobua), hydrophis, boa, surtoul dans les deux derniers. Elles occupent toute la longueur du corps sur la plupart des vertèbres, et ont la mème largeur dans toute leur hauteur; elles deviennent mème plus larges supérieurement chez les trigonocépliales, et s'atteignent par leurs extrémités antérieure et postérieure.

Chez les boas, elles ne correspondent partout qu’à environ la moitié postérieure du 
D) A ATOMIE COMPARÉF.

corps. Chez les autres, celles des vertèbres les plus antérieures sont plus courtes d'avant en arrière, et plus ou moins apointies.

Elles sont très-généralement petites aux vertèbres coccygiennes; néanmoins chez l'hydrophis, les épines supérieures et inférieures, qui, à la queue sont d'égale hauteur, y sont beaucoup plus étroites, mais bien plus longues que les autres. En effet, tandis que ces mêmes épines de la queue sont communément les plus courtes, la plupart d'entre elles offrent, chez les hyclrophis, le double de la hauteur des autres, quoique celle-ci soit même considérable, comme la remarque en a déjà été faite. La plupart des épines inférieures, en outre, ne sont pas bifurquées, ou du moins cette division est très-insensible.

Les apophyses épineuses inférieures n'existent, chez les rouleaux (tortrix), qu'ì peu près an septième antérieur du corps. Les vertèbres coccygiennes n'en offrent aucune trace.

Le genre éryx présente la même organisation; les épines antérieures y sont seulement plus longues, et il existe des rudimens d'épines inférieures courts, larges, non réunis dans la ligne médiane.

Les amphisbènes n'ont des épines inférieures. qu'aux huit verièbres cervicales antérieures, et aux vertèbres coccygiennes. Les premières di- 
minuent subitement de volume d'avant en arrière; les secondes sont divisées aux quatre premières verlèbres, et forment aux autres des arcs larges, percés d'une grande ouverture, et offrant à leur point de réunion un pédicule court, dirigé en bas.

Chez l'anguis fragilis, il n'y a, parmi les vertèbres les plus antérieures, que les premières cervicales qui soient munies de courles apophyses epineuses. Celles des vertèbres coccy'giennes ressemblent beaucoup à celles des amphisbènes; mais elles sont beaucoup plus alongées, par suite du développement extraordinaire de la pointe inférieure et moyenne.

La quatre-raies (coluber elaphis) présente, dans le dixième antérieur de sa colonne, des épines inféricures très.fortes, étroites, verticales, qui augmentent de longueur des parties antérieure et postérieure vers la partie moyenne.

Le coluber pluthonius offre la même disposition.

Chez les boas, ces épines existent à peu près sur le cinquieme antérieur du rachis.

Chez les maja et les vipères, et particulièrement chez les crotales, presque toutes les vertèbres sont munies d'épines inférieures considérables, étroites et longues, qui sout dirigées en arrière.

Dans plusieurs espèces de ces genres, en outre dans le genre trigonocéphale, les épines 
inférieures de la queve sont bifurquées en deux moitiés latérales, qui sont pour la plupart verticales, très-rapprochécs l'une de l'autre, et forment de larges plaques. Elles sont simples, presque insensibles chez les boas.

Les apophyses transverses sont ordinairement courtes, minces, élroites et alongées. Le bongarus semicinctus fait exception à cette règle; chez lui, ces apophyses forment des disques arrondis, très-larges d'avant en arrière, et de dedans en dehors, minces de haut en bas, qui ont presque toute la longueur du corps des verièbres.

Elles sont surtout développées dans les genres trigonocéphale, orvet et ophisaure; elles manquent au contraire tout-à-fait chez lès serpens d'eau (hydrophis), ou du moins ne sont indiquées qu'aux vertèbres antérieures par de petites saillies, à la partie supérieure des épines inférieures.

Simples pour la plupart, elles sont doubles aux six vertèbres antérieures chez les vipères et les ırigonocéphales, et aux quatre premières vertèbres du crolalus horridus. Il en existe aussi une rangée inférieure plus longue, et une supérieure plus courte chez le devin (boa constrictor); la dernière rangée disparaît chez les autres ophidiens.

Quelque particulière que paraisse cette disposition, elle n'est pourtant qu'un développe36. 
ment ultérieur de la forme des côtes; elle résulte de ce que la rangée supérieure des apophyses transverses colrespond à une apophyse située à l'extrémité postérieure des côtes qui est dirigéc en arrière, et qui s'alonge considérablemeut à la dernière còte. Elles sont com. munément au maximum de leur longueur aux vertèbres coccygiennes, et elles sont surtout considerables sur ces vertèbes dans les genres orvet et boa.

Généralement transversales sur ces vertèbres, elles se portent en dehors et en bas chez les boas et les couleuvres, tandis que chez le platurus fasciatus elles sont dirigées directement en bas.

La plupart des apophyses transverses sont simples, surlout celles qui supportent des còtes. Quelquefois cependant, surtout chez les couleuvres et les boas, celles qui sont siluées à l'origine de la queue sont bifurquées; disposition remarquable en ce que c'est un passage aux côtes qui confirme l'analogie qu'offrent ces os avec les apophyses transverses.

En effet, une des pointes postérieures de la dernière côte s'alonge à tel point, que celte còte paraît bifurquée. İa vertèbre qui suit ne supporte plus de côle, mais une apophyse transverse absolument bifurquée de la mème manière que la còte précédente, seulement jusqu'ì sa racine; apophyse dont la branche externe, qui est convexe en dehors et, concave en dedans, a seule- 
DัANATMH: COMPAREF.

ment plus de longueur; landis que la branche interne, qui se recourbe dans un sens opposé à lexterne, et embrasse avec elle une ouverture ronde, considérable, se porte plus en dedans. Cette disposition existe sur les trois ou quatre premières vertèbres coccygiennes, chez les trigonocéphales même sur six de ces vertèbres, et disparait insensiblement, la branche externe de l'apophyse devenant plus courte.

Cette description démontre que la côte se bifurque, se cunfond avec le corps de la vertèbre, et devient ainsi apophyse transverse. It n'y'a dans cette disposition rien qui se rapporte aux apophyses épineuses inférieures, qui existen't également, en partie bifurquées elles-mêmès, sur les vertebres coccygiennes antérieures.

Chez les serpens d'eau ( $/$ jodrophis) il semblerait; au contraire, exister une corrélation entre cette disposition et celle des apophyses épineuses inférieures, du moins d'après ce que j'ai cru remarquer sur un squelette du cabinet de paris.

Yers la queue, les côtes se renflent dans leur parlie interne, comme dans les espëcés préritéps; il en résulte à leur face supérieure uñe siillie qui, aux dernières còtes, se prólunge en inc apophyse propre, considérable. Aux vertélires coccygienries, la côte se soude avèc le corpis, se ports on dedans, et devient ainsi didbord me apophyse transverse, et consuite secule- 
ment apophyse épineuse inférieure, qui, aux dernières verlèbres seulement, se soude a celle du còté opposé, pour former un arc.

\section{S. $17 \%$}

Chez les ophidiens il y a aussi certaines vertèbres qui se distinguent des autres.

Cette remarque s'applique surtout aux deux premières, qui sont communément privées de côtes, disposition qui est aussi quelquefois offerte par la troisiène. Il est cependant plusieurs ophidiens, par exemple, le coluber elaphis, qui ont une petitesaillie costiforme à toutes les verièbres. La première des vertèbres esı, cn outre, plus petite que les autres, situées au devant de l'anus, et forme davantage un auneau simple, qui est absolument plus élroit et moins alongé. L'ouverture de cet anneau est plus considérable qu'aux autres vertèbres; la partie supérieure en est plus large que l'iuférieure, elle est saus apophyse épineuse; celle-ci est, au contraire, communément garuie d'une petile épine inférieure. La partie inférieure de l'anneau, correspondant au corps de la vertèbre, est ordiuairement aussi noyau osseux propre, même chez les sujets avancés en âge; elle est en outre toujours séparéc de la parlie supérieure par une saillie interne el un ligament transversal.

la partie supérieure seule loge la moelle épi- 
nière; l'inférieure reçoit l’apophyse odontoïde de l'axis.

Quelquefois la première verièlure se partage, d'une autre façon, en deux moitiés; l'anneau est dans ce cas formé de deux parlies symétriques, séparées dans la ligue médiane. Cette disposition existe chezles amphisbènes.

D'après mes recherches, cette vertèbre offre. vraisemblablement, chez les rouleaux (tortrix), une réunion des deux dispositions qui rieunent d'ètre indiquées; elle y est formée de trois pièces, savoir: de deux moitiés d'arc, séparées dans la ligne médiane, et d'une pièce moyenne, inférieure, qui est le corps.

Toutes ces particularités sont remarquables si l'on observe que la première ver tèbre des mammilères, et principalement celle de l'homme, sossifie alıssi plus tard que les autres, et que le point d'ossitication du corps surtout se forme si tardivement, comparé aux autres, que son existence a même été niée par de bons anatomistes.

La seconde vertèbre cervicale des ophidiens se distingue généralement des autres par son apophyse odontoide, saillie encroûtée de carti. lage par laquelle le corps de cette vertèbre se termine en avant, et qui dépasse l'arc. Cette apophyse, en général assez déprimée, est grosse et tres-large chez les amphisbènes; la seconde vertébre est en rapport, au moyen de cette 
saillie, avec la partie supérieure et moyenne de la facette articulaire postérieure de l'occipital.

\section{b. Cótes. \\ 5. 173}

Tous les ophidiens possèdent des côtes qui sont séparées des vertèbres. Elles sont en général légèrement convexes, courtes, et présentant une épaisseur assez égale partout, si ce n'est à leur base qui est un peu plus épaisse; on les trouve aussi alongées et apointies. La base de la plupart de ces côtes est aplatie d'avant en arrière, et large, et on remarque entre cette base, qui est très-courte, et la partie plus étroite de la côte, une petite apophyse dirigée en arrière.

Leur facette articulaire est légèrement concave. Les côtes antérieures et les postérieures, surtout celles-ci, sont en général plus courtes que les autres, qui offrent communément une longueur uniforme. On les trouve toujours sur un très-grand nombre de vertèbres; elles ne manquent d'ordinaire qu'anx deux, trois ou quatre premières vertébres cervicales et aux vertèbres coccygiennes. Constamment elles ne s'attachent qu'à une seule vertèbre, au moyen d'une apophyse transverse, courte. Filles ne se réunissent jamais entre elles, ni avec un sternum qui leur serait intermédiaire; mais elles supportent 


\section{D'ANATOMLE COMPARÉE.}

a leur extrémité inférieure un cartilage costal, court, dont l'existence est sans doute générale.

Chez les ophidiens, les côtes ne servent plus, comme chez les poissons, uniquement à la locomotion; elles sont à la fois organes de respiration et de locomolion; cependant elles remplissent encore la dernière fonction, car on peut s'assurer, par la vue et le toucher, que l'animal les soulève successivement en avant quand il rampe ( 1 ); fait très-curieux, parce qu'à l'analogie de forme qui existe entre les membres et les còtes, il unit une analogie de fonction. Elles remplissent la même fonction chez plusieurs sauriens à pieds très-courts, par exemple, les seps et les bimanes (chirotes), dont les poumons sont, courts, et dont les côtes très-nombreuses s'étendent jusqu'au bassin. Dans tous les genres de cet ordre le grand nombre des côtes et la briéveté des membres sont un indice qu'elles président à la locomotiou dans les degrés d'organisation qui précèdent.

Les côtes varierit en nombre, en volume et en forme, tant sous le rapport absolu que sous le rapport relatif.

Le genre orvet (anguis) en offre le moins de cótes sous l'un et l'autre rapport; les boas, les

(1) E. Home observations intended in show that the frogressive motion of snakes is partly performed by means of the ribs. Phil, trans., 1812 , p.' 163. 
trphlops, les scytales, les érix, les amphisbìnes en ont le plus.

Celles des amphisbènes sont les plus grosses; les apophyses dirigées en arrière y sont le pius marquées. Les raja ont, au contraire, les còtes les plus grêles.

Les dix-huit à vingt premières paires de cồtes dans ces derniers ophidiens, au lieu d'ètre convexes de dehors en dedans, sont planes daus celte direction et convexes en avant; elles sont aussi plus longues que les autres, à l'exception des quatre ou cinq les plus antérieures qui sont courtes, comme on les rencontre généralement; cette longueur plus considérable est en rapport avec l'expansion de la peau, qui, en celte région, s'épanouit en forme de manteau.

Chez les serpens d'eau (hydrophis), les côtes postérieures, sion en excepte lendernières paires. sont beaucoup plus longues que les aures.

L’augmentation de longueur et d'épaisseur des dernières còtes, qui a lieu dans plusieurs genres, et en particulier dans les boas, les couleuvres, les hydrophis, ainsi que la bifurcation subite de leur apophyse postérieure, ont déjà été mentionnées plus haut (1).

(i) Voy. p. 564. 
c. Sternum.

$$
\text { S. } 174 .
$$

Les ophidiens manquent très-généralement de toute irace de sternum; les orvets seuls, surtout l'anguis fragilis, offrent, immédiatement derrière la pièce claviculaire du membre antérieur, un os très-petit proportionellement au corps. Cet os est mince, alongé, triangulaire, un peu concave au milieu de son bord antérieur, situé transversalement, contigu par sá base à son congénère dans la ligne médiane; son diamètre transversal est considérablement plus long que les autres, il correspond évidem- . ment à l'os des batraciens situé à la mème place, et qui est également le sternum.

\section{Chéloniens.}

$$
\text { S. } 175
$$

Le squelette des chéloniens se distingue de celni des autres reptiles, et en général de celui de tous les autres vertébrés, d'une manière touta-fait particulière, par le grand développement des côtes et du slernum, qui détermine ici la forme de tout le corps.

Les signes raractéristiques de leur squelette 
et particulièrement du trone, sont: $1^{\circ}$ ce développement considérable des côtes et du stermum daus le sens de la longuenr et de la largeur; $2^{\circ}$ une soudure exacle, entièrement ou prosque entièrement immobile, des côtes avec les vertèbres thoraciques et entre elles, d'où résulte un bouclier dorsal ou une carapace plus ou moins convexe; $5^{0}$ une soudure andogue du sternum, développé en plastron, avec les còtes, par l'intermédiaire des cartilages costaux ossifiés; $4^{\circ}$ la séparation de la plupart des vertèbres, et spécialement de toutes les vertẻbres coccygieunes, dont il faut cependant excepter les plus postérieures, en leur corps et leur arc, qui ne sont unis que par une, suture, séparation qui persiste pendaut loute la duréc de la vie.

\section{a. Colonne vertébrale.}

$$
\text { S. } 176 .
$$

Les vertèbres des chéloniens peuvent toujours être distinguées facilement en vertèbres cervicales, thoraciques, sacrées et coccygicnnes.

Les vertèbres thoraciques offrent les conditions les plus dignes de remarque, qui sont en rapport immédiat avec la forme particulière de tout le corps, et d'une manière plus spéciale avec la soudure des verlèbres avec les côles.

Le corps de ces vertibres est lomjours fort 
n'anatomie comparté.

alongé, considérablement aplati d'un côté à lautre, de manière à ce qu'il se termine inférieurement par un bord plus ou moins tranchant, tandis que les deux extrémités en sont renflées. Il présente à sa face supérieure une gouttière d'une profondeur considérable, destinée à loger le cordon rachidien. Il se termine en avant et en arrière par une surface assez plane, appuyée sur le côté, par laquelle il s'arıicule d'une manière immobile avec le corps voisin et avec la côte, de telle sorte qu'il y a de chaque còté une facette articulaire pour deux côtes, formée:par deux corps de vertèbres contigus. La forme alongée et comprimée appartient surtout aux corps des vertèbres thoraciques, moyennes.

Les parties des vertèbres dorsales qui correspondent aux arcs sont au nombre de deux, une inférieure, verticale, peu élevée, et une supérieure, plate, horizontale, qui s'étend surtout de l'un et de l'autre côté en partant du bord supérieur de la première.

Le bord inférieur de la partie inférieure est garni de dentelures par lesquelles il s'articule arec le corps de la vertèbre; la pièce supérieure, au contraire, présente de semblables dentelures dans tout son pourtour; elles lui serventà s'unir en avant et en arrière à ses congénères et latéralement aux côtes.

Les plaques moyennes sont les plus larges; les antérieures et les postérieures sont plus alon-. 
gées. Il n'y a d'exception que pour la première et les deux dernières vertèbres dorsales; leur arc séparé du corps, comme de coutume, a la forme d'un anneau étroit, et ne s'épanouit pas de la manière qui vient d'être indiquée. Mais l'are de la dernière de ces vertèbres est recouvert d'une écaille large, correspondante à la partie large des autres, seulement plus alongée, et unie à l'avant-dernier arc par une suture, écaille qui est aussi articulée par suture avec la moitié inférieure du bord interne de la dernière côte. Il résulterait de cela que ces vertèbres ne seraient pas divisées en deux pièces seulement, comme les autres, mais en trois, à cause de la séparation des parties qui correspondent à l'apophyse épineuse et à l'apophyse transverse, d'avec le reste de l'arc.

Dans la tortue grecque (testudo graca) cette écaille est simple, et recouvre les deux dernières vertèbres thoraciques. Dans la tortue d'eau douce d'Europe (emys europara), elle est au contraire partagée en deux moitiés, dont chacune correspond à une vertèbre.

La première vertèbre dorsale ne présente point de pièce osseuse propre, mais une disposition analogue, en ce que la partie antérieure de la seconde piece de l'arc se recourhe en avant presque entièrement par dessus la première.

Il est digne de remarque que, contre la règle suivie chez d'autres animaux, il n'y a pas un 
1) ANATOME COMPARÉE.

corps pour chaque arc, ces deux parties s'engrenant de telle facon, que la moitié antérieure d'un corps est recouverte par la moitié postérieure du corps qui précède, et la moitié postérieure par la moitié antérieure du corps qui est en arrière; c'est-á-dire que chaque corps est surmonté de deux apophyses épineuses, qui se succèdent. Le dernier corps seul fait exception; il est situé, à lui tout seul, sous le dernier arc entier. Le prenier est aussi situé presque complètement sous le premier arc.

On remarque en outre quelques écailles considérables, impaires, qui sont beaucoup plus grandes que les autres, et terminent en avant et en arrière la série, en touchant à la fois par leurs bords externes aux côtes et aux cartilages de còtes voisines, et qui complètent ainsi le bouclier dorsal.

Il existe en avant chez les emycles, les tortues et les chélonées une plaque hexagonale; en arrière il y a deux plaques qui se succèdent d'avant en arrière. Elles ont toutes deux la forme d'un carré à côtés inégaux, de telle sorte que l'antérieure a son côté le plus étroit en avant, tandis que la plaque postérieure a son côté le moins long en arrière. Elles forment, en se réunissant, egalement un hexagone, mais qui n'est pas aussi grand que celui qui le précède.

Il n'est pas douteux qu'une comparaison trèsexacte ne puisse être établie entre ces trois 
plaques et des apophyses épineuses, supérieures; elles corréspondent vraicemblablement aux vertèbres coccygiennes et à celles des thoraciques, dont le corps ne s'articule pas, comme ceux des vertèbres thoraciques moyennes, avec des arcs ainsi développés en largeur. Elles sont déjà indiquées aux verièbres dorsales antérieures et aux postérieures, puisqu'on y voit au dessus des arcs qui sont petits, des plaques particulières dont nous avons donné la description.

\section{\$. 177 .}

Le sacrum est formé de deux à trois vertèbres courtes et larges, diminuant de volume d'avant en arriere, qui se distinguent de la plupart des vertèbres thoraciques par la largeur et l'aplatissement de leur corps, par la petilesse, la minceur et l'étroitesse des pièces qui correspondent aux arcs, et par la présence d'une troisième et d'une quatrième pièce alongée, renflée surtout à son extrémité externe. Chacune de ces pièces se détache de la partie latérale di corps et se porte en dehors où elle se soudle avec la pièce suivante; il en résulte une facette articulaire alongée, convexe en dehors, destinée à recevoir l'os coxal.

Ces pièces osseuses alongées correspondent parfaitement par leur forme et leur position au col des côtes, qui s'est développé en une apo- 


$$
\text { D'ANATOMIE COMPARÉ. }
$$

physe propre; il est par conséquent très-digne de remarque que déja eutre la dernière vertèbre thoracique et la dernière côte l'on trouve un semblable os isolé, à la place de l’apophyse costale qui existe ordinairement en cet endroit des còtes.

$$
\text { \$. } 178 .
$$

Les vertebres coccygiennes sont de toules les plus petites, quoiqu'elles ne le cèdent que peu aux vertèbres sacrées, surtout les premières; mais elles diminuent insensiblement de volume. d'avant en arrière. Elles sont toujours en beaucoup plus grand nombre que les autres, puisqu'il y en a près de vingt. Plus de la moitié de ces vertèbres sont composées de trois pièces au moins, d'un anneau moyen et de deux apophyses transverses; les vertèbres du tiers antérieur sont constituées par quatre pièces, l'anneau étaut partagé en un corps et en un arc. Ces derniers se confondent ensemble aux vertèbres du milieu de la colonne coccygienne; l'apophyse transverse se réduit en outre insensiblement à un noyau arrondi, très-petit. Les vertèbres coccygiemnes posterrieures sont des os pleins, quadrilatères et simples, dont le dernier est apointi.

Toutes ces vertèbres s'articulent entre elles d'une manière mobile. 


$$
\text { 5. } 179
$$

Les chéloniens ont vraisemblatblement tous huit vertebres cervicales. Je trouve du moins ex nombre chez la tortue franche (chelone mychas), la tortue d'ean douce d'lurope (emys suropaea) 'it la tortue grecque (testudo grecea). Elles ditferent considérablement des vertères thorariques, surtout par le mode de connexion, qui les unit à celles-ci et entre elles.

Au lieu d'ètre soudées entre elles, comme les vertèbres thoraciques, de manière a ne pas offrir la moindre mobilité, les cervicales sont extrêmement mobiles; absolument d'après le type ordinaire des vertèbres des ophidiens et des sauriens. Les faces antérieure et postérieure de leurs corps décrivent des segmens de sphère considérables qui s'engrènent avec les segmens correspondans des vertèbres voisines et permettent un mouvement de rotation étendu. La rlisposition des surfaces articulaires est la même dans les différens genres; mais les chélonées d'un côté, les émydes et les tortues de l'antre, different en ce sens, que chez les premières il y a de la substance fibro-cartilagineuse qui atlache l'une à l'autre daus toute leur élendue les deux faces opposées, tandis qu'elles soni libres chez les secondes et ne sont tenues ensemble qua i leur pourlour par une capsule synoviale, et qu il cxiste dans l'intérieur de celte capsule tout au 
D'A NTUMH COMPARER.

plus un ligament fibreux. libre et ètroit, se por tant d'avant en arrière d'une vertébre à l'autre.

Cette différence se rattache d'une manière remarquable arec la faculté, dont jouissent les deux derniers ordres, de recourber le cou a tel point quils peuvent le cacher profondément, ainsi que la tète sous la carapace, faculıé qui manque aux chélonées.

Les différentes vertèbres cervicales offrent $d u$ reste, également sous le rapport de la disposition des facettes articulaires des corps, des différences dignes de remarque.

La dernière ou la huitième présente deux facetles articulaires convexes; dont la postérieure qui est la plus petite, est unique, tandis quine petile éminence longitudinale partage l'antérieure en deux facettes latérales. Le corps de la septième, au contraire, offre à ses extrémités antérieure et postérieure, deux cavités latérales juxtaposées. La cinquième et la sixième ont en arrière une facette articulaire convexe, en avant une concave; les deux facettes ariculaires de la sixième et la postérieure de la cinquième sont partagées en deux moitiés latérales, l'antérieure de la cinquième étant unique. Les facettes articulaires du corjs de la quatrième sont toutes deux convexes, mais l'antérieure est beaucoup plus arrondie que la postérieure qui est transversale. Sur la troisième enfin la facette postérieure est concave, l'antérieure convexe. 
La disposition qui vient d'être décrite est surlout très-marquée chez les corlues; elle est cependant aussi distincte chez les autres.

La forme des vertèbres cervicales des chéloniens est fort alongée. Leurs corps offrent au milieu de leur face inférieure une crête longi. tudinale que l'on peut considérer comme une apophyse épineuse inférieure. La supérieure manque sur l'arc, à l'exception de la seconde vertèbre. En effet, la partie qui correspond à l'arc, déborde le corps en avant et en arrière, présente une profonde incisure aux bords antérieur et postérieur, et se prolonge des deux côtés; ces prolongemens forment leś deux avophyses articulaires, qui sont longues et divergentes.

Les deux premières vertèbres cervicales ressemblent beauconp à celles des ophidiens.

La seconde offre une apophyse odontoïde longue, large, supportant une pointe mousse supérieurement; la face supérieure de cette apophyse, qui est encroûtée de cartilage, est située transversalement en forme de toit.

L'atlas, qui est la plus courte de toutes les vertèbres cervicales, se compose de trois pieces, de deux moitiés d'arc et du corps, qui est petit; il reçoit dans la petite portion antérieure de son onverture la partie supérieure de l'apophyse odontoïde, et s'articule avec elle par deus ficelles articulaires qui correspondent à la face 
supérieure de cette apophyse, face qui est encroùtée de cartilage. Le corps de l'atlas est comprimé d'un coté à l'autre en forme de toit, de manière à présenter à sa face inférieurc une petite ćminence alongée.

\section{S. 180.}

Les différens ordres de chéloniens offrent, sous le rapport du rachis, plusieurs particularités assez intéressantes, dont les plus importantes sont celles que présente la colonne thoracique ou dorsale.

Les corps des vertèbres sont ouverts à leur face supérienre chez les chélonées et les émycles, et forment des demi-canaux qui sont complétés par la branche verticale des arcs qui leur est contiguë. Il se trouve entre enx, à peu près vers le milieu de la longueur du corps, unc ouverture arrondie qui donne passage aux vais. seaux et aux nerfs. Cette branche verticale de l'arc se compose par conséquent de deux lames, à la vérité très-rapprochées, mais entièrment. séparées l'une de l'autre, formant eu quelque sorte chacune une demi-gouttière qui occupe toute la longueur de l'arc.

La plupart des vertèbres thoraciques moyennes, depris la seconde jusqu’à la septième, se comportent tont autrement chez les lorkus, du moins rhez la corluc grecouc. 
Leurs corps sont non-seulement beaucoup plus comprimés d'un côté à l'auire que dans les deux autres ordres, mais ils se réunissent encore supérieurement dans la ligne médiane, tout-àfait indépeudamment de l'arc ; il en résulte dans leur intérieur un canal parfait, à parois minces, entouré supérieurement et inférieurement d'un bord tranchant, canal qui loge la colonne verté. brale. C'est pourquoi les corps de ces vertèbres sont : $1^{\circ}$ séparés des ares dans presque toute leur longueur par une grande lacune; $2^{\circ}$ ils offrent un trou rond qui donne passage aux nerfs ei aux vaisseaux, à l'endroit où les autres ont une echancrure; disposition certainement curieuse et presque sans exemple, qui dépend vraisem. bablement de la mème cause que la tendance, surtout très-prononcée dans cet ordre, à la soudure intime et solide des os de la colonne dorsale et lombaire entre eux.

Les außres vertèbres offrent des différences moins considérables.

Dans plusieurs espèces de chéloniens, du moins parmi les tortues, chez les testudo tabulata "t $t$. grecca, de même parmi les chélunées, chez la tortue franche (chelone myclas) et le cared (ch. imbricala), le sacrum est formé de trois verièbres, dont la plus postérieure offre l'apophyse uransverse la plus courte et la plus mince; chez les émydes, du moins les c'my's serrita et curopera, de mème chez le testrito clausa, qui sous 


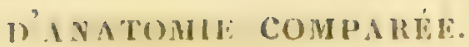

d'autres rapports a encore beancoup d'analogie Nu les einycles, le satrum n'est formé que de deux vertèbres.

Le nombre des vertèbres coccygiennes n'est pas le mème partout. Chez les chélunées il est en géucral moindre que chez les autres.

Les verlèbres cervicales n'offrent que peu ou point de particularités autres que celles indiyuces ci-dessus. Chez les chélonées et les toritr's, surtout les premiers de ces reptiles, elles sout plus petites que les vertèbres thoraciques muyennes; chez les émydes elles sont aussi gran. des que celles-ci; chez les chélonées beaucoup) moins alongées, courtes et épaisses, garniess dapophyses inférieures très-longues, qui som beaucuup plus petites chez les émydes et à peine visibles chez les tortues. J'ai trouvé dans mes re. cherches que l'apophyse odontoide porte, chez la turiue grecyue, à son extrémité postérieure, in petit noyau osseux, propre, que je ne découvris pas chez les autres; mais je ne sais si plus tard il n'aurait pas disparu.

Une différence sexuelle digue de remarque cst wferte, du moins par les tortues, dans la disposition de leur quene; celle des mâles, dans les especes $t$. labulata et $t$. gracea, a presque le druble de la longueur de celle des femelles. lin ontre, toutes les verièbres des mâles sont teatuconj plus grandes dans tous les sens, et rmberenent srparés les unes des anres, tandis 
que chez la femelle du t. tabulaur, les cinq ou six dernières sont sourlées entre elles et ne forment qu'une pièce, sur laquelle on reconnât cependant encore la composition primitive, surtout par la disposition des apophyses transverses, qui sont séparées. Chez la tortue grecque, aus contraire, c'est précisément le mâle qui a les six ou sept dernières vertèbres de la queue tout$\grave{a}$-fait confondues en un os unique, très-volumineux, large, plat, à pointe mousse, et non pourvu d'apophyses transverses; cet os forme le dernier quart de la queue. Toutes les vertèbres coccygiennes de la femelle sont entièrement séparées les unes des autres.

\section{Côtes.}

$$
\text { 5. } 181 \text {. }
$$

Les côtes offrent la disposition la plus particulière, qui est cependant déjà indiquée par la forme de la plus grande portion supérieure de l'arc des vertèbres respectives. Elles sont trèsgrandes, larges, épaisses, modérément convexes, soudées par leur extrémité postérieure, sur deux points, avec les vertebres thoraciques, et unies entre elles par des sutures daus une élendue plus ou moıns longue de leurs bords antérieur et postérieur.

Elles se bifurquent toujours au voisinage de leur extrémité postérieure, de telle sorte que la 
branche postérieure de la fourche qui en résulte, est, sous le rapport de la direction et de la larśeur, comme une continuation de la côte, et que l'antérieure, toujours plus étroite et déviant aussi par sa direction, se présente sous la forme d'une saillie de la face interne de la côte. Elles s'appliquent avec la première branche contre le bord latéral et dentelé de la lame horizon1ale de l'arc, de mánière à ce que chaque côte ne touche jamais à un seul arc, mais toujours à deux, quelquefois à trois, par la seconde elles touchent au point de réunion de deux corps de vertèbres. Leur extrémité antérieure se termine, soit dans la totalité, soit seulement dans une partie de sa largeur, par une pointe dentelée qui s'engrène avec un enfoncement existant sur le cartilage costal.

C'est donc chez ces animaux que la côte s'articule pour la première fois, sur deux points, avec les vertèbres, savoir : avec le corps et l'arc; il faut par conséquent, par analogie avec les animaux supérieurs, donner le nom de tubercule à la branche postérieure de la bifurcation des còtes, et celıi de tête et de col ì l'antérieure.

I.es côtes antérieures et les postérieures sont toujours les plus courtes, mais en revanche les plus larges.

Une autre particularité réside dans l'ossification, la déviation de forme et la connexion des. cartilages costaux. 
Ils sont loujours ossifies; leur forme est. comme celle des côtes, moins alongée de dolurors en dedars que chez les autres aniwaux.

Enfin, et c'est là la particularité la plus graude, mais qui est aussi indiquée déjà par les cûtes. ils s'articulent entre eux, d'avant en arrière, par. des sutures, de maniere à former une couronur. qui constitue la circonférence externe de la ca. rrpace ou bouclier dorsal.

\section{S. 182 .}

Les côtes des chéloniens offreut aussi des va. riélés, très-dignes de remarque, du type qui vient d'être décrit.

Ce sont les tortues qui s'éloignent le plus du type commun. Leurs còtes sont les plus larges: la face interne en est presque uniformément concave; elles sont unies entre elles dans toute leur longuenr; leurs borls offreut les dentelures les plus profondes; leurs extrémités antérienres sont le plus mousses et le moins dislinctes du reste de l'os.

Une chose digne de remarque dans cet ordre, c'est que plusieurs còles voisines changent de forme, en ce sens que l'une d'elles offre sa plus grande largeur en dedans, le plus d'étroi. lesse en dehors, landis que l'autre présente une disposition contraire. Les troisieme, cinquiène et sepliène sont surtoul trés-larges eil arrière, 
fort apointies en avant; les autres ont une disposition inverse. Celles qui sont plus larges à leur extrémité antérieure touchent à trois cartilages costaux, les autres touchent seulement à la région moyenne d'un seul cartilage.

La partie de ces côtes qui représente le col est extrèınement grèle et longue; l'espace compris entre elle et le tubercule alongé est fort considérable. Le testudo clausa, par sa largeur et son aplatissement, forme en quelque sorte le passage aux émydes.

Les cartilages costaux des tortues offrent leur plus grande longueur de dehors en dedans; ils sont semblables à des côtes, grands, offrent leur étendue la moins considérable d'avant en arrière, et sont tonjours contigus à deux, même à trois còtes. Ils forment toujours avec elles une plaque continue.

Les cinq premiers sont unis immobilement, par une sulure, avec les deux pièces moyennes du sternum.

Chez les émydes les còtes sout plus étroites, plus alongées, d'une largeur plus uniforme, ou offrant la même largeur partout, ou ayant le plus d'étroitesse à leur partie interne; leurs pointes terminales externes, ainsi que leurs apophyses supérieures et internes, sont beaucoup phus larges; les ruremières sont plus longues et se détachent plus brusquement. Les sutures sont morins composées que chez les tortues. 
Les cartilages costaux sont plus étruits de dehors en dedans, plus longs d'avant en arrière. Ils forment également avec les côtes une plaque non interrompue.

Les chélonées offrent une disposition bien différente, à laquelle conduisent cependant les émydes. Les côtes ont partout la mème largeur, ou sont le plus étroites dans leur partie interne; elles ne se rencontrent que par leur moitié interne, qui est la plus grande; cette union a lieu au moyen de sutures encore plus simples que chez les émydes. Leur partie antérieure correspond an milieu des côtes; on la voit, à partir du col de la côte, qui est encore plus court et plus large que celui des émycles, s'étendre sous la forme d'une tubérosité déprimée dans le milieu de toute la face interne de la côte; elle est très-étroite et par conséquent bien éloignée des parlies voisines de même nom; elle n'est contiguë qu“à un cartilage costal.

Les cartilages costaux sont bien plus alongés d'avant en arrière et beaucoup plus étroits de dehors en dedans que chez les émydes; il se trouve, entre eux et les parties antérieure et pos. térieure des côtes, des espaces quadrilatères. fort considérables, qui se succèdent d'avant en arrière.

La conformation des côté, est la mème dans les tortues molles (trionyx); la soudure encor: plus imparfaite du rachis et des còtes, que lion 
rencontre dans ce genre à leur extrémité auté rieure, conduit en effet des chélinées aux autres reptiles.

Dans ce dernier geure, comme chez tous les autres chéloniens, la carapace est encore complètele long de la ligne médiane, en ce sens que toutes les còtes se confondent avec les larges plaques dorsales; chez le trionyx subplanus il existe, au contraire, une lacune à la partie antérieure. I.a première vertèbre dorsale est tout-àfait libre sur les côtés, de façon que l'on voit d'en haut les deux os latéraux dans toute leur longueur : cette disposition résulte de c'e que la première côte ne se porte pas assez en avant pour alteindre la plaque osseuse également trèsétroite, qui termine en avant la série des plaques dorsales (1). Il existe peut-être à cet égard des différences individuelles, spécifiques ou des différences d'âge; car chez un sujet quatre fois plus grand je trouvai la lacune d'une petitesse absolue beaucoup plus prononcée : la première còte et la seconde plaque dorsale s'étendaient heaucoup plus en avant, de sorte qu'elles atteignaient toutes deux la plaque la plus antérieure, qu'il ne restait de chaque côté qu'une ouverture peu considérable, et que le corps de toute la première vertèbre dorsale était entièrement caché.

I.es parties externes et étroites des côtes n'ont

(1) Voycz pag. 575. 
du reste pas la mime longueur dans tous les individus du genre trionyx. Ceci n'est pas une différence d’âge, puisque je les ai lrouvées proportionnellement plus longues dans de grandes carapaces que dans d'autres plus petites.

Aucune des cinq carapaces que j'eus occasion d'examiner n'offrit du reste une trace de cartilages costaux; de sorte que, sous ce rapport aussi, ce genre forme le passage des chélonéc's, où ils commencent a disparaître, aux autres reptiles.

\section{Sternum.}

\section{S. 18.3}

I.e sternum des chéloniens dévie du iype ordinaire par sa longueur et sa largeur extraordinaires; il occupe toute la largenr et la longuem de la portion thoracique et abdominale du corps, et s'accorde en cela, d'une manière remarquahle, avec la colonne dorsale et les cotes. Mais on rencontre mème dans les autres animaux des rapprochemens de ceite conformation. On en trouve un indice, eu égard i: la longueur, dans le sternum postérieur ou abdominal du crocodile; dans les còtes abdominales du caméléon el du polychrus, qui sont réunies sur la ligne médiane; dans le sternum de plusieurs poissons: dans le sternum abdominal du phutagin qui s'étoud jusqu'à l'os du pubis. Te sternum est anssi 
1) ANATOMIE COMPAREE.

vrès-long et le plus souvent tout à la fois trèslitrge chez la plupart des oiseaux, et la largeur 't la longueur considérables du sternum inférieur chez le pipa, est aussi un indice de cette disposition des chéloniens ( 1 ).

Le stermim des chéloniens (2) est en général lormé d'un nombre considérable de pièces osseuses, plus ou moins solidement unies entre clles, qui ne sont pas seulement disposées bout a bout, comme chez la plupart des autres animaux. mais qui sont aussi placées les unes à

(1) La phllosophe axatomeve contient une comparaisorn lihs différences que présente le sternum dans diverses lasses de vertébrés, élablies sur l'évaluation proportionnelle: W. la part qu'y prend chaque élément en particulier. (Voyez la détermination et les désinnations de ces élémens dans la note des pages 357,358 de ce volume.)

( Note des traducteurs.)

(2) Le slernum de ces repiles a particulièrement fixé l'attention de M. Geoffroy-Santr-Hilayne sur l'ćtude de cet os, qui se trouvanl ché ces êtres au maximum de développement a donné à cet anatomiste la clef de la difficulté quil y avait a rapporter, pièce a pièce, les modifications (qu'éproure cel os dans les quatre classes supérieures. Lisez son Anatomie philosophique, tome Icr', p. 104 , vons y verrez le raport quoffre le développement proportionnel de cet os, et de chacun de ses élémens avec les fonclions auxquels il est appelé, soil dans les habitudes des animaux apjartenant aux principales divisions de lordue des chéloniens, snit dans les mours des aulres groupes d'animaux vertebris. Consuliez encore Annales du Muséum d'histoire nelurelle, t. XIV, I. I.

(Nole des traducleurs.) 
côté des autres dans le sens de la largeur; dis position qui se rattache incontestablement à la largeur considérable de l'os, mais qui n'est pas sans exemple, puisque chez les srumiens le sternum est séparé, quoique d'ane manière incomplète, en deux moitiés latérales, et qu'il se forme, chez l'komme, d'un nombre considérable de points d'ossification disposés deux à deux et les uns à côlé des autres.

Le sternumı des chéloniens se compose en général de neuf pièces osseuses, de huit qui sont paires et d'une impaire, moyenne. La pièce impaire est très-généralement d'une forme plus ou moins triangulaire; elle est contiguë par sa base qui est tournée en avant, au point de réunion et à la parie interne des bords postérieurs des deux pièces de la paire antérieure, et s'étend en arrière, par son sommel, dans une étendue plus ou moins grande, entre les deux pièces de la scconde paire. Les deux piéces de la première paire se rencontrent également d'une manière constante sur la ligne médiane.

Telles sont les conditions générales du plastron ou bouclier thoracique des chéloniens; mais il y a des différences suivant les sexes et les genres. On peut signaler en géréral, comme une différence sexuelle, la circonstance que le plastron des femelles est plane et uni, tandis que celui des mâles est considérablement concave dans la région de sa ligne médiane. Ie's diffé- 


\section{D'. N.ITOMIE COMPARÉ}

rens genres se distinguent sous le rapport de sa disposition, comme ils varient sous celui de la carapace.

1. Chez les chélonées et les trionyx il offre, a tous égards, le moins de déviation du type ordinaire. 11 est proportionnellement plus petit et moins large; il s'apointit en avant et surtout en arrière, et s'unit mobilement, au moyen d'une substance fibro-cartilagineuse, avec la carapace, par sa partie moyenne qui est relativement plus petite. Il a la forme d'une croix à cause de l'étroitesse de ses parties antérieure et postérieure.

2. La substance osseuse y est moins aboudante, car les pièces dont il est formé ne se touchent pas réciproquement dans une étendue considérable. Il n'y a que les deux pièces antérieures qui s'unissent entre elles dans toute leur hauteur; celles qui suivent ue s'unissent ni entre elles, ni avec la pièce impaire qui est proportionnellement très-petite et étroite. Les pièces de la troisieme paile ne sont unies que dans leur moitić postćrieure, et celles de la quatrième seulement dains la partie postérieure, qui est trés-petile. Il résulte de là deux lacunes moyennes, et de chaque côté autant de lacunes latérales, yui sont remplies de cartilage; les pièces osseuses du sternum sont par conséquent jusqu'is un certain point mobiles entre elles.

J)ans !e genre triony $x$ le sternum est: $\mathbf{r}^{0}$ proII 
portionnellement beaucoup plus volumineux, tant sous le rapport de la largeur que sous celui de la longueur; $2^{\circ}$ plus large que chez les chélonées, car il a autant de largeur que de lon. gueur, et il déborde même de beaucoup la carapace, qui est privée des cartilages costaux unis entre eux, lesquels sont déjà très-petits chez les chélonées; $3^{\circ}$ les deux pièces antérieures se prolongent chez les triony $x$, en avant et en dehors, en une longue branche, tandis que le bord antérieur est convexe chez les chélonées: $4^{\circ}$ la troisième pièce, qui est impaire, représente un grand arc, étroit, convexe en avant, qui s'applique sous les pièces antérieures, dans toute leur longueur, et qui est privée, en revanche, tout-à-fait de la pointe grêle, qui existe aussi chez les chélonées, où elle est même fort considérable; $5^{\circ}$ la seconde et la troisième paire sont moins hautes et beaucoup moins profondément bifurquées de dehors en dedans; $6^{\circ}$ les deux pièces de la paire postérieure sont beaucoup plus rapprochées l'une de l'autre que chez les chélonées.

Le sternum des triony:x a par conséquent une forme en croix très-prononcée, et la lacune est plus large en avant, plus étroite en arrière que chez les chélonées.

3. A cette conformation se rattache celle que l'on observe chez le chelys fimbriata. Le sternum est, dans cette dernière espèce, plus étroit, se 
D'ANATOMIE COMPARÉE.

prolonge de chaque côté, à sa partie moyenne seulement, en une apophyse latérale, qui fait à peu près un tiers de toute sa longueur, et qui présente aussi une lacune en dehors, au voisinage de ses extrémités antérieure et postérieure; mais il n'offre point d'autre lacune, si ce n'est dans le premier âge, et s'unit d'une manière tout-à-fait solide par son apophyse avec les cartilages costaux et en outre, en arrière, même 2vec l'os du pubis et de l'ischion.

4. Chez les émydes, il n'y a point de lacunes remplies de cartilage entre les pièces osseuses; toutes ces pièces sont unies entre elles par des sutures, mais qui sont faiblement dentées. Les pièces paires ont une forme plus ou moins quadrilatère. L'impaire est plus large dans sa partie antérieure, mais elle se prolonge en arrière, et devient une pointe longue et mince, par laquelle elle atteint presque l'extrémité postérieure des pièces de la seconde paire. La connexion avec la carapace a lieu par une substance cartilagineuse, au moyen des bords larges et assez égaux des seconde et troisième paires; elle est un peu nnobile comme chez les chélonées. Plus en avant qu'en arrière, le plastron est beaucoup plus large que chez les chélonées; sa forme est par conséquent fort alongée, et n'est pas celle d'une croix. Son bord postérieur, large, est un peu concave au milieu, l'antérieur l'est moins.

Le plastron de cet ordre est remarquable par 38. 
la laxité et la mobilité de l'articulation qui unit sa moitié antérieure, beaucoup plus petite avec la moitié postérieure, qui est beaucoup plus grande; la première est formée des deux paires antérieures et de la pièce moyenne, impaire; la seconde se compose des deux paires postérieures, qui sont proportionnellement beaucoup plus grandes que les premières. Cette disposition n'est nullement particulière au móle; du moins je la trouve également distincte dans les deux sexes de l'espèce cmys virgulata.

5. Chez les tortues on observe les particularités suivantes: $1^{\circ}$ les sutures sont beaucoup plus profondes; $2^{\circ}$ la pointe postérieure de la pièce impaire a disparu; celle-ci est par conséquent plus courte et plus arrondie, et les pices de la seconde paire s'unissent entre elles dans leur moitié postérieure qui est la plus grande; $3^{\circ}$ les deuxième et troisième paires s'articulent par des sutures, à dentelures profondes, avec cinq des cartilages costaux qui ont subil'ossification; $4^{\circ}$ tout le plastron est beaucoup plus large, la connexion est plus étendue en longueur, le bord postérieur, trés-large, est beaucoup plus profondémentéchancré au milieu. Le testudo claussa et des espèces voisines ont aussi un stornum composé de cỉeux moitiés unies entre ellès d'une manière mobile. 
4. Sauriens.

a. Colonne vertébrale.

$$
\text { S. } 184 \text {. }
$$

La plupart des sauriens ont, surtout à cause de la longueur considérable de leur queue, un très-grand nombre de vertèbres; mais il y a chez eux, par la même raison, entre le nombre des vertèbres antérieures et celui des vertèbres coccygiennes un rapport opposé à celui qui a lieu entre ces mèmes vertèbres chez les ophidiens. Les orvers se comportent, à cet égard, tout-à-fait à la manière des sauriens. La forme des vertẻbres est généralement alongée. Elles consistent ordinairement en une pièce unique: les crocodiles font une exception remarquable à cette règle; leurs vertèbres sont séparées en un arc et un corps, qui sont unis entre eux par des sutures. Mais ces deux pièces sont également soudées, chez eux, aux vertèbres coccygiennes.

Les corps des vertèbres ne sont pas unis chez tous les sauriens, suivant le même type. Chez quelques-uns, et vraisemblablement la plupart, par exemple, le monitor elegans et le $m$. bengalensis, les crocodiles, lesiguanes, les caméléons, les agames ( $a$. calotes), les lézards (tels les lacerla umeiva, jamaicensis, turcica, viridis), les 
stellions (le st. cordylus et le st. vulyaris), s'accordent, sous le rapport de cette connexion, avec les ophidiens et plusieurs batraciens; en effet, le corps de leurs vertèbres est fort convexe à son extrémité postérieure, et fort concave à l'extrémité antérieure, de sorte que deux vertèbres sont unies par énarthrose. D'autres, au contraire, mais un petit nombre seulement, les geckos, par exemple, présentent la disposition des mammifères ou mieux encore celle des poissons; le corps des vertèbres est creusé, en avant et en arrière, d'une cavité infundibuliforme, considérable, remplie de substance fibrocartilagineuse, ce qui le fait paraître comme composé de deux cônes:

Les apophyses épineuses supérieures des vertèbres sont, pour la plupart, plus considérables que chez les ophidiens, mais jamais bien fortes.

Elles sont au maximum de longueur chez l'agame ombre (agama umbra) et le porte-crête ou basilic d'Amboine (lacerla amboinensis); les plus courles se rencontrent chez les dragons et les cordyles. Les inférieures existent moins généralement que lessupérieures; mais on les trouve toujours sur la plupart des vertèbres coccygiennes antérieures, où elles sont souvent fort longues; chez les monitors elles ne sont guère plus courtes que les supérieures; chez les crocodiles elles sont au contraire plus longues. Ces apophyses épineuses inférieures des verièbres 
D) ANATOMIE COMPARÉ:

coccygiennes semblent être, comme chez les poissons, les côtes qui se seraient dirigées en bas et en dedans, pour se souder ensemble sur la ligne médiane. En effet: $1^{\circ}$ elles ne sont jamais des parties de vertèbres, mais sont appliquées seulement entre deux verièbres, auxquelles elles sont unies d'une manière mobile; $2^{\circ}$ les còtes latérales manquent depuis le point où l'on rencontre ces épines inférieures. Ces côtes semblent, a la vérité, remplacées par des apophyses transverses beaucoup plus courtes; mais comme celles-ci se trouvent également sur les vertèbres antérieures, où elles sont cependant moins développées, il est, sans contredit, plus raisonnable de regarder les épines en question comme des côtes plus développées.

Outre ces apophyses épineuses inférieures que l'on trouve aux vertèbres coccygiennes, il y a encore des épines inférieures, chez les croco. diles, sur les douze ou treize premières vertèbres, l'atlas excepté, chez les monitors, sur les sept premières, chez le grand lézard vert ocellé ( lacerla ocellata), aux six premières vertèbres; elles sont plus ou moins fortes et soudées avec le corps. Chez les crocodiles ces épines occupent l'extrémité antérieure de la vertèbre, et se por. tent un peu sous l'extrémité postérieure de la vertèbre précédente; chez les autres sauriens, où elles sont plus fortes, elles se trouvent à l'extrémité postérieure de la vertèbre. L'axis des 
monitors présenie en avant une seconde épine inférieure, plus petite, mais également dirigée en arrière.

Ces épines cervicales inférieures ont le plus de longueur chez les dragons.

Les apophysestransverses sont situées, aux vertèbres cervicales et aux thoraciques, près de l'extrémilé antérieure; aux vertèbres coccygiennes elles occupent le milieu du corps. Elles disparaissent ici déjà bien avant les apophyses épineuses; chez les iguanes elles n'existent déjà plus vers la fin du premier quart, tandis que les apophyses épineuses ne manquent tout-à-fait que dans le dernier tiers de la queue.

Il n'en existe que d'un ordre chez la plupart des sauriens; elles sont étroites et d'une hauteur considérable aux vertèbres dorsales antérieures, et s'étendent depuis la racine de l'apophyse articulaire antérieure jusqu’à la face inférieure du corps. Aux vertèbres postérieures elles sont situées plus haut et sont plus carrées, et en mème temps plus arrondies

Cette disposition varie chez les crocodiles. Toutes les vertc̀bres, hormis l'atlas, l'axis et les dernières verlèbres coccygiennes, sont munies d'apophyses transverses, qui sont, pour la plupart, appliquées sur la base de l'arc, à l'exception des vertébres sacrées où elles appartieunent au corps; elles augmentent considérablement de volume des vertebres antíricures ot 
D'ANATOME COMPARÉE.

601

des postérieures vers celles du milieu. Outre les apophyses transverses généralement existantes, il y en a d'autres plus petites en avant, sur le corps, depuis la troisième jusquà la onzième vertèbre, qui reçoivent les têtes des côtes. Les apophyses transverses simples des verlèbres situées derrière la onzième présentent deux facettes articulaires; celles des vertèbres antérieures n'en ont qu'une: cela tient à ce que, sur les premières, les apophyses transverses supérieures et inférieures sont confondues ensemble, tandis qu'elles sout séparées sur les secondes.

Les apophyses transverses des vertèbres antérieures, qui sont plus grandes chez les autres sauriens, correspondent sans contredit aux deux ordres de ces apophyses que présentent les crocodiles. Elles ue sont pas seulement alongées, mais encore, et surtoul les premières, sont rétrécies au milieu, quoique aussi encroûtées de cartilage en cet endroit.

\section{S. 185 .}

Chez tous les sauriens que j’ai eu occasion d'examiner, le sacrum se compose de deux vertèbres, qui se distinguent des autres par leur largeur considerable, par l'épaisseur et le renflement de leurs parties litérales, et qui sont soudées crisemble: non-seulement par leurs 
corps, mais en ontre par l'extrémité externe de leurs apophyses transverses, de telle sorte qu'il en résulte une surface articulaire pour l'os coxal. Les apophyses transverses de la première vertèbre sont situées transversalement ou un peu en arrière; celles de la seconde, un peu en avant; elles se regardent par conséquent.

Par suite de cette soudure qui unit l'extrémité externe des apophyses transverses, l'échancrure qui existe, sans cela, entre deux vertèbres, est convertie en une ouverture, le trou sacré.

Cette soudure offre divers degrés de solidité. Chez les geckos, par exemple, il est facile de séparer les deux vertèbres du sacrum; cela est absolument impossible chez le monitor elegans de l'archipel des Indes.

\section{\$. 186.}

La première et la seconde vertèbre s'accordent beaucoup avec celles de la plupart des ophidiens et chéloniens, à cela près que l'antérieure est communément plusétroite et offre une forme plus annulaire. L'apophyse odontoïde dé la seconde est très-généralement un point d'ossification propre. Chez les crocodiles, la première est formée de quatre pièces, savoir : le corps, les deux moitiés d'arc, et une petite pièce peu élevée qui appartient aussi à l'arc. 


\section{Côtes.}

S. 187 .

Le nombre des côtes est ordinairement fort considérable chez les sauriens; presque toutes les vertèbres situées au devant du sacrum, les plus antérieures exceptées, en sont pourvues. Toutes ces côtes ne s'attachent individuellement qu'à une vertèbre, notamment à sa partie antérieure. Les côtes antérieures aussi bien que les postérieures, celles-ci principalement, ont leur extrémité externe libre et plongée dans la chair. Celles du milieu, communément les moins nombreuses, mais qui sont beaucoup plus longues, s'attachent au sternum, au moyen de cartilages en partie très-considérables, qui surpassent mème en longueur les dernières de ces côtes moyennes. Cette attache est immédiate aux plus antérieures et médiate aux plus postérieures. Chez les crocodiles ces cartilages sont tout-à-fait partagés en une moitié antérieure plus grande, et une postérieure plus petite.

Dans quelques genres, notamment les caméléons, les marbrés (polychrus), la plupart des côtes postérieures, à l'exception des dernières qui sont très-courtes, sont unies à leurs congénères par de la substance ligamenteuse sur la ligne médiane, sans cependant être unies d'avant en arriere par un os analogue au ster- 
num. Le geclio frangé présente une organisation intermédiaire à cette disposition et à celle qui va être décrite chez le crocodile à l'uccasion du sternum. Parmi les dix-sept côtes que présente cette espèce de gecko, il n'y a que les quatre premières qui atteignent le sternum, lequel est court et large. Les treize paires postérieures se rencontrent sur la ligne médiane, et chacune d'elles détache, au point de réunion, une petite pointe antérieure, qui diminue de longueur d'avant en arrière et n'atteint pas la paire qui précède. Cette pointe manque à la dernière paire, qui est situće immédiatement devant l'os du pubis, mais en revanche cette paire présente un petit crochet sur son bord postérieur.

Une déviation remarquable du type ordinaire est offerte par les dragons; la plupart de leurs côtes, notamment les neuf côtes du milieu, depuis la septième jusqu'à la quinzième, se prolongent brusquement, tandis que la longueur des autres côtes diminue considérablement d'avant en arrière. Elles sout en mème temps fort convexes, non en dehors, mais en avant, et ne contribuent absolument en rien à la formation de la cavité viscérale, mais servent seulement à soulenir la membrane du vol, en s'intercalant entre les deux lames qui la composent. La cavité thoracique est formée par ces six premières côtes, qui s'attachent au sternum. 
La disposition des còtes présente une autre particularité chez les crocodiles.

Les dix-neut à vingt premières vertèbres supportent des cùtes. Celles-ci sont articulées par une racine simple avec l'atlas, l'axis et les' six vertèbres qui viennent après la treizième; cette articulation est immédiate aux deux premières, médiate aux autres, où elle se fait par une apophyse transverse, considérable, aplatie de haut en bas, qui se détache du corps de la vertèbre. Les autres còtes sont articulées par deux racines, dont l'une est interne et inférieure, l'autre externe et supérieure, de telle façon que celle-ci s’applique contre le sommet de l'apophyse trans. verse, qui existe aussi sur ces vertèbres, tandis que la racine interne s'applique, à compter de la troisième jusqu'à la onzième vertèbre, plus bas à la face latérale du corps, et, sur les autres vertèbres qui viennent après, à une petite saillie de lapophyse transverse, saillie qui est située plus en avant et en dedans. Entre les deux raciues il existe, depuis la troisième jusqu'à la onzième còte, une lacune triangulaire, large et profonde, qui est très-superficielle aux dernières vertèbres.

Les deux premières, la huitième et la neuvième, ainsi que la dix-neuvième côte, se terminent librement. La troisième et la septième sont unies entre elles par des apophyses dirigées en avant et en arrière, qui se détachent de leur 
bord inférieur; la dixième et les sept vertèbres qui la suivent s'attachent au sternum au moyen de cartilages.

I a troisième et la septième sont, à tous égards, les plus dignes de remarque. Elles se dirigent d'avant en arrière et se prolongent en une forte apophyse postérieure et en une antérieure, par lesquelles elles se touchent. Cette disposition limite considérablement la flexion latérale du cou; il en résulte la roideur connue de celte partie. On trouve un indice de cette disposition, sur les huitième, neuvième et dixième côtes, dans une apophyse antérieure, bien plus petite, qui disparaît insensiblement à partir de la huitième jusqu’à la dixième, et est située immédiatement au dessous du point de réunion des deux branches de la côte. La bifurcation de la plupart des côtes, à leur partie interne, en une branche supérieure et en une inférieure, par conséquent leur articulation avec deux points de la vertèbre, est, autant que je sache, particulière aux crocodiles; on ne la trouve que chez eux, mais dans toutes les espèces. Les première, huitième et dix-neuvième côtes ont à peu près le même volume; la seconde est plus petite; les autres, à l'exception de la troisième jusqu'à la septième, sont plus grandes, surtout celles du milieu.

Les cinq vertèbres situées au devant du sacrum sont sans côtes, même leurs apophyses trans. verses deviennent insensiblement plus petiles. 
Au lieu que tontes ou presque toutes les vertèbres antérieures au sacrum portent des côtes, on trouve ici, à la face abdominale, des cartilages costaux ossifés, qui, étant unis avec un cartilage situé dans la ligne médiane de la paroi thoracique antérieure, et étendu du sternum aux os du pubis, sont libres en haut et en dehors.

\section{Sternum.}

\section{\$. 188.}

Tous les sauriens ont un sternum plus ou moins développé, ordinairement court, mais large.

Il est composé communément de deux parties principales, une antérieure et une postérieure. L'antérieure est le plus souvent mince, alongée, étroite, prolongée, en avant, de chaque côté en une branche transversale dont l'extrémité externe atteint à peu près le milieu de la clavicule antérieure; postérieurement elle est considérablement plus mince et apointie, et s'étend plus ou moins en arrière au devant de la face inférieure de la partie postérieure du sternum. La Ipièce antérieure est, dans la plupart des animaux de cet ordre, plus ossifiée que la postérieure, celle-ci est communément rhomboïdale, plus grande et surtout beaucoup plus large; elle re. 
çoit, par son bord antérieur, qui est un peu con. cave et encroûté de cartilage, la partie inférieure du bord antérieur de la clavicule postérieure, à laquelle elle est unie au moyen d'une capsule lâche. A son bord postérieur s'attachent les cartilages costaux moyens. Le milieu de celte pièce postérieure est d'ordinaire seulement cartilagineux dans une étendue variable d'avaut en arrière; souvent on y trouve même une ouverture, qui est parfois plus ou moins complètement remplie par la pointe postérieure de la pièce antérieure du sternum, lorsque celle-ci se porte fort en arrière.

Ordinairement ces deux pièces sont troutà-fait séparées, d'autres fois elles n'en font qu'une, par exemple chez les geckos. Dans ce genre, la pièce antérieure est en mèrne temps plus large que de contume, et les branches transversales en sont moins distinctes et s'en détachent brusquement.

A ces deux pièces principales il s'en joint ordiuairement encore deux autres, plus petites, alongées, ayant la forme de cartilages costaux, séparées l'une de l'autre, mais appliquées près de la ligne médiane, par leur extrémité antérieure, sur l'extrémité postérieure de la seconde pièce principale; ces pièces se portent directement d'avant en arrière et présentent sur leur bord externe les cartilages des deux dernières côtes vraies. 
V'A TATOMIE COMPARÉ.

Les crocodiles présentent une disposition digne de remarque.

Leur sternum s'étend, en effet, dans toute la longueur de la cavité thoracique et abdominale. Sa partie antérieure qui reçoit les clavicules est la plus large; elle est rhomboïdale et se terrnine en avant par une pointe qui dépasse considérablement les clavicules. Il se rétrécit ensuite et reçoit les cartilages des côtes, à compter de la troisième jusqu'à la huitième. Au dessous du niveau de la huitième côte, dans la cavité abdominale, il devient encore plus étroit, mais s'élargit de nouveau considérablement vers son extrémité postérieure, et s'appuie par son bord postérieur sur le bord antérieur des os du pubis. Cette partie située en face des vertèbres lombaires, correspond sans doute à la dernière pièce sternale des autres sauriens, qui s'est ici plus fortement développée; elle supporte huit paires de cartilages costaux, qui se terminent librement en arrière et auxquels ne correspondent point de côtes vertébrales. Cette disposition rappelle, en partie, le développement considérable du sternum chez les chéloniens, surtout à cause de la réunion du sternum avec les os pelviens, chez le matamata; disposition qui existe en partie chez les batraciens urodèles supérieurs (1).

(1) Voyez pags. 552 et 595. 
D'après ce qui vient d'être dit, le sternum supérieur des sauriens se compose le plus souvent de trois pières qui se succèdent d'avant en arrière. La première de ces pièces correspond très-vraisemblablement aux trois premières pièces du sternum des chéloniens qui représentent ensemble sa forme générale; la seconde pièce correspond aux deux pièces suivantes des mêmes chéloniens, et les deux dernières pièces représentent la dernière paire de pièces de leur plastron.

B. OS DES MEMBRES.

$$
\text { S. } 189
$$

Tous les reptiles ont des membres, à l'exception de la plupart des ophidiens. Ce sont le plus souvent des membres antérieurs et postérieurs qui se subdivisent en leurs quatre portions ordinaires.

Dans quelques genres d'ophicliens supérieurs, qui ressemblent aux sauriens, il existe des traces d'un ou de deux membres, surtout la première portion qui est cachée sous la peau. Mais nous ne pouvons pas commencer par la description de ces parties, parce que d'un côté elles sont plus compliquées que les os qui leur correspondent chez les batraciens, et que de 
1) INATOMIE COMPARÉE.

l'autre elles imitent le type de l'organisation des sauriens.

1. Membres antérieurs.

$$
\text { S. } 190 \text {. }
$$

Les membres antérieurs des reptiles qui en offrent plus qu'un rudiment, se composent de quatre portions. La première consiste en une ceintur'e considerable, formée au moins de deux moitiés latérales, qui entoure presque entièrement la partie autérieure de la cavité thoracique, surtout inférieurement et des deux côtés : la continuité en est à la vérité interrompue en haut et en bas dans la ligne médiane; mais ses deux moitiés sont unies inférieurement, d'une manière plus ou moins solide, dans la ligne moyenne, soit entre elles, soit avec le sternum, soit de l'une et de l'autre façon; supérieurement elles ne sont unies ni entre elles, ni avec le rachis, ni avec le crâne, par des cartilages ou des ligamens, mais seulement par des muscles. Ces os forment l'épaule et représentent par leur partie supérieure l'omoplate, par l'inférieure la clavicule ou les clavicules.

La seconde portion du membre se compose généralement de l'humérus; os simple, plus ou moins alongé, convexe en haut et en bas, et pourvu de deux apophyses. 
La troisième est formée par l'os ou les os de l'avant-bras, le radius et le cubitus.

Les deux pièces de cette portion existent mème aux membres qui sont très-peu développés en longueur, comme, par exemple, dans le genre seps; mais il y a des variétés sous le rapport du nombre; séparés pour la plupart, ces deux os sont quelquefois confondus en un os unique, par exemple chez les batraciens anoures.

La quatrième portion ou la main, se divise distinctement en trois fractions, le carpe, le métacarpe et les doigts ; les os de la première fraction sont arrondis; ceux des deux autres plus ou moins alongés.

Nous avons considéré les os du tronc d'une manière générale dans chaque ordre, par plusieurs raisons, savoir $: 1^{\circ}$ parce qu'ils se déterminent réciproquement d'une manière très-exacle; $2^{\circ}$ que souvent ils se confondent ensemble; $3^{\circ}$ et qu'ils sont en etfet des parties et des répétitions les uns des autres. Quant aux membres, je crois, au contraire, plus convenable d'en suivre les régions déterminées, chaque fois, à travers tous les ordres de reptiles, d'autant plus qu'ils sont formés beaucoup plus que les o.s du tronc, d'après un même type. 
1. Os dé l'épaule.

\section{a. Batraciens.}

$$
\text { S. } 191
$$

Les us de l'épaule des batraciens sont plus simples que ceux de la plupart des autres reptiles, mais ils sont très-grands, à tel point qu'ils l'emportent à cet égard sur la plupart des autres reptiles, notamment sur les ophidiens, qui en sont pourvus, et les sauriens.

\section{Batraciens urodèles.}

$$
\text { S. } 192 \text {. }
$$

Les batraciens à queue présentent la disposition la plus simple; ils ont de chaque côté du corps une seule plaque, en majeure partie cartilagineuse, ossifiee seulement au centre. Chacune de ces plaques entoure tout le tronc deson côté ; celle du côté droit s'applique par son bord inLerne jjar dessus celle du côté gauche, mais en conservant sa mobilité.

Sa plus grande longueur est de haut en bas: elle présente un bord antérieur, convexe, se prolongeant au milieu en une apophyse plus ou moins longue, dirigée en avant; un bord postérieur qui est concave; un bord supérieur 
droit, et un inférieur également convexe. Un peu en avant de la jonction du bord postérieur avec l'inférieur, on trouve, à sa surface extérieure, très-près du bord postérieur, un enfoncement simple, arrondi, qui reçoit la tête de l'humérus. La partie inférieure, située au devant de cet enfoncement, est plus petite que la supérieure, mais elle est plus large qu'elle dans le sens antéro-postérieur.

L'os qui est toujours proportionnellement petit occupe la partie postérieure de la plaque cartilagineuse.

Les protées se distinguent des salamandres et des tritons par la forme générale de cette plaque, aussi bien que par le rapport qui existe entre l'os et le cartilage.

Chez eux les apophyses antérieures et la moitié supérieure qui est située derrière la cavité articulaire, sont beaucoup plus grandes et plus alongées, de sorte qu'elles semblent ètre bien plutôt des appendices de la partie antérieure et inférieure, qui est proportionnellement plus volumineuse.

Il n'y a de véritable os que daus la moitié supérieure; il est petit, mince et alongé, et ne prend aucune part à la formation de la cavité articulaire.

Chez les tritons et les salamandres, l'os qui est en proportion beaucoup plus considérable et qui occupe postérieurement toute la partie moyenne 


$$
\text { 1) ANATOMUE COMPARÉ. }
$$

de la plaque, imite la forme du tout par trois apophyses dirigées en avant, en haut et en bas, et il contient en entier la cavité articulaire.

Les sirènes offrent essentiellement là disposition qui vient d'être décrite (1).

\section{Batraciens anoures.}

Les batraciens sans queue présentent une conformation plus compliquée, qui ressemble davantage à celle des reptiles supérieurs, dont elle se rapproche aussi par une ossification beaucoup plus avancée.

Il existe très-généralement, de chaque côté, quatre pièces osseuses unies, sur la ligne médiane, entre elles, et, en partie, aussi avec celles du côté opposé, savoir :

$1^{\circ}$ Une grande pièce supérieure, irrégulièrement quadrilatère, cartilagineuse dans sa partie supérieure: une bandelette cartilagineuse, étroite, unit son bord inférieur, qui est droit, avec la pièce suivante, d'une manière plus on moins mobile;

$2^{\circ}$ Une seconde pièce étroite, ayant la forme d'un carré àlongé, un peu resserrée au milieu, se recourbant de haut en bas et de dehors en dedans, qui se bifurque, à son extrémité infé-

(1) Cnvier, Mémoire sur les reptiles douteux. Paris, 1817, p. 20. 
rieure, en une branche antérieure et en une postérieure, et forme ici la majeure partie de la surface articulaire de l'épaule, c'est-à-dire qu'elle en constitue les parties inférieure et postérieure.

Ces deux pièces forment la partie supérieure de la ceinture osseuse; les deux qui suivent en constituent l'inférieure;

$3^{\circ}$ et $4^{\circ}$. Ces dernières sont alongées; l'antérieure est, dans la plupart de ces animaux, plus mince que la postérieure, laquelle devient plus large de dehors en dedans. Elles sont situées transversalement, se rencontrent en dehors entre elles et a vec la seconde, pour former la partie interne et inférieure, qui est la plus petite, de la surface articulaire de l'épaule, et divergent ensuite en se dirigeant de dehors en dedans. Les pièces de mème nom, des deux còtés, se rapprochent dans la ligne médiane, tellement que l'antérieure et interne, au moins, est unie par une harmonie étroite avec sa congénère, et que les deux postérieures se touchent également. La pièce antérieure et la postérieure sont de même unies entre elles par du cartilage, de telle facon que l'ouverture qui existe entre elles est fermée en dedans, el que par conséquent le grand interstice transversal qui est entouré par les pièces antérieure et postérieure des deux côtés, est partagé par une cloison moyenne en deux moitiés arrondies. 


\section{\$. 193.}

Les divers genres de batraciens anoures offrent, sous quelques rapports, plusieurs modifications considérables de cette disposition.

Les crapauds se rapprochent le plus des $b a-$ traciens urodèles; chez eux la masse cartilagineuse est plus abondante que chez les grenouilles. Chez le pipa surtout, la première pièce, qui est très-grande et large, est presque entièrement cartilagineuse et ossifiée seulement dans son bord antérieur et son extrémité externe. Chez les autres crapauds, elle est cartilagineuse, au moins dans sa moitié supérieure. Chez les grenouilles et les raines (hyla), les pièces antérieures et les postérieures se rencontrent par leurs extrémités internes, et sont exactement retenues des deux côtés par une harmonie étroite. Chez le pipa et les crapauds, au contraire, il n'y a que les antérieures qui soient unies d'une manière immédiate, mais lâche; elles sont unies aux postérieures par une strie cartilagineuse, dirigée d'avant en arrière, qui est plus large chez. le pipa, plus étroite chez les crapauds. Le pipa fait encore ici le passage des autres crapauds aux grenouilles; en effet, la strie cartilagineuse de chaque côté, est non seulement tres-étroite chez les crapauds, mais elle y est aussi séparée de l'autre, en ce que celle du côté droit 
est placée d'une manière libre et mobile sous la gauche, tandis que chez le pipa, elles se confondent toutes deux sur la ligne médiane.

D'autres différences portent sur le volume et la forme de ces os.

Chez le pipa toute cette collection d'os est furmée des os les plus grands. Cela s'applique surtout à la première et à la quatrième piece, moins à la troisième; la quatrième est au contraire beaucoup plus petite que partout ailleurs; petitesse d'où dépend en partie le volume considérable de la première. La première et la secrnde surtout sont aussi très-larges.

Chez les grenouilles, la troisième pièce est en proportion la plus mince, la quatrième est d'une largeur considérable, et son union avec la troisième est plus intime qu'avec les autres.

\section{b. Chéloniens.$$
\text { ఏ. } 19 j \text { (bis). }
$$

Les chéloniens, que je considère immédiatement après les batraciens, parce qu'ils en sunt le plus rapprochés par la disposition de leurs membres antérieurs, se distinguent des autres reptiles: $I^{0}$ parce que les os de leurs membres supérieurs sout unis au rachis par des ligamens, mode d'articulation qui peut ètre comparé à celui qui existe chez les poissons, et quils ne 


\section{D'ANATOHE COMPAREE.}

sont unis entre eux que par le moyen du sternum; $2^{\prime \prime}$ que les os de l'épaule et du bras se trouvent entre la carapace et le plastron, et leur sont par conséquent sous-jacens, tandis qu'ils sont situés ordinairement à la face externe des os qui correspondent à ces boucliers.

Il y a de chaque côté, dans la région de l'épaule, deux os au lieu de quatre. L'antérieur se compose de deux moitiés réunies sous un angle obtus : d'une supérieure, plus longue, prismatique, qui descend à peu près verticalement, et d'une inférieure, plus horizontale, ou dirigée de haut en bas et d'arrière en avant, qui est beaucoup plus courte, aplatie de haut en bas et un peu plus large à sa partie antérieure. Les deux moitiés inférieures convergent des deux côlés en avant. A l'endroit de réunion de la moitié supérieure avec l'inférieure, l'os présente à sa face externe une surface articulaire encroûtée de cartilage, qui forme la partie antérieure de la cavité d'articulation qui reçoit l'humérus. L'extrémité supérieure de cet os est articulée mobilement avec la première côte, à l'endroit oi le col de celle-ci se continue avec son corps.

Le second os se rencontre avec le premier, à l'endroit où ses deux moitiés passent l'une dans lautre, et forme la partie antérieure de la cavité articulaire de l'épaule; il est large, en forme de pelle, s'élargit et s'aplatit considérablement en dedans, converge avec son congé- 
nère du côté opposé, en dedans et en arrière, mais ne se soude pas à lui.

La disposition qui vient d'être décrite fait voir clairement la grande analogie qui existe entre" les batraciens et les chéloniens, et qui justifie leur rapprochement immédiat.

L'os antérieur représente évidemment, au moins la seconde et la troisième pièce de l'épaule des batraciens, peut-être même la première pièce. Mais il est bien possible aussi que la première soit ici sortie de la série des os de l'épaule et forme la plaque moyenne et antérieure de la carapace, qui a été décrite (1).

L'os postérieur représente le plus sûrement le quatrième os de l'épaule des batraciens.

\section{S. 195}

Ces os ne sont pas formés d'après le même type chez tous les chéloniens. Chez les tortues, la branche verticale du premier os est beaucoup plus longue que l'horizontale, et l'os postérieur est encore plus court, quoique beaucoup plus large que celui-ci. Chez les érnydes, les deux dernières pièces augmentent de volume, et cela s'applique encore bien plus à la seconde pièce chez les chélonées, chez lesquelles la première devient considérablement plus petite, à tel point que la seconde de ces pièces est bien plus longue

(1) Voycz pag. 575 . 
que chaque branche de la première pièce en particulier. Mais en outre la pièce postérieure est beaucoup plus étroite chez les chélonées que chez les autres, parmi lesquels les tortues la présentent surtout très-considérable.

\section{c. Ophidiens.}

S. 196.

Nous avons déjà dit que les ophidiens supérieurs seuls possèdent des traces de membres. L'orvet (anguis fragilis) présente de chaque côté trois os du membre antérieur; ils sont situés immédiatement au devant du coeur et derrière la tète, et se réunissent de chaque côté en un demi-anneau alongé, concave en avant, convexe en arrière. De ces trois pièces les deux postérieures s'unissent entre elles plus exactement qu'avec la troisième.

La piece supérieure et postérieure est beaucoup plus petite que toutes les autres; elle a la forme d'un carré alongé, et représente, sans contredit, l'épaule.

L'inférieure est alongée et quadrilatère, dirigée d'arrière en avant et de dehors en dedans; elle se rapproche de sa congénère par son extrémité antérieure, sans cependant s'unir à elle. Elle est percée, vers son extrémité externe, par une ouverture considérable, alongée et arrondie. L'anneau qui entoure celte ouverture est 
bien plus mince dans sa partie antérieure que dans les autres partics de son contour. Cet os s'articule par tout son bord inférieur et interne, avec le bord externe de la moitié du sternum qui est de son côté.

Cet os correspond incontestablement à la clavicule des batraciens.

L'os antérieur est alongé, en forme de $S$, fort convexe en arrière à sa partie supérieure, en avant à l'inférieure; il est situé au devant de l'extrémité inférieure de l'omoplate et descend jusqu'à la ligne médiane, de manière à attcindre ici celui du côté opposé. La même conformation existe chez le bipes Pallasii.

On trouve essentiellement la même disposition chez l'ophisaurus ventralis; seulement les os y sont en proportion un peu plus grands, et l'antérieur est plus alongé et plus droit. Mais en revanche il n'existe ici aucune trace de sternum. Les rudimens du membre antérieur se comportent d'une manière fort analogue chez plusieurs sauriens qui n'ont que des membres postérieurs.

Le bipes lepidopus présente, par opposition avec l'ophisaure, un sternum, mais nulle trace de l'os antérieur, tandis que chez ce dernier, les autres os ont proportionnellement le plus de volume.

Chez l'acontias meleagris, au contraire, il n'y a point de vestige desmembres antérieurs et pos- 
térieurs, mais chez lui les côtes antérieures sont unies entre elles par leurs cartilages (1).

\section{d. Sauriens.}

\section{S. 197.}

Les os de l'épaule des sauriens ne sont pas partout également composés, mais ils consistent toujours, de chaque côté, au moins en un os supérieur et en un inférieur, qui forment à leur bord posiérieur, par lequel ils sont en contact, la face articulaire de l'épaule. Les crocodiles présentent la conformation la plus simple. Les deux os ont à peu près la même forme et le même volume. Ils sont alongés, un peu plus minces au milieu qu'aux extrémités; le supérieur est cartilagineux dans son quart supérieur. où il est libre; l'inférieur touche par son bord iuterne et inférieur au bord latéral du sternum, à l'endroit de la plus grande largeur de cet os.

Mais ordinairement il y a beaucoup plus de complication; les deux os mentionnés se divisent en trois et même en quatre autres plus composés, et il s'en forme encore un antérieur, de sorte que celte région est formée, des deux côtés, de cinq paires d'os.

Le plus supérieur de ces os résulte de l'am-

(1) Cuvier, Règne animal, t. II , p. 6 . 
pliation et de la bifurcation de l'os supérieur des crocodiles. Il est le plus large, mais mince, osseux seulement dans sa partie inférieure, cartilagineux dans la supérieure, irrégulièrement quadrilatère, en haut beaucoup plus large qu'en bas; il correspond incontestablement à la première pièce des batraciens anoures.

Le second os, la partie inférieure de l'os supérieur des crocodiles, est plus petit et plus alongé, mais plus épais, et forme la partie postérieure de la surface articulaire de l'épaule. C'est la seconde pièce des batraciens anoures.

Le troisième correspond vraisemblablement à leurs troisième et quatrième pièces. Il se prolonge en avant et en dedans par trois branches, dont la postérieure est la plus large, la moyenne la plus étroite et la plus longue. Son bord postérieur offre deux échancrures dont la supérieure et externe, plus large, forme la partie antérieure de la surface articulaire de l'épaule, laquelle est par conséquent encore, dans ces animaux, seulement composée de deux os. Il existe ordinairement duns cet os, entre les deux branches internes, une petite ouverture arrondie, et en outre, entre ces deux branches et la troisième, eutre celle.ci et le second os, trois incisures très-profondes.

Cet os correspond très-vraisemblablement aux troisième et quatrième os des batraciens, qui sont soudés ensemble. Celte conjecture me paraît 
du moins fort plausible, puis qu'il est divisé en brauches dirigées en avant et en dedans, et que lui seul prend part à la formation de la surface articulaire de l'épaule, à laquelle est étranger le cinquième os qui va être décrit.

Cette manière de voir est encore confirmée davantage par la présence et la disposition du quatrième os. Celui-ci, fort mince, plus cartilagineux, constitue une plaque alongée, pourvue d'un bord interne, convexe, et d'un externe, concave, laquelle s'étend de l'extrémité interne de l’apophyse postérieure du troisième os; d̀ l'extrémité interne de l'os moyen, et convertit ainsi en ouverture la lacune qui sépare ces deux os. Il s'applique, par son bord interne, contre le bord externe de la moitié antérieure de la jièce sternale moyenne; moitié qui est la plus grande.

Cette pièce est évidemment le cartilage longitudinal, intermédiaire, qui existe chez les crapauds et le pipa.

I a cinquième paire, qui, déjà chez l'ophisaure et l'orvet fragile, s'ajoutait aux pièces osseuses primitives, est très-alongée, mince, concave en avant, convexe en arrière; elle est la plus antérieure et s'étend de haut en bas et de dehors en dedans, de l'extrémité interne et inférieure du bord antérieur du premier os, au devant du second, de sorte qu'il reste, entre eux, une lacunce considérable; elle rencontre en dedans; 
par son extrémité interne, l'os de même nom du côté opposé, en arrière la pièce la plus antérieure du sternum.

Cette disposition se trouve dans les genres lézard, monitor, iguane. Chez le gecko la forme extérieure est la même, mais lì seconde et la troisième pièce ne sont pas séparées l'une de l'autre.

2. Humérus.

$$
\text { S. } 198 \text {. }
$$

Chez les batraciens et surtout les grenouilles proprement dites, l'humérus, dont les conditions générales ont déjà été examinées plus haut, est plus volumineux que les os de l'avant-bras; sa tête articulaire supérieure est sphérique. Au dessous de cette tête on voit naître, chez le protée, une petite ligne âpre, antérieure, et deux chez les salamandres, une antérieure et une postérieure; chez les batraciens anoures, il n'y en a qu'une antérieure, bien plus longue, qui est prononcée au moindre degré chez le pipa, qui est plus marquée chez les crapauds, et qui ne l'est nulle part ailleurs autant que chez le rana latrans (1). Cette grenouille possède aussi la ligne postérieure, qui existe chez les salamandies et manque aux autres batraciens anoures: elle y est tellement développée en largeur et en

(1) Steffen, De ranis nonnullis. Berlin, 1815 ; fig 2 et 5. 


$$
\text { D'ANATOME COMPARÉE. }
$$

longueur, que l'humérus en paraît transformé en une espèce de plaque.

L'extrémité inférieure de l'humérus des bàtraciens, soit urodèles, soit anoures, se renfle en une forte tète, ronde, à côté de laquelle il existe en dedans, séparée d'elle par un espace profond, une saillie longitudinale également haute, qui empêche l'os de l'avant-bras de s'échapper dans cette direction.

Chez les sauriens, la différence de longueur entre l'os du bras et ceux de l'avant-bras est en général moindre que chez les batraciens; l'humérus est très-ordinairement droit, sans lignes âpres distinctes, assez fortement renflé à ses deux extrémités. L'inférieure se termine fort généralement par deux éminences arrondies, dont l'interne et antérieure supporte le radius, l'externe et postérieure servant d'appui au cubitus, et dont le volume proportionnel est tellement. variable, que c'est tantôt l'externe, comme, par exemple, chez le monitor, tantôt l'interne, comne chez le crocodile, qui est la plus grande.

Chez les chéloniens, cet os est le plus petit proportionnellement au reste du corps et aux os de l'épaule, en faisant toutefois exception des sauriens a petites pattes; il est, au contraire, chez. les mèmes chéloniens, par rapport aux os de l'avant-bras, plus considérable que chez tous les autres reptiles. Il est plus ou moins aplati de haui con bas. La tète ronde de cet os est sup40. 
portée par un col considérablement plus large, qui se renfle en une éminence antérieure et en une postérieure, ou en une inférieure et une supérieure. Le corps en est, toute proportion gardée, fortement contracté; mais vers la partie inférieure il s'élargit de nouveau considérablement, et s'articule avec les deux os de l'avantbras par une surface articulaire, transverse et convexe.

Chez les chélonées, l'humérus est beaucoup plus court que chez les autres chéloniens, il est à sa plus grande longueur chez les tortues. Il est chez les premières, droit et fort aplati; chez les secondes, au contraire, il est fortement recourbé en avant et arrondi; là ses apophyses sont faibles, ici elles sont fort développées. I Les émycles tiennent encore le milieu en cela, comme de coutume. L'humérus de tous les chéloniens est tellement contourné autour de son axe, que la face de cet os à la quelle s'attachent les muscles extenseurs, est tournée en avant, et celle des muscles fléchisseurs en arrière. La surface articulaire, inférieure, est simple et convexe chez les chélonées, légèrement concave à son milieu chez les tortues; l'humérus des émydes a une tète arrondie, à côté de laquelle se trouve une saillie étroite, mais assez considérable, qui cn est séparée par un sillon profond. 
5. Os de l'avant -bras.

$$
\text { \$. } 199
$$

L'avant-bras de la plupart des reptiles, notamment des balraciens urodèles, des chéloniens, des sauriens, et parmi ceux-ci, même celui des s'ps, du moins du seps tridactylus, se compose de deux os, nonobstant le développement peu considérable de leurs membres. De ces deux os, celui qui est généralement situé en av̌ant et qui correspond constamment au pouce est le radius, le postérieur est le cubitus, dont l'olécrane dépasse très-généralement le premier dans une étendue variable.

On ne sait pas encore si les sauriens bimanes (chirotes, Guv.) ont également les membres anıérieurs aussi parfaitement développés. M: Cuvier (1) parle bien du développement parfait des os de l'épaule et des doigts, mais non des os des autres régions. Cependant j’ai trouvé positivement chez le chirotes propus, un humérus et deux os de l'avant-bras.

Les batraciens anoures, au contraire, n'ont qu'un os à l'avant-bras, qui est assez large. On voit cependant, à ses extrémités supérieure et inférieure, surtout à celle-ci, un sillon longitudinal plus ou moins marqué, visible sur les deux

(1) Règne animal, 1817, t. II, pag. 57 . 
faces de l'os, qui le partage en deux et an fond duquel la substance de l'os est plus mince. Quoique la continuité ne soit pas rompue à l'extérieur, la cavité de l'intérieur de l'os est pourtant divisée dans sa partie inférieure, qui est la plus grande, en deux cavités médullaires entièrement distinctes, et mèrne la cloison intermédiaire est en majeure partie double. C'est là la disposition naturelle de cet os; mais il n'est pas exact de dire que la partie moyenne de l'os est sans cavité médullaire, et qu'il y a également deux de ces cavités dans la partie supérieure de l'os (1). Il est creux partout, mais supérieurement la cavité en est unique; elle est double inférieurement, comme il vient d'être indiqué. La largeur de cet os est, au reste, un autre indice de l'analogie qui existe entre lui et les deux os de l'avant-bras des autres reptiles.

Cet os simple est supérieurement beaucoup plus long à la face des muscles extenseurs qu'à celle des fléchisseurs; il forme à cette extrémité une espèce de crochet, l'olécrane, qui s'engrène a vec un enfoncement existant à la face d'extension de l'humérus. Chez le pipa la division de la région inférieure de l'os de l'avant-bras est beaucoup moins distincte que chez les crapauds; elle est marquée au plus haut point chez les grenouilles.

(I) Blumenbach's Handbuch der vergleichenden Anatomie, p. $9^{8}$. 


$$
\text { V'ANATOMHE COMPARÉ. }
$$

I a surface articulaire inférieure se compose toujours de deux éminences; de ces éminences celle qui correspond au radius est plus grande, transversale et un peu plus déprimée que celle du cubitus.

Les os de l'avant-bras des chéloniens offrent une disposition fort particulière à plusieurs égards. Ils sont plus ou moins épais et plats, courts, larges, unis entre eux immobilement en haut et en bas, le plus souvent dans une étendue très-considérable; conditions par lesquelles ces os se rapprochent évidemment beaucoup de celle des batraciens. Ils offrent le plus d'épaisseur chez les chélonées, le plus d'aplatissement et le plus de briéveté chez les tortues, le plus de longueur et le plus de gracilité chez les émydes. Chez les tortues, ils sont unis entre eux, à leurs deux extrémités, dans la plus grande étendue de leur longueur; cette union n'existe que dans la plus courte position de cette même longueur chez les émydes, chez lesquelles ils ne sont pas même unis inférieurement.

Partout le radius descend plus bas et le cubitus monte plus haut; chez les émydes et les tortues ces deux os ont à peu près la même longueur. Les chélonées offrent à cet égard une exception digne de remarque: le radius est beaucoup plus long, il descend bien plus bas que le cubitus, tandis que celui-ci ne le dépasse guère en haut.

Une autre chose digne de remarque chez les 
chéloniens, est la déviation graduelle que présentent ces os de leur position. ordinaire. Chez tous, le radius n'est pas situé au devant, mais plus ou moins en arrière du cubitus; l'hurnérus est déjà contourné autour de son axe, à tel point que la face de ses muscles extenseurs est diricréc en avant, celle des fléchisseurs en arrière. Celte disposition est prononcée au moindre degré chez les chélonées. Le radius est encore situé au devant du cubitus; mais celui-ci se glisse déjà un peu vers la partie antérieure du premier. Cisez les émydes, les deux os sont déjà presque entièrement sur un même plan, chez les tortues, ce nivellement est parfait; le bord tranchant du cubitus, qui est communément postérieur, est ici tourné en avant.

Les faces articulaires, inférieures, sont convexes; chez les tortues et les émydes très-larges aux deux os, dans le sens transversal; il n'y a de surface articulaire inférieure qu'au cubitus chez les chélonées; le radius de celles-ci se terminant par une petite tête fort arrondie.

Les os de l'avant-bras des sauriens et des batraciens urodeles sont assez droits; ils ont à peu près la même grosseur, seulement le cubitus est un peu plus épais que le radius. La surface articulaire, supérieure, de l'un et de l'autre, est concave de dedans en dehors et d'avant en arrière; plus longue dans le dernier sens que dans le premier; celle du cubilus devient plus large en arrière. 
I.es deux os se réunissent ici pour former une surface arliculaire, commune, et s'unissent cutre enx par des surfaces encroûtées de cartilagge. Ils s'écartent, au contraire, à leur extrémité inférieure, et, chez plısieurs d'entre eux, par exemple, le monitor, il y a même un petit os dåus lintervalle qui les sépare. La surface articulaire, inférieure, du radius ressemble ordinairement a la supérieure; elle est concave et alongée; celle du cubitus, aı contraire, est souvent, si ce n'est toujours (il en est ainsi du moins chez les crocodiles, les monitors, les iguanes, les lézarls), renflée en une tête, qui est légèrement comprimée sur les parties latérales, fortement convexe d'un côté à l'autre et d'avant en arrière, surtout dans cette dernière direction; disposition particulière, qui est surtout remarquable, parce que par là le cubitus ressemble aux os du métacarpe et des doigts; ce qui explique le nombre, en partie, médiocre des os du carpe, en ce que cette extrémité inférieure du cubitus semble s'èlre formée aux dépens d'un os du carpe.

$$
\text { S. } 200 \text {. }
$$

Outre la différence qui vient d'étre indiquée entre les batraciens anoures et les autres repliles, le nombre des os de l'avant-bras présente, chez plusieurs de ces animaux une autre différence tout-à-fait opposée. Il s'ajoute quelquefois aux deux os de l'avant-bras un troisième os ap- 
pliqué sur l'extrémité supérieure du cubitus. C'est la totalité de lolécrane ou la partie supérieure de cet os qui ne s'est pas réunic au reste du cubitus; on peut lui donner le nom de rotule du coude,par analogie avec les membres inférieurs des autres vertébrés chez lesquels on trouve un semblable os propre, la rolule du genou.

M. Rudolphi a, le premier, démontré la présence de cet os chez le pipa. On le rencontre, en outre, chez plusieurs autres reptiles, surtout d'après mes recherches, parmi les sauriens, chez l'iguane à col ǹu (iguana delicatissima), les marbrés (polychrus marmoratus), le grand lézard-crocodile (lacerta ocellata) et le lézard vert ( $l$. viridis); parmi les chéloniens, chez la tortue grecque; parmi les batraciens, chez la grenouille verte (rana esculenta); il est par conséquent assez général, puisqu'on le trouve dans plusieurs genres de tous les ordres.

\section{Os de la main.}

$$
\text { S. } 20 \mathrm{I} \text {. }
$$

Des trois fractions de la main, la troisième, qui est formée par les doigts, est sans contredit toujours la plus longue. Le carpe est la fraction la plus courte, mais moins généralement; quoique cette remarque s'applique à la pluralité de ces animaux.

Le rapport de la main entière avec les autres 
portions n'est pas le même chez tous les repiiles. Ces différences se rapportent moins au caractère de l'ordre qu'au mode de locomotion et au milieu habité par ces animaux. Ainsi, chez les tortues, la main forme à peine le quart, chez quelques-unes seulement le cinquième de toute la longueur des trois portions extérieures du membre, tandis que, chez les chélonées, elle est bien plus longue que les leux portions situées immédiatement au devant d'elle.

Elle est considérable chez la plupart des sauriens, et présente à peu près la même longueur que l'avant-bras et le bras.

On peut dire la même chose des batraciens. Chez les tortues, la main est à beaucoup près la plus petite portion du membre.

$$
\begin{aligned}
& \text { a. Carpe. } \\
& \text { S. } 202 .
\end{aligned}
$$

Le carpe se compose de plusieurs os courts, arrondis, unis d'une manière peu mobile entre eux et avec les os métacarpiens; leur articulation avec les os de l'avant-bras permet, au contraire, des mouvemens plus étendus. Ces os carpiens se succèdent du bord radial au bord cubital en formant une rangée, ou plus ordinairement se disposant suivant plusieurs rangées transversales; quelquefois aussi ils doublent, mais sur quelques points seulement, dans la direction de la face palmaire à la fáce dorsale. 


\section{a. Batraciens.}

1. Batraciens urodèles.

$$
\text { S. } 203 .
$$

Le protée, parmi les batraciens ì queue, pré. sente, autant que j’ai pu mien assurer par les recherches les plus attentives, trois os carpiens, plats, tout-à-fait cartilagineux, qui sont disposés suivant deux rangées les uns derrière les autres. La première rangée est formée de deux os alongés, dont l'un correspond au radius, l'autre au cubitus. Le premier est plus long et plus étroit, le dernier plus court et plus large. $\Lambda u$ devant du dernier on trouve un autre os transversal, arrondi, qui forme la seconde rangée et ne correspond pas tout-à-fait à tout le bord antérieur de la première. Cet os supporte entièrement l’os métacarpien du doigt médian, et en outre une partie de la base des premier et troisième doigts, qui, touchent en outre aux deux os carpiens de la première rangée.

M. Rusconi, dans son ouvrage (1), ne décrit pas du tout les os des membres du protée, et renvoie seulement aux planches, où l'on voit figurés cinq de ces os pour le carpe (2); mais il

(1) Monogr. del proteo anguino. Pavia, 1819, p. 47.

(2) Tab. IV , fig. 3, ๆ. 
est extrêmement vraisemblable que l'un de ces os n'est que l'épiphyse cartilagineuse, inférieure du radius, tout-à-fait semblable à celle que présente le cubitus qui est seulement plus petite, et que les deux os antérieurs de la première rangée ne sont que le résultat d'une séparation artificielle du troisième os.

L'état cartilagineux de ces os, chez le protée, est très-digne de remarque, à cause de l'ossification plus tardive de ces os chez les animaux supérieurs.

Chez les autres batraciens urodèles, du moins les tritons et les salamandres, d'une part ils sont osseux, d'autre part ils sont plus nombreux.

Ces reptiles, d'après M. Cuvier (1), en posséderaient huit, disposés par trois rangées; cependant je n'en ai pu trouver que sept, et ne crois pas m'être trompé, parce que j'ai fait mes recherches avecle plus grand soin.

Ils sont disposés, en effet, sur trois rangées. La première en contient deux, savoir : un antérieur, petit et arrondi, qui correspond seulement au radius, et un postérieur, beaucoup plus grand et plus large, qui vient après le radius et le cubitus. Le premier est purement cartilagineux chez les salamandres; il est osseux chez les tritons. La seconde rangée en renferme trois, ayant à peu près le même volume, qui, sous ce rap-

(3) Leçons d'analomie comparéce, vol. I, pag. 516. 
port "et sous celui de la forme, ressemblent au premier de la première rangée.

Les deux antérieurs supportent le premier os de la troisième rangée, le postérieur supporte le second de la même rangée, et en outre le quatrième os métacarpien.

La rangée antérieure est formée de deux os, un antérieur beaucoup plus grand, et un postérieur bien plus petit. L'antérieur supporte le premier et le second os du métacarpe, celui-ci supporte le troisième de ces os.

Tous ces os sonl courts et arrondis.

2. Batraciens anoures.

$$
\text { \$. } 204 \text {. }
$$

M. Cuvier donne aux batraciens sans quene le mème nombre d'os carpiens qu'aux batraciens à queue; mais je le dois encore contredire en cette proposition. Chez la grenouille commune (rana esculenta) et la grenouille rousse (1. temporaria), je ne trouve pas huit os, mais seulement cinq, qui sont disposés, non en trois rangées, mais seulement en deux. La première rangée est formée par trois os, dont le moyen est le plus volumineux, et semble par conséquent correspondre au second os, résultant de la division du deuxieme os des batraciens urodèles. La seconde rangée se compose de deux 


\section{D'ANATOMIE COMPARÉE.}

os, l'un arrondi et très-petit, situé entre l'antérieur de la première rangée et l'os inétacarpien du premier doigt, et un autre, plus large, offrant trois facettes articulaires, très-convexes, qui supportent les trois autres os métacarpiens.

Chezles jeunes sujets même, qui présentent ces os à un état plus cartilagineux, on ne peut représenter les huit os, indiqués par $\mathbf{M}$. Cuvier, quien employant la force.

Les crapauds offrent absolument la même dis. position; je m'en suis assuré chez le crapaud gris (bufo cinereus), le crapaud brun (b. fuscus), et surtout chez le $b$. agua.

La disposition n'est pas la même chez le pipa; mais il n'y a non plus chez lui que six os, disposés sur deux rangées. La première en contient deux; l'antérieur est, à beaucoup près, le plus grand; il est large, court, et semble résulter de la réunion du premier et du second os de la première rangée avec le premier de la seconde des. autres batraciens anoures, puisqu'il supporte l'os métacarpien du premier doigt.

Des quatre os de la seconde rangée l'avantdernier est le plus grand; le quatrième est situé hors de la rangée; le premier, le second et le troisième portent les deuxième, troisième et quatrième os métacarpiens. 
b. Chéloniens.

$$
\text { S. } 205 \text {. }
$$

Les chéloniens, par la disposition de leur main en général, ainsi que par celle de leur carpe en particulier, offrent plusieurs particularités dignes de remarque, qui sont en rapport avec la disposition de leurs membres antérieurs.

Chez les tortues, et spécialement la torlue grecque, il y a trois os dans la première rangée. Deux de ces os, dont le premier est le plus large, sont situés au devant du radius et du cubitus. Le troisième, moyen, qui est le plus petit, sc trouve entre le radius et le cubitus.

Dans la seconde rangée il y a quatre os, dont le plus antérieur, qui est le plus large et situé trausversalement, correspond au pouce et à l'index; les autres correspondant aux trois doigts externes. Le quatrième est le plus petit.

Chez les émycles, les deux os les plus antérieurs de la première rangée se partagent en deux, savoir : l'os qui correspond au radius, en un pelit os antérieur et en un postérieur plus grand; et l'os qui correspond au cubitus, en une moitié antérieure, plus grande. et en une postérieure, plus petite, de sorte que la première rangée se compose de cinq os. Il y en a quatre dans la seconde, mais ils sont autre- 


$$
\text { Vis ITOML: COMPARIR: }
$$

munt disposés que dans la première. A chacun des trois premiers os métacarpiens correspond un os particulier, tandis que le quatrième et le cinquième sont supportés par un os carpien, unique, plus large; différence qui se rattache sans doute au développement extrêmement incomplet du pouce chez les tortues. Il existe par conséquent ici neuf os carpiens.

Chez les chélonées, le nombre des os du carpe est encore plus considérable, et cette fraction de la main est aussi proportionnellernent plus grande.

Il y en a dix. Deux de ces os, qui sont juxtaposés, sont placés à l'extrémité inférieure du cubilus, et forment la première rangée, conjointement avec un troisième os antérieur, qui esı beaucoup plus petit et qui tait suite au radius.

Jes sept autres constituent la seconde rangée. Cing d'entre eux, dont les deux externes sont les plus gros, correspondent chacun à un os mé. tacarpien; le sixième est situé entre celui du second métacarpien et la prenière rangée; le septieme, qui est heaucoup plus grand que les autres de cet ordre et aussi grand que les plus volunineux du premier ordre, est appligué sur le sixieme; il sort entièrement de la rangée et augmente cousidérablement la largeur de la main dans cette région. 
c. Sauriens.

S. 206.

Les sauriens possedent en général un assizz grand nombre de petits os, courts et larges, disposés sur deux rangées; mais ils n'en ont pas autant que les chéloniens. Ordinairement ceux de la première rangée sont moins nombreux, mais plus volumineux que les suivans. L'os qui correspond au cubilus est le plus grand; il est plus ou moins concave en arrière, pour recevoir la tête du cubitus, en avant il est convexe et pourvu de plusieurs replis. Lios qui correspond au radius offe, vers le bord radial, un pelit enfoncement qui loge la petite tête du radius.

Cette organisation existe dans les genres mo. nitor et iguane. Les os de la première rangée sont plus nombreux chez les monitors. Il y en a cinq; quatre d'entre eux correspondent aux cinq os du métacarpe, de telle façon que le troisième, qui est le plus grand, soutient le troisieme et le quatrième de cesos, landis que chacun des autres en porte un, et que le cinquième est situé entre le premier et le second os carpien de celte rangée et l'os radial de la première rangée.

Chez les iguanes, ce cinquième os manque ou est confondu avec le second de la rangée antirieure; du reste la disposilion est la mème. 
1)après M. Cuvier (1), on pourrail présumer quil existe, pour les crocodiles, une disposition insolire, tout autre; il ne leur attribue que quatre os, dunt deux scraient silués hors de la rangée sur le còlé radial; mais il en existe réellement sept. Trois d'entre eux occupent la première rangée. Les deux supérieurs sont beaucoup plus considérables que l'autre, surtout le plus antérieur, qui succède au radius. Ils se distinguent. tous deux des os analogues des autres sauriens particulièrement par leur forme alongée et apla(ie, qui les fait beaucoup ressembler à des os métacarpiens. Au dessus de celui qui correspond au cubitus, il existe un petit os pisiforme.

I. a seconde rangée contient quatre os dont trois corresponilent chacun à un os métacarpien; le second, qui est le plus grand, supporte le deuxième et le troisième os du métacarpe.

\section{b. Métacarpe.}

$$
\text { \$. } 207 .
$$

Les us di mélacarpe soni presque loujours renflés aux deux extrémités; ils ressemblent beancoup à ceux des nageoires des poissons cartilagineux, à cela près qu'ils sont bien moins nombreux et correspondent le plus souvent au

(1) Anctomie romparée, vol. I, p. 316 .

$$
4:
$$


nombre des doigts. Celui du pouce est urdinairement plus court et plus gros que celui des autres doigt.s. Cette disposition est surtout prononcée chez les chélonées, moins chez les autres reptiles et mêrne chez les émydes, principalement sous le rapport de la largeur et de l'épaisseur. Le premier et le dernier os métacarpien ont tontà-fait la même longueur chez plusieurs reptiles, par exemple, les sauriens, ce qui n'est pas sans intérêt sous le rapport de la symétrie. Chez le pipa, les os du métacarpe ont une longueur et une ténuité extraordinaires; ils sont au minimum de la lonugeur chez les chélonicns, particulierement chez les lortues d'eau douce ou émy's.s.

Cette fraction de la main existe si commuriément, et son absence a si peu été signalée, à ce que je sache, que lorsque je la vis manquer d'abord dans quelques reptiles, je doulai moimême de ce que je voyais, jusqu'ì ce que des recherches réitérées el faites avec la plus grande attention, me donnassent une conviclion pleine et entière de l'absence de cette fraction.

Je ne connais que deux espèces de reptiles où le métuctirpe n'existe pas, savoir: la tortue grecque, à laquelle se rattachent peut-ètre la plupart des tortues, et le protée, quoique la chose ne soit pas encore absolument hors de doute pour celui-ci. Voici la disposilion qui existe chez ce dernier: Au devant des os carpiens décrits ci-dessus, il ne se trouve, pour. chaque doigt, que trois pha- 
D'ANATOMLE COMPARÉT.

langes, qui diminnent de volume de dedans en dehors, et se ressemblent du reste par leur forme. Les deux extérieures, en d'autres termes la seconde et la troisième phalange, sont libres, la première est entourée de muscles et cachée sous la peau. Il est possible par conséquent que ce dernier os forme le métacarpe, d'où il s'ensuivrait que les doigts ne seraient composés que de deux phalanges. Mais chez la tortue greeque, la rangée antérieure des os du carpe est suivie immédiatement de la première phalange; or comme ces os carpiens et cette phalange ressemblent absolument aux os de mème nom qui existent chez les émydes, que les chélonées mêmes n'ont que trois phalanges, bien que leurs doigts soient tres-longs, que la tortue grecquie n'a que deux os dans le pouce, tandis que les émydes en ont trois, il fant bien admettre que les parties, qui manquent sont les os métacarpiens. Je n'ai pas besoin d'insister sur ce que cette absence coincide d'une manière très-remarquable avec l'élat rabougri de la main.

Une différence sexuelle digne de remarque est celle qu'offrent ceux des batraciens, chezlesquels le prouce du mâle se gonfle à l'époque des amours. Chez ceux-ci, le premier os du métacarpe non-seulement est deux fois plus gros et plus large que chez la femelle, prolongé en une crète tranchante sur son bord radial, convese is sa face drorsale, el concave à la palmaire; 
mais il existe à son exırémilé inférieure, surson côté radial, un os mince et pointu, d'un tiers aussi long que l'os principal, pelit os dont la femelle n'offre pas de trace; c'est sans doute un rudiment de pouce.

$$
\begin{aligned}
& \text { c. Doigts. } \\
& \text { \$. } 208 \text {. }
\end{aligned}
$$

Les reptiles, qui sont en général pourvus de membres antérieurs, visibles à l'extérieur, ont aussi généralement des doigts, ainsi que les os qui servent de base à celux-ci et en déterminent la forme, quoique ces parties offrent des différences très-considérables sous le rapport de leur nombre, de celui de leurs phalinges, de leur forme et de leur volume.

Sous le rapport du nombre, on peut dire en général, que la plupart des reptiles ont cinq doigts, dont chacun, ou le plus grand nombre, surlont ceux du milieu, sont formés de trois phalanges pour le moins, qui sont unics plus ou moins mobilement entre elles et avec les os métacarpiens, dont le nombre correspond au leur.

Leur volume varie. Ils forment en général la partie la plus considérable de la main, quelquefois même la plus grande porlion du meubre; ensemble ils surpassent communément en volume le métacarpe. Le rapport qui existe entre leurs phalanges ne présente pas de différences 


$$
\text { D'Anatome COMPARÉ. }
$$

fort notables. Elles diminuent ordinairement de volume des postérieures aux antérieures.

Dans le plus grand nombre des cas, elles sont alongées. légèrement concaves à leur extrémité postérieure, convexes à l'extrémité antérieure, de telle façon qu'il en résulte une poulie ou trochlée soit simple, soit partagée en deux moitis latérales par un léger enfoncement.

\section{Batraciens.}

\section{209}

Les baumciens à queue, aussi bien que ceux sans queıte, n’ont en général que quatre doigts parfaiss; il y en a cependant parmi les anoures, du moins les grenouilles et les crapauds, mais non le pipa, qui ont, en outre, un rudiment de pouce extrèmement petit. Le prolée et la siréne font exception relativement au nombre des doigis.

Le protée n'a que trois doigts, tandis que la sirène en à cinq, qui se sont formés peut-être aux dépens clu nembre postérieur qui 'n'existe plus chez elle.

Le second et le troisième doigt du protée ne se composent que de deux phalanges; le troisième n'en a qu'une.

Chez les tritons, je trouve deux phalanges dans le premier doigt, wne seule chez les salcman- 
dres. Chez les uns et les autres, les second et quatrième doigts ont deux phalanges, le troisième en a trois. L'ensemble des phalanges des doigts est un peu plus long que les os du mélacarpe; cette disposition est le plus prononcée chez le protée, le moins chez les salamandres. Chacune des trois phalanges est, au contraire, plus courte. La phalange ungueale est toujours la plus petite. Elles sout grèles et longues chez les tritons et le protée; très-larges, plates et courtes chez les salamandres.

Chez les grenouilles et les crapa":ds, le rudiment du pouce, caché tout-à-fait sous la peau, est à peine sẹnsible et formé d'une seule phalange. Les second et troisième doigts sont en général formés de deux phalanges; les deux doigts exlernes, de trois phalanges. Mais le pipa a trois phalanges anx trois doigts interues, et seulement deux au doigt le plus externe. Constamment chaque phalange, surtout l'unguéale, est beacoup plus petite que l'os métacarpien, quoique toute la fraction digitale soit plus grande que le métacarpe. Le pipa fait exception is celte règle, à cause de la longueur extraordinaire de ses os mélacarpiens, qui ue sont pas unis entre eux par la peau.

Elles diminuent le plus souvent de volume de la première à la troisième; chez les raines (hyla), les deux premieres phalanges des troisième et quatrième doights ont lat mème longueur. 
l) ANATOMIE COMPARÉ.

En géliéral le second doigt parfait (à proprement parler, le (roisième) est beaucoup plus court que les autres, celui qui le suit de dedans en dehors est lc plus long; chez le pipa, au contraire, le second est le plus long.

Les phalanges sout généralement alongées, droites et étroites; les plus petites et les plus étroites sont celles des pipa, les plus grosses et les pius courles celles des crapauds.

\section{Chéloniens.}

\section{S. 210}

Les chéloniens ont en général cinq doigts, dont les trois du milieu sont de plus composés de trois phalanges; le pouce et le petit cioigt en ont Irois chez les chelonées et chez les émydes; chez les tortues, au contraire, du moins la tortue. grecque, ces doigts n'ont que deux phalanges, ce qui s'accorde avec le peu de développement des membres, qui est déjà si bien indiqué par l'absence du métacarpe. Les doigis sont loujours la fraction la plus longue de la main.

Ils sont, chez les chélonées, irès-longs, minces, alongés, aplatis de haut en bas. Les trois doigts üu milieu sunt considérablement plus longs que le pauce et le cinquième doigt; de ces doigts, le moyen offre le plus de longueur, le rroisieme est un yeu plus court, le second est beaur(oup plus court encore. Le pouce est de tous le plus 
court, mais aussi le plus gros. Les doigts du milieu, dont la longueur est considérable, rendent la fraction digitale plus longue que le reste de la main. Les phalanges unguéales sorıt généralement les plus courtes; celle du second doigt seulement esi plus longrie que les antres philanges de ce doigt, dont la deuxième est plus courte que la première. Les phalanges considérées individutllement sont aussi plus pelites que les os métacarpiens, à l'exception de la seconde phalange des truisième el quatrième doigls, qui nou seulement l'emporte de beaucoup en volume sur les autres phalanges, mais encore sur les os métacarpiens correspondans; ces deux phalanges sont surtout la cause de la longueur considérable de la main.

Chez les émydes, les os rles doigts sont beaucoup plus courts et plus gros, plus aplatis d'un côté à l'autre. Ceux du milieu sont encore ici les plus longs. Les phalanges unguéales sont un peu plus longues que les os métacarpiens et les deux autres phalanges, qui ont à peu près la même grandeur et sont un peu plus courtes que les os du métacarpe.

Les phalanges postérieures sont encore plus courtes et plus grosses chez les tortues; elles sont en mêne temps très-larges de deliors en dedans, de sorte qu'elles sont, dans ce sens même, plus grandes que dans le sens longitudinal. 


\section{D'ANATOMIE COMPARÉ.}

Lorsqu'il y en a deux, elles ont à peu près le mème volume. Les phalanges unguéales sont, au contraire, trois à quatre fois plus longues, très-fortes, aplaties d'un côté à l'autre, et se terminent en pointe. La longueur de ces dernières phalanges et l'absence des os du métacarpe font que les doigts des tortues sont, nonobstant la briéveté des premières phalanges, la fraction la plus grande de la main.

\section{Sauriens.}

$$
\text { S. } 211 .
$$

Les sauriens se distinguent sous le rapport de la disposition des doigts entre eux plus que les autres ordres de reptiles.

Chez les scinques, ils sont, aussi bien que la main en général, très-petits. Leur numbre est en mème temps considérablement réduit, comme, par exemple, dans les genres seps, chalcide; quelquefois il n'y en a qu'un. Les genres plus élevés ont, au contraire, cinq doigts d'une longueur considérable, qui sont plus grands que le reste de la main, et qui égalent au moins le volume du bras et de l'avant-bras.

Chez le seps tridactylus, je trouve le premier doigt formé de deux phalanges; les deux doigts externes en ont trois, alongées, très courtes, qui offrent entre elles a peu pres la mème lon- 
gueur. Ie doigt moyen est le plus long, l'interne le plus court.

Dans la plupart des genres plus élevés, le doigt du milieu est également le plus long; après lui vient le quatrième, puis le second, ensuite le cinquième, enfin le plus interne.

Chaque phalange isolée est en général beaucoup plus conrte que les os métacarpiens; mais ensemble elles sont considérablement plus longues que ceux-ci. Llles diffèrent, scus ce rapport, très peu los unes dos autres. Sonvent les postérieures sont plus courtes que les antérieures; les premières et la phalange unguéale sont, dans la plupart des cas, les plus longues. Cela s'applique surtout anx troisieme et quatrième doigts.

Ce scut les seuls repriles ou le nombre des phalanges des doights s'éléve au dessus de trois.

I.e pouce en a ordinairement deux, les second et cinquieme doigts en ont chacun trois. Ise troisieme, au contraire, a quatre phalanges, et le quatrieme en a cinq, ce qui est d'autaut plus digue de remarque, que le quatrième est ordinairement, si ce n'est généralement, plus court que le troisième.

La forme des phalanges est alongée, comprimée latéralenient. La phalange unguéale est, dans le plus grand nombre des cas, fortement recourbée, et se termine en avant par une pointe fort aiguë. 
1) ANATOMIE COMPARIE.

Le cuméléon diffire, d'une manière remarquable, de tous les autres reptiles, par la circonstance que ses trois doigts internes sont séparés des lenx externes, d'où résulte une pince, tandis que cliez les autres reptiles les doigts sont disposés dans une mème rangée, de dehors en dedans.

II. MFMBRES POSTÉRIEURS.

$$
\text { S. } 212 \text {. }
$$

Les membres postérieur's des reptiles sont toujours unis au rachis, notamment au sacrum, si ce riest chez le typhlops crocotatus qui fait exception à cette règle générale (I). L'état le plus incomplet est présenté par les typhlops, l'orvet fragile, les ophisaures et les chirotes. Ces reptiles ne possèdent que quelqus-uns des os pelviens. Cependant je ne commencerai pas la description par les ophidiens, mais par les batraciens, et cela pour les mèmes raisons que j’ai déjà exposées ci-dessus en traitant du membre antérieur. Chez tous les autres reptiles il s'est ajouté au bassin une série d'os qui constituent la base de la cuisse, de la jamle et du pied.

1. Os pelviens.

$$
\text { S. } 215 .
$$

L. bassin est Loujours composé de deux moi-

(1) Voyez la note pag. 658. 
tiés latérales qui se rejoiguent sur la ligne médiane, excepté chez les orvets, les ophisaures, les chirotes. Le nombre des pièces qui composent le bassin n'est pas toujours le mème. Hormis les genres mentionnés, il y en a, autant que je sache, toujours au moins dcux pièces, le plus souvent trois. L'os supérieur, articulé avec le sacrum, est l'os iléon (os ilium); l'antérieur et inférieur, est l'os du pubis (os pubis s. pectinis); le postérieur et inférieur, est l'ischion (os ischii). Lorsqu'il n'y a qu'un seul os inférieur, il représente les deux derniers. L'os supérieur et les os inférieurs, ou l'os inférieur, sont toujours unis entre eux pour former la cavité articulaire qui est destinée à recevoir le fémur.

L'os des îles est plus alongé; l'os inférieur ou les os inferieurs, quand il y en a deux, sont le plus souvent plats et larges. L'iléon n'est uni que lâchement, et d'une manière mobile, au moyen d'un ligament capsulaire, avec la face latérale du sacrum.

\section{A. BATRACIENS.}

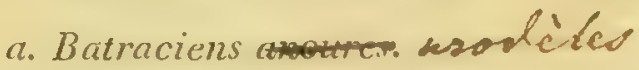

$$
\text { S. } 214 .
$$

Parmi les batraciens, ceux qui sont munis d'une queue n'ont que deux os de chaque côté, 
savoir : un petit os, alongé, dirigé d'arrière en avant, et de haut en bas, c'est l'os des iles; et un inférieur, quadrilatère, horizontal, trèslarge, qui sunit dans toute sa longueur avec l'os de mème nom, du côté opposé. Chez le protée, l’os des îles est très-petit, alongé, tandis quel'os qui représente l'ischion et le pubis, l'ischio-pubien, est très-développé, surtout dans le sens de sa longueur; le bord interne en est le plus long. Le milieu de son bord externe, qui est convexe, se rencontre avec l'os iléon, et forme avec lui la cavilé cotyloïde. Chez les autres batraciens urodèles, il est proportionnellement beaucoup plus large et plus court. Cette forme, plus alongée chez le protée, se rattache vraisemblablenuent à l'absence de l'us abdominal qui a éte décrit plus haut chez les salamandres. L'os iléon est osseux partout; l'ischio-pubien l'est seulement chez les salamandres; chez le protée, il est absolument cartilagineux. Chez les salamandres et les tritons, on voit un rapprochement du protée, en ce que la partie antérieure de cet os est cartilagineuse. Cette partie cartilagineuse est surtout considérahle chez les salamandres, où l'os inférieur est aussi plus alongé que chez les tritons. 
656 TRAITÉ GÉNERAT.

\section{b. Batraciens anoures.}

$$
5.215
$$

Chez les batraciens anoures, la disposition est extrèmement singulière. Les os des îles sont fort alongés, tranchans supérieurement, un peu plus épais en bas, là convexes, ici concaves; leur partic postérieure cst la plus grosse; clle se prolonge en une apophyse antéricure et inférieure, et en ure postérieure et supéricure. 1ls forment la partie moyenne, qui est la plus grande, de la cavité colyloïle, et sont soudés par leur bord postéricur, non-seulement avec l'os du pubis et l'ischion, mais mème entre eux. L'ischion est un os ayant la forme d'un carré alongé, pourru d'un bord inférieur convexe, situé sous la partie postérieure de l'articulation des ilćons, et qui se confond dans toute sa hauteur avec son congénère du còté opposé, de manière à ne former qu'un os. Le pubis est situé sous la parlie antérieure de l'articulation des iléons; il est aplati d'un cốé à l'autre, également soudé par sa face interne avec celui du còté opposé dans toute sa hauteur; il se recourbe en dehors, dans son milieu, pour former la partie antérienre, qui est la plus petite, de la cavité cotyloïde et s'articule supérieurement avec l'os tles îles, inférieurement avec l'ischion. 
Ces trois os se touchent partout, excepté dans la partie moyenne de la cavité cotyloïde, où l'on trouve une ouverture considérable, qui élablit une communication entre l'une de ces cavités et l'autre; ce qui semble être un indice de la séparation des os du pubis et de l'ischion, qui est constante chez les reptiles supérieurs, quoique ces trois os réunis forment une cavité cotyloïde, dont le plancher est exactement fermé.

La connexion antérieure des os du bassin n'est opérée ici que par les os des îles et les ischions; chezles grenouilles, ceux-ci n'y prennent part que d'une manière à peine sensible; ce ne sont pas en effet eux-mêmes, mais un petit os, alongé et arrondi, qui est situé entre eux et l'extrémité postérieure de l'articulation des iléons; chez les crapauds, et plus encore chez le pipa, les ischious forment, au contraire, toute la moitié postérieure de cette connexion.

L'os rlu pubis n'est qu'un os très-subordonné.

Tout le bassin des batraciens a par conséquent la forme d'un V, à branches très-longues, qui est fort apointi en bas et en arrière, auquel correspond la pièce coccygienne, unique et longue, située au-dessus.

Toutes les diverses pièces osseuses, qui occupent le même côlé du bassin, ne se soudent jamais entre elles. 
2. Ophidiens.

$$
\text { S. } 216 .
$$

Les ophidiens n'ont généralement aucune trace de bassin (1); il n'y en a des rudimens que dans les genres supérieurs de cel ordre, qui pourraient

(1) Suivant M. MAYRR, professenr à Bonn, l'ergot que l'on remarque des deux côtés de l'anus chez le boa constrictor et chez d'autres boas, est un véritable ongle dans la cavité duquel existe un petit os demi-cartilagineux, ou phalange unguéale, s'articulant avec un autre os, beanconp plus fort, qui est cachésousla peau. Ce second os est arrondi; son corps est recourbé et présente vers sa partie moyenne une apophyse relativement considérable; il s'articule, par un condyle épais, avec la phalange unguéale, et par une apophyse plus petite avec une autre piece du squelette; c'est l'os du mítutarse. La pièce avec laquelle cet os du mćtalarse s'articule par son condyle interne est beaucoup plus longue; la surface, an moyen de laquelle cette articulation a lieu, est encroûtée de cartilage et est en rapport avec deux apophyses, dont l'une, située en dehors, est parfaitement ossifiée; dont l'autre, interne, est en partie cartilagineuse: entre ces apophyses il en existe une troisième qui est absolument cartilagineuse; ce grand os se courbe en forme de sabre et se termine par un condyle cartilagineux. Cet os principal est le libia; les deux apophyses sont des os tarsiens. Des muscies distincts concourent à déterminer ces os.

Cette extrémité est indlépendante dubassin et siluée sous le muscle transverse de l'abdomer, entre ce muscle et le peritoine; il n'y a de libre que le tarse, le métatarse et la phalange. Les genres érix, pithon, clotonia, tortrix sont dans le même cas; it y a seulement plus de difficultés à apercevoir 


\section{D'ANATOMIE COMPAREE.}

ètre rangess, avec le mème droit, parmi les sauriens ( 1 ):

la partie libre du membre postérieur. Ces genres sont des pHÉxoponfs; d'autres ophidiens ont le membre postérieur caché sous la peau (les cryptopodes). L'orvet (anguis fragilis) et l'ophisaure (angutisvontralis) ont été seuls étudiés. Sous ce rapport M. Mayer ajoule à la description qui sera donnée plus loin, que l'os simple dont il fait mention est en rapport, au moyen de fibres ligamenteuses, avec l'apophyse transverse de la vertébre la plus proche de la dernière côte, qu'il présente une granulation cartilagineuse à son extrémité tournée du còté del'anus; il en conclut que ces parties sont analogues aux membres postérieurs du boa, et qu'elles ne sont pas un rudiment du bassin, mais bien un rudiment de ces membres.

Le genre typlilops est rapproché des genres précédens. Il en est de mème des amphisbènes; dans l'amplisbcena alba il existe un petit os blanc reconrbé, assez fort, situé sous la peau et entouré par le nuscle peaussier. Cet os est garni, en arrière vers l'anus, d'un rudiment cartilagineux, celui de l'ongle, mais qui est aussi caché sous la peau. Cet os est plus faible et coloré en brun chez l'amphisbcena fuliginosa. Longle moins distinct y existe.

Enfin les familles des CHONDROPODEs et aPODEs ne présentent au plus qu'un filament carlilaginea $x$ ou nulle trace de ce membre postérieur. La couleurre changeante (coluber pullatus) présente un filament cartilagineux, courbé, assez fort. Les espèces venimeuses ne le présentent pas. M. Maxfr observe que d'une manière générale on peut considérer l'existence d'un rapport inverse entre le développement de la queue et des membres postérieurs. (Voyez Annales des sciences naturelles, t. VII, P. 170. Voyez premier volume de cetle traduclion, 1). 488.)

(Noce des traducteurs.)

(ij Les naturalistes français placent, en effet, les ophisaures, les orvets, les chirotes parmi les sauriens. 
Il se présente sous dẹx formes. Ou il est un développement du rachis auquel il est alors uni, et c'est la disposition ordinaire, ou il se forme vis-à-vis de la colonne vertébrale, à la face abdominale.

La première forme est présentée par l'orvet (anguisfragilis). Le rudiment du membre postérieur consiste, de chaque côré, en un os unique, alongé, courbe, se terminant par une apophyse à la partie supérieure de son bord postérieur. Cet os est situé dans la rangée des autres côtes, et n’en diffère que parce qu'il est un peu plus volumineux que les côtes immédiatement placees au devant de lui, qu'il est plus gros et plus large que toutes les autres, et est loin d'atteindre son congénère du côté opposé.

Il correspond, en avant, à la clavicule et au scapulum, en arrière à l'os coxal des animaux supérieurs.

Les ophisaures et les chirotes offrent une disposition absolument semblable.

Les typhlops, du moins le $t$. crocotatus, nous offrent l'exemple de la seconde forme. On voit à la place des membres postérieurs, en avant de l'orifice anal, immédiatement sous la peau, deux os étroits, beaucoup plus petits en proportion du corps entier, dont l'antérieur est un peu plus long que le postérieur. Les deux os antérieurs des deux côtés se dirigent d'arrière en avant et de debors en dedans, et s'unissent 
entre eux à leur extrémité antérieure, sous un angle peu aigu; les postérieur's sont presque parallèles l'un à l'autre, et se portent directement d'avant en arrière.

Ces os sont entièrement séparés et même fort éloignés de la colonne vertébrale; celle-ci a'offre aucun indice de sacrum ou d'os coxal.

\section{Sauriens.}$$
\$ .217 .
$$

Les trois os pelvieus décrits existent constamment chez les sauriens, mais ils restent également séparés les uins des autres pendant toute la durée de la vie.

L'os des îles est en général le plus fort; il a une forme alongée, aplatie d'un côlé à l'autre, apointie d'avant en arrière; il dépasse, par son extrémité postérieure, le sacrum, auquel il s'attache, et descend un peu d'arrière en avant.

I'os du pubis a presque la même forme, mais il s'apointit en avant et en dedans, est convexe supérieurement, concave inférienrement. Son bord externe et antérieur donne naissance ordinairement ì une apophyse qui se dirige en avant, surtout chez le monitor élégant et le marbré (polychrus marmor.); elle est très-faible chez le premier, trés-longue, au contraire, chez le secoud. Lischion se porte plus en arrière et en 
dedans, et se déploie de dehors en derkns aussi bien que d'avant en arrière. Les os du pubis, aussi bien que les ischions, s'unisent eutre eux des deux côtés, sur la ligne médiane, dans toule leur hauteur; les sauriens ont par.conséquent une symphyse du pubis et une de l'ischion. Mais il existe entre ces deux symphyses une ouverture arrondie, considérable, cordiforme en avant; l'os pelvien inférieur, large et unique chez les salamandres, s'est par conséquent ici partagé en deux. Le genre seps est le seul, à ce que je sache, qui fasse exception à cette règle; la symphyse du pubis est complètement ferniće chez les individus de ce genre. Les ischions convergent en avant, mais sont distans l'un de l'autre d'une demi-ligne et sont beaucoup plus étroits que de coutume.

Chez les crocodiles, l’os des îles n'atteint pas l'os du pubis; mais l'ischion donue naissance à une apophyse qui s"intercale entre eux. Les os du pubis ne s'unissent pas non plus d'une manière immédiate; mais il y a entre eux, de chaque côté, un cartilage considérable, aplati, qui s'unit à celui du côté opposé, et se continue en avant ávec l'os abdominal.

Les symphyses du pubis et de l'ischion sont unies sourent par une substance ligamenteuse qui se dirige d'avant en arrière.

Ce même cartilage est peut-ètre un développement ultérieur de lapophyse du pubis des 
monilors et des marbrés (polychrus); ils sont vraisemblablement l'un et l'autre l'analogue, d'un cûté, du cartilage situé au devant des os du pubis des salamandres, de l'autre còté, de l'os marsupial des animaux didelphes.

\section{Chéloniens.}

\section{S. 218.}

Les os latéraux du bassin des chéloniens sont essentiellement formés d’après le type des sauriens; mais ils en diffèrent néanmoins sous le rapport de leurs position, forme et connexion.

Ils sont entourés de la partie postérieure de la carapace et du plastron, de la mème manière que les os de l'épaule le sont par la partie antérieure de ces boucliers. L'iléon descend verticalement, comme le scapulum, chez les tortues et les émydes: dans les chélonées il descend en avanı. L'os du pubis et de l'ischion ont, au contraire, une position horizontale, et s'unissent dans la cavité cotylö̈de, sous un augle droit, avec l'iléon.

Les os des iles des tortues et des émydes sont des os trés-longs, tout-à-fait cyliudriques, un peu couvexes en dehors, concaves en dedans; chez les chélonées, ils sont, au contraire, courts et épais; chez celles - ci proportionnellement plus courts, chcoz celles-là beaucoup plus longs yue 
chez les autres reptiles; ils ressemblent d'une manière frappante aux omoplates.

Les os du pubis et de l'ischion sont tous deux très-plats et larges; ils offrent les uns et les autres une forte tubérosité, les premiers en arrière, les seconds en avant. La tubérosité ischiadique ne manque que chez les chélonées. Les os du pub is sont, dans toutes les dimensions, beaucoup plus développés que les os de l'ischion, surtout chez les chélonées, oủ les derniers sont très-petits.

C'est chez les tortues que les ischions offrent le plus de largeur, et que les tubérosités du pubis sont les plus longues et les plus étroites. ('hez elles et les énydes, la partie interne des os du pubis se prolonge en avant en une pointe considérable, qui est la plus forte chez les émydes, et est remplacée chez les chélonées par un cartilage court et large. Cette pointe est située en général dans le plan des autres os; mais chez le matamata elle est dirigée d'arrière en àvant, tandis que l'os principal descend verticalement de haut en bas.

Les os des îles s'unissent avec les deux vertèbres sacrées et les deux dernières vertèbres lombaires, de la manière accoutumée, savoir : par une articulation mobile en général, qui a été considérée, à tort, comme une particularité propre à cet ordre de reptiles (1). Il y a une

(1) Cuvier. Leçons d'Anat. comp., vol. I, p. 38/4. 
exception offerte par le matamata, dont les os iléons très-courts, larges et épais, sont soudés d'une manière solide, par de la substance fibrocartilagineuse, avec la face interne des deux dernières còtes.

La syinphyse du pubis et celle de l'ischion existent constamment. Un voita la derriière une forte crète longitudinale faire saillie en bas, et qui est surtout développée chez les émydes et les tortues. Ces deux genres possèdent, en outre, une symphyse ischio-pubienne qui manque aux chélonées; en effet, dans ces genres, les deux os du pubis et de l'ischion sont unis entre eux solidement dans leur partie moyenne. Cette dis. position n'a pas lieu chez les chélanées, pas plus chez le caret (ch. imuricata), que chez les tortues franches (ch. mydas); il n'existe dans l'ou: verture, entre les os du pubis et de l'ischion, qu'une substance cartilagineuse, mince, qui occupe, chez les autres, les deux ouvertures séparées l'une de l'autre par le pont osseux.

On voit par là que les chéloniens présentent, pour la plupart, à l'état parfait, la réunion de l'os du pubis et de l'ischion, qui était déjà indiquée chez les sauriens; et la conformation des chéloniens peut être envisagée comme intermédiaire à celle des batraciens et des sauriens.

Les os pelviens sont partout séparés du sternum, excepté chez le malamala.

Dans ce dernier, $1^{\circ}$ la lubćrosité ischia- 
dique s'unit solidement avec le bord postérieur de la dernière pièce sternale, sans que cela ait lieu, du moins dans le jeune âge, pour la symphyse ischiadique, car il existe entre elle et le steruum un petit intervalle; $2^{\circ}$ la tubérosité externe du pubis s'applique, en avant de la tu. bérosité ischiadique, contre le bord externe de l'avant-dernière pièce sternale. Dans ce dernier cas, la symphyse du pubis proprement dite est, du reste; a aussi éloignée du sternum qu'on le rencontre ordinairement; il existe, outre la tubérosilé externe du pubis qui est tournée en bas, la unbérositè interne et horizontale dont il a déjà été question, qui est dirigée en avant. Celle-ci se réunit, comme cela a lieu communément, à quel. que distance au dessus du sternum, dans la ligne médiane, avec la tubérosité congénère du còté opposé, 'tandis que les ischions s'unissent'sur un point, entre eux et aveo le sternum. Cette disposition est très-digne de remarque; en ce qu'elle augmente considérablement l'analogie qui existe ęntre les os du męribre antérieur et céux du postérieur, et entre ces os et kes còtes.

\section{Fémur.}

\section{S. 219.}

- Le fémur est toujours uin os alongé, terminé supérieurement par une téle ronde, inférieurement par une extrémité large el transversale, 


\section{D'ANATOMIE COMPARÉ.}

s'unissant d'une manière mobile, à l'aide d'une capsule, en haut avec les os des îles, en bas avec les os de la jambe.

$$
\text { S. } 220 \text {. }
$$

Parmi les batraciens urodèles, le protée a un fémur très-long et étroit, cylindrique, droit et simple, à l'exception d'une petite apophyse si. tuée à sa face interne, immédiatement au rlessous de la tête. Cet os est plus court chez les sialumandres, où la tète est unie an corps de l'os, ì l'aide d'un col qui a la mème directión que la tète. La moilié supérieure de l'os estmunie, surtout chez les tritorns, d'une crète inférietire, träichante, qui le rend considérablement plus large d'avant en àrrière; la moitié inférieure est fort large dans la direction opposée. Ge qui frappe chez les salamzondres, c'est sirtotit la grossein considérable de la' itêtè.

La face articulaire inférieure est plane:

Le fémur des batraciens anoures ést beancoup" plus long, très-arrondi, sans crète, il èst concave' en avant et convexe en arrière à sa partie supét rieure; à l'inférieure il est recourbé dans la direction opposée; supérieuremént il se termine par une têle ronde, inférieuremént par dèx enfoncemens latéraux, peu profonds.

Les balraciens urodeles nont ni ligameus propres, ni cartilages intermédiaires dans l'articula- 
tion de la hanche et du genou; les anoures ont, au contraire, dans celle du genou, deux ligamens croisés, intérieurs et larges, qui s'étendent de l'os de la cuisse à l'os de la jambe, et en outre deux anneaux tendineux, situés plus près de la circonférence entre les deux os, et qui sont un indice des cartilages falciformes que présentent les sauriens.

Le fémur des sauriens est conforméde la même manière, à cela près que la tête en est moins arrondie, mais beaucoup plus large de dedans en dehors que d'avant en arrière. Cela est surtout distinct chez les crocodiles. Les sauriens ont une apophyse ou poulie inférieure; ils ont aussi, ainsi que les iguanes, plusieurs ligamens fibrocartilagineux dans l'intérieur de l'articulation coxo-fémorale. L'extrémité inférieure de l'os est légèrement concave, au milieu, dans la direction de la face à laquelle s'attachent les muscles extenseurs à celle des muscles fléchisseurs.

Dans l'articulation du genou, il existe deux forts ligamens qui se croisent et deux fibrocartilages annulaires, correspondant aux deux apophyses, et se réunissant au milieu de l'articulation, qui sont unis à leur pourtour avec le ligament capsulaire; ces fibro-cartilages ont leur plus grande minceur au centre, où ils sont même percés.

Chez les chéloniens, la tête du fémur est en proportion beaucoup plus volumineuse et unie 


$$
\text { I'ANATOMYY COMPARÉE. }
$$

aे la diaphyse sous un angle droit. Au point de réunion il s'ajoute, chez les émydes, de chaque còté, deux fortes saillies dirigées en arrière, les trochanters supérieur et inférieur, qui, séparés ici par une fosse profonde, forment, chez les autres chéloniens, une éminence unique qui est considérable.

Chez les tortues et les émydes, le fémur est, dans toute sa longueur, fortement recourbé en avant; chez les chélonées il est plus droit.

Il n'y a point de ligament intérieur dans l'articulation coxo-fémorale.

Dans l'articulation du genou je ne trouve, du moins chez la tortue grecque, qu'un cartilage articulaire falciforme, externe, très-petit et mince, avec lequel est confondu le rudiment, simple et postérieur, des ligamens croisés, qui s'insère à la fosse inter-trochantérienne du fémur.

3. Os de la jambe.

$$
\text { \$. } 221 .
$$

La jambe des reptiles, les batraciens anoure excepiés, se compose du libia et du péroné, qui sont entièrement séparés l'un de l'autre et s'articulent l'un à côté de l'autre, à la même hauteur, avec le fémur. Ils ont ordinairement la mème longueur, et sont plus ou moins renflés à leurs exırémités supérieure et inférieure. L'ex- 
trémité supérieure du tibia et l'inférieure du péroné ont cornmunément le plus d'épaisseur. Le tibia est, pour la plupart, un peu plus gros que le péroné; le premier do ces os correspond au côté du gros orteil, c'est-ád-dire à la partie interne du pied, le second au petit orteil, ou à la partie externe du pied. Chez lessalanandres, le péroné est plus fort que le tibia.

Chez ces dernières et les chéloniens, surtout les chéloncés, il y a moins de différence sous le rapport de l'épaisseur des deux os de la jambe. Cette différence est beaucoup plus considérable chez les sauri:ns. Ces os sont en général arrondis, cylindriquies, droits; chezles tritons ils sont, au contraire, plats.

Chez les cuméléons, le péroné est fortement recourbé en dehors, surtout daus sa partie inférieure.

Parmi tous les reptiles, cette portion du membre postérieur est proportiomellement la pius courte chez les batracions anoures, et après eux chez les chélonées; elle est la plus longue chez la plupart des sauriens, surlout les calotes, et chez les balraciens anoures. Les extrémilés supéricure et inféricure sont en général des surfaces: plates, simples.

$$
\text { \$. } 222 .
$$

Les batiaciens anoures nont quiu os à la 
jambe; cet os est le plus long, le plus arrondi et le plus étroit chez les grenouilles, le plus court et le plus large chez le pipa. Il présente déjà exlérieurement, comme celui de l'avant-bras, à ses extrémités supérieure et inférieure, une tendauce à se diviser en tibia el en péroné; on voit cela non-seulement par son extension en largeur, mais encore par un sillon longitudinal profoul, qui est creusé en haut et en bas à ses faces externe el interne. Celte tendance est encore bien plus marquée à l'intérieur; sa cavité médullaire est pariagée supérieurement et iuférieurement, jusque vers le milieu, en deux moitiés, par une cloison qui part des sillons mentionnés, et se compose dans sa partie terminale, qui est la plus grande, de deux lames adossées l'une à l'autre.

Cette tendance à la division est la plus faible chez les grenouilles proprement dites, parce que chez elles l'os de la jambe est le plus étroit, surtout à sa partie moyenne, que les sillons et les cloisons correspondantes manquent dans le tiers tnoyen de l'os, et que la cavité médullaire est par conséquent simple; celte tendance est, au contraire, prononcée au dernier degré chez le pipa, où l'os est aussi très-large dans sa partie moyenne, et que les sillons, quoique plus superficiels dans ce genre, se continuent néanmoius dans toute la longuérir de l'os. Chez les crapauds et les grenouilles, surtout les deruieres, 
il existe, à peu près vers la partie moyenne de l'os, un trou qui pénètre de la face externe jusqu'à l'interne, et au milieu une cloison transversale qui partage réellement l'os de la jambe en une moitié supérieure et une inférieure. L'extrémité inférieure de l'os se termine par une surface articulaire, légèrement concave de dehors en dedans et d'avant en arrière. L'ouverture moyenne manque chez le pipa, la cloison se porte plus vers le milieu, où l'os n'est pas solide mais contient une cavité simple.

\section{S. 223 .}

Jamais il ne s'ajoute à ces os un troisième os, ou une rotule.

4. Pied.

$$
\text { S. } 224 \text {. }
$$

La disposition du pied varie beaucoup, $\tan t$ sous le rapport de sa longueur, relative aux autres portions du membre postérieur, à la main, et aux différentes fractions dont il est composé, que sous le rapport du nombre et de la forme des parties qui se succèdent de dehors en dedans.

Le pied le plus grand, considéré relativement aux autres portions du membre postérieur, se 
D'ANATOMIE COMPARÉ.

rencontre chez les sulamand!es, les sauriens en scimiral, par exemple, chez les agames, plus cucore chez les iguanes, puis chez quelques lialraciens, principalement le pipa, les crapiruds, et chez les chélonées; il est plus petit chez les grenouilles, les émydes, et au minimum le développement chez les tortues.

Sun rapport avec la main est très-varié. Chez une partie des batraciens, lẹs émydes, les sauriens. le pied est considérablement plus grand que la main; chez les tortues, ces deux parties ont ì peu près le mème volume; le pied des chélorées, entièrement constitué d'après le type des poissons et conformément à leur mode de locomotion, est beaucoup plus petit.

Le rapport des portions du pied entre elles offre également des différences considérables. Chez les salamandres, les crapauds, les grenouilles, parmi les batraciens, chez la majeure partie des sauriens et dans tous les chéloniens, la fraction digitale est la plus longue; tous les orteils, ou du moins quelques-uns, surpassent considérablement les autres parties sous le rapport de la longueur. Le pipa fait exception; chez lui le métatarse forme la fraction la plus longue du pied, et le métacarpe est aussi la fraction la plus longue de:sa main.

() uant au nombre des parlies qui composenl le pied de dedruns en drohors, on de la face tibiale ¿l la fice péronćale, on distingue surtout les os 
du métatarse et des orteils. La disposition la plus générale est la présence de cinq os métatarsiens et de ciug orteils, comme chez la plupart des batraciens, chez les émydes et les chélonées, parmi les chéloniens, et chez la plupart des sauriens.

On trouve des exceptions à cette règle, parmi lesbatraciens, chez le protée, qui n'a que deux os métatarsiens, et chez le plus grand nombre des batraciens anoures, qui possèdent à la partie interne du premier orteil, qui est parfait, un rudiment de sixième orteil, extrèmement petit, et appliqué sur le premier os métatarsien, qui n'est lui-même pas considérable. On rencontre encore une autre exception, parmi les chéloniens, chez les tortues, du moins la tortue grecque, parmi les sauriens, chez les crocodiles, et surtont les chirotes, qui sont si peu développés; il n'existe chez tous ces reptiles que quatre os métatarsiens et quatre orteils.

\section{S. 225.}

Les principales conditions que puisse déter. miner lanatomie sont: la forme des fractions qui se succèdent d'avant en arrière, en général et en particulier, et le nombre des os qui composent ces fractions. 
1. Tarse.

\$. 226 .

Le tarse est formé très-généralement, comme la main, de plusieurs os courts, arrondis, disposés cominunément sur deux rangées qui se succèdent d'avant en arrière.

a. Batraciens.

S. 227

Tous les batraciens ont un tarse considérable.

Les batraciens urodèles s'accordent entre eux par la largeur proportionnelle du tarse, et sa composition de plusieurs os courts et plats; mais ils diffèrent aussi les uns des autres sous plusieurs rapports.

Il est étroit et formé de deux rangées chez le protée. Il y a deux os dans la rangée supérieure; ces os sont alongés et les plus volumineux; l'un correspond au tihia, l'autre au péroné. Les tritons ont, dans trois rangées, huit os assez semblables sous le rapport du volume. et qui ont la forme d'un carré arrondi. Les trois supérieurs touchent aux deux os de la jambe; l'anlérieur touche aussi à l'os antérieur de la troisirrnce rangée. Lá seconde rangée est formée de deux us, la troisième de trois, parmi lesquels 
celui du milieu supporte l'os métatarsien moyen, chacum des deux autres supportant deux os du mélalarse. Chez les salamandres, Los de la rangée antérieure, qui correspond au péroné, est partagé en deux.

Il est digne de remarque que tous ces os sont 'complètement ossifiés chez les trilons, tandis que chez les salamandres, du moins le s. maculata, et chez le protée, ils sout à l'état cartilagineux, tous les autres os du pied élant du reste distinctement ossifiés.

Le tarse offre des différences considérables chez les batraciens anoures. Chez tous, il semble entièrement formé d'après le type des os de la jambe de beaucoup d'animaux, savoir: de deux us superposés, très-longs, planes ou convexes en dehors, fortement concaves en dedans, qui sont unis entre eux, à leurs extrémités, d'une manière plus on moins solide suivant l'âge des individus; mais cette identité de type n'existe réel. lement que dans sa majeure partie. On trouve, chez le pipa, à l'eudroit où ces os s'articulent avec l'os de la jambe, à la face postérieure, dans le tendon du muscle gastrocnémien, un os considérable, alongé, ressemblant à une rotule, et dont je ne découvre awcune trace chez les autres genres de cet ordre.

MM. Cuvier et Schneider considèrent les deux os les plus postérieurs du pied, qui sont longs et constans, comme des os métatarsieus, 


$$
\text { D'ANATOMIE COMPARÉE. }
$$

et le dernier surtout comme l'astragale et le calcanéum, qui se seraient confondus et alongés. MI. Rudolphi (1), au contraire, les prend pour un tibia et un péroné accessoires; mais il pense aussi que l'on pourrait les considérer comme un astragale, qui serait double, tandis que l'os postérieur ou les deux os postérieurs, qui existent chez le pipa, pourraient être envisagés comme un calcanéum également double.

La première vue de M. Rudolphi, fondée uniquement sur la ressemblance extérieure, est d'autant moins admissible qu'il existe, chez ces batraciens, une jambe très-distincte, et que les os du tarse et du métacarpe des oiseaux sont formés d'après un type tout-à-fait semblable.

L'os postérieur ou les deux os postérieurs, ne sont peut-être que des os accessoires, de grands os sésamoüdes; je crois cependant plus vraisemblable qu'ils représentent la partie postérieure du calcanéum, qui, même chez l'homme, a son point d'ossification propre, mais se confond avec le reste de l'os.

Des deux os longs, l'interne est l'astragale, l'externe le calcanéum, qui, conformément à la largeur considérable de l'os de la jambe, sont juxtaposés, et dont le premier s'articule aussi, chez les chélonées, aveć le tibia, le dernier avec le péroné.

(1) Obs, de raná pipá, p. 11 . 
Les autres os sont beaucoup plus petits. Ils sont toujours situés au devant de l'os tibial de la grande rangée postérieure, tandis que celle. ci est immédiatement contiguë, par l'os péronéal, aux quatrième et cinquième os métatarsiens.

La disposition n'en est pas exactement lat même partout.

Chez les crapauds, ils sont réduits à l'état de petitesse et d'imperfection le plus extrême; chez le bufo agua, une partie d'entre eux est toutà-fait cartilagineuse.

L'os externe est plat et plane chez les crapauds et les grenouilles, étroit chez le pipa; il a sa plus grande longueur de la face dorsale à la face plantaire, est fort concave en haut, convexc en bas. Il correspond aux second et troisième os métatarsiens.

Celui qui vient aprèsce dernier est beaucoup plus petit.

Le troisième, situé derrière le premier os du métatarse, est le plus considérable chez les crapauds, tandis que chez le pipa et les grenouilles il est le moyen pour la grosseur.

On trouve ensuite un os arrondi, plus petit, faisant saillie au bord tibial; il supporie un petit os, arrondi chez le pipa et les crapauds, alongé et apointi chez les grenouilles, qui se remarque déjà extérieurement, sous la forme d'une saillie au bord interne du pied, et que je regarde 


$$
\text { n'ANATOMIE COMPARÉE. }
$$

comme un rudiment de sixième orteil, auquel appartiendrait alors peut-être le quatrième os, qui, dans ce cas, ne serait plus un os tarsien, mais ou un métatarsien ou la première phalange de cet orteil surnuméraire.

Le pipa se distingue des autres bartaciens anoures d'une manière digne de remarque, non-seulement par l'os tarsien le plus supérieur qui a déjà été mentionné, mais en outre, $1{ }^{\circ}$ par la briéveté et la grosseur des deux grands os supérieurs; $2^{\circ}$ par le volume et l'ossification parfaite des os antérieurs; $3^{\circ}$ par la présence de deux autres os, de sorte qu'il y a six os dans le carpe. Un de ces os, celui qui est beaucoup plus considérable que l'autre, est situé derrière l'extrémité supérieure des quatrième et cinquième os métatarsiens, et au dessous de l'extrémité de l'os de la rangée supérieure qui correspond au péroné, mais de manière à ce qu'il n'empêche pas le contact de cet os avec les os métatarsiens. Le second os, étroit et alongé, se trouve derrière le troisième de la rangée antérieure au bord tibial.

Par cette disposition encore le pipa se rapproche des chéloniens.

\section{b. Chéloniens.}

$$
\text { S. } 228 \text {. }
$$

Chez celly-ci, le nombre des os du tarse est 
loujours considérable; il y en a six chez les tortues (t. grecque) et la tortue d'eau douce d'Eu(e. europra), et sept chez les chélonées (ch. mydas ).

Ils sont partout disposés sur deux rangées.

Chez les tortues et les émydes, la première rangée ne consiste qu'en un os, qui est de tous le plus grand; chez les premières, il surpasse même en volume tous les autres réunis; chez les chélonées, il est en proportion plus petit et divisé en deux, de sorte que chez elles lis première rangée est formée de deux os, dont le péronéal est beaucoup plus petit, et correspond a la moitié externe de l'extrémité inférieure du péroné.

La rangée antérieure contient partout cinq os, dont chacun supporte un os metatarsien; une partie du cinquième correspond aussi au quatrième os du métatarse. Chez les tortues, dont le cinquième orteil est rudimentaire, le cinquième os du tarse correspond, en outre, presque enlièrément au quarrième os mélatarsien, et le quatrième os tarsien est presque tout-à-fait rcfoulé en arrière.

Le second et le troisieme sont toujours les plus petits; après eux vient le plus interne. Chez. les chélonées, c'est le cinquième; chez les autres le quatrième, qui est le phus grand.

Tous sont courts et épais che\% les torlues, minces et plats chez les chélonies. Les imyales 


$$
\text { 1) ANATOME COMPARE. }
$$

681 Lienuent encore le milieu eu cela. Chez les chélonée's, le cinquième dépasse de beaucoup tous les atutres, et est situé tout-á-fait transversalement; il élargit ainsi le pied en manière de nageoire.

Le tarse des chélonées est proportionnellement le plus petit; celui de la tortue grecque est le plus grand.

\section{c. Sauriens.}

$$
\text { S. } 229 \text {. }
$$

I.es sauriens ont, d'après mes recherches, moins d'os au tarse que les autres reptiles.

Ces os sont disposés sur deux rangées.

La premiére se compose d'un os ou de deux qui sont superposés; l'un recevant le tibia, l’autre le péroné.

Je n'en trouve qu'un chez le monitor élégant, deux chez les iguanes et les crocodiles.

Liantérieur de ces os, ou sa partie tibiale, lorsqu'il u'y en a qu'un, supporte le premier os du métatarse.

Il $\mathrm{y}$ a trois os dans la rangée antérieure chez le monitor, seulement deux chez les crocodiles et les iguanes; ils correspondent aux deux ou trois os métatarsiens externes. Chez les crocodiles et les iguanes, ils ont à peu près le même volume; chez le monilor, au contraire, ils diminuent considérablement de volume de la face pérouciale à la faco tibiale. 
Le plus externe de ces os, chez le monilor, est surtout grand, plat, long en avant, se prolongeant en une apophyse articulaire arrondie, et a beaucoup de ressemblance avec l'os tarsien le plus externe des chélonées.

\section{Métatarse.}

\section{S. 230.}

Les os métatarsiens sont plus longs que les os du tarse chez les batraciens urodèles et le pipa, ainsi que chez les chélonées et les sauriens; chez les batraciens anoures ils sont, au contraire, plus courts, en outre beaucoup plus étroits que ces mêmes os, et deviennent plus ou moins alongés. Leur longueur respective diminue dans les différens ordres et genres, dans l'ordre suivant :

Salamandres et tritons, $3 \mathrm{e}, 2^{\mathrm{e}}, 4^{\mathrm{e}}, 1^{\mathrm{cr}}, 5^{\mathrm{e}} \mathrm{os}$.

Grenouilles, $4^{\mathrm{e}}, 3^{\mathrm{e}}, 5^{\mathrm{e}}, 2^{\mathrm{e}}, \mathrm{I}^{\mathrm{er}}$.

Crapauds, $4^{\mathrm{e}}, 5^{\mathrm{e}}, 5^{\mathrm{e}}, 2^{\mathrm{e}}, \mathrm{I}^{\mathrm{er}}$.

Pipa, $2^{\mathrm{e}}$ et $4^{\mathrm{e}}$ us égaux; $3^{\mathrm{e}} ; 1^{\mathrm{er}}$ et $5^{\mathrm{e}}$ égaux.

Emydes, 3e, $4^{\mathrm{e}}, 2^{\mathrm{e}}, 1^{\mathrm{er}}, 5^{\mathrm{e}}$.

Tortues, $3^{\mathrm{e}}, 2^{\mathrm{c}}, 4^{\mathrm{e}}, 1^{\mathrm{er}}$.

Chélonées, $3^{\mathrm{e}}, 4^{\mathrm{e}}, \mathrm{F}^{\mathrm{e}}, 5^{\mathrm{e}}, 1^{\mathrm{er}}$.

Iguana delic., $4^{\mathrm{c}}, 3^{\mathrm{e}}, 2^{\mathrm{e}}, 1^{\mathrm{er}}, 5^{\mathrm{e}}$.

Polychrus marm., $3^{\mathrm{e}}, 4^{\mathrm{e}}, 2^{\mathrm{e}}, 5^{\mathrm{e}}, \mathrm{1}^{\mathrm{er}}$.

Crocodilus sclerops, $3^{\mathrm{c}}, 2^{\mathrm{c}}, 4^{\mathrm{e}}, \mathrm{I}^{\mathrm{er}}, 5^{\mathrm{c}}$.

Calotes vulg. $3^{\mathrm{e}}, 2^{\mathrm{e}}, 4^{\mathrm{e}}, 1^{\mathrm{er}}, 5^{\mathrm{e}}$. 
1) ANATOMIE COMPARÉE.

Agama asperce, $3^{\mathrm{e}}, 4^{\mathrm{c}}, 2^{\mathrm{e}}, 1^{\mathrm{cr}}, 5^{\mathrm{c}}$.

Lacerta ocellala, $4^{\mathrm{c}}, 3 \mathrm{e}, 2^{\mathrm{e}}, 1^{\mathrm{er}}, 5 \mathrm{c}$.

Monitor elegans, $3^{\mathrm{c}}, 4^{\mathrm{e}}, 2^{\mathrm{e}}, \mathbf{1}^{\mathrm{er}}, 5^{\mathrm{e}}$.

Scincus ocellatus, $3^{\mathrm{e}}, 4^{\mathrm{e}}, 2^{\mathrm{e}}, 5 \mathrm{e}, 1^{\mathrm{cr}}$.

Gecko fuscicularis, $3^{\mathrm{e}}, 2^{\mathrm{e}}, 5^{\mathrm{c}}, 4^{\mathrm{e}}, \mathrm{1}^{\mathrm{e}}$.

La différence de longueur est peu de chose chez le pipa; chez les grenouilles, les crapauls, les tortues, les os métatarsiens des quatre doigts externes se suivent à peu prés sous le rapport du volume; celui du gros orteil est, au contraire, tout à coup considérablement plus court quie les autres.

Cette fraction du pied manque également tout-à-fait chez la tortue grecque, comme je m’en suis assuré par des recherches exactes; elle est, au contraire, très-considérable chez les émydes et les chélonées, particulièrement chez celles-ci.

Les os du métatarse sont, pour la plupart, alongés et minces; chez les chélonées, le premier, qui appartient au gros orteil, est à la vérité plus court que les trois suivans, mais très-large et plat, de sorte qu'il ressemble beaucoup à l'os le plus externe du tarse, dont il diffère cependant par sa direction. Le cinquième est à peu près aussi long que le premier; et il est aussi plus court, plus large et plus plat que les autres, mais pas à beaucoup près autant que le premier.

Ils sout fort alongés chez less sauriens. 


\section{Orteils.}

$$
\text { S. } 23 \mathrm{I}
$$

Les orleils offrent sous le rapport du nombre, du volume et de la forme, à peu près les mèmes différences que les doigts.

Ils sont souvent plus nombreux que ces derniers; le protée fait exception en cela, il n'a que deux orteils. Chez les chéloniens et les sauriens, le nombre des doigts et des orteils est le plus souvent le même; mais le cinquième orteil manque chez les tortues, et se montre aussi fort rabougri chez les émydes.

Parmi les batraciens urodèles, le protée n'a que deux phalanges, à peu près d'égale longueur aux deux orteils.

Chez les tritons et les salamandres, les deuxième et cinquieme orteils sont composés de deux phalanges; le troisième et le quatrième, chacun de quatre.

Parmi les batraciens anoures, les premier et deuxième orteils parfiits du pipa, des grenouilles, des raines, sont formés de deux phalanges; les troisième et cinquième de trois; le quatrième de quatre phalanges, qui diminuent de volume de la première à la łernière. Je ne connais aucune exception à cette règle. Le ru- 
1) ANATOMIE COMPARÉE.

685

diment lorteil, le plus interne, est seulement unique.

Le quatrième orteil est généralement le plus long, ce qui s'accorde avec son nombre plus considerable de phalanges. Après lui viennent le troisième te le cinquième, puis le second, enfin le premier.

Chez le pipa, le troisième orteil est cependant le plus long.

Chez les chéloniens le premier orteil, et aussi le cinquième, s'il exisı, sont beancoup plus courts que les autres. Le nombre de leurs phalanges n'excède jamais trois, et n'est pas inférieur à deux.

Chez les chélonées et les émydes, les phalanges diminuent de volume, à partir du métatarse; chez les tortues, au contraire, elles aug-. mentent, surlout la phalange unguéale.

Les chélonées ont, sous tous les rapports, les orteils les plus parfaits. Les quatre externes sont de beaucoup plus longs que l'interne, et sont formés de trois phalanges, qui diminnent plus ou moins de longueur, à partir du métatarse. Il riy a que la seconde phalange de l'orteil moyen qui soit considérablement plus longue. que les autres. Le premier orteil n'a que deux phalanges. Toutes sont fort alongées et plates.

Elles sont plus longues, plus épaisses, plus arrondies et comprimées latéralement chez les érrycles. J.e premier et le second orteil se com_ 
posent chacun seulement de deux phalanges.

Chez les tortues, elles sont très-courtes, larges et épaisses, à tel point que quelquesunes sont même plus larges que longues. La phalange unguénale fait exception; e!le est longue.

I.es phalanges des sauriens, sont alongées et comprimées latéralement; ensemble elles sont beaucoup plus longues que le métatarse, mais chacune en particulier est plus courte. Elles suivent, pour la plupart, sous le rapport de la longueur, l'ordre suivant: le $4^{\mathrm{e}}, 3^{\mathrm{e}}, 2^{\mathrm{e}}, 5^{\mathrm{e}}$, et le $\mathrm{i}^{\mathrm{cr}}$. Chez les crocodiles, ce rapport est ainsi : le $5^{\mathrm{e}}, 2^{\mathrm{e}}, 4^{\mathrm{e}}, 1^{\mathrm{e}} \mathrm{r}$; chez les agames, $4^{\mathrm{e}}, 5^{\mathrm{e}}, 2^{\mathrm{e}}, 1^{\mathrm{er}}, 5^{\mathrm{e}}$.

Les deux externes sont ordinairement beaucoup plus courtes que les autres.

Les premières phalanges sont généralement les plus longues.

Le quatrième orteil a communément ciny phalanges, le troisième quatre, le second et le cinquième trois, le premier deux.

C. OS DE LA TETE.

$$
\text { S. } 232 \text {. }
$$

les conditions générales et particulières que présente la tête osseuse des reptiles, considérée dans son ensemble, seront exposées après avoir fait l'histoire des os particuliers qui la composent, et que nous allons étudier en détail, 
comme nous avons fait pour les poissons, dans les différens ordres de la classe des reptiles.

\section{BATRACIENS.}

1. Os basilaire.

$$
\text { S. } 233 \text {. }
$$

L'os basilaire est toujours formé, chez les batraciens, de plusieurs pièces qui se succèdent d'arrière en avant, et de dedans en dehors.

On trouve généralement dans ces animaux : I" une pièce inférieure, considérable, alongée, mince et plate, plus ou moins apointie en avant; c'est le corps qui est l'un des plus grands os de la tète, et même communément le plus grand;

$2^{\circ}$ Une ou deux paires de pièces latérales, une postérienre et une antérieure, dont une ou toutes deux sont assises sur le bord latéral du corps, et dont la postérieure représente la pièce latérale et supérieure de l'occipital, l'antérieure représentant la pièce latérale du sphénoïde.

Chez les batraciens urodèles, le corps de l'os est p'roportionnellement le plus volumineux; il diminue uniformément de largeur et d'épaisseur, d'arrière en avant.

Il est le plus considérable chez le protée, ou il se montre même plus compliqué que chez tous les autres batraciens; il supporte, en effet, 
a son bord postérieur, comme il a déjà été dit (1), un anneau dirigé en haut et en avant, vraisemblablement l'atlas qui s'est soudé à lui.

Chez les batraciens anoures, il est plus petit, mais il est beaucoup plus large dans sa parlie postérieure, et beaucoup plus étroit dans l'antérieure. Il consiste, chez les grenouilles, en une branche longitudinale postérieure, située transversalement, et en une antérieure. moyenne, qui à son milieu déborde la première, sous la forme d'une petite saillie dirigée en arrière, de sorte que cel os ressemble a une croix. Cibez le pipa, la partie postérieure est une plaque large et arrondie.

Il est digne de remarque que, dans ce genre, le corps de l'os basilaire, unique sans cela, est partagé en une partie antérieure et en une poslérieure, cunéiforme, beaucoup plus petie, siluée entre les deux parties articulaires ou condyloïdienne, et qui esı peut-être déjà indiquiè par la pointe postérieure que présentent les gyenouilles; il en résulte que, chez le pipa, le corps de la portion occipitale est séparé de celui de la portion sphénoïdale.

Le corps simple de l'os basilaire ne s'étend pas jusqu'à l'extrémilé postérieure du crâne, si ce n'est chez le protée, où il présente postérieurement deux facettes articulaires, alongées,

(1) Voycz page 5,46 
D'ANATOME COMPARÉE.

transversales, peu élevées, pour s'articuler avec la première vertèbre cervicale libre.

Les paires de pièces latérales et postérieures, ou les pièces condyloïdiennes qui correspondent à l'arc de la vertèbre, sont pour la plupart des os simples, courts, quadrilatères, convexes ì la face externe, concaves à l'interne, qui sont appliqués en arrière sur le corps de l'os basilaire. Ils se rencontrent en dedans sur la ligne médiane, et s'atteignent même inférieurement dans la mème ligne moyenne, excepté chez le protée et le pipa, chez lesquels ils sont séparés les uns des autres par le corps de l'occipital.

Chez le pipa, ils sont beaucoup plus grands que chez les autres batraciens; ils sont en effet les plus grands os de la tête chez le pipa; dans ce genre une lame transversale, longue, a été surajoutée à la partie qui existe constamment.

Chez les salamandres, ils se soudent au rorher, de manière à ne former qu'un os.

Les condyles arrondis se voient partout à leur partie postérieure et inférieure, si ce n'est chez le protée, par la raison exposée ci dessus.

Les paires de pièces latérales et antérieures ne sont pas toujours en même nombre, et n'ont pas toujours la même forme.

Il est singulier qu'il existe chez les batraciens urodèles une paire de pièces qui manque aux anoures. C'est de chaque côté une lame alongée, s'ćlevant de la partie inférieure du bord latéral 
du corps du sphénoïde, plane ou légèrement convexe à sa face externe, et contiguë postérieurement au rocher, antérieurement au frontal, supérieurement au pariétal.

Il existe à sa place, chez les batraciens anoures, une lacune remplie seulement par du cartilage.

Cette pièce est sans doute la grande aile du sphénoïde, seule ou unie à la pelite aile.

Les pièces osseuses qui viennent d'être décrites, et qui s'unissent toutes entre elles d'une manière solide, font de l'os basilaire un anneau ou une vertèbre parfaitement close postérieurement, et ouverte en haut à sa partie antérieure.

La dernière paire de pièces latérales, qui existe partout , descend de dedans, en dehors. de haut en bas, et d'arrière en avant; elle s'étend depuis la partie postérieure du corps du sphénoïde et le rocher, en passant devant l'os carré, jusqu'au bord interne de la mâchoire supérieure et à l'os palatin. Elle est, dans la plupart de ces animanx, alongée et convexe à sa surface externe, si ce n'est chez les salamandres, du moins le s. maculata, où elle est triangulaire: ordinairement simple, elle est divisée, chez les grenouilles, en une portion supérieure, transversale, et en une inférieure, longitudınale.

Cet os doit être considéré incontestablement comme l'analogue de l'aile inférienre du sphénoüde. 
2. Temporal.

$$
\text { S. } 234 \text {. }
$$

Le temporal des batraciens se compose de la porlion crinienne et de la portion articulaire.

La portion crânienne est, chez les batraciens urodeles et les grenouilles, un os carré, arrondi, formé d'une paroi supérieure et d'une inférieure, d'une antérieure et d'une postérieure, dont la cavité, qui est considérable, est dirigée librement en dedans dans la cavité du crâne.

Il forrue, chez le pipa, une plaque mince, transversale, alongée, légèrement concave en arrière.

Chez le même pipa, les grenouilles et le protée, cet os est séparé de la portion condyloidienne de l'occipital; chez les salamandres et les tritons il lui est, au contraire, soudé entièrement.

La porlion articulaire consiste en deux pièces chez les batraciens urodèles, en une seulement chez les grenouilles.

La supérieure s'applique contre la pièce articulaire de l'occipital, et s'unit à l'inférieure par une suture. L'inférieure s'articule avec la mâchoire inférieure. Chez les grenouilles, il s'intercale entre la portion arliculaire et la mâchoire inférieure un petit os étroit, transversal, 
qui rencontre en avant l'extrémité postérieurc de la mâchoire supérieure, et qui est, sans doute, sil'on en juge par cette counexion, l'os malaire, qui peut-être forme de plus la moitié inférieure de la portion articulaire.

Chez les batraciens anoures, les deux os forment en général ensemble une plaque mince et alongée. Chez les grcnouilles, l'os unique a la forme d'un marteau; il est formé d'une branche supérieure, transversale, et d'une inférieure, longitudinale. Il est simple chez les crapauds, la branche supérieure, transversale, étant beauboup plus courte. Chez le pipa, il est divisé en deux pièces, comme chez les batraciens urodèles; ces deux pièces réunies sont très-déprimées, courtes et carrées. La pièce supérieure, ici devenue antérieure, est courte, large, fortement convexe en avant, de sorte qu'il en résulte une cavilé presque fermée; elle est concave postérieurement, et contient l'os auditif, qui est volumineux et situé transversalement. La seconde pièce, quadrilatère, plane, beaucoup plus épaisse, est couverte en haut par une écaille de la pièce antérieure; elle s'articule eu bas avec la mâchoire inférieure.

Ici cet os se montre par conséquent distinctement être la portion tympanique du temporal.

La pièce supérieure esı très-grande chez le protée; chez les tritons elle offre à peu près le míme volume que la pièce inférieure; chez les 
salamandres elle constitue une plaque extrêmement mince, à peine sensible.

L'os carré ne s'articule pas partout de la inême manière avec la portion crânienne du temporal.

Chez le protée, la moitié postérieure, située en haut et plus large de la pièce supérieure, s'applique à côté de la portion crânienne et même de la partie latérale de l'occipital; elle y est assez solidement unie, sans trace de surface articulaire. Chez les salamandres et les tritons, elle s'attache, dans une petite étendue, au bord externe du rocher, au moyen d'une facette encroûtée de cartilage, qui est plus large chez les tritons et permet des mouvemens plus libres.

Chez les batraciens urodèles, l'articulation de cet os avec le rocher n'a lieu, le plus souvent, que par une partie supérieure et postérieure, proportionnellement petite, surtout chez les grenouilles, et cela à l'aide d'une facette articulaire, supérieure et concave. Chez le pipa, l'os supérieur s'articule, par un cartilage, pour ainsi dire immobilement, dans tout son bord interne qni est mince, avec le bord externe du rocher et de l'occipital latéral.

3. Pariétal.

$$
\text { S. } 235 \text {. }
$$

Les pariéıaux sont toujours quadrilatères, 
moins alongés inférieurement, séparés des os frontaux, du moins, parmi les batraciens urodèles, chez les sulamandres et les tritons, tandis qu'ils sont soudés à ces os chez les anoures. Ils sont ordinairement convexes à leur face supérieure; chez le pipa ils y sont fort concaves, et beaucoup plus longs à leur bord externe qu'à leur bord interne.

Ils se rencontrent très-généralement dans toute leur longueur sur la ligne médiane; chez les raines, le bufo igneus et le $b$ gibbus, ils sont, au contraire, séparés par une Irès-grande fontanelle. Chez plusieurs crapauds, notamment le crapand brun (b. fuscus) et le crapaud gris ( $b$. griseus;, il y a un faible indice de celle disposition.

4. Ethmoïde.

$$
\text { S. } 236 .
$$

L'ethmoïde n'existe pas généralennent; je ne l'ai pu trouver réellement que chez les grenouilles; peut-être existe-t-il chez le pipa. C'est un canal ayant la forme d'un carré long, plus large que haut, nuvert en avant et en arrière, simple dans sa moitié postérieure, partagé dans l'antérieure en deux moités latérales par une cloison. La moitié postérieure s'apointit d'arrière cu avant, et communique avec l'antérieure par 
deuxonvertures arrondies, qui donnent passage au nerf olfactif.

La partie moyenne de la face supérieure de cet os est située à découvert, chez les grenouilles, entre les os nasaux et les os pariéto-frontaux.

Chez le pipa il se trouve, en cet endroit, deux plaques triangulaires, larges, qui touchent postérieurement à l'os pariéto-frontal, et que je regarde comme un rudiment de l'os ethmoïde, parce qu'il y a un os nasal.

\section{Coronal.}

$$
\text { S. } 237 \text {. }
$$

Les os frontaux ou coronaux sont des plaques considérables, alongées, qui se rencontrent dans la ligne médiane, sans se souder, et ont ḋ peu près la même forme et le même volume que les pariélaux; ils n'existent isolément que chez les batraciens zurodeles, car chez les anoures ils sont soudés avec les os pariétaux.

\section{Maxillairé supérieut.}

$$
\text { S. } 238 .
$$

L'os maxillaire supérieur se divise très-généralement en pièce sus-maxillaire postérieure, ou piece sus-maxillaire proprement dite, qui 
est toujours beancoup plus grande, et en pièce sus-maxillaire antérieure, ou pièce inter-maxillaire.

La pièce sus-maxillaire du protée et du pipa est une lame simple, seulement un peu plus haute en avant; chez les salumandres et les Iritons la partie antérieure est beaucoup plus haute et triangulaire. Chez eux et les grenouilles, il s'élève de sa face interne une portion palatale, transverse et horizontale.

L'os inter-maxillaire qui est soudé en avant avec le sus-ınaxillaire, ne représente, chez le pipa, de chaque côté, qu'une petite plaque, simple, quadrilatère et mince; chez les autres il est formé d'une branche supérieure et d'une inférieure.

Les deux os inter-maxillaires sont toujours séparés l'un de l'autre, si ce n'est chez les tritons, où ils sont soudés dans la ligne médiane de la tête.

\section{ๆ. Vomer.}

\section{S. 239 .}

Derrière l'os inter-maxillaire il y a, de chaque côté, un os mobile, plus large en avant qu'en arrière, qui ne rencontre pas son congénère dans la ligne médiane, mais qui en est fort éloigné.

Il est très-considérable chez les salamandres, se prolongeant, en arrière, par un crochet long et étroit, qui est dirigé en dehors; il est beaucoup 


\section{D'ANATOME COMPARÉl.}

plus petit chez les grenouille's. Je ne l'ai pas pu découvrir chez le pipa.

Comme le crochet n'existe pas chez les grenouilles, et que celles-ci ont un os palatin qui manque aux salamandres, pourvues d'ailleurs d'un crochet fort développé, je regarde la partie postérieure de cet os, chez les salainandres, comme l'os palatin.

\section{Palatin.

$$
\text { S. } 240 .
$$

C'est, chez les grenouilles, un petit os, mince el transversal, situé entre l'extrémité postérieure dı vomer, l'antérieurc du corps du sphénoïde, la face inférieure de l'ethmoïde, le maxillaire supérieur, à peu près à partir du commencement de son quart antérieur, et de l'extrémité antérieure de l'os ptérygoïde.

\section{Nasal.}

\section{S. 241.}

Chez les salamandres, les grenouilles et les crapauds, l'os nasal est situé au devant de l'ethmoïde et du frontal, si celui-ci existe, derrière el au dessus de linter-maxillaire, en dedans et en haut du sus-maxillaire. Il a la forme d'une 
plaque quadrilatère, qui atteint sa congénère du còté opposé, sans se souder à elle, et qui, chez les grenouilles, se prolonge en dehors par un crochet.

Chez le pipa, l'os nasal me paraît être un petit os, alongé, situé en avant sur l'os sus-maxillaire, et dirigé en dedans et en haut.

10 et 11 . Malaire et unguis.

$$
\text { S. } 2: 2 \text {. }
$$

Ces deux os manquent généralement, à moins que l'os mentionné plus haut (1) ue puisse ètre considéré comme un os malaire.

12. Maxillaire inférieur.

$$
\text { S. } 243 .
$$

La mâchoire inférieure, qui est longue, mince et peu élevée, et dont les deux moitiěs latérales ne sont unies entre elles, dans la ligne médiane, que par un cartilage large qui permet la mobilité, se compose toujours, de chaque còté, de deux pièces osseuses, dout la postérieure s'applique dans une assez grande étendue contre la face interne de la pièce antérieure.

La postérieure est plus épaisse et plus élevée; il se trouve une cavité articulaire simple à son extrémité postérieure.

(1) Voyez pag. 692. 
D'ANATOMIE COMPARÉ.

Liantérieure est de beaucoup la plus grande chez les batraciens urodèles; c'est l'inverse chez les anoures.

Jamais lá postérieure ne porte de dents; elle est par conséquent la pièce articulaire; l'antérieure est la pièce dentaire.

II. CHéLONIENS.

1. Basilaire.

$$
\text { \$. } 244 .
$$

Chez tous les chéloniens on trouve la portion occipitale composée, du moins pendant un long espace de temps, de quatre pièces, peut-être mème de six, savoir : le corps, la partie squameuse, deux pièces latérales supérieures, et deux pièces latérales inférieures.

Le corps est plat, court, se prolonge d'avant en arrière en une pointe mousse, et se termine. par un condyle arrondi ou triangulaire, creusé * +1 godet à son centre, pour s'articuler avec la première des vertèbres cervicales; cette surface articulaire est complétée en haut et sur les côtés par les parties articulaires ou condyloïdiennes.

Les parties condy lö̈diennes qui se recourbent de haut en bas el de dedans en dehors sont assez éloignées l'une de l'autre en haut et en bas; elles sont à leur partie supérieure plus minces rt plus pointues qu'à l'inférieure. 
Sur les parties articulaires repose la partic squameuse, qui présente deux racines inférieurement, et est comprimée supérieurement dans la ligne médiane, d'un côlé à l'autre, pour former une crête occipitale; elle déborde en arrière les parties articulaires.

Au devant et au dessus de ces dernières, et en même temps plus en dehors, on trouve les os indéterminés, dont il a déjà été question au chapitre des poissons (1); sans ces os, la pièce squameuse déborderait trop, en avant, la pièce articulaire, et serait trop peu appuyée; ils appartiennent à l'occipilal; ce sont, du moins chez les chélonées, de petits os triangulaires, touchant en dehors à l'os mastoïde, el recevant la partie postérieure du labyrinthe.

La portion sphénoïdale de l'os basilaire se compose de trois pièces.

Le corps en est court, triangulaire, très-large en arrière, très-étroil en avant, plus ou moins concave à sa face inférieure, convexe à la supérieure d'avant en arrière, el creusé en demicanal d'un côté à l'autre.

A ses côtés existent deux os considérables, plats et alongés, qui lui sont unis intimement par une suture, et le débordent un peu en arrière et en avant. Ils sont limités en dedaus par un bord conrexe, en dehors par un bord con cave, se divisent antérieurement en une bran-

(1) Voyez pag. 479, 480. 
che externe et une interne, et donnent naissance en haut, vers le milieu de leur face supérieure, a une autre branche, mais qui est plus courte. Cette branche constitue, sans contredit, à la fois l'apophyse inférieure et la grande apophyse; ses connexions indiquent cette analogie; elle touche au pariétal, et sa partie inférieure est située entre le temporal, le corps du sphénoïde et l'os palatin.

De ces os, plusieurs se soudent ensemble de boune heure, du moins chez la tortue d'eau douce d'Europe (emys europcea), surtout les deux pièces du corps et les deux pièces latérales supérieures de l'occipital (nouveau motif pour les considérer comme des parties de l'occipital); ils se soudent du moins plutôt entre eux qu'avec les os ptérygoïdes et la pièce squameuse; cette soudure a lieu aussi plutôt qu'entre d'autres pièces osseuses, notamment entre le temporal et la mâchoire inférieure.

C'est là sans doute un rapprochement vers l'organisation des oiseaux et des mammiferes, et en même temps une déviation de la loi générale, d'après laquelle les arcs des vertèbres se soudent entre eux plutôt qu'avec les corps.

La soudure s'opère, du reste, d'après mes recherches, d'abord entre le corps de l'occipital et la pièce occipiłale, latérale et inférieure, ou les pièces articulaires de cet os. 
2. Temporal.

$$
\text { S. } 24.5 .
$$

Le temporal des chéloniens se compose certainement de quatre pièces, peut-être même de cinq, savoir : le rocher, la pièce mastoüdienne, la pièce écailleuse, le tympan et la pièce du cercle du tympan, dont les trois premières forment la portion crânienne, les deux autres la portion articulaire.

Le rocher est petil, triangulaire, creux it l'intérieur, convexe à l'extérieur. Chez les émydes, il se soude aussi de bonne heure avec les parties latérales et les parties inférieures de l'os basilaire.

La pièce mastoüdienne est triangulaire, convexe en dehors, concave en dedans. Elle est extrêmement petite chez les tortues, ne forme qu'une petite écaille mince à l'extrémité postérieure du tympan; chez les chélonées, au contraire, elle est grande, plate, fortement creusée en arrière dans le sens de la longueur, et offrant seulement une légère concavité en dedans et en avant; elle est très-grande chez les émydes; elle y présente des parois minces; elle est creuse, complète la paroi postérieure du tympan qui manque, et agrandit considérablement par sa cavité celle de cette portion de l'organe acoustique. 


\section{D'ANATOME COMPARÉE.}

Au devant de la pièce mastoïdienne, on trouve la pièce écailleuse, os alungé, pourvu d'une apophyse qui prend naissance en arrière de sou bord inférieur; cet os est contigu en haut au pariétal, en avant au córonal, en bas à l'os malaire, ou en même temps à cet os et au cercle du tympan.

Le tympan est convexe en avaǹt, concare en arrière; il présente à sa partie inférieure une grande facette articulaire, transversale, deslinée à recevoir la mâchoire inférieure.

11 est en proportion plus petit chez les chélonées, et sá cavité est peu considérable; chez les émydes et les tortues il est très-grand; chez les premières la paroi postérieure en est percée, chez les secondes elle est complète.

En avant et en dehors du tympan il existe un os plat, alongé, convexe en avant, concave en arrière, qui descend jusqu'à la facette articulaire. Il correspond sans doute à l'os grêle, dont l'existence a déjà été constatée dans les grenouilles (1), et peut appartenir au temporal aussi bien qu’à l'os malaire; dans le premier cas, ce serait la partie inférieure de la portion articulaire ou le cercle du tympan, dans le second, ce serait l'os malaire postérieur.

Les lortues se distinguent des émydes et des chélonées surtout par la disposition de la partie écáilleuse du temporal. Elle est trés-étroite d'a-

(i) Voyez pag. $69^{2}$ 
vant en arrière, par conséquent alongée, et descend de haut en bas, sous la forme d'une strie osseuse gui n'est unie au tympan que par le cinquième os, et nullement avec la pièce mastoïdienne.

\section{Pariétal.}

$$
\text { S. } 246 .
$$

Le pariélal est toujours un os considérable, large, alongé et presque le plus volumineux des os de la tête chez les chéloniens. Il est formé d'une lame supérieure, plus longue, légèrement convexe, horizontale, et d'une lame interne, étroite, verticale, qui se détache de la partie inférieure de la première sous un angle droit. $\mathrm{La}$ dernière concourt à la formation de la cavité du crâne; la première fait voûte en dehors et est contiguë à la partie écailleuse, soit par tout son bord externe, soit seulement par la partie antérieure de ce bord.

Chez les chélonées, la lame verticale, proportionnellement étroite et élevée, est située près du bord interne de l'horizontale; chez les émydes et les tortues, elle est située très-en dehors, de sorte qu'elle est ici la continuation insensible de la lame supérieure, ou mieux, le pariétal est, dans les deux derniers genres, un os simplement convexe, formé d'après le type ordinaire des animaux supérieurs. 
D'ANATOMF: COMPARÉ.

Cinnstamment les deux pariélaux s'atteignent daus la ligne médiane, mais ne se sourlent pas.

\{. Eithmoïde.

$$
\text { S. } 247 \text {. }
$$

L'ellunoüde est considérable, plus grand que le frontal, très-semblable au pariétal; il est formé d'une pièce supérieure, horizontale, plus grande, et d'une inférieure, verticale. De mème que le pariétal forme par sa branche inférieure, la majeure partie de la paroi latérale de la cavité crânienne; de mème l'ethmoïde représente, par la même branche, la paroi postérieure de la cavité olfactive, et entoure l'espace par lequel le nerf olfactif passe dans cette cavité.

\section{Frontal.}

$$
\text { S. } 248 .
$$

Les frontaux sont des os triangulaires courts, ien général petits, plats, ou légèrement convexes à leur face supérieure, offrant de faibles sillons longitudinaux à l'inférieure, pour loger le nerf colfactif. Il se trouve, chez l'emys serrata, à la partie antérieure du bord externe, un petit os semi-lunaire, peut-être l'os sus-orbitaire, ou l'os unguis. 
6. Maxillaire supérieur.

$$
\text { \$. } 249 .
$$

L'os maxillaire supéricur n'est pas très-volımineux. Il se divise toujours en pièce susmaxillaire et en pièce inter-maxillaire; la première forme unie plaque verticale, qui est en avant beaucoup plus élevée qu'en arrière, et envoie en dedans une saillie horizontale; la seconde ne consiste qu'en une branche horizontale, inférieure. Le bord inférieur de tout le maxillaire supérieur est fort tranchant. Les pièces inter-maxillaires se rencontrent toujours sur la ligne médiane, mais ne se soudent en général pas. Chez les tortues à gueule (chelys) et les tortues molles (triony $x$ ) il n'y a, par exception, qu'une pièce inter-maxillaire, impaire.

Les pièces sus-maxillaires sont séparées l'une de l'autre dans toute leur longueur par les pièces inter-maxillaires, le vomer et les os palatins; elles divergent considérablement d'avant eu arrière.

7. Vomer.

$$
\text { S. } 250 \text {. }
$$

Le vomer est toujours impair, alongé, plus large en avant qu'en arrière, comprimé d'un 


$$
\text { n'ANATOMIE COMPARÉE. }
$$

cơté à l'autre. 11 n'atteint pas l'extrémité antérieure du corps du sphénoïde, du moins chez les chélonées; ces os sort en effet séparés par les extrémités antérieures et internes des os ptérygoïdes qui s’intercalent entre eux.

$$
\begin{aligned}
& \text { 8. Palatin. } \\
& \text { S. } 251 .
\end{aligned}
$$

Les os palatins sont des os courts, alongés, larges et plats, situés entre le vomer, l'os ptérygoïde, le maxillaire supérieur et l'os malaire.

9 et 10 . Nasal et unguis.

$$
\text { S. } 252 \text {. }
$$

Je ne trouve aucune trace d'un os nasal et d'un os unguis propre; ces os manquent, ou sont confondus avec l'ethmoïde; l'un d'eux, surtout los lacrymal, est représenté peut-être par l'os qui existe quelquefois près du frontal.

$$
x \text { r. Os malaire. }
$$

$$
\$ 253 \text {. }
$$

Jios malaire est certainement un os considérable, large, surtout chez les chélonées et les émydes, plus étroit chcz les tortues; il est convexe en dehors et en arrière, concave en avant et en dedans, situé entre le maxillaire supérieur, 
l’os palatin, et la pièce écailleuse du temporal. Il faut vraisemblablement lui adjoindre la petite écaille, décrite plus haut comıne pièce du cercle du tympan( (1), qui est située entre lui et la portion articulaire de l'os temporal; de sorte que l'os malaire serait ici divisé en une moitié antéricure, . plus grande, et une postérieure, plus petite.

Cette manière de voir me semble, en effet, plus près de la vérité que celle qui regarde cette petite écaille comme appartenant à la portion articulaire du temporal, parce qu'elle ne con. court pas à la formation de la face articulaire destinée à ètre en rapport avec la mâchoire inférieure.

12. Maxillaire inférieur.

$$
\text { S. } 254 \text {. }
$$

La mâchoire inférieure des chéloniens est toujours peu élevée, apointie en avant, formée d'une moitié antérieure, plus grande et un peu plus élevée, et d'une moitié postérieure, plus petite et plus déprimée; la première monte d'avaut en arrière, la seconde d'arrière en avant. Le point de réunion des deux moitiés est le plus élevé de cette mâchoire; il forme une pointe mousse. ILes deux moitiés latérales sont toujours, en avant, confondues sur la ligne médiane. La face

(1) Voyez pag. 703. 


$$
\text { n'ANATOMIE COMPARÉ. }
$$

articulaire, plate, pour le temporal, se trouve fout-a-fait en arrière, et descend de haut en bas et d'avant en arrière.

La mâchoire inférieure du malamata se rapproche de celle des batraciens, sous le rapport de sa ténuité, de son peu de hauteur et de sa forme demi-circulaire.

La mâchoire inférieure est aussi, dans les chéloniens, généralement composée d'une moitié antérieure et d'une postérieure.

L'antérieure, formée par les deux moitiés lalérales qui sont soudées ensemble dans la ligne médiane, constitue la majeure partie de la mâchoire; sa face supérieure est large et concave, pour recevoir la lame cornée du hec inférieur; elle est percée d'une foule d'ouvertures qui donnent passage aux vaisseaux et aux nerfs; sa face interne est aussi fort concave et très-généralement formée d'une seule pièce, qui est la pièce alvéolaire. Le matamata seul fait exception à cette règle, autant que je sache; les deux moitiés latérales ne sont pas soudées chez lui, ce qui est remarquable comme une analogie de plus qu'il présente avec les grenouilles.

La moitié postérieure se compose de cinq pieces :

$1^{\circ}$ Une petite pièce, déprimée, triangulaire, alongée, qui touche en haut et en árrière à la pièce alvéolaire et forme la partie la plus élevée de la mâchoire, c’est la piéce coronoüde; 
$2^{\circ}$ Une pièce mince, fort alongée, contiguc̈ à la pièce alvéolaire supérieurement et postérieurement, qui constitue, en partie, la parui interne de la mâchoire inférieure : c'est la piécé supplémentcire antéricure el inférieure;

$3^{\circ}$ Une autre plus grande, située à côté de la précédente, mais de même forme, qui constitue la partie postérieure et supérieure de la paroi interne de la mâchoire inférieure, et qui est la pièce supplémentaire, postéricure et supérieure, que l'on peut aussi nommer la pièce articulaire ou cundylö̈dienne interne, puisqu'elle forme, par la partie postérieure de son bord supérieur, le bord interne de la surface articulaire.

$4^{\circ}$ En dehors de la pièce précédente, il en existe une autre arrondie, légèrement concave supérieurement, et formant avec la précédente la partie postérieure de la mâchoire inférieure, se continuant en avant avec un cartilage long et apointi: c'est la pièce terminale ou la pièce condylö̈dienne, moyenne, ou enfin la pièce angulaire.

$5^{\circ}$ La paroi externe et postéricure est formée par une plaque considérable, concave en arrière, qui s'applique contre la quatrième pièce, s'appuie sur la partie postérieure de la pièce alvéolaire, et touche en avant à la pièce coronoïde: c'est la pièce condyloüdienne proprement dite ou pièce condyloïdienne exter e.

Entre ces cinq pièces, on trouve, dans la di- 


$$
\text { D'ANATOMIE COMPARÉE. }
$$

vision antérieure de la moitié postérieure de la màchoire, un intervalle haut et étroit, qui conduit d'en haut, où il est ouvert, à la gouttière considérable qui existe à la face interne de la pièce alvéolaire.

\section{OPHIDIENS.}

$$
\text { S. } 255 \text {. }
$$

Quoique les ophidiens ne diffèrent pas peu entre eux par la forme des os de la tête, ils fofrent cependant, sous ce rapport, plus de concordance entre eux qu'avec les autres ordres de reptiles. Il y en a tout au plus quelques-uns, parmi les ophidiens supérieurs, surtout les orvets, qui aient une grande analogie arec la disposition des sauriens.

\section{Basilaire.}

$$
\text { S. } 256 \text {. }
$$

L'os basilaire est formé, long-temps du moins, d'un nombre assez considérable de pièces. Le corps de la portion occipitale est toujours séparé de celui de la sphénoüdale; il est court, large, arrondi, pentagonal, légèrement concave vers la cavité crânienne, terminé au milieu de son bord postérieur par un condyle unique, arrondi.

Il supporte sur son bord latéral et postérieur 
les pièces articulaires ou condylö̈licunes qui sont recourbées, el dont les faceites articulaires touchent postériourement et en dedans à la facette articulaire, moyenne, du corps qu'elles servent à compléter.

La partie squameuse est déprimée, ordinairement Iransversale, plus alongée chez les amphisbènes, surmontée d'une petite crètedans son milieu.

Le corps de la portion sphénoüdale est beaucoup plus grand que celui de la portion occipitale, et se compose d'une moitie postérieure, plus large, et d'une antérieure, plus étroite, formant une épine longue; ces moitiés se détachent plus brusquement l'une de l'autre chez les serpens ordinaires que chez les autres, notamment les amphisbènes. Les orvets présentent, sous ce rapport, la disposition des serpens.

La moitié postérieure est munie, à la parie antéricure de son bord externe, d'une saillie plus ou moins longue, indice de son articulation avec l'apophyse ptérygoïde. Chez les orvets, cette saillie est considérablement plus forte; elle a une facette articulaire contre laquelle s'applique la partie moyenne de l'os plérygoïde. Une disposition digne de remarque, qui se rencontre chez plusieurs serpens proprement dits, et qui paraît surlout chez les venimeux, du moins les zipères, les naja, les crolales, cousiste dans l'exis- 
tence d'un fort crochet situé à la face inférieure du corps du sphénuïle, s'étendant d'avant en arrière, et dont la pointe se dirige en arrière; c'est une véritable apophyse épineuse inférieure, qui rappelle les apophyses épineuses inférieures, dont sont armées certaines autres de leurs vertèbres.

Chez les serpens ordinaires, il y a deux pièces ptérygoüdes, constituant toutes deux des os alungés.

La postérieure, beaucoup plus grande, est une plaque considérable, mais étroite, convexe en dedans, concave en dehors, qui s'etend de l'extrémité inférieure de l'os carré et de la postérieure de la mâchoire inférieure, en avant et en dedans, sans s'unir à la plaque du côté opposé. Elle est contiguë par son extrémité antérieure à la postérieure de l'os palatin, et supporte, un peu plus en arrière, sur son bord externe, la pièce ptérygoide antérieure qui est beaucoup plus petite.

Celle-ci s'élargit de dedans en dehors, se porte dans cette direction en avant et s'applique par son extrémité externe contre l'extrémité postérieure du bord interne du maxillaire supérieur.

C'est chez les serpens venimeux, surtout les vipères, les riaja, les crotales, que cette pièce osseusc a proportionnellement le plus de volume; re qui s'accorde avec la mobilité plus grande 
de leur face et la petitesse du maxillaire supérieur. Chez les éryx, au contraire, clle commence à disparaître d'une manière frappante; elle est en proportion beaucoup plus petite et plus étroite que chez les serpens ordinaires non venimeux. La pièce postérieure et interne est, en outre, très-grande, large, fort convexe à sa face inférieure, concave à la supérieure.

Chez les orvets, les rouleaux, les amplhisbènes, la pièce antérieure ou externe, comme os propre, est toul-a-fait disparue, ct les deux os n'en font plus qu'un, mais qui, surtout chez les orvets et les rouleaux, se divise en cleux branches, à l'endroit même où la pièce postérieure supporte l'antérieure, chez les autres serpens, de sorte que la forme des deux pièces s'est du moins conservée.

D’après cela la branche interne peut être désignée sous le nom de pièce ou lame ptérygoüde interne, et l'autre, sous celui de pièce oulame ptérygoüde externe.

Chez lcs orvets, il s'ajoutc aux pièces qui viennent d'ètre décrites une autre paire de pièces, dont il n'existe point de traces chez les serpens ordinaires.

Elle se trouve à peu près sur le milieu de la face supérieure de l'os ptérygoïde, derrière sa bifurcation; elle est mince, alongée, plane, et s'applique, en montant verticalement, contre la face extcrne du milieu de l'os pariétal. 


$$
\text { D'ANATOMIE COMPARÉL. }
$$

Comme il existe déjà chez les poissons et les salamandres une grande aile du sphénoide, l'os dont il vient d'être question est, sans doute, la grande aile du sphénoïde.

D'après tout cela, les rouleaux et les amphis. bènes forment évidemment le passage de la con. formation ordinaire des serpens à la conformation supérieure; chez eux, en effet, les deux pièces ptérygoïdes inférieures se soudent, mais il a'y a pas encore de grande aile. Les orvets conduisent de ces derniers aux sauriens, chez lesquels on trouve très-généralement la grande aile, qui est du reste conformé de la même manière que chez les orvets.

Les différentes pièces de l'occipital me semblent avoir une grande tendance à se souder entre elles. J'ai du moins trouvé cela souvent, tant chez les couleuvres que chez les orvets.

2. Temporal.

$$
\text { \$. } 257 \text {. }
$$

Le temporal est toujours formé de sa portion crânienne et de sa portion articulaire.

La partie pétrée, ou le rocher, est petite, arrondie, convexe en dehors, concave en dedans.

La partie écailleuse est beaucoup plus petite, alongée, convexe en arrière, concave en avant, et est située à l'extrémité antérieure du parić- 
tal, derrière l'orbite; elle est entièrement libre eu bas et en avant. Du moins, je prends cet os pour la partie écailleuse et non pour l'os malaire; je me fonde en cela sur l'analogie existant entre les poissons et les chéloniens, et sur ce que l'os malaire est moins important que la partie écailleuse du temporal.

La pièce mastoüdienne est un os alongé, mince, plat, situé d'avant en arrière, le long de la face externe du pariétal, au dessus du rocher et au dessous de la circonférence supérieure de l'occipital, et s'articulant mobilement avec tous ces os; en arrière il déborde considérablement l'occipital, et peut glisser d'avant en arrière dans un sillon creusé à la partie inférieure du pariétal.

A la partie postérieure dı bord externe de la pièce mastoïdienne touche la pièce articulaire, qui est simple, fort alongée, dirigée de haut en bas et d'avant en arrière, un peu renflée à ses deux extrémités, surtout à la supérieure, et qui ne mérite ici nullement le nom d'os carré.

Cette disposition existe chez la plupart des serpens, surtout les serpens proprement dits. On la trouve aussi dans le genre éryx; mais ici la pièce mastö̈dicnne est petite, tandis que l'écailleuse est plus dévéloppée et s'étend en bas jusqu'au maxillaire supérieur. Les orvets, non-seulement ont la piece mastoïdienne, qui est ici plus mince et recourbée, apointie en avant et très-alongée, mais ils ont en outre, entre cette 
D'ANATOML COMPARÉE.

pièce el la pièce récalleuse, qui s'étend aussi plus en longueur et touche inférieurement à l'os malaite, un petit os, alongé, qui se place sous la partie postérieure de là pièce écailleuse.

Cilız les rouleaux, les amphisbènes et, autant que j'ai pu m'en assurer, aussi chez les typhlops, il ny a, au contraire, aucune trace de pièce mastö̈dienne isolée, et la pièce articulaire du temporal s'articule immédiatement avec le temporal et l'occipilal, qui sont unis entre eux, et aver les autres os du crâne, d'une manière immobile, au moyen de sutures.

En mème temps, tous ces ophidiens, qui se rapprochent davantage des sauriens, ont la pièce articulaire plus courte et plus épaisse, moins mobile que chez les serpens proprement dits; chez les amphisbènes elle se dirige très-obliquement d'arrière en avant et de haut en bas; chez les autres, elle est, au contraire, verticale.

5. Pariétal.

$$
\text { S. } 258 \text {. }
$$

Le parićlal des ophidiens est, d'après mes recherches, ct couformément à la forme étroite et alongée de leur tête, un os simple, fort considérable, surtout très-développé chez les amphisbènes et les orvets.

Il est formé, chez les serpens proprement dits, d'une lame verticale, petite, et d'une ho- 
rizontale, plus grande, qui déborde la première en avant et en arrière par des apophyses courtes et mousses.

Cet os est très-alongé chez les amphisbènes, formé de deux moitiés qui se réunissent sur la ligne médiane, de manière à représenter un toit; ces deux moitiés sont séparées l'une de l'autre postérieurement par un petit intervalle, comprenant environ le quart du tout.

Cette organisation forme le passage aux orvets, chez lesquels le pariétal n'est qu'une lame horizontale, se terminant postérieurement par deux apophyses divergentes, très-longues.

4. Ethmoïde.

$$
\text { S. } 259 \text {. }
$$

L'ethmö̈de se compose de deux petites moitiés latérales, triangulaires, non réunies, percées par l'ouverture considérable du nerf olfactif; elles sont unies mobilement avec les os voisins, et sont situées au devant du frontal, sous les os nasaux, derrière l'os inter-maxillaire.

\section{5. frontal.}

$$
\text { S. } 260 .
$$

Le frontal n'offre pas partout les mèmes dispositions. Souvent il est formé de deux moitiés latérales, qui s'alteignent dans la ligne médiane, 


\section{I) ANATOME COMPARÉE.}

par exemple, chez les couleuvres, tes vipères, les amphisbines; c'est, au contraire, un os simple chez les orvets.

11 est, chez les serpens communs, beaucoup plus petit en proportion que chez les orvets; il est également très-petit chez les amphisbènes.

Le frontal des serpens communs, du moins des couleurres, forme un canal, de chaque côté de son extrémité antérieure. Ce canal résulte de l'union de ses parois supérieure et inférieure, au moyen d'une lame verticale, courte. La réunion de ces deux lames qui s'atteignent sur la ligne médiane, produit une cloison courte. Les nerfs olfactifs sortent de ces ouvertures.

Chez les amphisbènes, ces os ont, comme chez les serpens communs, une forme alongée; ils sont fort convexes en dehors, concaves en dedans; mais en avant on ne trouve pas la lame qui transforme le demi-canal en un canal entier.

Chez les orvets, le frontal est une lame horizontale, simple, ayant la forme d'un carré long.

6. Maxillaire supérieur.

$$
\text { S. }{ }_{2} 61:
$$

Les os maxillaires supérieurs, postérieu! et antéricur, ou le sus-maxillaire et l'inter-maxillaire sunt toujours séparés l'un de l'autre. Ils sont surtout trés-distans l'un de l'autre chez les serpens communs. 
La pièce sus-maxillaire est toujours beaucoup plus grande que l'inter-maxillaire; elle est en général alongée, et se dirige tout droit d'avant en arrière. Chez les serpens venimeux, elle est courte, épaisse, et se dirige obliquement de haut en bas et d'avant en arrière. Chez les amphis. bènes et les orvets, il est en avant considérablement plus haut qu'en arrière.

La pièce inter-maxillaire est constamment simple; elle consiste en une partie verticale, moyenne, et en une trausversale, inférieure, qui est armée de dents.

Chez les éryx, j'ai trouvé celte pièce en proportion beaucoup plus grande que dans les autres genres.

\section{Vomer.}

\section{\$. 262.}

Le vomer est divisé en deux os non unis, et même séparés le plus souvent l'un de l'autre dans la ligne médiane par un intervalle; ils sont alongés, comprimés d'un côté à l'autre, percés au milieu d'une ouverture considérable, au moins chez les serpens proprement dits, et situés entre l'os ethmoïde, l'inter-maxillaire et le palatin. 


\section{Os palatin.}

$$
\text { S. } 263 .
$$

Les os pulatins existent constamment; ils sont simples, alongés, étroils, peu hauts, séparés entre eux et des maxillaires supérieurs par un très-large intervalle; ils sont appuyés sur l'extrémité antérieure de la pièce ptérygoïde inierne, et convergent d'arrière en avant, où ils se terminent librement.

\section{Os nasal.}

$$
\text { S. } 264 \text {. }
$$

Les os nasaux sont minces, quadrilatéres ou iriangulaires, se rencontrent dans la ligne médiane et sont situés au devant des frontaux, qui ont constamment plus de volume et leur sont unis d'une manière mobile. Ils consistent en une lame supérieure, transversale, large, et en une inférieure et interne, étroite.

Ils sont surtout considérables chez les éryx.

10. Os malaire.

$$
\text { S. } 265 \text {. }
$$

L'os malaire manque très-généralement chez les rophicliens, si l'exposé que nous avons donné 
plus hant de la disposition du temporal est exact; mais les orvels ont un os, peut-ètre deux, qui représentent le malaire.

Indépendamment des os précédemment décrits, comme appartenant au temporal ( 1 ), il $y$ a d'abord mu os trés-mince, alongé, concave a sit face supérieure, convexe à l'iuférieure, situé au dessous de l'ceil, et qui forme le bord inférieur de l'orbite. Comme il s'étend depuis les deux os antérieurs, décrits ei-dessus, jusqu'au maxillaire supérieur, c'est, sans contredit, l'os jugal on malaire.

Il y a en outre, plus en dehors et en bas, entre l'extrémité inféricure de la portion articulaire du temporal et le maxillaire supérieur, un os beaucoup plus voluminenx, alongé et plane. Cet os est-il une pièce malaire ou la pièce articulaire inférieure du temporal? Mon opinion n'est pas fixée. Il ne se montre osseux que cher les individus plus âgés; il est tendineux chez les jeunes sujets. Sa position, la ressemblance de sa forme avec celle de l'os malaire des oiseaux, militent évidemment pour la première opinion.

11. Os lacrymal.

S. 266 .

Liunguis est toujours un os triangulaire on (1) Voyez page $716,71 \%$ 
D'ANATOMHE COMPIRÉ.

quadrilatere, considérable, convexe en avant, concave en bas, percé inférieurement d'une ouverture longitudinale, assez grande; il est situé entre l'extrémité ancérieure du frontal et là partie antérieure du maxillaire supérieur et du palatin, et ferme la cavité de l'orbite en avant.

12. Nlaxillaire inférieur.

$$
\text { §. } 267 .
$$

Leś déux móitiés de la màchoire infériéure sont constamment séparées sur fa ligne médiane; elles sont en outre, dans la plupart des cas, plu's ou moins mobilement unies ensemble: Elles sont surtout très-distantes en avant, chez lés serpens proprement dits, et unies entre elles, tout au phis, par un cartilage mince, très-long, peu élevé. Chez les éryx, leurs extrémités antérieures sont aussi fort éloignées l'ure de lautre; chez les rouleaux (tortrix), elles sont sur le point de se rencontrer; chez les orvets et les amphislienes, elles sé rencontrent tout-à-fait sur la ligne médiane, mais sans se souder comme chez les chéloniens. Elles sont alóngées chez leś serpens proprement dits, peu élevées, presque sans courbure, et composées d'une piècé antérieure, un peu plus petite, la dentuire, et d'une postćrieure, plus grande, l'articulaire.

Chez les serpens venimenx, elles sont tres if 6 . 
minces, grêles, convexcs à la face interne, concaves à l'externe.

Che\% les amphisbènes, elles sont courtes, hautes et composées de quatre pièces, savoir: I" de la pièce dentaire, qui est chez ces ètres plus grande que les autres; $2^{\circ}$ de la pièce arliculaire; $3^{\circ}$ de la pièce coronoïde, qui est triangulaire et plus pelite que la précédente, contre laquelle ell: s'applique; 4" d'une pièce mince et alongée qui s'applique en bas et en dedans à la pièce dentaire.

Chez les éry $x$, je ne trouve que trois pièces, savoir : les deux pièces communes et la qua. trième des amphisbènes; cette disposition s'accorde avec la forme géuérale, alongée, des éry $x$, qui se rapproche de la conformation des serpens ordinaires.

Chez les rouleaux (tortrix), il n'existe pas mème la quatrième quèce des amplisbènes. La disposition des genres éryx et cortrix est curieuse, en ce sens que les éryx se rapprochent davantage, d'une part, des amphisbènes, par la séparation de la troisième pièce d'avec la pièce deritaire, er, d'autre part, les serpens proprement dits, par la forme alongée de la mâchoire inférieure, tandis que les rouleaux se rapprnchent des premières par la forme des pièces en question, et des derniers, par le nombre de ces pièces.

Jad disposition chez les orvets est encore plus 


$$
\text { n'ANATOMIF COMPARÉE. }
$$

composée que che\% les amphisbènes; chaquer moitié de mâchoire, qui est entièrement séparée de celle du còté opposé, se compose de six pièces.

La pièce articulaire, qui est alongée, en est la plus grande. Après elle vient la pièce dentaire. Entre elles se trouvent la pièce coronoïde, qui est iriangulaire, un autre os, qui est situé au côté interne de la pièce articulaire, et deux os alongés, l'un au dessus de l'autre, au côté interne de la pièce dentaire.

\section{SAURIENS.}

$$
\text { S. } 268 \text {. }
$$

La rète des sauriens présente, dans l'essentiel, la mênoe conformation que celle des orvets parmi les ophidiens, et qui a été décrite; les crocodiles seuls offrent, sous ce rapport, quelques déviations dignes de remarque.

1. Os basilaire.

$$
\text { S. } 269 \text {. }
$$

La portion occipitale de l'os basilaire est formée constamment, au moins, des quatre os ordinaires. Le corps est en général court, large. hexagonal. Les parties latérales se prolongent les deux côtés en une forte apophyse transversalc, el we rontribuent que peu ou point à la 
formation de la surface articulaire, moyenme et arrondie, gani a la forme d'un condyle. La piece squameuse est peu élevée large.

Chez les c'ocodiles (1), 1 le curpsest très-étroit;

(1) Le crocodile est l'animal que $\mathbf{1 1}$. Georfroy-Sant-HıLAIRE a particulièrement choisi pour objet de ses observations; les molifs de ce chạix sorıl: $1^{0}$ l'exiguité de la capacité crâniennc, en rapport avec une cxirême petilesse des centres nerveux, el avec une sorte d'uniformité de volume des ćminences qui les constituent; d'où résulte la moindre étendue des ceintures osseuses qui cmbrassent ces centres nerveux, et la concordance de forme qu'offrent avec les vertibres les parties osseuses du crâne; $2^{0}$ l'exircine développement de la face, qui permet de voir à l'état d'une plus grande multiplicité, et sur une échelle plus considérable, les diverses pieces qui la composent; $3^{\circ}$ le rapport transitoire établi par les crocodiles entre les vertébrés supérieurs et les poissons. C'est dans la lête du crocodile que cet anatomiste a parliculièrement détełminé les pièces qui entrent, suivant lui, dans la composition de loute tìte osseusc. Ce que M. Мескец désigne comme basilaire lu cráne de cet animal a spécialement offert quątre pieces à M. Groffroy-SantHıLarre, dans sa portion inférieure et médiane. Ces pièces sont les cycléaux. des quạtre vertèbres postérieures du crâne. Il appelle ces cycléun, en procédant du rachis vers la face, le basisplénal, ou cycléal de la vertẻbre cérébelleuse; l'otosphénal, ou cycleal de la ventèbre auruculatre; l'hyposphénal, ou cycléal de la iertèbre ouaniuda le; l'entosphénal, on cycléal de la vertébre cérébrale. I.e corps du basiláre propı ement dit ne se compose quo des deux premiers noyaux oø̣seux; leз deux seconds constituent le corps du sphénoïde, ou de la portion sphénoüdale du basilaire, comme l'indique M. Meckex. La séparation des deux premiers noyaux ssseux, c'est-à-dire du lusisphénal el de l'otosphénal a étí 
1'ANATOMLE COMPARÉ.

$2^{\circ}$ le nombre des pièces scmble augmenté, parce yque la partie squaneuse separtage en une pièce antérieure et en une postérieure; de ces deux pièces, la dernière est formée d'une moitié infèrieure, verticale, et d'une supérieure, horizontale; la première est un os quadrangulaire, unique, qui s'étend vers le bord postérieur de l'orbite. Celte manière de voir n'est cependant pas exacte; car l'os antérieur est le pariétal.

Toute cette portion occipitale est très-petile. vertébriforme et communément séparée du pariétal, qui est situé au devant d'elle, par un large iniervalle, de sorte qu'elle ne lui est contiguë que par les extrémiles externes de ses parties latérales, transverses; ce qui n'a pas lieu chez les crocodiles.

Le corṇs de la portion sphénoüdale est court, carré, et se prolonge communément, en avant. en un os court, pointu, impair et moyen; di-

découverte par H. Geoffroy-Sant-Hilaing sur plusieurs fœtus humains monstrueux. Ce que M. MECKe nomme portion squameuse se décompose en irois élémens de chaque cêté; ces élémen's sont, en procćdant d'arrière en avant, $1^{\circ}$ loccipital latéral, ou exoccipital, qui est le périal du corps. le plus postéricur du basilaire ou du basisphénal;

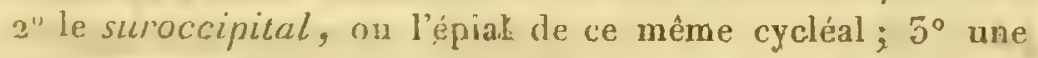
troisième partie de l'occipital, l'inter-pariétal, épial du second cycleai, que fournit le basilaire, en comptant d'arrière en avant. Les exoccipitaux, les suroccipitaux, unis au basilaire. concourent à constituer le contour du trou ocripitat.

(N. des Tr.) 
rigé en haut, yui quelquefois n'est pas uni all reste de l'os principal; à sa face supérieure, ce même corps se prolonge, à son milieu, en deux os plus petits et encore plus dirigés en haut; a sa face inférieure, an contraire, il se termine en avant el en arrière en deux autres os, dont ceux du devant sont les plus considérabies.

Les piècesplérygoüdes et les grandes ailes (1) du sphénoide se composent, en partie, comme chez les orvets, en partie, comme chez les serpens communs; en effet, l'aile supérieure du sphénoïle est un os alongé, les apophyses ptérygoildes externe et interne sont séparées l'une de l'autre.

Les dernières sont très-petites chez les geckos, les premières sont éloignées l'une de l'autre par une distance extraordinaire, ce qui est une grande analogie avec la conformation des grenouilles.

Chez les crocodiles, les pièces ptérygoïdes inférieures présentent des exceptions considérables au type des autres sauriens.

Elles consistent, il est vrai, comme chez ces derniers, en une pièce postérieure et interne, plus grande, et en une antérieure et externe, plus petite; mais ces pièces, surtout les internes, sont:

(1) Les premières renferment les hérissémux, cataanx de la vertèbre céríbrale, les secondes sont les ptéréaux ou épiaux de la mème vertébre.

(N. des Tr.) 
$1^{\circ}$ Beaucoup moins alongées et plus larges que chez les autres reptiles;

20 Elles se rencontrent dans la ligne médiane et en haut avec le corps du sphénoïde;

$5^{\circ}$ Les antérieures et externes ne s'appliquent pas seulement à l'extrémité postérieure du maxillaire supérieur, mais aussi à la région moyenne de l'os malaire.

Les gavials présentent une particularilé; c'est le renflement subit de toute la partie du sphénoide située au dessus des os palatins; elle se transforme en une grosse vésicule osseuse, qui se porte en haut et en dehors, et est entièrement séparée de celle du côté opposé, à l'extérieur. Quand on ouvre ces vésicules, on voit qu'elles ne forment qu'une cavilé, car il n'y a point de cloison. Ce sont évidemment des dilatations considérables de la cavité nasale. Je n'en ai trouvé aucune trace sur le crâne d'un individu très-jeune; je les ai vues, au contraire, chez tous les sujets adultes. Les autres crocodiles noffrent qu'un léger indice de cette dispositıou.

Chez plusieurs sauriens, notamment les iguanes, les monitors, les crocodiles, il y a, au devant. au dessus et en dedans de la grande aile du sphéuoinde, à la paroi antérieure du crâne et derriére l'orbite, un petit os, plat, convergent aver: cetui du côté opposé; je le considèr. 
comme l'uile anlérieure ou da pelice aile du spluinoide (1).

\section{Temporal}

$$
\text { S. } 270 .
$$

Le temporal (2) est formé très grénéralement du rocher, de la partie mastoüdic'nne, de l'écailleus" et de l'articulaire.

(1) Ingrassial de M. Geoffroy-Sannt-Hatare; il est du double plus étendu que le ptéréal on grande aile; il le précède, et vers le plafond du crâue il atteint le pariétal en ar. rière et le frontal err avant.

( $N$. des $T r$.)

(2) L'autour de la. Philosophie anatomique ne reconnait pas de portion mastoidienne; elle constitue son temporti. et quant à la portion écailleuse de M. Mескен, elle forme l'os jugal de M. Georfroy. Les connexions de ce temporal avec le jugal, le paricial et l'adorbital, pour embrasser cette grandelacune que l'on rencontre a lá tête du crocodile, et qui n'est autre que la fosse temporale, appuient ces déterminations. Le rupéal, sans y comprendre les osselets de l'ouie, dant il ne sera question que lorsque M. Meck El. traitera des organes de l'audition, „est un autre élérnent qui fait partie de ce qui est appelé ici rocher. Les rupéaux, con-sistent endeux sphères contiguës, ouvertes transversalemen de part en part; ils sont converts chacun par une plaque qui les embrasse, et qui ést empruntée à l'occipital supuriers. La porlion articulaire est spécialement formée par le servial et le tympanal. Il faut ajouter à ces os, pour constituer le: caisse du tympan, le cotyléal. Le premier de ces os, le serrial, est la grosse tubérosité din cercle du tympan, it ha troisiène, ou te cotyléal, nn élénent usseux qui avait eté disigné sous te nom de temporal par M. Covmer. A ces piece's sajonke cucore, un detrors, le strthical. Le cotrlial, dans le 


$$
\text { D'ANATOMIE COMPARÉE. TJ }
$$

Le rocher forme, chez les monitors, wuce branche antérieure, large, longitudinale, une postérieure, transversale et une partie moyenue, petite, concave à la lace interne, convexe à l'externe. Chez les iguanes et les crocodiles, il ressemble à celui des batraciens, c'est-à-dire, il est beaucoup plus simple.

La parlie mastoüdienne est communément petite, mince, alongée; elle ne saille pas en arrière, mais se porte de l'extremité supérieure de la partie articulaire en avant. Chez les croco. diles, elle est située à la face supérieure du crârie, dont elle constiue la partie postérieure et exterue; elle est triangulaire et se termine par une apophyse dirigée en arrière, en dehors et en bas, qui s'appuie sur la partie condylö̈dienue de l'occipital.

Elle est suivie, en avant, de quelques pièces, qui semblent appartenir à la partie écailleuse du temporal, et dont l'antérieure, qui est la plus grande, s'applique latéralement contre le pariétal.

Chez les lézurcls, du moins le lézard vert (l. voridis; e te lézard occellé (l. ocelluta), sur-

crocodile, est une lame alongée, employée comme un anneáu d'articulation, et sert a fixer le maxillaire supérieur sur les parties de la caisse; cette union, qui est mediate, s'opère par le moyen de l'aclorbilal qui est ca devant, et en arrière. par la grosoc tuberositi qui tient lieu de condyle maxillaire.

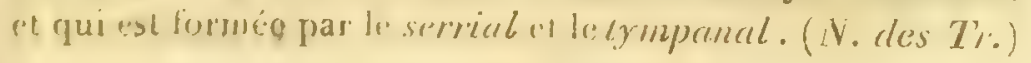


tout chez celui-ci, la pièce antérieure est fort considérable et large, ce qui est une analogice avec les chélonées.

L'os carré est d'ordinaire considérable, vertical, fort concave en arrière dans le sens de sa longueur, convexe en avant, garni inféricurement d'une surface articulaire large, transverse. concave au centre. Chez les crocodiles, il est situé, en majeure partie, sous la pièce condyloïdienne de l'occipital, et descend obliquement d'avant en arrière, de haut en bas ct de dedans en dehors : immédiatement au devant de l'os carré (1), on trouve un os arrondi, tamiôt plus alongé, tantôt plus plat, qui touche en avant à l'os malaire, en haut, du moins souvent, à la partie écailleuse, et dont il a déjà été question chez les chéloniens.

Les crocodiles se distinguent des autres sinuriens par la suture immobile de la partie articulaire avec le reste du temporal, tandis que chez ceux-ci, elle y est unie mobilement, d'après le type offert par les orvets.

\section{Pariétal.}

$$
\text { S. } 271 .
$$

Le parieial(2) est ordinairement simple, volu-

(1) Cet os est le cotyléal de M. Georfrox. ( $N$. des $T r$.)

(2) Le pariétal est également partagé en deux moitiés chez les jcunes crocurliles, il existe mème une fontanelle. Grofr.

( $N$. des $\operatorname{Tr}$.) 


$$
\text { D'ANATOME COMPARÉ. }
$$

mineux, prolongé en arrière en deux branches latérales, qui sont contiguës à l'extrémité externe les parties condyloïdiennes de l'occipital.

Il est pelit chez les crocodiles, plus large en avant qu'en arrière; il ne présente pas ces apophyses, ou elles ne sont que faiblement indiquées par une concavité du bord posterieur, à laquelle correspond la partie squameuse de l'occipital. Il n'existe pas d'intervalle entre lui et les autres os.

Chez les geclios il est parlagé, dans la ligne médiaue, en deux moitiés latérales.

4. Ethmoïde.

$$
\text { S. } 27^{2}
$$

Liethmoïde ( 1 ) se compose, dans la plupart des cas au moins, de deux moitiés latérales, dont chacune est formée d'une lame verticale, plus étroite, et d'une sipérieure, large, plus grande.

(1) Le cosps de l'ethmoïde forme le cycléal de la verténRE oCtLAJRE; la lame ethmoïdale est un cycléal; les cornets sujuérieurs forment des élémens latéraux distincts : ce sont ces cornets supérieurs, désignés par M. Georrroy, sous le nom riethrnophysaux, qui avaient été nommés frontaux antérieurs. Les connexions de ces os, chez le crocodile, sont valles des os de l'homme, c'est-à-dire qu'ils sont enourés intérieurement des apophyses antérieures du fronlal, in deliors du lacrymal, en avant du nasal. Geoff.

$$
\text { ( } i \text {. des } T r .)
$$


Les premières consituent les parois laréraies de liz cavile nasale, et sont unies inférienrement par du cartilage, et séparees supéricurement pour le passage du nerf olfactif. Les secondes sont situées librement à la face supérieure du crâne, entre le coronal. l'unguis ef le nasal, du moins chez les crocodiles.

Cet os saccorde parfaitement avec celui qui a été décrit chez less chéloniers. (1); il en résulte que cenx-ci, ou n'ont pas d'os lacrymal et de nasal, ou que ces os sont confondus avec l'ethmoïle : cette dernière circonstance est d'autant moins vraisemblable, que les crocudiles, outre leur ethmoïde qui est absolument comme celui des chéloniens, ont encore un os nasal et un un. guis très-distincls et bien développés.

\section{Coronal.}

$$
\text { 5. } 273 .
$$

Le coronal (2) est grand, alongé, quadrilatère, plus large en arrière qu'cn avant, Iriangulaire chez les crocodiles, toujours unique, plus considérableque le pariétal. II est, daus la plupart des cas, aplati, horizontal, irès-étroit chez les

(1) Sons le nom de frontal, cet os est constitué par deux moitiés latérales chez les jeunes crocodiles. It n'rxiste, suivant M. Geofrnoy, qu'un frontal unique chez ladulle, ciest le frontal antérieur.

( $N$. des $T r_{r}$ )

(2) Voyez pag., 05. 
D'anatomie COMParé.

rocodiles, fort concave le long de sa ligne médiiune, relevé sur les côtés, où il est intercalé entre la lame supérieure de l'ethmoide, sous laquelle il marche loin en avant.

Au bord exierue du frontal il y a, surtoutchez les crocudiles, et particulièrement chez le c. sclerops, des pièces osseuses formant une voûte orbitaire transversale, qui semblent appartenir au frontal et se trouvent déjà indiquées chez les poissons (1). J'en comple quat'e chez le cro:odilus palpebrosus; les deux antérieures, trianzulatires, sont les plus grandes; la postérieure et interne est la plus petite. Elles sumissent entre elles par des sutures, mais sont séparées au milieu par in intervalle rond.

6. Maxillaire supérieur.

$$
\text { S. } 274 \text {. }
$$

Ia pièce sus-maxillaire (2) est constamment

(1) Voyez pag. 195.

(2) Cette prièce sus-maxiilaircest formćc de trois portions, I la dentaire, nommée addental; l'addental est le maxiliaire proprement dit, en rapport sur la ligne médiane, et on bas avec son congénère; en haut avec le palatal, en arricere et en dedans avec le lacrymal, en arrière et plas en lehors avec l'adorbital, en arrière et en bas avec l'adgustal; $3^{\circ}$ la palatine, désignée par le mot d'adgustal. Celle portion du maxillaire s'étend te l'arcadc maxillaire sur l'arcade pa- 
beaucoup plus considérable que la pièce inler. maxillaire; elle est courte, triangulaire, communément plus haute en arrière qu'en avant. Cés deux pièces sont unies entre elles par des sutures; mais les pièces sus-maxillaires sont séparees ordinairement l'une de l'autre dans la ligne médiane par le vomer et les os palatins. Le plus souvent elles ne touchent que par une petite partie de leur bord interne, en arrière à l'os palatin, en avant au vomer, d'où résulte de chaque côté, entre ces trois os, un intervalle alongé, très-considérable. Chez les crocodiles elles se réunissent cependant dans la grande partie antérieure de leur longueur.

La pièce (1) inter-maxillaire est communément unique, comme chez les serpens; chez les crocodiles, au contraire, où elle est aussi proportionnellement plus grande, elle est formée de deux pièces latérales, unies en majeure partie par une suture dans la ligne médiane.

latine; $3^{\circ}$ l'orbitaire, à laquelle a été imposé le nom d'acorbital. Cet adorbital est intermédiaire au maxillaire proprement dit, ou addental, au lacrymal, au jugal, dont les autres anatomistes que M. Grofrroy la considerent comme l'analogue, enfin au cotyléal.

\section{(N. des $T r_{0}$ )}

(1) L'inter-maxillaire porte le nom d'adnasal; les adnasaux, réunis aux addentaux, forment les deux tiers de la face. Les addentaux: sont les plus antérieurs; ils sont, comme partout, séparés par les nasaux. (N. des Tr.) 
D’A Natome COMPaRÉ.

Il existe cher quelques crocodiles, notamment les cä̈mcins, au bord externe de la face inférieure, deux enfoncemens considérables, dont l'antérieur se trouve immédiatement derrière l'extrémité antérieure de l'inter-maxillaire, la postérieure étant située au point de réunion de cet os avec le sus-maxillaire ; ces enfoncemens reçoivent les première et quatrième dents de la mâchoire inférieure, qui sont de toules les plus longues; il n'existe qu'une échancrure à la place de ces cavités, chez les crocodiles proprement dits et les gavials.

\section{Vomer.}

$$
\text { S. } 275 \text {. }
$$

Le vomer ( I) est divisé en deux moitiés latérales, sur la ligne moyenne; il est alongé, plus large en avant, plus étroit dans sa partie postérieure, qui est la plus grande; il semble manquer quelquefois comme os propre, par exemple anx crocodiles.

(1) Le vomer est identiquement lè voméral; il en existe un de chaque cóté dans le crocodile. Les voméraux sont unis par un rebord intérieur et vertical au rhinosphénal; leur engrenage avec celui-ci est favorisé par une.scissure produite par le protosphénal, à son union avec le rhinosphénrıl. Lisez!Ann. des sciences naturelles, t. III, p. 241. ( $N$. des $\boldsymbol{T r}_{r}$ ) 


$$
\therefore 276
$$

Les os palutins (1) existent constamment. Ce sont, comme de coutume, des plaques alongées, divisées antérieurement, dans une étendue plus ou moins considérable, en une branche externe et en une interne; elles soilt séparées l'une de l'autre, sur la ligne médiane, dans toute leur longueur; cela est surtout frappant chez les geckos. Chez les crocodiles, au contraire, elles se touchent sur la ligne médiane dans toute leur longueur.

$$
\text { 9. Os nasal. }
$$

$$
\text { \$. } 277 \text {. }
$$

Les os nasaux (2) sont toujours doubles, alongés, arliculés par des sutures immobiles aver: tous les os voisins.

(1) L'os palatin est le palatal de M. Geoffroy-SaintHuLAIRE.

(2) Le nasal, auquel cette dénomination est conservir. Les nasaux séparent les adnasaux.

( $N$. des $T r$.) 


$$
\text { S. } 278
$$

L'os mulaire (I) est communément mince, alongé, convexe à sa face inférieure, concave à la supérieure, qui forme le bord inférieur de l'orbite.

11 est d'un volume et d'une largeur extraordinaires chez les crocodiles, bifurqué postérieurement en une branche supérieure, verticale et en nue inférieure, horizontale. Il manque toutitrait aux geckos.

$$
\begin{aligned}
& \text { II. Os l.crymal (2). } \\
& \text { S. 279. }
\end{aligned}
$$

Ciest un us considérable, situé au haut de la

(1) Set os est l'adorbital. M. Geofrror considère cette pièce comme un des élémens du maxillaire supérieur prolongé fost en arrière, et non comme l'os malcire ou jugal. I.e jugal est, suivant lui, en avant dn temporal; il ne concourt que preu à constituer l'orbite. Ce jugal a été désigné sous le num de frontal postérieur. Опек a désigné sous le nom de jugaux plusieurs pièces déterminées autrement par II. GeOFFROY. ( $N$. des $T r$.)

(2) Le lacrymal conserve son nom dans le systeme crânien établi par M. Geofroy. On sait que c'est le mème os 
face, au devant de l'orbite, en haut et en arrière du maxillaire supérieur, entre cet os, le malaire, le frontal et l'ethmoïde; il est percé d'un trou d'avant en arrière.

12. Maxillaire inférieur.

$$
\text { S. } 180 .
$$

Le maxillaire inférieur (1) des sauriens est, dans la plupart de ces ètres, alongé et peu élevé. Ordinairement l'angle dépasse un peu en arrière la face articulaire; chez les crocodiles, il la dépasse de beaucoup. Il existe à la face articulaire un enfoncement musculaire, dont on voit s'élever chez ces animaux, dont il faut excepter les crocodiles, une saillie ordinairement considérable; saillie qui est l'apophyse coronoïde.

que l'unguis. Lies autres pièces du système crânien font partie de divers autres appareils qui ne sont pas encore décrits par l'auteur ici traduit.

Pour rapporter chacune des pièces dont il s'est agi ici à l'appareil vertébral dont elle fait partie, voyez les notes précédentes, indiquant la composition de chacune des sept verièbres de la tête. Lisez, Annales du Muséum, tom. XI, et Annales des Sciences naturelles, tom. III, pag. I73, 24 I et 490 .

( $N$. des $T r_{0}$ )

(1) Les pièces qui existent de chaque côté du maxillaire inférieur ont été indiquées ci-dessus. Voyez la note de la page 445.

(N. des Tr.) 
Il est essentiellement composé du même nom. bre de pièces que le maxillaire inférienr des ché. loniens et des orvets. La différence principale qui distingue les sauriens des chéloniens se réduit à ce que, chez les premiers, les deux pièces antérieures, quoique unies par une suture immobile, ne sont pas confondues, tandis que, chez les chéloniens, elles sont toujours soudées et ne constituent qu'une seule pièce.

L'os qui recouvre en dedans la pièce dentaire est ici beaucoup plus long, et s'avance tellement qu'il en occupe presque toute la longueur, s'étend presque jusqu'à l'extrémité antérieure de cet os, et transforme par là le sillon existant chez les chéloniens, en un canal qui chemine sous les dents; disposition conforme à la longueur de tout le maxillaire inférieur et surtout de sa partie antérieure, et en rapport avec la présence des dents. Chez les geckos, je n'ai pas pu séparer cette pièce d'avec la pièce dentaire; mais j'en ai trouvé un rudiment très-petit à la face interne du maxillaire inf'érieur. La pièce supplémentaire postérieure ne concourt pas à la formation de la face articulaire.

Ordinairement il n'offre de trou nulle part; mais chez les crocodiles on y voit généralement fort en arrière $: 1^{\circ}$ une ouverture alongée, considérable, située dans la paroi externe, conduisant à la fosse, qui existe entre la pièce dentaire et celle qui la recouvre; $2^{\circ}$ plus en avant, 
une ouverture plus petite, également alongrée, dans la paroi interne. Ces ouvertures proviennent, sans doute, de la longueur considérable de la mâchoire inférieure, qui empèche l'ossification complète.

D. İ TÊTK DES HHPTLES CONSHUKKE EN CRNÁRAL.

\section{S. 281 .}

La tète osseuse des rep̧tiles diffère de celle des poissons, en général, par un nombre moindre et une articulation moins mobile des pièces dont elle est formée. En même temps, la partie supérieure, ou le crâne, est proportionnellement plus grande que l'inférieure, ou la face.

1. Balraciens.

$$
\text { \$. } 282 \text {. }
$$

Les caractères les plus généraux de la tète des batraciens sont l'aplatissement de haut en bas, l'étroitesse, le peu de hauteur et la longueur du crâne, la briéveté de la voûte palatine, la présence à l'occipital de deux condyles, convergeant de haut en bas et de dehors en dedans, une union non interrompue de l'orbite et de la fosse temporale, l'absence complete du plan cher et de la paroi postérieure de l'urbite, et enfiu la peritesse des cavités olfactives. 
Chez les batracicns urodeles, particulièrement. lin sirine et le procée, la tète est benucoup plus alongée que chez les anoures; chez le protée, elle est plus étroile. apointie en avant, tandis qu'elle est, chez les aurres, plus large, par conséquent plus semblable id celle des batraciens anoures. Il existe chez la sirène, en haut entre le pariétal et loccipital, un intervalle considérable, qui manque chez les autres.

Chez les batraciens anoures, la tête est encore pius plate que chez les urodeles; mais clle est en proportion beaucoup plus courte, de sorte qu'elle est plus large que longue. Cela est surtout frappant chez le pipa et les crrpauds, et particulièrement les agua, où elle forme un demi-cercle, dont le bord antérieur et convexe est formé par la face, et le bord postérieur, qui est droil, par la partie postérieure du crâne. La face supérieure du crâne des grenouilles est, le plus souvent, plane ou légèrement convexe; chez les crapauds et le pipa, elle est plus ou moins concare; chez quelques-uns, par exemple les bufo igncus et gibbus, ainsi que les raines, tlle est percée d'une fontanelle, qui occupe la majeure partie de sa longueur. Cet intervalle est su!lout fort considérable entre la partie supúrieure de la tête, du crâne particulièrement, et la partie inférieure el externe; ce qui licoul a l'ampleur des orbiles. 
2. Chéloniens.

$$
\text { S. } 283 .
$$

Les chéloniens se rattachent aux batraciens atussi par la disposition de leur tête.

Elle est, en général, considérablement plus haute dans toutes ses parlies, moins plate, pourvue d'une crête plus ou moins développée, qui déborde en arrière le trou occipital, est fortement comprimée d'un côté à l'autre, convexe à sa face supérieure, et concave à l'inférieure. Le condyle de l'occipital est simple, arrondi ou triangulaire, le sommet étant dirigé en bas. La voûte palatine est plus longue que chez les grenouilles, les orifices postérieurs des narines sont par conséquent plus en arrière.

A côté de la cavité crânienne, les os pariétaux et temporaux se recourbent, en général, derrière l'orbite, par-dessus la fosse temporale pour former un anneau, très-long et large chez les chélonées, un peu étroit chez les émydes, très-étroit chez les torlues. Cet anneau a, chez les premières, la forme d'un toit long, qui ferme l'orbite du moins en dehors. La partie antérieure de ce toit représente la partie postérieure du cercle orbitaire; sa partie inférieure et postérieure représente l'arcade zygomalique.

Lorsquil y a moins de substance osseuse ac- 
D'ANATOME COMPAREE.

cumulée, ces deux parties sont séparées en deux branches, qui passent l'une dans l'autre sous un angle droit. L'orbite est très - grand, fermé en avant et en bas par des os, séparé de celui du còté opposé seulement par du cartilage, et confondu particulièrement avec la fosse temporale.

Tous les os sont unis entre eux par des sutures; il n'y a une articulation mobile qu'entre la mâchoire inférieure et le temporal.

Le chelys fimbriata conduit aux batraciens; les trionyx conduisent aux batraciens, aux ophidiens et aux sauriens.

Le crâne de la première espèce est très-large, court, peu élevé; le toit de la fosse temporale est petit. Il existe, à l'occipital, deux condyles qui ne sont unis qu'à la partie supérieure, où ils se continuent avec la pièce squameuse. L'occipital n'est pas presque entièrement caché sous les pariétaux, comme cela existe ordinairement chez les autres chéloniens; il est, au contraire, presque tout-à-fait libre.

Les triony $x$ ont le crâne plus plat et plus alongé que les chéloniens communs; leur occipital est plus libre et le pont de la fosse temporale très-étroit. 
3. Ophidiens.

$$
\text { S. } 284 \text {. }
$$

Les ophidiens offrent des différences très-considérables entre eux.

Chez la plupart, le crâne est proportionnellement petit, plat, de la forme d'un carré long; le condyle de l'occipital est simple, arrondi ou en forme de feuille de trèfle; sa face supérieure est plane ou légèrement convexe; il est un peu renflé postérieurement, sur les côtés, dans la région temporale; en avant, il présente un orbite, incomplet en bas et en arrière, et entièrement séparé de celui de l'autre côté par une cloison osseuse. La fosse temporale est entièrement ouverte. Les os nasaux, les inter-maxillaires et le vomer sont unis entre eux d'une manière immobile. Leur articulation avec les os circonvoisins est, au contraire, avec mourement.

Le maxillaire supérieur, l'os palatin et l'apophyse ptérygoïde forment une seconde collection d'os, unie mobilement avec les os voisins; celte réunion d'os touche, en arrière, à l'extrémité postérieure de la mâchoire inférieure et se meut, avec elle, sur la partie articulaire du tem. poral, qui est aussi unie mobilement au reste du crâne. L'areade zygomatique manque. Ia voûte palatine est, à la vérité, très-longue, mais large- 


$$
\text { D'ANATOML CUMPARÉ. }
$$

ment fendue dans toute sa longueur. Comme les ar's, non réunis, de la voute palatine et les os ptérygoidies sont mobiles, celte fente est suscep. tible de varier considérablement sous le rapport de la forme et de l'étendue.

Chez les ophictiens supérieurs, qui se rapprochent des sauriens, le crâne est proportionnellement plus grand, et les os, qui, chez les ophidiens inférieurs, sont unis mobilement avec leurs voisins, sont immobiles. Cela s'applique surtout aux amplisbénes, où tous les os de la partie.supérieure de la tète et les deux moitiés de la mâchoire inférieure sont soudés. Chez les amphisbènes et les rouleaux, il n'y a pas de trace de paroi postérieure de l'orbite. Chez les typlilops, au contraire, il y a une trace de l'anneau postérieur, sous forme d'une petite saillie supérieure, qui, chez les éryx et les orvets, descend jusqu'au maxillaire supérieur. Ise plancher de l'orbite, qui manque chez les autres ophidiens, existe chez les éry $x$, en partie aussi chez les amphisbènes. L'arcade zygomalique n'existe chez aucun.

La voûte palatine est ordinairement partagée en deux moiliés latérales, mais qui sont peu mobiles, si ce n'est chez les orvels; elle manque ioul-à-fait chez les typlilops. 
4. Sauriens.

\section{ஒ. 285 .}

Les orvets conduisent aux sauriens par la conformation de la tête osseuse, comme par toutes les autres conditions de leur organisation. La forme générale de leur crâne offre des différences considérables relativement à la prédominance des diverses dimensions; différences qui se remarquent déjà à l'extérieur. Presque toujours la partie du crâne qui entoure l'encéphale est arrondie et courte. Le condyle de l'occipital est simple et arrondi. Ce qu'il y a de remarquable, ce sont plusieurs lacunes, en partie considérables, qui existent seules ou conjointement avec celles qui ont été décrites chez les chélonées et les ophidiens, et d'oü résulte le pont de la fosse temporale ou l'arcade orbitaire et l'arcade zygomatique; ces intervalles donnent à la tête un aspect percé à jour, qui est singulier, surtout chez les caméléons.

La lacune la plus constante est une postérieure, triangulaire, située de chaque côté, entre l'extré. mité postérieure et externe, alongée, du pariétal, l'os occipital et l'os mastö̈dien; elle est unie à celle du côté opposé, par exemple, chez les zeckos, les monitors, les iguanes, les marbrés, chez les premiers surtout, par une lacune trans- 
versale, qui s'étend entre le pariétal et l'occipital. Il résulte de cette disposition que l'occipital est presque entièrement séparé des autres os, de telle sorte qu'il représente plus qu'à l'ordinaire l'organisation vertébrale, même sous le rapport de la connexion, puisqu'il n'est uni au reste du crâne que d'une manière très-lâche.

Cette lacune est la seule qui existe chez les geckos.

En général considérable, elle est petite chez les crocodiles, très-cachée et transformée, par suite de la briéveté du sphénoïde, de la largeur de la portion articulaire de l'occipital et de l'os mastoïde, en un conduit long et étroit, descendant obliquement de dedans en dehors et d'avant en arrière.

Il s'y joint très-généralement une lacune considérable, fermée en dehors par l'arcade zygomatique, la fosse temporale, qui est ouverte chez les geckios, et fermée par un pont simple chez la plupart des sauriens, excepté les crocodiles, où il y a deux ponts, un supérieur et un inférieur. Le pont supérieur, qui est le plus ordinaire, est formé en bas et en avant par l'os malaire, aı milieu par la partie écailleuse du temporal, en arrière par sa partie mastoïdienne. La lacune entre ce pont et la paroi externe du crâne n'est trés-considérable que dans les cas où il n'y a qu'un pont ; lorsqu'il y en a un second, comme chez les crocodiles, elle est en général petite, surtout 
choz le crocodilus palpebrosus, chez lequel dle est à peine sensible dans le jeune âge, et disparaît tout-à-fait dans l'àge avancé, en ce que le pariétal et le temporal s'atteignent presque toutà-fait, par suile du développement général du crâne. Les gavials forment le passage des autres sauriens aux autres crocodiles; chez rux, celle ouverture est énorme, présente partolıt la mème largeur, et ne se récrécit pas de haut en bas, comme chez les deruiers, en maniere d'entonnoir. Chez, les autres crocodiles, elle est constamment plus petite que chez les autres sauriens.

Le crocodilus palpebrosus se ratıche évidemment par sa disposition aux chelonées; les autres crocodiles forment le passage de lui et de cellesci aux sauriens communs; en effet, chez eux, l'ouverture, qui vient d'être indiquée à la face supérieure du crâne, résulte de l'amoindrissement de la lame horizontale et supérieure du pariétal des chélonées.

Le second pont, l'inférieur, est réellement une deuxième arcade zygomatique; il est formé par une apophyse horizontale inférieure, qui, se détachan de l'os malaire, consitue presque un arc simple lequel va rejoindre liextrémité inférieure de l'os carré.

Quelques-uns, notamment les calotes el l'asama aspera, ont, en outre, dans la ligne médiane, une petite fontanelle quadrangulaire, intermédiaire au pariétal et au frontal; elle rap- 


$$
\text { DiNATOME: COMPARE: } 751
$$
pelle celle qui exsste cloc les batrancions, mais ant beaucoup plus petite; ce qui est conforme an denré d'organisation plus partail des situricins.

FIN DU SECOND VOLUME. 


\section{ERRATA DU II VOLUME.}

Page 534, ligne 8 de la note, au lieu de au-dessous, lisez au-dessus. Page 534, ligne 9 de la note, au lieu de au-dessus, lisezau-iessous. Page 535, ligne 30 de la note, au lieu de mobilité, lisez motilité.

Page 536, ligne 21 de la note, au lien de un excmple, tisez des exemples. Page 536, ligne 21 de la note, après mollusques, lisez et chez les araigneés.

Page 536, ligne 25 de la note, après deux, lisez dernicres.

Page 537, ligne 3 de la note, aprés perd, lisez de.

Page 537 , dernière ligne de la note, au lieu de et locomotrices, lisez. devenues organcs de locomotion aérienne.

$\mathrm{P}$ age 654 , ligne 23, au lieu de anoures, lisez urodiles. 





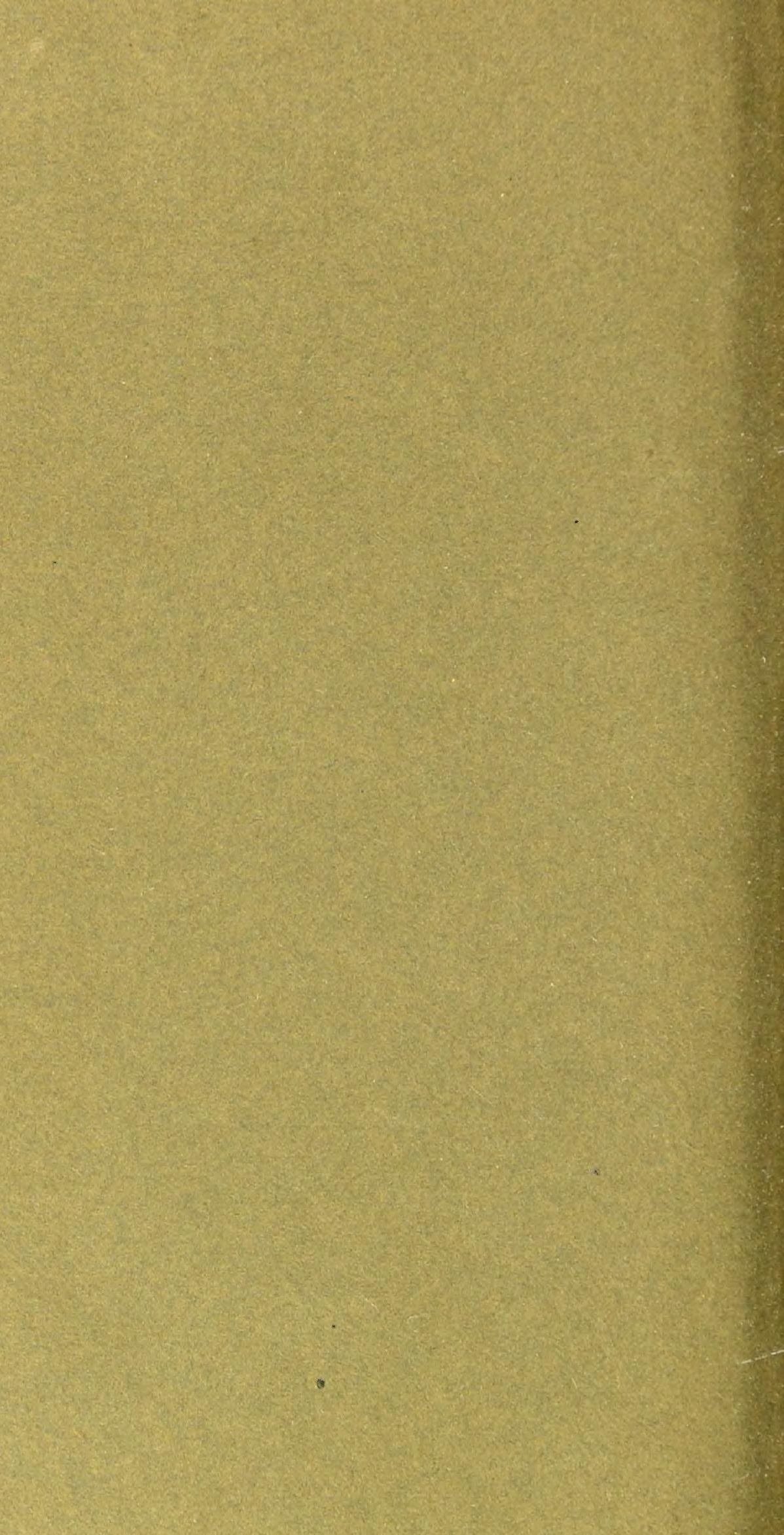




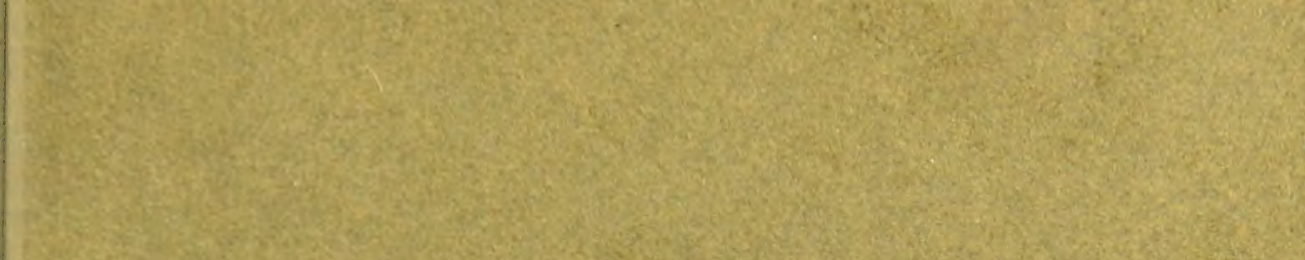


